


\title{
Health at a Glance 2019
}

\author{
OECD INDICATORS
}


This work is published under the responsibility of the Secretary-General of the OECD. The opinions expressed and arguments employed herein do not necessarily reflect the official views of OECD member countries.

This document, as well as any data and map included herein, are without prejudice to the status of or sovereignty over any territory, to the delimitation of international frontiers and boundaries and to the name of any territory, city or area.

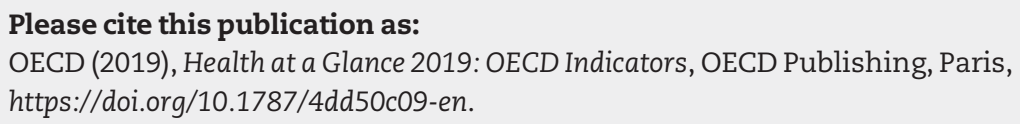

Photo credits: Cover @ kudla/Shutterstock.com; Images - Health status: ๑ Thitiporn taingpan/Shutterstock.com, Risk factors for health: @ Radachynskyi Serhii/Shutterstock.com, Access to care: @ onoky -Fotolia.com, Quality and outcomes of care: ๑ YAKOBCHUK VIACHESLAV/Shutterstock.com, Health Expenditure: @ Doubletree Studio/ Shutterstock.com, Health Workforce: @ Flamingo Images/Shutterstock.com, Health care activities: @ NaruFoto/ Shutterstock.com, Pharmaceutical sector: @ Fahroni/Shutterstock.com, Ageing and long-term care: ๑Thinkstock/ iStockphoto.com.

Corrigenda to publications may be found on line at: www.oecd.org/about/publishing/corrigenda.htm.

The use of this work, whether digital or print, is governed by the Terms and Conditions to be found at http://www.oecd.org/ termsandconditions. 


\section{Foreword}

Health at a Glance compares key indicators for population health and health system performance across OECD members, candidate and partner countries. This 2019 edition presents the latest comparable data across 80 indicators, reflecting differences across countries in health status, risk factors and health-seeking behaviour, access, quality of care, and the financial and physical resources available for health. Alongside indicator-byindicator analysis, an overview chapter summarises the comparative performance of countries and major trends, including how much health spending is associated with staffing levels, access, quality, and health outcomes. This edition also includes a special chapter on patient-reported outcomes and experiences.

The production of Health at a Glance would not have been possible without the contribution of national data correspondents from OECD countries. The OECD gratefully acknowledges their effort in supplying most of the data contained in this publication, as well as their detailed feedback to a draft of the report. Special acknowledgement is extended to members of the Patient-reported Indicator Surveys (PaRIS) Working Groups on mental health, breast cancer care, and hip and knee replacement for their contribution to Chapter 2, especially those individuals from countries, registries and health care organisations that facilitated the provision of patient-reported data. The OECD also recognises the contribution of other international organisations, notably the World Health Organization and Eurostat, for providing data and comments. The European Union provided financial and substantive assistance for work related to PaRIS, but the opinions expressed and arguments employed herein do not necessarily reflect the official views of the OECD member countries or the European Union.

This publication was prepared by the OECD Health Division under the coordination of Chris James. Chapter 1 was prepared by Chris James and Alberto Marino; Chapter 2 by Luke Slawomirski, Ian Brownwood, Emily Hewlett and Rie Fujisawa; Chapter 3 by Chris James, Viviane Azaïs, Eileen Rocard, Yuka Nishina and Emily Hewlett; Chapter 4 by Cristian Herrera, Jane Cheatley, Gabriel Di Paolantonio, Yuka Nishina and Michael Padget; Chapter 5 by Chris James, Michael Mueller, Viviane Azaïs, Alberto Marino and Marie-Clémence Canaud; Chapter 6 by Frédéric Daniel, Michael Padget, Eliana Barrenho, Rie Fujisawa, Luke Slawomirski and Ian Brownwood; Chapter 7 by David Morgan, Michael Mueller, Emily Bourke, Luca Lorenzoni, Alberto Marino and Chris James; Chapter 8 by Karolina SochaDietrich, Gaëlle Balestat, Gabriel Di Paolantonio, Emily Bourke and Emily Hewlett; Chapter 9 by Chris James, Gabriel Di Paolantonio, Gaëlle Balestat, Alberto Marino and Caroline Penn; Chapter 10 by Valérie Paris, Ruth Lopert, Suzannah Chapman, Martin Wenzl, MarieClémence Canaud and Michael Mueller; Chapter 11 by Elina Suzuki, Leila Pellet, MarieClémence Canaud, Thomas Rapp, Eliana Barrenho, Michael Padget, Frédéric Daniel, Gabriel Di Paolantonio, Michael Mueller and Tiago Cravo Oliveira Hashiguchi. The OECD databases used in this publication are managed by Gaëlle Balestat, Emily Bourke, Ian Brownwood, 
Marie-Clémence Canaud, Frédéric Daniel, David Morgan, Michael Mueller and Michael Padget.

Detailed comments were provided by Frederico Guanais and Gaétan Lafortune, with further useful inputs from Francesca Colombo, Mark Pearson, Stefano Scarpetta and Sarah Thomson. Editorial assistance by Lucy Hulett, Lydia Wanstall and Marie-Clémence Canaud is also gratefully acknowledged. 


\section{Table of contents}

Executive summary $\ldots \ldots \ldots \ldots \ldots \ldots \ldots \ldots \ldots \ldots \ldots \ldots \ldots \ldots \ldots \ldots \ldots \ldots \ldots \ldots, \quad 9$

Reader's guide. ............................................. 13

Chapter 1. Indicator overview: comparative performance of countries and major

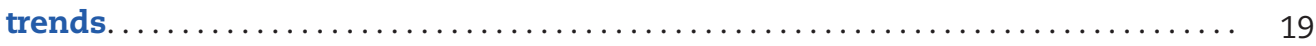

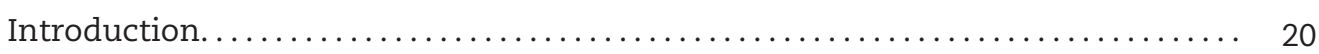

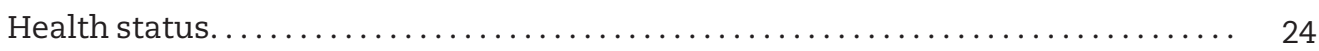

Risk factors for health..................................... 26

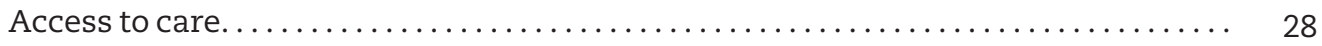

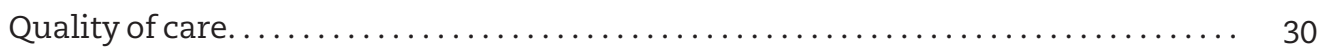

Health care resources........................................... 32

To what extent does health spending translate into better access, quality and health outcomes, and more health professionals?..................... 34

Chapter 2. Measuring what matters for people-centred health systems........... 39

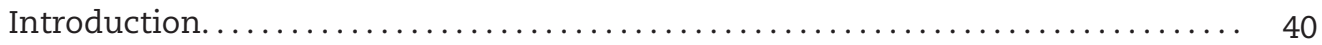

A people-centred health system needs to measure what matters to patients ..... 40

Joint replacement rates are rising but are patients reporting improvement?..... 44

Better information on breast cancer care outcomes helps patients facing difficult

treatment choices ....................................... 50

Existing mental health measures say little about experiences and outcomes of

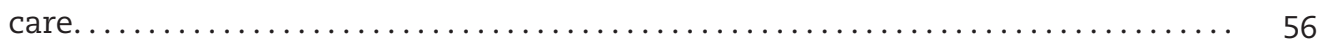

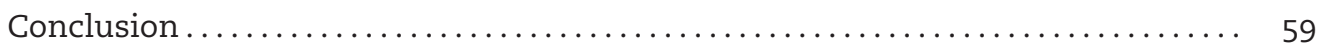

Chapter 3. Health status. ........................................ 65

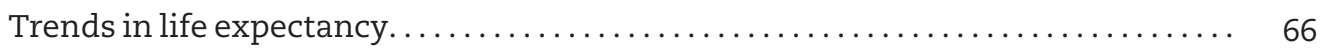

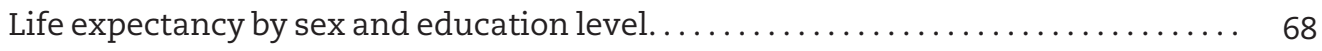

Main causes of mortality.......................................... 70

Avoidable mortality (preventable and treatable) $\ldots \ldots \ldots \ldots \ldots \ldots \ldots \ldots \ldots \ldots, 72$

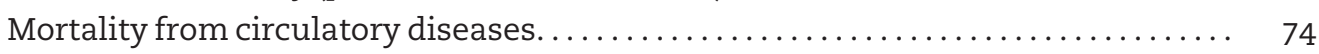

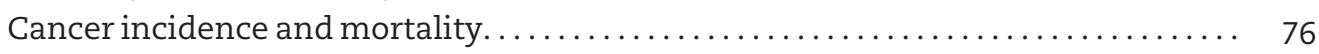

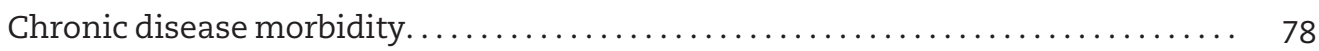

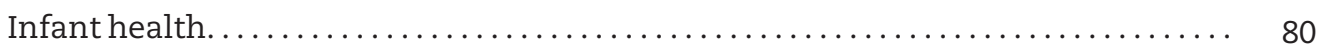

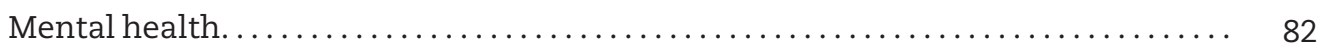

Self-rated health............................................. 84

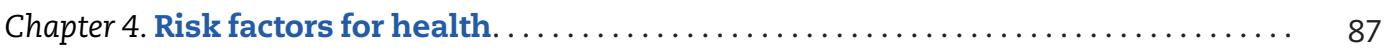

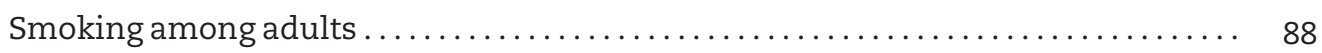

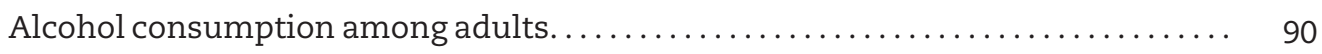

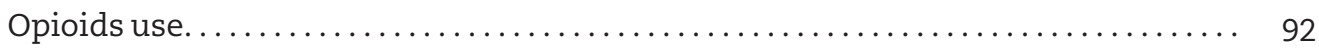




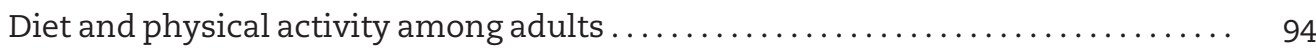

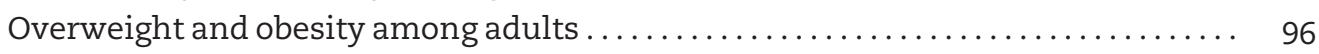

Overweight and obesity among children ...................... 98

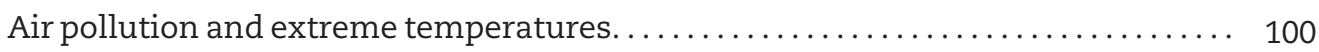

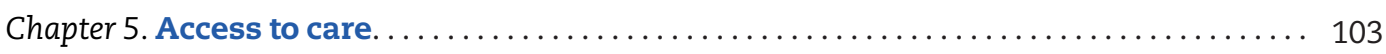

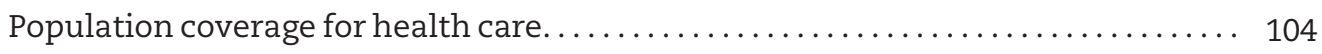

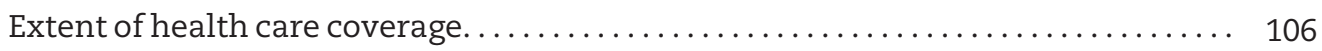

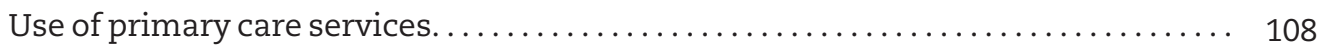

Unmet need for health care . . . . . . . . . . . . . . . . . . . . . . . 110

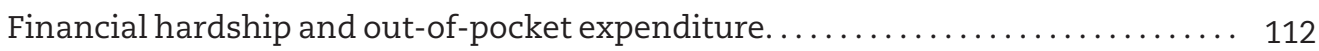

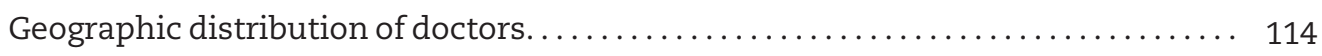

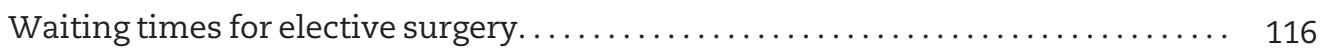

Chapter 6. Quality and outcomes of care .......................... 119

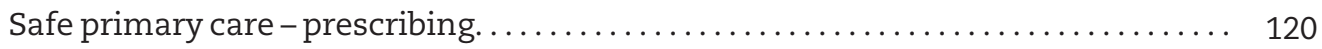

Safe acute care-surgical complications and health care-associated infections. ... 122

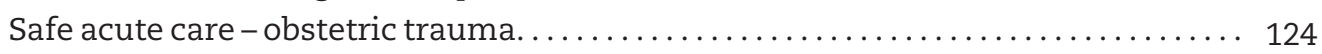

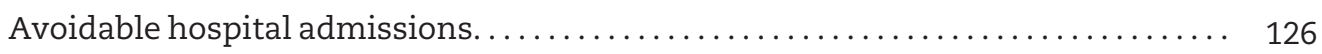

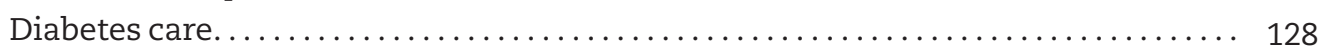

Mortality following ischaemic stroke . . . . . . . . . . . . . . . . . . 130

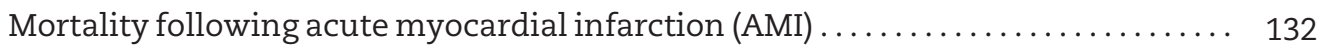

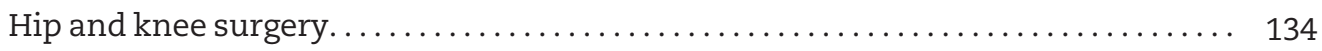

Care for people with mental health disorders. . . . . . . . . . . . . . . . 136

Breast cancer outcomes. ............................... 138

Screening and survival for colorectal cancer $\ldots \ldots \ldots \ldots \ldots \ldots \ldots \ldots \ldots \ldots \ldots \ldots . \ldots$

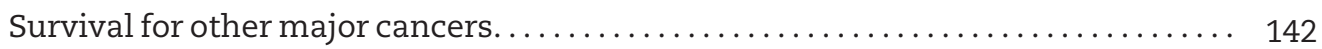

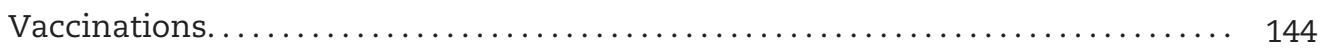

Patient experiences of ambulatory care $\ldots \ldots \ldots \ldots \ldots \ldots \ldots \ldots \ldots \ldots \ldots \ldots$

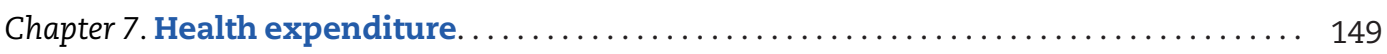

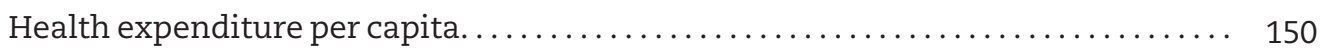

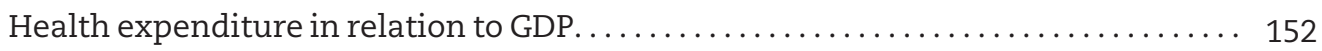

Prices in the health sector. . . . . . . . . . . . . . . . . . . . . . . . 154

Health expenditure by financing scheme. . . . . . . . . . . . . . . . 156

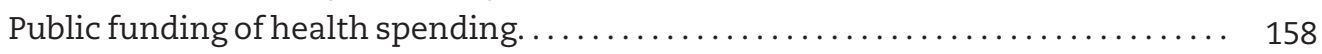

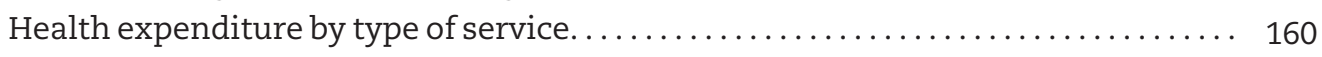

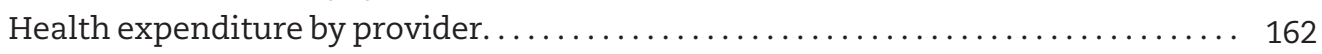

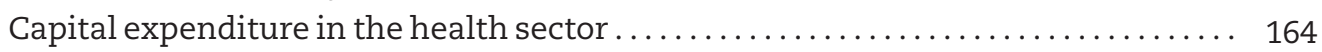

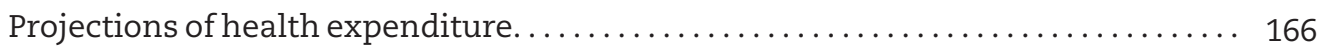

Chapter 8. Health workforce. ................................ 169

Health and social care workforce . . . . . . . . . . . . . . . . . . . 170

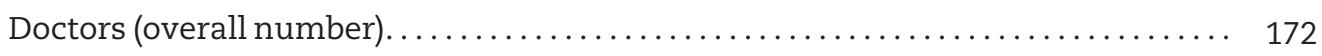

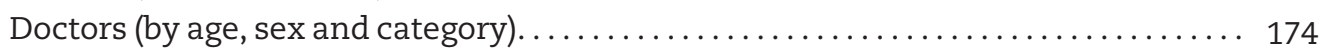

Remuneration of doctors (general practitioners and specialists) . . . . . . . 176

Nurses......................................... 178

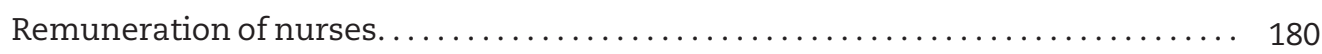

Medical graduates......................................... 182 


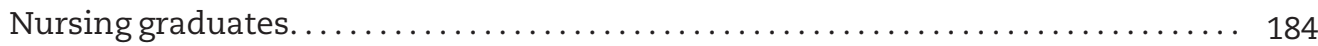

International migration of doctors and nurses. $\ldots \ldots \ldots \ldots \ldots \ldots \ldots \ldots \ldots \ldots$

Chapter 9. Health care activities. . . . . . . . . . . . . . . . . . . . . . . 189

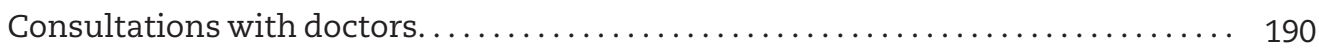

Medical technologies........................................ 192

Hospital beds and discharge rates. . . . . . . . . . . . . . . . . . . 194

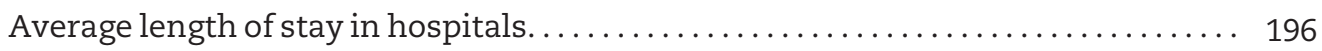

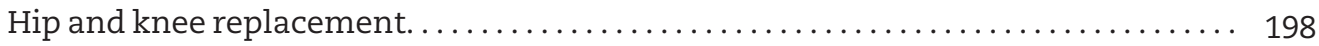

Caesarean sections. . . . . . . . . . . . . . . . . . . . . . . . . . . . . . . . 200

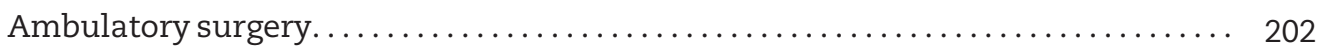

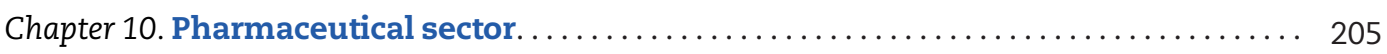

Pharmaceutical expenditure. ............................... 206

Pharmacists and pharmacies........................... 208

Pharmaceutical consumption. .......................... 210

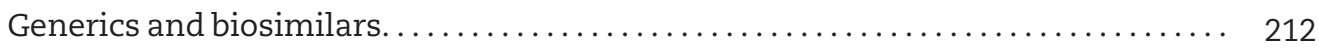

Research and development in the pharmaceutical sector. . . . . . . . . . 214

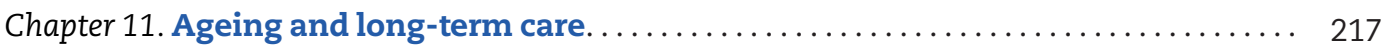

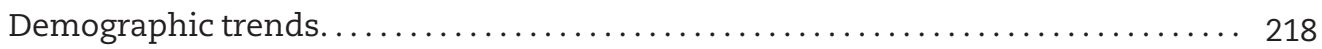

Life expectancy and healthy life expectancy at age $65 \ldots \ldots \ldots \ldots \ldots \ldots \ldots \ldots \ldots$

Self-rated health and disability at age 65 and over. . . . . . . . . . . . . 222

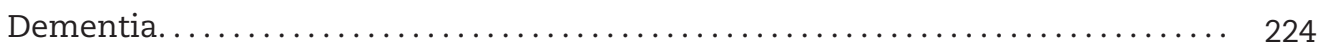

Safe prescribing in older populations. . . . . . . . . . . . . . . . . . 226

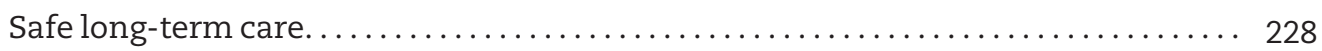

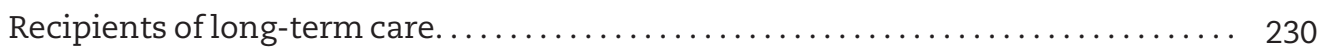

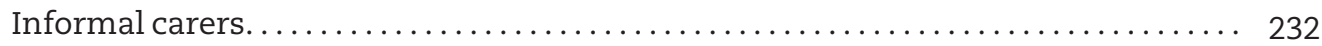

Long-term care workers. .............................. 234

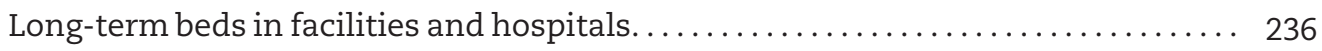

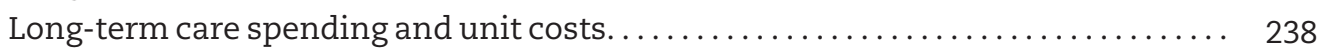




\section{Follow OECD Publications on:}

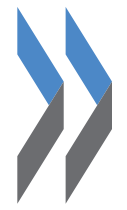

http://twitter.com/OECD_Pubs

1 http://www.facebook.com/OECDPublications

in. http://www.linkedin.com/groups/OECD-Publications-4645871

Yoube http://www.youtube.com/oecdilibrary

OECD

http://www.oecd.org/oecddirect/

This book has...

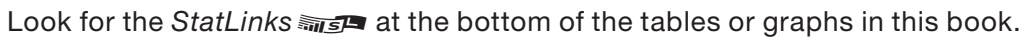
To download the matching Excel® spreadsheet, just type the link into your Internet browser, starting with the $h t t p: / / d x$. doi.org prefix, or click on the link from the e-book edition. 


\section{Executive summary}

Health at a Glance 2019 provides the latest comparable data and trends over time on population health and health system performance across OECD members, candidate and partner countries.

\section{Gains in longevity are stalling; chronic diseases and mental ill health affect more and more people}

- On average across OECD countries, a person born today can expect to live almost 81 years. But life expectancy gains have slowed recently across most OECD countries, especially in the United States, France and the Netherlands. 2015 was a particularly bad year, with life expectancy falling in 19 countries.

- The causes are multifaceted. Rising levels of obesity and diabetes have made it difficult to maintain previous progress in cutting deaths from heart disease and stroke. Respiratory diseases such as influenza and pneumonia have claimed more lives in recent years, notably amongst older people.

- In some countries the opioid crisis has caused more working-age adults to die from drugrelated accidental poisoning. Opioid-related deaths have increased by about $20 \%$ since 2011, and have claimed about 400000 lives in the United States alone. Opioid-related deaths are also relatively high in Canada, Estonia and Sweden.

- Heart attacks, stroke and other circulatory diseases caused about one in three deaths across the OECD; and one in four deaths were related to cancer. Better prevention and health care could have averted almost 3 million premature deaths.

- Almost one in ten adults consider themselves to be in bad health. This reflects in part the burden of chronic diseases - almost a third of adults live with two or more chronic conditions. Mental ill health also takes its toll, with an estimated one in two people experiencing a mental health problem in their lifetime.

\section{Smoking, drinking and obesity continue to cause people to die prematurely and worsen quality of life}

- Unhealthy lifestyles - notably smoking, harmful alcohol use and obesity - are the root cause of many chronic health conditions, cutting lives short and worsening quality of life.

- Whilst smoking rates are declining, $18 \%$ of adults still smoke daily.

- Alcohol consumption averaged 9 litres of pure alcohol per person per year across OECD countries, equivalent to almost 100 bottles of wine. Nearly $4 \%$ of adults were alcohol dependent. 
- Obesity rates continue to rise in most OECD countries, with $56 \%$ of adults overweight or obese and almost one-third of children aged 5-9 overweight.

- Air pollution caused about 40 deaths per 100000 people, across OECD countries. Death rates were much higher in partner countries India and China, at around 140 deaths per 100000 people.

\section{Barriers to access persist, particularly amongst the less well-off}

- An estimated one in five adults who needed to see a doctor did not do so, with worse access for the less well-off. Uptake of cancer screening is also lower amongst poorer individuals, even though most OECD countries provide screening programmes at no cost.

- Direct payments by households (out-of-pocket payments) make up just over a fifth of all health spending on average, and over $40 \%$ in Latvia and Mexico. Cost concerns lead people to delay or not seek care, with the least well-off three times more likely than wealthier individuals to have unmet need for financial reasons.

- Waiting times and transportation difficulties hinder access in some countries. For example, waiting times for a knee replacement were over a year in Chile, Estonia and Poland.

- Such access constraints occur despite most OECD countries having universal or nearuniversal coverage for a core set of services. Parts of the explanation are high cost sharing, exclusion of services from benefit packages or implicit rationing of services. Limitations in health literacy, imperfect communication strategies and low quality of care are also contributing factors.

\section{Quality of care is improving in terms of safety and effectiveness, but more} attention should be placed on patient-reported outcomes and experiences

- Patient safety has improved across many indicators, but more needs to be done. For example, $5 \%$ of hospitalised patients had a health-care associated infection.

- Strong primary care systems keep people well and can treat most uncomplicated cases. They also relieve pressure on hospitals: avoidable admissions for chronic conditions have fallen in most OECD countries, particularly in Korea, Lithuania, Mexico and Sweden.

- In terms of acute care, fewer people are dying following a heart attack or stroke, with Norway and Iceland having low case-fatality rates for both conditions. Alongside adherence to evidence-based medicine, timely care is critical.

- Survival rates for a range of cancers have also improved, reflecting better quality preventive and curative care. Across all OECD countries, for example, women diagnosed early for breast cancer have a $90 \%$ or higher probability of surviving their cancer for at least five years.

- A deeper understanding of quality of care requires measuring what matters to people. Yet few health systems routinely ask patients about the outcomes and experiences of their care. Preliminary results show improvements in patient-reported outcomes. For 
example, following hip replacement, an individual's quality of life - in terms of mobility, self-care, activity, pain and depression - improved on average by around $20 \%$.

Countries spend a lot on health, but they do not always spend it as well as they could

- Spending on health was about USD 4000 per person (adjusted for purchasing powers), on average across OECD countries. The United States spent more than all other countries by a considerable margin, at over USD 10000 per resident. Mexico spent the least, at around USD 1150 per resident.

- Health expenditure has largely outpaced economic growth in the past, and despite a slowdown in recent years, is expected to do so in the future. New estimates point to health spending reaching $10.2 \%$ of GDP by 2030 across OECD countries, up from $8.8 \%$ in 2018. This raises sustainability concerns, particularly as most countries draw funding largely from public sources.

- Reforms to improve economic efficiency are critical. Increased use of generics has generated cost-savings, though generics only represent around half of the volume of pharmaceuticals sold across OECD countries. Increases in day surgery, lower hospitalisation rates and shorter stays may also indicate a more efficient use of expensive hospital resources.

- In OECD countries, health and social systems employ more workers now than at any other time in history, with about one in every ten jobs found in health or social care. Shifting tasks from doctors to nurses and other health professionals can alleviate cost pressures and improve efficiency.

- Population ageing increases demand for health services, particularly for long-term care. This places more pressure on family members, particularly women, with around $13 \%$ of people aged 50 and over providing informal care at least once a week for a dependent relative or friend. By 2050, the share of the population aged 80 and over will more than double. 



\section{Reader's guide}

Health at a Glance 2019: OECD Indicators compares key indicators for population health and health system performance across the 36 OECD member countries. Candidate and partner countries are also included where possible - Brazil, People's Republic of China (China), Colombia, Costa Rica, India, Indonesia, the Russian Federation (Russia) and South Africa. On 25 May 2018, the OECD Council invited Colombia to become a Member. At the time of preparation of this publication, the deposit of Colombia's instrument of accession to the OECD Convention was pending and therefore Colombia does not appear in the list of OECD Members and is not included in the OECD zone aggregates.

Data presented in this publication come from official national statistics, unless otherwise stated.

\section{Conceptual framework}

The conceptual framework underlying Health at a Glance assesses health system performance within the context of a broad view of the determinants of health (Figure 1). It builds on the framework endorsed by the OECD work stream on health care quality and outcomes, which recognises that the ultimate goal of health systems is to improve people's health.

Many factors outside the health system influence health status, notably income, education, the physical environment in which an individual lives, and the degree to which people adopt healthy lifestyles. The demographic, economic and social context also affects the demand for and supply of health services, and ultimately health status.

At the same time, the performance of a health care system has a strong impact on a population's health. When health services are of high quality and are accessible to all, people's health outcomes are better. Achieving access and quality goals, and ultimately better health outcomes, depends critically on there being sufficient spending on health. Health spending pays for health workers to provide needed care, as well as the goods and services required to prevent and treat illness. However, these resources also need to be spent wisely, so that value-for-money is maximised.

\section{Structure of the publication}

Health at a Glance 2019 compares OECD countries on each component of this general framework. It is structured around eleven chapters. The first chapter presents an overview of health and health system performance, based on a subset of core indicators from the report. Country dashboards shed light on the relative strengths and weaknesses of OECD countries' health systems, alongside OECD-wide summary data. Linkages between how much a country spends on health and outcomes that matter to people are also illustrated. 
Figure 1. Mapping of Health at a Glance indicators into conceptual framework for health system performance assessment

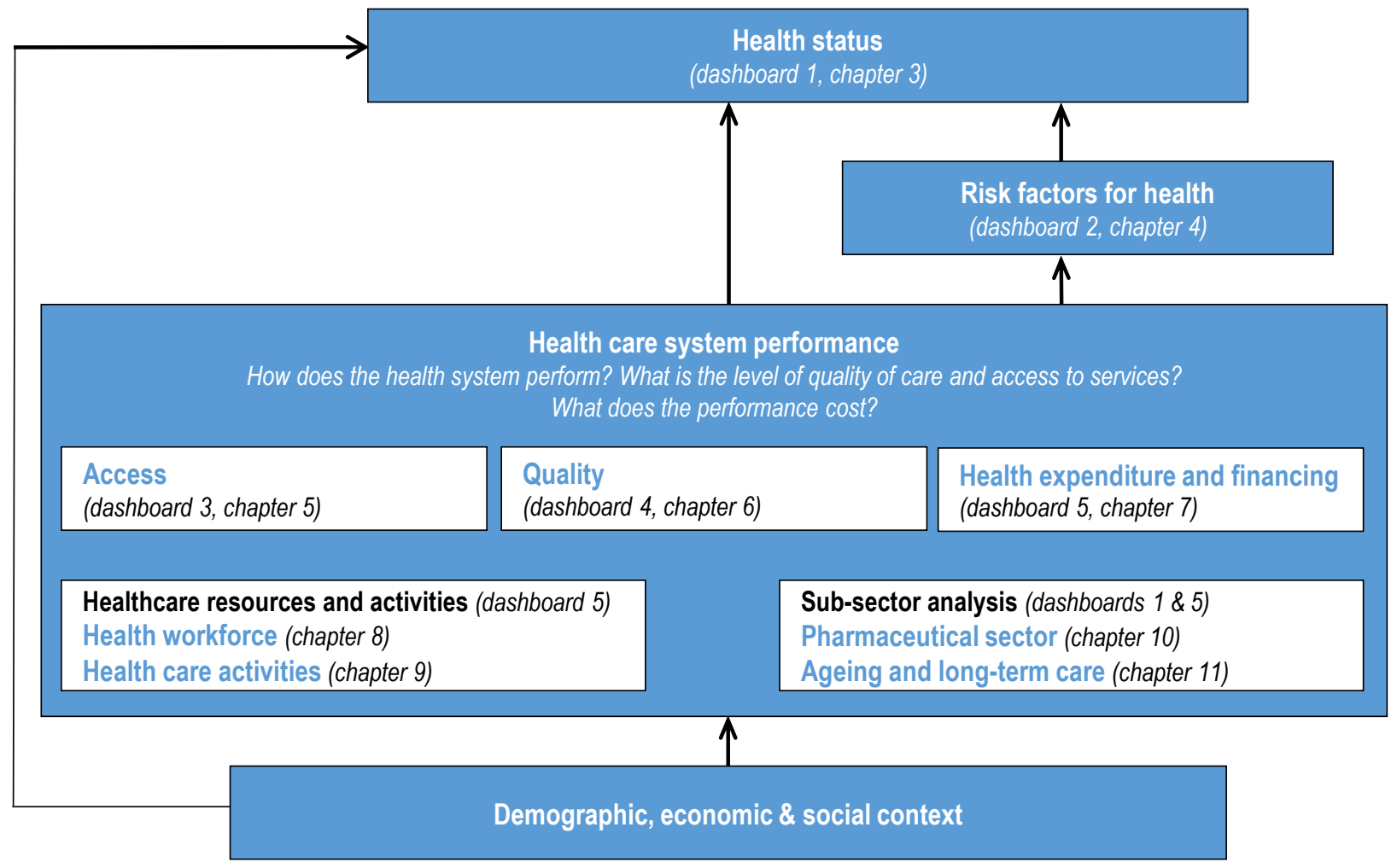

Source: Adapted from Carinci, F. et al. (2015), "Towards Actionable International Comparisons of Health System Performance: Expert Revision of the OECD Framework and Quality Indicators", International Journal for Quality in Health Care, Vol. 27, No. 2, pp. 137-146.

The second chapter provides a special focus on patient-reported outcomes and experiences, indicators that offer better measures of what matters to patients. It describes the rationale for collecting and using information reported by patients. It also provides preliminary results from a small number of countries in three clinical areas: elective hip and knee replacement; breast cancer care; and mental health.

The next nine chapters then provide detailed country comparisons across a range of health indicators, including where possible time trend analysis and data disaggregated by demographic and socioeconomic characteristics.

Chapter 3 on health status highlights variations across countries in life expectancy, the main causes of mortality, disease incidence and other indicators of population health. This chapter also includes measures of inequality in health status by education and income level for key indicators such as life expectancy and self-assessed health.

Chapter 4 analyses risk factors for health. The focus is on an individual's health-related behaviours, most of which effective public health and prevention policies can modify. These include the major risk factors for non-communicable diseases of smoking, alcohol and obesity; and new data on opioids use. Healthy lifestyles and population exposure to air pollution and extreme temperatures are also analysed.

Chapter 5 on access to care investigates the extent to which people can access needed services, with special attention paid to socioeconomic inequalities. Overall measures of 
population coverage are also presented, as are the financial consequences for households of accessing services.

Chapter 6 assesses quality and outcomes of care in terms of patient safety, clinical effectiveness and the person responsiveness of care. Indicators across the full lifecycle of care are included, from prevention to primary, chronic and acute care. This includes analysis of prescribing practices, management of chronic conditions, acute care for heart attacks and stroke, mental health, cancer care and prevention of communicable diseases.

Chapter 7 on health expenditure and financing compares how much countries spend on health per person and in relation to GDP. It then analyses differences in prices paid, the extent to which countries finance health through prepayment schemes or household outof-pocket payments, and the public-private funding mix. Spending by type of service and health provider are also explored. Finally, projections estimate spending to 2030 under different policy scenarios.

Chapter 8 examines the health workforce, particularly the supply and remuneration of doctors and nurses. The chapter also presents data on the number of new graduates from medical and nursing education programmes. Indicators on the international migration of doctors and nurses compare countries in terms of their reliance on foreign-trained workers.

Chapter 9 on health care activities describes some of the main characteristics of health service delivery. It starts with the number of consultations with doctors, often the entry point of patients to health care systems. The chapter then compares the use and supply of hospital services, in terms of discharges, number of beds and average length of stay. Utilisation of medical technologies, common surgical procedures, and the increased use of ambulatory surgery are also analysed.

Chapter 10 takes a closer look at the pharmaceutical sector. Analysis of pharmaceutical spending gives a sense of the varying scale of the market in different countries, as does spending on research and development. The number of pharmacists and pharmacies, consumption of certain high-volume drugs, and the use of generics and bio-similars, are also compared.

Chapter 11 focuses on ageing and long-term care. It assesses key factors affecting the demand for long-term care, such as demographic trends and health status indicators for elderly populations. Dementia prevalence and the quality of dementia care is compared, as is the safety of care for elderly populations. Recipients of long-term care, and the formal and informal workers providing care for these people, are also assessed, along with trends in spending and unit costs.

\section{Presentation of indicators}

With the exception of the first two chapters, indicators covered in the rest of the publication are presented over two pages. The first page defines the indicator, highlights key findings conveyed by the data and related policy insights, and signals any significant national variation in methodology that might affect data comparability. A few key references are also provided.

On the facing page is a set of figures. These typically show current levels of the indicator and, where possible, trends over time. Where an OECD average is included in a figure, it is the unweighted average of the OECD countries presented, unless otherwise 
specified. The number of countries included in this OECD average is indicated in the figure, and for charts showing more than one year this number refers to the latest year.

\section{Data limitations}

Limitations in data comparability are indicated both in the text (in the box related to "Definition and comparability") as well as in footnotes to figures.

\section{Data sources}

Readers interested in using the data presented in this publication for further analysis and research are encouraged to consult the full documentation of definitions, sources and methods presented in the online database OECD Health Statistics on OECD.Stat at https:// oe.cd/ds/health-statistics. More information on OECD Health Statistics is available at http:// www.oecd.org/health/health-data.htm.

\section{Population figures}

The population figures used to calculate rates per capita throughout this publication come from Eurostat for European countries, and from OECD data based on the UN Demographic Yearbook and UN World Population Prospects (various editions) or national estimates for non-European OECD countries (data extracted as of early June 2019). Mid-year estimates are used. Population estimates are subject to revision, so they may differ from the latest population figures released by the national statistical offices of OECD member countries.

Note that some countries such as France, the United Kingdom and the United States have overseas territories. These populations are generally excluded. However, the calculation of GDP per capita and other economic measures may be based on a different population in these countries, depending on the data coverage.

\section{OECD country ISO codes}

\begin{tabular}{llll}
\hline Australia & AUS & Korea & KOR \\
Austria & AUT & Latvia & LVA \\
Belgium & BEL & Lithuania & LTU \\
Canada & CAN & Luxembourg & LUX \\
Chile & CHL & Mexico & MEX \\
Czech Republic & CZE & Netherlands & NLD \\
Denmark & DNK & New Zealand & NZL \\
Estonia & EST & Norway & NOR \\
Finland & FIN & Poland & POL \\
France & FRA & Portugal & PRT \\
Germany & DEU & Slovak Republic & SVK \\
Greece & GRC & Slovenia & SVN \\
Hungary & HUN & Spain & ESP \\
Iceland & ISL & Sweden & SWE \\
Ireland & IRL & Switzerland & CHE \\
Israel & ISR & Turkey & TUR \\
Italy & ITA & United Kingdom & GBR \\
Japan & JPN & United States & USA \\
\hline
\end{tabular}




\section{Partner country ISO codes}

\begin{tabular}{llll}
\hline Brazil & BRA & India & IND \\
China (People's Republic of) & CHN & Indonesia & IDN \\
Colombia & COL & Russia & RUS \\
Costa Rica & CRI & South Africa & ZAF \\
\hline
\end{tabular}





\section{Chapter 1}

\section{Indicator overview: comparative performance of countries and major trends}

This chapter analyses a core set of indicators on health and health systems. Country dashboards shed light on how OECD countries compare across five dimensions: health status, risk factors for health, access, quality and outcomes, and health care resources. OECD snapshots summarise the extent of variation in performance across countries, as well as time trends. Finally, quadrant charts illustrate how much health spending is associated with staffing, access, quality and health outcomes. 


\section{Introduction}

Health indicators offer a useful 'at a glance' perspective on how healthy populations are and how well health systems perform. This introductory chapter provides a comparative overview of OECD countries across 20 core indicators. It also explores how much health spending is associated with staffing, access, quality and health outcomes.

Such comparative analysis does not indicate which countries have the best performing health systems overall. Rather, it identifies some of the relative strengths and weaknesses of different OECD countries. This can help policymakers determine priority action areas for their country, with subsequent chapters in Health at a Glance providing a more detailed suite of indicators, organised by topic area.

Five dimensions of health and health systems are analysed in this chapter, covering core aspects of population health and health system performance. For each of these dimensions, four summary indicators are analysed (Table 1.1). These indicators are selected from the publication based on how relevant and actionable they are from a public policy perspective; as well as the more practical consideration of data availability across countries.

Table 1.1. Population health and health system performance: summary indicators

\begin{tabular}{|c|c|}
\hline Dimension & Indicator \\
\hline $\begin{array}{l}\text { Health status } \\
\text { (chapters } 3 \text { and 11) }\end{array}$ & $\begin{array}{l}\text { Life expectancy - years of life at birth } \\
\text { Avoidable mortality - deaths per } 100000 \text { people (age standardised) } \\
\text { Chronic disease morbidity - diabetes prevalence (\% adults, age standardised) } \\
\text { Self-rated health - population in poor health (\% population aged } 15+\text { ) }\end{array}$ \\
\hline $\begin{array}{l}\text { Risk factors for health } \\
\text { (chapter 4) }\end{array}$ & $\begin{array}{l}\text { Smoking - daily smokers (\% population aged } 15+\text { ) } \\
\text { Alcohol-litres consumed per capita (population aged } 15+\text { ) } \\
\text { Overweight/obese - population with BMl>=25 } \mathrm{kg} / \mathrm{m} 2(\% \text { population aged } 15+\text { ) } \\
\text { Air pollution - deaths due to pollution (per } 100000 \text { population) }\end{array}$ \\
\hline $\begin{array}{l}\text { Access to care } \\
\text { (chapter 5) }\end{array}$ & $\begin{array}{l}\text { Population coverage - population eligible for core services (\% population) } \\
\text { Financial protection - expenditure covered by prepayment schemes (\% total expenditure) } \\
\text { Service coverage, primary care - needs-adjusted probability of visiting a doctor (\% population aged 15+) } \\
\text { Service coverage, preventive care - probability of cervical cancer screening (\% population aged } 15+\text { ) }\end{array}$ \\
\hline $\begin{array}{l}\text { Quality of care } \\
\text { (chapter6) }\end{array}$ & $\begin{array}{l}\text { Safe prescribing - antibiotics prescribed (defined daily dose per } 1000 \text { people) } \\
\text { Effective primary care -avoidable asthma/COPD admissions (per } 100000 \text { people, age-sex standardised) } \\
\text { Effective secondary care - 30-day mortality following AMI (per } 100000 \text { people, age-sex standardised) } \\
\text { Effective cancer care - breast cancer 5-year net survival (\%, age-standardised) }\end{array}$ \\
\hline $\begin{array}{l}\text { Health care resources } \\
\text { (chapters 7-10) }\end{array}$ & $\begin{array}{l}\text { Health spending - per capita (US dollars based on purchasing power parities) } \\
\text { Health spending share - as a \% of GDP } \\
\text { Doctors - number of practising physicians (per } 1000 \text { people) } \\
\text { Nurses - number of practising nurses (per } 1000 \text { people) }\end{array}$ \\
\hline
\end{tabular}

Note: $\mathrm{AMI}$ = acute myocardial infarction (heart attack); $\mathrm{BMI}$ = body mass index; COPD = chronic obstructive pulmonary disease.

Based on these indicators, country dashboards are produced for each of these five dimensions. These compare a country's performance to others and to the OECD average. Country classification for each indicator is into one of three colour-coded groups: 
- Blue, when the country's performance is close to the OECD average

- Green, when the country's performance is considerably better than the OECD average

- Red, when the country's performance is considerably worse than the OECD average

The only exception to this grouping is for the dashboard on health care resources (Table 1.6), where indicators cannot be strictly classified as showing better or worse performance. For this reason, the colour coding in this dashboard uses a lighter and darker shade of blue to signal that a country has considerably less or more of a given health care resource than the OECD average.

OECD snapshots provide accompanying summary statistics for each of these indicators. They complement the country dashboards by providing an OECD-wide overview for each indicator. Highest and lowest values per indicator, alongside the OECD average, provide a general sense of the degree of cross-country variation. Countries with comparatively large improvements over time in a given indicator are also shown.

Finally, quadrant charts illustrate basic associations between how much countries spend on health and how effectively health systems function. That is, they show the extent to which spending more on health translates into better health outcomes, higher quality of care and improved access to care, across OECD countries; whilst also recognising the importance of major risk factors. The relationship between spending and the number of health professionals is also explored. These quadrant charts only show simple associations at a macro level between indicators rather than causal relationships. That is, their purpose is to stimulate deeper discussions on policy priority setting, by highlighting areas where countries could potentially do better. The centre of each quadrant chart is the OECD average, with health expenditure on the $x$-axis and the other variable of interest on the $y$ axis. Figure 1.1 shows the basic interpretation of each quadrant, taking health outcome variables as an example.

Figure 1.1. Interpretation of quadrant charts: Health expenditure and health outcome variables
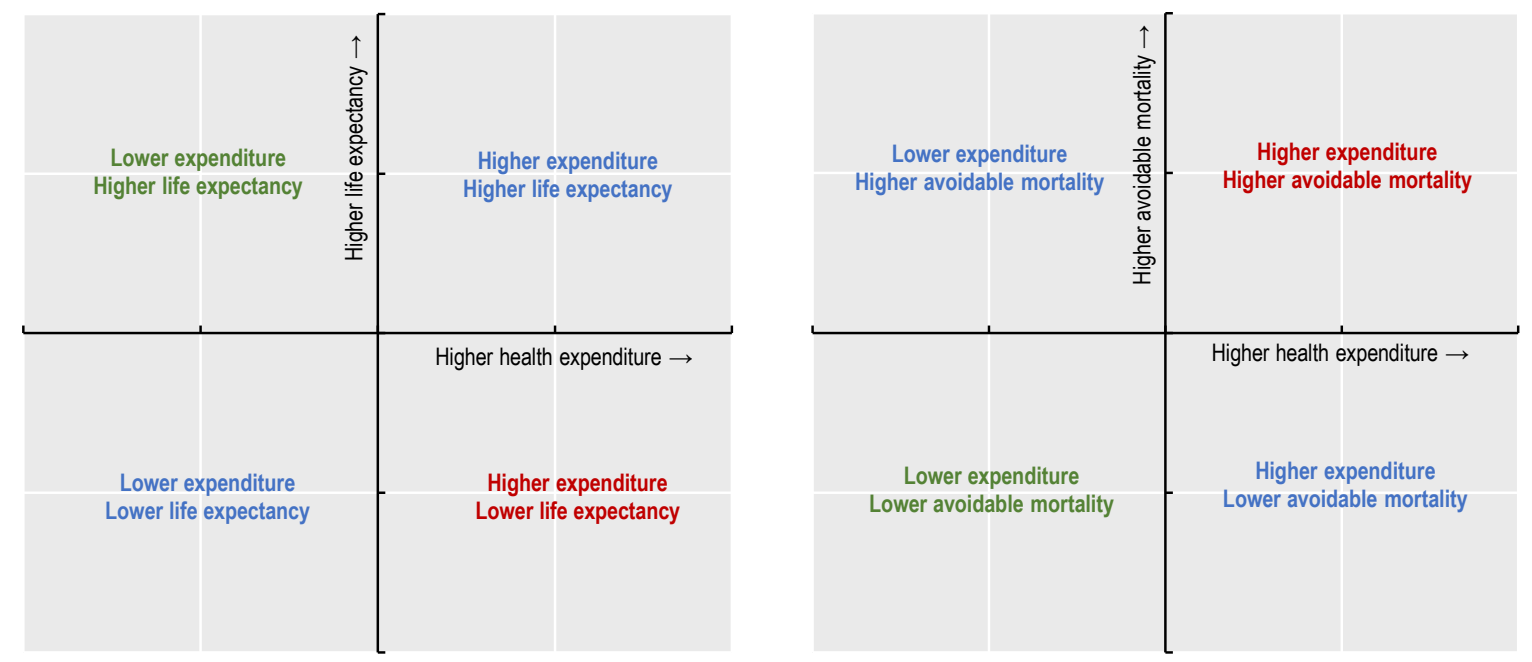



\section{Methodology, interpretation and use}

\section{Country dashboards}

The classification of countries being close to, better or worse than the OECD average is based on an indicator's standard deviation (a common statistical measure of dispersion). This method is preferred to using a fixed percentage or fixed number of countries per category, since it reflects the degree of variation, i.e. how far a country is from the OECD average. Countries are classified as "close to the OECD average" (blue) whenever the value for an indicator is within one standard deviation from the OECD average for the latest year. Particularly large outliers (values larger than three standard deviations) are excluded from the calculation of the standard deviation in order to avoid statistical distortions. These exclusions are noted under the relevant dashboards.

For a typical indicator, about $65 \%$ of the countries (24-25 countries) will be close to the OECD average, with the remaining 35\% performing significantly better (green) or worse (red). When the number of countries that are close to the OECD average is higher (lower), it means that cross-country variation is relatively low (high) for that indicator. For example, for obesity rates, 27 countries are close to the OECD average. In contrast, for avoidable mortality, only 16 countries are close to the OECD average.

\section{OECD snapshots}

For each indicator, the OECD average, highest and lowest values for the latest available year are shown, corresponding to the data presented in the main chapters of the publication. Countries with comparatively large improvements over time in a given indicator are also shown.

\section{Quadrant charts}

Quadrant charts plot health expenditure per capita against another indicator of interest (on health outcomes, quality of care, access and physical resources). These show the percentage difference of each indicator as compared with OECD averages. The intersection of the axes represents the OECD average for both indicators, so deviations from the midpoint show countries that perform above or below average compared to the OECD average. A simple correlation line is also included. Each country is colour-coded based on a simple (unweighted) risk factors index averaging smoking, alcohol and obesity variables (with blue, green and red having the same interpretation as in country dashboards).

Data from the latest available year are used for both variables in a given quadrant chart. A limitation of this approach is that lagged effects are not taken into account - for example, it may take a few years before higher health spending translates into longer life expectancy, or risk factors translate into higher avoidable mortality rates. 


\section{Health status}

Four health status indicators reflect core aspects of both the quality and quantity of life. Life expectancy is a key indicator for the overall health of a population; avoidable mortality focuses on premature deaths that could have been prevented or treated. Diabetes prevalence shows morbidity for a major chronic disease; self-rated health offers a more holistic measure of mental and physical health. Figure 1.2 provides a snapshot on health status across the OECD and Table 1.2 provide more detailed country comparisons.

Figure 1.2. Snapshot on health status across the OECD

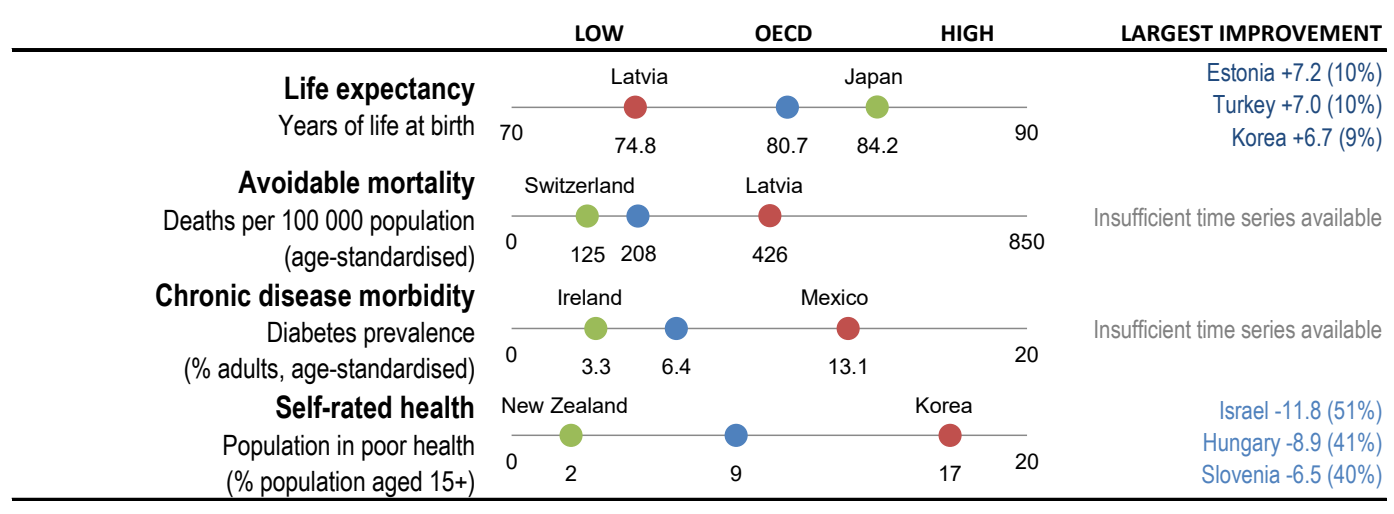

Note: Largest improvement shows countries with largest changes in value over time (\% change in brackets). Source: OECD Health Statistics 2019.

Across these indicators, Japan, Spain, Switzerland and the Netherlands generally have the best overall health outcomes. Hungary, Latvia, Mexico, Poland and the Slovak Republic are consistently below the OECD average for these indicators. Stronger health systems contribute to gains in health outcomes, by offering more accessible and higher quality care. Differences in risk factors such as smoking, alcohol and obesity also explain cross-country variation in health outcomes. Wider determinants of health matter too, notably rising incomes, better education and improved living environments.

Japan, Switzerland and Spain lead a large group of 26 OECD countries in which life expectancy at birth exceeds 80 years. A second group, including the United States and a number of central and eastern European countries, has a life expectancy between 77 and 80 years. Latvia, Lithuania, Mexico and Hungary have the lowest life expectancy, at less than 76 years in 2017. Across the OECD, whilst life expectancy has increased steadily over time, there has been a slowdown in longevity gains in recent years.

Avoidable mortality rates (from preventable and treatable causes) were lowest in Switzerland, Iceland, Japan, Sweden and Norway, where less than 300 per 100000 people died prematurely. Latvia, Lithuania and Hungary had the highest avoidable mortality rates, at over 800 premature deaths per 100000 people.

Diabetes prevalence is highest in Mexico, Turkey and the United States, with over $10 \%$ of adults living with diabetes (age-standardised data). Age-standardised diabetes prevalence rates have stabilised in many OECD countries, especially in Western Europe, but increased markedly in Turkey. Such upward trends are due in part to rising rates of obesity and physical inactivity, and their interactions with population ageing.

Almost $9 \%$ of adults consider themselves to be in bad health, on average across the OECD. This ranges from over $15 \%$ in Korea, Lithuania, Latvia and Portugal to under $4 \%$ in 
Table 1.2. Dashboard on health status

\begin{tabular}{|c|c|c|c|c|c|c|c|c|}
\hline \multirow[b]{3}{*}{ OECD } & \multirow{2}{*}{\multicolumn{2}{|c|}{\begin{tabular}{|l} 
Life expectancy \\
Years of life at birth
\end{tabular}}} & \multirow{2}{*}{\multicolumn{2}{|c|}{$\begin{array}{c}\text { Avoidable mortality } \\
\begin{array}{c}\text { Deaths per } 100000 \text { people } \\
\text { (age-standardised) }\end{array}\end{array}$}} & \multirow{2}{*}{\multicolumn{2}{|c|}{$\begin{array}{c}\text { Chronic disease morbidity } \\
\text { Diabetes prevalence } \\
\text { (\% adults, age-standardised })\end{array}$}} & \multirow{2}{*}{\multicolumn{2}{|c|}{$\begin{array}{c}\text { Self-rated health } \\
\begin{array}{l}\text { Population in poor health } \\
\text { (\% population aged } 15+)\end{array}\end{array}$}} \\
\hline & & & & & & & & \\
\hline & 80.7 & ○ & 208 & ○ & 6.4 & ○ & 8.7 & ○ \\
\hline Australia & 82.6 & ○ & 145 & $\nabla$ & 5.1 & ○ & 3.7 & $\bar{\nabla}$ \\
\hline Austria & 81.7 & ○ & 175 & ○ & 6.4 & $\odot$ & 8.1 & ○ \\
\hline Belgium & 81.6 & ○ & 172 & ○ & 4.3 & $\nabla$ & 8.6 & ○ \\
\hline Canada & 82.0 & ○ & 176 & ○ & 7.4 & ○ & 3.2 & $\nabla$ \\
\hline Chile & 80.2 & ○ & 206 & ○ & 8.5 & $凶$ & 6.6 & ○ \\
\hline Czech Republic & 79.1 & ○ & 245 & ○ & 6.8 & ๑ & 10.7 & ○ \\
\hline Denmark & 81.2 & ○ & 184 & ○ & 6.4 & $\odot$ & 7.5 & ○ \\
\hline Estonia & 78.2 & 凶 & 297 & 凶 & 4.0 & $\nabla$ & 14.6 & 凶 \\
\hline Finland & 81.7 & ○ & 184 & ○ & 5.8 & $\odot$ & 5.7 & ○ \\
\hline France & 82.6 & ○ & 154 & $\nabla$ & 4.8 & ○ & 8.3 & ( ) \\
\hline Germany & 81.1 & ○ & 186 & ○ & 8.3 & $凶$ & 8.4 & ○ \\
\hline Greece & 81.4 & ○ & 187 & ○ & 4.6 & $\nabla$ & 10.4 & ○ \\
\hline Hungary & 75.9 & 凶 & 388 & $凶$ & 7.6 & $\odot$ & 11.9 & ○ \\
\hline Iceland & 82.7 & ○ & 140 & $\nabla$ & 5.3 & ๑ & 6.4 & ○ \\
\hline Ireland & 82.2 & ○ & 172 & ○ & 3.3 & $\nabla$ & 3.4 & $\nabla$ \\
\hline Israel & 82.6 & ○ & 134 & $\nabla$ & 6.7 & $\odot$ & 10.9 & ○ \\
\hline Italy & 83.0 & ○ & 143 & $\nabla$ & 4.8 & $\odot$ & 5.8 & ○ \\
\hline Japan & 84.2 & $\nabla$ & 138 & $\nabla$ & 5.7 & $\odot$ & 14.1 & 凶 \\
\hline Korea & 82.7 & ○ & 159 & ○ & 6.8 & $\odot$ & 17.0 & 凶 \\
\hline Latvia & 74.8 & 凶 & 426 & 凶 & 4.9 & $\odot$ & 15.5 & 凶 \\
\hline Lithuania & 75.6 & 凶 & 385 & $凶$ & 3.7 & $\nabla$ & 16.4 & 凶 \\
\hline Luxembourg & 82.2 & ○ & 152 & $\nabla$ & 4.4 & $\nabla$ & 9.3 & ○ \\
\hline Mexico & 75.4 & $凶$ & 367 & $\otimes$ & 13.1 & $凶$ & - & \\
\hline Netherlands & 81.8 & ○ & 153 & $\nabla$ & 5.3 & $\odot$ & 4.6 & $\nabla$ \\
\hline New Zealand & 81.9 & ○ & 178 & ○ & 8.1 & $\odot$ & 2.3 & $\nabla$ \\
\hline Norway & 82.7 & ○ & 145 & $\nabla$ & 5.3 & ○ & 7.2 & ○ \\
\hline Poland & 77.9 & 凶 & 268 & 凶 & 5.9 & $\odot$ & 13.6 & 凶 \\
\hline Portugal & 81.5 & 0 & 180 & ○ & 9.9 & $凶$ & 15.3 & $凶$ \\
\hline Slovak Republic & 77.3 & $凶$ & 323 & $\otimes$ & 7.3 & $\odot$ & 11.3 & ○ \\
\hline Slovenia & 81.1 & ○ & 210 & ○ & 7.3 & $\odot$ & 9.7 & ○ \\
\hline Spain & 83.4 & $\nabla$ & 146 & $\nabla$ & 7.2 & $\odot$ & 6.6 & ○ \\
\hline Sweden & 82.5 & 0 & 144 & $\nabla$ & 4.8 & ○ & 5.7 & ○ \\
\hline Switzerland & 83.6 & $\nabla$ & 125 & $\nabla$ & 5.6 & ○ & 4.1 & $\nabla$ \\
\hline Turkey & 78.1 & 凶 & 257 & ○ & 12.1 & $凶$ & 9.4 & ○ \\
\hline United Kingdom & 81.3 & ○ & 189 & ○ & 4.3 & $\nabla$ & 7.1 & ○ \\
\hline United States & 78.6 & 0 & 262 & 凶 & 10.8 & 凶 & 2.6 & $\nabla$ \\
\hline
\end{tabular}

Note: $\nabla$ Better than OECD average; $\bigcirc$ Close to OECD average; $₫$ Worse than OECD average. Hungary, Latvia and Lithuania excluded from the standard deviation calculation for avoidable mortality, while Mexico and Turkey excluded from diabetes prevalence.

New Zealand, the United States, Canada, Ireland and Australia. However, socio-cultural differences, the share of older people and differences in survey design affect cross-country comparability. People with lower incomes are generally less positive about their health as compared with people on higher incomes, in all OECD countries. 


\section{Risk factors for health}

Smoking, alcohol consumption and obesity are the three major risk factors for noncommunicable diseases, contributing to a large share of worldwide deaths. Air pollution is also a critical non-medical determinant of health. Figure 1.3 provides a snapshot on risk factors for health across the OECD and Table 1.3 provides more detailed country comparisons.

Figure 1.3. Snapshot on risk factors for health across the OECD

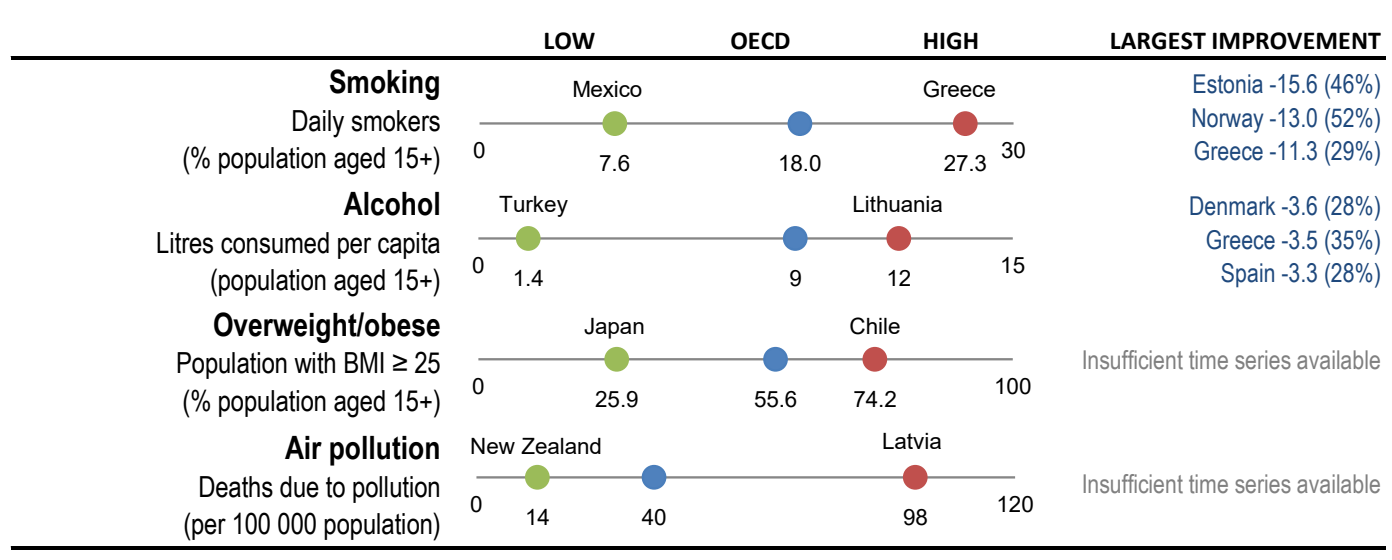

Note: Largest improvement shows countries with largest changes in value over time (\% change in brackets). Source: OECD Health Statistics 2019, WHO Global Health Observatory.

Norway and Sweden perform well across these indicators. Smoking causes multiple diseases - the World Health Organization estimates tobacco smoking kills 7 million people in the world every year. Smoking rates range from over $25 \%$ in Greece, Turkey and Hungary, to below $10 \%$ in Mexico and Iceland. Daily smoking rates have decreased in most OECD countries over the last decade, from an average of $23 \%$ in 2007 to $18 \%$ in 2017 . In the Slovak Republic and Austria, though, smoking rates have risen slightly.

Alcohol use is a leading cause of death and disability worldwide, particularly in those of working age. Measured through sales data, Lithuania reported the highest consumption (12.3 litres of pure alcohol per person per year), followed by Austria, France, the Czech Republic, Luxembourg, Ireland, Latvia and Hungary, all with over 11 litres per person. Turkey, Israel and Mexico have comparatively low consumption levels (under 5 litres). Average consumption fell in 27 OECD countries since 2007. Harmful drinking is of particular concern in certain countries, notably Latvia, Hungary and the Russian Federation.

Obesity is a major risk factor for many chronic diseases, including diabetes, cardiovascular diseases and cancer. Obesity rates have been increasing in recent decades in almost all OECD countries, with an average of $56 \%$ of the population being overweight or obese. Obesity rates are considerably higher than the OECD average in Chile, Mexico, the United States, Finland, Portugal and New Zealand. Obesity is lowest in Japan, Korea, and Switzerland. The measure reported here for overweight (including obese) adults is based on both measured and self-reported data. Caution should be taken when comparing countries with reporting differences, since measured data are generally higher.

Air pollution is not only a major environmental threat, but also worsens health. OECD projections estimate that outdoor air pollution may cause 6 to 9 million premature deaths a 
Table 1.3. Dashboard on risk factors for health

\begin{tabular}{|c|c|c|c|c|c|c|c|c|}
\hline \multirow[b]{3}{*}{ OECD } & \multicolumn{2}{|c|}{ Smoking } & \multicolumn{2}{|c|}{ Alcohol } & \multicolumn{2}{|c|}{ Overweight/obese } & \multicolumn{2}{|c|}{ Air pollution } \\
\hline & \multicolumn{2}{|c|}{$\begin{array}{c}\text { Daily smokers } \\
\text { (\% population aged } 15+)\end{array}$} & \multicolumn{2}{|c|}{$\begin{array}{l}\text { Litres consumed per capita } \\
\quad \text { (population aged } 15+\text { ) }\end{array}$} & \multicolumn{2}{|c|}{$\begin{array}{l}\text { Population with } \mathrm{BMI} \geq 25 \\
\text { (\% population aged } 15+\text { ) }\end{array}$} & \multicolumn{2}{|c|}{$\begin{array}{l}\text { Deaths due to pollution } \\
\text { (per } 100000 \text { people) }\end{array}$} \\
\hline & 18.0 & ○ & 8.9 & ○ & 55.6 & ○ & 39.6 & ○ \\
\hline Australia & 12.4 & $\nabla$ & 9.4 & ( ) & 65.2 & ○ & 16.8 & $\nabla$ \\
\hline Austria & 24.3 & 凶 & 11.8 & 凶 & $46.7^{*}$ & ○ & 38.7 & ○ \\
\hline Belgium & 18.9 & ○ & 10.4 & $\odot$ & 51.0 & ○ & 39.4 & ○ \\
\hline Canada & 12.0 & $\nabla$ & 8.1 & ○ & 59.1 & $\odot$ & 14.7 & $\nabla$ \\
\hline Chile & 24.5 & 凶 & 7.9 & ○ & 74.2 & 凶 & 34.8 & ○ \\
\hline Czech Republic & 18.4 & ○ & 11.6 & 凶 & 55.0 & ○ & 64.3 & $凶$ \\
\hline Denmark & 16.9 & ○ & 9.1 & ○ & $51.0^{*}$ & $\odot$ & 30.4 & ○ \\
\hline Estonia & 17.2 & ○ & 10.3 & ○ & 51.3 & ○ & 59.9 & 凶 \\
\hline Finland & 14.0 & ○ & 8.4 & ○ & 67.6 & 凶 & 18.7 & $\nabla$ \\
\hline France & 25.4 & 凶 & 11.7 & 凶 & 49.0 & ○ & 25.2 & ○ \\
\hline Germany & 18.8 & ○ & 10.9 & ○ & 60.0 & $\odot$ & 45.3 & ○ \\
\hline Greece & 27.3 & $凶$ & 6.5 & ○ & $55.0^{*}$ & ○ & 76.7 & 凶 \\
\hline Hungary & 25.8 & 凶 & 11.1 & ○ & 62.3 & $\odot$ & 82.7 & 凶 \\
\hline Iceland & 8.6 & $\nabla$ & 7.7 & ○ & $65.4^{*}$ & ○ & 16.9 & $\nabla$ \\
\hline Ireland & 17.0 & ○ & 11.2 & ○ & 62.0 & ○ & 20.2 & $\nabla$ \\
\hline Israel & 16.9 & ○ & 2.6 & $\nabla$ & 50.9 & ○ & 23.2 & ○ \\
\hline Italy & 19.9 & ○ & 7.6 & ○ & $46.0^{*}$ & $\odot$ & 48.7 & ○ \\
\hline Japan & 17.7 & ○ & 7.2 & ○ & 25.9 & $\nabla$ & 42.9 & ○ \\
\hline Korea & 17.5 & ○ & 8.7 & ○ & 33.7 & $\nabla$ & 35.0 & ○ \\
\hline Latvia & 24.1 & 凶 & 11.2 & ○ & 54.6 & ○ & 97.8 & $凶$ \\
\hline Lithuania & 20.3 & ○ & 12.3 & 凶 & $53.3^{*}$ & ○ & 82.1 & 凶 \\
\hline Luxembourg & 14.5 & ○ & 11.3 & ○ & 58.1 & ○ & 22.6 & ○ \\
\hline Mexico & 7.6 & $\nabla$ & 4.4 & $\nabla$ & 72.5 & 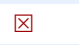 & 33.0 & ○ \\
\hline Netherlands & 16.8 & ○ & 8.3 & ○ & $47.3^{*}$ & ○ & 31.3 & ○ \\
\hline New Zealand & 13.8 & ○ & 8.8 & ○ & 66.6 & $凶$ & 13.6 & $\nabla$ \\
\hline Norway & 12.0 & $\nabla$ & 6.0 & $\nabla$ & $46.0^{*}$ & ○ & 18.7 & $\nabla$ \\
\hline Poland & 22.7 & ○ & 10.6 & ○ & $53.3^{*}$ & ○ & 76.3 & 凶 \\
\hline Portugal & 16.8 & ○ & 10.7 & ○ & 67.6 & $凶$ & 28.3 & 0 \\
\hline Slovak Republic & 22.9 & ○ & 9.7 & ○ & 51.5 & ○ & 59.1 & 凶 \\
\hline Slovenia & 18.9 & ○ & 10.1 & ○ & $55.6^{*}$ & ○ & 56.8 & ○ \\
\hline Spain & 22.1 & ○ & 8.6 & ○ & $53.0^{*}$ & ○ & 27.1 & ○ \\
\hline Sweden & 10.4 & $\nabla$ & 7.1 & ○ & $48.2^{*}$ & ○ & 18.5 & $\nabla$ \\
\hline Switzerland & 19.1 & ○ & 9.2 & ○ & $41.8^{\star}$ & $\nabla$ & 25.2 & ○ \\
\hline Turkey & 26.5 & 凶 & 1.4 & $\nabla$ & 64.4 & ○ & 46.2 & ○ \\
\hline United Kingdom & 17.2 & $\odot$ & 9.7 & ○ & 64.3 & ○ & 32.1 & ○ \\
\hline United States & 10.5 & $\nabla$ & 8.9 & ○ & 71.0 & 凶 & 24.1 & ○ \\
\hline
\end{tabular}

Note: $\nabla$ Better than OECD average; $\bigcirc$ Close to OECD average; $₫$ Worse than OECD average. Hungary, Latvia and Lithuania excluded from standard deviation calculation for air pollution. * Likely under-estimate of obesity as selfreported.

year worldwide by 2060. Death rates in 2016 ranged from over 80 deaths in Latvia, Hungary and Lithuania, to 15 deaths or less per 100000 people in New Zealand and Canada. 


\section{Access to care}

Ensuring equitable access is critical for inclusive societies and high performing health systems. Population coverage, measured by the share of the population eligible for a core set of services, offers an initial assessment of access to care. The share of spending covered by prepayment schemes provides further insight on financial protection. The probability of visiting a doctor, adjusted for need, and the share of women aged 20-69 screened for cervical cancer measure use of needed services. Figure 1.4 provides a snapshot on access to care across the OECD and Table 1.4 provides more detailed country comparisons.

\section{Figure 1.4. Snapshot on access to care across the OECD}

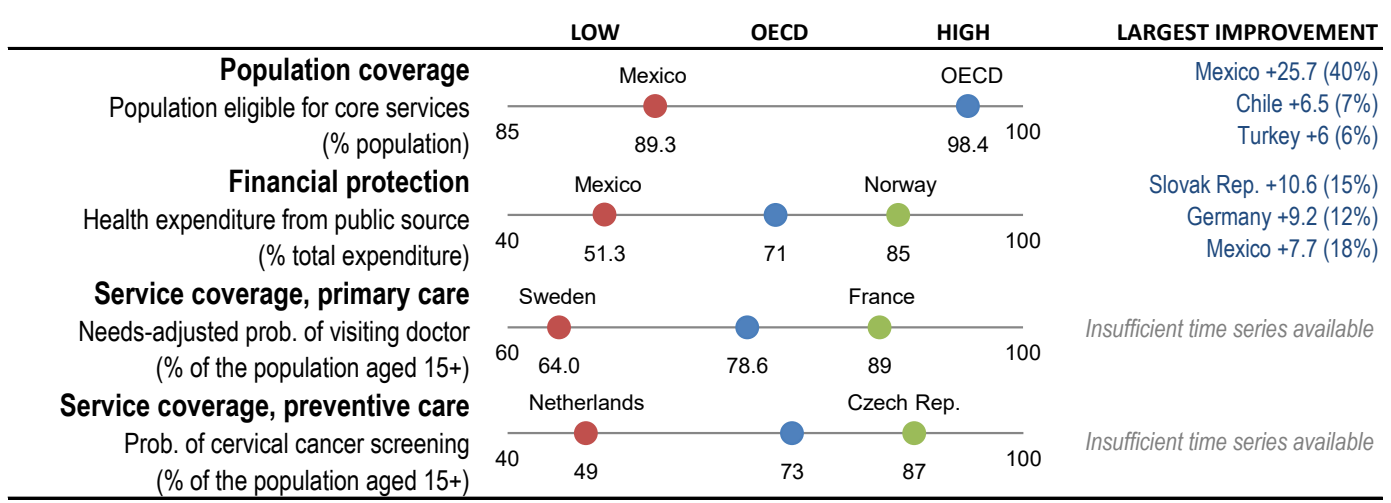

Note: Largest improvement shows countries with largest changes in value over time (\% change in brackets).

Source: OECD Health Statistics 2019.

Austria, the Czech Republic, France, Germany and Luxembourg perform well across these indicators, In terms of population coverage, most OECD countries have achieved universal (or near-universal) coverage for a core set of services. However, in seven countries coverage remains below 95\% - Chile, Estonia, Hungary, Mexico, Poland, the Slovak Republic and the United States.

Population coverage, though, is not sufficient by itself. The degree of cost sharing applied to those services also affects access to care. Across the OECD, almost three-quarters of all health care costs are covered by government or compulsory health insurance schemes. However, in Mexico, Latvia and Korea less than $60 \%$ of all costs are covered by publicly mandated schemes. Mexico, though, has significantly expanded population coverage and financial protection over the last decade.

One in five people report not seeing a doctor despite having medical need. Crosscountry differences in utilisation are large, with need-adjusted probabilities of visiting a doctor ranging from around $65 \%$ in Sweden and the United States to $89 \%$ in France. Excepting Denmark and the Slovak Republic, wealthier individuals are more likely to see a doctor than individuals in the lowest income quintile, for a comparable level of need.

Uptake of cancer screening is also lower amongst the less well-off. This is despite most OECD countries providing screening programmes at no cost. Overall uptake of cervical cancer screening ranged from just under $50 \%$ of women aged 20 to 69 in the Netherlands, to over $85 \%$ in the Czech Republic and Austria. 
Table 1.4. Dashboard on access to care

\begin{tabular}{|c|c|c|c|c|c|c|c|c|}
\hline \multirow[b]{3}{*}{ OECD } & \multicolumn{2}{|c|}{ Population coverage } & \multicolumn{2}{|c|}{ Financial protection } & \multicolumn{2}{|c|}{$\begin{array}{l}\text { Service coverage, primary } \\
\text { care }\end{array}$} & \multicolumn{2}{|c|}{$\begin{array}{l}\text { Service coverage, } \\
\text { preventive care }\end{array}$} \\
\hline & \multicolumn{2}{|c|}{$\begin{array}{l}\text { Population eligible for core } \\
\text { services (\% population) }\end{array}$} & \multicolumn{2}{|c|}{$\begin{array}{l}\text { Health expenditure from public } \\
\text { sources (\% total expenditure) }\end{array}$} & \multicolumn{2}{|c|}{$\begin{array}{l}\text { Needs-adjusted prob. of } \\
\text { visiting doctor (\% pop 15+) }\end{array}$} & \multicolumn{2}{|c|}{$\begin{array}{l}\text { Prob. of cervical cancel } \\
\text { screening (\% pop } 15+\text { ) }\end{array}$} \\
\hline & 98.4 & $\odot$ & 71.2 & $\odot$ & 78 & 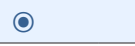 & 73 & ○ \\
\hline Australia & 100 & $\circ$ & 68.6 & ○ & - & & - & \\
\hline Austria & 99.9 & ○ & 74.0 & $\odot$ & 86 & $\nabla$ & 87 & $\nabla$ \\
\hline Belgium & 98.7 & $\odot$ & 77.2 & ○ & 86 & $\nabla$ & 76 & ○ \\
\hline Canada & 100 & $\odot$ & 73.0 & $\odot$ & 75 & ○ & 76 & ○ \\
\hline Chile & 94.0 & $凶$ & 50.1 & $凶$ & - & & 72 & $\odot$ \\
\hline Czech Republic & 100 & $\odot$ & 82.1 & $\odot$ & 85 & $\nabla$ & 87 & $\nabla$ \\
\hline Denmark & 100 & $\odot$ & 84.0 & $\nabla$ & 81 & ○ & 64 & 凶 \\
\hline Estonia & 94.1 & $凶$ & 74.7 & ○ & 75 & ○ & 58 & 凶 \\
\hline Finland & 100 & $\odot$ & 76.7 & $\odot$ & 74 & ○ & 79 & ○ \\
\hline France & 99.9 & $\circ$ & 77.1 & $\odot$ & 89 & $\nabla$ & 82 & $\nabla$ \\
\hline Germany & 100 & $\odot$ & 77.7 & $\odot$ & 86 & $\nabla$ & 81 & $\odot$ \\
\hline Greece & 100 & ○ & 60.8 & ○ & 76 & ○ & 76 & ○ \\
\hline Hungary & 94.0 & $凶$ & 68.7 & $\odot$ & 84 & $\circ$ & 71 & ○ \\
\hline Iceland & 100 & ○ & 81.8 & ○ & 75 & ○ & 80 & ○ \\
\hline Ireland & 100 & $\odot$ & 73.3 & ○ & 75 & ○ & 69 & ○ \\
\hline Israel & 100 & ○ & 63.6 & ○ & - & & - & \\
\hline Italy & 100 & ○ & 73.9 & ○ & 80 & $\circ$ & 68 & ○ \\
\hline Japan & 100 & $\circ$ & 84.0 & $\nabla$ & - & & - & \\
\hline Korea & 100 & $\circ$ & 57.4 & $凶$ & - & & - & \\
\hline Latvia & 100 & ○ & 57.2 & $凶$ & 76 & ○ & 78 & ○ \\
\hline Lithuania & 98.1 & $\circ$ & 65.5 & $\circ$ & 76 & $\circ$ & 62 & 凶 \\
\hline Luxembourg & - & & 84.9 & $\nabla$ & 88 & $\nabla$ & 84 & $\nabla$ \\
\hline Mexico & 89.3 & $凶$ & 51.3 & 凶 & - & & - & \\
\hline Netherlands & 99.9 & ○ & 81.5 & ○ & 75 & ○ & 49 & $凶$ \\
\hline New Zealand & 100 & 0 & 78.6 & 0 & - & & - & \\
\hline Norway & 100 & ○ & 85.5 & $\nabla$ & 77 & ○ & 66 & ○ \\
\hline Poland & 92.6 & 凶 & 69.0 & ○ & 80 & $\odot$ & 72 & $\odot$ \\
\hline Portugal & 100 & ○ & 66.3 & ○ & 86 & $\nabla$ & 71 & ○ \\
\hline Slovak Republic & 94.6 & 凶 & 79.9 & $\odot$ & 74 & ○ & 69 & ○ \\
\hline Slovenia & 100 & ○ & 71.8 & ○ & 71 & $凶$ & 78 & ○ \\
\hline Spain & 99.9 & $\odot$ & 70.6 & ○ & 84 & ○ & 69 & $\odot$ \\
\hline Sweden & 100 & ○ & 83.7 & $\nabla$ & 64 & $凶$ & 81 & ○ \\
\hline Switzerland & 100 & $\odot$ & 30.5 & 凶 & - & & - & \\
\hline Turkey & 99.2 & $\odot$ & 77.7 & ○ & - & & - & \\
\hline United Kingdom & 100 & ○ & 79.4 & ○ & 76 & ○ & 63 & $凶$ \\
\hline United States & 90.8 & 凶 & 50.2 & 凶 & 65 & 凶 & 80 & ○ \\
\hline
\end{tabular}

Note: $\nabla$ Better than OECD average; $\bigcirc$ Close to OECD average; $₫$ Worse than OECD average. 


\section{Quality of care}

Good quality care requires health services to be safe, appropriate, clinically effective and responsive to patient needs. Antibiotics prescriptions and avoidable hospital admissions for asthma and chronic obstructive pulmonary disease (COPD) measure the safety and appropriateness of primary care. 30-day mortality following acute myocardial infarction (AMI) and breast cancer survival are indicators of clinical effectiveness of secondary and cancer care. Figure 1.5 provides a snapshot on quality and outcome of care across the OECD and Table 1.5 provides more detailed country comparisons.

\section{Figure 1.5. Snapshot on quality of care across the OECD}

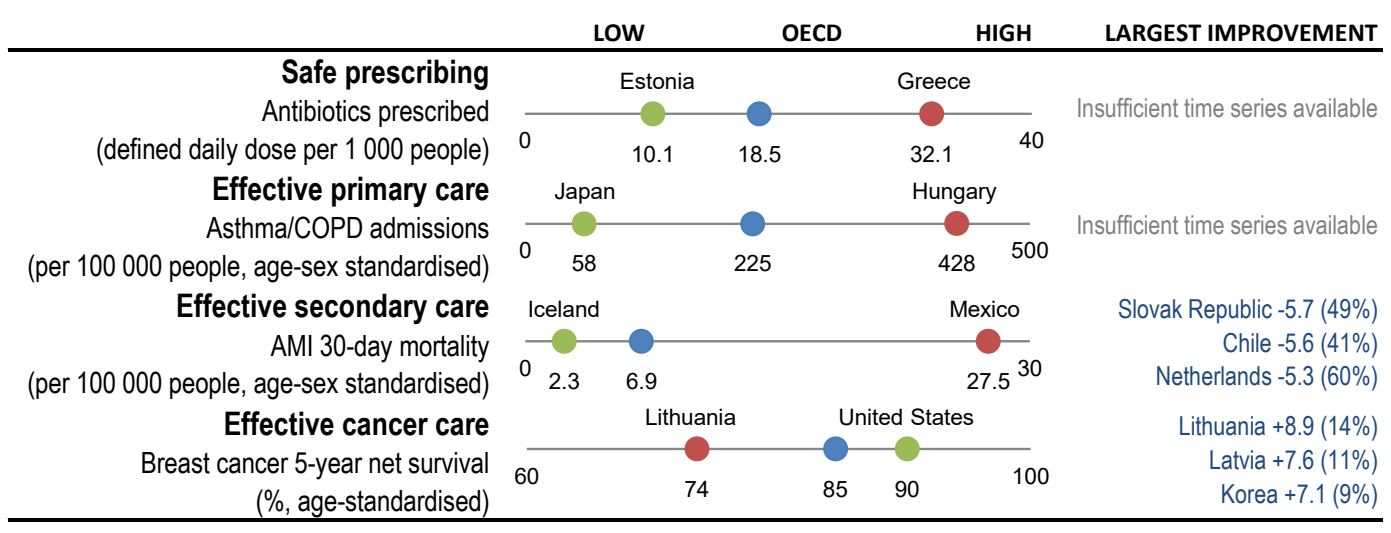

Note: Largest improvement shows countries with largest changes in value over time (\% change in brackets).

Source: OECD Health Statistics 2019.

The overuse, underuse or misuse of antibiotics and other prescription medicines contribute to increased antimicrobial resistance and represent wasteful spending. Total volumes of antibiotics prescribed vary more than three-fold across countries, with Estonia and Sweden reporting the lowest volumes, whereas Italy and Greece report the highest volumes. Across the OECD, the number of antibiotics prescribed has increased slightly over time.

Asthma and COPD are conditions for which effective treatment at the primary care level is well established - and hospital admissions for these conditions may signal quality issues in primary care. Admission rates for asthma vary 12-fold across countries with Mexico, Italy, and Colombia reporting the lowest rates and Latvia, Turkey and Poland reporting rates over twice the OECD average. International variation in admissions for COPD is 15 -fold across OECD countries, with Japan, Italy and Mexico reporting the lowest rates and Hungary, Turkey and Australia the highest rates. Combined, there is a lower 7-fold variation across countries for these two respiratory conditions.

Mortality following acute myocardial infarction (AMI) is a long-established indicator of the quality of acute care. It has been steadily declining since the 1970s in most countries, yet important cross-country differences still exist. Mexico has by far the highest 30-day mortality following AMI (28 deaths per 100 admissions); rates are also relatively high in Latvia, Japan, Korea and Estonia. The lowest rates are found in Iceland, Denmark, Norway, Netherlands, Australia and Sweden (all $4 \%$ or less).

Breast cancer survival is an important measure of clinical effectiveness, with generally high survival across the OECD. Some of the best survival rates are found in Australia, Japan 
Table 1.5. Dashboard on quality of care

\begin{tabular}{|c|c|c|c|c|c|c|c|c|}
\hline \multirow[b]{3}{*}{ OECD } & \multicolumn{2}{|c|}{ Safe prescribing } & \multicolumn{2}{|c|}{ Effective primary care } & \multicolumn{2}{|c|}{ Effective secondary care } & \multicolumn{2}{|c|}{ Effective cancer care } \\
\hline & \multicolumn{2}{|c|}{$\begin{array}{c}\text { Antibiotics prescribed } \\
\text { (defined daily dose per } 1000 \\
\text { people) }\end{array}$} & \multicolumn{2}{|c|}{$\begin{array}{l}\text { Avoidable asthma/COPD } \\
\text { admissions (per } 100000 \\
\text { people, age-sex standardised) }\end{array}$} & \multicolumn{2}{|c|}{$\begin{array}{l}\text { 30-day mortality following } \\
\text { AMI (per } 100000 \text { people, age- } \\
\text { sex standardised) }\end{array}$} & \multicolumn{2}{|c|}{$\begin{array}{l}\text { Breast cancer 5-year ne } \\
\text { survival (\%, age- } \\
\text { standardised) }\end{array}$} \\
\hline & 17.8 & ○ & 225 & ○ & 6.9 & ○ & 84.5 & ○ \\
\hline Australia & 23.5 & ○ & 403 & 凶 & 3.8 & $\nabla$ & 89.5 & $\nabla$ \\
\hline Austria & 12.1 & $\nabla$ & 248 & ○ & 6.2 & ○ & 84.8 & ○ \\
\hline Belgium & 15.9 & ○ & 291 & ○ & 6.8 & ○ & 86.4 & ○ \\
\hline Canada & 14.8 & $\odot$ & 253 & $\odot$ & 4.8 & ○ & 88.6 & ○ \\
\hline Chile & - & & 98 & $\nabla$ & 8.2 & ○ & 75.5 & $凶$ \\
\hline Czech Republic & 19.6 & $\odot$ & 174 & $\odot$ & 6.2 & ○ & 81.4 & ○ \\
\hline Denmark & 13.9 & $\odot$ & 325 & $凶$ & 3.2 & $\nabla$ & 86.1 & $\odot$ \\
\hline Estonia & 10.1 & $\nabla$ & 122 & $\nabla$ & 9.6 & $凶$ & 76.6 & $凶$ \\
\hline Finland & 12.6 & ○ & 182 & $\odot$ & 8.0 & $\odot$ & 88.5 & ○ \\
\hline France & 23.0 & ○ & 150 & ○ & 5.6 & ○ & 86.7 & ○ \\
\hline Germany & 12.3 & $\nabla$ & 289 & $\odot$ & 8.5 & ○ & 86.0 & $\odot$ \\
\hline Greece & 32.1 & $凶$ & - & & - & & - & \\
\hline Hungary & 13.4 & ○ & 428 & 凶 & - & & - & \\
\hline Iceland & 24.6 & $凶$ & 201 & ○ & 2.3 & $\nabla$ & 89.1 & ○ \\
\hline Ireland & 24.6 & $凶$ & 329 & $凶$ & 5.4 & ○ & 82.0 & ○ \\
\hline Israel & 20.5 & ○ & 214 & 0 & 5.5 & ○ & 88.0 & ○ \\
\hline Italy & 28.3 & $凶$ & 64 & $\nabla$ & 5.4 & ○ & 86.0 & ○ \\
\hline Japan & - & & 58 & $\nabla$ & 9.7 & $凶$ & 89.4 & $\nabla$ \\
\hline Korea & 26.5 & $凶$ & 263 & ○ & 9.6 & $凶$ & 86.6 & ○ \\
\hline Latvia & 12.1 & $\nabla$ & 242 & ○ & 13.4 & $凶$ & 76.9 & $凶$ \\
\hline Lithuania & 13.6 & $\odot$ & 263 & ○ & 8.6 & ○ & 73.5 & $凶$ \\
\hline Luxembourg & 25.3 & 凶 & 203 & ○ & 8.5 & ○ & - & \\
\hline Mexico & - & & 85 & $\nabla$ & 27.5 & $凶$ & - & \\
\hline Netherlands & 14.3 & ○ & 236 & ○ & 3.5 & $\nabla$ & 86.6 & $\circ$ \\
\hline New Zealand & 25.8 & $\otimes$ & 363 & 凶 & 4.7 & ○ & 87.6 & ○ \\
\hline Norway & 14.6 & ○ & 244 & ○ & 3.5 & $\nabla$ & 87.2 & ○ \\
\hline Poland & 23.8 & ○ & 236 & ○ & 4.1 & $\nabla$ & 76.5 & $凶$ \\
\hline Portugal & 16.4 & ○ & 90 & $\nabla$ & 7.3 & ○ & 87.6 & ○ \\
\hline Slovak Republic & 23.6 & ○ & 209 & ○ & 5.9 & ○ & 75.5 & $凶$ \\
\hline Slovenia & 19.0 & ○ & 128 & ○ & 4.1 & $\nabla$ & 83.5 & ○ \\
\hline Spain & 12.6 & ○ & 210 & ○ & 6.5 & $\odot$ & 85.3 & $\odot$ \\
\hline Sweden & 10.2 & $\nabla$ & 169 & ○ & 3.9 & $\nabla$ & 88.8 & ○ \\
\hline Switzerland & - & & 138 & ○ & - & & 86.2 & $\odot$ \\
\hline Turkey & 16.6 & ○ & 425 & 凶 & 6.8 & ○ & 82.1 & ○ \\
\hline United Kingdom & 17.5 & $\odot$ & 281 & ○ & 7.0 & ○ & 85.6 & ○ \\
\hline United States & - & & 268 & ○ & 5.0 & 0 & 90.2 & $\nabla$ \\
\hline
\end{tabular}

Note: $\square$ Better than OECD average; $\bigcirc$ Close to OECD average; $₫$ Worse than OECD average. Mexico excluded from standard deviation calculation for AMI mortality.

and the United States, while rates significantly below the OECD average are found in Chile, Estonia, Latvia, Lithuania, Poland and the Slovak Republic. 


\section{Health care resources}

Having sufficient health care resources is critical to a well-functioning health system. More resources, though, do not automatically translate into better health outcomes - the effectiveness of spending is also important. Health spending per capita and as a share of GDP summarise overall resource availability. The number of practising doctors and nurses provide further information on the supply of health workers. Figure 1.6 provides a snapshot on health care resources across the OECD and Table 1.6 provide more detailed country comparisons.

Figure 1.6. Snapshot on health resources across the OECD

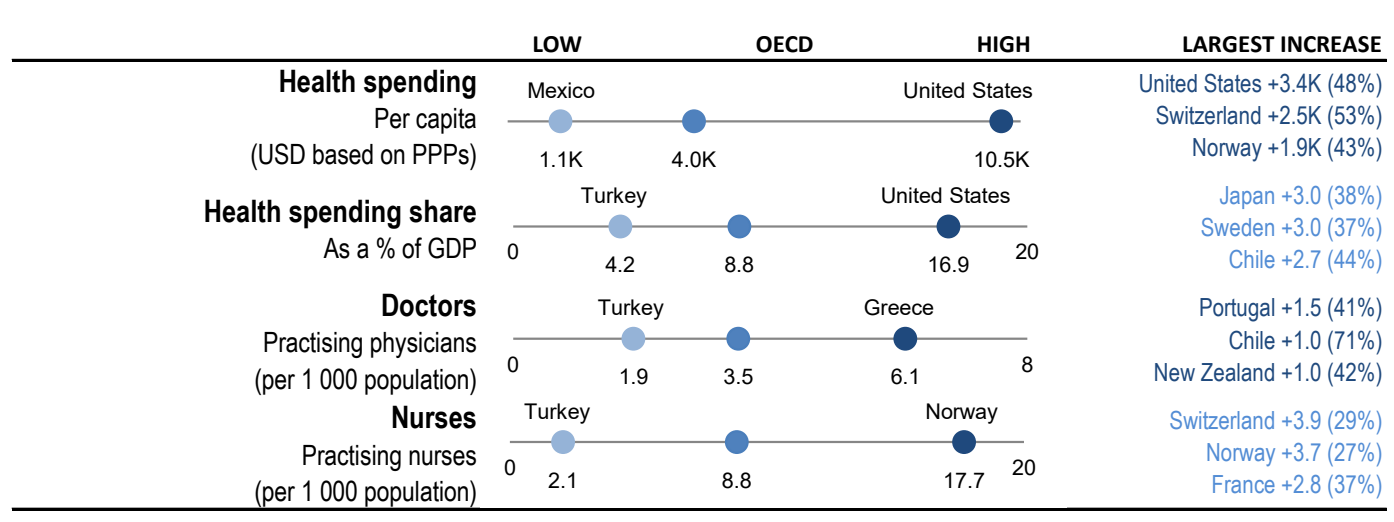

Note: Largest improvement shows countries with largest changes in value over time (\% change in brackets).

Source: OECD Health Statistics 2019.

Overall, countries with higher health spending and higher numbers of health workers and other resources have better health outcomes, quality and access to care. However, the absolute amount of resources invested is not a perfect predictor of better outcomes efficient use of health resources and the wider social determinants of health are also critical. The next section will further investigate the associations between health spending and staffing, access, quality and health outcomes.

The United States spends considerably more than any other country (over USD 10000 per person, adjusted for purchasing power). Health care spending per capita is also high in Switzerland, Norway and Germany. Mexico and Turkey spend the least, at around a quarter of the OECD average. Health spending has grown consistently across most countries over the past decades, other than a temporary slowdown following the 2008 financial crisis. Rising incomes, new technologies and ageing populations are key drivers of health spending growth.

In terms of health spending as a share of GDP, the United States spends by far the most on health care, equivalent to $16.9 \%$ of its GDP - well above Switzerland, the next highest spending country, at $12.2 \%$. Germany, France, Sweden and Japan devote the next highest shares of GDP to health. A large group of OECD countries spanning Europe, but also Australia, New Zealand, Chile and Korea, spend between $8-10 \%$ of GDP. A few OECD countries spend less than $6 \%$ of their GDP on health care, including Mexico, Latvia, Luxembourg, and Turkey at $4.2 \%$.

A large part of health spending is translated into wages for the workforce. The number of doctors and nurses in a health system is therefore an important way of monitoring how resources are being used. The number of doctors ranged from about two per 1000 
Table 1.6. Dashboard on health resources

\begin{tabular}{|c|c|c|c|c|c|c|c|c|}
\hline \multirow[b]{3}{*}{ OECD } & \multicolumn{2}{|c|}{ Health spending } & \multicolumn{2}{|c|}{ Health spending share } & \multicolumn{2}{|c|}{ Doctors } & \multicolumn{2}{|c|}{ Nurses } \\
\hline & \multicolumn{2}{|c|}{$\begin{array}{l}\text { Per capita (USD based on } \\
\text { purchasing power parities) }\end{array}$} & \multicolumn{2}{|c|}{$\begin{array}{l}\text { As a \% of Gross Domestic } \\
\text { Product (GDP) }\end{array}$} & \multicolumn{2}{|c|}{$\begin{array}{l}\text { Practising physicians (per } \\
1000 \text { population) }\end{array}$} & \multicolumn{2}{|c|}{$\begin{array}{l}\text { Practising nurses (per } 1000 \\
\text { population) }\end{array}$} \\
\hline & 3994 & ○) & 8.8 & ○) & 3.5 & ○ & 8.8 & ○ \\
\hline Australia & 5005 & ○) & 9.3 & ( ) & 3.7 & ○ & 11.7 & ( ) \\
\hline Austria & 5395 & ○ & 10.3 & ○ & 5.2 & i & 6.9 & 0 \\
\hline Belgium & 4944 & ○ & 10.4 & ○ & 3.1 & $\odot$ & 11.0 & ○ \\
\hline Canada & 4974 & ○ & 10.7 & ○ & 2.7 & $\circ$ & 10.0 & ○ \\
\hline Chile & 2182 & 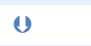 & 8.9 & ○ & 2.5 & $\boldsymbol{U}$ & 2.7 & 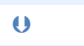 \\
\hline Czech Republic & 3058 & ○ & 7.5 & ○ & 3.7 & ○ & 8.1 & ○ \\
\hline Denmark & 5299 & ○ & 10.5 & ○) & 4.0 & ๑ & 10.0 & ○ \\
\hline Estonia & 2231 & () & 6.4 & c) & 3.5 & ○ & 6.2 & ○ \\
\hline Finland & 4228 & $\odot$ & 9.1 & ○ & 3.2 & ○ & 14.3 & i \\
\hline France & 4965 & ○) & 11.2 & 0 & 3.2 & ○ & 10.5 & ○ \\
\hline Germany & 5986 & 0 & 11.2 & 0 & 4.3 & ○ & 12.9 & 0 \\
\hline Greece & 2238 & (1) & 7.8 & ○ & $6.1^{*}$ & 0 & 3.3 & $\boldsymbol{U}$ \\
\hline Hungary & 2047 & (1) & 6.6 & e) & 3.3 & ○ & 6.5 & ○ \\
\hline Iceland & 4349 & ○ & 8.3 & ○) & 3.9 & ○ & 14.5 & 0 \\
\hline Ireland & 4915 & ○ & 7.1 & ○ & 3.1 & ○ & 12.2 & ○ \\
\hline Israel & 2780 & ○ & 7.5 & 0 & 3.1 & ○ & 5.1 & ○ \\
\hline Italy & 3428 & ○ & 8.8 & ○ & 4.0 & ○ & 5.8 & ○ \\
\hline Japan & 4766 & ○ & 10.9 & 0 & 2.4 & $U$ & 11.3 & ○ \\
\hline Korea & 3192 & ○ & 8.1 & ○ & 2.3 & (1) & 6.9 & ○ \\
\hline Latvia & 1749 & 0 & 5.9 & 0 & 3.2 & ○ & 4.6 & $\omega$ \\
\hline Lithuania & 2416 & 0 & 6.8 & 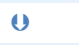 & 4.6 & O & 7.7 & ○ \\
\hline Luxembourg & 5070 & 0 & 5.4 & c) & 3.0 & 0 & 11.7 & 0 \\
\hline Mexico & 1138 & 0 & 5.5 & c) & 2.4 & $\theta$ & 2.9 & $\theta$ \\
\hline Netherlands & 5288 & ○ & 9.9 & ○ & 3.6 & ○ & 10.9 & ○ \\
\hline New Zealand & 3923 & ○ & 9.3 & ○ & 3.3 & ○ & 10.2 & ○ \\
\hline Norway & 6187 & 0 & 10.2 & 0 & 4.7 & i & 17.7 & 0 \\
\hline Poland & 2056 & 0 & 6.3 & $\omega$ & 2.4 & 0 & 5.1 & 0 \\
\hline Portugal & 2861 & 0 & 9.1 & ○ & $5.0^{*}$ & 0 & 6.7 & 0 \\
\hline Slovak Republic & 2290 & 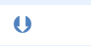 & 6.7 & 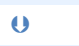 & 3.4 & ○ & 5.7 & ○ \\
\hline Slovenia & 2859 & ○ & 7.9 & ○ & 3.1 & ○ & 9.9 & ○ \\
\hline Spain & 3323 & ○ & 8.9 & ○ & 3.9 & ○ & 5.7 & ○ \\
\hline Sweden & 5447 & ○) & 11.0 & 0 & 4.1 & ○ & 10.9 & ( ) \\
\hline Switzerland & 7317 & 0 & 12.2 & 0 & 4.3 & $\odot$ & 17.2 & i \\
\hline Turkey & 1227 & (1) & 4.2 & c) & 1.9 & U & 2.1 & U) \\
\hline United Kingdom & 4070 & $\odot$ & 9.8 & ○ & 2.8 & $\odot$ & 7.8 & ○ \\
\hline United States & 10586 & 0 & 16.9 & 0 & 2.6 & ○ & 11.7 & ( ) \\
\hline
\end{tabular}

Note: $\uparrow$ Above OECD average; $\bigcirc$ Close to OECD average; $₫$ Below OECD average. United States excluded from standard deviation calculation for both health expenditure indicators. *Includes all doctors licensed to practice, resulting in a large over-estimation.

population in Turkey, Japan, Chile, and Korea, to five or more in Portugal, Austria, and Greece. However, numbers in Portugal and Greece are over-estimated as they include all doctors licensed to practise. There were just under nine nurses per 1000 population in OECD countries in 2017, ranging from about two per 1000 in Turkey to more than 17 per 1000 in Norway and Switzerland. 
To what extent does health spending translate into better access, quality and health outcomes, and more health professionals?

Quadrant charts plot the association between health spending and another variable of interest. They illustrate the extent to which spending more on health translates into stronger performance across four dimensions: health outcomes, quality of care, access, and more health professionals. Note, though, that only a small subset of indicators for these four dimensions are compared against health spending. Quadrant charts also show pure statistical correlations, they do not imply causality.

The midpoint of a quadrant chart represents the OECD average, with dots the relative position of countries across health spending and the given indicator analysed. Each country is also colour-coded, based on a simple risk factors index (RFI) of smoking, alcohol and obesity indicators. Green dots indicate countries with a relatively low RFI (e.g. Israel, Norway), blue dots countries with a RFI close to the OECD average, and red dots countries with a relatively high RFI (e.g. Chile, Hungary). The RFI is an unweighted average of these three risk factors. Hence, the United States, for example, is coloured blue despite having high obesity rates, because of relatively low smoking rates and alcohol consumption. See box on "methodology, interpretation and use" for further methodological details.

\section{Health spending and health outcomes}

These quadrant charts illustrate the extent to which countries that spend more on health have better health outcomes (noting such associations do not guarantee a causal relationship).

There is a clear positive association between health spending per capita and life expectancy (Figure 1.7). Amongst the 36 OECD countries, 17 countries spend more and have higher life expectancy than the OECD average (top right quadrant). A further 10 countries spend less and have lower life expectancy at birth (bottom left quadrant).

Of particular interest are countries that deviate from this basic relationship. Eight countries spend less than average but achieve higher life expectancy overall (top left quadrant). These countries are Italy, Korea, Portugal, Spain, Slovenia, Greece, Israel and New Zealand. The only country in the bottom right quadrant is the United States, with much higher spending than in all other OECD countries, but lower life expectancy than the OECD average.

Most countries with high overall risk factors (red dots) have lower life expectancy than the OECD average. They are also typically below the trend line, which shows the average spending to life expectancy ratio across OECD countries. The converse generally holds for countries with low risk factors (green dots).

For avoidable mortality, there is also a clear association in the expected direction (Figure 1.8). Amongst 36 countries with comparable data, 16 countries spend more and have lower avoidable mortality rates (bottom right quadrant). A further nine countries spend less and have more deaths that could have been avoided (top left quadrant). Seven countries spend less than average but achieve lower avoidable mortality rates - for example, Italy, Israel and Spain (bottom left quadrant). The United States spends more than the OECD average and has worse avoidable mortality rates. Consistent with life expectancy, countries with higher (lower) risk factors (respectively in red and green dots) typically have higher (lower) avoidable mortality rates. 
Figure 1.7. Life expectancy and health expenditure

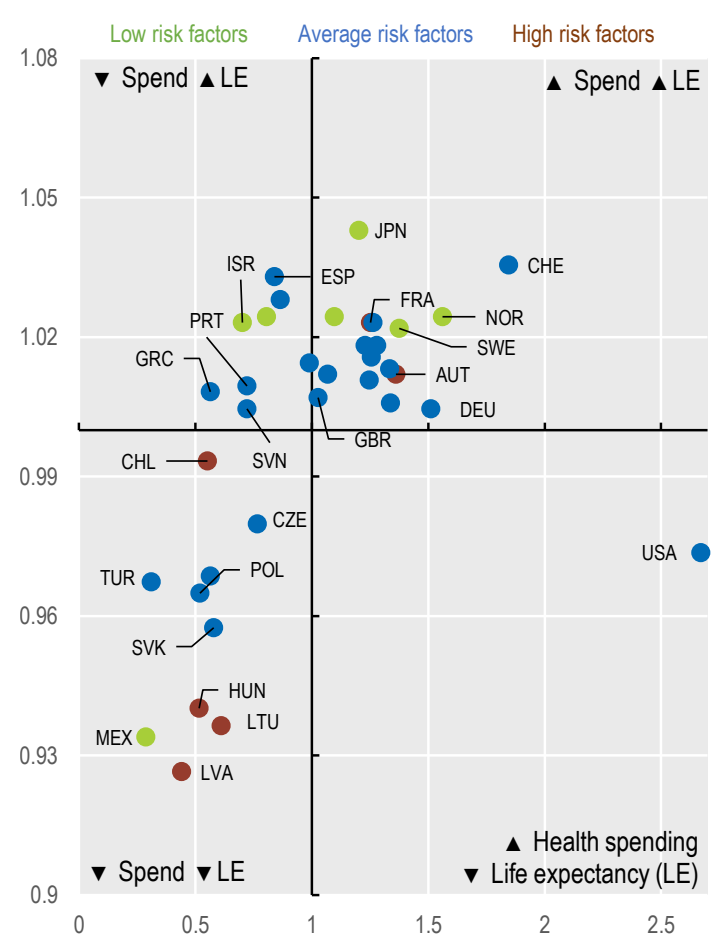

Figure 1.8. Avoidable mortality (preventable and treatable) and health expenditure

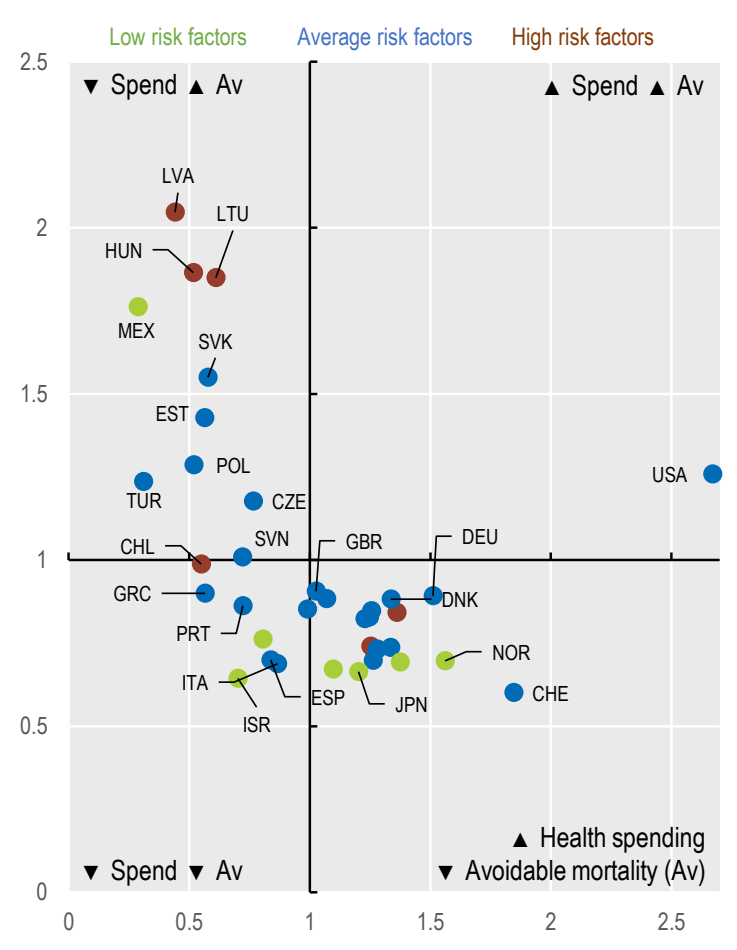

\section{Health spending, access and quality of care}

These quadrant charts illustrate the extent to which countries that spend more on health deliver more accessible and better quality care (noting such associations do not guarantee a causal relationship).

In terms of access, Figure 1.9 shows that universal (or near-universal) coverage of a core set of services can be achieved even with comparatively low health spending levels for example, Turkey and Latvia spend under USD 2000 per person (less than half the OECD average) and still achieve universal population coverage.

Still, six of the seven countries with population coverage rates below $95 \%$ do spend relatively less - Mexico, Poland, Chile, Estonia, the Slovak Republic and Hungary (bottom left quadrant). The one OECD country with high spending levels and lower population coverage is the United States. Replacing health expenditure per person with spending by government or compulsory insurance, or spending as a share of GDP, results in very similar patterns.

In terms of quality of care, Figure 1.10 shows the relationship between health spending and breast cancer five-year net survival (an indicator reflecting the quality of both prevention and curative care). There is a clear positive association: among 32 OECD countries, 16 countries spend more on health and have better net survival (top right quadrant); and nine countries spend less and have lower net survival (bottom left quadrant). Six countries have relatively high breast cancer survival despite spending less than the OECD average (Israel, Italy, Korea, Portugal, New Zealand and Spain). In Ireland, health spending is almost $25 \%$ higher than the OECD average, yet net survival is slightly below the OECD average. 
Figure 1.9. Population coverage for a core set of services and health expenditure

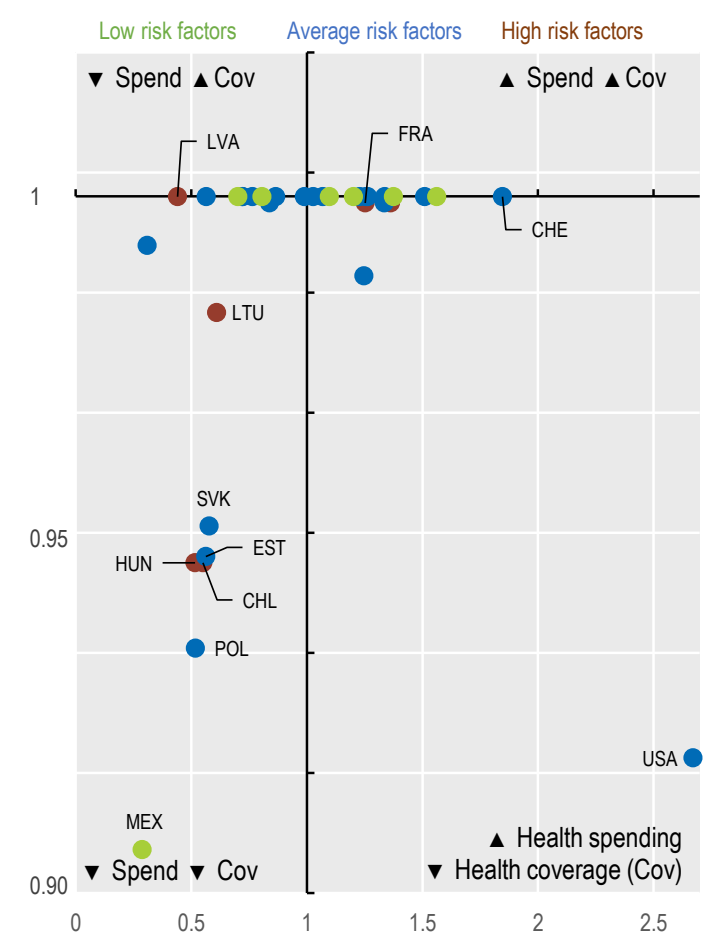

Figure 1.10. Breast cancer survival and health expenditure

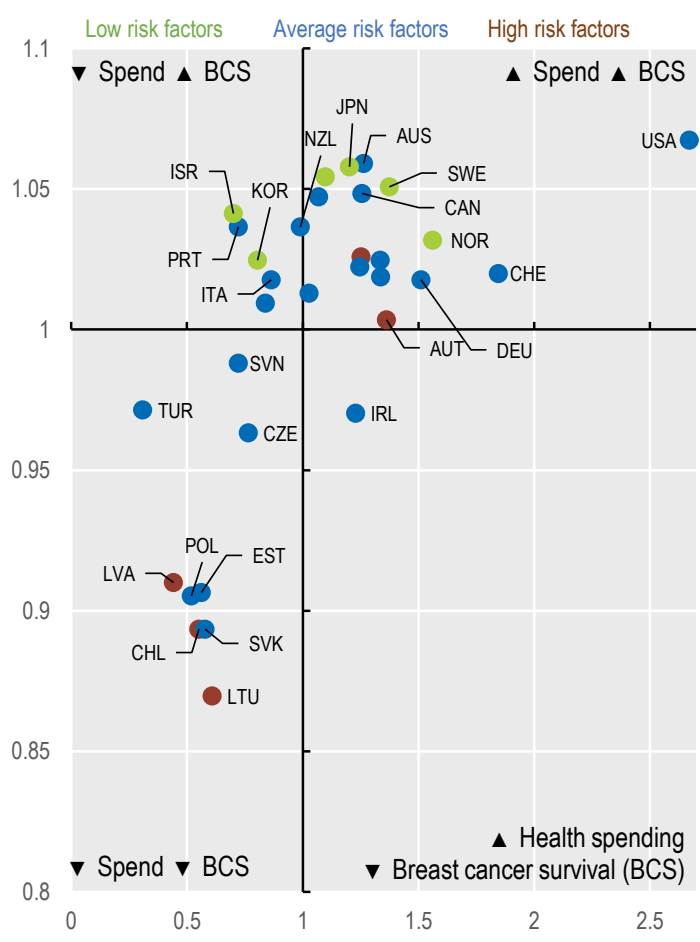

\section{Health spending and number of health professionals}

These quadrant charts illustrate the extent to which countries that spend more on health also have more doctors and nurses (noting such associations do not guarantee a causal relationship).

There is only a weak positive association between spending on health and number of doctors (Figure 1.11). Nine countries spend more than the OECD average yet have fewer doctors (e.g. Canada, Luxembourg, United States); a further six countries spend relatively little yet have more doctors than average (Czech Republic, Greece, Italy, Lithuania, Portugal, Spain). However, numbers in Portugal and Greece are over-estimated as they include all doctors licensed to practise. Such divergences may also reflect differences in remuneration levels, staff composition and the prominence given to nurse practitioners and other health professionals (as compared with doctors).

The positive association between health spending and number of nurses is much more clear-cut (Figure 1.12). Amongst the 36 OECD countries, 16 countries spent more than the OECD average and also had more nurses per 1000 people. Likewise, 16 countries spent relatively little and had fewer nurses. Only two countries spent less than the OECD average and had more nurses (Slovenia and, to a lesser extent, New Zealand). Two other countries had comparatively high spending but fewer nurses (Austria and the United Kingdom). 
Figure 1.11. Number of doctors and health expenditure

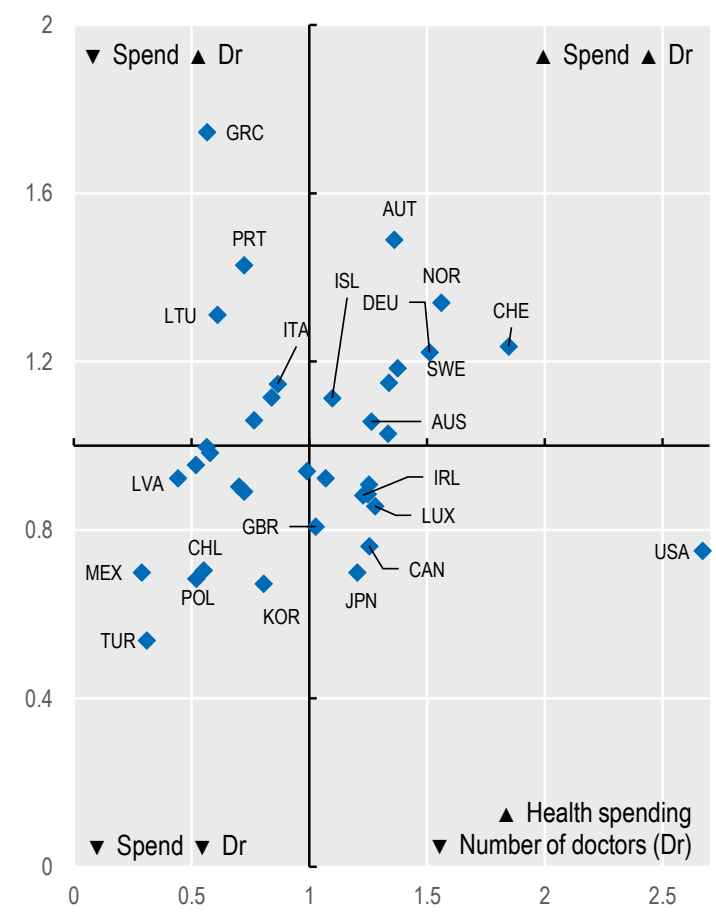

Figure 1.12. Number of nurses and health expenditure

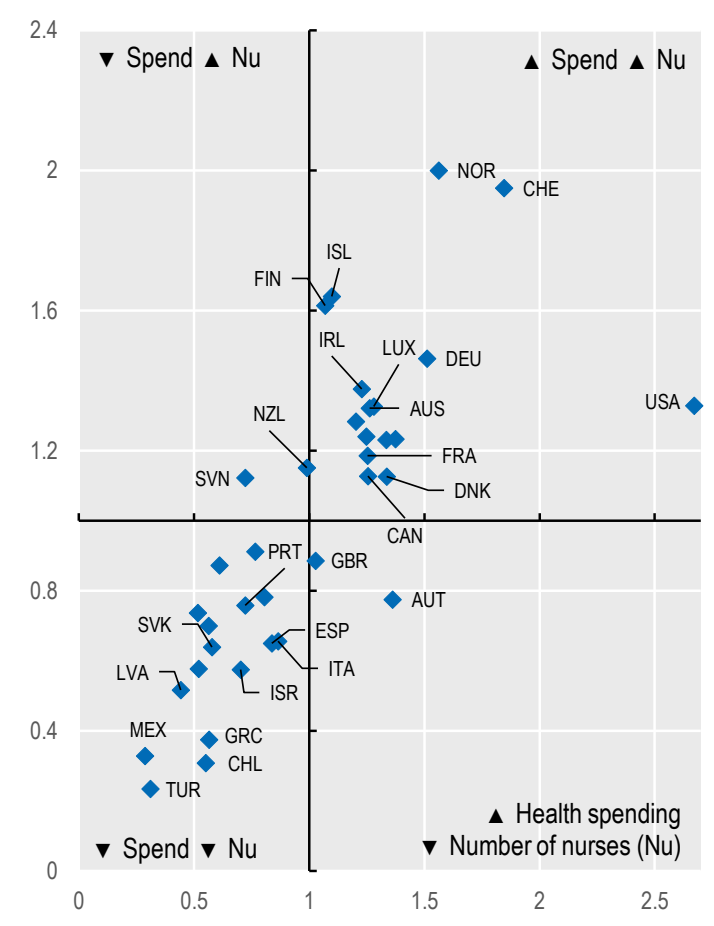





\section{Chapter 2}

\section{Measuring what matters for people- centred health systems}

The key objective of a health system is to improve the health of patients and populations. However, few health systems routinely ask patients about the outcomes and the experience of their care. This chapter presents patient-reported outcomes following hip and knee replacement, and breast cancer surgery, as well as patient-reported experiences of people with mental health problems, from a subset of OECD countries. Patients who underwent joint replacement surgery reported, on average, improved function and quality of life with hip replacements generating slightly higher gains. Women who underwent autologous breast reconstruction surgery reported, on average, better outcomes than women who underwent implant reconstruction. Meanwhile results of a 2016 Commonwealth Fund survey of 11 countries suggest that people with a mental health problem report a worse experience in some aspects of care. Such information is valuable for other health service users, for clinicians, providers, payers and policymakers.

The statistical data for Israel are supplied by and under the responsibility of the relevant Israeli authorities. The use of such data by the OECD is without prejudice to the status of the Golan Heights, East Jerusalem and Israeli settlements in the West Bank under the terms of international law. 


\section{Introduction}

The primary objective of any health system, service or organisation is to maximise the health of the individuals and populations they serve, and to do so in an equitable way within budgetary parameters.

Good health is not just important in its own right. It also promotes personal, social and economic well-being. Healthy people create healthy communities and contribute towards a well-functioning, prosperous and more productive society. For example, good health can enhance a person's lifetime earnings by up to 25\% (OECD, 2017[1]; OECD, 2018[2]).

Yet very few health systems assess their impacts on health and well-being from the perspective of the people they serve. While the concept of health-related quality of life (QoL) has existed for almost three decades, it is not measured or reported systematically. Performance metrics in health tend to focus principally on inputs and outputs. Outcomes such as life expectancy are important, but they are silent on a range of other things valued by patients, including pain, function and QoL as well as the experience of care itself. This means that the picture of health care and health system performance is missing an essential part.

The patient perspective on the outcomes and experience of their care is essential in driving continuous quality improvement of health services. It is also increasingly relevant in overcoming the broader demographic, epidemiological and economic challenges faced by all health systems. The rise of chronic conditions as the main source of disease burden, coupled with better but also more expensive technologies to manage them and prolong life, heightens the need for a more people-centred approach to both policy and practice. But people-centred health systems remain an empty promise without more information on how health care and health policy actually affect the lives of individual patients.

This chapter presents the results of a preliminary data collection on patient-reported outcomes from a sample of OECD countries. The areas covered are joint replacement surgery and breast cancer surgery. The next section discusses the importance of using patient-reported data in mental health. These areas of work are part of a broader OECD initiative - the Patient-Reported Indicator Surveys (PaRIS) - which aims to promote systematic use of these important metrics in health systems (see https://www.oecd.org/ health/paris.htm).

\section{A people-centred health system needs to measure what matters to patients}

People's assessment of their health, and the outcomes of their care, go beyond whether they survive a disease or medical intervention. A range of inter-related physical and mental health domains including pain, mobility, fatigue, anxiety and depression all contribute to person's health-related QoL. Patients also value their care experience, which includes having one's autonomy respected, feeling invited and empowered to participate in decisions about one's care, and if organisational aspects of the care pathway are well coordinated or disjointed and burdensome. 
It makes sense to capture this knowledge in a way that is systematic and useful for decision-making. Yet the health sector has been remiss at measuring the effects of its activities on outcomes and experiences as reported by patients. Forward thinking provider organisations, disease registries and in some health systems have been collecting this information for some conditions or procedures. However, coherent and systematic patient reporting across the entire range of health system activities and interventions is not yet in place.

\section{Outputs provide only a partial picture of health system performance}

Processes and activities, on the other hand, are routinely collected and reported. While these are an important part of the overall picture, in isolation they reveal quite little about performance, quality and value. For example, the average rate of total knee replacement in OECD countries doubled between 2000 and 2016 (Figure 2.1). Rates also vary up to 5-fold between and within countries (OECD, 2014[3]). Are the increased rates and the variation warranted? Do these operations make a difference to people's lives, or are some of them performed unnecessarily? What is the effect of waiting times for knee replacement, and patient's age at surgery? Are some patients better off choosing other treatments for their symptoms?

Figure 2.1. Total knee replacement rates have doubled since $\mathbf{2 0 0 0}$

Total knee replacement rates per 100000 population - adjusted for population ageing - selected countries and OECD average

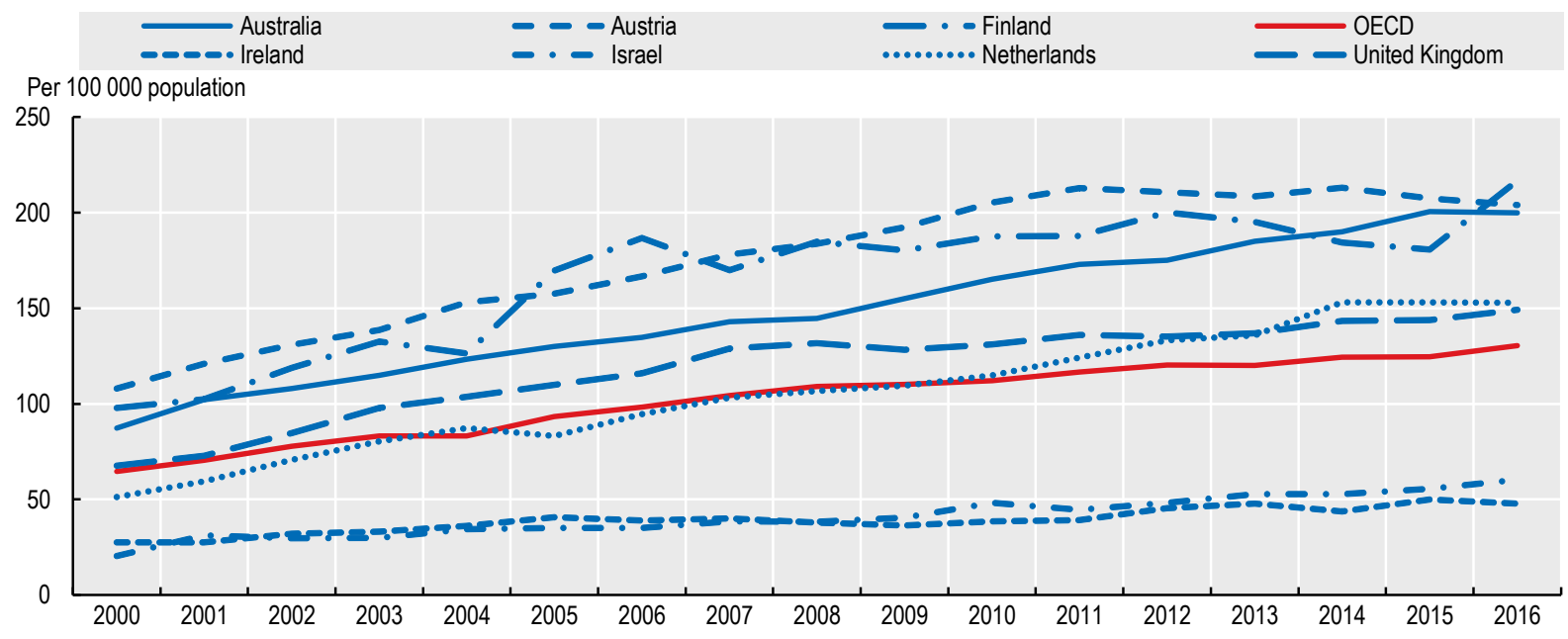

Note: OECD is age-adjusted rate of countries submitting data. Countries chosen based on data availability over this period. Source: OECD Health Statistics 2019.

Such questions cannot be answered without knowing care outcomes. Case fatality or hospital re-admission are useful measures but are becoming rare in routine procedures such as joint replacement. They are also silent on other outcomes valued by these patients such as reduction in pain, and increase of mobility and function.

\section{We know how medicine treats diseases but what about the patient's quality of life?}

Traditional outcome measures like survival or mortality will remain useful but cannot capture more subtle yet important effects. For example, people diagnosed with cancer 
value survival highly, but therapeutic success entails more than just survival (Abahussin et al., 2018[4]). Survival and mortality say little about nausea, pain, sleep quality, body image, sexual function, independence and time spent with loved ones. Also, for some conditions, mortality and survival are now similar between OECD countries (Figure 2.2), with little separating the 'best from the rest'. This hinders continued learning about best therapeutic approaches, techniques and interventions (Donovan et al., 2016[5]; Hamdy et al., 2016[6]).

Figure 2.2. Cancer survival is similar between countries

Breast cancer age-standardised five-year survival of patients diagnosed from 2010-2014

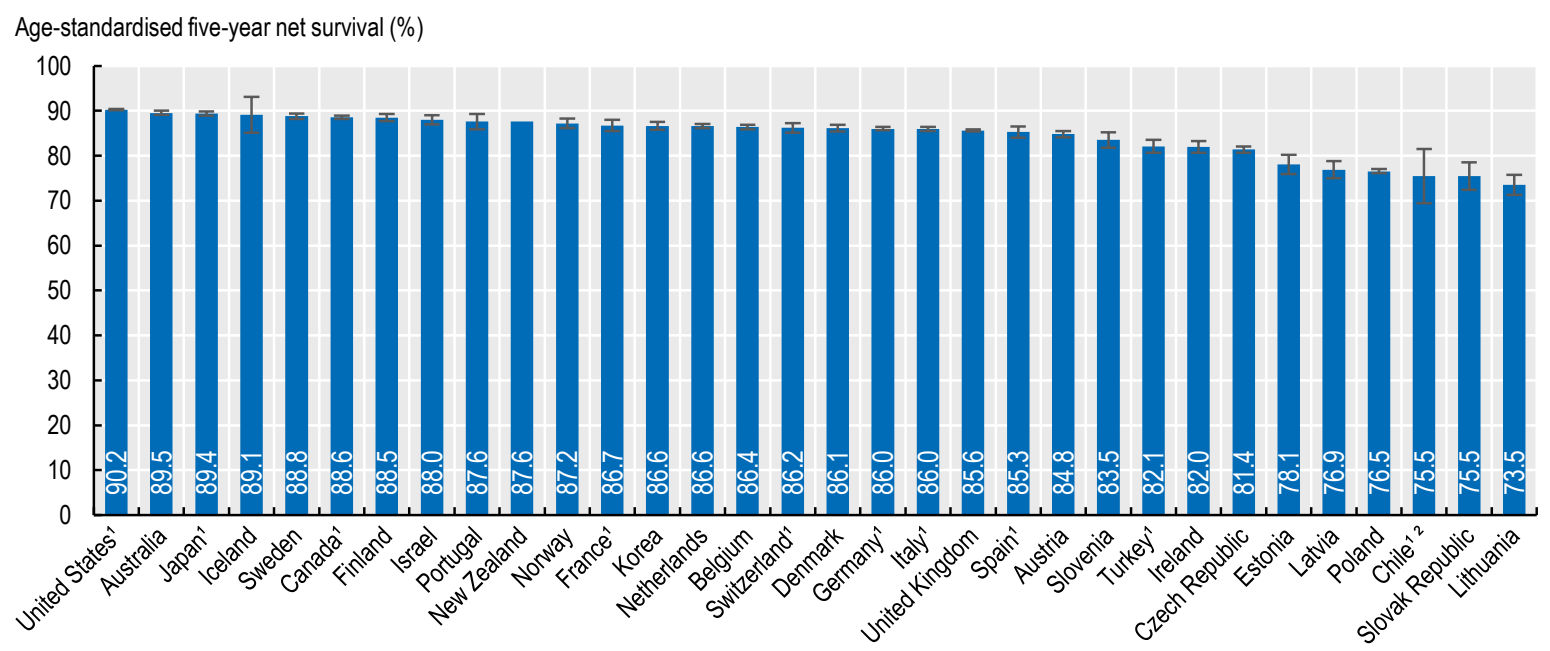

Note: $\mathrm{H}$ lines show $95 \%$ confidence intervals. 1. Coverage less than $100 \%$ of national population. 2. Survival estimates considered less reliable.

Source: CONCORD programme, London School of Hygiene and Tropical Medicine.

StatLink (iाls https://doi.org/10.1787/888934014593

That medicine has become quite successful at treating disease should be celebrated. However, continual improvement must include assessment of the impact treatments have on people's lives. This makes outcomes valued by patients a key indicator of success. Men diagnosed with prostate cancer are now very likely to survive this condition. Beyond survival they also highly value preserving erectile function and avoiding incontinence (Nag et al., 2018[7]) - outcomes of significant interest to patients, providers as well as policymakers.

\section{A good care experience contributes to better outcomes and is also an end in itself}

In addition to outcomes, how people are treated also matters. This includes being treated with respect and compassion and being supported, listened to and involved in decision-making. It also means that care is better integrated across teams who communicate well with each other and with the patient.

A positive care experience is a strong signal of quality care and is instrumental in outcomes achieved, especially for those who manage multiple chronic conditions (Stein et al., 2014[8]; Trzeciak et al., 2016[9]; Luxford, Safran and Delbanco, 2011[10]). In mental health, for example, a positive care experience influences the relationship with the care team, manifesting in better communication, therapeutic continuity, adherence and health outcomes (Wong et al., 2019[11]). But it is also an important end in itself. All patients expect 
and deserve to be treated with respect. In some sectors, such as palliative care, being cared for with compassion and dignity are among the most important components of care.

Yet despite considerable progress in some specific cases, the care experience is not captured systematically. This needs to change, given the growing importance of this dimension of service delivery.

\section{Shared decision making requires patient-reporting}

In the clinical setting, measuring patient-reported metrics helps to focus the health care interaction on the needs of the individual. The discussion moves from 'what's the matter with you?' to 'what matters to you?' - a critical first step in shared decision making, a core principle of people-centred care. Aggregated patient-reported outcomes can inform care decisions and help choose the right therapeutic option where various interventions (including 'watchful waiting') are available (Veroff, Marr and Wennberg, 2013[12]). People see what the most likely outcomes of an intervention may be and can decide accordingly.

Regular reporting by patients throughout their care journey adds structure and rigour to assessment, decision-making and action. Care can be better tailored to individual needs, and enables a rapid and accurate response to clinical deterioration. For example, reporting of symptoms by patients during chemotherapy has been found to significantly prolong survival and reduce hospitalisation (Basch, 2017[13]; Basch et al., 2017[14]).

Knowledge derived from patient-reported data can be used to develop decision aids and update clinical practice guidelines. It also informs providers on how their work affects patient health and well-being. Patient-reported outcome measures, for example, provide a way to measure clinical progress more objectively. They can complement other metrics to provide a fuller assessment of performance of therapies and services. If implemented well, benchmarking and even public reporting can be a powerful driver of quality improvement (Greenhalgh et al., 2017[15]).

Data generated by patients can also contribute towards assessing the performance of medical products, combination therapies, care pathways, health services and the health system as a whole. Combined with other data, these can furnish researchers, regulators, health technology agencies, payers, researchers and policy makers with the knowledge to make more informed decisions to maximise health system performance, and meet the expectations of patients, citizens and communities (Calvert, O'Connor and Basch, 2019[16]).

\section{Patient-reported measures are robust and reliable}

The ability to elicit information from individuals on their health status, quality of life and care experience is now decades old. The available instruments and surveys have undergone rigorous psychometric testing and statistical validation, with results published in the peer-reviewed literature. The field is mature and evidence supports that these instruments reliably measure what is intended (Black, 2013[17]). Box 2.1 outlines the different types of patient-reported outcome and experience measures (PROMs and PREMs) as well as some of the technical aspects of how these are collected, interpreted and used.

In the end, no single data source can provide information for a complete assessment of how a highly complex, adaptive health system performs. Patient-reported data need to be interpreted in the context of other metrics on health system activity and performance. They are not meant to supplant but to complement existing data that are collected in an effort to avoid tunnel vision and generate a more complete picture of performance for all involved: patients, providers, regulators and policy makers. In order for patient-reported 


\section{Box 2.1. Measuring patient-reported outcomes and experiences of care}

Instruments to elicit information from patients on self-reported health status, outcomes and experiences of care typically comprise questionnaires of varying length and format. These are administered in a range of ways (verbally, electronically or on paper). The two main categories of patient-reported outcome measures (PROMs) are condition-specific PROM instruments and health-related Quality of Life (QOL) instruments commonly termed 'generic' PROMs.

\section{Condition-specific PROMs}

These are designed specifically for a condition (e.g. osteoarthritis) or a procedure (e.g. joint replacement). These PROMs are tailored to the symptoms of a specific condition, or those that a specific procedure tries to address. As such their advantage is sensitivity and specificity. Their key limitation is a lack of generalisability - that is, their results cannot be directly compared with results from instruments designed for other conditions or procedures, unless validated mapping algorithms ('crosswalks') exist to convert scores between one and the other.

\section{Health-related QoL instruments ('generic' PROMs)}

'Generic' PROMs instruments attempt to capture a broader range of physical and psychosocial domains that are considered important determinants of health-related QoL. Their advantage is that they can be compared across different conditions, procedures and interventions. For this reason they are often used in cost-utility analysis and health technology assessment (HTA).

\section{Patient-reported experience measures (PREMs)}

The patient experience is also measured using surveys or questionnaires. These can be administered in various ways and a number of approaches and questions have been developed. Questions can be tailored to a certain setting (e.g. primary, hospital, long-term care) or assess a specific aspect of care (e.g. continuity, autonomy, information provision). PREMs are now sophisticated and anchored to objective events, having moved well beyond the more subjective patient 'satisfaction' surveys of the past. They elicit scaled data across a range of dimensions including accessibility, communication, continuity and confidence. These data are now used to inform assessment and international comparisons of health systems (Schneider W, 2017[18]).

\section{Collecting and using patient-reported data}

A range of factors influence the outcomes of care as reported by patients. These factors include behaviour, adherence, age and comorbidities. But more traditional outcome measures such as readmission and mortality are subject to the same confounding variables. All data, whether patient-reported or not, have limitations and should be interpreted with the necessary caution. Like any outcome data that are used for benchmarking, confounders for patient-reported indicators should usually be adjusted in order to enable meaningful comparisons (Nuttall, Parkin and Devlin, 2013[19]).

measures to fulfil their promise in service provision, research and policy, standardisation of methods for data collection, analysis and reporting are essential. This relies heavily on international collaboration (Calvert, O'Connor and Basch, 2019[16]).

\section{Joint replacement rates are rising but are patients reporting improvement?}

Each year, over 2.2 million people undergo an elective hip or knee replacement in OECD countries. Knee replacement rates have doubled since the year 2000 (Figure 2.1), while hip replacements have increased by $30 \%$. Inter- and intra-country variation in rates can be as high as 5-fold (OECD, 2014[3]).

Patients typically undergo these procedures to manage symptoms of osteoarthritis such as pain and loss of mobility and function, which have a considerable impact on health-related QoL. Both procedures are invasive and, like all surgery, involve a degree of risk. They require a long period of rehabilitation. They are also expensive. In Australia, for example, they account for over $2 \%$ of total health expenditure. ${ }^{1}$ 
Given that alternative non-surgical ways of managing hip and knee pain exist (physical therapy, exercise and medication) patients should be able to base their decision to proceed with surgery on the expected outcomes including pain, mobility and capacity to perform daily activities following a period of recovery. Payers should expect that the procedures represent value compared to the alternatives.

The orthopaedic community has been among the most active in encouraging the collection of patient-reported data. Nevertheless, national-level reporting is the exception. Most patient-reported data collections are part of regional and local programmes, or voluntary registries covering a subset of a country's providers and hospitals.

A range of instruments measuring dimensions such as pain, function and QoL are in use around the world. Questionnaires are typically completed by the patient pre-surgery and then at a specified time point after the operation (usually 6 or 12 months). The numerical difference between the pre-operative and post-operative scores is the key value of interest.

The OECD has been working with a range of stakeholders and experts, including patients and clinicians, to collect PROM data internationally. Ten programmes across eight countries contributed to a recent pilot data collection. These included national initiatives (England, Netherlands, Sweden), regional (Canada - Alberta and Manitoba, Switzerland Geneva), sub-national registries (the Australian Clinical Outcomes Registry - ACORN which collects data from providers in two States) and single hospitals (Coxa hospital, Finland; ${ }^{2}$ the Galeazzi Institute Italy). Various PROM instruments are used among the contributing programmes, and the post-operative data were collected at either 6 or 12 months.

Adult patients with a diagnosis of osteoarthritis ${ }^{3}$ who underwent a unilateral, primary elective total replacement procedure were included in the data collection. The three most recent years of data were collected and aggregated to provide one result per participating programme.

\section{On average, hip replacement patients reported improvement}

Hip replacement results derived from the generic instruments (EQ-5D-3L, EQ-5D-5L and SF12) are presented on a common scale - the EQ-5D-3L index with a United Statesderived valuation (Shaw JW, 2005[20]). The maximum score on the EQ-5D scale, is 1.0 (denoting optimal health-related QoL) while a negative score suggests health-related QoL rated as worse than death (Box 2.2).

Figure 2.3 presents the average difference between the pre- and post-operative scores ${ }^{4}$ - i.e. the mean change in QoL - adjusted for patients' age, sex and pre-operative score (Box 2.2). ${ }^{5}$ Results suggest that the average patient in each programme reported improvement in their health-related QoL following a hip replacement. The average mean adjusted change across the programmes was +0.23 , which equates to approximately $21 \%$ improvement on this index at the respective post-operative time points of 6 or 12 months. ${ }^{6,7,8}$

The adjusted changes between pre-and post-operative scores derived from conditionspecific instruments (Oxford Hip Score, HOOS-PS) ${ }^{9}$ are presented in Figure 2.4. These need to be displayed on separate axes because algorithms to convert scores from one to the other are not available at present. The Oxford scale ranges from 0 to 48, the HOOS-PS from 0 to 100. In both cases a higher value represents a more desirable outcome. ${ }^{10}$ Results suggest, on average, improvement of similar magnitude in all programmes. The average adjusted 
Figure 2.3. Hip replacement: adjusted mean change between pre- and post-operative EQ-5D-3L scores (US valuation), 2013-16 (or nearest years)

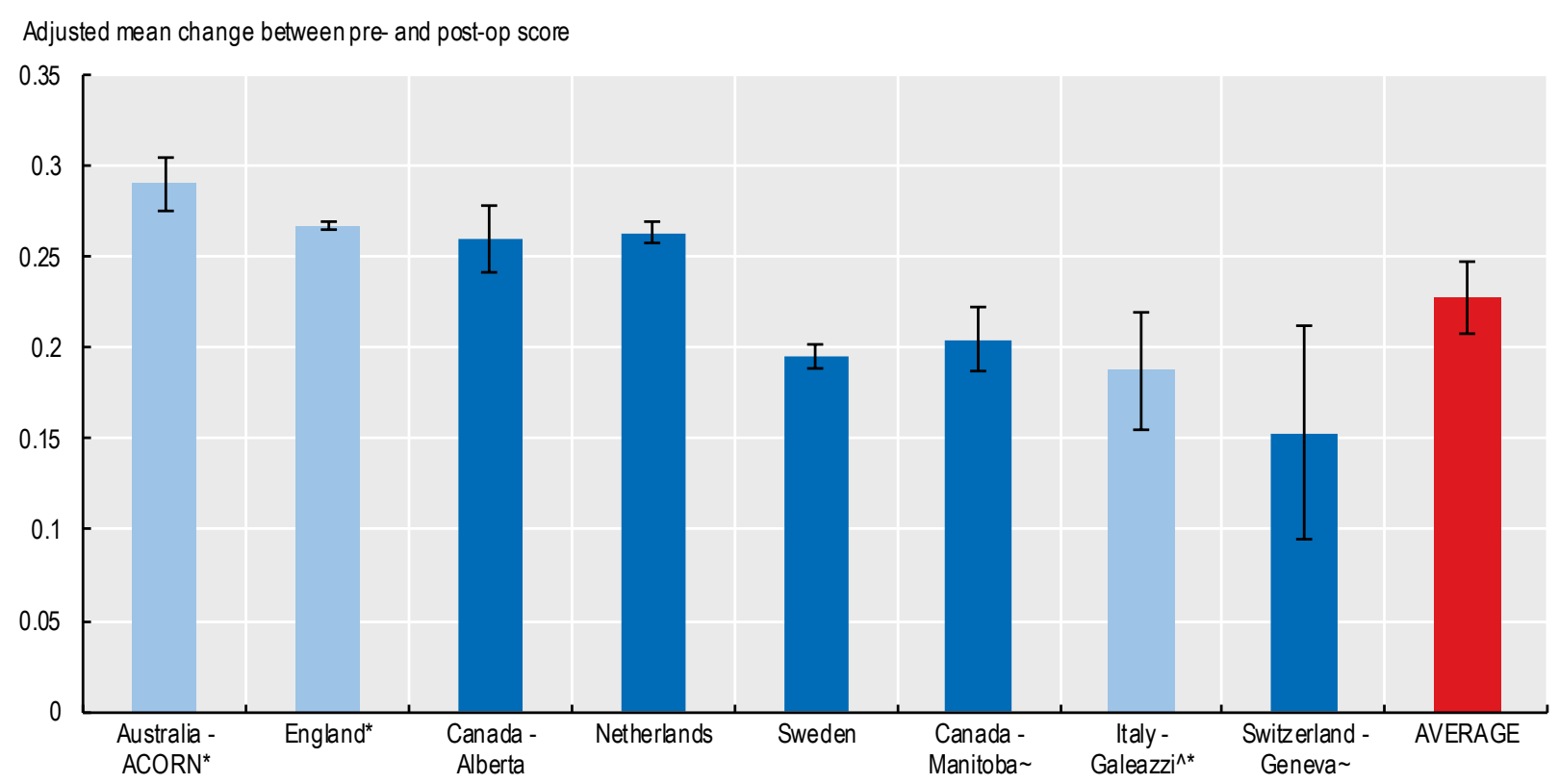

Note: ^ results converted from SF-12v1 instrument; converted from SF-12v2 instrument; ${ }^{6} 6$-month post-op collection - lighter shade blue (all others are 12 months). H lines show $95 \%$ confidence intervals.

Source: PaRIS Hip/Knee Replacement Pilot Data Collection.

mean change (not shown) was +23 on the Oxford scale and +32 on the HOOS-PS scale, which equates to about $48 \%$ and $32 \%$ improvement respectively. ${ }^{11}$ More condition-specific results are provided in Chapter 6 .

\section{Improvements reported following knee replacement were more modest}

The adjusted changes between pre-and post-operative knee replacement scores derived from condition-specific instruments are presented in Figure 2.5 (the scales are the same as for hip replacement). On average, patients in each programme reported improvement of similar magnitude. The average adjusted mean change (not shown) was +17 on the Oxford scale and +22 for KOOS-PS, ${ }^{12}$ or $36 \%$ and $22 \%$ improvement respectively (the corresponding values for hip replacement were $48 \%$ and $32 \%$ ). ${ }^{13}$

Knee replacement results derived from generic instruments are presented using the EQ-5D-3L index with US valuation (see Box 2.2). Data derived from EQ-5D-5L and SF-12 scales were converted using validated algorithms (van Hout et al., 2012[21]; Sullivan and Ghushchyan, 2006[22]; Le, 2013[23]). Figure 2.6 shows the mean changes between pre- and post-op scores, adjusted for age, sex and pre-operative score (Box 2.2). On average, patients in each programme reported improvement ranging from +0.08 to +0.22 . The average adjusted mean change across all programmes was +0.18 (about $16 \%$ improvement). ${ }^{14}$ In comparison, as shown above, the hip replacement equivalent value was $+0.23(21 \%)$, a statistically significant difference at the $95 \%$ level.

The EQ-5D results suggest that - all other things being equal - the average 65-year-old patient undergoing a knee replacement in the contributing programmes gained an additional (incremental) 3.3 quality adjusted life years (QALYs). ${ }^{15}$ In other words, the gain 


\section{Box 2.2. The common EQ-5D index and data standardisation}

Different instruments and measures of health-related QoL are used in the participant programmes and countries. Here, data derived from the EQ-5D-5L, and the SF-12 version 1 and version 2 instruments were converted to the EQ-5D-3L scale using validated mapping algorithms (van Hout et al., 2012[21]; Sullivan and Ghushchyan, 2006[22]; Le, 2013[23]).

\section{The EQ-5D instrument}

The EQ-5D health-related QoL instrument comprises questions covering five dimensions: mobility, selfcare, usual activities, pain/discomfort and anxiety/depression. The patient rates each from 1-3 (on the 3L version) or 1-5 (on the 5L version) with 1 being best and 3 or 5 worst. The output is a five-digit 'health state' e.g. 11111 (perfect health), 33333 or 55555 (worst possible state for 3L and 5L respectively) and a range of permutations in between.

The health states are converted to a single index by referring to so-called valuations specific to a population or country. These valuations have been determined by asking a sample of that population about how they would rate a particular health state against being in perfect health (1.0) and death (0) using a method called time trade-off (TTO). The resulting function is called a valuation or value set. Currently, over a dozen national valuations exist for the 3L version, but fewer have been completed for the newer 5L. The functions can differ considerably between countries (Zhuo et al., 2018[24]). Some remain above zero, others decline into negative values at the worst possible health states. This means respondents rated these states as worse than death, and were willing to trade off time in good health to avoid that health state.

The EQ-5D was designed to generate quality-adjusted life years (QALYs) - a measure that combines morbidity and mortality and is often used assess the effectiveness of medical interventions. For example, living in a health state of 0.8 on the index for 10 years equates to 8 QALYs.

\section{The EQ-5D-3L index (US valuation) as the common scale}

The EQ-5D-3L index was chosen as the common metric because (a) the majority of countries use this instrument; (b) algorithms exist to convert - or map - scores from other generic instruments to the EQ-5D-3L. Score conversions were conducted using patient-level data.

'Native' EQ-5D-3L health state valuations (see above) exist for most participation programmes. A single valuation, rather than a mix of respective native value sets, is preferred because it goes some way to mitigate cultural, demographic, socio-economic and other confounders of self-reported health status (Devlin, 2019[25]). It de facto presents results consistent with their underlying health state, and removes the additional variability created by a country's unique valuation of these states.

The choice of the US valuation was pragmatic. It was the only 'end point' of the available algorithms to generate EQ-5D-3L scores from the other instruments used by the contributing programmes (van Hout et al., 2012[21]; Sullivan and Ghushchyan, 2006[22]; Le, 2013[23]).

\section{Standardising results to enhance comparability}

To enhance comparability and mitigate the effect of demographic and other variables, results shown (derived from both generic and condition-specific tools) were adjusted for age, sex as well as the reported pre-operative PROM score, to a population based on the pooled data of the contributing programmes. Three age categories and two pre-operative score categories were used. Differences between crude and adjusted results were small in the majority of cases. Results were not adjusted for co-morbidity or socio-economic status due to the lack of consistent data.

was the equivalent of 3.3 years with 'full' health-related QoL over the expected remainder of their life compared to the pre-operative status quo (i.e. a 'no intervention alternative). The corresponding figure for hip replacement is higher at 4.3 QALYs (Figure 2.7). ${ }^{16}$ The difference between the procedures is consistent with existing literature (Konopka et al., 2018[26]). It should be noted, however, that knee replacement procedures typically have a longer recovery period than hip replacements. This may explain some of the difference. 
Figure 2.4. Adjusted mean change between pre- and post-operative Oxford Hip Score and HOOS-PS scores, 2013-16 (or nearest years)
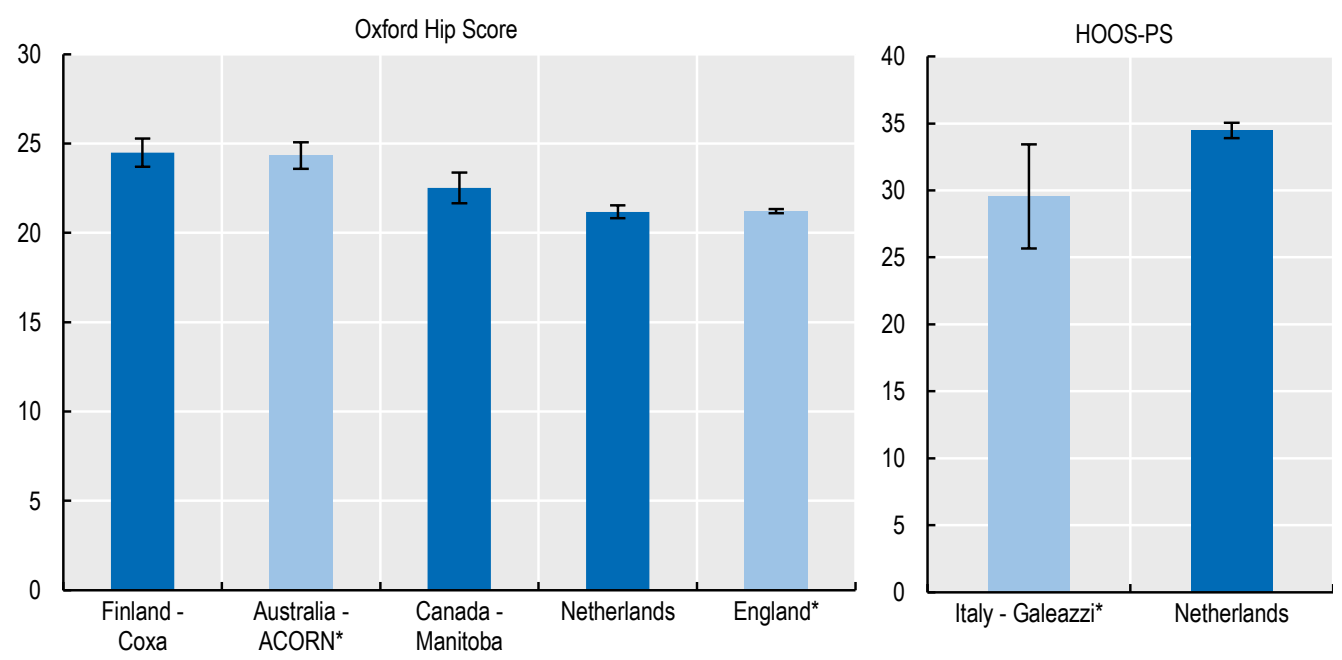

Note: *post-op collection at 6 months - lighter shade blue (all others at 12 months); Scales: Oxford 0-48; HOOS-PS 0-100. $\mathrm{H}$ lines show $95 \%$ confidence intervals.

Source: PaRIS Hip/Knee Replacement Pilot Data Collection.

StatLink क्ञाज https://doi.org/10.1787/888934014631

\section{Figure 2.5. Adjusted mean change between pre- and post-operative Oxford Knee Score and KOOS-PS scores, 2013-16 (or nearest years)}
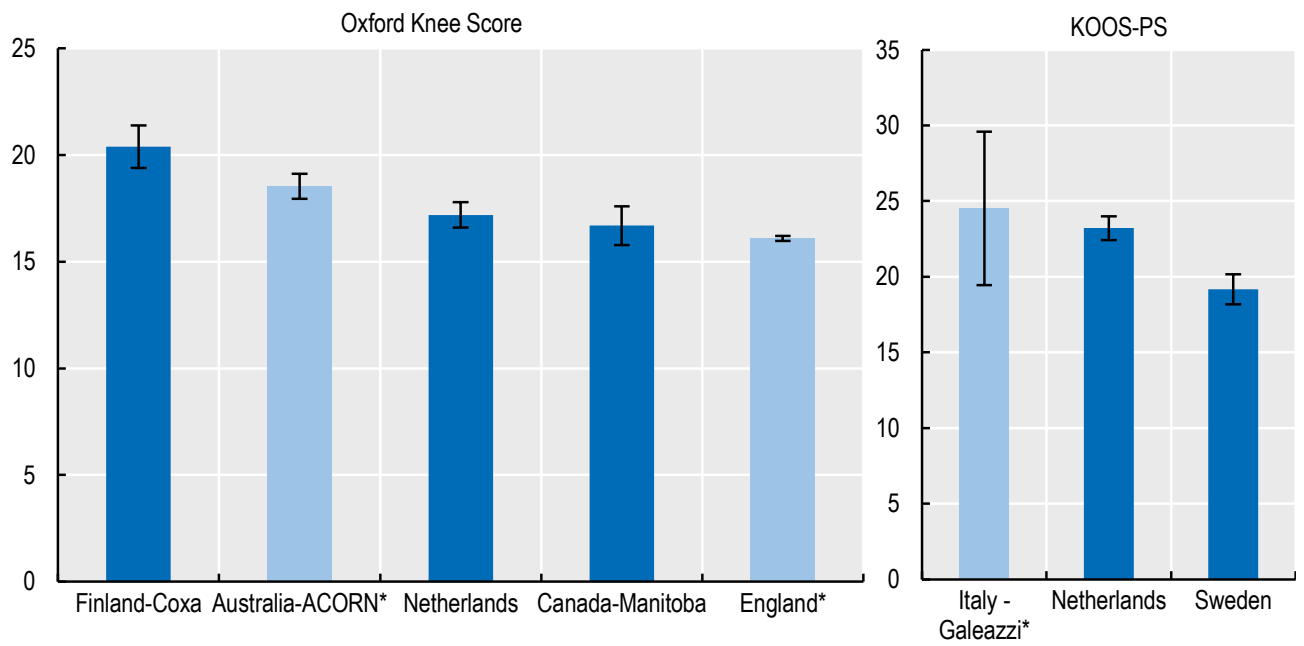

Note: *post-op collection at 6 months - lighter shade BLUE (all others at 12 months); Scales: Oxford 0-48; KOOS-PS 0-100. H lines show $95 \%$ confidence intervals.

Source: PaRIS Hip/Knee Replacement Pilot Data Collection.

StatLink काIst https://doi.org/10.1787/888934014650

\section{Results should be interpreted with caution}

On average, patients undergoing hip or knee replacement procedures in the participating programmes reported an improvement in their symptoms and health-related QoL. This does not mean that all patients improved. In fact, a small but significant proportion reported no change or a worsening in their symptoms and health-related QoL for both procedures across the participating programmes. While this may still be a better 
Figure 2.6. Knee replacement: adjusted mean change between pre- and post-operative EQ-5D-3L scores (US valuation), 2013-16 (or nearest years)

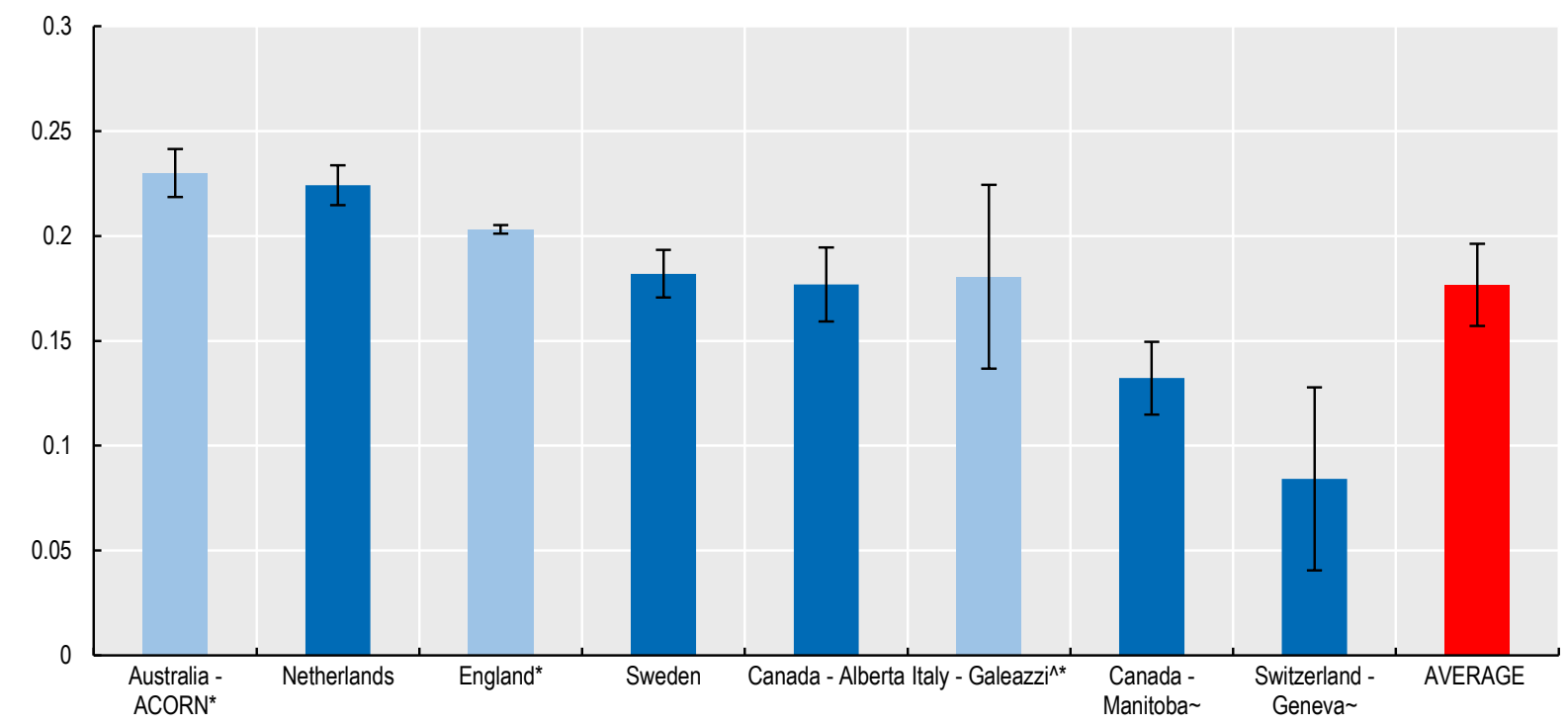

Note: ^ results converted from SF-12v1 instrument; converted from SF-12v2 instrument; *6-month post-op collection - lighter shade green (all others are 12 months). H lines show $95 \%$ confidence intervals. Source: PaRIS Hip/Knee Replacement Pilot Data Collection.

\section{Figure 2.7. Both hip and knee replacements generate additional QALYs for patients}

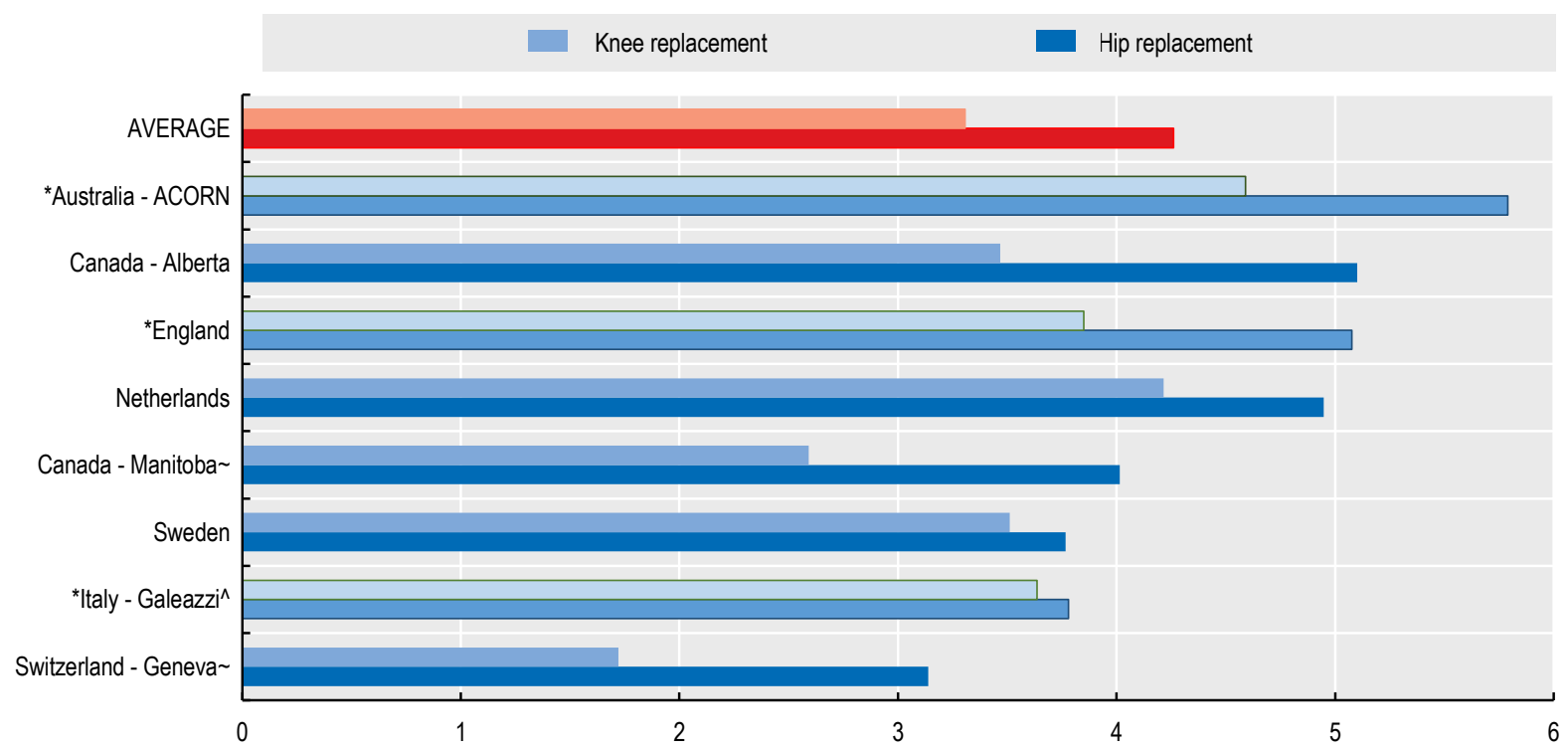

Note: ^ results converted from SF-12v1 instrument; converted from SF-12v2 instrument; *6-month post-op collection (all others are 12 months).

Source: PaRIS Hip/Knee Replacement Pilot Data Collection.

outcome compared to the counterfactual (the status quo), receiving no intervention is unlikely given the availability of other treatment modalities in most health systems.

Results presented here are, in fact, silent on how the outcomes of hip and knee replacement surgery compare with other, more conservative surgical or non-surgical 
treatments including exercise therapy and pharmaceuticals. This would require expanding the study cohort to patients who choose non-surgical therapy for joint pain. The literature suggests that non-surgical interventions indeed improve joint pain and function in people suffering from osteoarthritis, although joint replacement (followed by exercise therapy) results in greater patient-reported improvement (Skou, Bricca and Roos, 2018[27]; Skou et al., 2018[28]). However, joint replacement is associated with a higher number of serious adverse events such as infection than non-surgical treatment (Skou et al., 2015[29]).

Although results were standardised for age, sex and pre-operative score, a number of programme-specific variables limit their comparability. The number of patients differ considerably in each programme. Some of the contributing programmes collect postoperative scores at 6 months, others at 12 months. The latter is considered to be the optimal time for post-operative assessment as full recovery is expected 1 year after surgery. It is unknown how outcomes change beyond the respective time points when data are collected post-operatively. Programmes also deploy different modes of collecting data (paper, electronic, telephone) which is known to influence results. The response rates vary between programmes. Despite adjustment for pre-operative score, differences in wait times between countries may also influence results. Finally, results from three programmes were converted from, EQ-5D-5L and SF-12 to the EQ-5D-3L index (US valuation), which may bias the final results.

In addition, results have not been adjusted for casemix and co-morbidities because consistent data were not available across all programs. A range of cultural, demographic and socio-economic factors influence self-reported health status and will also influence the comparability of results, even when a common index and valuation are used.

\section{Better information on breast cancer care outcomes helps patients facing difficult treatment choices}

Breast cancer is the most prevalent form of cancer in women worldwide, with about 2.1 million newly diagnosed cases in 2018 accounting for almost 1 in 4 cancer cases among women (Bray et al., 2018[30]). While an increase in the incidence of breast cancer over the past decade has been observed, mortality has declined in most OECD countries. Early diagnosis as well as improved treatments have contributed to this result, with most OECD countries now having 5-year net survival rates of $80 \%$ (see earlier discussion and Figure 2.2).

Although surgery is the preferred local treatment for the majority of early breast cancer patients, a range treatment options exist when considering the specific approach for each women's care. For example, primary systemic treatment with chemotherapy or hormonal therapy can improve surgical options by reducing tumour size before surgery. Post-surgical radiation therapy, chemotherapy, and/or hormonal therapy can lower the risk of recurrence of the cancer.

The three main surgical interventions for breast cancer are:

- Breast conserving therapy (BCT) involves a surgical operation to remove the cancer while leaving as much of the breast as possible - commonly an option in early-stage cancer. This is the primary surgical choice for breast cancer, with $60 \%-80 \%$ of newly diagnosed cancers amenable to breast conservation at diagnosis or after primary systemic therapy for women in Western Europe (Cardoso et al., 2019[31]).

- Mastectomy involves complete removal of the breast surgically and is often undertaken when a woman cannot be treated with breast conserving therapy. However, a woman 
may prefer a mastectomy over a breast conserving therapy and women at very high risk of getting a second cancer sometimes have both breasts removed.

- Breast reconstruction may be chosen by women who have had mastectomy of their breast to rebuild the shape and look of the breast. The two main types of breast reconstruction are: 1) implant reconstruction surgery which involves the insertion of a silicone implant after the removal of the woman's breast tissue; and 2) autologous reconstruction surgery, which uses tissue from other parts of the woman's body, such as her belly, back, thighs, or buttocks to rebuild the breast shape. This form of reconstruction is generally considered to look more natural and behave more like natural breast tissue than breast implants.

The choice of treatment and outcomes for women with cancer are influenced by a number of factors including the size and location of the tumour, biology or type and characteristic of the tumour, age, general health status, service availability, related health risks and patient preferences.

As such, the choice of surgical approach can influence a woman's subsequent quality of life. Women diagnosed with breast cancer can therefore face difficult decisions when considering treatment options. While factors such as age, general health status and the size and location of primary tumour are important to clinical decision making, the preferences of the patient are also central to the choice of treatment strategy (Cardoso et al., 2019[31]).

Beyond the overarching objective to stay alive, QoL is also a key consideration. In weighing treatment options, information about the outcomes of other women who have been in similar circumstances can potentially be of great help in the decision making process and ongoing reflection of progress during and after treatment and into survivorship.

\section{The collection and use of PROMs in breast cancer care is growing}

Motivated providers and patients across OECD countries are increasingly measuring patient-reported care outcomes to help inform difficult clinical decisions. The utility of such measurement is increasingly appreciated. For example, in the Netherlands breast cancer has been identified as one of the possible priority areas as part of a current national policy effort to measure patient-reported outcomes systematically and implement 'valuebased' care (van Egdom et al., 2019[32]). Nevertheless, a variety of different PROM instruments are used, making comparability of outcomes more difficult. In addition, the scale of uptake is still largely localised and isolated to specific initiatives and clinical champions at specific sites.

In an effort to address this emerging priority, the OECD worked with a group of experts (including patients, clinicians, policymakers and industry representatives) and collaborating organisations to understand the current state of the art in breast cancer PROMS and to explore opportunities for international data collections and comparisons.

These efforts have culminated in a preliminary international data collection involving 11 clinical sites from 8 countries (Flinders Medical Centre, Adelaide, Australia; Angers Cancer Centres, Nantes, France; Charité - Universitätsmedizin Berlin, Germany; Erasmus Medical Center, Rotterdam, Netherlands; Capio St Göran Breast Unit, Södersjukhuset Bröstcentrum and Karolinska Univ.sjukhuset Bröst Endokrin och Sarkom, Stockholm, Sweden; Universitätspital Basel, Basel, Switzerland; Manchester University Hospitals NHS Foundation Trust, Manchester, UK; Memorial Sloan Kettering Cancer Center, New York, US and Brigham and Women's Hospital, Boston, US). 
The postoperative breast satisfaction scale of the breast conserving therapy and breast reconstruction modules of the Breast $Q$ tool was used. This is an internationally validated instrument used to measure breast surgery outcomes reported by patients (Pusic et al., 2009[33]) (Box 2.3).

The data collection involved women aged 15 years and older who received unilateral breast conserving therapy or a breast reconstruction following a mastectomy during the primary treatment of breast cancer. Women undergoing bilateral breast surgery were excluded, given the possible differential impact this surgery may have on breast satisfaction.

\section{Box 2.3. Breast Q Postoperative Breast Satisfaction Scales}

The Breast Q suite of tools is one of the more widely used amidst the range of instruments currently in use internationally to measure patient-reported outcomes from breast cancer surgery (Tevis et al., 2018[34]).

The breast satisfaction scales of the Breast $Q$ tools measure body image in terms of a woman's satisfaction with her breasts and asks questions regarding how comfortably bras fit and how satisfied a woman is with her breast area both clothed and unclothed. Postoperative items ask about breast appearance (e.g., size, symmetry, softness), clothing issues (e.g., how bras fit; being able to wear fitted clothes) and location and appearance of scars. There are separate modules for lumpectomies, mastectomies and reconstructions, with each module consisting of multiple separate scales covering such issues as psychosocial wellbeing, sexual wellbeing, physical wellbeing, satisfaction with breasts and satisfaction with care. There are also implant-specific items, including the amount of rippling that can be seen or felt.

The scores from each scale of the breast conserving therapy and reconstruction scales, along with the other Breast Q scales can be transformed to an Equivalent Rasch Transformed Score of 1-100 to allow direct comparison between scales.

See http://qportfolio.org/breast-q/breast-cancer/ for more details.

\section{Results suggest higher breast satisfaction outcomes after breast conserving therapy in some, but not all sites}

The crude (unadjusted) outcomes scores at 6-12 months following breast conserving therapy, breast reconstruction, and the aggregate of the two are provided in Figure 2.8. Results are from relatively small samples and are not intended to be representative of the outcomes of breast cancer patients across each country. However, they demonstrate the capacity for metrics of this kind to be reported internationally.

Crude data from sites that reported scores for breast conserving therapy and reconstruction suggest that women in most sites may have higher breast satisfaction outcomes after breast conserving therapy, aligning with conventional wisdom in this area (for example (Flanagan et al., 2019[35])). However, in some sites women may have higher satisfaction scores for reconstruction. Further work and more extensive data collection are needed to validate this observation and consider the generalisability of the data outcomes, but these early observations may provide some basis for further sharing and learning of outcomes across sites. For example, follow up beyond 6-12 months may be warranted, given the timing of outcomes for women can vary as a result of differences in the duration and impact of the usual treatment pathways for BCT and breast reconstruction.

A number of personal factors can influence a woman's postoperative satisfaction with the outcomes of her breast cancer surgery, including age, smoking, obesity, tumour burden, education level, cultural background and overall satisfaction with breasts and physical health before surgery. For example, smoking and obesity can impair tissue healing and 


\section{Figure 2.8. Crude PROM scores for breast cancer point to variations in surgical outcomes}

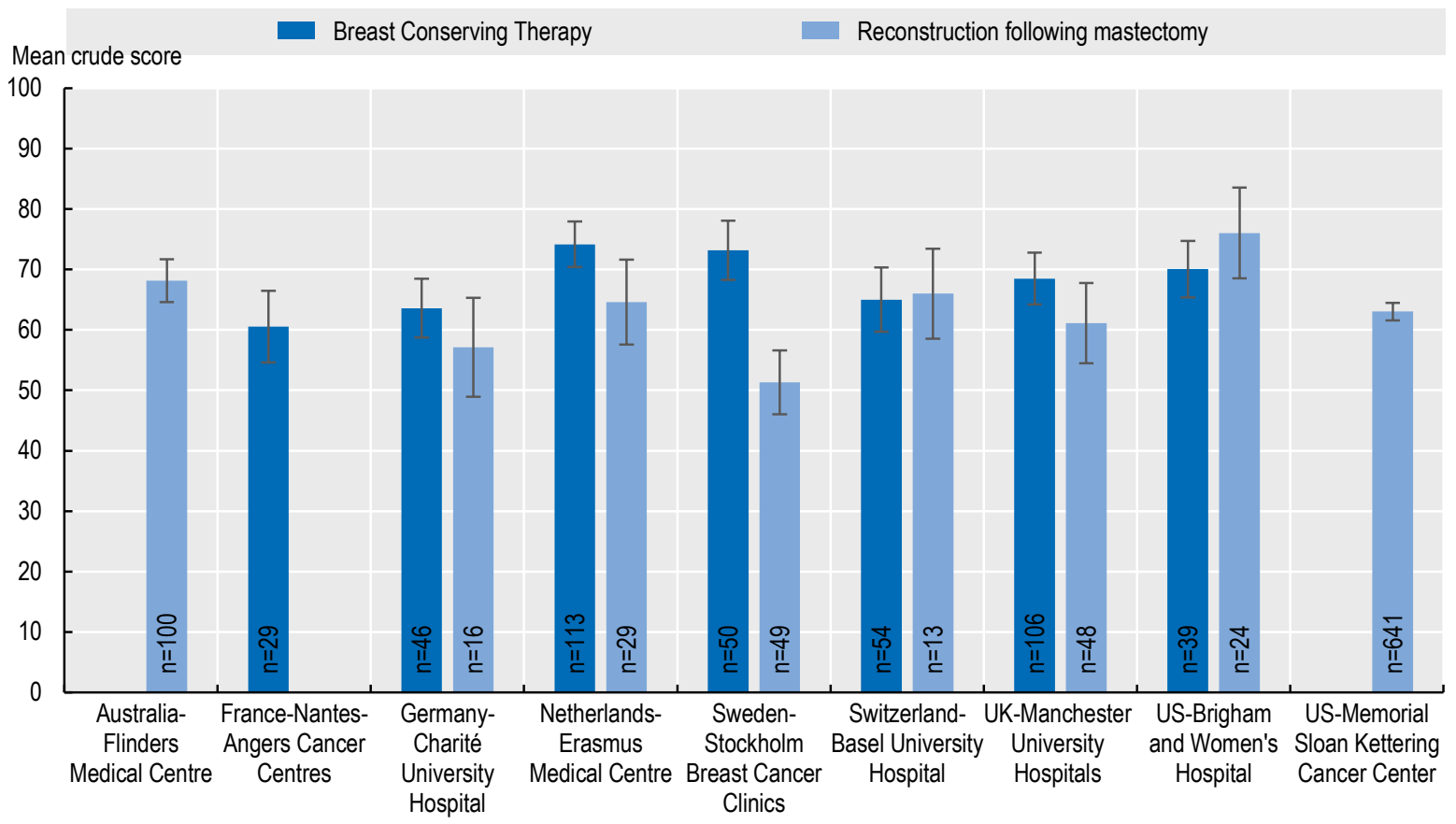

Note: Measurement extended beyond 12 months after surgery for sites in both Sweden and Switzerland. The data labels at the base of the histogram refer to the sample size at each site. $\mathrm{H}$ lines show $95 \%$ confidence intervals.

Source: PaRIS Breast Cancer PROMS Pilot Data Collection, 2019.

StatLink 에s https://doi.org/10.1787/888934014707

have a negative impact on implant reconstruction results, including aesthetic outcomes (Kern et al., 2015[36]). These factors are largely outside of the health service's direct influence and their impact should ideally be taken into account when comparing the quality of care across sites. Data were collected from participating sites on key patient variables, including age, smoking and obesity but limitations on sample size and incomplete capacity for reporting by all sites prevented risk-adjusting results for the time being.

\section{Women report slightly more satisfaction following autologous than implant breast reconstruction}

Consolidated crude scores from the participating sites indicate that women are $6 \%$ more satisfied with their breasts after autologous reconstruction surgery than women after a breast implant (Figure 2.9). This result aligns with existing evidence (Matros et al., 2015[37]) and can be an important consideration where choice of surgical intervention is possible.

It follows that the variation in breast satisfaction scores presented in Figure 2.8 may be influenced, among other factors, by the proportion of women undergoing autologous reconstruction surgery. Table 2.1 presents the sample size of women and the proportion undergoing autologous reconstruction reported by each site. The proportion ranges from $100 \%$ of women receiving autologous reconstructions (Dutch and Swiss sites) to $0 \%$ in the Swedish site, where all women would have received implant reconstructions. However, it is likely some sites have not included all women undergoing reconstruction. For example, data may have been only provided by the plastics surgery unit in some sites and so not include the implant reconstructions performed by the breast surgeons and vice versa. 


\section{Figure 2.9. Crude patient-reported outcomes for implants and autologous reconstructions}

Self-reported satisfaction with breasts by type of reconstruction surgery, 2017-18 (or nearest years)

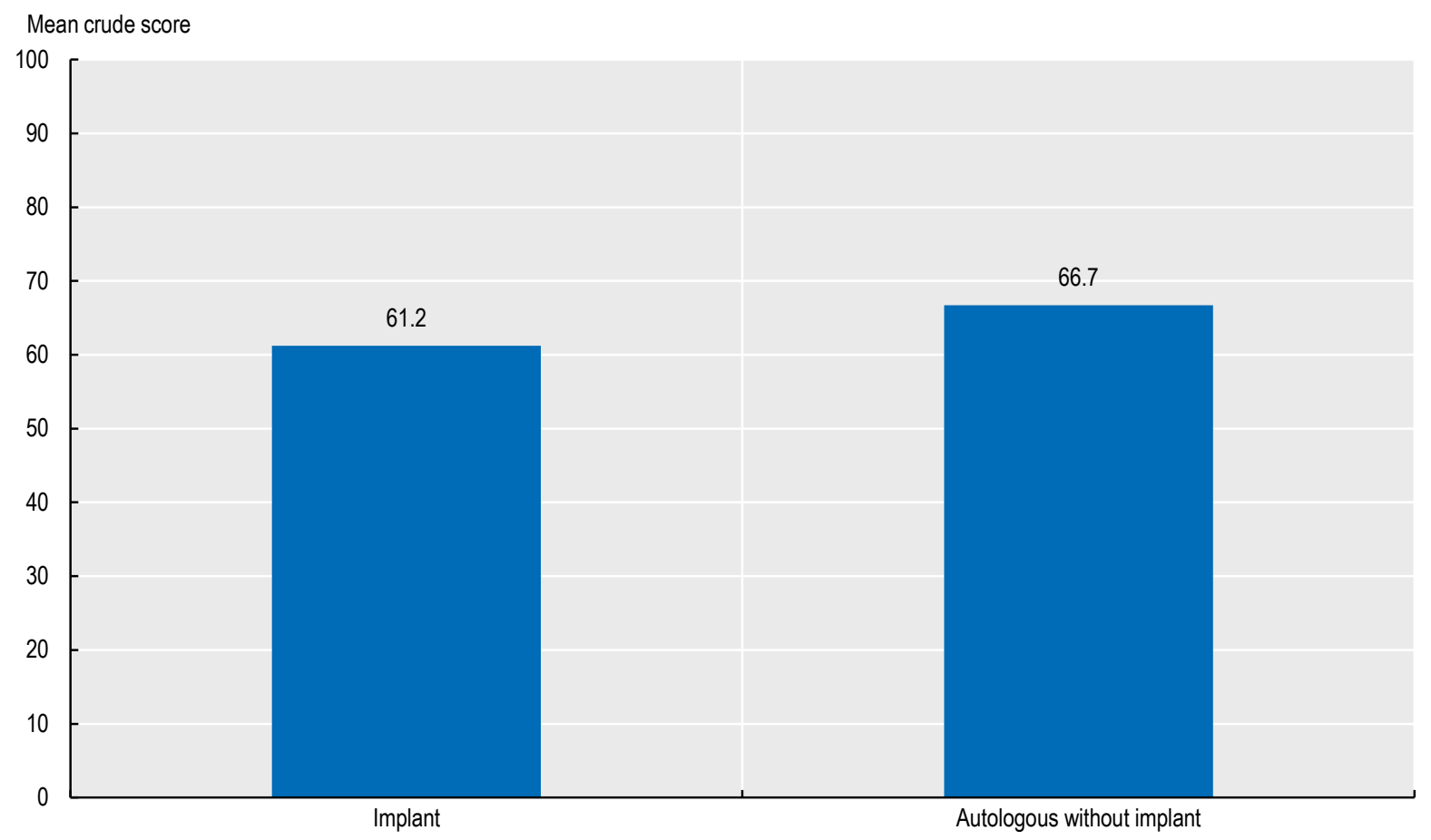

Note: Derived from consolidated data from all 11 participating sites. Source: PaRIS Breast Cancer PROMS Pilot Data Collection, 2019.

While no clear relationship between the proportion of women undergoing autologous reconstruction and the overall crude outcomes scores (Figure 2.8) is apparent, further consideration of the factors contributing to the observed wide variation across sites may be warranted, particularly given the conventional wisdom regarding care outcomes. For example, the role of each site within the broader service arrangements for women with breast cancer or the representativeness of the sites' data.

Table 2.1. Total breast reconstructions and the proportion of autologous reconstructions by site

\begin{tabular}{l|c|c}
\hline & Total breast reconstructions & Autologous reconstructions without implant \\
\hline Australia-Flinders Medical Centre & No. of Women & $\%$ of total reconstructions \\
Germany-Charité University Hospital & 100 & $57 \%(57)$ \\
Netherlands-Erasmus Medical Centre & 16 & $19 \%(3)$ \\
Sweden-Stockholm Breast Cancer Clinics & 29 & $100 \%(29)$ \\
Switzerland-Basel University Hospital & 49 & $0 \%(0)$ \\
UK-Manchester University Hospitals & 13 & $100 \%(13)$ \\
US-Brigham and Women's Hospital & 48 & $25 \%(12)$ \\
US-Memorial Sloan Kettering CancerCenter & 641 & $38 \%(9)$ \\
\hline
\end{tabular}

Source: PaRIS Breast Cancer PROMS Pilot Data Collection, 2019. 


\section{Recent use of PROMs indicates that autologous reconstruction may be cost- effective}

Significant variation in treatment pathways and practices persists for women with breast cancer, including the use of different surgical approaches, even in the face of established clinical practice guidelines (Cardoso et al., 2019[31]; OECD, 2013[38]). Figure 2.10 presents the rates, setting and mix of breast conserving therapy and mastectomy surgery across OECD countries. This Figure suggests that different treatment patterns are evident, even across countries showing a very similar level of cancer incidence. Data need of course to be interpreted cautiously as patients' cancer stages, comorbidity and pre-operative patient performance status may also vary.

\section{Figure 2.10. Breast cancer surgery type and setting (2017) and incidence (2012) per 100000 women}

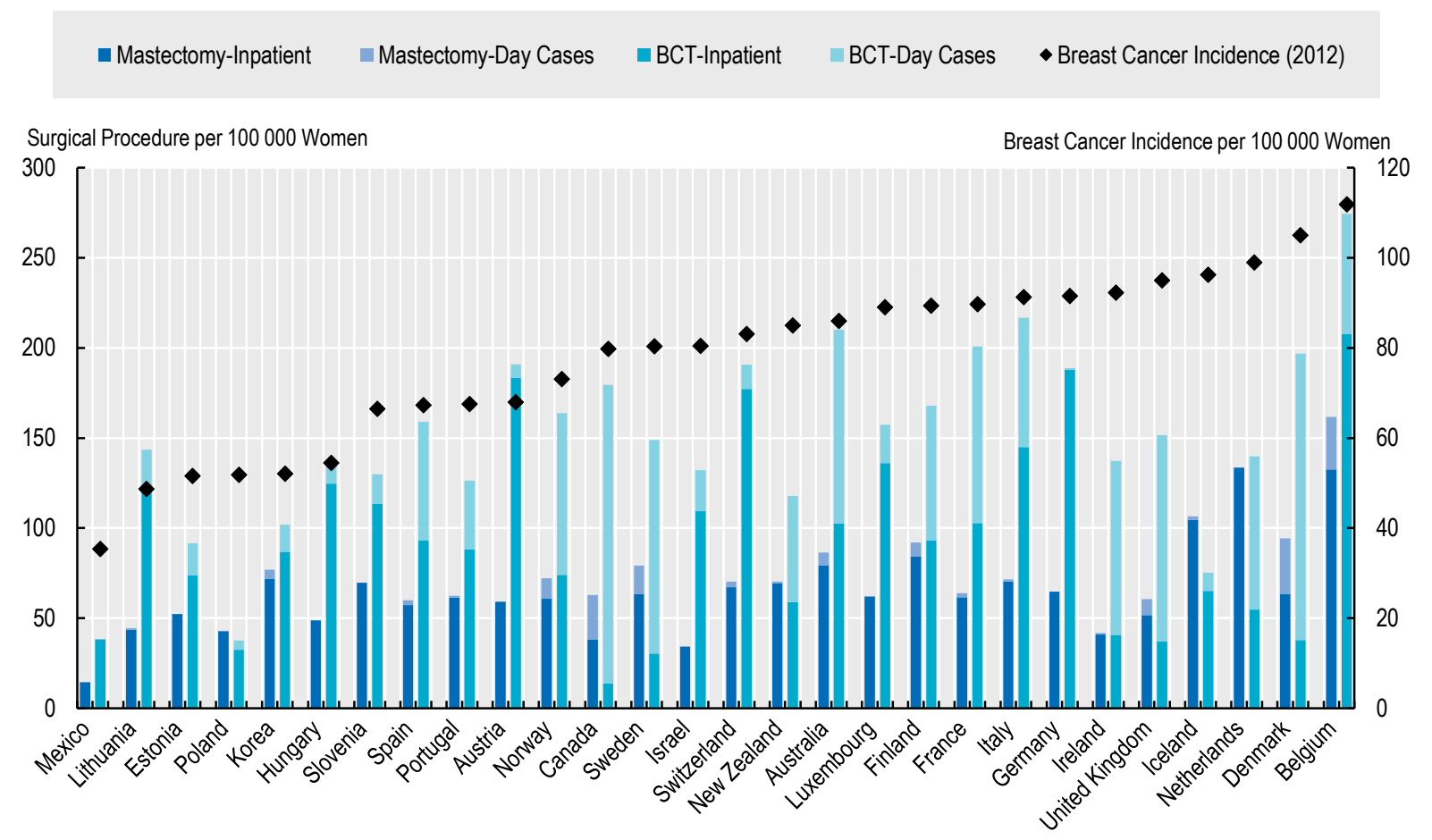

Source: OECD Health Statistics 2019.

Variation in the treatment patterns can also be affected by a number of other factors. For example, regional differences in breast reconstruction surgery in Sweden have recently been attributed to variation in patient information, availability of plastic surgery services and the involvement of women in decision-making (Frisell, Lagergren and de Boniface, 2016[39]).

Treatment choices made by patients in consultation with their clinical teams have not only consequences for survival and QoL, but also financial implications. For example, after a mastectomy a woman faces the choice of whether to have breast reconstruction (as an immediate or delayed procedure) or not and if she proceeds with breast reconstructive surgery, what type of reconstruction she should have. While the outcomes in terms of survival of having a breast reconstruction or not after a mastectomy are generally 
comparable (Platt et al., 2015[40]), the choice of reconstruction can lead to different outcomes that are important to women, such as quality of life or satisfaction with breasts as well as different costs faced by the women and the health system.

While autologous reconstructions appear to result in better patient outcomes than implant surgery, they tend to be more complex and expensive, raises questions about value for money (Scurci et al., 2017[41]). A recent study in the United States compared the Breast $Q$ scores of patients who had implant and those who underwent autologous reconstructions and calculated the average additional cost for obtaining 1 year of perfect breast-related health for a unilateral autologous reconstruction at just under USD 12000 in 2010, compared with implant reconstruction, with lower additional costs for younger patients and earlier stage breast cancer (Matros et al., 2015[37]).

Although society's value for a year of perfect breast-related quality of life is unknown, a threshold of USD 50000 to USD 100000 for a year in perfect overall health is commonly been used to classify interventions as cost-effective and considered as acceptable for adoption of new technologies or techniques in OECD countries (Cameron, Ubels and Norström, 2018[42]). On this basis, further consideration of the relative cost-effectiveness of autologous reconstructions may be warranted, along with broader economic evaluation of both BCT and breast reconstruction surgery.

Routine collection of data on outcomes that matter for breast cancer patients is useful not only for direct patient care but also for system improvement through better understanding of the impact of different care pathways. They complement traditional measures such as survival, mortality, complications and readmissions. Bringing measures of what matters to patients into the equation creates potential to evaluate alternative modes of treatment both in terms of outcome and value for patients, policy makers and third party payers (Cardoso et al., 2019[31]).

\section{Existing mental health measures say little about experiences and outcomes of care}

Mental health is a vital component of individual well-being as well as social and economic participation. However, many OECD countries consider that their mental health care is inadequate. It is estimated that about one in five people experience a mental health problem in any given year, while every second individual will experience a mental health problem in their lifetime (Institute for Health Metrics and Evaluation, 2019[43]). The most common mental health problems are anxiety disorder (5.1\% of the population), followed by depressive disorders (4.5\%), and drug and alcohol use disorders (2.9\%) (ibid.).

The economic and social costs of mental ill-health are significant. Direct spending on mental health services was estimated to account for around $13 \%$ of total health spending or 1.3\% of GDP - across EU countries in 2015 (OECD/EU, 2018[44]). But larger costs are also borne outside of the health system. Lower employment rates and productivity of people with mental health issues incur economic impact equivalent to $1.6 \%$ of GDP in EU countries; with greater spending on social security programmes, such as disability benefits or paid sick leave, accounting for a further 1.2\% of GDP (OECD/EU, 2018[44]).

Comparable cost estimates have been established in OECD countries beyond the EU. In Australia, for example, the total costs of mental ill-health amount to $4 \%$ of GDP, $45 \%$ of which are indirect costs (Australian Government - National Mental Health Commission, 2016[45]), Similar figures are reported in Canada and Japan (Sado et al., 2013[46]; Sado et al., 2013[47]; Mental Health Commission of Canada, 2012[48]). 
The impact of mental health problems on individuals' lives, and on societies and economies, can be addressed through more effective policies and interventions to prevent and manage them. However, understanding of the impact that mental health care makes on service users' lives is still weak; there is a pressing need to measure the effects and impact of prevention and treatment approaches more consistently and methodically.

Traditional measures say little about the lasting impact that mental health care has on the patient. For example, inpatient suicide is a critical safety measure which indicates when something has gone terribly wrong (Figure 2.11), and is one of the limited measures of care quality that can currently be reported internationally. Thankfully inpatient suicide is very rare, which means for the vast majority of psychiatric patients we do not have a meaningful insight into their experience or outcomes of care.

Figure 2.11. Inpatient suicide among patients with a psychiatric disorder, 2015-2017

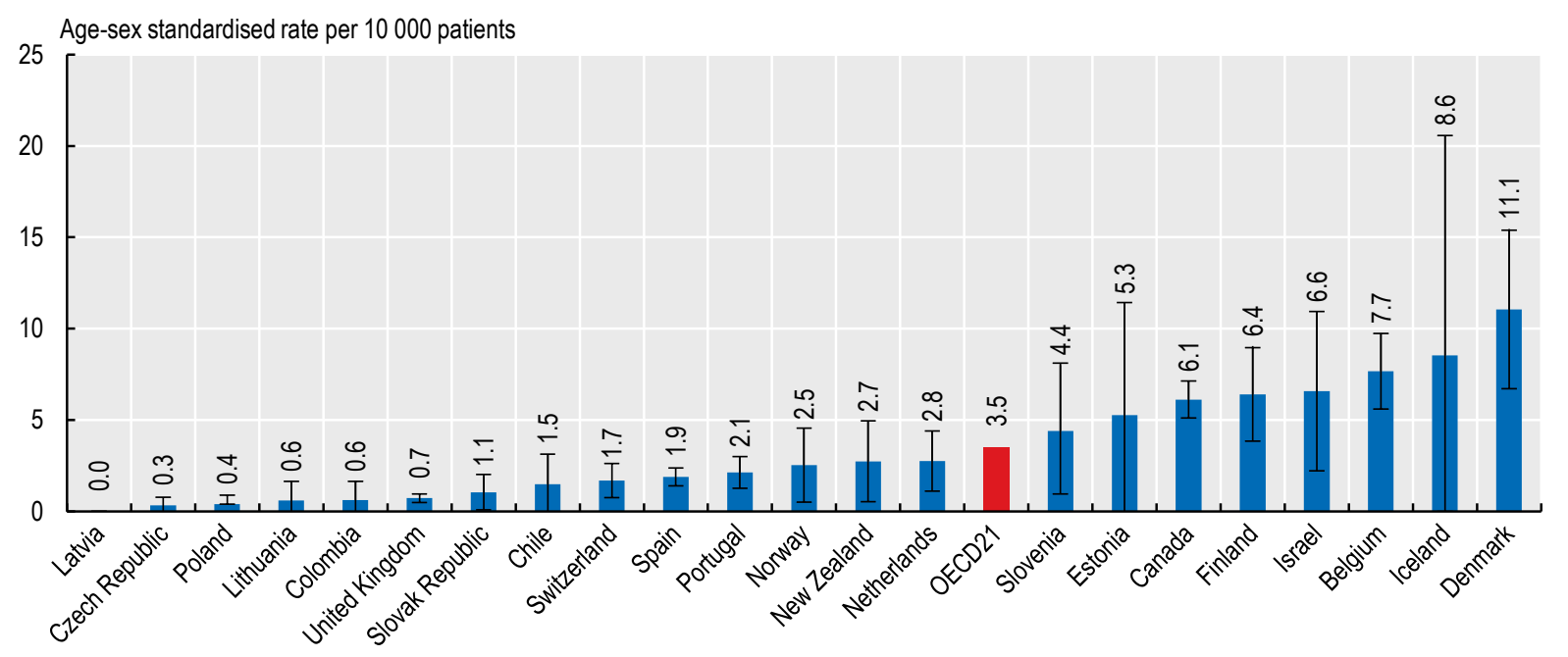

Note: $\mathrm{H}$ line shows $95 \%$ confidence intervals. Three year average except for New Zealand. Source: OECD Health Statistics 2019.

StatLink क्ञाs https://doi.org/10.1787/888934014783

Patient-reported measures are a critical tool for improving policy and practice in mental health care. An example of how patient-reported measures (in this case PREMs) can shed light on potential problems with mental health care is provided in Box 2.4, which report survey data on the care experience of people who report having been told by a doctor that they have a mental health condition, compared to those who have not.

\section{Collaboration to enhance patient-reporting in mental health}

Given the health and economic impact of mental ill-health, it is important to assess the quality and outcomes of care in this area. Existing outcome and process indicators while very useful in some circumstances - do not provide the entire picture of quality and performance. This information gap impedes efforts to improve care, practice and policy.

However, patient-reporting in mental health is still at a relatively nascent stage. Data collection is patchy, and routine reporting and use of the information is far from the norm. As of 2018, only five of the twelve countries surveyed (Australia, Israel, Netherlands, Sweden, United Kingdom - England) reported that PROMs and PREMs were collected regularly in the mental health setting. Only Australia, the Netherlands and England 


\section{Box 2.4. The Commonwealth Fund International Health Policy Survey of Adults}

The Commonwealth Fund 2016 International Health Policy Survey of Adults (The Commonwealth Fund, 2016[49]) was conducted in 11 countries - Australia, Canada, France, Germany, the Netherlands, New Zealand, Norway, Sweden, Switzerland, the United Kingdom, and the United States - with a total of 26863 adults interviewed by phone about their experiences with their country's health care system, their health and well-being.

The survey included the question "Have you ever been told by a doctor that you have depression, anxiety or other mental health problems". While there are some methodological challenges in using the survey in this way, including around comparability of response groups and sample sizes, comparing responses across all the survey questions for respondents who answered 'yes' with those who responded 'no' to the mental health question can shed light on how people who manage a mental health condition in the participating countries experience their health care journey.

Respondents who answered 'yes' reported similar experiences to the remaining respondents in some areas of care. In others, their reported care experience appears to be inferior. In several countries, for example, people with a mental health problem were significantly more likely to report having received conflicting information from different health care professionals (Figure 2.12). The differences were most pronounced in Australia, Sweden and France.

\section{Figure 2.12. People who have been told by a doctor that they have depression, anxiety or other mental health problems are more likely to report receipt of conflicting information from health care professionals}

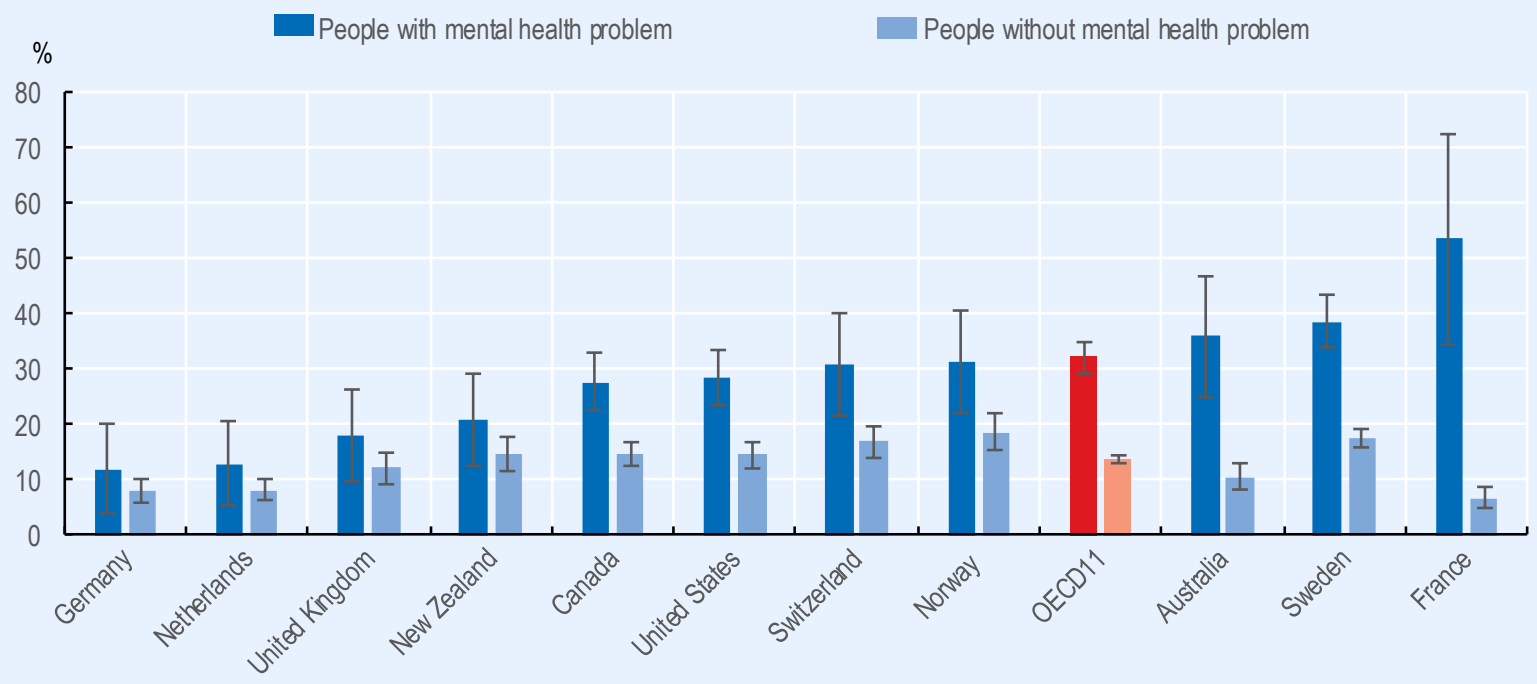

Note: 'People with a mental health problem' are the respondents who answered "yes" to the question "thinking about the past 2 years, when receiving care for a medical problem, was there EVER a time when you received conflicting information from different doctors or health care professionals?" Data limitations. The number of respondents in the 11 countries ranged from 1000 (Germany) to 7124 (Sweden). Lowest response rates were observed in Norway (10.9\%), Sweden (16.9\%), and the United States (18.1\%) and the highest were in the New Zealand (31.1\%), the Netherlands (32.4\%) and Switzerland (46.9\%). The sample sizes of respondents who answered 'yes' to the mental health question were therefore small, which is reflected in the large confidence intervals (H refers to $95 \%$ confidence intervals). In addition, the mental health survey question does not permit distinguishing between individuals who were suffering from a mental health problem at the time of the survey, and those who had experienced mental ill-health in the past but have since recovered. Cultural and linguistic differences in how the question was interpreted could also influence responses. Results have not been risk-adjusted for co-morbidities and socio-economic status.

Source: OECD analysis based on Commonwealth Fund 2016 International Health Policy Survey (The Commonwealth Fund, 2016[49]).

StatLink जाञाय https://doi.org/10.1787/888934014802

reported collecting and routinely reporting both. As such, a limited pool of national data exists that are not readily comparable at an international level. 
This needs to change, and the OECD has been working with patients, clinicians and policymakers and other experts from 13 countries to develop PREM and PROM data collection standards in mental health to enable international reporting, and foster the capacity to collect and use this important information in OECD countries.

\section{Conclusion}

A fundamental objective of health care is to improve the health and wellbeing of patients and populations. Yet, collecting information from patients on how successful health systems are in this endeavour is not the norm. In addition, emerging demographic, epidemiological and financial challenges are increasing the need to orient health systems around the needs of people and communities. This will not be possible without knowledge sourced directly from patients themselves to complement existing information on health system performance.

Results from preliminary data on patient-reported outcomes were presented in the areas of hip/knee replacement and breast cancer care, while work is underway in the area of mental health.

Over 2.2 million patients undergo a hip or a knee replacement each year in OECD countries. Since 2000, age-adjusted knee replacement rates have doubled in OECD countries, while hip replacement rates have grown by a third. The international landscape for collecting outcomes data from people undergoing hip or knee replacement is varied. Nevertheless, ten programmes from eight OECD countries contributed data reported by adult patients following an elective hip or knee replacement procedure. Results suggest that:

- In each country, both hip and knee replacement surgery improved the pain, function and health-related QoL as reported by patients, with results adjusted for age, sex and preoperative score.

- Greater gains were reported by patients who underwent a hip replacement. If performed at age 65, hip replacement would, on average, generate an additional 4.3 QALYs compared to of 3.3 QALYs for the average knee replacement (although the longer recovery period following knee replacement surgery must be noted).

- Inter-country variation was modest, suggesting that methods to collect and analyse the pilot data were sound.

Public knowledge of these types of results are very important as a way to improve informed decision-making by patients, and to calibrate patients' goals and expectations when deciding to undergo elective procedures. Results also enable policy decisions and assessing the cost-effectiveness, cost-utility and value from the patient perspective. More patient-reported data will enable solid, temporal analysis and inter-country comparisons in the future. It is important that countries harmonise their data collection at national level.

Breast cancer is the most prevalent form of cancer in women worldwide. While an increase in the incidence has been observed over the past decade, most OECD countries display 5 -year net survival rates of $80 \%$ or higher. A range of surgical interventions can be deployed to treat breast cancer but relatively little is known about their outcomes valued by women such as pain, breast satisfaction and QoL. Ten sites spanning 7 countries participated in a pilot collection of patient-reported outcomes data for women undergoing surgical breast cancer treatment. The preliminary results from this data collection - which have not been risk-adjusted - generate the following tentative observations: 
- Postoperative breast satisfaction of women may vary by type of surgery (whether this be a mastectomy or breast conserving therapy) and by the site of surgery, with some sites reporting higher scores for lumpectomies and others higher scores for reconstructions. This may offer additional opportunities for sharing and learning across sites and countries.

- Of the women who had a breast reconstruction after a mastectomy, the women who underwent autologous breast reconstruction surgery reported, on average, slightly better outcomes to women who underwent implant reconstruction. This aligns with conventional wisdom, providing women with potentially greater assurance in the use of such information to help assess treatment options.

- Autologous reconstruction may be a cost-effective alternative to implant surgery, when the additional costs for an additional year of perfect breast-related health is compared with broadly accepted cost-effectiveness thresholds.

A number of clinical factors need to be taken into account when considering these observations and ongoing data collection and analytical refinement is required explore their veracity. However, these results illustrate how this type of information derived directly from patients can potentially be very useful for other women when making difficult decisions and trade-offs on the optimal treatment pathway for their individual needs and preferences, providers when assessing the 'success' of various interventions, and payers and policymakers when considering the comparative cost-effectiveness and cost-utility of various treatments.

Mental ill-health exerts a considerable health and economic burden across the world, but systematic collection of patient-reported outcomes and experiences in mental health is at a nascent stage. Despite limitations in the data, the 2016 Commonwealth Fund survey of 11 countries suggests that people with a mental health problem report a worse care experience than those without mental health problems in some aspects of health care, such as receiving consistent information from providers. The OECD is working with international stakeholders including patients, clinicians and policymaker to advance measurement of mental health outcomes and experiences.

Overall, these results demonstrate that presenting valid and comparable results from patient-reported data at international level is eminently possible. However, capacity within and among countries must be increased to collect and report these data in a consistent and harmonised way. OECD will continue to work with countries to promote consistent collection and reporting of these data, in partnership with national and international stakeholders including patients and health care professionals.

\section{Notes}

1. Based on 45600 hip replacements and 49500 knee replacements reported in 2016 and 2017 respectively, at a 'national efficient price' (NEP) -- the official price paid by the national payer for conducting these procedures in the public sector. The 2019-20 NEP is just under AUD 20000 for each procedure (https://www.ihpa.gov.au/publications/national-efficient-price-determination-2019-20). The overall national figure is likely to be higher because approximately half of procedures are carried out in the private sector where higher prices are typically paid.

2. Coxa hospital has a patient catchment covering an entire region of Finland.

3. With the exception of Galeazzi, which included all principal diagnoses.

4. The value is derived by subtracting the pre-operative score from the post-operative score. A positive value therefore represents an improvement in QoL. 
5. Charts showing the average pre- and post-operative results for each participating programme are presented in Chapter 6 (Section: Hip and knee surgery).

6. The degree of improvement was statistically significant at the $95 \%$ confidence level in all programmes and in aggregate.

7. The generic and condition-specific scales are not linear - i.e. a change from 0.2 to 0.3 is not necessarily the same magnitude in terms of health-related QoL than 0.7 to 0.8 . The percentage improvements are provided for illustrative purposes and should be interpreted cautiously.

8. This does not mean that a joint replacement results in greater health gain than other, more conservative interventions for joint pain, which may be equivalent or even superior in this regard for some patients and on average. This comparison is beyond the scope of this chapter (Section: A good care experience contributes to better outcomes and is also an end in itself).

9. HOOS-PS: Hip disability and Osteoarthritis Outcome Score-Physical Function Shortform.

10. An alternative scoring system exists for both instruments where a lower value represents a better result.

11. See 6 and 7.

12. KOOS-PS: Knee injury and Osteoarthritis Outcome Score-Physical Function Shortform.

13. See 6 and 7 .

14. See 6 and 7.

15. As valued by a US population sample (Shaw JW, 2005).

16. The incremental QALYs are derived by multiplying the adjusted mean change by 20.5 years -- the average life expectancy at age 65 in the countries of the contributing programs, minus one year to account for recovery and rehabilitation (OECD, 2019[50]).

\section{References}

[4] Abahussin, A. et al. (2018), "PROMs for Pain in Adult Cancer Patients: A Systematic Review of Measurement Properties", Pain Practice, Vol. 19/1, pp. 93-117, http://dx.doi.org/10.1111/papr.12711.

[45] Australian Government - National Mental Health Commission (2016), Media Release: The Impact of Poor Mental Health: An Economic Issue, http://www.mentalhealthcommission.gov.au/media/181670/ Economics\%20of\%20Mental\%20Health\%20in\%20Australia\%20MR\%20(3).pdf.

[13] Basch, E. (2017), “Patient-Reported Outcomes - Harnessing Patients' Voices to Improve Clinical Care", New England Journal of Medicine, Vol. 376/2, pp. 105-108, http://dx.doi.org/10.1056/ nejmp1611252.

[14] Basch, E. et al. (2017), "Overall Survival Results of a Trial Assessing Patient-Reported Outcomes for Symptom Monitoring During Routine Cancer Treatment”, JAMA, Vol. 318/2, p. 197, http:// dx.doi.org/10.1001/jama.2017.7156.

[17] Black, N. (2013), "Patient reported outcome measures could help transform healthcare", BMJ, Vol. 346, p. f167.

[30] Bray, F. et al. (2018), "Global cancer statistics 2018: GLOBOCAN estimates of incidence and mortality worldwide for 36 cancers in 185 countries”, CA: A Cancer Journal for Clinicians, Vol. 68/6, pp. 394-424, http://dx.doi.org/10.3322/caac.21492.

[16] Calvert, M., D. O'Connor and E. Basch (2019), "Harnessing the patient voice in real-world evidence: the essential role of patient-reported outcomes", Nature Reviews Drug Discovery, http://dx.doi.org/ 10.1038/d41573-019-00088-7.

[42] Cameron, D., J. Ubels and F. Norström (2018), "On what basis are medical cost-effectiveness thresholds set? Clashing opinions and an absence of data: a systematic review", Global Health Action, Vol. 11/1, p. 1447828, http://dx.doi.org/10.1080/16549716.2018.1447828.

[31] Cardoso, F. et al. (2019), "Early breast cancer: ESMO Clinical Practice Guidelines for diagnosis, treatment and follow-up”, Annals of Oncology, http://dx.doi.org/10.1093/annonc/mdz173.

[25] Devlin, N. (2019), Can We Really Compare and Aggregate PRO Data Between People and Settings? Implications for Multi-Country Clinical Trials and HTA.

[5] Donovan, J. et al. (2016), "Patient-Reported Outcomes after Monitoring, Surgery, or Radiotherapy for Prostate Cancer", New England Journal of Medicine, Vol. 375/15, pp. 1425-1437, http://dx.doi.org/ 10.1056/nejmoa1606221. 
[35] Flanagan, M. et al. (2019), “A Comparison of Patient-Reported Outcomes After Breast-Conserving Surgery and Mastectomy with Implant Breast Reconstruction", Annals of Surgical Oncology, Vol. 26/10, pp. 3133-3140, http://dx.doi.org/10.1245/s10434-019-07548-9.

[39] Frisell, A., J. Lagergren and J. de Boniface (2016), "National study of the impact of patient information and involvement in decision-making on immediate breast reconstruction rates", British Journal of Surgery, Vol. 103/12, pp. 1640-1648, http://dx.doi.org/10.1002/bjs.10286.

[15] Greenhalgh, J. et al. (2017), "How do aggregated patient-reported outcome measures data stimulate health care improvement? A realist synthesis", Journal of Health Services Research \& Policy, Vol. 23/1, pp. 57-65, http://dx.doi.org/10.1177/1355819617740925.

[6] Hamdy, F. et al. (2016), "10-Year Outcomes after Monitoring, Surgery, or Radiotherapy for Localized Prostate Cancer", New England Journal of Medicine, Vol. 375/15, pp. 1415-1424, http://dx.doi.org/ 10.1056/nejmoa1606220.

[43] Institute for Health Metrics and Evaluation (2019), , http://www.healthdata.org/.

[36] Kern, P. et al. (2015), "Impact of Age, Obesity and Smoking on Patient Satisfaction with Breast Implant Surgery - A Unicentric Analysis of 318 Implant Reconstructions after Mastectomy", Geburtshilfe und Frauenheilkunde, Vol. 75/06, pp. 597-604, http://dx.doi.org/10.1055/s-0035-1546171.

[26] Konopka, J. et al. (2018), "Quality-Adjusted Life Years After Hip and Knee Arthroplasty”, JBJS Open Access, Vol. 3/3, p. e0007, http://dx.doi.org/10.2106/jbjs.oa.18.00007.

[23] Le, Q. (2013), "Probabilistic mapping of the health status measure SF-12 onto the health utility measure EQ-5D using the US-population-based scoring models", Quality of Life Research, Vol. 23/2, pp. 459-466, http://dx.doi.org/10.1007/s11136-013-0517-3.

[10] Luxford, K., D. Safran and T. Delbanco (2011), “Promoting patient-centered care: a qualitative study of facilitators and barriers in healthcare organizations with a reputation for improving the patient experience", International Journal for Quality in Health Care, Vol. 23/5, pp. 510-515, http://dx.doi.org/ 10.1093/intqhc/mzr024.

[37] Matros, E. et al. (2015), “Cost-Effectiveness Analysis of Implants versus Autologous Perforator Flaps Using the BREAST-Q", Plastic and Reconstructive Surgery, Vol. 135/4, pp. 937-946, http://dx.doi.org/ 10.1097/prs.0000000000001134.

[48] Mental Health Commission of Canada (2012), Making the Case for Investing in Mental Health in Canada, https://www.mentalhealthcommission.ca/sites/default/files/2016-06/ Investing_in_Mental_Health_FINAL_Version_ENG.pdf.

[7] Nag, N. et al. (2018), "Development of Indicators to Assess Quality of Care for Prostate Cancer", European Urology Focus, Vol. 4/1, pp. 57-63, http://dx.doi.org/10.1016/j.euf.2016.01.016.

[19] Nuttall, D., D. Parkin and N. Devlin (2013), "Inter-provider comparison of patient-reported outcomes: Developing an adjustment to account for differences in patient case mix", Health Economics, Vol. 24/1, pp. 41-54, http://dx.doi.org/10.1002/hec.2999.

[50] OECD (2019), "Health status", OECD Health Statistics (database), https://dx.doi.org/10.1787/data-00540en.

[2] OECD (2018), A Broken Social Elevator? How to Promote Social Mobility, OECD Publishing, Paris, https:// dx.doi.org/10.1787/9789264301085-en.

[1] OECD (2017), Preventing Ageing Unequally, OECD Publishing, Paris, https://dx.doi.org/ 10.1787/9789264279087-en.

[3] OECD (2014), Geographic Variations in Health Care: What Do We Know and What Can Be Done to Improve Health System Performance?, OECD Health Policy Studies, OECD Publishing, Paris, https://dx.doi.org/ 10.1787/9789264216594-en.

[38] OECD (2013), Cancer Care: Assuring Quality to Improve Survival, OECD Health Policy Studies, OECD Publishing, Paris, https://dx.doi.org/10.1787/9789264181052-en.

[44] OECD/EU (2018), Health at a Glance: Europe 2018: State of Health in the EU Cycle, OECD Publishing, Paris/ European Union, Brussels, https://dx.doi.org/10.1787/health_glance_eur-2018-en.

[40] Platt, J. et al. (2015), "Does Breast Reconstruction after Mastectomy for Breast Cancer Affect Overall Survival? Long-Term Follow-Up of a Retrospective Population-Based Cohort", Plastic and Reconstructive Surgery, Vol. 135/3, pp. 468e-476e, http://dx.doi.org/10.1097/prs.0000000000001054.

[33] Pusic, A. et al. (2009), "Development of a New Patient-Reported Outcome Measure for Breast Surgery: The BREAST-Q", Plastic and Reconstructive Surgery, Vol. 124/2, pp. 345-353, http://dx.doi.org/ 10.1097/prs.0b013e3181aee807. 
[46] Sado, M. et al. (2013), "The cost of schizophrenia in Japan", Neuropsychiatric Disease and Treatment, p. 787, http://dx.doi.org/10.2147/ndt.s41632.

[47] Sado, M. et al. (2013), "Cost of anxiety disorders in Japan in 2008: a prevalence-based approach", BMC Psychiatry, Vol. 13/1, http://dx.doi.org/10.1186/1471-244x-13-338.

[18] Schneider W, E. (2017), Mirror Mirror 2017: International Comparison Reflects Flaws and Opportunities for Better U.S. Health Care, https://interactives.commonwealthfund.org/2017/july/mirror-mirror/.

[41] Scurci, S. et al. (2017), “Abstract P8”, Plastic and Reconstructive Surgery - Global Open, Vol. 5, p. 107, http://dx.doi.org/10.1097/01.gox.0000516665.93739.ee.

[20] Shaw JW, J. (2005), "US valuation of the EQ-5D health states: development and testing of the D1 valuation model", Medical Care, Vol. 43/3, pp. 203-20.

[27] Skou, S., A. Bricca and E. Roos (2018), "The impact of physical activity level on the short- and longterm pain relief from supervised exercise therapy and education: a study of 12,796 Danish patients with knee osteoarthritis", Osteoarthritis and Cartilage, Vol. 26/11, pp. 1474-1478, http://dx.doi.org/ 10.1016/j.joca.2018.07.010.

[28] Skou, S. et al. (2018), "Total knee replacement and non-surgical treatment of knee osteoarthritis: 2year outcome from two parallel randomized controlled trials", Osteoarthritis and Cartilage, Vol. 26/9, pp. 1170-1180, http://dx.doi.org/10.1016/j.joca.2018.04.014.

[29] Skou, S. et al. (2015), “A Randomized, Controlled Trial of Total Knee Replacement”, New England Journal of Medicine, Vol. 373/17, pp. 1597-1606, http://dx.doi.org/10.1056/nejmoa1505467.

[8] Stein, S. et al. (2014), "Patients' Perceptions of Care Are Associated With Quality of Hospital Care", American Journal of Medical Quality, Vol. 30/4, pp. 382-388, http://dx.doi.org/ 10.1177/1062860614530773.

[22] Sullivan, P. and V. Ghushchyan (2006), "Mapping the EQ-5D Index from the SF-12: US General Population Preferences in a Nationally Representative Sample”, Medical Decision Making, Vol. 26/4, pp. 401-409, http://dx.doi.org/10.1177/0272989x06290496.

[34] Tevis, S. et al. (2018), "Patient-Reported Outcomes for Breast Cancer", Annals of Surgical Oncology, Vol. 25/10, pp. 2839-2845, http://dx.doi.org/10.1245/s10434-018-6616-1.

[49] The Commonwealth Fund (2016), International Health Policy Survey.

[9] Trzeciak, S. et al. (2016), “Association Between Medicare Summary Star Ratings for Patient Experience and Clinical Outcomes in US Hospitals”, Journal of Patient Experience, Vol. 3/1, pp. 6-9, http://dx.doi.org/10.1177/2374373516636681.

[32] van Egdom, L. et al. (2019), "Implementation of Value Based Breast Cancer Care”, European Journal of Surgical Oncology, Vol. 45/7, pp. 1163-1170, http://dx.doi.org/10.1016/j.ejso.2019.01.007.

[21] van Hout, B. et al. (2012), "Interim Scoring for the EQ-5D-5L: Mapping the EQ-5D-5L to EQ-5D-3L Value Sets”, Value in Health, Vol. 15/5, pp. 708-715, http://dx.doi.org/10.1016/j.jual.2012.02.008.

[12] Veroff, D., A. Marr and D. Wennberg (2013), "Enhanced Support For Shared Decision Making Reduced Costs Of Care For Patients With Preference-Sensitive Conditions", Health Affairs, Vol. 32/2, pp. 285-293, http://dx.doi.org/10.1377/hlthaff.2011.0941.

[11] Wong, E. et al. (2019), "Associations between provider communication and personal recovery outcomes”, BMC Psychiatry, Vol. 19/1, http://dx.doi.org/10.1186/s12888-019-2084-9.

[24] Zhuo, L. et al. (2018), “Time Trade-Off Value Set for EQ-5D-3L Based on a Nationally Representative Chinese Population Survey", Value in Health, Vol. 21/11, pp. 1330-1337, http://dx.doi.org/10.1016/ j.jual.2018.04.1370. 

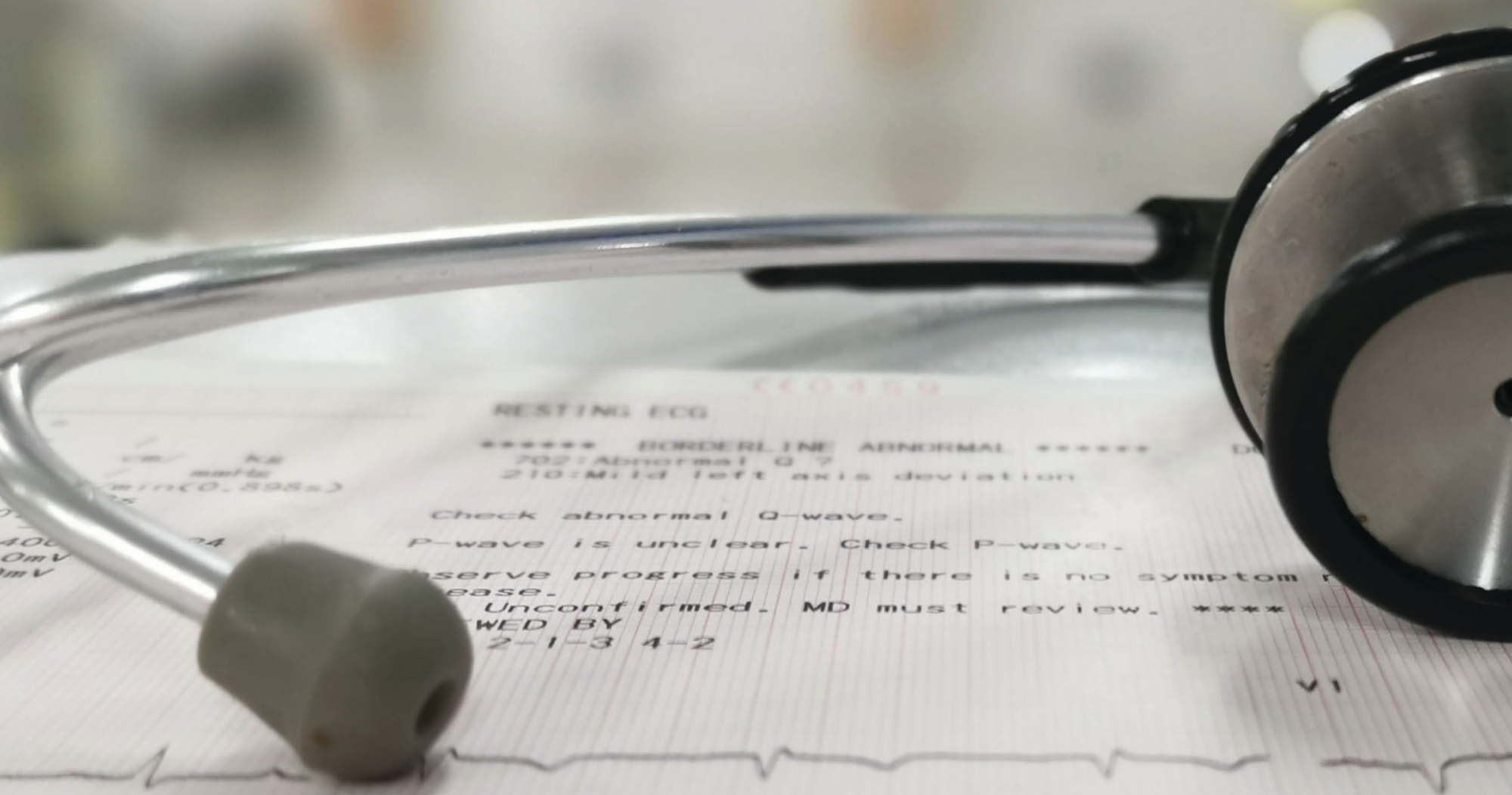

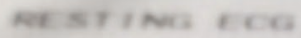

$\cdots+\cdots$

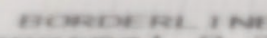

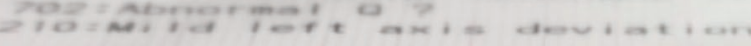

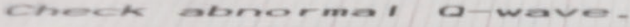

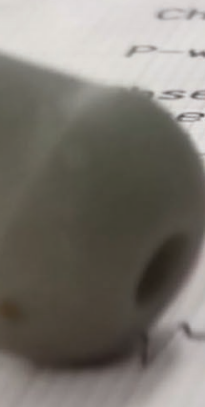

\section{.}

$a+2$

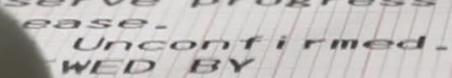
WID W $\mathrm{CO}, \mathrm{C}$
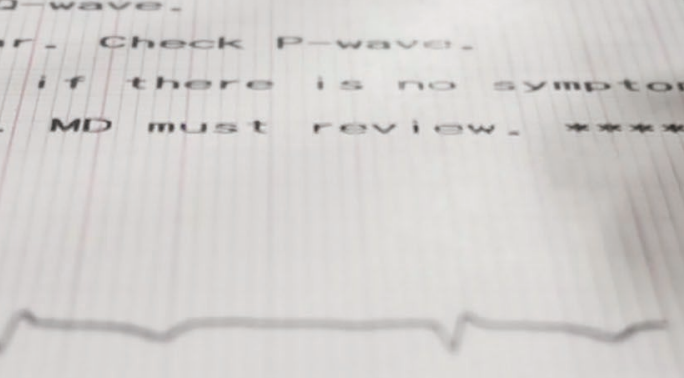

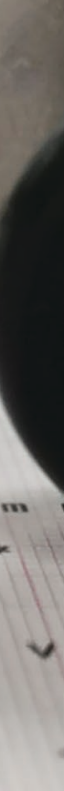

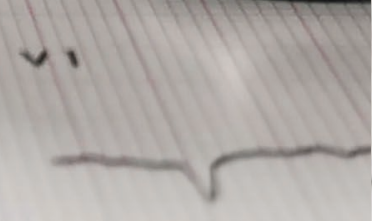

$+4$

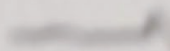

L

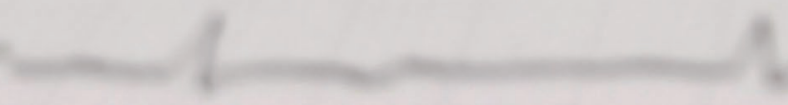


Nerstines eres

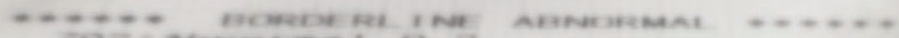

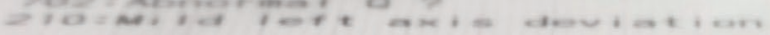
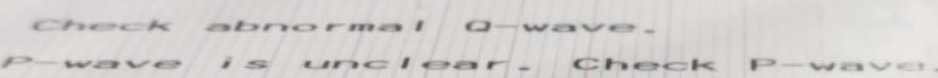

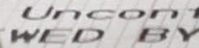

$2-1,3,2+2$

\section{HEALTH STATUS}

Trends in life expectancy

Life expectancy by sex and education level

Main causes of mortality

Avoidable mortality (preventable and treatable)

Mortality from circulatory diseases

Cancer incidence and mortality

Chronic disease morbidity

Infant health

Mental health

Self-rated health

The statistical data for Israel are supplied by and under the responsibility of the relevant Israeli authorities. The use of such data by the OECD is without prejudice to the status of the Golan Heights, East Jerusalem and Israeli settlements in the West Bank under the terms of international law. 
Life expectancy has increased in all OECD countries over the last few decades, although gains have slowed in recent years. In 2017, life expectancy at birth was 80.7 years on average across OECD countries, over 10 years higher than it was in 1970 (Figure 3.1).

Japan, Switzerland and Spain lead a large group of 26 OECD countries in which life expectancy at birth exceeds 80 years. A second group, including the United States and a number of central and eastern European countries, has a life expectancy between 77 and 80 years. Latvia, Mexico, Lithuania and Hungary have the lowest life expectancy, at less than 76 years in 2017.

Among OECD countries, Turkey, Korea and Chile have experienced the largest gains since 1970, with increases of 24,20 and 18 years respectively. Stronger health systems have contributed to these gains, by offering more accessible and higher quality care. Wider determinants of health matter too - notably rising incomes, better education and improved living environments. Healthier lifestyles, influenced by policies within and beyond the health system, have also had a major impact (James, Devaux and Sassi, 2018[1]).

In partner countries, life expectancy remains well below the OECD average except in Costa Rica. Still, levels are converging rapidly towards the OECD average, with considerable gains in longevity since 1970 in India, China, Brazil, Indonesia, Colombia and Costa Rica. There has been less progress in the Russian Federation, due mainly to the impact of the economic transition in the 1990s and a rise in risky health behaviours among men. South Africa has also experienced slow progress, due mainly to the HIV/AIDS epidemic, although longevity gains over the last decade have been more rapid.

A closer look at trends in life expectancy at birth shows a considerable slowdown in gains in recent years. Comparing the last five years (2012-17) with a decade earlier (2002-07), 27 OECD countries experienced slower gains in life expectancy (Figure 3.2). This slowdown was most marked in the United States, France, the Netherlands, Germany and the United Kingdom. Longevity gains were slower for women than men in almost all OECD countries.

Indeed, life expectancy fell on average across OECD countries in 2015 - the first time this has happened since 1970. Nineteen countries recorded a reduction, widely attributed to a particularly severe influenza outbreak that killed many frail elderly people and other vulnerable groups (Figure 3.3). Most of these were European countries, with the exception of the United States and Israel. The largest reductions were in Italy (7.2 months) and Germany (6 months).

The causes of this slowdown in life expectancy gains are multifaceted (Raleigh, 2019[2]). Principal among them is slowing improvements in heart disease and stroke. Rising levels of obesity and diabetes, as well as population ageing, have made it difficult for countries to maintain previous progress in cutting deaths from such circulatory diseases. Respiratory diseases such as influenza and pneumonia have claimed more lives in recent years - most notably in 2015, but also in the winters of 2012-13 and 2016-17. In some countries, particularly the United States and Canada, the opioid crisis has caused more working-age adults to die from drug-related accidental poisoning.

More broadly, economic recessions and related austerity measures, as in the 2008 global economic crisis, have been linked to deteriorating mental health and increased suicide rates, but with a less clear-cut impact on overall mortality (Parmar, Stavropoulou and Ioannidis, 2016[3]). What is clear is that continued gains in longevity should not be taken for granted, with better protection of older people and other atrisk populations paramount to extending life expectancy.

Higher national income is generally associated with greater longevity, particularly at lower income levels. Life expectancy is also, on average, longer in countries that invest more in health systems - although this relationship tends to be less pronounced in countries with the highest health spending per capita (see Chapter 1 for further analysis).

\section{Definition and comparability}

Life expectancy at birth measures how long, on average, people would live based on a given set of agespecific death rates. However, the actual age-specific death rates of any particular birth cohort cannot be known in advance. If age-specific death rates are falling (as has been the case over the past few decades), actual life spans will be higher than life expectancy calculated with current death rates.

Data for life expectancy at birth comes from Eurostat for EU countries, and from national sources elsewhere. Life expectancy at birth for the total population is calculated by the OECD Secretariat for all OECD countries, using the unweighted average of life expectancy of men and women.

\section{References}

[1] James, C., M. Devaux and F. Sassi (2017), "Inclusive Growth and Health", OECD Health Working Papers, No. 103, OECD Publishing, Paris, https://doi.org/10.1787/93d52bcd-en.

[3] Parmar, D., C. Stavropoulou and J. Ioannidis (2016), "Health Outcomes During the 2008 Financial Crisis in Europe: Systematic Literature Review", British Medical Journal, p. 354, https://www.bmj.com/content/354/bmj.i4588.

[2] Raleigh, V. (2019), "Trends in life expectancy in EU and other OECD countries: why are improvements slowing?", OECD Health Working Papers, No. 108, OECD Publishing, Paris, https://doi.org/10.1787/223159ab-en. 
Figure 3.1. Life expectancy at birth, 1970 and 2017 (or nearest year)

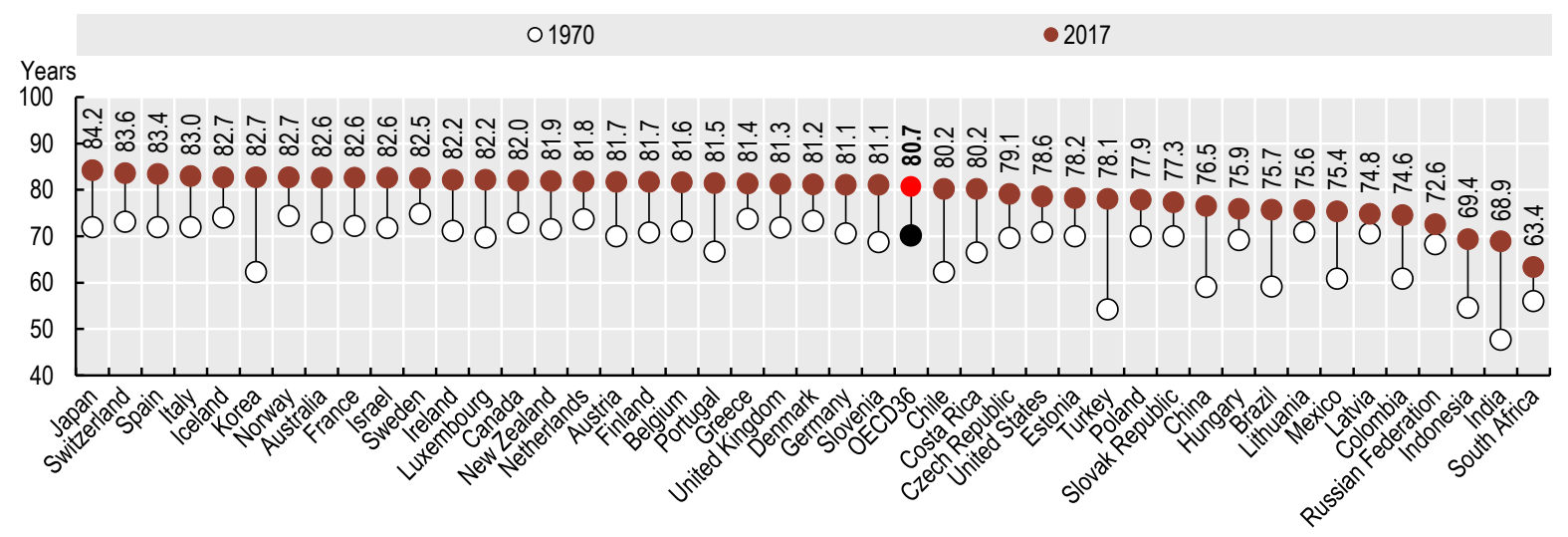

Source: OECD Health Statistics 2019.

StatLink ils https://doi.org/10.1787/888934014821

Figure 3.2. Slowdown in life expectancy gains, 2012-17 and 2002-07

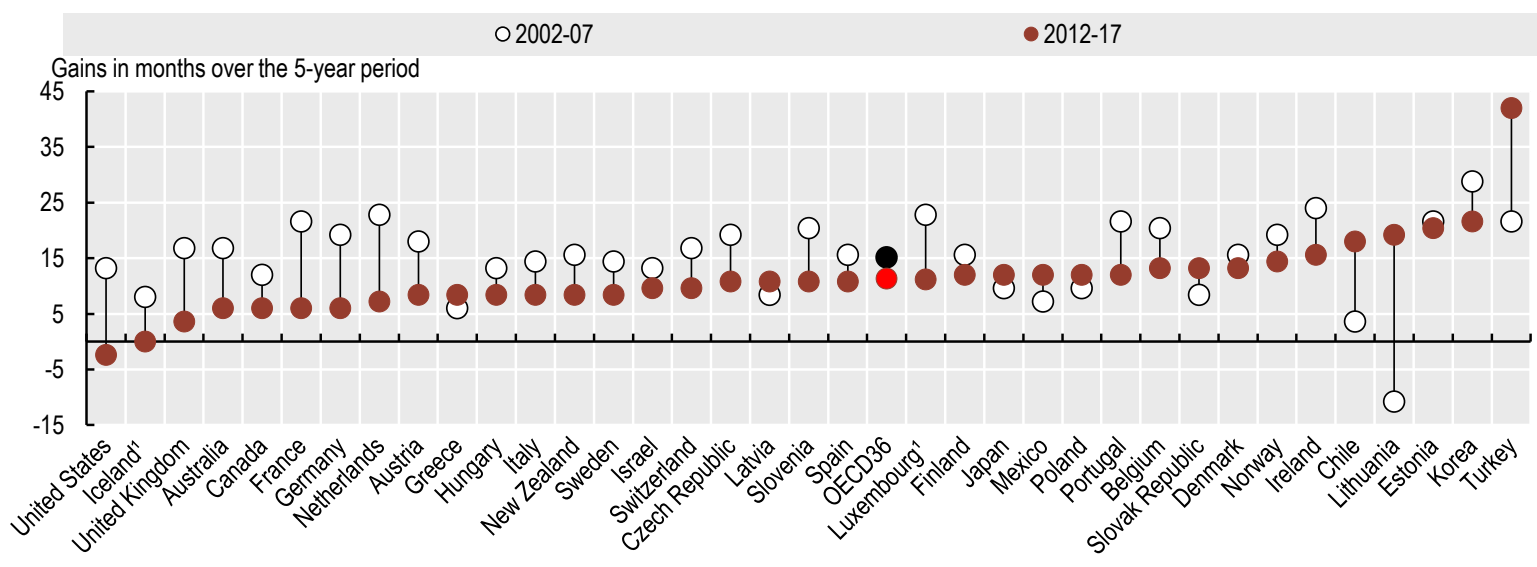

1. Three-year average.

Source: OECD Health Statistics 2019.

Figure 3.3. Change in life expectancy at birth, 2014 to 2015

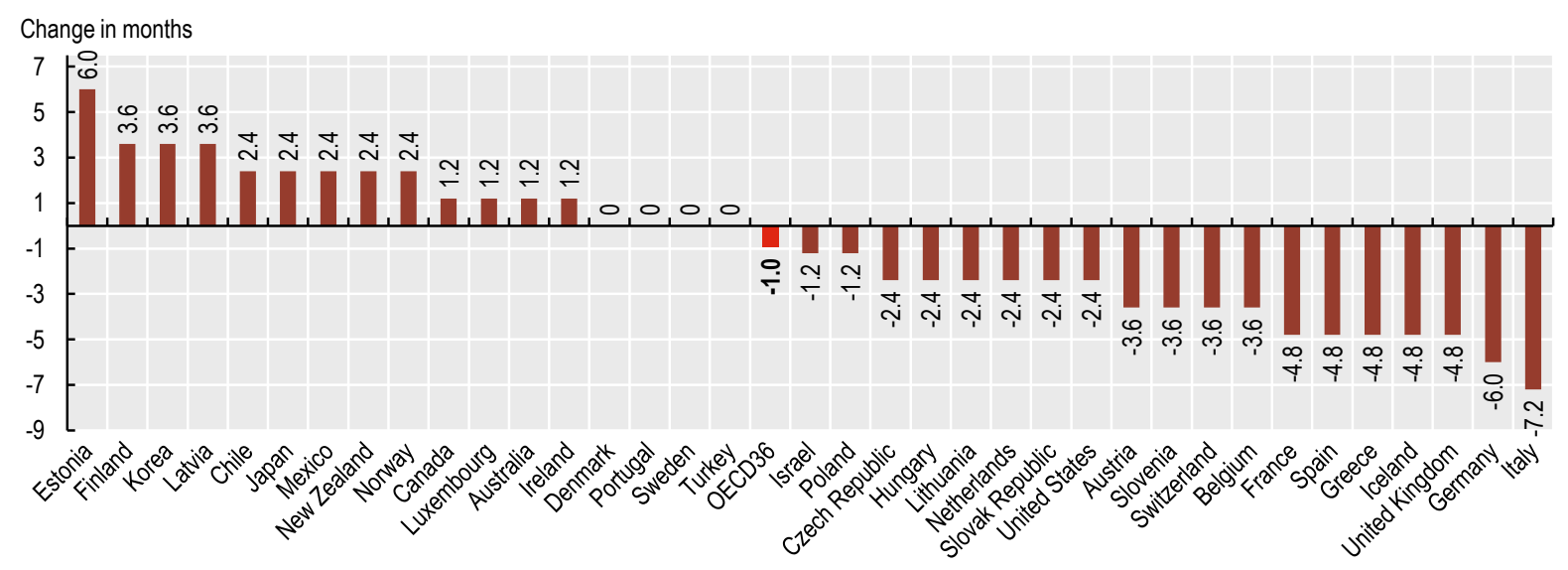

Source: OECD Health Statistics 2019. 
Women live longer than men do in all OECD and partner countries. This gender gap averaged 5.3 years across OECD countries in 2017 - life expectancy at birth for women was 83.4 years, compared with 78.1 years for men (Figure 3.4). The gender gap in life expectancy, though, has narrowed by one year since 2000, reflecting more rapid gains in life expectancy among men in most countries.

In 2017, life expectancy at birth for men in OECD countries ranged from around 70 years in Latvia and Lithuania to 81 years or higher in Switzerland, Japan, Iceland and Norway. For women, life expectancy reached 87.3 years in Japan, but was less than 80 years in Mexico, Hungary and Latvia.

Gender gaps are relatively narrow in Iceland, the Netherlands, Norway, Sweden, New Zealand, Ireland, the United Kingdom and Denmark - at less than four years. However, there are large gender differences in many central and eastern European countries, most notably in Latvia and Lithuania (around ten years), Estonia (around nine years) and Poland (around eight years). In these countries, gains in longevity for men over the past few decades have been much more modest. This is partly due to greater exposure to risk factors among men - particularly greater tobacco use, excessive alcohol consumption and less healthy diets resulting in more deaths from heart diseases, cancer and other diseases. For partner countries, the gender gap is around ten years in the Russian Federation, and just over seven years in Colombia, Brazil and South Africa. China and India have small gender gaps, of about three years.

Socioeconomic inequalities in life expectancy are also evident in all OECD countries with available data (Figure 3.5). On average among 26 OECD countries, a 30-year-old with less than an upper secondary education level can expect to live for 5.5 fewer years than a 30-year-old with tertiary education (a university degree or equivalent). These differences are higher among men, with an average gap of 6.9 years, compared with an average gap of 4.0 years among women.

Socioeconomic inequalities are particularly striking among men in many central and eastern European countries (Slovak Republic, Hungary, Poland, Czech Republic, Latvia), where the life expectancy gap between men with lower and higher education levels is over ten years. Gaps in life expectancy by education are relatively small in Turkey, Canada and Sweden.

More deaths amongst prime-age adults (25-64 years) with lower education levels drive much of this education gap in life expectancy. Mortality rates are almost four times higher for less educated prime-age men, and about twice as high for less educated prime-age women, compared to those with tertiary education (analysis based on data from 23 OECD countries). Differences in mortality rates among older men and women, while less marked, remain higher among the less educated, driven mainly by more deaths from circulatory diseases and cancer (Murtin et al, 2017[1]).

Higher smoking rates amongst disadvantaged socioeconomic groups is an important contributor to gaps in life expectancy by education or other measures of socioeconomic status. Other risk factors are also more prevalent among disadvantaged groups, notably excessive alcohol consumption among men, and higher obesity rates for men and women (see indicators in Chapter 4 on "Risk factors for health").

\section{Definition and comparability}

Life expectancy at birth measures how long, on average, people would live based on a given set of agespecific death rates. Data on life expectancy by sex comes from Eurostat for EU countries, and from national sources elsewhere.

For life expectancy by education level, data were provided directly to the OECD for Australia, Austria, Belgium, Canada, Chile, France, Iceland, Israel, Latvia, Mexico, Netherlands, New Zealand, Switzerland, Turkey and the United Kingdom. Data for the remaining European countries were extracted from the Eurostat database. The International Standard Classification of Education (ISCED) 2011 is the basis for defining education levels. The lowest education level ISCED 0-2 - refers to people who have not completed their secondary education. The highest education level - ISCED 6-8 - refers to people who have completed a tertiary education (a university degree or equivalent).

Not all countries have information on education as part of their deaths statistics. In such cases, data linkage to another source (e.g. a census) containing information on education is required. Data disaggregated by education are only available for a subset of the population for Belgium, the Czech Republic and Norway. In these countries, the large share of the deceased population with missing information about their education level can affect the accuracy of the data.

\section{References}

[1] Murtin, F. et al (2017), "Inequalities in Longevity by Education in OECD Countries: Insights from New OECD Estimates", OECD Statistics Working Papers, No. 2017/02, OECD Publishing, Paris, http://dx.doi.org/10.1787/6b64d9cf-en.

[2] OECD (2019), Health for Everyone? Social Inequalities in Health and Health Systems, OECD Publishing, Paris, https://doi.org/ 10.1787/3c8385d0-en. 


\section{Figure 3.4. Life expectancy at birth by sex, 2017 (or nearest year)}

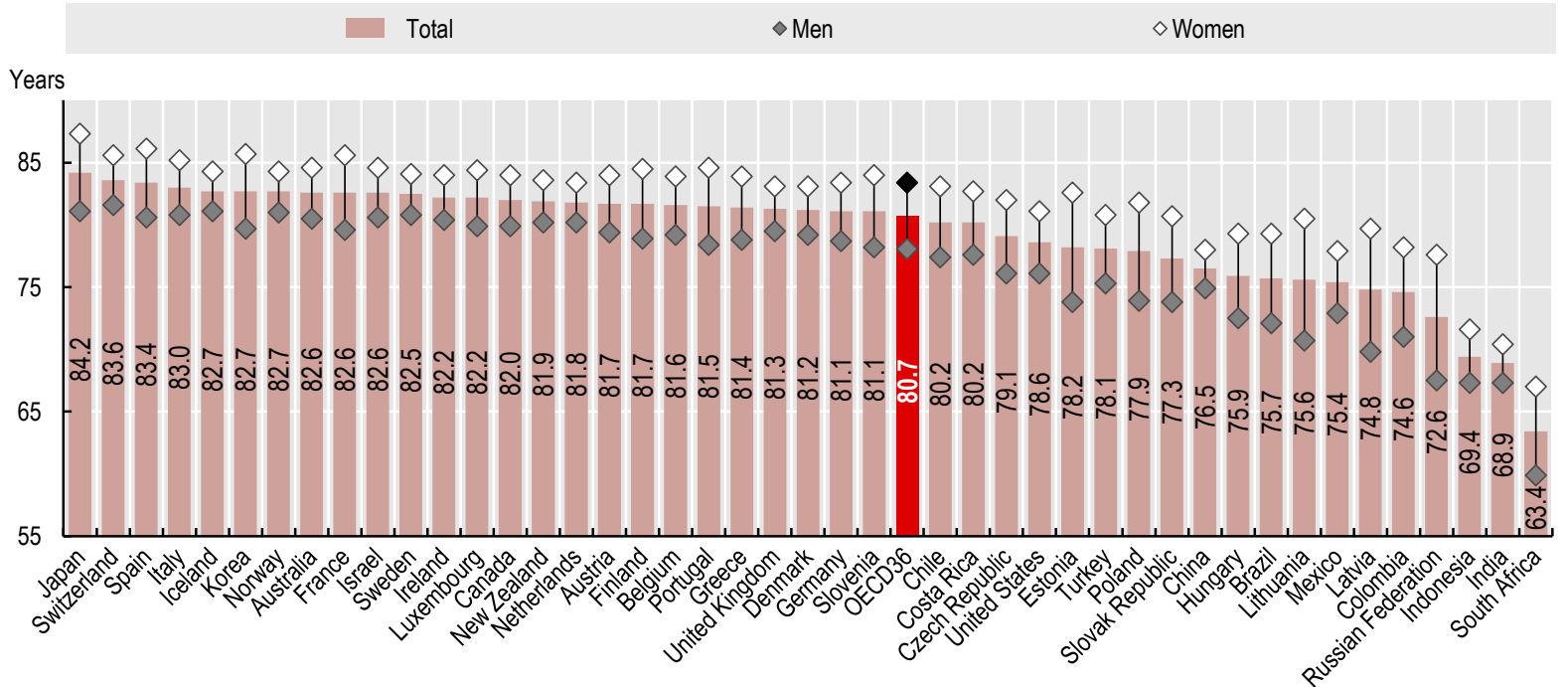

Source: OECD Health Statistics 2019.

StatLink क्गाड़ https://doi.org/10.1787/888934014878

Figure 3.5. Gap in life expectancy at age 30 between highest and lowest education level, by sex, latest available year
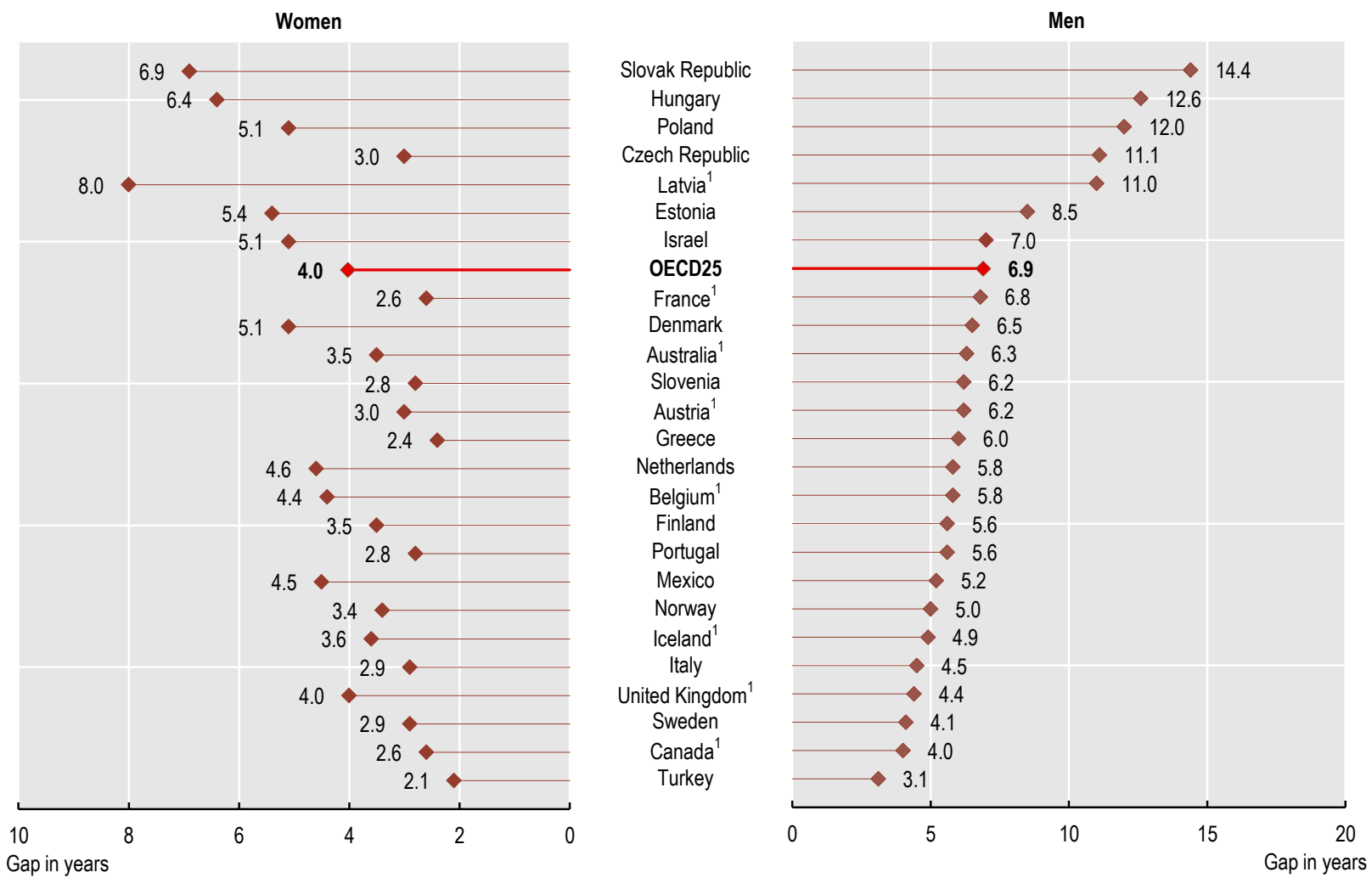

1. 2010-12 data. All other data are from 2015-17.

Source: OECD Health Statistics 2019. 
Over 10 million people died in 2017 across OECD countries, equivalent to about 800 deaths per 100000 population (Figure 3.6). All-cause mortality rates ranged from under 600 deaths per 100000 in Japan to over 1100 deaths per 100000 in Latvia, Hungary and Lithuania (age-standardised rates). Among partner countries, mortality rates were highest in South Africa and the Russian Federation (1 940 and 1417 per 100000 deaths respectively).

Age-standardised mortality rates were 50\% higher for men than women across OECD countries (997 per 100000 population for men, compared with 655 for women). In Lithuania, Latvia and Hungary there were about 1500 deaths per 100000 men. For women, mortality rates were highest in Hungary, Chile and Latvia. Among partner countries, male mortality rates were around 2400 deaths per 100000 in South Africa and almost 2000 in the Russian Federation. These countries also had the highest female mortality rates. Gender gaps are partly due to greater exposure to risk factors - particularly smoking, alcohol consumption and less healthy diets - alongside intrinsic gender differences. Accordingly, men had higher death rates from heart diseases, lung cancers and injuries, among other diseases.

Diseases of the circulatory system and cancer are the two leading causes of death in most countries. This reflects the epidemiological transition from communicable to noncommunicable diseases, which has already taken place in high-income countries and is rapidly occurring in many middle-income countries (GBD 2017 Causes of Death Collaborators, 2018[1]). Across OECD countries, heart attacks, strokes and other circulatory diseases caused about one in three deaths; and one in four deaths were related to cancer in 2017 (Figure 3.7). Population ageing largely explains the predominance of deaths from circulatory diseases - with deaths rising steadily from age 50 and above.

Respiratory diseases were also a major cause of death, accounting for $10 \%$ of deaths across OECD countries. Chronic obstructive respiratory disease (COPD) alone accounted for $4 \%$ of all deaths. Smoking is the main risk factor for COPD, but occupational exposure to dusts, fumes and chemicals, and air pollution in general are also important risk factors.

External causes of death were responsible for $6 \%$ of deaths across OECD countries, particularly road traffic accidents and suicides. Road traffic accidents are a particularly important cause of death among young adults, whereas suicide rates are generally higher among middle-aged and older people.
Looking at other specific causes, Alzheimer's and other dementias accounted for $9 \%$ of all deaths, and were a more important cause of death among women. Diabetes represented $3 \%$ of all deaths across OECD countries. The main causes of death differ between socio-economic groups, with social disparities generally larger for the most avoidable diseases (Mackenbach et al., 2015[2]).

\section{Definition and comparability}

Mortality rates are based on the number of deaths registered in a country in a year divided by the population. Rates have been directly age-standardised to the 2010 OECD population (available at http://oe.cd/ mortality) to remove variations arising from differences in age structures across countries and over time. The source is the World Health Organization (WHO) Mortality Database.

Deaths from all causes are classified to ICD-10 codes A00-Y89, excluding S00-T98. The classification of causes of death defines groups and subgroups. Groups are umbrella terms covering diseases that are related to each other; subgroups refer to specific diseases. For example, the group diseases of the respiratory system comprises four subgroups: influenza, pneumonia, chronic obstructive pulmonary diseases and asthma. Charts are based on this grouping, except for Alzheimer's and other dementias. These were grouped together (Alzheimer's is classified in Chapter G and other dementias in Chapter F).

\section{References}

[1] GBD 2017 Causes of Death Collaborators (2018), “Global, regional, and national age-sex-specific mortality for 282 causes of death in 195 countries and territories, 1980-2017: a systematic analysis for the Global Burden of Disease Study 2017", The Lancet, Vol. 392/10159, pp. 1736-1788.

[2] Mackenbach, J. et al. (2015), "Variations in the relation between education and cause-specific mortality in 19 European populations: A test of the 'fundamental causes' theory of social inequalities in health", Social Science and Medicine, Vol. 127, pp. 51-62. 
Figure 3.6. All-cause mortality rates, by gender, 2017 (or nearest year)

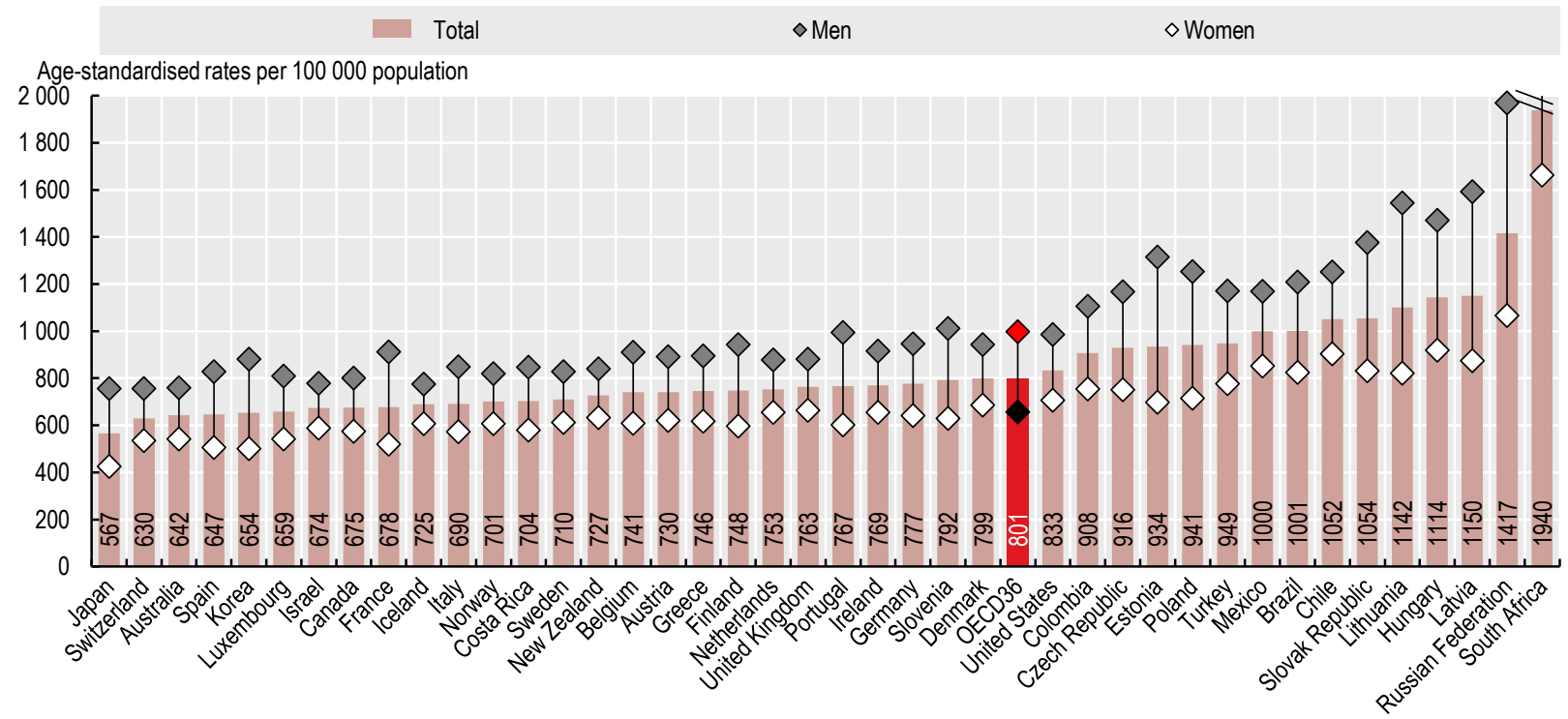

Source: OECD Health Statistics 2019.

Figure 3.7. Main causes of mortality across OECD countries, 2017 (or nearest year)

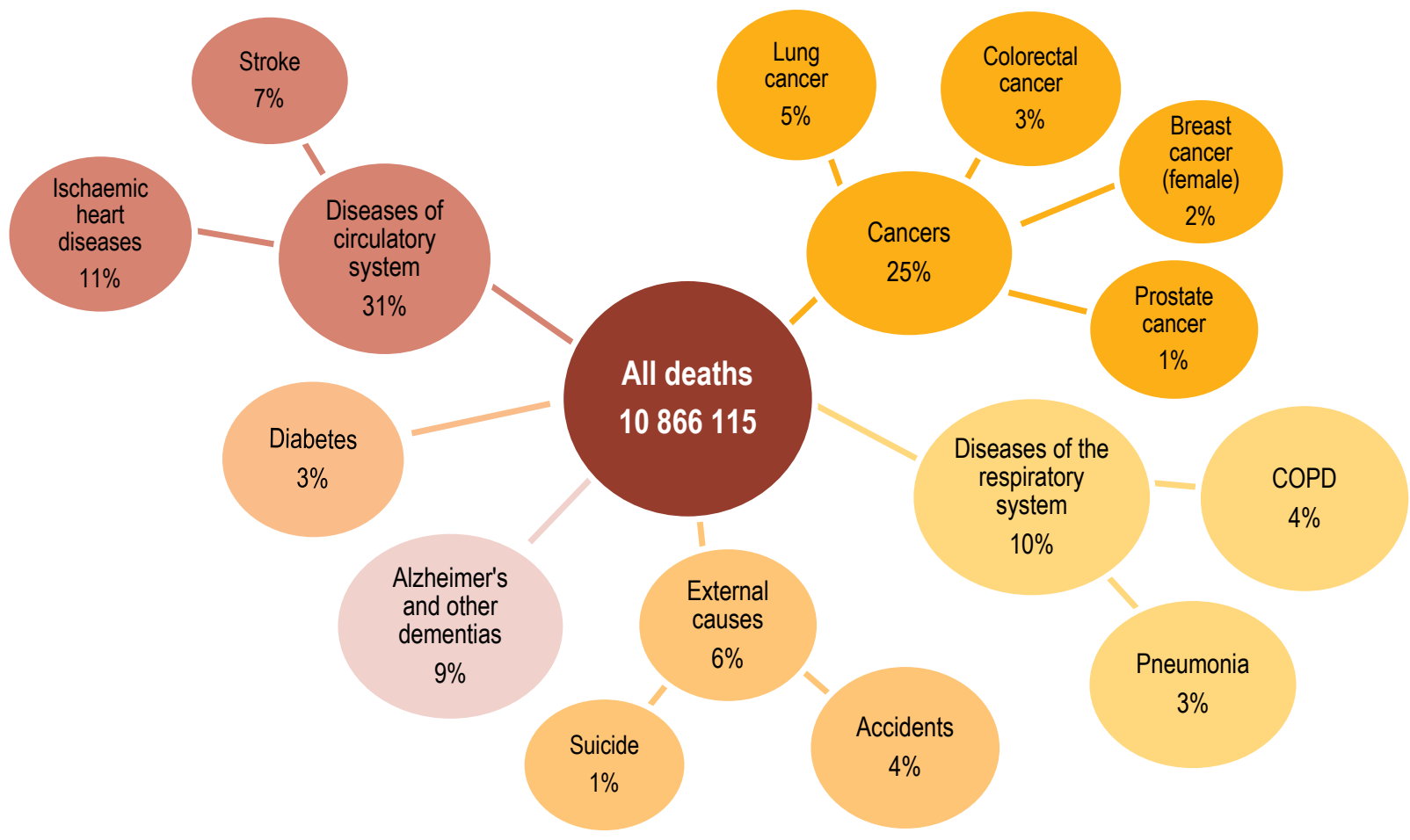

Note: Other causes of death not shown in the figure represent $15 \%$ of all deaths. Source: OECD Health Statistics 2019. 
Indicators of avoidable mortality can provide a general "starting point" to assess the effectiveness of public health and health care systems in reducing premature deaths from various diseases and injuries. However, further analysis is required to assess more precisely different causes of potentially avoidable deaths and interventions to reduce them.

In 2017, almost 3 million premature deaths across OECD countries could have been avoided through better prevention and health care interventions. This amounts to over one quarter of all deaths. Of these deaths, about 1.85 million were considered preventable through effective primary prevention and other public health measures, and over 1 million were considered treatable through more effective and timely health care interventions.

Some cancers that are preventable through public health measures were the main causes of preventable mortality (32\% of all preventable deaths), particularly lung cancer (Figure 3.8). Other major causes were external causes of death, such as road accidents and suicide (25\%); heart attack, stroke and other circulatory diseases (19\%); alcohol and drug-related deaths (9\%); and some respiratory diseases such as influenza and chronic obstructive pulmonary disease (8\%).

The main treatable cause of mortality is circulatory diseases (mainly heart attack and stroke), which accounted for $36 \%$ of premature deaths amenable to treatment. Effective, timely treatment for cancer, such as colorectal and breast cancers, could have averted a further $26 \%$ of all deaths from treatable causes. Diabetes and other diseases of the endocrine system (9\%) and respiratory diseases such as pneumonia and asthma (9\%) are other major causes of premature deaths that are amenable to treatment.

The average aged-standardised mortality rate from preventable causes was 133 deaths per 100000 people across OECD countries. Premature deaths ranged from under 96 per 100000 in Israel, Switzerland, Japan, Italy, Spain and Sweden to over 200 in Latvia, Hungary, Lithuania and Mexico (Figure 3.9). Higher rates of premature death in these countries were mainly due to much higher mortality from ischaemic heart disease, accidents and alcohol-related deaths, as well as lung cancer in Hungary.

The mortality rates from treatable causes across OECD countries was much lower, at 75 per 100000 population. It ranged from less than 50 in Switzerland, Iceland, Norway, Korea, France and Australia, to over 130 deaths per 100000 people in Latvia, Mexico, Lithuania and Hungary. Ischaemic heart diseases, strokes and some types of treatable cancers (e.g. colorectal and breast cancers) were the main drivers in Latvia, Lithuania and Hungary, countries with some of the highest treatable mortality rates.

Preventable mortality rates were 2.6 times higher among men than among women across OECD countries (197 per
100000 population for men, compared with 75 for women). Similarly, mortality rates from treatable causes were about $40 \%$ higher among men than women, with a rate of 87 per 100000 population for men compared with 62 for women. These gender gaps are explained by higher mortality rates among men, which are in part linked to different exposure to risk factors such as tobacco smoking (see indicator Main causes of mortality).

\section{Definition and comparability}

Based on the 2019 OECD/Eurostat definitions, preventable mortality is defined as causes of death that can be mainly avoided through effective public health and primary prevention interventions (i.e. before the onset of diseases/injuries, to reduce incidence). Treatable (or amenable) mortality is defined as causes of death that can be mainly avoided through timely and effective health care interventions, including secondary prevention and treatment (i.e. after the onset of diseases, to reduce case-fatality).

The two current lists of preventable and treatable mortality were adopted by the OECD and Eurostat in 2019. The attribution of each cause of death to the preventable or treatable mortality category was based on the criterion of whether it is predominantly prevention or health care interventions that can reduce it. Causes of death that can be both largely prevented and also treated once they have occurred were attributed to the preventable category on the rationale that if these diseases are prevented, there would be no need for treatment. In cases when there was no strong evidence of predominance of preventability or treatability (e.g. ischaemic heart disease, stroke, diabetes), the causes were allocated on a $50 \%-50 \%$ basis to the two categories to avoid doublecounting the same cause of death in both lists. The age threshold of premature mortality is set at 74 years for all causes (OECD/Eurostat, 2019[1]).

Data come from the WHO Mortality Database and the mortality rates are age-standardised to the OECD 2010 Standard Population (available at http://oe.cd/mortality).

\section{References}

[1] OECD/Eurostat (2019), “Avoidable mortality: OECD/Eurostat lists of preventable and treatable causes of death", http:// www.oecd.org/health/health-systems/Avoidable-mortality-2019Joint-OECD-Eurostat-List-preventable-treatable-causes-ofdeath.pdf. 


\section{Figure 3.8. Main causes of avoidable mortality, OECD countries, 2017 (or nearest year)}

Preventable causes of mortality

1856450 premature deaths

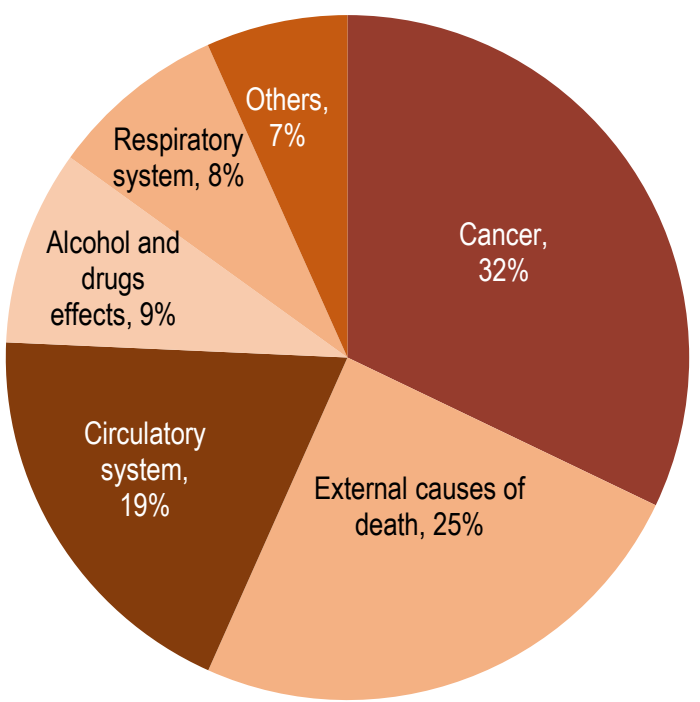

Treatable causes of mortality

1051184 premature deaths

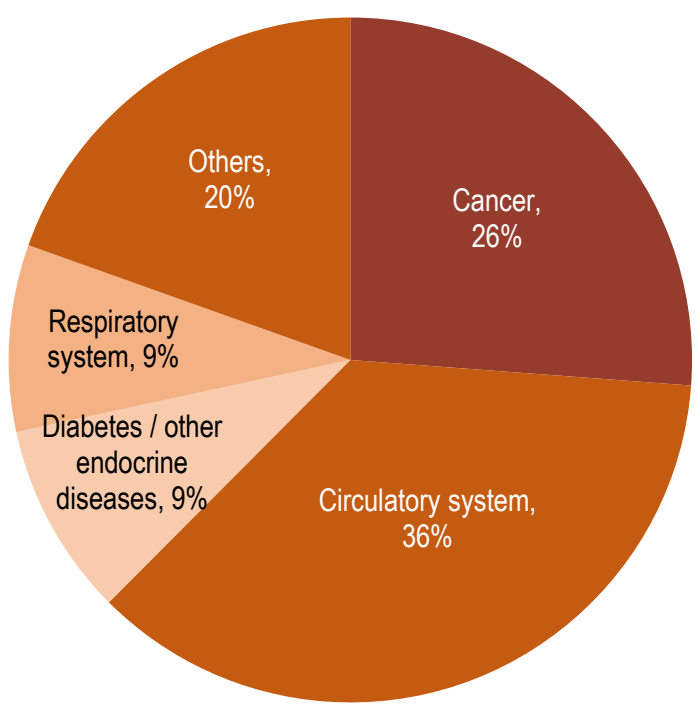

Note: The 2019 OECD/Eurostat list of preventable and treatable causes of death classifies specific diseases and injuries as preventable and/or treatable. For example, lung cancer is classified as preventable; whereas breast and colorectal cancers are classified as treatable. Source: OECD calculations, based on WHO Mortality Database.

Figure 3.9. Mortality rates from avoidable causes, 2017 (or nearest year)

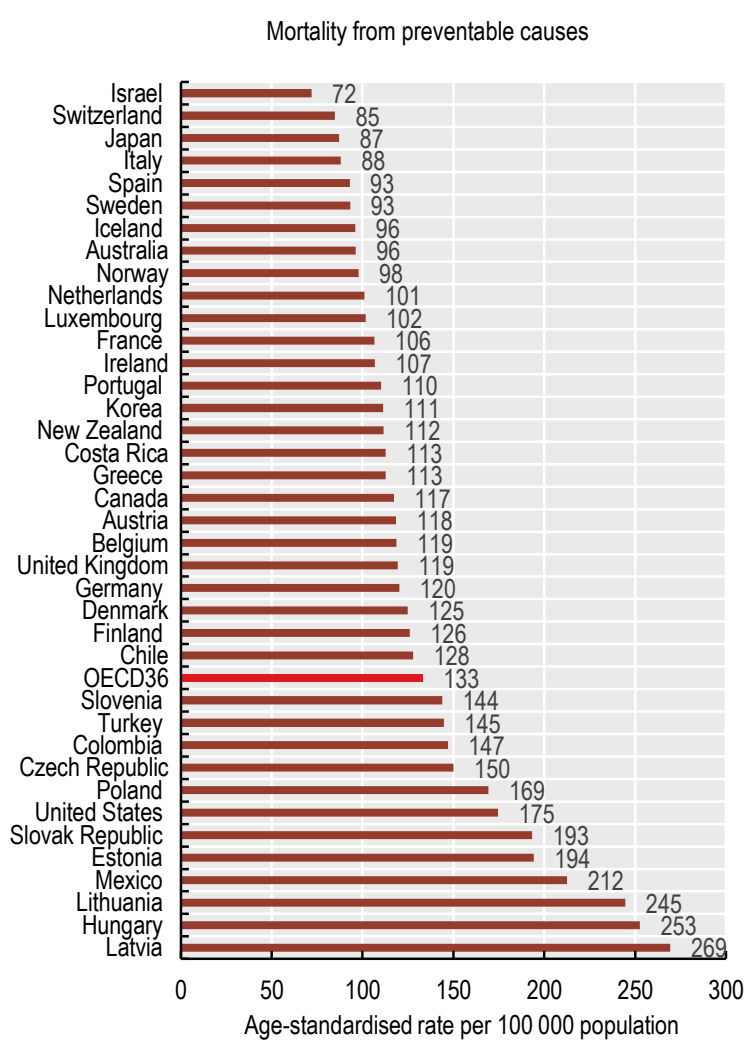

Source: OECD calculations, based on WHO Mortality Database.

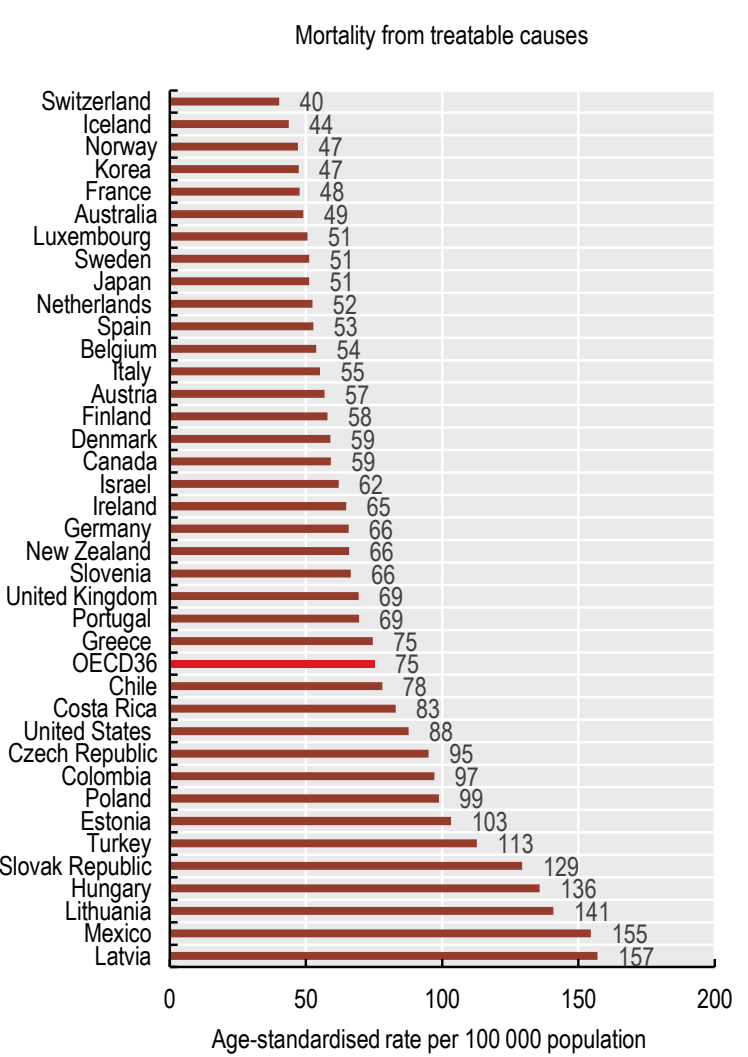

StatLink क्ञाs https://doi.org/10.1787/888934014973 
Circulatory diseases - notably heart attack and stroke remain the main cause of mortality in most OECD countries, accounting for almost one in three deaths across the OECD. While mortality rates have declined in most OECD countries over time, population ageing, rising obesity and diabetes rates may hamper further reductions (OECD, 2015[1]). Indeed, slowing improvements in heart disease and stroke are one of the principal causes of a slowdown in life expectancy gains in many countries (Raleigh, 2019[2]).

Heart attacks and other ischaemic heart diseases (IHDs) accounted for 11\% of all deaths in OECD countries in 2017. IHDs are caused by the accumulation of fatty deposits lining the inner wall of a coronary artery, restricting blood flow to the heart. Mortality rates are $80 \%$ higher for men than women across OECD countries, primarily because of a greater prevalence of risk factors among men, such as smoking, hypertension and high cholesterol.

Among OECD countries, central and eastern European countries have the highest IHD mortality rates, particularly in Lithuania where there are 383 deaths per 100000 people (age-standardised). Rates are also very high in the Russian Federation. Japan, Korea and France have the lowest rates among OECD countries, at about one quarter of the OECD average and less than a tenth of rates in Lithuania and the Russian Federation (Figure 3.10).

Since 2000, IHD mortality rates have declined in nearly all OECD countries, with an average reduction of $42 \%$. Declines have been most marked in France, Denmark, the Netherlands, Estonia and Norway, where rates fell by over $60 \%$. Mexico is the one country where IHD mortality rates have increased; this is closely linked to increasing obesity rates and diabetes prevalence. Survival rates following a heart attack are also much lower in Mexico than in all other OECD countries (see indicator on "Mortality following acute myocardial infarction" in Chapter 6).

Stroke (or cerebrovascular disease) was the underlying cause of $7 \%$ deaths across the OECD in 2017. Disruption of the blood supply to the brain causes a stroke. As well as causing many deaths, strokes have a significant disability burden. Mortality rates are particularly high in Latvia and
Lithuania, at over double the OECD average. Rates are also high in the partner countries such as South Africa and the Russian Federation (Figure 3.11).

Mortality rates from stroke have fallen in all OECD and partner countries since 2000, with an average reduction of $47 \%$. Declines have been slower in the Slovak Republic and Chile, however, at less than $15 \%$. For strokes, as for IHD, a reduction in certain risk factors - notably smoking - has contributed to fewer deaths, alongside improved survival rates following an acute episode, reflecting better quality of care (see indicators on "Mortality following ischaemic stroke" and "Mortality following acute myocardial infarction (AMI)" in Chapter 6).

\section{Definition and comparability}

Mortality rates are based on numbers of deaths registered in a country in a year divided by the size of the corresponding population. The rates have been directly age-standardised to the 2010 OECD population (available at http://oe.cd/mortality) to remove variations arising from differences in age structures across countries and over time. The source is the WHO Mortality Database.

Deaths from ischaemic heart disease are classified to ICD-10 codes I20-I25, and cerebrovascular disease to I60-I69.

\section{References}

[1] OECD (2015), Cardiovascular Disease and Diabetes: Policies for Better Health and Quality of Care, OECD Publishing, Paris, http://dx.doi.org/10.1787/9789264233010-en.

[2] Raleigh, V. (2019), "Trends in life expectancy in EU and other OECD countries: Why are improvements slowing?", OECD Health Working Papers, No. 108, OECD Publishing, Paris, https://doi.org/10.1787/223159ab-en. 
Figure 3.10. Heart attacks and other ischaemic heart disease mortality, 2017 and change 2000-17 (or nearest year)
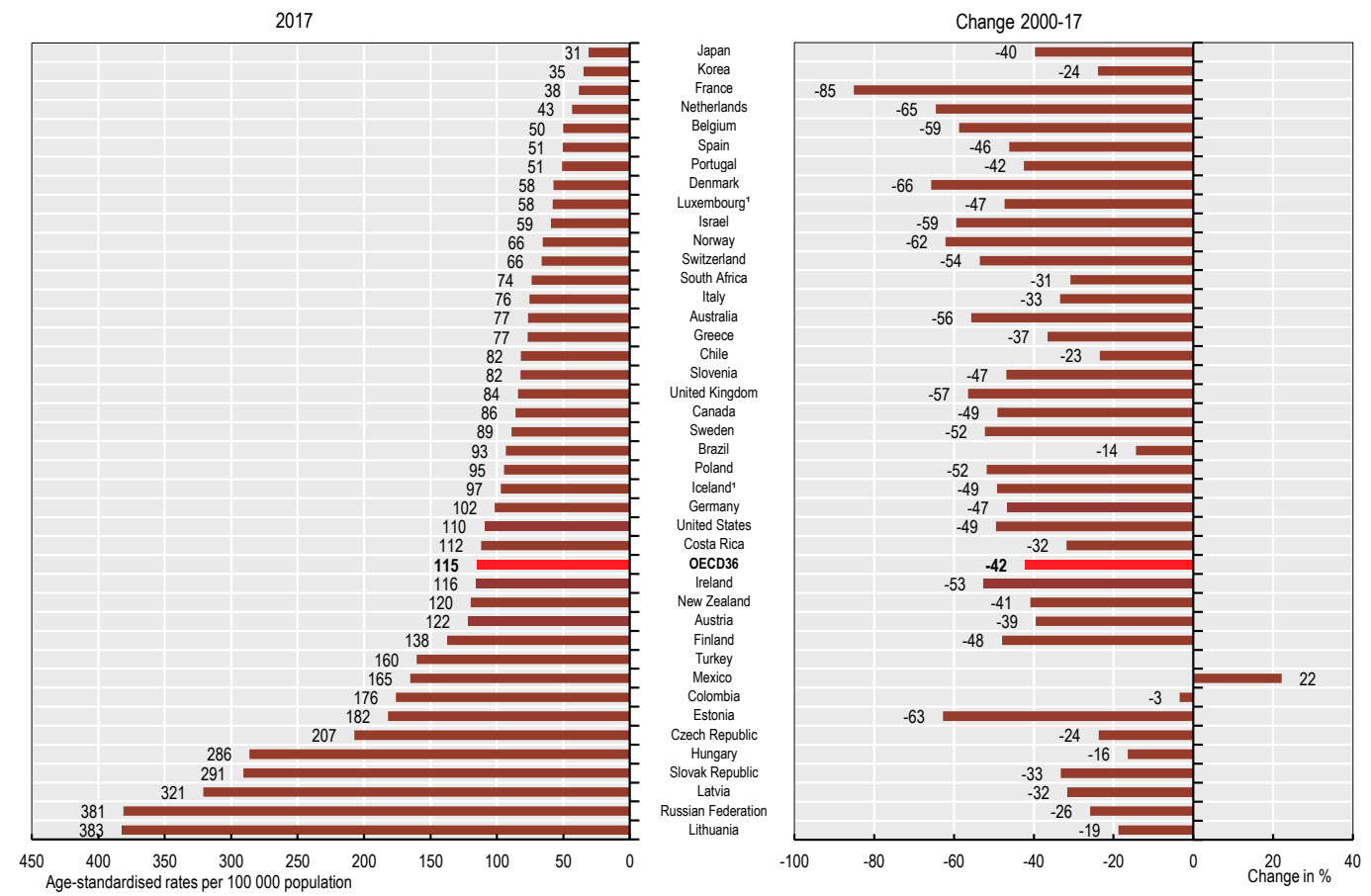

1. Three-year average.

Source: OECD Health Statistics 2019.

Figure 3.11. Stroke mortality, 2017 and change 2000-17 (or nearest year)
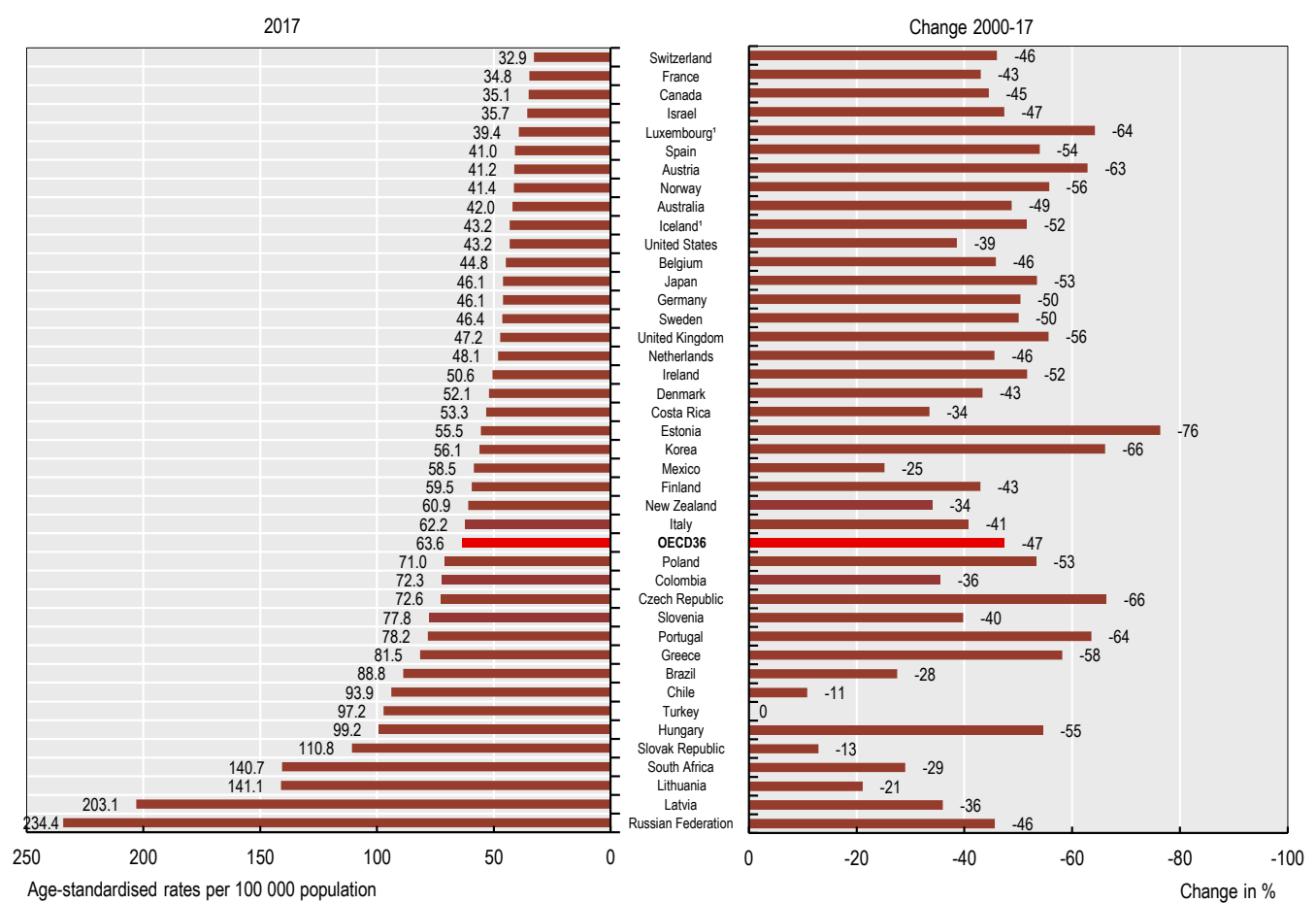

1. Three-year average.

Source: OECD Health Statistics 2019. 
Cancer is the second leading cause of mortality in OECD countries after circulatory diseases, accounting for $25 \%$ of all deaths in 2017. Further, there was an estimated 7.5 million newly diagnosed cases of cancer across the OECD. Common cancers are lung cancer (21.5\%), colorectal cancer (11\%), breast cancer (14.5\% among women) and prostate cancer (9.4\% amongst men). These four represent more than $40 \%$ of all cancers diagnosed in OECD countries. Mortality rates from cancer have fallen in all OECD countries since 2000, although across the OECD the decline has been more modest than for circulatory diseases.

Cancer incidence rates vary across OECD countries, from over 400 new cases per 100000 people in Australia and New Zealand, to around 200 cases or fewer in Mexico and Chile (Figure 3.12). Cancer incidence is also comparatively low in all partner countries. Cross-country variations in incidence rates, though, reflect differences not only in new cancers occurring each year but also differences in national cancer screening policies, quality of cancer surveillance and reporting. High rates in Australia and New Zealand are mainly driven by the high incidence of non-melanoma skin cancer.

Mortality rates from cancer averaged 201 deaths per 100000 people across the OECD (Figure 3.13). They were highest in Hungary, the Slovak Republic and Slovenia (above 240); lowest in Mexico, Turkey and Korea (165 or less). Among partner countries with comparable data, cancer mortality rates were also comparatively low in Colombia, Costa Rica and Brazil.

Earlier diagnosis and treatment significantly increase cancer survival rates. This partly explains why, for example, Australia and New Zealand have below average mortality rates despite having the highest rates of cancer incidence. In both countries, five-year net survival from common cancers is also above the OECD average (see various indicators on survival following cancer in Chapter 6).

Cancer incidence rates are higher for men than women in all OECD and partner countries; cancer mortality rates are also higher for men except in Mexico, Iceland, Indonesia and India. Greater prevalence of risk factors among men notably smoking and alcohol consumption - drive much of this gender gap in cancer incidence and mortality.

Lung cancer is the main cause of death for both men and women, with smoking the main risk factor. It accounts for $25 \%$ of cancer deaths among men and $17 \%$ among women (Figure 3.14). Colorectal cancer is a major cause of death for men and women (second main cause for men and third for women, accounting for about $10 \%$ of cancer-related deaths for each sex). Apart from age and genetic factors, a diet high in fat and low in fibre, lack of physical activity, obesity, smoking and alcohol consumption all increase the risk of developing the illness.

Breast cancer is the second most common cause of cancer mortality in women (14.5\% of deaths). While incidence rates for breast cancer have increased over the past decade, mortality has declined or stabilised, indicative of earlier diagnosis and treatment, and consequently higher survival rates (see indicator on "Breast cancer outcomes" in Chapter 6). Prostate cancer is the third most common cause of cancer mortality among men, accounting for just over $10 \%$ of all cancer-related deaths.

\section{Definition and comparability}

Cancer incidence rates are based on numbers of new cases of cancer registered in a country in a year divided by the population. Differences in the quality of cancer surveillance and reporting across countries may affect the comparability of data. Rates have been agestandardised based on Segi's world population to remove variations arising from differences in age structures across countries and over time. Data come from the International Agency for Research on Cancer (IARC), GLOBOCAN 2018. These data may differ from national estimates due to differences in methodology. The incidence of all cancers is classified to ICD-10 codes C00-C97.

Mortality rates are based on numbers of deaths registered in a country in a year divided by the size of the corresponding population. The rates have been directly age-standardised to the 2010 OECD population (available at http://oe.cd/mortality). The source is the WHO Mortality Database.

Deaths from all cancers are classified to ICD-10 codes C00-C97. The international comparability of cancer mortality data can be affected by differences in medical training and practices as well as in death certification across countries.

\section{References}

[1] GLOBOCAN (2018), Cancer Today, https://gco.iarc.fr/today/home.

[2] OECD (2013), Cancer Care: Assuring Quality to Improve Survival, OECD Health Policy Studies, OECD Publishing, Paris, https:// doi.org/10.1787/9789264181052-en. 
Figure 3.12. All cancer incidence by sex, 2018 (estimated)

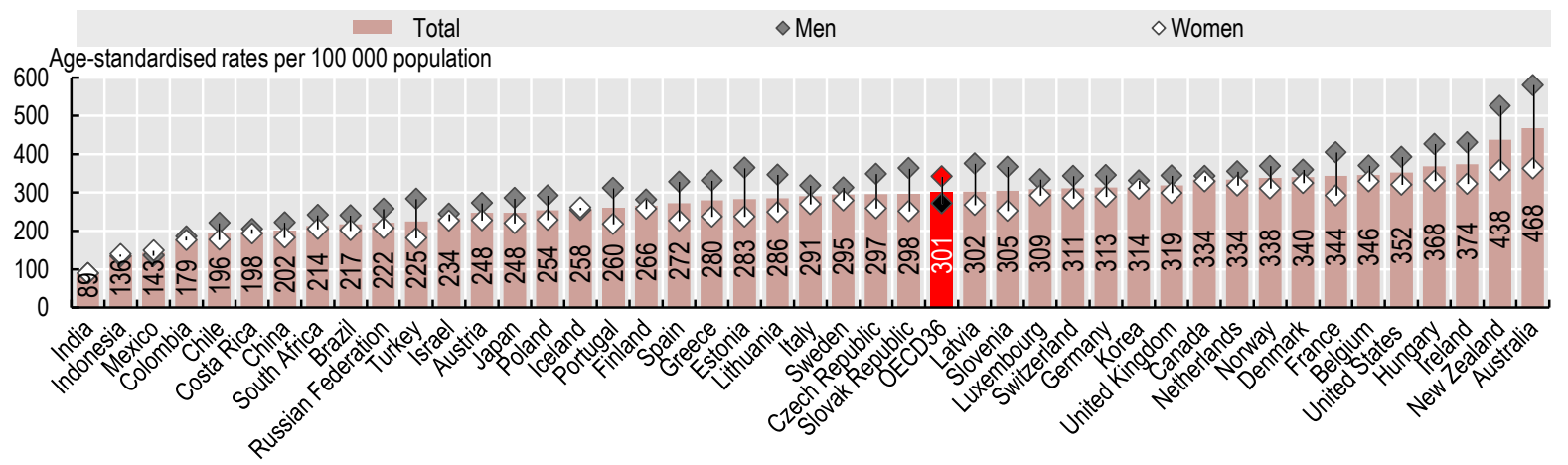

Source: International Agency for Research on Cancer (IARC), GLOBOCAN 2018.

Figure 3.13. Cancer mortality, by sex, 2017 (or nearest year)

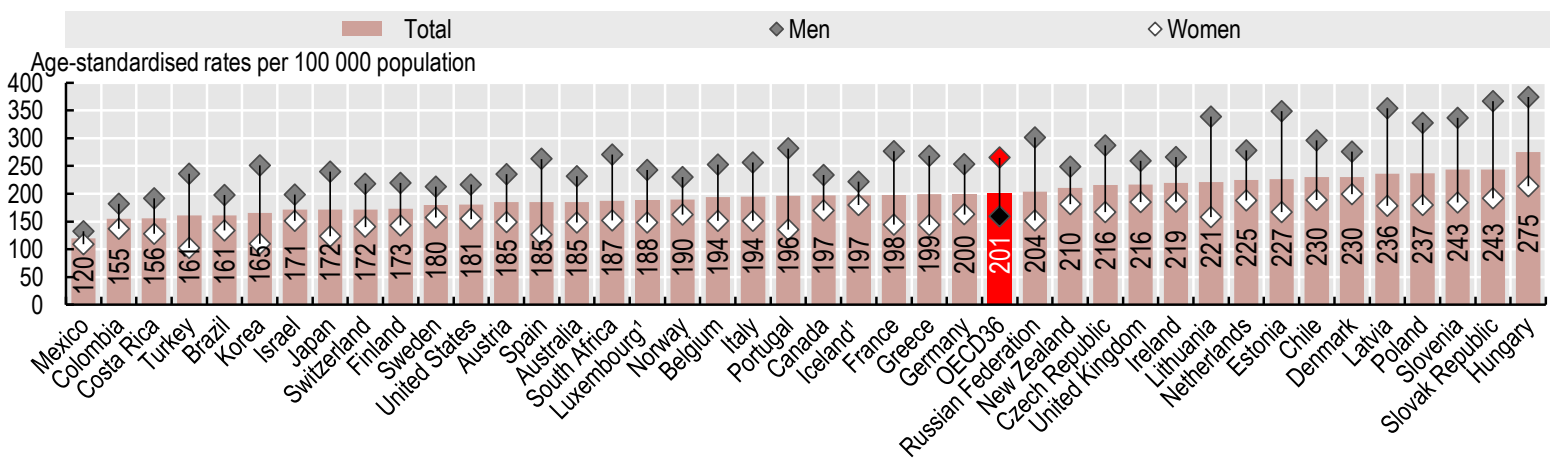

1. Three-year average.

Source: OECD Health Statistics 2019.

StatLink तीारम https://doi.org/10.1787/888934015049

Figure 3.14. Main causes of cancer mortality across OECD countries, by sex, 2017 (or nearest year)
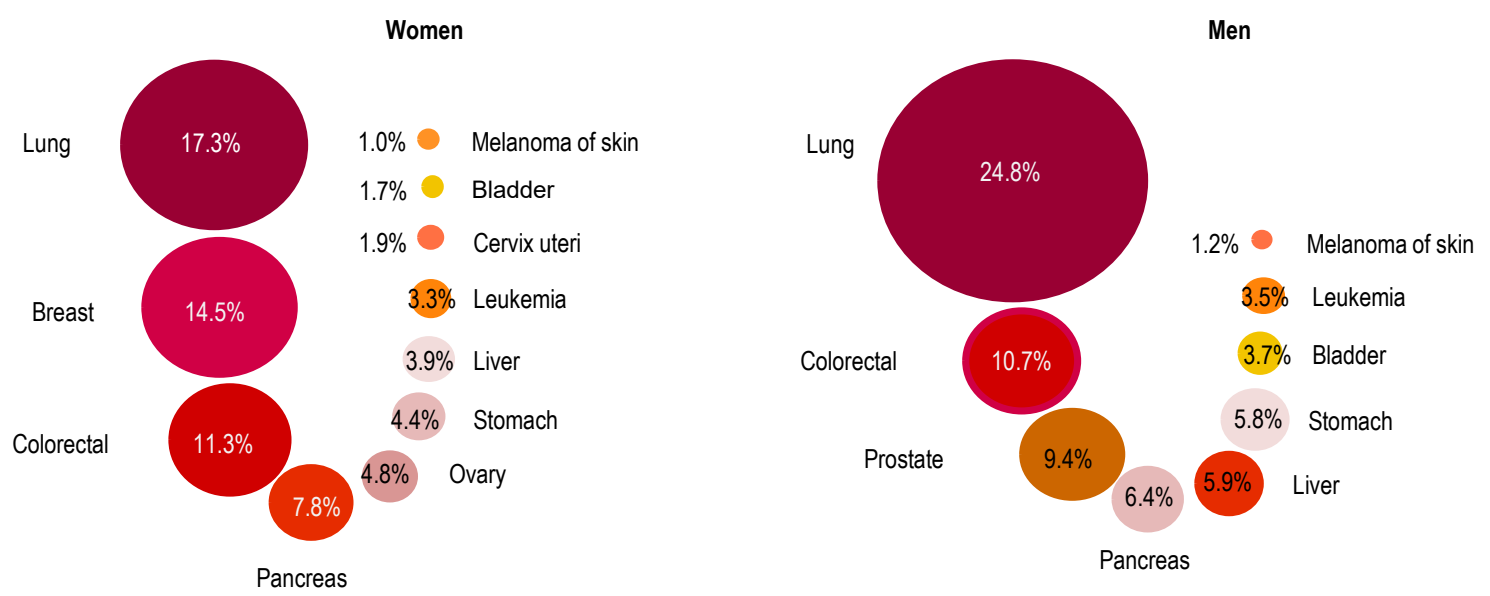

Note: Proportion of the sums of cancer-related deaths across OECD countries, by sex.

Source: OECD Health Statistics 2019. 
Chronic diseases such as cancer, heart attack and stroke, chronic respiratory problems and diabetes are not only the leading causes of death across OECD countries. They also represent a major disability burden amongst the living. Many chronic diseases are preventable, by modifying major risk factors such as smoking, alcohol use, obesity and physical inactivity.

Almost one third of people aged 15 years and over reported living with two or more chronic conditions, on average across 27 OECD countries (Figure 3.15). In Germany and Finland, this figure rises to almost one in two. Multimorbidity is far more common among older age groups - on average, $58 \%$ of adults aged 65 or over reported living with two or more chronic diseases, and this figure rises to $70 \%$ or more in Portugal, Poland, Hungary, the Slovak Republic and Germany. This compares with $24 \%$ for people aged less than 65 years reporting two or more chronic conditions.

Socioeconomic disparities are also large: on average across OECD countries, $35 \%$ of people in the lowest income quintile report two or more chronic conditions, compared with $24 \%$ of people in the highest income quintile (Figure 3.16). This income gradient is largest in Hungary, Slovenia and Latvia.

Diabetes is a chronic condition with a particularly large disability burden, causing cardiovascular disease, blindness, kidney failure and lower limb amputation. It occurs when the body is unable to regulate excessive glucose levels in the blood. In 2017, about 98 million adults - or $6.4 \%$ of the adult population - were living with diabetes across OECD countries (Figure 3.17). In addition, a further 39 million adults were estimated to have undiagnosed diabetes (International Diabetes Federation, 2017[1]).

Among OECD countries, diabetes prevalence is highest in Mexico, Turkey and the United States, with over $10 \%$ of adults living with diabetes (age-standardised data). For partner countries, diabetes prevalence is also high in India and China, at around $10 \%$.

Age-standardised diabetes prevalence rates have stabilised in many OECD countries, especially in western Europe, but have increased markedly in Turkey and most partner countries. Such upward trends are due in part to rising rates of obesity and physical inactivity, and to their interactions with population ageing (NCD Risk Factor Collaboration, 2016[2])

Diabetes is much more common among older people, and slightly more men than women have the condition. Diabetes also disproportionately affects those from disadvantaged socio-economic groups. The economic burden of diabetes is substantial. In OECD countries an estimated USD 572 billion was spent on treating diabetes and preventing complications (International Diabetes Federation, 2017[1]).

\section{Definition and comparability}

Data on multiple chronic diseases come from three different sources: Eurostat's European Health Interview Survey (EHIS-2) for European countries; the Medical Panel Expenditures Survey (MEPS) 2016 for the United States; and the Canadian Community Health Survey (CCHS) 2015-16 for Canada. The following chronic diseases and conditions are available in each survey:

- EHIS-2: asthma (1), chronic bronchitis/COPD/ emphysema (2), heart attack and chronic consequences (3), coronary heart disease (4), hypertension (5), stroke and chronic consequences (6), arthrosis, low back disorder (7), neck disorder (8), diabetes (9), allergy (10), cirrhosis of the liver (11), urinary incontinence (12), kidney problems (13) and depression (14).

- MEPS and CCHS: (1) - (6), (9) and (14).

As fewer conditions are available for both Canada and the United States, multi-morbidity prevalence is mechanically lower for these countries, and thus not comparable with European data.

Sources and methods used by the International Diabetes Federation (IDF) are outlined in the Diabetes Atlas, 8th edition (International Diabetes Federation, 2017). The IDF produces estimations based on a variety of sources that met several criteria for reliability. The majority were national health surveys and peerreviewed articles. Age-standardised rates were calculated using the world population based on the distribution provided by the WHO. Adult population here covers those aged between 20 and 79 with Type 1 or Type 2 diagnosed diabetes.

\section{References}

[1] International Diabetes Federation (2017), IDF Diabetes Atlas, 8th edition, International Diabetes Federation, Brussels.

[2] NCD Risk Factor Collaboration (2016), "Worldwide trends in diabetes since 1980: a pooled analysis of 751 populationbased studies with 4.4 million participants", Lancet, Vol. 387, pp. 1513-1530, http://dx.doi.org/10.1016/S0140-6736(16)006 18-8. 
Figure 3.15. People living with two or more chronic diseases, by age, 2014

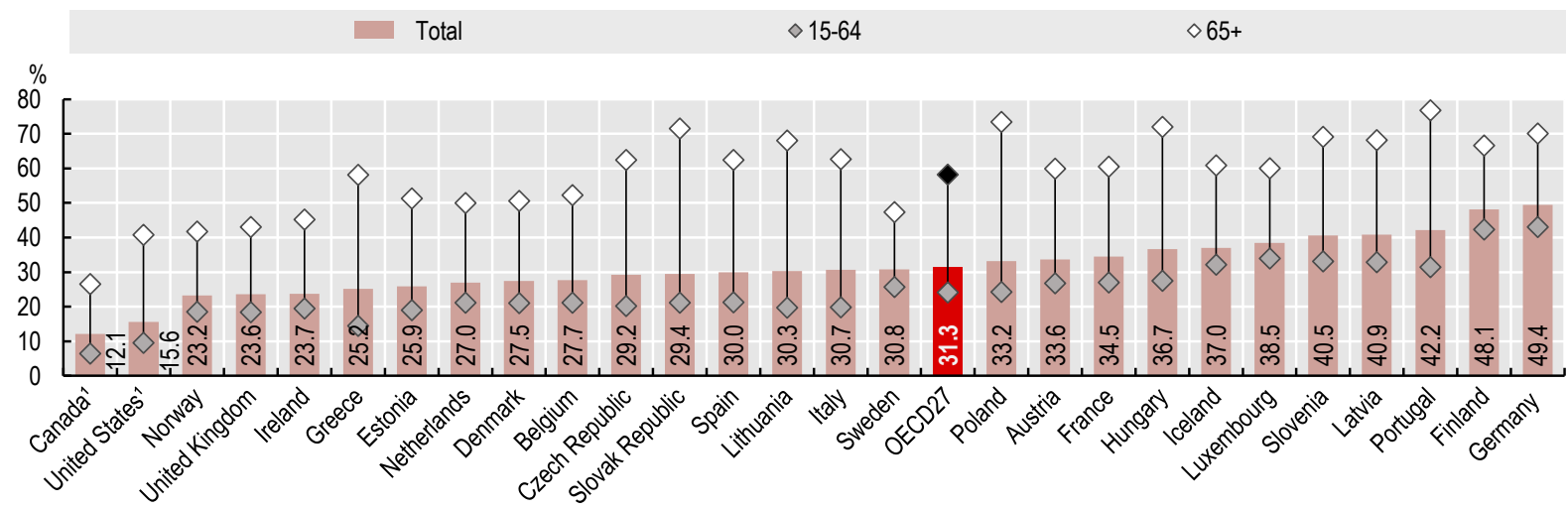

1. Results not directly comparable with those for other countries, due to differences in the variable definition ( 8 chronic conditions considered instead of 14), resulting in a downward bias.

Source: EHIS-2 2014 and other national health surveys.

StatLink क्ञाज https://doi.org/10.1787/888934015087

Figure 3.16. People living with two or more chronic diseases, by income level, 2014

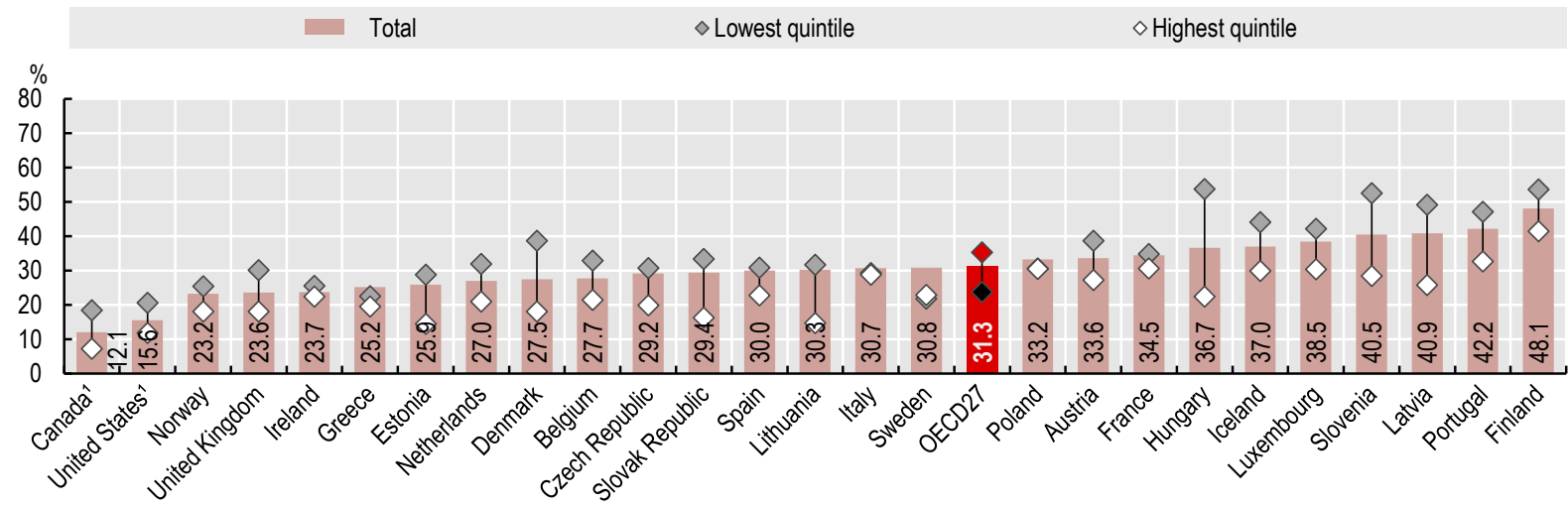

1. Results not directly comparable with those for other countries (see note in Figure 3.15).

Source: EHIS-2 2014 and other national health surveys.

Figure 3.17. Type I and II diabetes prevalence among adults, 2017 (or nearest year)

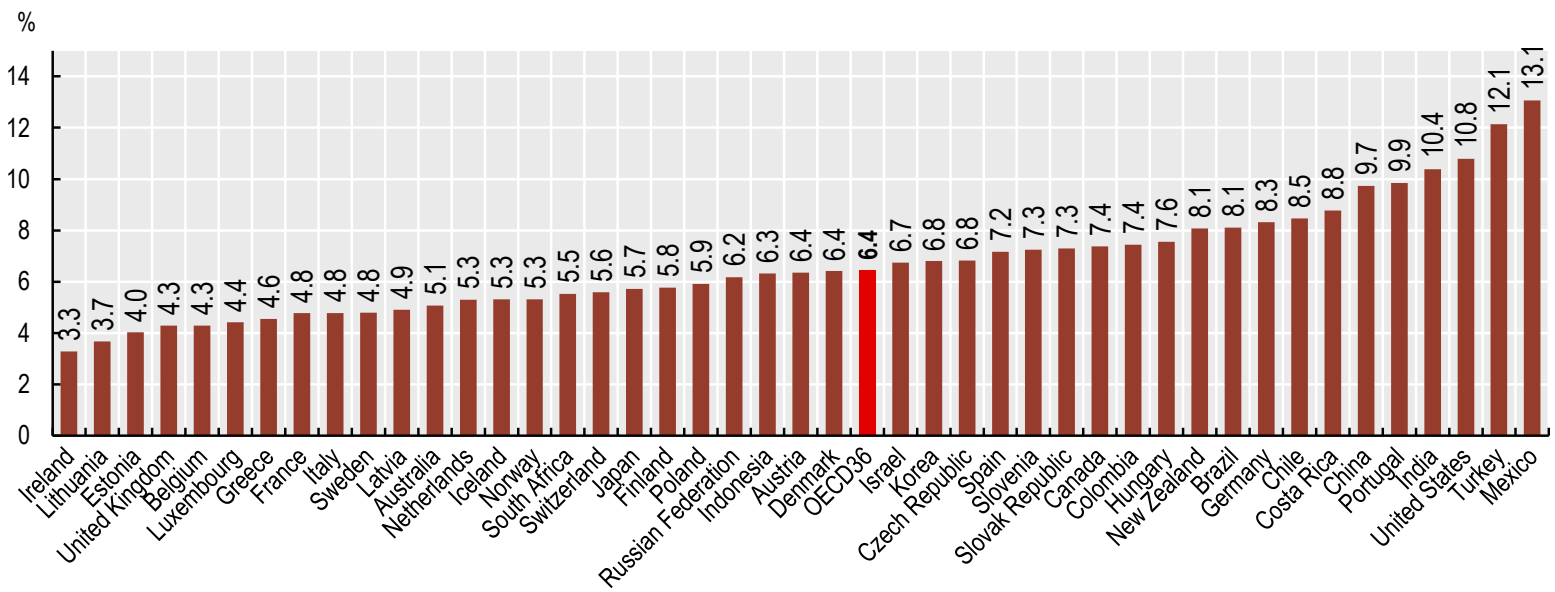


Inadequate living conditions, extreme poverty and socioeconomic factors affect the health of mothers and newborns. However, effective health systems can greatly limit the number of infant deaths, particularly by addressing life-threatening issues during the neonatal period. Around two-thirds of deaths during the first year of life occur before an infant reaches 28 days (neonatal mortality), primarily from congenital anomalies, prematurity and other conditions arising during pregnancy. For deaths beyond these first critical weeks (post-neonatal mortality), there tends to be a greater range of causes - the most common being Sudden Infant Death Syndrome (SIDS), birth defects, infections and accidents.

Infant mortality rates are low in most OECD countries, at less than five deaths per 1000 live births in all countries except Mexico, Turkey and Chile (Figure 3.18). Within OECD countries, though, infant mortality rates are often higher among indigenous populations and other vulnerable groups - as observed in Australia, Canada, New Zealand and the United States (Smylie et al., 2010[1]). In partner countries, infant mortality remains above 20 deaths per 1000 live births in India, South Africa and Indonesia, and above ten deaths in Colombia and Brazil. Infant mortality rates have fallen in all OECD and partner countries since 2000, with reductions generally largest in countries with historically the highest rates.

Despite this progress in reducing infant deaths, the increasing numbers of low birthweight infants are a concern in some OECD countries. On average, one in 15 babies born in OECD countries (6.5\% of all births) weighed less than 2500 grammes at birth in 2017 (Figure 3.19). Low birthweight infants have a greater risk of poor health or death, require a longer period of hospitalisation after birth, and are more likely to develop significant disabilities later in life. Risk factors for low birthweight include maternal smoking, alcohol consumption and poor nutrition during pregnancy, low body mass index, lower socio-economic status, having had in-vitro fertilisation treatment and multiple births, and a higher maternal age. The increased use of delivery management techniques such as induction of labour and caesarean delivery, which have contributed to increased survival rates of low birthweight infants, may also explain the rise in their numbers.

Japan, Greece and Portugal have the greatest share of low birthweight infants among OECD countries. There are fewer low birthweight infants in the Nordic (Iceland, Finland, Sweden, Norway, Denmark) and Baltic (Estonia, Latvia, Lithuania) countries. In 23 of the 36 OECD countries, the proportion of low birthweight infants has increased since
2000, most markedly in Korea. Among partner countries, Indonesia and Colombia have a high share.

\section{Definition and comparability}

The infant mortality rate is the number of deaths of children under one year of age per 1000 live births. Some of the international variation in infant mortality rates may be due to variations in registering practices for very premature infants. While some countries register all live births including very small babies with low odds of survival, several countries apply a minimum threshold of a gestation period of 22 weeks (or a birth weight threshold of 500 grammes) for babies to be registered as live births (Euro-Peristat, 2018[2]). To remove this data comparability limitation, data presented in this section are based on a minimum threshold of 22 weeks' gestation period (or $500 \mathrm{~g}$ birth weight) for a majority of OECD countries that have provided these data. However, the data for ten countries (Australia, Canada, Greece, Ireland, Italy, Lithuania, Luxembourg, Mexico, Norway and Portugal) continue to be based on all registered live births (i.e. with no minimum threshold of gestation period or birthweight), resulting in potential over-estimation.

Low birth weight is defined by WHO as the weight of an infant at birth of less than $2500 \mathrm{~g}$ (5.5 pounds) irrespective of the gestational age. This threshold is based on epidemiological observations regarding the increased risk of death to the infant. Despite the widespread use of this $2500 \mathrm{~g}$ limit for low birth weight, physiological variations in size occur across different countries and population groups, and these need to be taken into account when interpreting differences (Euro-Peristat, 2018[2]). The number of low weight births is expressed as a percentage of total live births.

\section{References}

[2] Euro-Peristat (2018), European Perinatal Health Report: Core indicators of the health and care of pregnant women and babies in Europe in 2015.

[1] Smylie, J. et al. (2010), "Indigenous birth outcomes in Australia, Canada, New Zealand and the United States - an overview", Open Womens Health, Vol. 4, pp. 7-17. 
Figure 3.18. Infant mortality, 2017 (or nearest year)

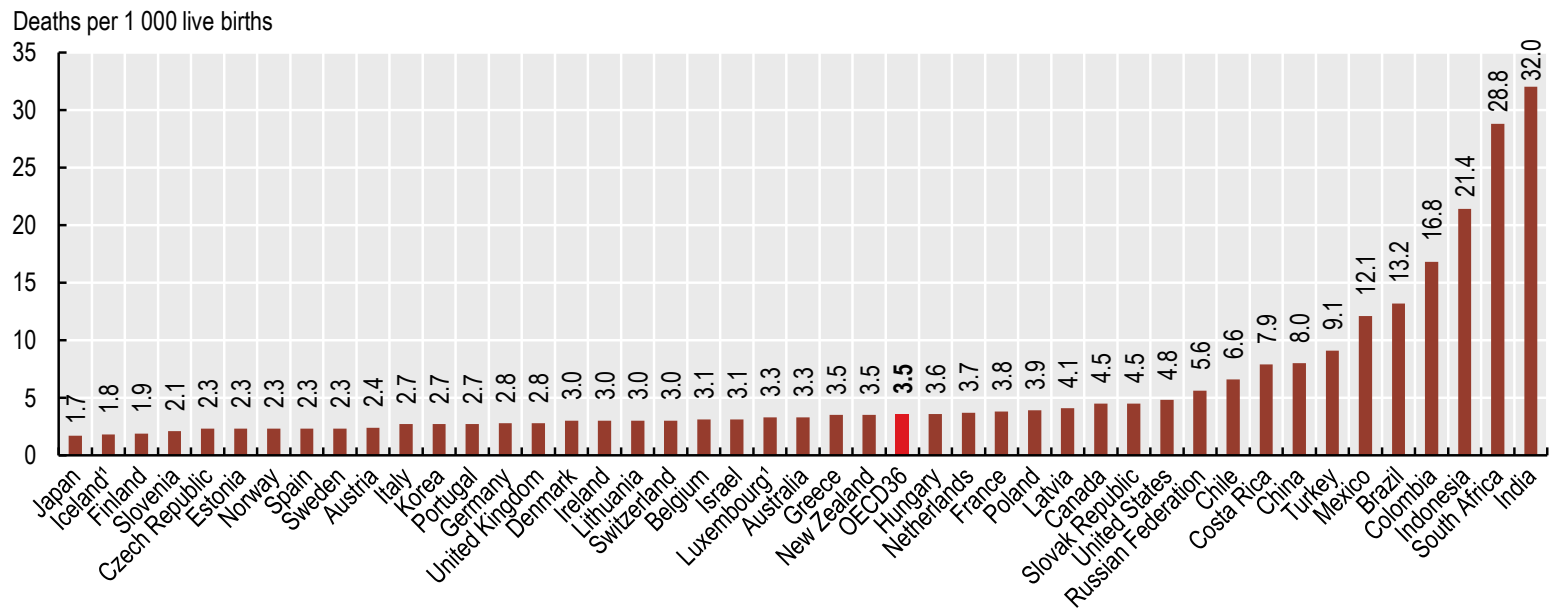

Figure 3.19. Low birthweight infants, 2017 and change 2000-17 (or nearest year)

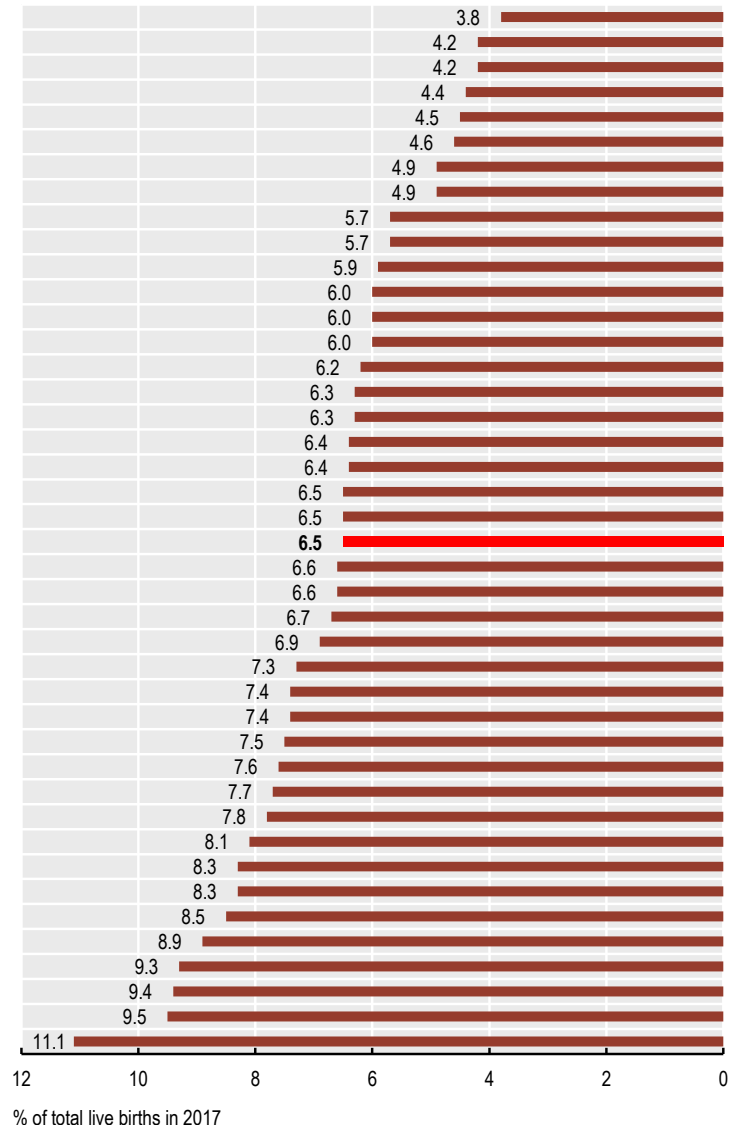

Source: OECD Health Statistics 2019

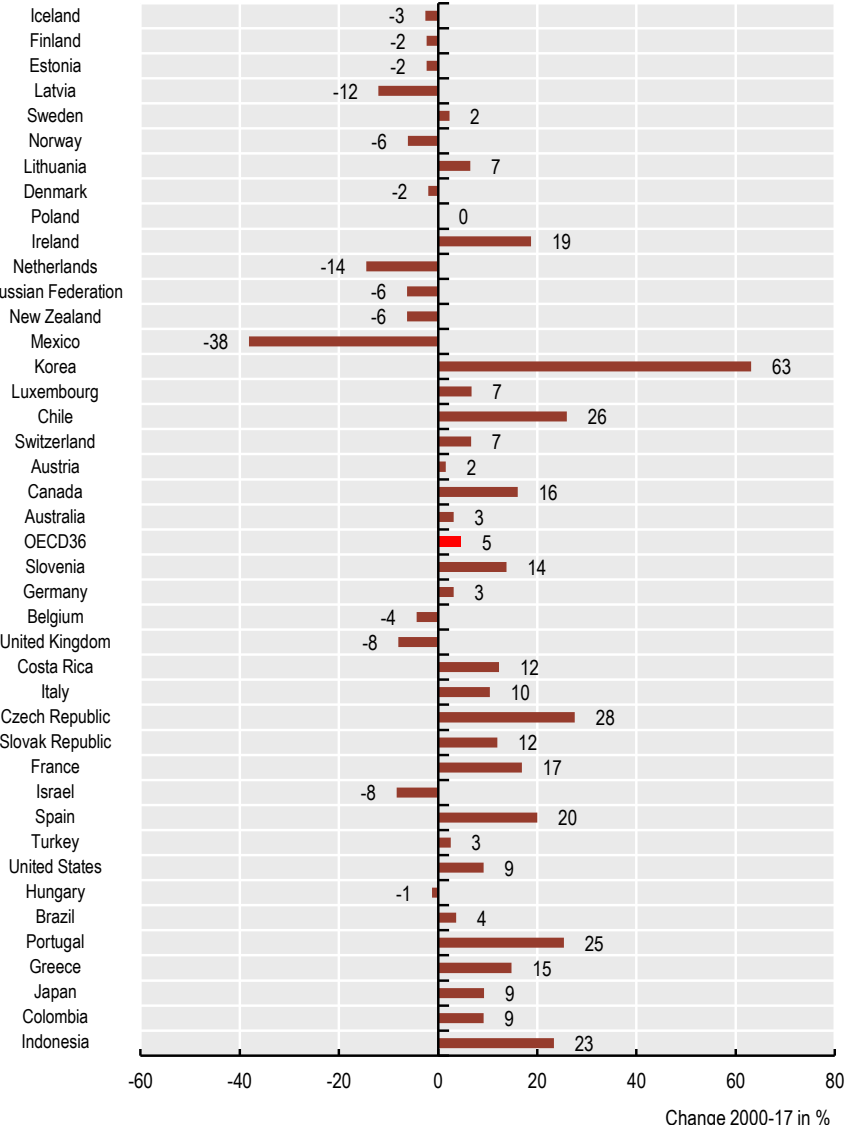

StatLink क्ताs https://doi.org/10.1787/888934015163 
Good mental health is vital for people to be able to lead healthy, productive lives, but an estimated one in two people experience a mental health problem in their lifetime (OECD, 2015[1]). When people are living with a mental health problem it can have a significant impact on their daily life, contributing to worse educational outcomes, higher rates of unemployment, and poorer physical health. Figure 3.22 shows the impact of peoples' health on their daily activities and ability to work; people who reported a mental health problem were significantly more likely to say that their health had a negative impact on their daily life. In Norway and France, more than $50 \%$ of respondents who had been told by a doctor that they had a mental health problem felt that their ability to work or daily activities were limited. More can be done to help people participate in activities that matter to them, even if they have a mental health problem, including promoting timely access to treatment and integrating mental health and employment services.

Without effective treatment or support, mental health problems can have a devastating effect on people's lives, and can even lead to death by suicide. While there are complex social and cultural reasons affecting suicidal behaviours, suffering from a mental health problem also increases the risk of dying from suicide (OECD/EU, 2018[2]). A higher suicide rate also contributes to a significantly higher rate of overall mortality for people with serious mental disorders, as discussed in Chapter 6. In 2017, there were 11.2 deaths by suicide per 100000 population in OECD countries. Figure 3.20 shows that suicide rates were lowest in Turkey and Greece, where there were fewer than five deaths by suicide per 100000 population in 2017. Korea and Lithuania had the highest suicide rate, with 24.6 and 24.4 deaths per 100000 population, respectively. The rate of suicide was higher among men than women in all countries; in Lithuania, the suicide rate among men was more than five times higher than that for women.

Suicide rates have decreased in almost all OECD countries, falling by more than 30\% between 1990 and 2017. In some countries, the declines have been significant, including in Finland, Switzerland and Slovenia, where suicide rates have fallen by more than $40 \%$. Other countries such as Chile and Korea saw suicide peaks in the past decade followed by a decline in more recent years (Figure 3.21). In Switzerland, suicide has fallen by $48 \%$ since 1990 ; rates of 'assisted suicide' are rising, mainly in older people, but since 2009 assisted suicides have been excluded from overall suicide data, explaining the sharp decline the year the reporting changed. Switzerland has taken steps to reduce deaths by suicide, such as introducing a suicide prevention action plan in 2016 that included providing fast access to mental health support, seeking to reduce stigma around suicide, and raising awareness of suicide risks. Finland, where a particularly significant decline in suicide was seen in the early 1990s, has recently moved away from stand-alone suicide prevention plans and includes suicide reduction in broader mental health strategies, focusing on improving treatment for mental illness, and implementing a network for coordinating suicide prevention (OECD/EU, 2018[2]).

\section{Definition and comparability}

The registration of suicide is a complex procedure, affected by factors such as how intent is ascertained, who is responsible for completing the death certificate, and cultural dimensions including stigma. Caution is therefore needed when comparing rates between countries. Age-standardised mortality rates are based on numbers of deaths divided by the size of the corresponding population. The source is the WHO Mortality Database; suicides are classified under ICD-10 codes $\mathrm{X} 60-\mathrm{X} 84, \mathrm{Y} 870$.

Figure 3.22 uses data from the Commonwealth Fund 2016 International Health Policy Survey of Adults. It is possible to identify adults who responded "yes" to "Have you ever been told by a doctor that you have depression, anxiety or other mental health problems" and track their responses to other survey questions. This figure shows the rate of responses to the question "Does your health keep you from working full-time or limit your ability to do housework or other daily activities?". Respondents who answered "yes" to this question are identified as "with a mental health problem" and those who responded "no" as "no mental health problem". Respondents identified as "no mental health problem" may have another health problem. The data have shortcomings, including some low response rates and a limited sample size (see also Box 2.4 in Chapter 2). Interpretation of questions may be different across countries; further, it is not known whether respondents were living with a mental health problem at the time of responding, and self-reported prevalence can be affected by stigma around mental health problems. The rate at which respondents reported having been told they had a mental health problem was fairly consistent with national prevalence estimates except for France, where respondents were significantly less likely to report a mental health problem than other national estimates suggest. 
Figure 3.20. Suicide rates, 2017 (or nearest year)

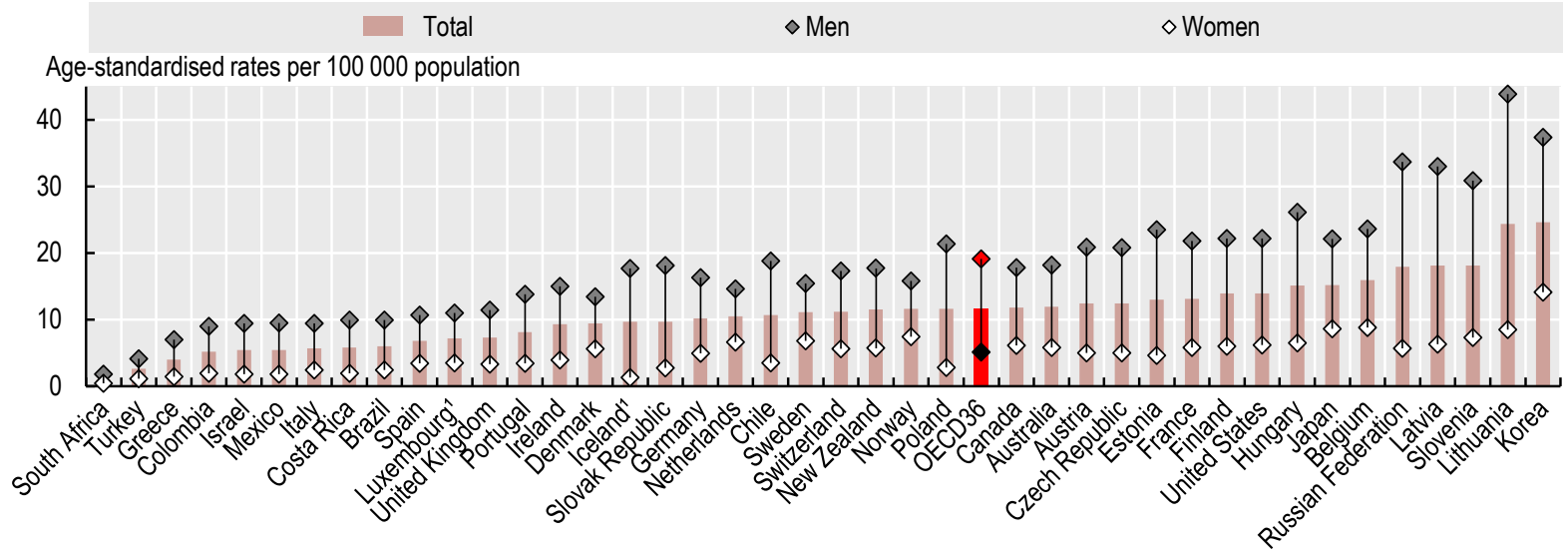

1. Three-year average.

Source: OECD Health Statistics 2019.

Figure 3.21. Trends in suicide, selected OECD countries, 1990-2017 (or nearest year)
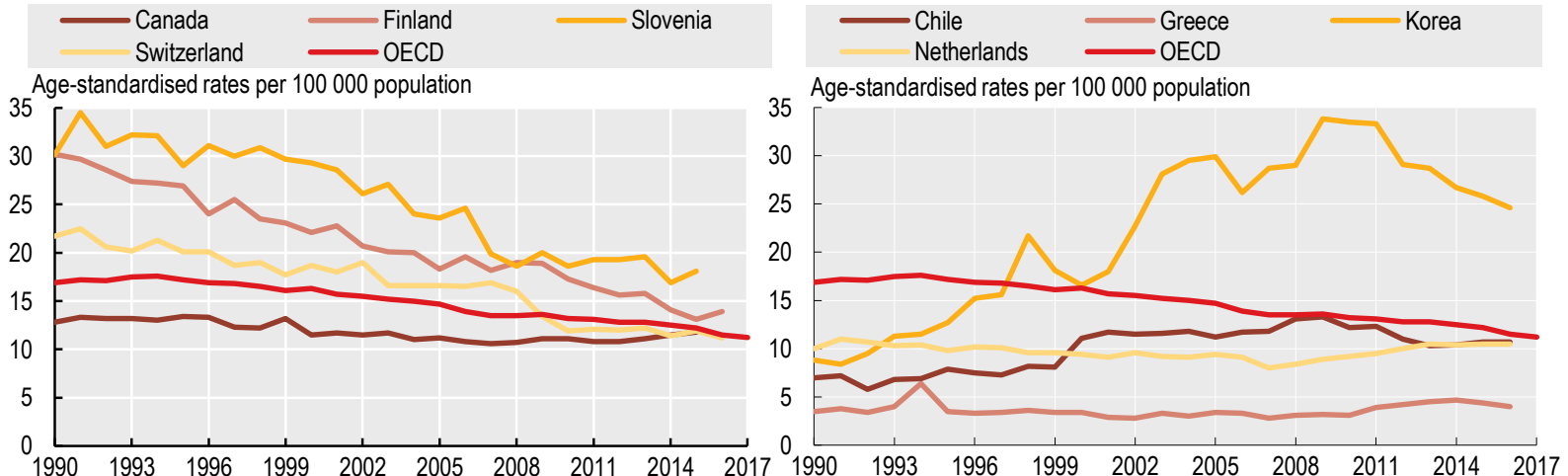

Source: OECD Health Statistics 2019.

StatLink क्ञाs ht htps://doi.org/10.1787/888934015201

Figure 3.22. People whose health kept them from working full-time or limited their daily activities, 2016

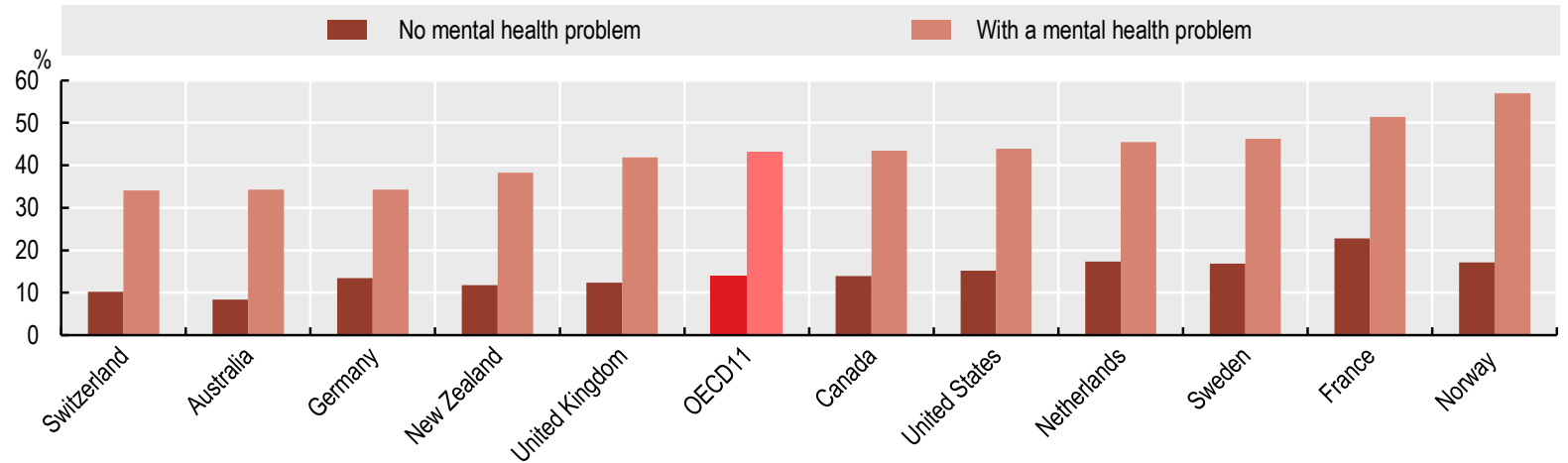

Source: Commonwealth Fund International Health Policy Survey 2016. 
How individuals assess their own health provides a holistic overview of both physical and mental health. By adding such a perspective on quality of life, it complements life expectancy and mortality indicators that only measure survival. Further, despite its subjective nature, self-rated health has proved to be a good predictor of future health care needs and mortality (Palladino et al., 2016[1]).

Most OECD countries conduct regular health surveys that include asking respondents how, in general, they would rate their health. For international comparisons, socio-cultural differences across countries may complicate cross-country comparisons of self-assessed health. Differences in the formulation of survey questions, notably in the survey scale, can also affect comparability of responses. Finally, since older people generally report poorer health and more chronic diseases than younger people do, countries with a larger proportion of elderly people are likely to have a lower proportion of people reporting that they are in good health.

With these limitations in mind, almost $9 \%$ of adults consider themselves to be in poor health, on average across OECD countries (Figure 3.23). This ranges from over 15\% in Korea, Lithuania, Latvia and Portugal to under 4\% in New Zealand, the United States, Canada, Ireland and Australia. However, the response categories used in OECD countries outside Europe and Asia are asymmetrical on the positive side, which introduces a comparative bias to a more positive selfassessment of health (see the box on "Definition and comparability"). Korea, Japan and Portugal stand out as countries with high life expectancy, but relatively poor selfrated health.

People with lower incomes are generally less positive about their health than people on higher incomes, in all OECD countries (Figure 3.24). Almost $80 \%$ of adults in the highest income quintile rate their health as good or very good, compared with just under $60 \%$ of adults in the lowest income quintile, on average across the OECD. Socioeconomic disparities are particularly marked in Latvia, Estonia, the Czech Republic and Lithuania, with a percentage point gap of 40 or more between adults on low and high incomes. Differences in smoking, harmful alcohol use and other risk factors are likely to explain much of this disparity in these countries. Socio-economic disparities are relatively low in New Zealand, Greece, Italy, Australia and France, at less than 10 percentage points.

Self-rated health tends to decline with age. In many countries, there is a particularly marked decline in how people rate their health when they reach their mid-forties, with a further decline after reaching retirement age. Men are also more likely than women to rate their health as good.

\section{Definition and comparability}

Self-rated health reflects an individual's overall perception of his or her health. Survey respondents are typically asked a question such as: "How is your health in general?". Caution is required in making crosscountry comparisons of self-rated health for at least three reasons. First, self-rated health is subjective, and responses may be systematically different across and within countries because of socio-cultural differences. Second, as self-rated health generally worsens with age, countries with a greater share of older people are likely to have fewer people reporting that they are in good health. Third, there are variations in the question and answer categories used in survey questions across countries. In particular, the response scale used in the United States, Canada, New Zealand, Australia and Chile is asymmetrical (skewed on the positive side), including the response categories: "excellent, very good, good, fair, poor". In most other OECD countries, the response scale is symmetrical, with response categories: "very good, good, fair, poor, very poor". This difference in response categories may introduce a comparative bias to a more positive self-assessment of health in those countries that use an asymmetrical scale.

Self-rated health by income level is reported for the first quintile (lowest $20 \%$ of income group) and the fifth quintile (highest 20\%). Depending on the surveys, the income may relate to either the individual or the household (in which case the income is equivalised to take into account the number of people in the household).

\section{References}

[2] Lumsdaine, R. and A. Exterkate (2013), "How survey design affects self-assessed health responses in the survey of health, ageing and retirement in Europe", European Economic Review, Vol. 63, pp. 299-307.

[1] Palladino, R. et al. (2016), “Associations between multimorbidity, healthcare utilisation and health status: evidence from 16 European countries", Age and Ageing, Vol. 45, pp. 431-435. 


\section{Figure 3.23. Adults rating their own health as bad or very bad, 2017 (or nearest year)}

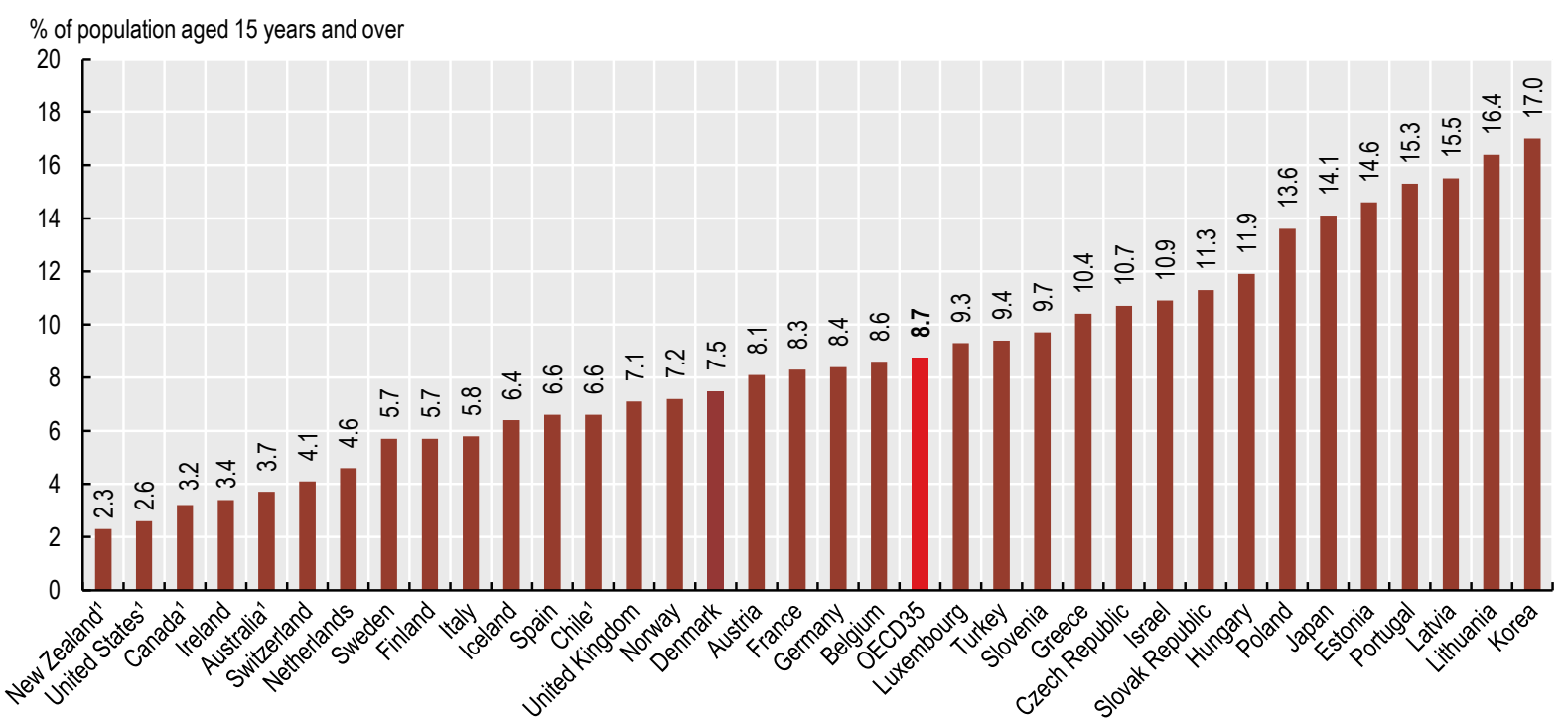

1. Results for these countries are not directly comparable with those for other countries, due to methodological differences in the survey questionnaire resulting in a bias towards a more positive self-assessment of health.

Source: OECD Health Statistics 2019 (EU-SILC for European countries)

StatLink 겐 https://doi.org/10.1787/888934015239

Figure 3.24. Adults rating their own health as good or very good, by income quintile, 2017 (or nearest year)

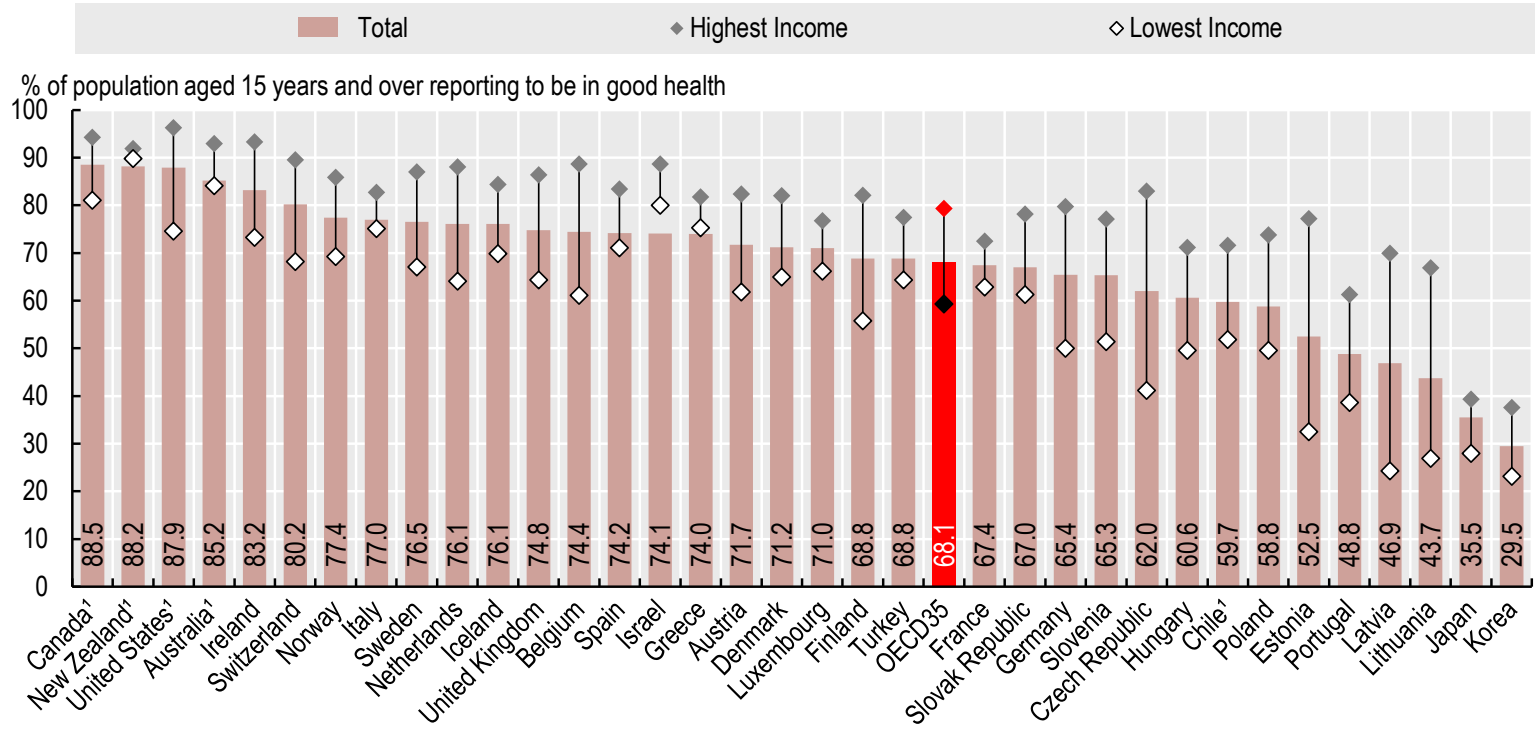

1. Results for these countries are not directly comparable with those for other countries, due to methodological differences in the survey questionnaire resulting in a bias towards a more positive self-assessment of health.

Source: OECD Health Statistics 2019 (EU-SILC for European countries) 



\section{RISK FACTORS FOR HEALTH}

Smoking among adults

Alcohol consumption among adults

Opioids use

Diet and physical activity among adults

Overweight and obesity among adults

Overweight and obesity among children

Air pollution and extreme temperatures

The statistical data for Israel are supplied by and under the responsibility of the relevant Israeli authorities. The use of such data by the OECD is without prejudice to the status of the Golan Heights, East Jerusalem and Israeli settlements in the West Bank under the terms of international law. 


\section{RISK FACTORS FOR HEALTH}

\section{Smoking among adults}

Smoking is a leading cause of multiple diseases, including cancers, heart attacks and stroke, and respiratory diseases such as chronic obstructive pulmonary disease. Smoking among pregnant woman increases the risk of low birth weight and premature delivery. The WHO estimates that tobacco smoking kills 7 million people in the world every year, of which more than 1.2 million deaths are due to second-hand smoke and 65000 are children (WHO, 2017[1]). Of these deaths, just over half took place in four countries China, India, the United States, and the Russian Federation. Over recent decades, smoking caused the largest share of overall years of healthy life lost in 15 OECD countries, and ranked second in further 16 OECD countries (Forouzanfar et al., 2016[2])

Across OECD countries, $18 \%$ of adults smoke tobacco daily (Figure 4.1). Smoking rates range from over $25 \%$ in Greece, Turkey, Hungary and France to below 10\% in Mexico and Iceland. In key partner countries, rates are very high in Indonesia (40\%) and the Russian Federation (30\%); and 10\% or less in Costa Rica. Men smoke more than women in all countries except Iceland - on average across the OECD, 23\% of men smoke daily compared with $14 \%$ among women. The gender gap in smoking rates is comparatively high in Korea and Turkey, as well as in Indonesia, China and the Russian Federation. Among men, rates are highest in Indonesia (76\%), the Russian Federation (50\%), China (48\%) and Turkey (40\%); and below $10 \%$ in Costa Rica and Iceland. For women, rates are the highest in Austria, Greece, Chile, France and Hungary (over $20 \%$ ). Less than $5 \%$ of women smoke in China, India, Costa Rica, Korea, Mexico and Indonesia.

Daily smoking rates have decreased in most OECD countries over the last decade, from an average of $23 \%$ in 2007 to $18 \%$ in 2017 (Figure 4.2). In the Slovak Republic and Austria, though, smoking rates have risen slightly. Smoking rates also increased in Indonesia. Greece reduced smoking rates the most, followed by Estonia, Iceland and Norway.

People with a lower education level are more likely to smoke in all countries except Greece, with an average gap of 8 percentage points in 2017 (Figure 4.3). Education gaps are largest in Estonia and Hungary (about 16 percentage points), and relatively small in Portugal, Bulgaria, Lithuania, and Turkey (less than 2 percentage points).
Raising taxes on tobacco is one of the most effective ways to reduce tobacco use. Tobacco prices in most OECD countries contain more than $50 \%$ of taxes. Health warnings on packages, bans on promotional and misleading information, and restricted branding are other key tobacco control policies. Awareness raising and support for smokers, including nicotine replacement treatment and smoking cessation advice, also help reduce smoking.

\section{Definition and comparability}

The proportion of daily smokers is defined as the percentage of the population aged 15 years and over who report smoking tobacco every day. Other forms of smokeless tobacco products, such as snuff in Sweden, are not taken into account. This indicator is more representative of the smoking population than the average number of cigarettes smoked per day. Most countries report data for the population aged 15 and older, but there are some exceptions as highlighted in the data source of the OECD Health Statistics database.

Data for differences in daily smoking by education level comes from the European Health Interview Survey in 2014 in EU countries. The United States and Canada reported the data respectively from the Medical Expenditure Panel Survey (MEPS) in 2016 and Canadian Community Health Survey (CCHS) 2015-2016. The latter reflects only daily cigarette smoking.

\section{References}

[2] Forouzanfar, M. et al. (2016), “Global, regional, and national comparative risk assessment of 79 behavioural, environmental and occupational, and metabolic risks or clusters of risks, 1990-2015: a systematic analysis for the Global Burden of Disease Study 2015", The Lancet, Vol. 388/10053, pp. 1659-1724, http://dx.doi.org/10.1016/ s0140-6736(16)31679-8.

[1] WHO (2017), WHO report on the global tobacco epidemic, 2017. 
Figure 4.1. Adult population smoking daily by sex, 2017 (or nearest year)

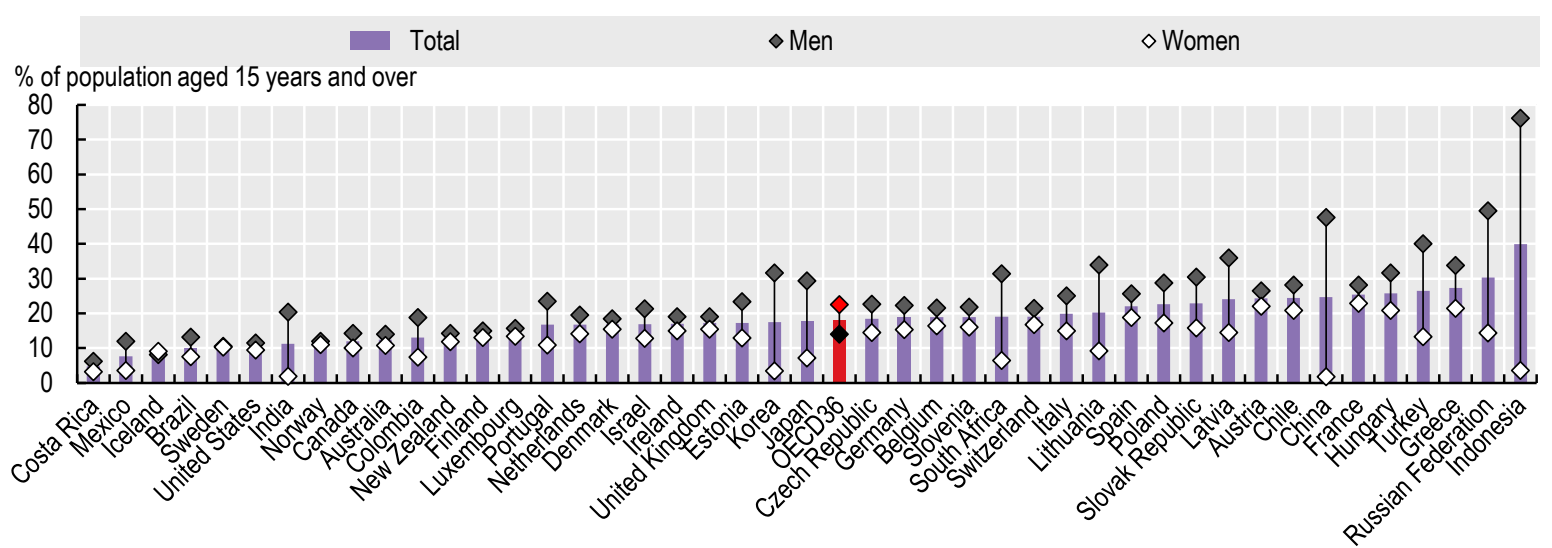

Source: OECD Health Statistics 2019.

Figure 4.2. Adult population smoking daily, 2007 and 2017 (or nearest years)

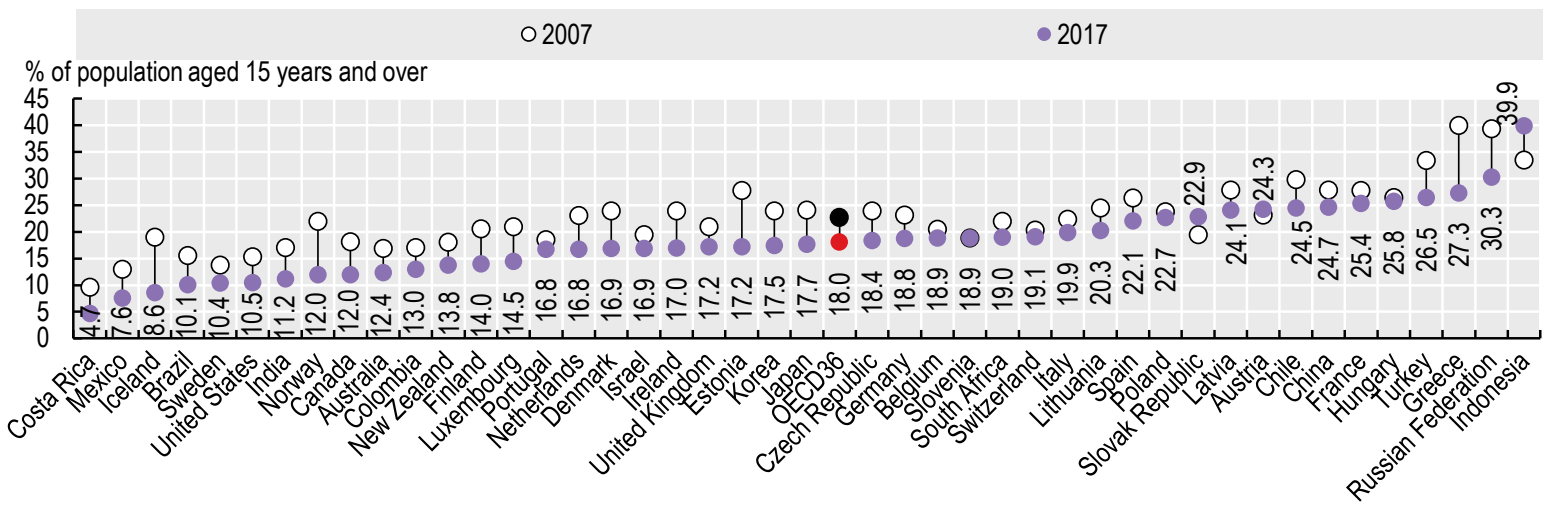

Source: OECD Health Statistics 2019.

StatLink 시내 https://doi.org/10.1787/888934015296

Figure 4.3. Difference in daily smoking between highest and lowest education level, 2016 (or nearest year)

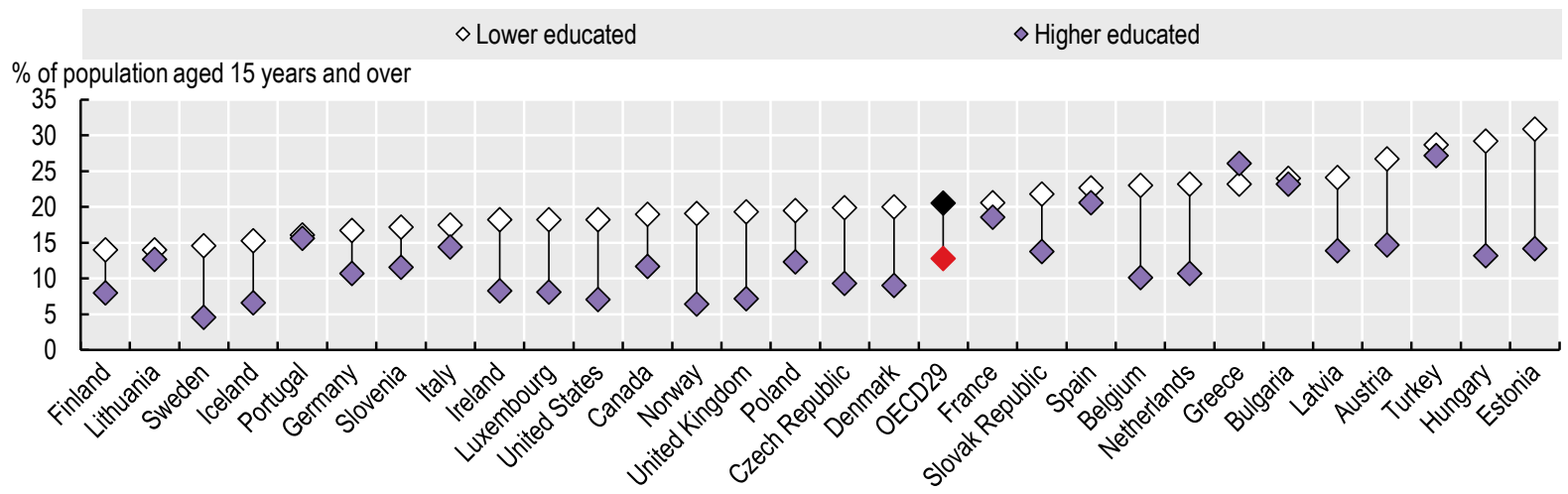

Source: EHIS 2014 for Europe; MEPS 2016 for the United States; and CCHS 2015-2016 for Canada. 
Alcohol use is a leading cause of death and disability worldwide, particularly in those of working age. It accounted for an estimated $7 \%$ of male and $2 \%$ of female deaths worldwide in 2016 (Griswold et al., 2018 $[1]$ ). High alcohol intake is a major risk factor for heart diseases and stroke, liver cirrhosis and certain cancers, but even low and moderate alcohol consumption increases the long-term risk of these diseases. Alcohol also contributes to more accidents and injuries, violence, homicide, suicide and mental health disorders than any other psychoactive substance, particularly among young people.

Measured through sales data, overall alcohol consumption averaged 8.9 litres per person across OECD countries in 2017, down from 10.2 litres in 2007 (Figure 4.4). Lithuania reported the highest consumption (12.3 litres), followed by Austria, France, the Czech Republic, Luxembourg, Ireland, Latvia and Hungary, all with over 11 litres per person. Turkey, Israel and Mexico have comparatively low consumption levels (under 5 litres per person). Among key partners, consumption was relatively high in the Russian Federation (11.1 litres) and low in Indonesia, India, Costa Rica and Colombia (less than 5 litres). Average consumption fell in 27 OECD countries between 2007 and 2017, with the largest reductions in Israel, Estonia, Greece and Denmark (by 3 litres or more). Consumption also fell markedly in the Russian Federation (by 7 litres). However, alcohol consumption increased by more than 1 litre per person in China and India, and by over 0.5 litres per person in Chile.

While overall consumption per capita helps assess longterm trends, it does not identify sub-populations at risk from harmful drinking patterns. Heavy drinking and alcohol dependence account for an important share of the burden of disease. On average across OECD countries, $3.7 \%$ of adults were alcohol dependent in 2016 (Figure 4.5). In all countries, men are more likely to be alcohol dependent, with $6 \%$ of men and $1.6 \%$ of women alcohol dependent on average. Dependence is most common in Latvia, Hungary, and Russian Federation (more than 9\% of adults). In these three counties, gender gaps are also high, with the share of alcohol dependent men about five times higher than for women.

The share of dependent drinkers does not always correlate with overall alcohol consumption levels, reflecting differences in consumption patterns and diagnosis of alcohol dependence. France, for instance, had the third highest alcohol consumption in 2017, yet rates of alcohol dependence below the OECD average. Conversely, the United States has a high share of alcohol dependence in $2016(7.7 \%)$, but recorded consumption is at the OECD average.

Policies addressing harmful alcohol use include broad-based strategies and ones that target heavy drinkers. All OECD countries apply taxes to alcoholic beverages, but the level of taxes differs greatly. In addition, some countries have implemented new forms of pricing policies, such as minimum pricing of one alcohol unit in Scotland. Advertising regulations exist in most OECD countries, but law enforcement and the forms of media included in these regulations (e.g. printed newspapers, billboards, the internet and TV) varies. In Norway, Lithuania and Sweden, for instance, there are complete bans on TV adverts, including on social media, while other countries set partial limitations. Controls on the physical availability, drinking age and hours of sale; and drink-driving rules are other commonly used policies (OECD, 2015 $\left.{ }_{[1]}\right)$.

\section{Definition and comparability}

Recorded alcohol consumption is defined as annual sales of pure alcohol in litres per person aged 15 years and over (with some exceptions highlighted in the data source of the OECD Health Statistics database). The methodology to convert alcohol drinks to pure alcohol may differ across countries. Official statistics do not include unrecorded alcohol consumption, such as home production. In Estonia, data adjust for tourist consumption and unrecorded consumption. In some countries (e.g. Luxembourg), national sales do not accurately reflect actual consumption by residents, since purchases by non-residents may create a significant gap between national sales and consumption. Alcohol consumption in Luxembourg is thus estimated as the mean of alcohol consumption in France and Germany.

Alcohol dependence is coded as F10.2 in ICD-10 among adults aged over 15 years old during a given calendar year. The numerator is the number of adults between 18 and 65 years with a diagnosis of F10.2 during a calendar year. The denominator is the mid-year resident population over 15 years during the same calendar year. The WHO also reports alcohol use disorders among people aged 15 years and over as a prevalence over 12 months, which includes both alcohol dependence and harmful use of alcohol coded as F10.1 in ICD-10.

\section{References}

[2] Griswold, M. et al. (2018), "Alcohol use and burden for 195 countries and territories, 1990-2016: a systematic analysis for the Global Burden of Disease Study 2016", The Lancet, Vol. 392/10152, pp. 1015-1035, http://dx.doi.org/10.1016/ S0140-6736(18)31310-2.

[1] OECD (2015), Tackling Harmful Alcohol Use: Economics and Public Health Policy, OECD Publishing, Paris, https://dx.doi.org/ 10.1787/9789264181069-en.

[3] WHO (2018), Global status report on alcohol and health. 


\section{RISK FACTORS FOR HEALTH}

\section{Alcohol consumption among adults}

Figure 4.4. Recorded alcohol consumption among adults, 2007 and 2017 (or nearest year)

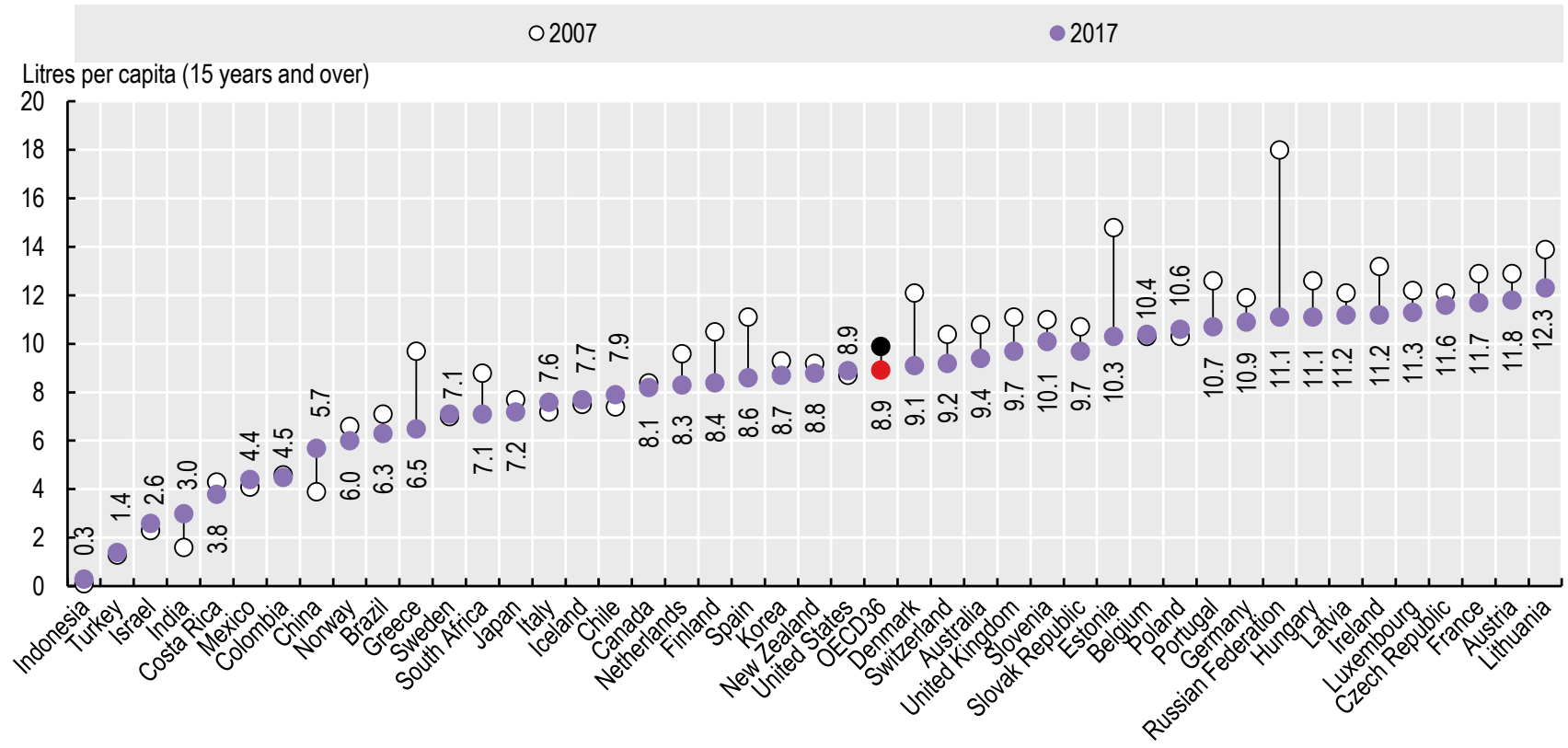

Source: OECD Health Statistics 2019

Figure 4.5. Share of dependent drinkers, by sex, 2016

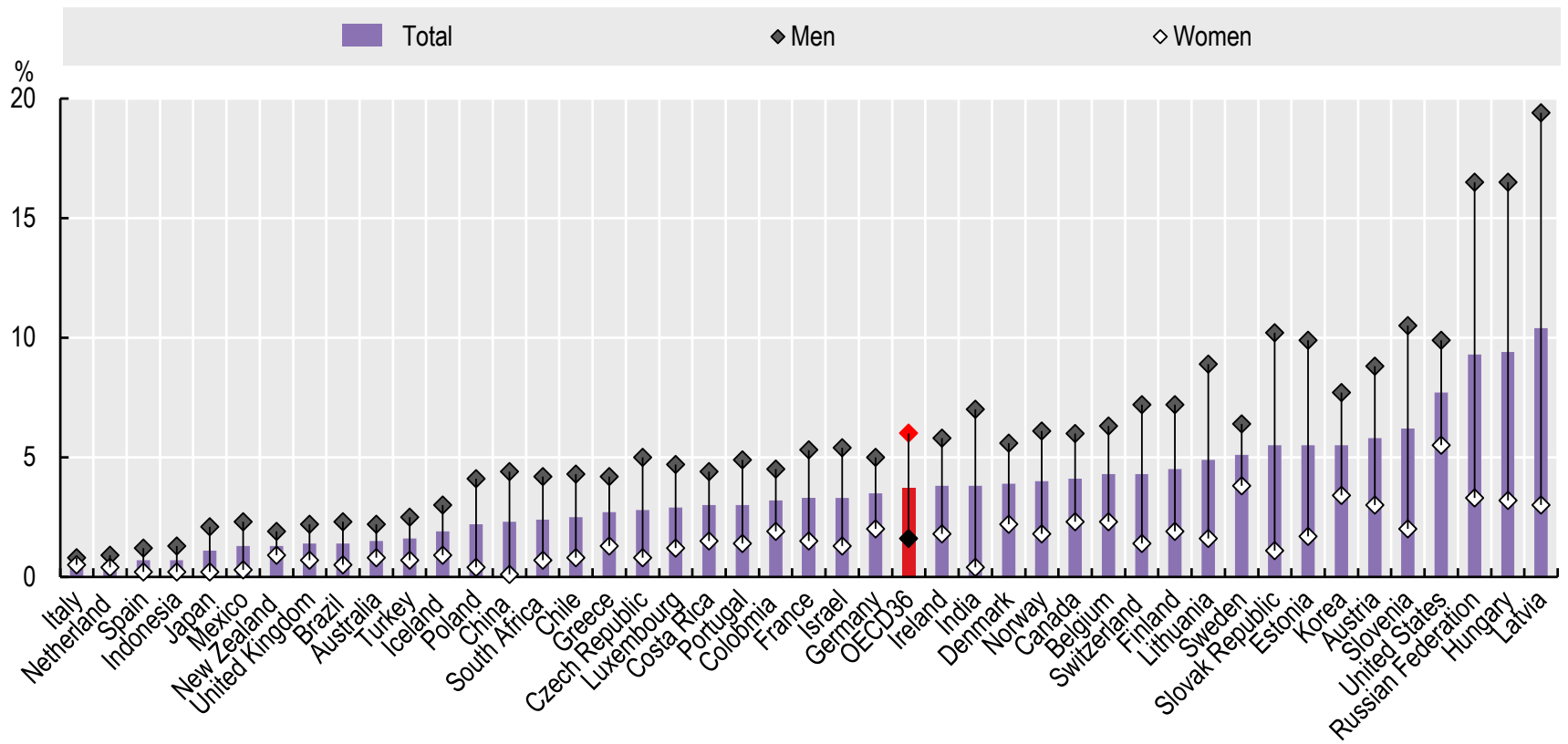

Source: Global Status Report on Alcohol and Health, WHO 2018 
Opioids are a narcotic pain medication that have become the cornerstone therapy for treatment of moderate to severe pain in many high-income countries. In parallel, illicit opioid use for nonmedical purposes has created illegal, increasingly commercialised global markets. Canada and the United States have experienced an opioid crisis in recent years, fuelled by growth in the consumption of synthetic opioids such as fentanyl and carfentanil. Problematic opioid use is also spreading in Australia and some European countries, due to growing prescription rates (see indicator on "Safe primary care - prescribing" in Chapter 6) and the development of a dynamic illegal drug supply market (OECD, 2019[1]).

For prescription opioids, whilst there is insufficient access in many low- and middle-income countries, the reality in OECD countries is quite different, where the availability of analgesic opioids has been steadily growing. The United States has the highest availability of analgesic opioids among OECD countries, followed by Germany and Canada, while Mexico, Chile and Colombia show the lowest numbers. The sharpest increases occurred in the 2000s: between 2002-04 and 2005-07 analgesic opioids availability grew on average by $59 \%$ and over the decade by almost $110 \%$. More recently, the growth rate dropped to $5.4 \%$ on average between 2011-13 and 2014-16. In absolute terms, availability per person increased the most in Israel, the United Kingdom, Germany; the sharpest falls were in the United States, Denmark and Luxembourg (Figure 4.6).

Opioid-related deaths is a key indicator that reflects the impact of problematic use of the drug, both of legally prescribed drugs and illegal drugs (e.g. heroin). On average across 25 OECD countries for which data are available, there were 26 opioid-related deaths per million inhabitants in 2016 (Figure 4.7). However, death rates were over five times higher in the United States (131 opioid-related deaths), followed closely by Canada (120). Opioid-related deaths have increased by about $20 \%$ since 2011, with large increases in the United States, Sweden, Canada, England and Wales, and Lithuania. In the United States, almost 400000 people died from an opioid overdose between 1999 and 2017, with the opioid crisis contributing to the first decline in life expectancy observed in over half a century.

Countries are implementing several strategies to address the problematic use of opioids, with comprehensive approaches across different sectors, covering health, social services, law enforcement, data systems and research. Countries have aimed to improve opioid prescribing through evidence-based clinical guidelines, training, surveillance of opioid prescriptions, and regulation of marketing and financial relationships with opioid manufacturers. Educational materials and awareness interventions have been developed for both at-risk patients and the general public. For patients with opioid use disorder, there has been increased coverage for long-term medication-assisted therapy combined with specialised services for infectious diseases and psychosocial interventions. Many countries have also implemented harm minimisation interventions such as overdose reversal medications, needle and syringe programmes and medically supervised consumptions centres. Research initiatives to boost innovation in pain relief and opioid use disorders treatments have also been launched (OECD, 2019[1]).

\section{Definition and comparability}

Availability of analgesic opioid is defined as amounts that each country's competent national authority estimates are needed and used annually, including reporting of medicines destroyed, losses during manufacture, etc. This information is verified by the International Narcotics Control Board using data from export and import notifications. The S-DDD is a technical unit of measurement. It is not a recommended prescription dose. It recognises that no internationally agreed standard doses exist for opioid medicines and therefore provides a rough measure to rank opioid use of countries. Levels of use, expressed in S-DDD per million inhabitants per day, are calculated with the following formula: annual use divided by 365 days, divided by the population in millions of the country or territory during the year, divided by the defined daily dose (Berterame et al., 2016[2]). Analgesic opioids include codeine, dextropropoxyphene, dihydrocodeine, fentanyl, hydrocodone, hydromorphone, morphine, ketobemidone, oxycodone, pethidine, tilidine and trimeperidine. It does not include illicit opioids. Those data do not directly reflect the consumption of analgesic opioids in countries, but the general availability for different purposes, of which the largest component is for medical use.

Opioid-related deaths for European countries are collected and shared with the OECD by the European Monitoring Centre for Drugs and Drug Addiction (EMCDDA). This was complemented with data contributed directly from countries to the OECD using an adapted version of the EMCDDA's data questionnaire.

\section{References}

[2] Berterame, S. et al. (2016), "Use of and barriers to access to opioid analgesics: a worldwide, regional, and national study", The Lancet, Vol. 387/10028, pp. 1644-1656, http:// dx.doi.org/10.1016/S0140-6736(16)00161-6.

[1] OECD (2019), Addressing Problematic Opioid Use in OECD Countries, OECD Health Policy Studies, OECD Publishing, Paris, https://dx.doi.org/10.1787/a18286f0-en. 
Figure 4.6. Availability of analgesic opioids, 2011-13 and 2014-16

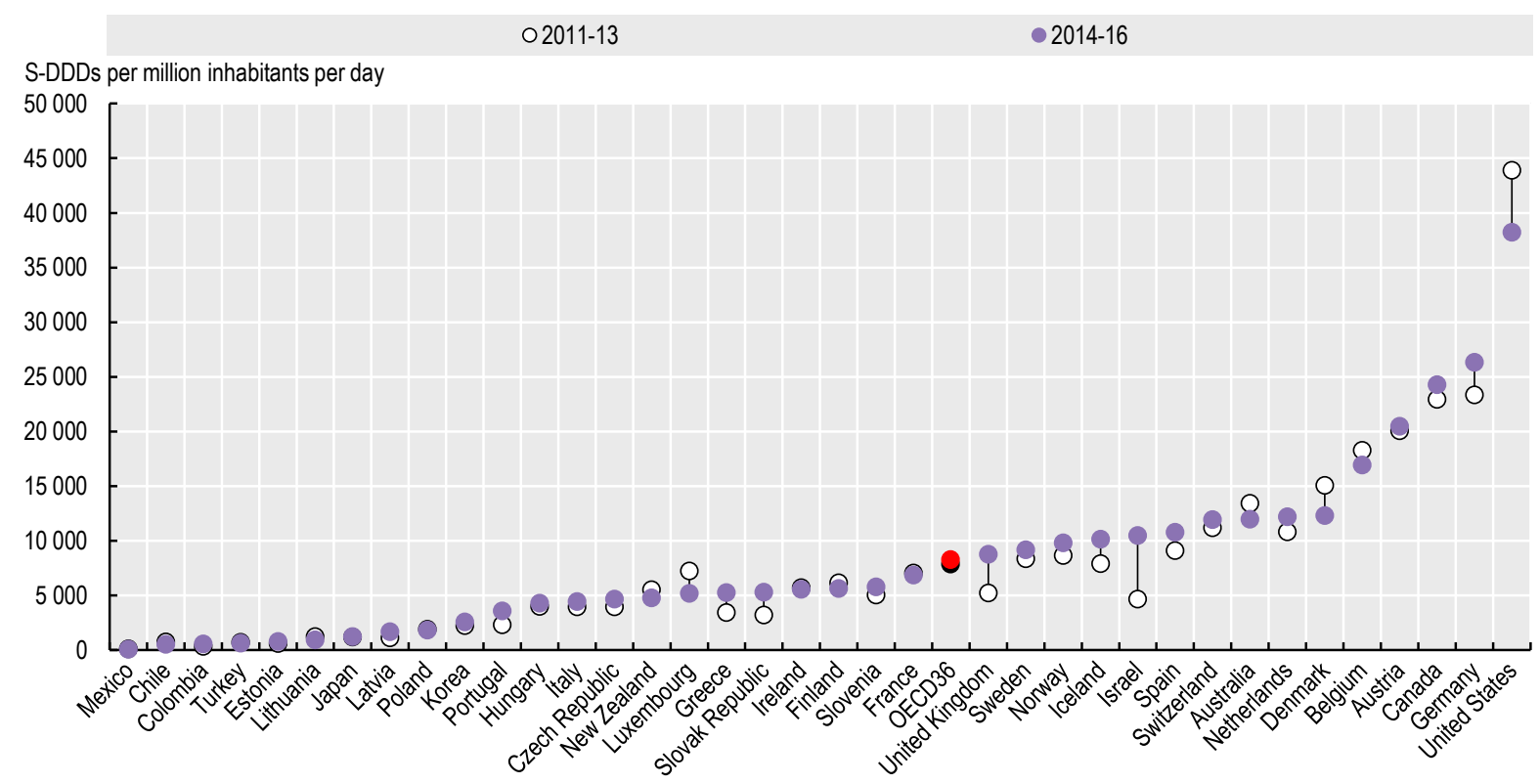

S-DDD: Defined daily doses for statistical purposes.

Source: INCB 2018.

Figure 4.7. Opioid-related deaths, 2011 and 2016 (or nearest year)

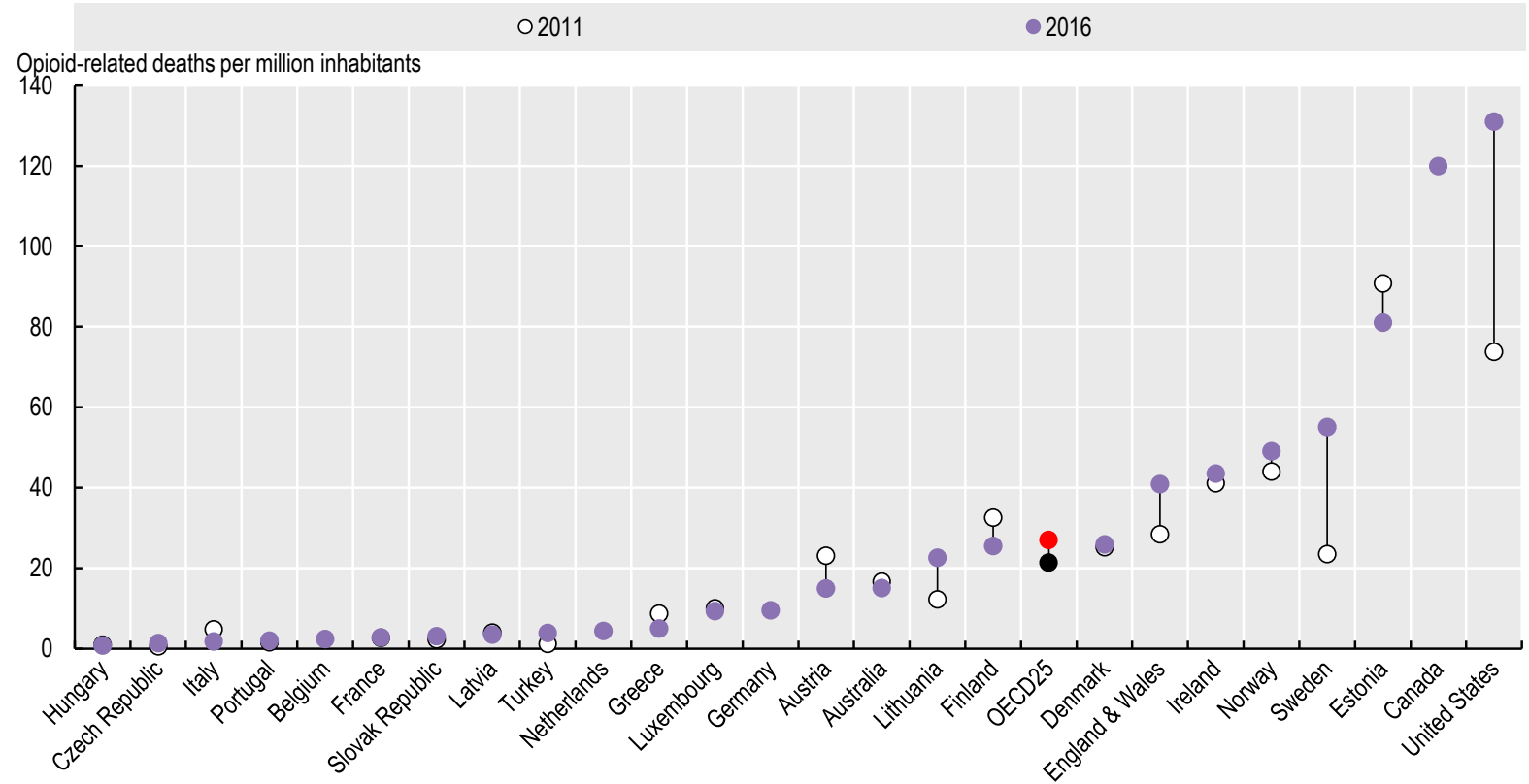

Note: Canada's data corresponds to 2018.

Source: EMCDDA for European countries and country responses to OECD opioid data questionnaire 2018. 


\section{RISK FACTORS FOR HEALTH}

\section{Diet and physical activity among adults}

A healthy diet is associated with improved health outcomes. Adults who follow a diet rich in fruits and vegetables and low in fat, sugars and salt/sodium are at a lesser risk of developing one or more cardiovascular diseases and certain types of cancer (Graf and Cecchini, 2017[1]). Healthy diet may also reduce the likelihood of being overweight or obese. In 2017, inadequate fruit and vegetable consumption led to an estimated 3.9 million deaths worldwide (Global Burden of Disease Collaborative Network, 2018[2]).

On average across OECD countries, over half (57\%) of all adults consumed at least one piece of fruit per day in 2017 (Figure 4.8). Values for this metric are highest in Australia, Spain, New Zealand and Italy (greater than 75\%). Conversely, Chile, Finland and Latvia recorded values below $40 \%$. In all countries except Spain, women are more likely to consume fruit daily. This gender gap in fruit consumption was largest in Finland and Austria, with over a 20 percentage point difference.

The share of populations consuming vegetables daily was similar: $60 \%$ of adults, on average across the OECD. Countries with the highest rate of vegetable consumption are Australia, Korea, New Zealand and the United States, all of which recorded values greater than 90\% (Figure 4.9). At the other end of the spectrum, this figure fell below $35 \%$ in Germany and the Netherlands. As with fruit consumption, women are more likely than men to eat at least one portion of vegetables per day (65\% of women v $54 \%$ of men, on average). Daily vegetable consumption was higher among women than men in all countries other than Korea and the United States (where gender differences were minimal).

Physical activity is also important for leading a healthy lifestyle. Regular physical activity is associated with significant benefits such as improved bone and functional health, and reduced risk of various non-communicable diseases and depression (Warburton and Bredin, 2017[3]). Advances in technology in areas such as transport, communication and entertainment have contributed to declines in physical activity (Graf and Cecchini, 2017[1]).

About two in three adults (66\%) meet the recommended guidelines for moderate physical activity, on average across 23 OECD countries (Figure 4.10). Adults are most likely to be sufficiently active in Sweden, Iceland, Norway and Denmark (over $75 \%$ of adults). Conversely, less than half of the adult population in Italy and Spain engage in the recommended amount of moderate physical activity. Other than Denmark, men are more likely to be physically active than women in all 23 OECD countries with comparable data.

\section{Definition and comparability}

Fruit and vegetable consumption are defined as the proportion of adults who consume at least one fruit or vegetable per day, excluding juice and potatoes. Estimates for fruit and vegetable consumption are derived from national health surveys and are selfreported (with some differences in reporting periods, see country-specific notes in OECD.Stat on definitions, sources and methods for further details).

Data for Australia, Korea and New Zealand are derived from quantity-type questions. Values for these countries may therefore be overestimated. Most countries report data for the population aged 15 years and over, with some exceptions as highlighted in the data source of the OECD Health Statistics database.

The indicator of moderate physical activity is defined as completing at least 150 minutes of moderate physical activity per week. Estimates of moderate physical activity are based on self-reports from the European Health Interview Survey 2014, combining work-related physical activity with leisure-time physical activity (bicycling for transportation and sport). Walking for transportation is not included.

\section{References}

[2] Global Burden of Disease Collaborative Network (2018), Global Burden of Disease Study 2017 (GBD 2017) Results, Seattle, United States: Institute for Health Metrics and Evaluation (IHME).

[1] Graf, S. and M. Cecchini (2017), "Diet, physical activity and sedentary behaviours: Analysis of trends, inequalities and clustering in selected OECD countries", OECD Health Working Papers No. 100, OECD Publishing, Paris, https://doi.org/ 10.1787/54464f80-en.

[3] Warburton, D. and S. Bredin (2017), "Health benefits of physical activity", Current Opinion in Cardiology, Vol. 32/5, pp. 541-556, http://dx.doi.org/10.1097/hco.0000000000000437. 
Figure 4.8. Daily fruit consumption among adults by sex, 2017 (or nearest year)

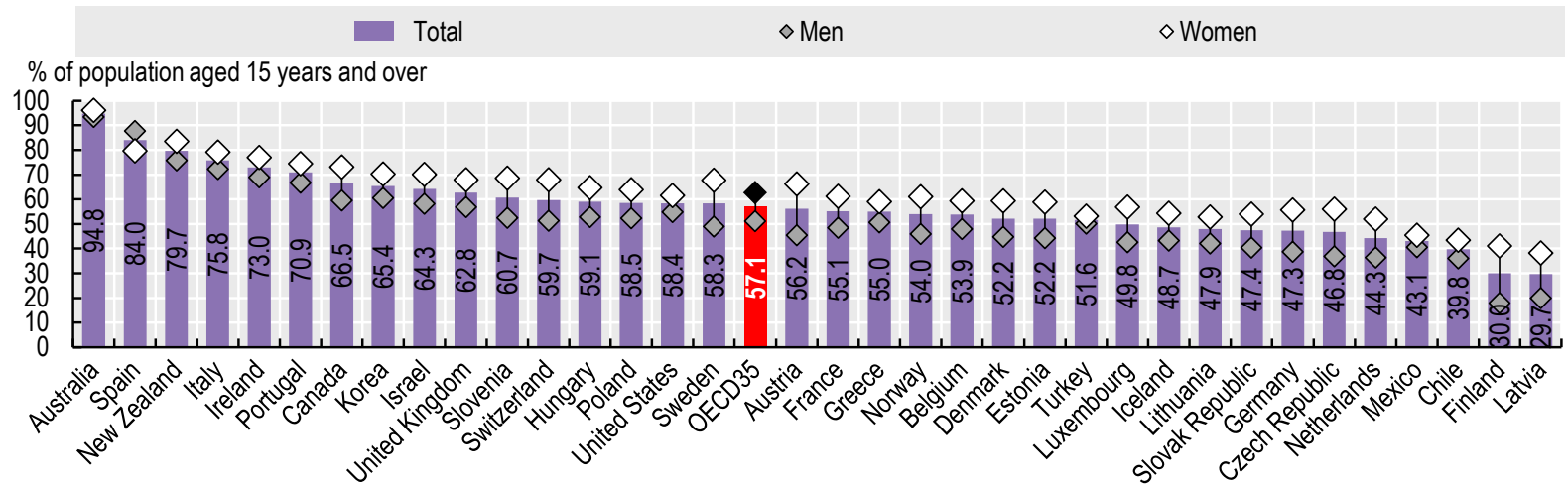

Source: OECD Health Statistics 2019.

StatLink तiाsட https://doi.org/10.1787/888934015410

Figure 4.9. Daily vegetable consumption amongst adults by sex, 2017 (or nearest year)

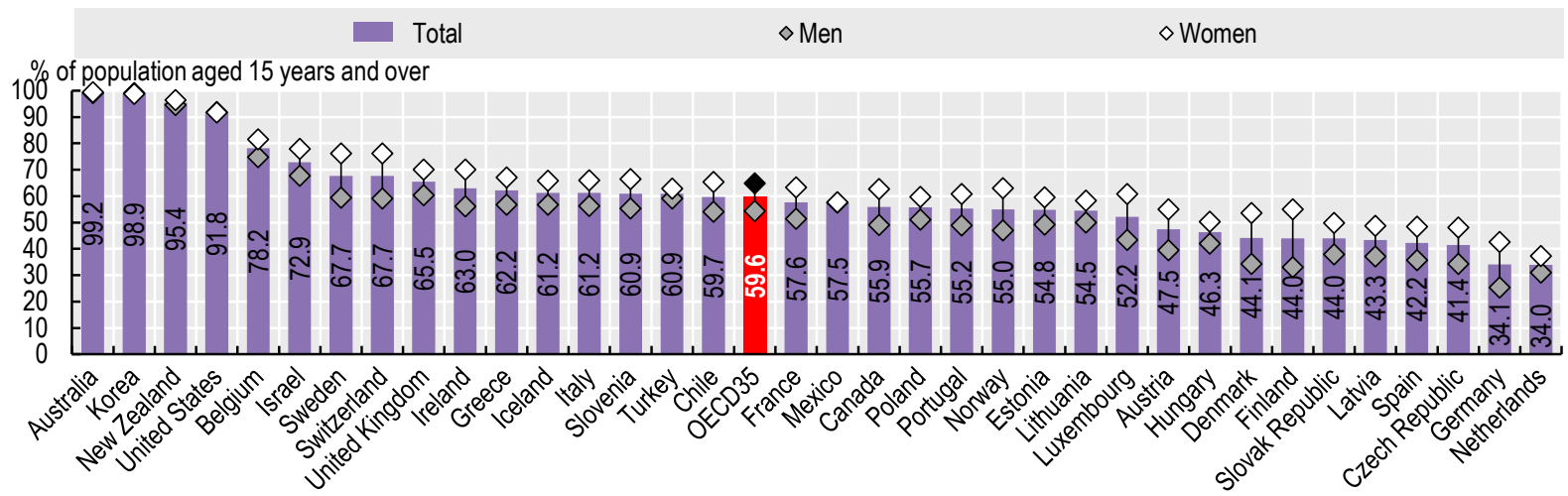

Source: OECD Health Statistics 2019.

StatLink 에내 https://doi.org/10.1787/888934015429

Figure 4.10. Moderate weekly physical activity among adults by sex, 2014

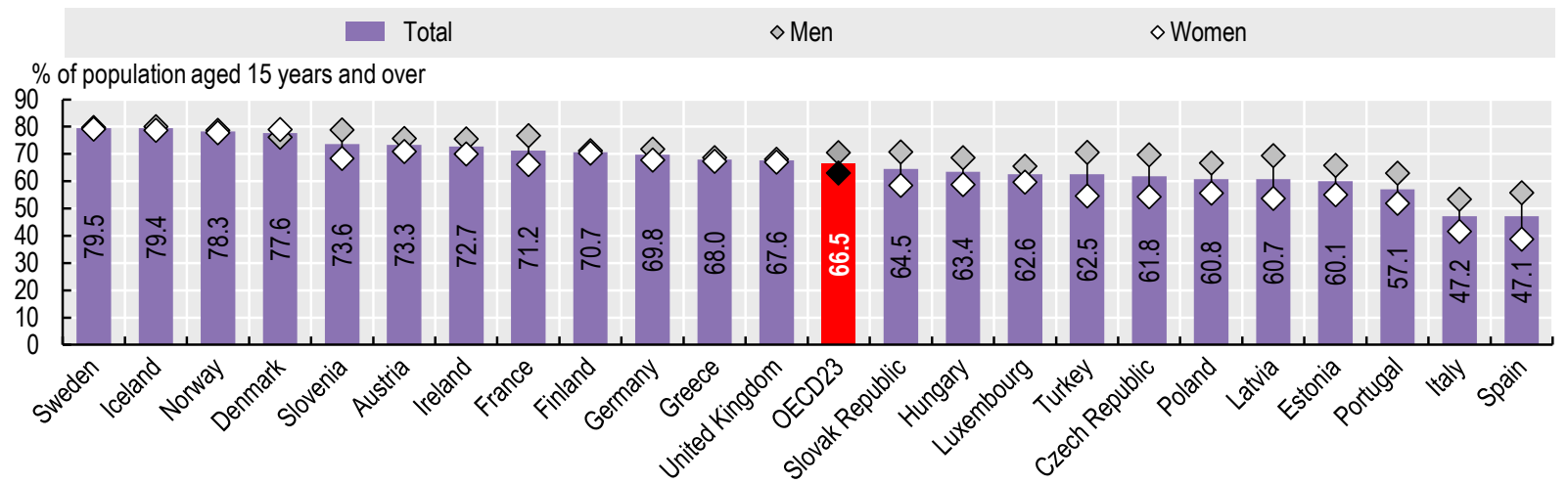

Source: Eurostat EHIS 2014. 


\section{RISK FACTORS FOR HEALTH}

\section{Overweight and obesity among adults}

Being overweight, including pre-obesity and obesity, is a major risk factor for various non-communicable diseases including diabetes, cardiovascular diseases and certain cancers. High consumption of calories-dense food and increasingly sedentary lifestyles have contributed to growing global obesity rates. The rate of growth has been highest in early adulthood and has affected all population groups, in particular women and those with lower levels of education (Afshin et al., 2017[1]). High body mass index (BMI) has been estimated to cause 4.7 million deaths worldwide (Global Burden of Disease Collaborative Network, 2018[2])

Based on measured data, $58 \%$ of adults were overweight or obese in 2017 on average across 23 OECD countries with comparable data (Figure 4.11). For Chile, Mexico and the United States this figure exceeds $70 \%$. Conversely, in Japan and Korea, less than $35 \%$ of adults were overweight or obese. The remaining 13 OECD countries include self-reported data, with rates ranging from $42 \%$ in Switzerland to $65 \%$ in Iceland. These estimates, though, are less reliable and typically lower than those based on measured data. For both measured and self-reported data, men are more likely than women to be overweight.

The proportion of overweight adults has been gradually increasing in most OECD countries since the early 2000s, including in countries where rates are relatively low (Figure 4.12). In Japan and Korea, this proportion has increased by 2.1 and 4.2 percentage points, respectively, between 2000 and 2017. In countries with relatively high rates of adults overweight, this figure ranged from 2.3 percentage points in Canada to 11.9 in Chile.

Adults with a low level of education are more likely to be overweight than those with a tertiary education level or above in all 27 OECD countries examined (Figure 4.13). The difference in the proportion of overweight adults by education level was greatest in Luxembourg, Spain and France, where the gap was greater than 15 percentage points.

OECD member countries have implemented a suite of regulatory and non-regulatory initiatives to reduce overweight population rates. Prominent examples include mass media campaigns to promote the benefits of healthy eating; promotion of nutritional education and skills; 'sin' taxes on energy-dense food and drink items to discourage consumption; food labelling to communicate nutritional value; and agreements with the food industry to improve the nutritional value of products. Policymakers are also exploring initiatives that address the social determinants of being overweight. For example, the Healthy Food Financing
Initiative in the United States aims to improve access to healthy foods in underserved areas. Despite these efforts, the overweight epidemic has not been reversed, highlighting the issue's complexity (OECD, 2019[3]).

\section{Definition and comparability}

Overweight is defined as abnormal or excessive accumulation of fat, which presents a risk to health. The most frequently used measure is body mass index (BMI), which is a single number that evaluates an individual's weight in relation to height (weight/ height $^{2}$, with weight in kilograms and height in metres). Based on WHO classifications, adults over age 18 with a BMI greater than or equal to 25 are defined as pre-obese, and those with a BMI greater than or equal to 30 as obese. Data come from national sources - in a few instances these may differ from data shown in the OECD 2019 report on obesity, which uses data from the WHO Global Health Observatory, with agestandardised estimates and other methodological differences. Overweight includes both pre-obesity and obesity. BMI measurements are the same for both genders and adults of all ages. Data for BMI can also be collected using self-reported estimates of height and weight. BMI estimates based on self-reported data are typically lower and less reliable than those based on measured data.

For Figure 4.13, the lowest level of education refers to people with less than a high-school diploma, while the highest refers to people with a university or other tertiary diploma.

\section{References}

[1] Afshin, A. et al. (2017), "Health Effects of Overweight and Obesity in 195 Countries over 25 Years.", The New England Journal of Medicine, http://dx.doi.org/10.1056/NEJMoa1614362.

[2] Global Burden of Disease Collaborative Network (2018), Global Burden of Disease Study 2017 (GBD 2017) Results, Seattle, United States: Institute for Health Metrics and Evaluation (IHME).

[3] OECD (2019), The Heavy Burden of Obesity: The Economics of Prevention, OECD Publishing, Paris, https://doi.org/ 10.1787/67450d67-en. 
Figure 4.11. Overweight including obesity among adults by sex, measured and self-reported, 2017 (or nearest year)

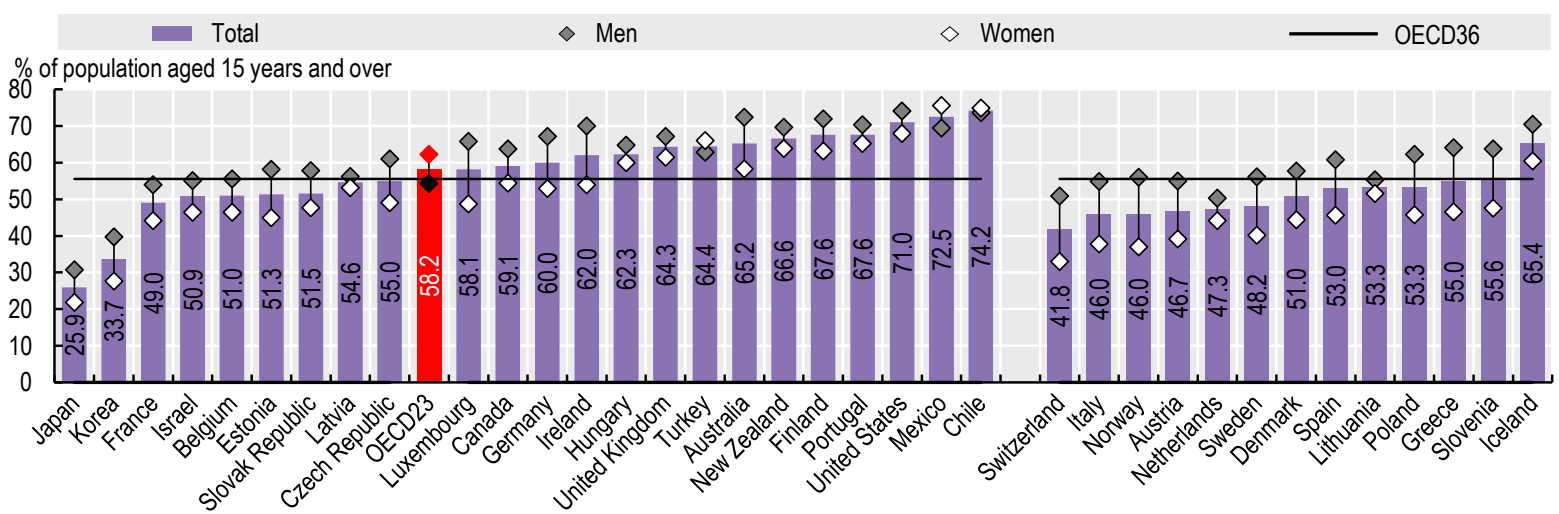

Note: Left- and right-hand side estimates utilise measured and self-reported data, respectively. OECD36 average includes both data types. Source: OECD Health Statistics 2019.

Figure 4.12. Evolution of overweight including obesity in selected countries, measured, 2000-17 (or nearest year)
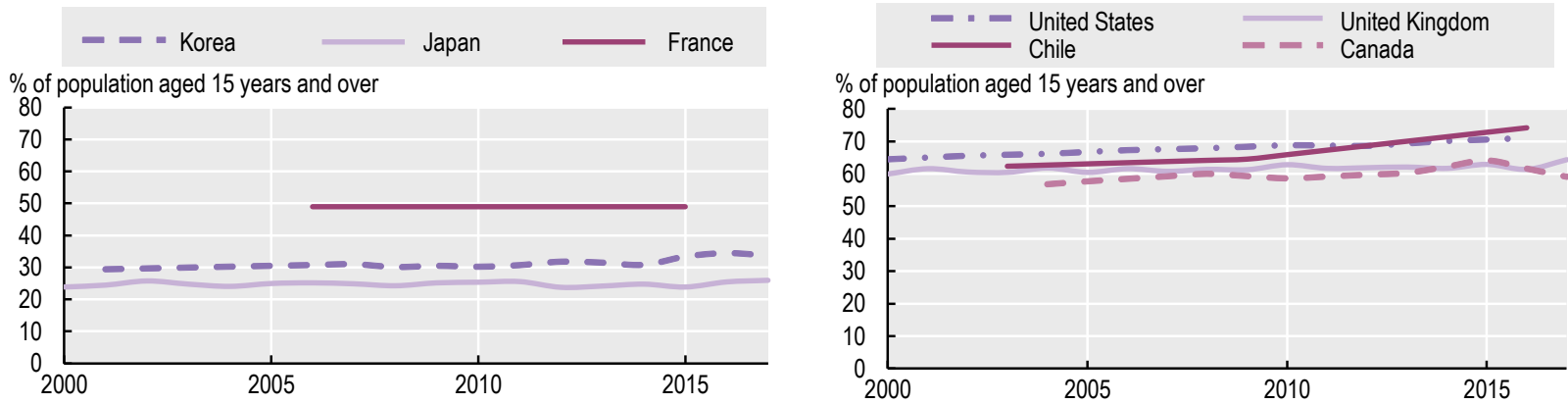

Note: Linear interpolation was used to impute values where data was missing. Source: OECD Health Statistics 2019.

Figure 4.13. Difference in overweight including obesity by education level, self-reported, 2014

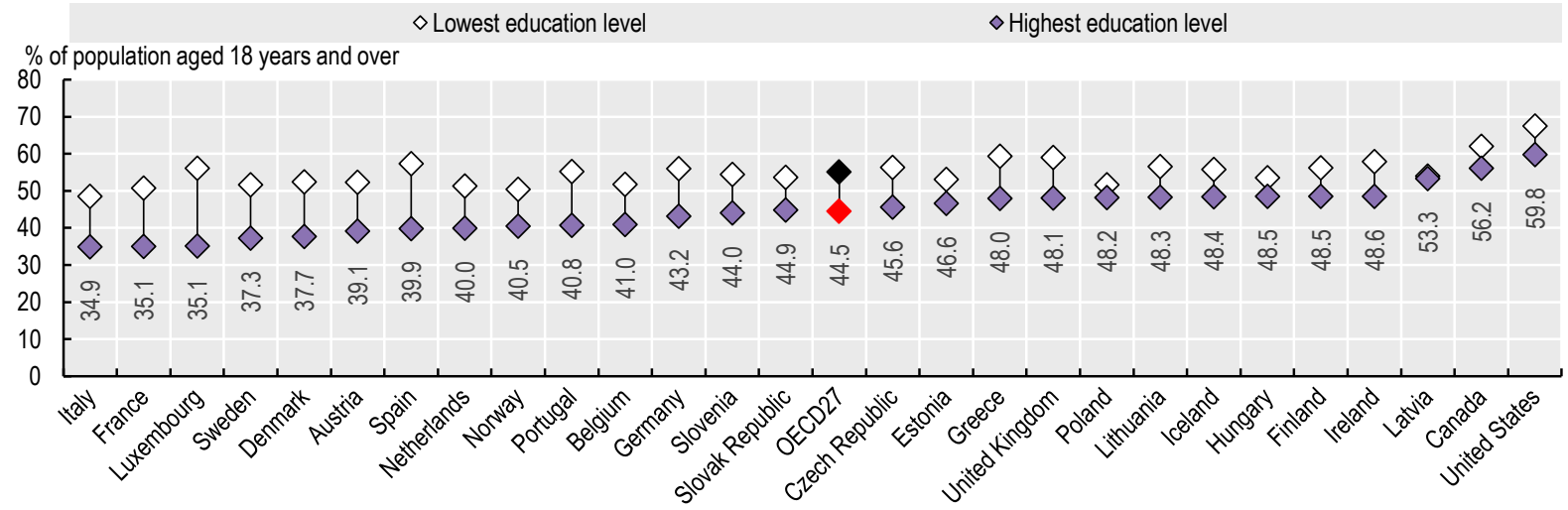

Source: EHIS2 and OECD estimates based on national health survey data. 


\section{RISK FACTORS FOR HEALTH}

\section{Overweight and obesity among children}

Childhood overweight rates, including pre-obesity and obesity, have been growing worldwide. Environmental factors, lifestyle preferences, genetic makeup and culture all can cause children to be overweight. Obese children are at greater risk of developing hypertension and metabolic disorders. Psychologically, obesity can lead to poor selfesteem, eating disorders and depression. Further, obesity may act as a barrier for participating in educational and recreational activities. Childhood obesity is particularly concerning as it is a strong predictor of obesity in adulthood, which is linked to diabetes, heart disease and certain types of cancer (Bösch et al., 2018[1]; OECD, 2019[2]).

Almost one-third (31\%) of children aged 5-9 years living in OECD countries are overweight (Figure 4.14). In the United States, Italy, New Zealand and Greece this figure exceeds $40 \%$. Conversely, in Japan, Estonia, Lithuania, Switzerland and Latvia, rates are below 25\%. The proportion of overweight boys exceeds that of girls in 38 of the 43 OECD and partner countries examined. Countries with the greatest disparity between genders are China, Korea, Poland, the Czech Republic and the Slovak Republic (above a 10 percentage point difference). The gap between boys and girls is small in Portugal and the United Kingdom (less than 1 percentage point).

The rate of overweight children increased from $20.5 \%$ to $31.4 \%$ across 35 OECD countries between 1990 and 2016 (Figure 4.15). Only in Belgium did this rate fall, albeit marginally. Growth was greatest in Hungary, Poland, Turkey, Slovenia and the Slovak Republic whose rates increased by more than $100 \%$. At the other end of the spectrum, Sweden, Israel, Iceland, Japan and Denmark recorded growth rates at or below $25 \%$. Similar trends were found in non-OECD countries. Growth in these countries was typically higher, which reflects their relatively low starting value. For example, the proportion of overweight and obese children in Indonesia, South Africa and India grew by over $600 \%$; however, their starting values were just $2.4 \%, 2.3 \%$, and $1 \%$, respectively.
Childhood obesity is a complex issue and its causes are multi-faceted. Consequently, the response has been to implement a suite of complementary policies involving government, community leaders, schools, health professionals and industry. Commonly used policies to alter individual behaviours or the obesogenic environment include tightened regulation of advertising of unhealthy foods and drinks targeted at children; improved access to parks and playgrounds; food reformulation policies; and price interventions to promote a healthy lifestyle (OECD, 2019[2]).

\section{Definition and comparability}

Childhood overweight and obesity rates were calculated using body mass index (BMI). BMI is calculated by dividing weight in kilograms by height in metres squared.

A child is considered overweight if their BMI is one standard deviation above the median, according to the World Health Organization child growth standards. A child whose BMI is two standard deviations above the median is classified as obese.

\section{References}

[1] Bösch, S. et al. (2018), Taking Action on Childhood Obesity, World Health Organization \& World Obesity Federation, Geneva, https://apps.who.int/iris/bitstream/handle/10665/274792/WHONMH-PND-ECHO-18.1-eng.pdf?ua=1.

[2] OECD (2019), The Heavy Burden of Obesity: The Economics of Prevention, Organisation for Economic Cooperation and Development, Paris, https://doi.org/10.1787/67450d67-en.

[3] OECD (2017), Obesity Update, OECD, Paris, https:// www.oecd.org/els/health-systems/Obesity-Update-2017.pdf. 
Figure 4.14. Overweight including obesity among 5-9 year olds by sex, 2016

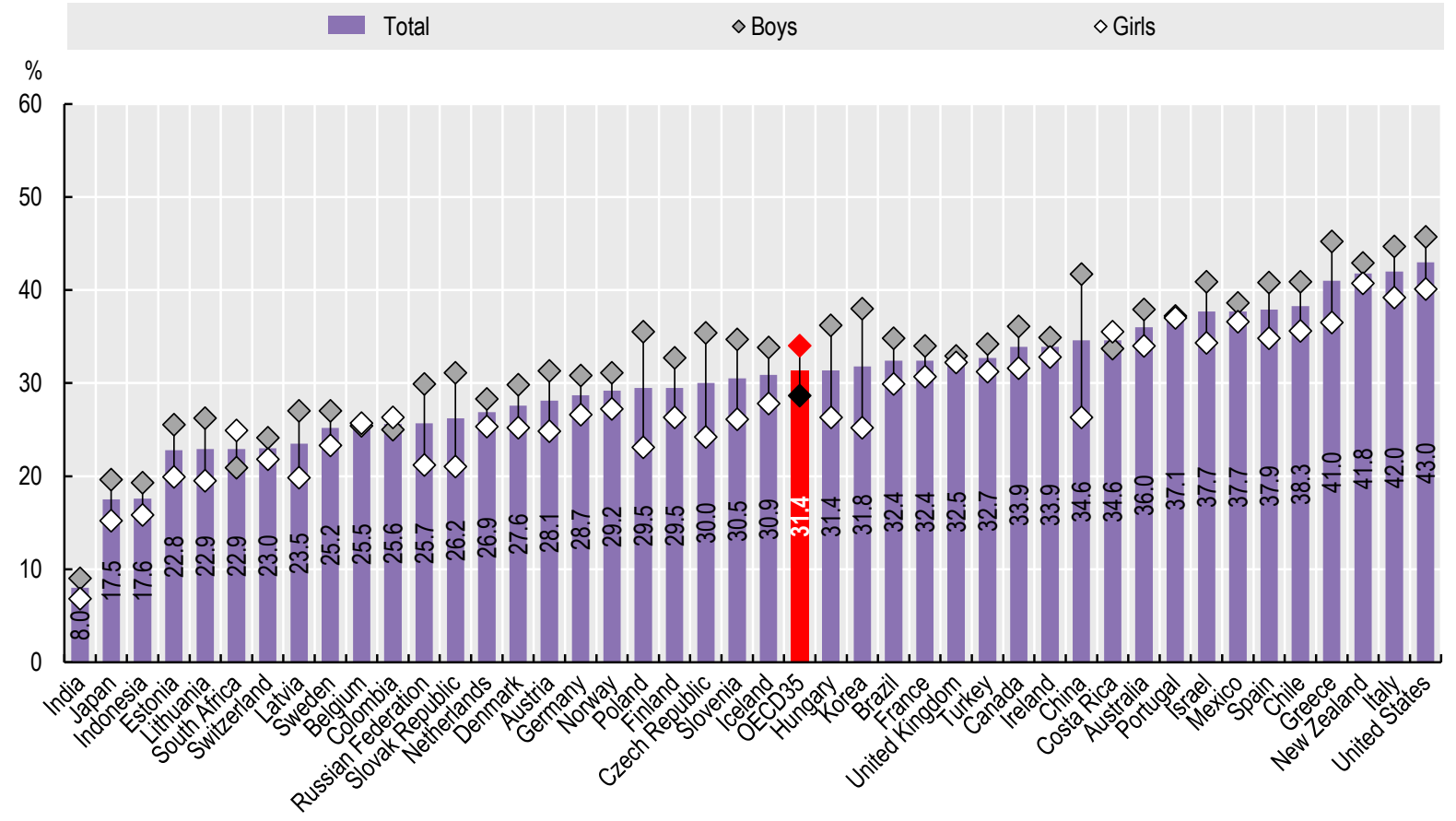

Source: WHO Global Health Observatory.

StatLink क्ञाजय https://doi.org/10.1787/888934015524

Figure 4.15. Change in overweight including obesity among 5-9 year olds, 1990-2016

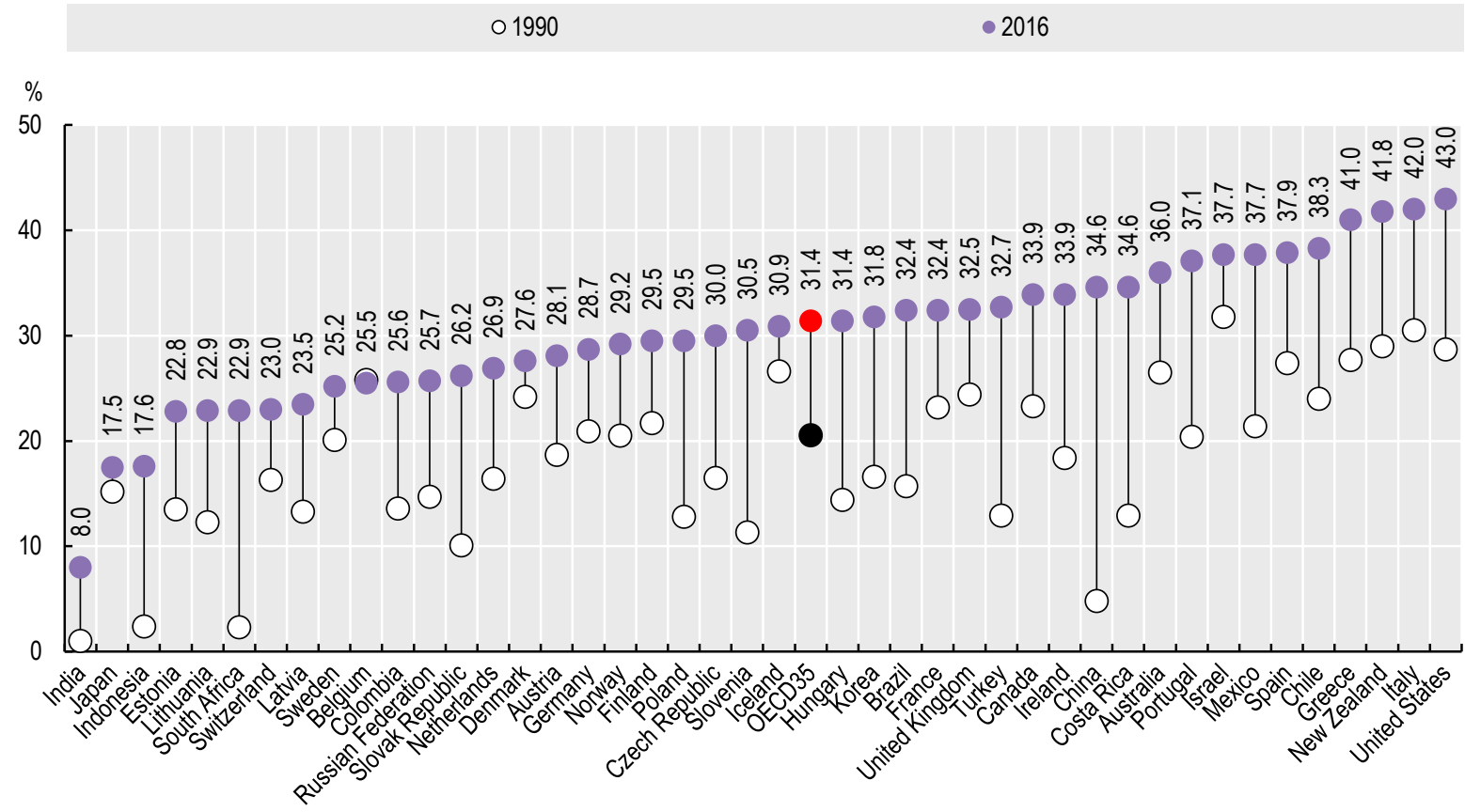

Source: WHO Global Health Observatory. 
Climate change is one of the biggest challenges of present and future generations. It is linked to different types of environment distress, including air pollution and extreme temperatures. Air pollution is already a major cause of death and disability today, and its future impact is likely to be even greater without adequate policy action. Projections have estimated that outdoor air pollution may cause 6 to 9 million premature deaths a year worldwide by 2060 , and cost $1 \%$ of global GDP as a result of sick days, medical bills and reduced agricultural output (OECD, 2015[1]).

Among OECD countries, ambient (outdoor) and household (indoor) air pollution caused about 40 deaths per 100000 people in 2016 (Figure 4.16). Death rates ranged from over 80 deaths per 100000 in Latvia, Hungary and Lithuania, to 15 deaths or less in New Zealand and Canada. In partner countries, death rates were particularly high in India and China (around 140 deaths per 100000 people), and also higher than most OECD countries in the Russian Federation and Indonesia.

Extreme temperatures are also a consequence of climate change. Both extreme heat and cold can cause health problems and lead to death, as has been experienced in some OECD countries in recent decades. Extreme cold has generally had a greater impact on mortality than heatwaves, particularly in Eastern Europe and Nordic countries. Still, heatwaves have caused significant numbers of deaths in certain years. For instance, the record warm summer of 2003 caused around 80000 deaths in Europe and the heatwaves in the summer of 2015 caused more than 3000 deaths in France alone.

Death rates due to cold extreme temperatures are far higher in Lithuania, Latvia and Estonia than other OECD countries, with over 1400 deaths per million people since 2000 (Figure 4.17). Although these high death rates are clearly linked to the naturally cold climates in these countries, they should not be viewed as inevitable - for example, Canada, Iceland and Norway had less than 80 deaths per million people over the same period. Evidence suggests that these deaths might be also linked to excessive alcohol use. For instance, in Finland among the deaths due to extreme cold in $2015-2017,46 \%$ of men and $24 \%$ of women were alcoholintoxicated.

Extreme heat caused 82 deaths per million people in Japan, followed by rates of 39 in France, 28 in Belgium and 21 in the United States since 2000. Whilst the total number of deaths due to cold temperatures has remained relatively stable since 2000, deaths from extreme heat have been on an upward trend, with two peaks in 2003 and 2010 (Figure 4.18).

Inter-sectoral policies are needed to address the impact of climate change. Countries can start planning to address pollution and its impacts on health, for instance, by creating partnerships with various international, national and local stakeholders, including local city authorities and ministries of industry, environment, transport, and agriculture. Bottled gas, for instance, can be used to replace solid fuels for cooking in order to address indoor pollution deaths. Reducing crop burning and lowering emissions from motor vehicles and industries would lower ambient air pollution. Health systems can also contribute, by preparing for new diseases that can develop with new climate conditions; promoting consumption of sustainably grown and sourced food; and reducing the carbon footprint of health facilities. In addition, health providers can reduce the environmental footprint in hospitals and in nursing homes by encouraging healthier food consumption, waste reduction and efficient energy use (Landrigan et al., 2018[2]; OECD, 2017[3]).

\section{Definition and comparability}

Household (indoor) air pollution results from polluting fuel used mainly for cooking. Ambient (outdoor) air pollution results from emissions from industrial activity, households, cars and trucks, which are complex mixtures of air pollutants, many of which are harmful to health. Of all of these pollutants, fine particulate matter has the greatest effect on human health. Polluting fuels include solid fuels such as wood, coal, animal dung, charcoal, crop wastes and kerosene. Attributable mortality is calculated by first combining information on the increased (or relative) risk of a disease resulting from exposure, with information on how widespread the exposure is in the population (e.g. the annual mean concentration of particulate matter to which the population is exposed). Applying this fraction to the total burden of disease (e.g. cardiopulmonary disease expressed as deaths or DALYs), gives the total number of deaths that results from exposure to household or ambient air pollution.

Data on fatalities due to extreme temperature events come from national registries on deaths by cause collected in the WHO Mortality Database. Deaths due to exposure to excessive natural heat (ICD code X30) and exposure to excessive natural cold (X31) were selected.

Note that for both air pollution and deaths from extreme temperatures, data are based on WHO estimates, which may differ from national data.

\section{References}

[2] Landrigan, P. et al. (2018), "The Lancet Commission on pollution and health", The Lancet, Vol. 391/10119, pp. 462-512, http://dx.doi.org/10.1016/s0140-6736(17)32345-0.

[3] OECD (2017), Healthy people, healthy planet - The role of health systems in promoting healthier lifestyles and a greener future, Organisation for Economic Cooperation and Development, OECD, Paris, http://www.oecd.org/health/healthy-peoplehealthy-planet.htm.

[1] OECD (2015), The Economic Consequences of Climate Change, OECD Publishing, Paris, https://dx.doi.org/ 10.1787/9789264235410-en. 
Figure 4.16. Ambient and household air pollution attributable death rate, 2016

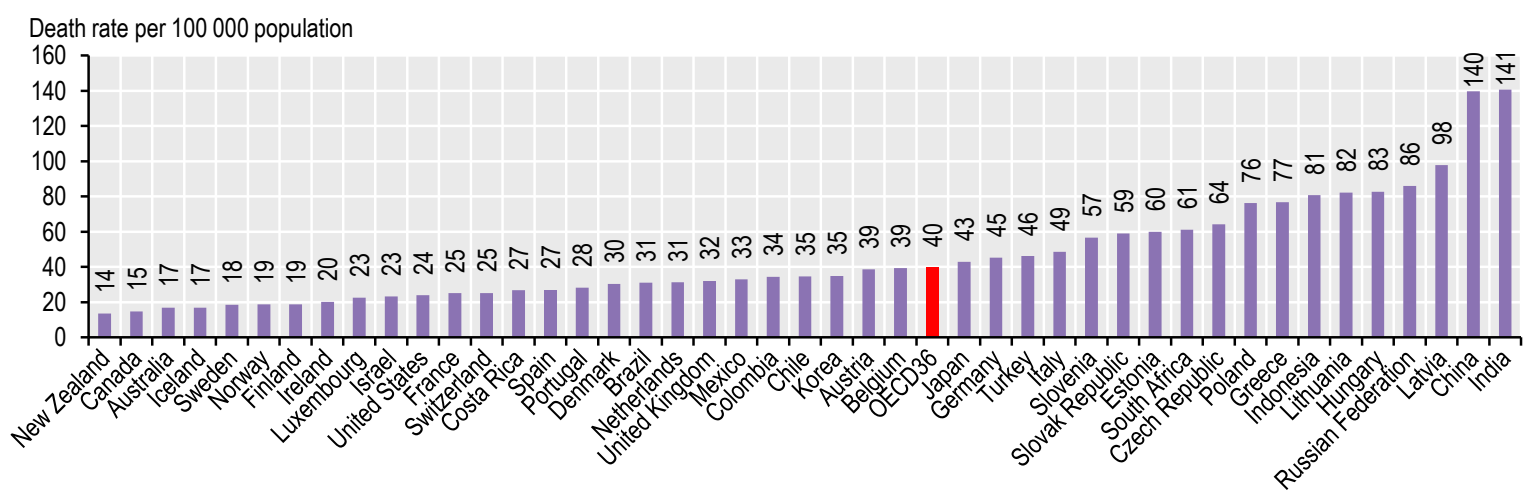

Source: Global Health Observatory data repository, WHO.

StatLink iㅣㄴ https://doi.org/10.1787/888934015562

Figure 4.17. Cumulative death rate due to extreme heat and extreme cold temperatures, 2000-17

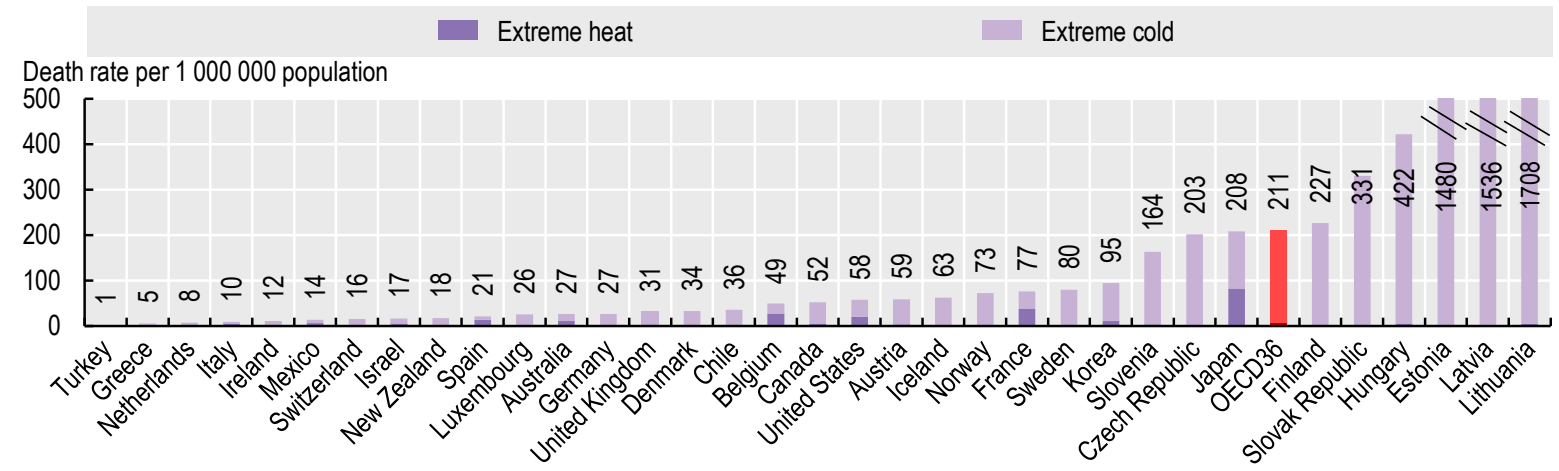

Note: Lithuania, Latvia and Estonia show cumulative death rates higher than 500 per 1000000 . The graph is truncated at this level to allow better comparability.

Source: WHO Mortality Database.

StatLink iㅔs https://doi.org/10.1787/888934015581

Figure 4.18. Number of deaths due to extreme heat and extreme cold temperatures in OECD36, 2000-16

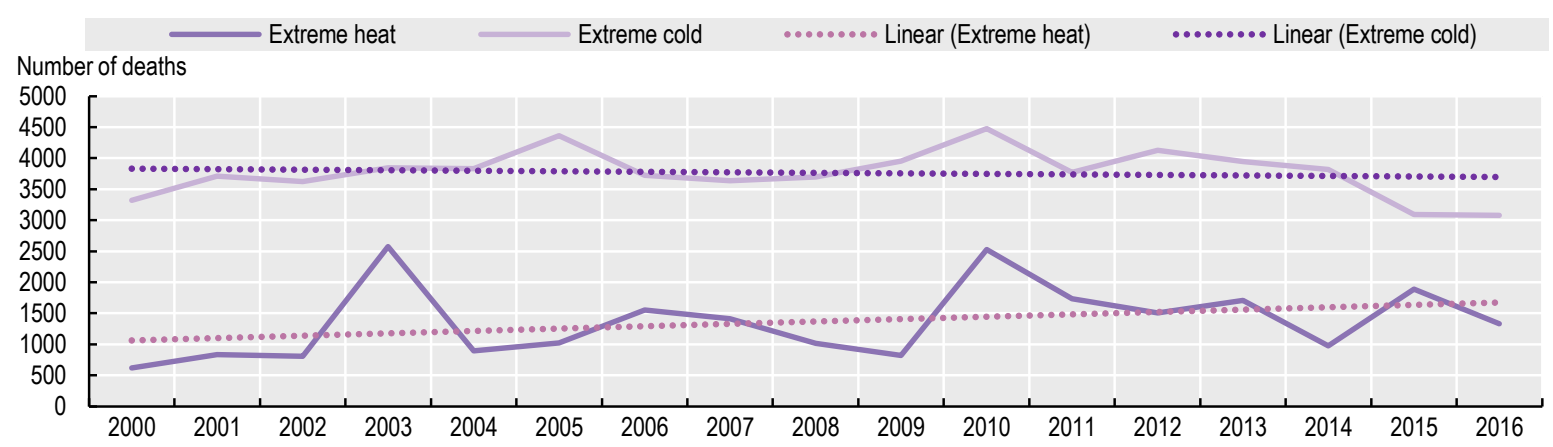

Source: WHO Mortality Database. 

The share of a population covered for a core set of health services offers an initial assessment of access to care and financial protection. However, it is only a partial measure of accessibility and coverage, focusing on the number of people covered. Universal health coverage also depends on the range of services covered and the degree of cost sharing for these services. Such services also need to be of sufficient quality. Indicators in this chapter focus on access and different dimensions of coverage, while Chapter 6 provides indicators on quality and outcomes of care.

Most OECD countries have achieved universal (or nearuniversal) coverage for a core set of health services, which usually include consultations with doctors, tests and examinations, and hospital care (Figure 5.1). National health systems or social health insurance have typically been the financing schemes for achieving universal health coverage. A few countries (the Netherlands, Switzerland) have obtained universality through compulsory private health insurance - supported by public subsidies and laws on the scope and depth of coverage. In Greece, a new law in 2016 closed the coverage gap for the $10 \%$ of the population who were previously uninsured.

Population coverage for core services remains below $95 \%$ in seven OECD countries, and is lowest in Mexico, the United States and Poland. Mexico has expanded coverage since 2004, but gaps remain. In the United States, the uninsured tend to be working-age adults with lower education or income levels - the share of people uninsured decreased sharply from about 13\% in 2013 to 9\% in 2015 (United States Census Bureau, 2018[1]), but has remained relatively unchanged since then. In Poland, the majority of uninsured are citizens living abroad. In Ireland, though coverage is universal, less than half of the population are covered for the cost of GP visits.

In some countries, citizens can purchase additional health coverage through voluntary private insurance. This can cover any cost sharing left after basic coverage (complementary insurance), add further services (supplementary insurance) or provide faster access or larger choice of providers (duplicate insurance). Eight OECD countries have additional private insurance coverage for over half of the population (Figure 5.2). In France, nearly all of the population (96\%) have complementary insurance to cover cost sharing in the social security system - with public subsidies making it free or at reduced rates for poor households. Complementary insurance is also widely used in Belgium, Slovenia and Korea. Israel and the Netherlands have the largest supplementary market (over $80 \%$ of the population), whereby private insurance pays for dental care, physiotherapy, certain prescription drugs and other services not publicly reimbursed. Duplicate private health insurance, providing faster private sector access to medical services where there are waiting times in public systems, are largest in Ireland and Australia. In the United States, $8 \%$ of the population has complementary private health insurance. This is in addition to the $55 \%$ of the population with primary private health insurance.

Over the last decade, the population covered by additional private health insurance has increased in 18 of 27 OECD countries with comparable data, though these increases have often been small. Changes have been most marked in Korea, Denmark, Slovenia and Finland (Figure 5.3). Note that in Slovenia increases were mainly due to one insurance company adding free supplementary health insurance to its insurance portfolio. Several factors determine how additional private health insurance evolves, notably the extent of gaps in access to publicly financed services and government interventions directed at private health insurance markets.

\section{Definition and comparability}

Population coverage for health care is defined here as the share of the population eligible for a core set of health care services - whether through public programmes or primary private health insurance. The set of services is country-specific but usually includes consultations with doctors, tests and examinations, and hospital care. Public coverage includes both national health systems and social health insurance. On national health systems, most of the financing comes from general taxation, whereas in social health insurance systems, financing typically comes from a combination of payroll contributions and taxation. Financing is linked to ability-to-pay. Primary private health insurance refers to insurance coverage for a core set of services, and can be voluntary or mandatory by law (for some or all of the population). Additional private health insurance is always voluntary. Private insurance premiums are generally not income-related, although the purchase of private coverage may be subsidised by government.

\section{References}

[3] OECD (2016), OECD Reviews of Health Systems: Mexico, OECD Publishing, Paris, https://doi.org/10.1787/9789264230491-en.

[2] OECD/European Observatory on Health Systems and Policies (forthcoming), Greece, Ireland, Poland, Country Health Profiles 2019, State of Health in the EU.

[1] United States Census Bureau (2018), Health Insurance Coverage in the United States. 
Figure 5.1. Population coverage for a core set of services, 2017 (or nearest year)

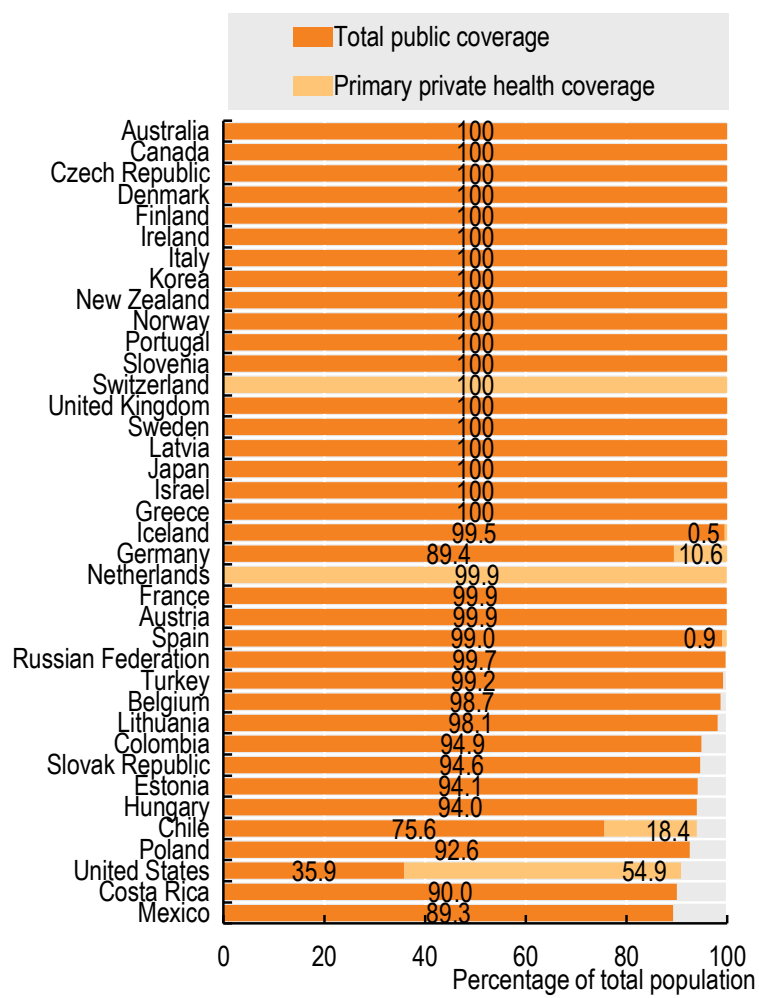

Source: OECD Health Statistics 2019.

StatLink काIs https://doi.org/10.1787/888934015619
Figure 5.2. Voluntary private health insurance coverage by type, 2017 (or nearest year)

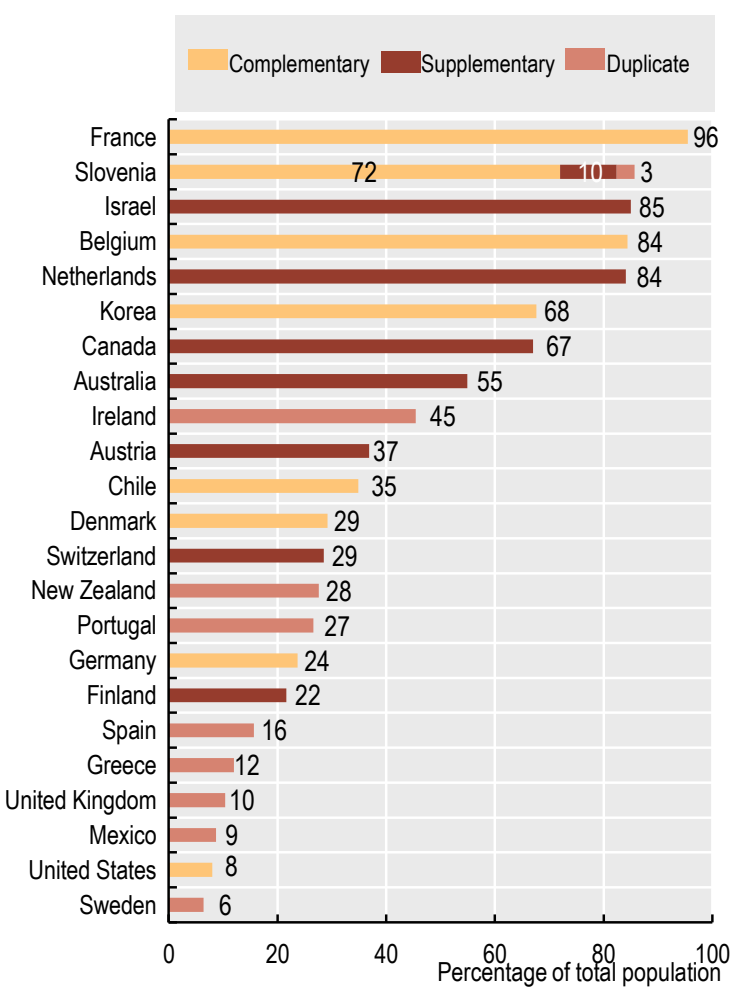

Note: Private health insurance can be both duplicate and supplementary in Australia; complementary and supplementary in Denmark and Korea; and duplicate, complementary and supplementary in Israel and Slovenia. In the United States, $55 \%$ of the population also has primary private health insurance.

Source: OECD Health Statistics 2019.

StatLink AाIs https://doi.org/10.1787/888934015638

Figure 5.3. Trends in private health insurance coverage, 2007 and 2017 (or nearest year)

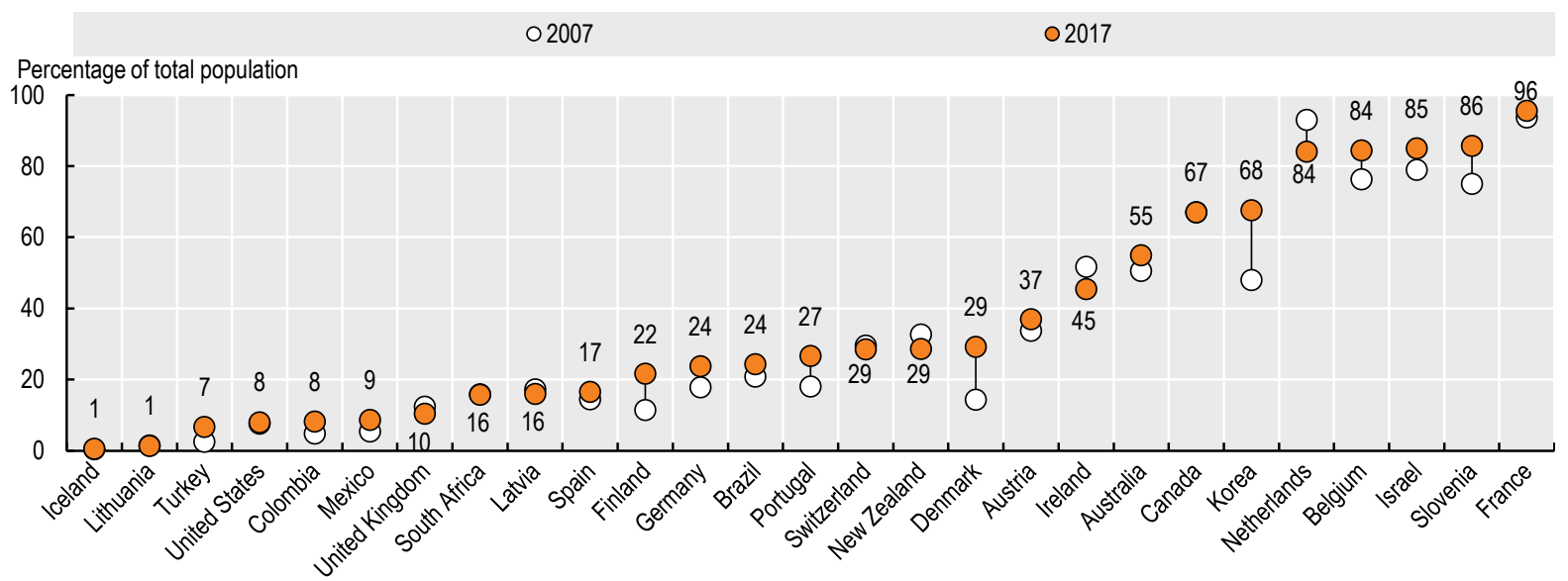

Source: OECD Health Statistics 2019. 
In addition to the share of the population entitled to core health services, the extent of health care coverage is defined by the range of services included in a publicly defined benefit package and the proportion of costs covered. Figure 5.4 assesses the extent of overall coverage, as well as coverage for selected health care services, by computing the share of expenditure covered under government schemes or compulsory health insurance. Differences across countries in the extent of coverage can be due to specific goods and services being included or excluded in the publicly defined benefit package (e.g. a particular drug or medical treatment); different cost-sharing arrangements; or some services only being covered for specific population groups in a country (e.g. dental treatment).

On average across OECD countries, almost three-quarters of all health care costs were covered by government or compulsory health insurance schemes. This share rose above $80 \%$ in ten countries (Norway, Germany, Japan, Denmark, Luxembourg, Sweden, France, the Czech Republic, Iceland, the Netherlands). However, in Mexico, Latvia and Korea less than $60 \%$ of all costs are covered by publicly mandated schemes. Coverage is also comparatively low in the Russian Federation.

Inpatient services in hospitals are more comprehensively covered than any other type of care. Across the OECD, $88 \%$ of all inpatient costs are borne by government or compulsory insurance schemes. In many countries, patients have access to free acute inpatient care or only have to make a small copayment. As a result, coverage rates are near $100 \%$ in Sweden, Norway, Iceland and Estonia. Only in Korea, Mexico, Greece, Australia and Ireland is the financial coverage for the cost of inpatient care $70 \%$ or lower. In some of those countries, patients frequently choose treatment in private facilities where coverage is not (fully) included in the public benefit package.

More than three-quarters of spending on outpatient medical care in OECD countries are borne by government and compulsory insurance schemes (77\%). Coverage ranged from under $60 \%$ in Korea and Italy, to over $90 \%$ in the Slovak Republic, Denmark and the Czech Republic. Outpatient primary and specialist care are generally free at the point of service, but user charges may still apply for specific services or if non-contracted private providers are consulted. This is for example the case in Denmark, where $92 \%$ of total costs are covered but user charges exist for visits to psychologists and physiotherapists, and the United Kingdom (85\%), where care provision outside of NHS commissioned services are not covered.

Public coverage for dental care costs is far more limited across the OECD due to restricted service packages (frequently limited to children) and higher levels of costsharing. On average only around $30 \%$ of dental care costs are borne by government schemes or compulsory insurance. More than half of dental spending is covered in only three OECD countries (Japan, Germany and the Slovak Republic). In Greece and Spain, dental care costs for adults without any specific entitlement are not covered. Voluntary health insurance may play an important role in providing financial protection when dental care is not comprehensively covered in the benefit package (e.g. the Netherlands).

Coverage for pharmaceuticals is also typically less comprehensive than for inpatient and outpatient care: across the OECD, around $57 \%$ of pharmaceutical costs are covered by government or compulsory insurance schemes. This share is less than $40 \%$ in Lithuania, Iceland, Poland, Canada and Latvia. Coverage is most generous in Germany (84\%), followed by France (80\%) and Ireland (78\%). Over-thecounter medications - which by their nature are not usually covered by public schemes - play an important role in some countries (see indicator "Pharmaceutical Expenditure" in Chapter 10).

\section{Definition and comparability}

Health care coverage is defined by the share of the population entitled to services, the range of services included in a benefit package and the proportion of costs covered by government schemes and compulsory insurance schemes. Coverage provided by voluntary health insurance and other voluntary schemes such as charities or employers is not considered. The core functions analysed here are defined based on definitions in the System of Health Accounts 2011. Hospital care refers to inpatient curative and rehabilitative care in hospitals, outpatient medical care to all outpatient curative and rehabilitative care excluding dental care, pharmaceuticals to prescribed and over-the-counter medicines including medical non-durables.

Comparing the shares of the costs covered for different types of services is a simplification. For example, a country with more restricted population coverage but a very generous benefit basket may display a lower share of coverage than a country where the entire population is entitled to services but with a more limited benefit basket. 
Figure 5.4. Extent of coverage in OECD countries, 2017 (or nearest year)

Government and compulsory insurance spending as proportion of total health spending by type of care

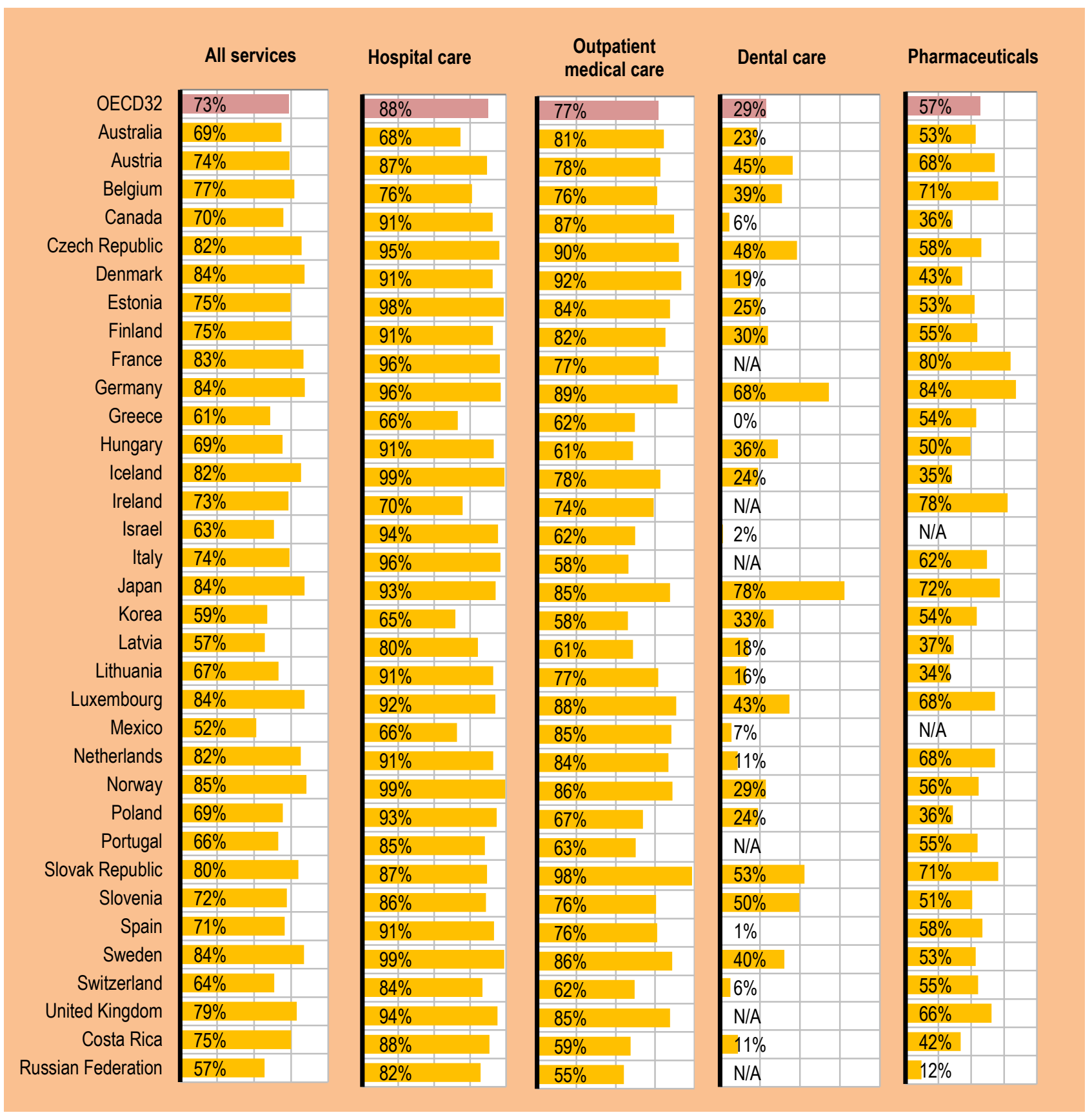

Source: OECD Health Statistics 2019. 
Primary care services are the main entry point into health systems. Indicators on the use of such services therefore provide a critical barometer of accessibility, with data disaggregated by income illustrating the degree of inequalities in access.

In terms of access to a doctor, on average just under $80 \%$ of individuals aged 15 or over reported visiting a doctor in the past year, adjusting for need (Figure 5.5). Note that need is modelled, rather than measured directly (see definition and comparability box). Furthermore, the probability of visiting a doctor may be lower in some countries because people make greater use of other types of health professionals, such as nurses. This is the case, for example, in Sweden, where other licensed healthcare professionals see patients that do not have a clear medical need for a doctor. Notwithstanding these issues, cross-country differences in utilisation are large, with need-adjusted probabilities of visiting a doctor ranging from around 65\% in Sweden and the United States to $89 \%$ in France.

Socioeconomic inequalities in accessing a doctor are evident within almost all OECD countries. Excepting Denmark and the Slovak Republic, wealthier individuals are more likely to see a doctor than individuals in the lowest income quintile, for a comparable level of need. Pro-rich inequalities in doctor access are highest in Finland and the United States (over 15 percentage-points difference) but practically nonexistent in the United Kingdom, Ireland and the Netherlands. Income inequalities in accessing doctors are much more marked for specialists than for general practitioners (OECD, $2019_{[1]}$ ).

For dental care, only $63 \%$ of individuals aged 15 or over reported visiting a dentist in the past year, on average across 27 OECD countries (Figure 5.6). This is partly due to benefit design: public coverage for dental care is much lower than for hospital care or doctor consultations in many OECD countries (see indicator on "Extent of health care coverage"). Overall access to dental care ranged from $41 \%$ of people visiting a dentist in the United States, to $93 \%$ in Ireland. Socioeconomic disparities are large - on average, there is an almost 20 percentage-point difference in visits between high and low-income groups ( $72 \%$ of wealthier individuals visited a dentist, compared with $54 \%$ among those from the lowest income quintile). Inequalities are largest in Canada, Portugal and the United States (over 30 percentage-point difference); but almost zero in Ireland.

Uptake of cancer screening is also lower amongst the less well-off. This is despite most OECD countries providing screening programmes at no cost. For example, on average $79 \%$ of wealthier women had a Pap smear test for cervical cancer, as compared with $65 \%$ amongst women from the lowest income quintile (Figure 5.7). Wealthier people also have greater access to screening for both breast and colorectal cancer, though inequalities are less marked than for cervical cancer. Screening for cervical cancer is disproportionately low among the bottom income group in Sweden and Norway (over 30 percentage-point gap between income quintiles), but relatively equal in Ireland, Chile and Iceland. Overall uptake of cervical cancer screening ranged from just under $50 \%$ in the Netherlands, to over $85 \%$ in the Czech Republic and Austria. This applies to women aged 20 to 69 with a screening interval of three years. Note that some countries (e.g. the Netherlands) offer screening amongst a narrower age group and less frequently. This may result in lower screening rates but not necessarily worse performance. Countries offering nationwide populationbased screening programmes have more equal access, as compared with countries where cancer screening happens in a more ad-hoc manner (Palencia, 2010 $0_{[2]}$ )

Such observed problems in accessing health services, particularly for the less well-off, occur despite most OECD countries having universal or near-universal coverage for a core set of services (see indicator on "Population coverage for health care"). Part of the explanation are high cost sharing, exclusion of some services from benefit packages or implicit rationing of services. Limitations in health literacy, imperfect communication strategies, and low quality of care are also contributing factors.

\section{Definition and comparability}

The health care module of the European Health Interview Survey (EHIS) and of national surveys allows respondents to report on their utilisation of health care services, whether they have visited a GP, specialist or dentist in the past year, as well as their use of various screening services.

The probability of visiting a doctor is defined as having seen a GP or a specialist in the past year. However, the volume of care a person receives in itself does not accurately measure access, as people have varying health care needs. Need is not measured directly. Rather, predicted needs are modelled, and then the probability of visiting a doctor is adjusted by this value (see O'Donnell $\left(2008_{[3]}\right)$ for further methodological details). Here, four categorical variables are used to model predicted need: age, sex, self-rated health and activity limitations.

Cervical cancer screening is defined as the proportion of women aged 20-69 who have undergone a Pap smear test in the past 3 years.

\section{References}

[4] Moreira, L. (2018), "Health literacy for people-centred care: Where do OECD countries stand?", OECD Health Working Papers, No. 107, OECD Publishing, Paris, https://doi.org/ 10.1787/d8494d3a-en.

[3] O'Donnell, O. (2008), Analyzing Health Equity Using Household Survey Data: A Guide to Techniques and Their Implementation, World Bank Group, Washington D.C., http://dx.doi.org/ 10.1596/978-0-8213-6933-3.

[1] OECD (2019), Health for everyone? Social Inequalities in Health and Health Systems, OECD Health Policy Studies, OECD Publishing, Paris, https://doi.org/10.1787/3c8385d0-en.

[2] Palencia, L. (2010), "Socio-economic inequalities in breast and cervical cancer screening practices in Europe: influence of the type of screening program", International Journal of Epidemiology, Vol. 39/3, pp. 757-765. 
Figure 5.5. Need-adjusted probability of visiting a doctor, by income, 2014

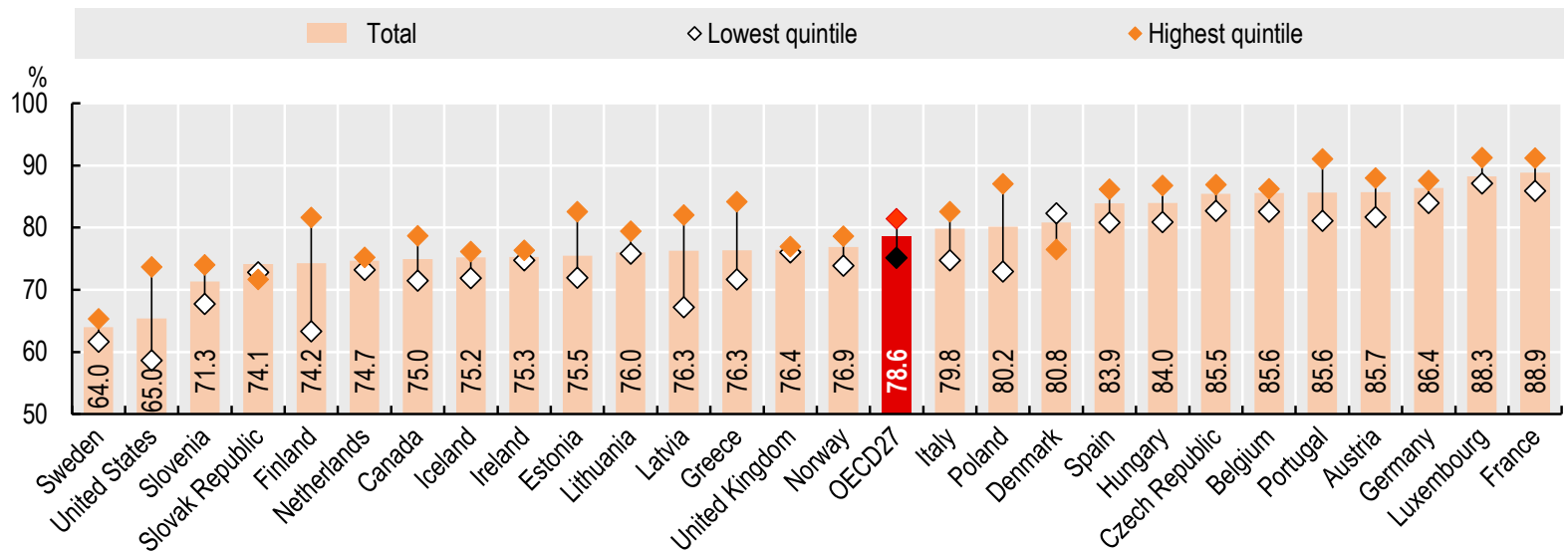

Source: OECD estimates based on EHIS-2 and other national survey data. In Sweden, the low number is explained in part by patients often visiting other healthcare professionals rather than doctors in primary care.

Figure 5.6. Share of the population who visited a dentist, by income, 2014

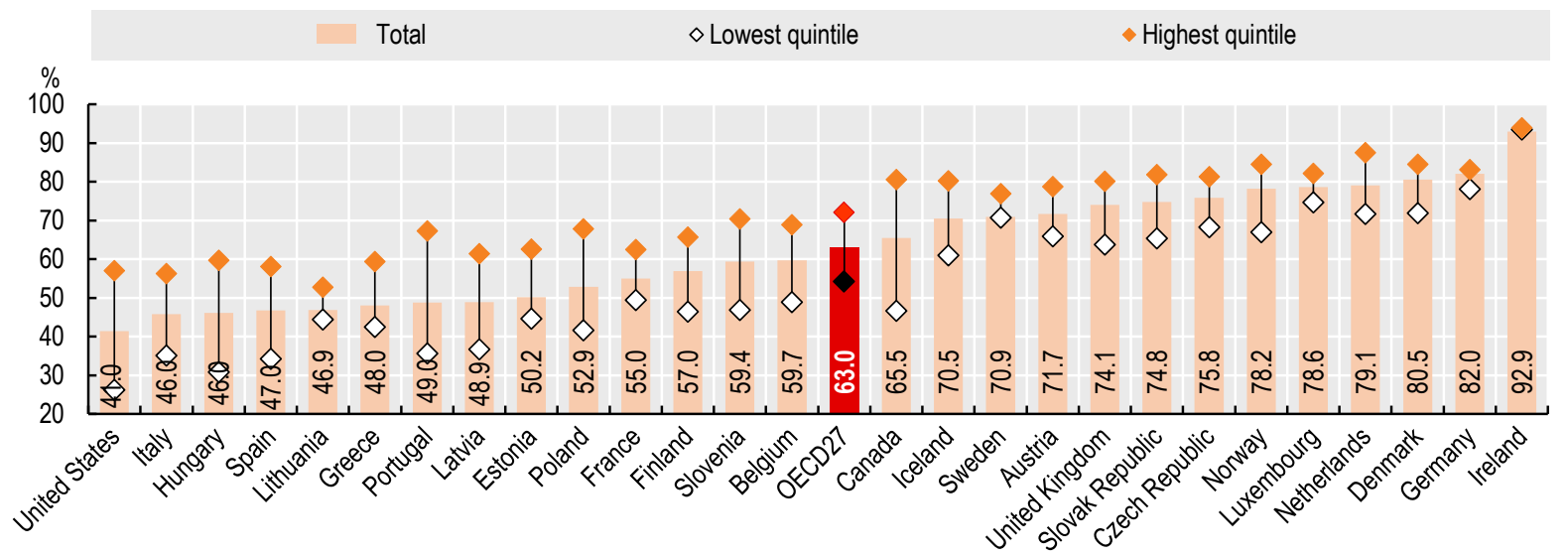

Source: OECD estimates based on EHIS-2 and other national survey data.

StatLink क्गाड़ https://doi.org/10.1787/888934015714

Figure 5.7. Share of women aged 20-69 screened for cervical cancer, by income, 2014

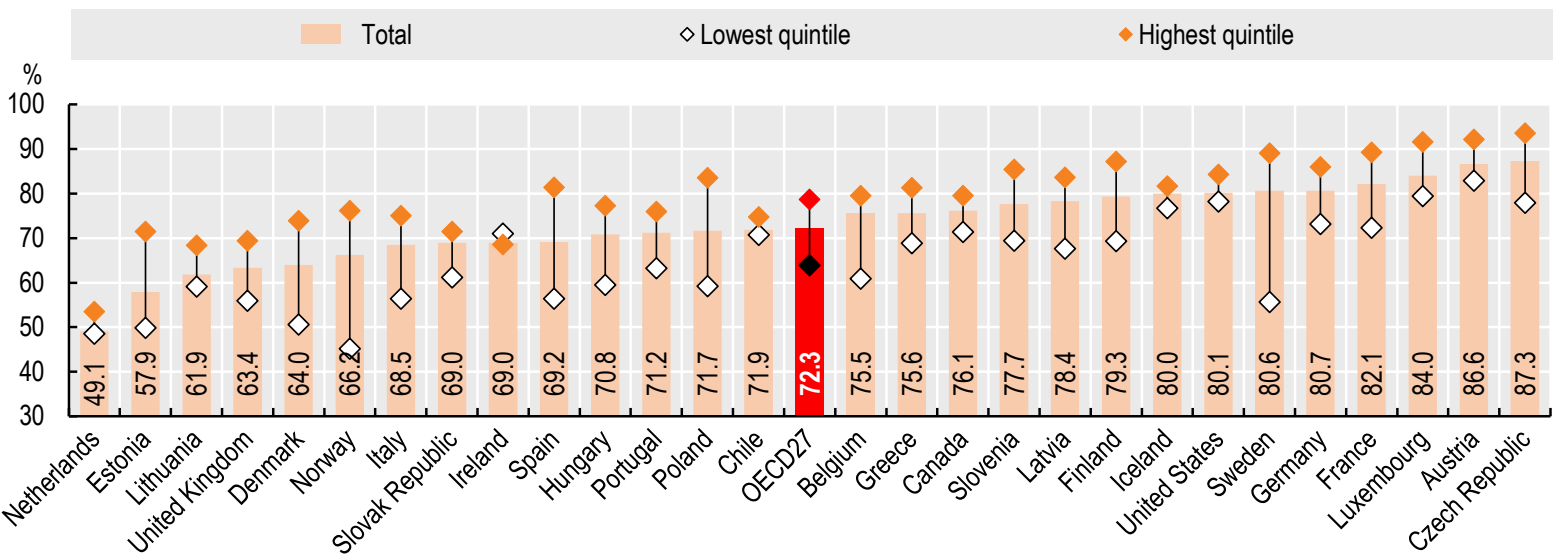

Source: OECD estimates based on EHIS-2 and other national survey data. 
People should be able to access health services when they need to, irrespective of their socio-economic circumstances. This is a fundamental principle underpinning all health systems across the OECD. Yet a quarter of individuals aged 18 or older report unmet need (defined as forgoing or delaying care) because limited availability or affordability of services compromise access, on average across 23 OECD countries. People may also forgo care because of fear or mistrust of health service providers. Strategies to reduce unmet need, particularly for the less well-off, need to tackle both financial and non-financial barriers to access (OECD, 2019[1]).

Looking specifically at availability of services, just over $20 \%$ of respondents reported unmet need due to waiting times and/or transportation difficulties (Figure 5.8). The share of the population delaying or forgoing care is comparatively high in Luxembourg, Italy, Ireland and Iceland (above 30\%); but much lower in Norway (5\%) and the Slovak Republic (7\%). In response to this accessibility constraint, telemedicine initiatives are becoming more popular in many OECD countries (Hashiguchi Cravo Oliveira, forthcoming[2]). Socioeconomic disparities are significant: on average, $23 \%$ of people from the lowest income quintile report availabilityrelated unmet need compared with $18 \%$ for richer individuals. This income gradient is largest in Finland, Italy and Portugal. In Slovenia, Poland and Estonia, richer individuals report slightly more unmet need than the less well-off, with results driven by the better-off being more likely to report waiting times as a cause of unmet need.

In terms of affordability, $17 \%$ of respondents delayed or did not seek needed care because the costs were too high for them (Figure 5.9). Across countries, unmet need due to such financial reasons ranged from less than $7 \%$ of the population in the Netherlands, the Czech Republic, the United Kingdom and Norway, to over $30 \%$ in Estonia, Ireland and Latvia. Affordability-related inequalities are more marked than inequalities related to availability of services. On average, $28 \%$ of people in the lowest income quintile forgo care for financial reasons compared with $9 \%$ for richer individuals. That is, the least well-off are three times more likely than the better-off to have unmet need for financial reasons.

Amongst people aged 65 or older, affordability constraints are slightly less marked than for the population as a whole. The proportion of cost-related reported unmet need is lower among older people, on average (14\% compared to $17 \%$ across the OECD) and in most countries (17 out of 23). Income inequalities are also less marked among older individuals. Although older people from the top income quintile report similar levels of forgone care to the overall top quintile ( $8 \%$ and $9 \%$ respectively), older people from the bottom income quintile report significantly lower levels on average ( $20 \%$ compared to $27 \%$ ).

\section{Definition and comparability}

The health care module of the European Health Interview Survey (EHIS) and of national surveys allows respondents to report on their utilisation of health care services, as well as potential barriers experienced when trying to access these services. The probability of reporting an unmet need due to availability issues is based on two of the available variables: unmet need due to long waiting lists or to physical accessibility (distance or transportation). The probability to report forgone care due to financial reasons aggregates unmet need for four different types of service (medical, dental and mental health services, and prescription drugs). Respondents who reported not having a health care need in the past 12 months were excluded from the sample. Probabilities thus reflect the proportion of people reporting an unmet need, among individuals that have reported a need, satisfied or not (rather than the total population surveyed). This leads to higher estimates than surveys where unmet needs are calculated as a share of the total population - as is done, for example, with the EU-SILC survey.

\section{References}

[2] Hashiguchi Cravo Oliveira, T. (forthcoming), "Is telemedicine leading to more cost-effective, integrated and peoplecentred care in the OECD?", OECD Health Working Papers, OECD Publishing, Paris.

[1] OECD (2019), Health for Everyone? Social inequalities in health and health systems, OECD Publishing, Paris, https://doi.org/ 10.1787/3c8385do-en. 
Figure 5.8. Population forgoing or postponing care because of limited availability, by income, 2014

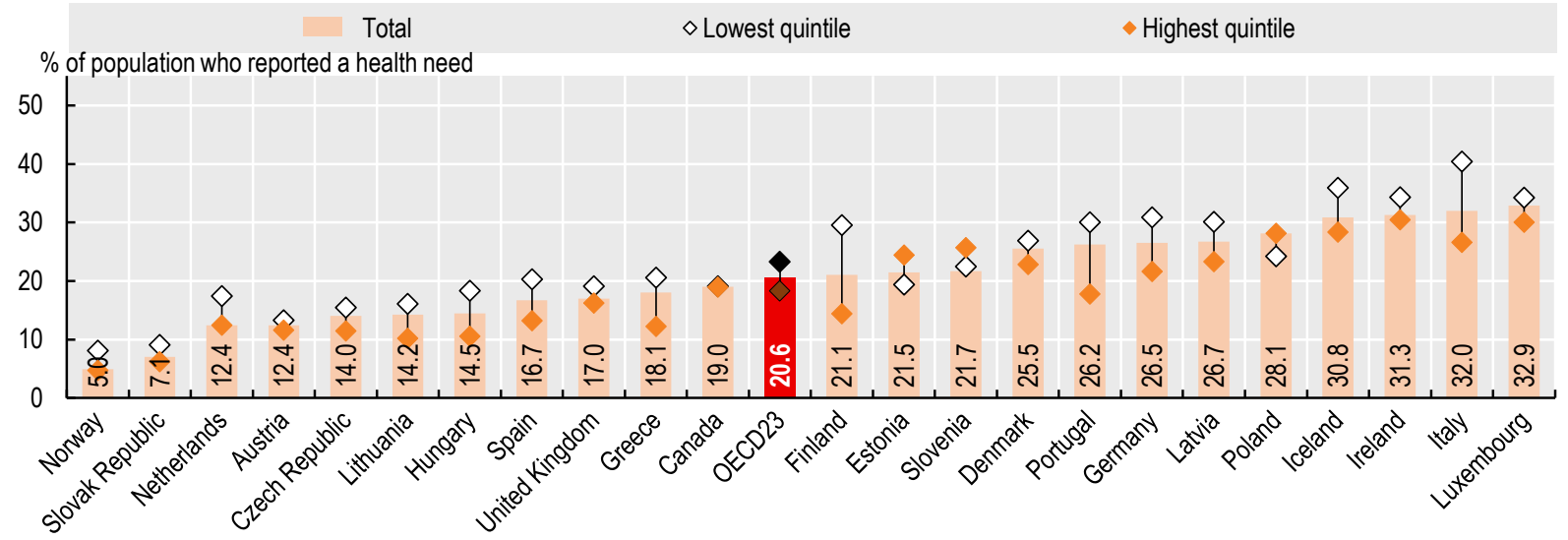

Source: OECD estimates based on EHIS-2 and other national health survey data.

Figure 5.9. Population forgoing care because of affordability, by income, 2014

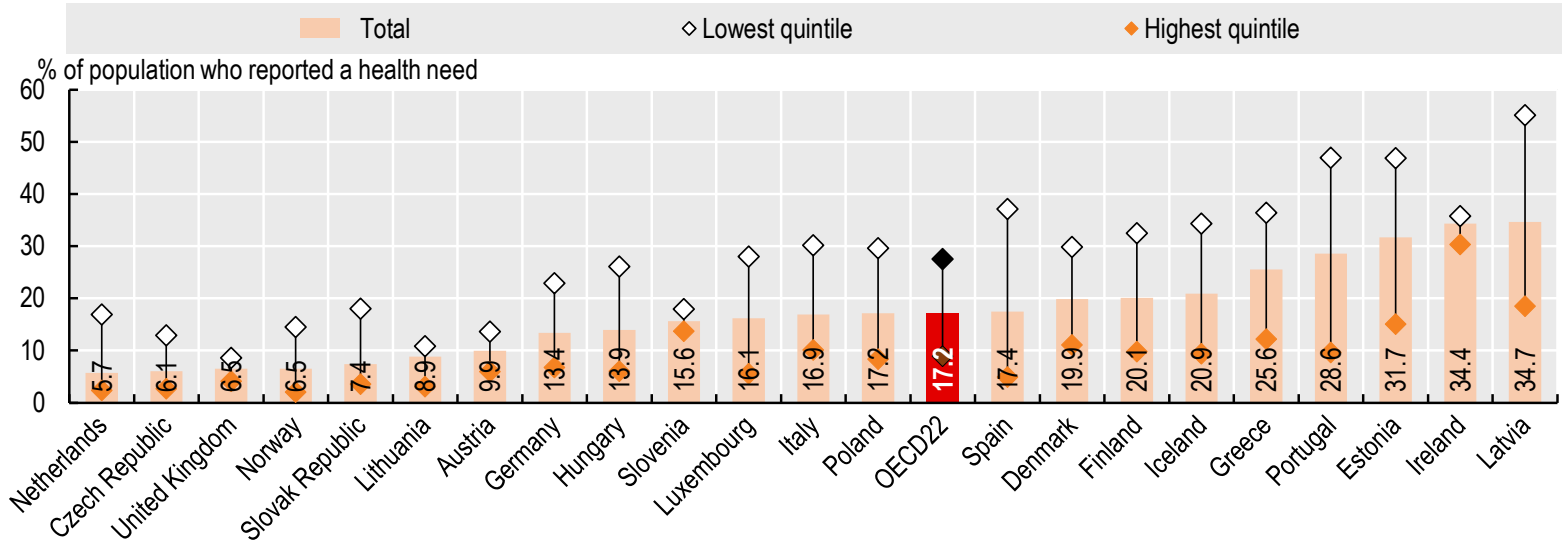

Source: OECD estimates based on EHIS-2.

Figure 5.10. Adults over 65 forgoing or postponing care because of affordability, by income, 2014

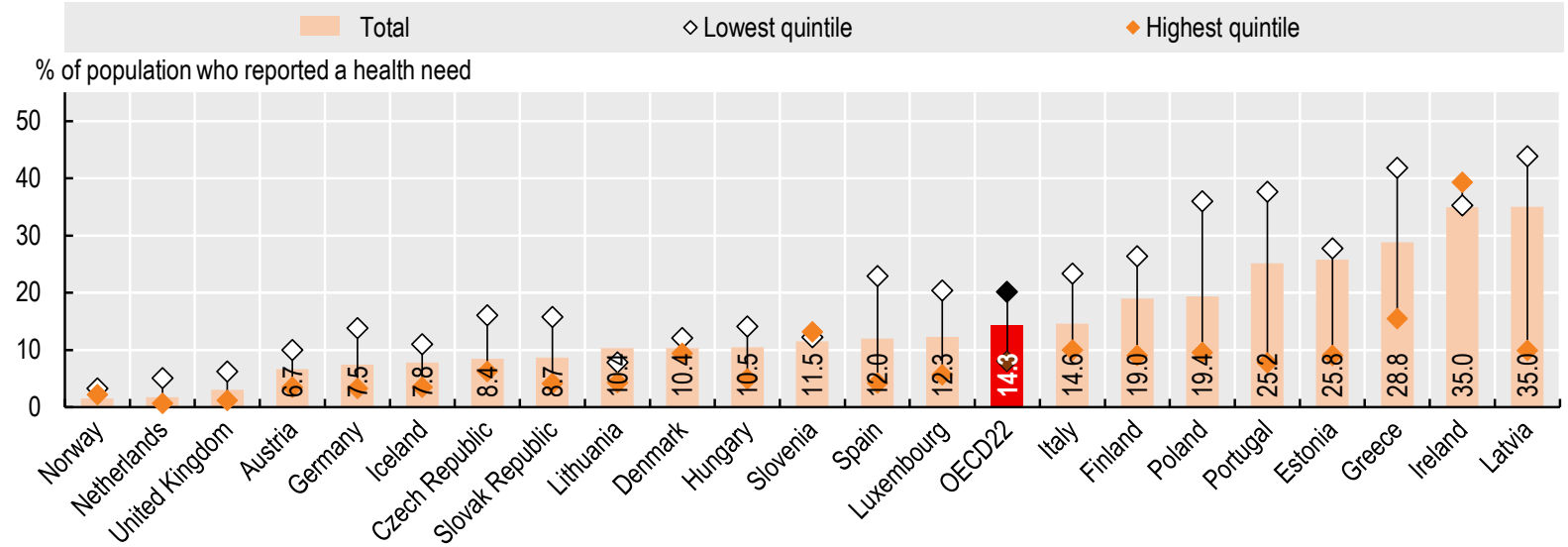

Source: OECD estimates based on EHIS-2. 
Where health systems fail to provide adequate financial protection, people may not have enough money to pay for health care or meet other basic needs. As a result, lack of financial protection can reduce access to health care, undermine health status, deepen poverty and exacerbate health and socio-economic inequalities. On average across OECD countries, just over a fifth of all spending on health care comes directly from patients through out-of-pocket (OOP) payments (see indicator "Financing of health care"). People experience financial hardship when the burden of such OOP payments is large in relation to their ability to pay. Poor households and those who have to pay for long-term treatment such as medicines for chronic illness are particularly vulnerable.

The share of household consumption spent on health care provides an aggregate assessment of the financial burden of OOP expenditure. Across OECD countries, about 3\% of total household spending was on health care goods and services, ranging from around $2 \%$ in France, Luxembourg and Slovenia, to more than $5 \%$ in Korea and nearly $7 \%$ in Switzerland (Figure 5.11).

Health systems in OECD countries differ in the degree of coverage for different health goods and services (see indicator "Extent of health care coverage"). Household spending on pharmaceuticals and other medical goods was the main health care expense for people, followed by spending on outpatient care (Figure 5.12). These two components typically account for almost two-thirds of household spending on health care. Household spending on dental care and long-term health care can also be high, averaging $14 \%$ and $11 \%$ of OOP spending on health respectively. Inpatient care plays only a minor role (9\%) in the composition of OOP spending.

The indicator most widely used to measure financial hardship associated with OOP payments for households is the incidence of catastrophic spending on health (Cylus et al., 2018[1]). This varies considerably across OECD countries, from fewer than $2 \%$ of households experiencing catastrophic health spending in France, Sweden, the United Kingdom, Ireland, the Czech Republic and Slovenia, to over $8 \%$ of households in Portugal, Poland, Greece, Hungary, Latvia and Lithuania (Figure 5.13). Across all countries, poorer households (i.e. those in the bottom consumption quintile) are most likely to experience catastrophic health spending, despite the fact that many countries have put in place policies to safeguard financial protection.

Countries with comparatively high levels of public spending on health and low levels of OOP payments typically have a lower incidence of catastrophic spending. However, policy choices are also important, particularly around coverage policy (WHO Regional Office for Europe, 2018[2]). Population entitlement to publicly financed health care is a prerequisite for financial protection, but not a guarantee of it. Countries with a low incidence of catastrophic spending on health are also more likely to exempt poor people and frequent users of care from co-payments; use low fixed co-payments instead of percentage co-payments, particularly for outpatient medicines; and cap the co-payments a household has to pay over a given time period (e.g. Austria, the Czech Republic, Ireland and the United Kingdom).

\section{Definition and comparability}

Out-of-pocket (OOP) payments are expenditures borne directly by a patient where neither public nor private insurance cover the full cost of the health good or service. They include cost-sharing and other expenditure paid directly by private households and should also ideally include estimations of informal payments to health providers.

Catastrophic health spending is an indicator of financial protection used to monitor progress towards universal health coverage (UHC). It is defined as OOP payments that exceed a predefined percentage of the resources available to a household to pay for health care. Household resources available can be defined in different ways, leading to measurement differences. In the data presented here, these resources are defined as household consumption minus a standard amount representing basic spending on food, rent and utilities (water, electricity, gas and other fuels). The threshold used to define households with catastrophic spending is $40 \%$. Microdata from national household budget surveys are used to calculate this indicator.

\section{References}

[1] Cylus, J., Thomson, S., Evetovits, T (2018), "Catastrophic health spending in Europe: equity and policy implications of different calculation methods", Bulletin of the World Health Organization, Vol. 96 No. 9, http://dx.doi.org/10.2471/ BLT.18.209031.

[2] WHO Regional Office for Europe (2019). "Can people afford to pay for health care? New evidence on financial protection in Europe", WHO Regional Office for Europe, Copenhagen. 
Figure 5.11. Out-of-pocket spending as share of final household consumption, 2017 (or nearest year)

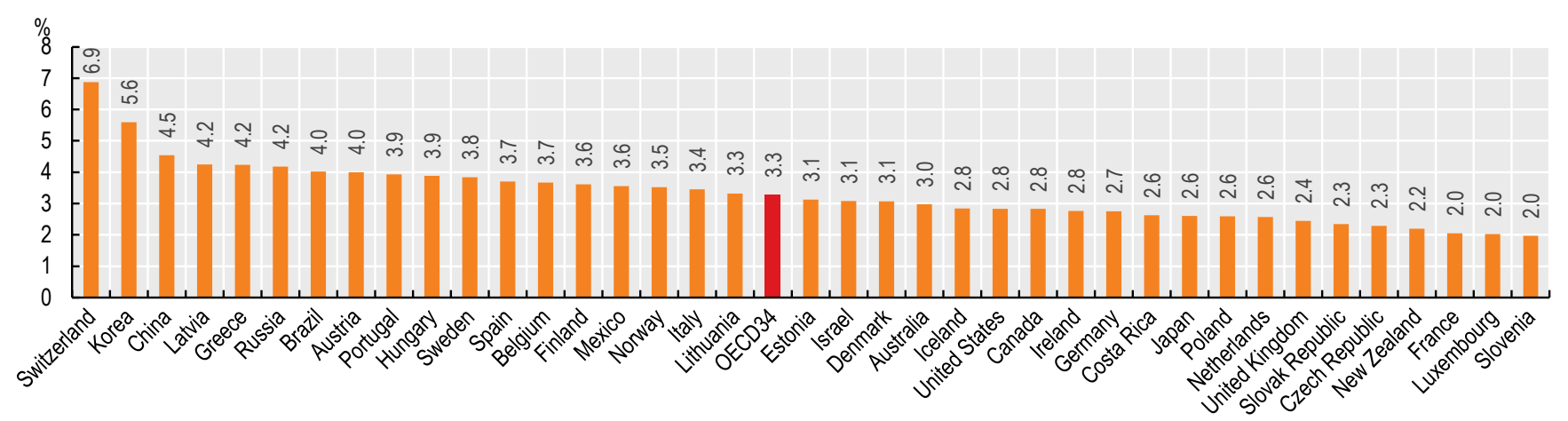

Source: OECD Health Statistics 2019, OECD National Accounts Database.

StatLink क्ञाज https://doi.org/10.1787/888934015809

Figure 5.12. Out-of-pocket spending on health, by type of services, 2017 (or nearest year)

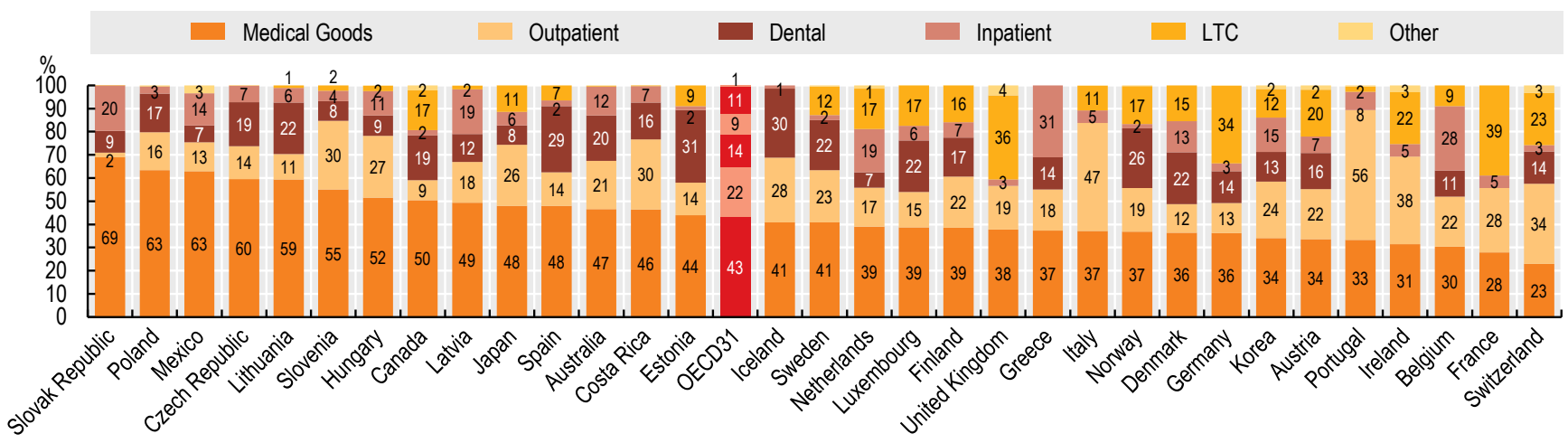

Note: The "Medical Goods" category includes pharmaceuticals and therapeutic appliances. The "Other" category includes preventive care, administrative services and services unknown.

Source: OECD Health Statistics 2019.

StatLink 에s https://doi.org/10.1787/888934015828

Figure 5.13. Share of households with catastrophic health spending by consumption quintile, latest year available

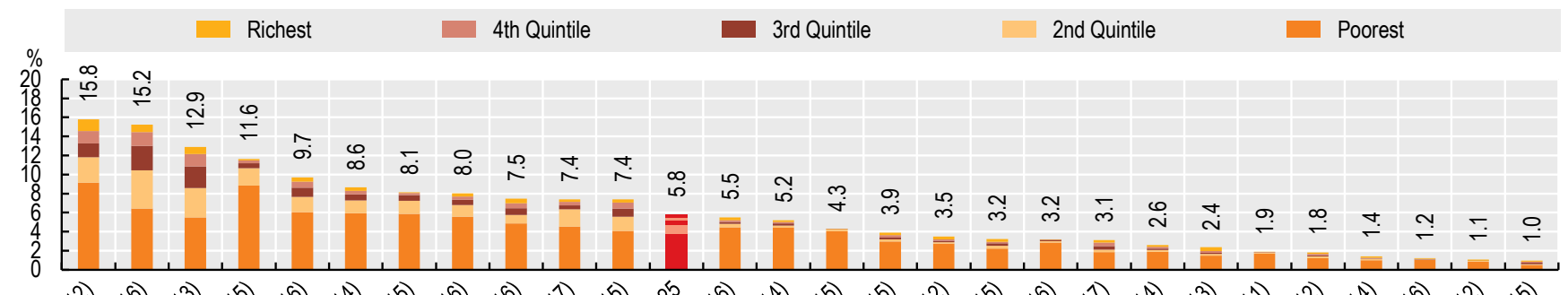

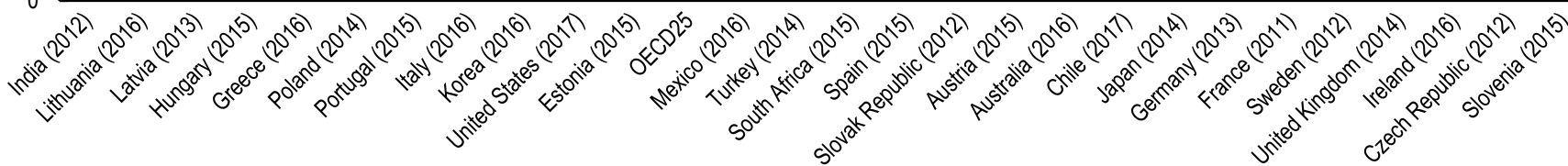

Source: WHO Regional Office for Europe, 2019. 
Access to medical care requires an adequate number and equitable distribution of doctors in all parts of the country. Concentration of doctors in one region and shortages in others can lead to inequities in access such as longer travel or waiting times. The uneven distribution of doctors and the difficulties in recruiting and retaining doctors in certain regions is an important policy issue in most OECD countries, especially in countries with remote and sparsely populated areas, and those with deprived rural and urban regions.

The overall number of doctors per capita varies widely across OECD countries from around two per 1000 population in Turkey, Korea and Poland, to five or higher in Portugal, Austria and Greece (see indicator on "Doctors" in Chapter 8). Beyond these cross-country differences, the number of doctors per capita also varies widely across regions within the same country. The density of physicians is consistently greater in urban regions, reflecting the concentration of specialised services such as surgery, and physicians' preferences to practice in urban settings. Differences in the density of doctors between urban regions and rural regions are highest in the Slovak Republic, Hungary and Portugal, notwithstanding differential definition of urban and rural regions across countries. The distribution of physicians between urban and rural regions was more equal in Japan and Korea, but there are generally fewer doctors in these two countries (Figure 5.14). Growing urbanisation will likely further widen existing geographic disparities in access to doctors.

Within predominantly urban areas, capital cities are typically capturing most of the physician supply (Figure 5.15). This is particularly evident in Austria, the Czech Republic, Greece, Portugal, the Slovak Republic and the United States. Differences between the capital region and the second region with highest density are largest in the United States and the Slovak Republic, with Washington D.C. and the Bratislava region having nearly twice as many physicians per capita as Massachusetts and East Slovakia (the second most dense), respectively. This usually results in higher dispersion between small regions for these countries, with the United States showing a nearly five-fold difference in physician density; and almost three-fold differences for the Slovak Republic and Greece. In contrast, Australia, Belgium and Korea show only around a $20 \%$ difference in physician densities between regions.

Doctors may be reluctant to practice in rural regions due to concerns about their professional life (including their income, working hours, opportunities for career development, isolation from peers) and social amenities (such as educational options for their children and professional opportunities for their spouse). A range of policy levers can be used to influence the choice of practice location of physicians. These include: 1) the provision of financial incentives for doctors to work in underserved areas; 2) increasing enrolments in medical education programmes of students coming from specific social or geographic backgrounds or decentralising the location of medical schools; 3 ) regulating the choice of practice location of doctors (for new medical graduates or foreign-trained doctors); and 4) re-organising service delivery to improve the working conditions of doctors in underserved areas.

Many OECD countries provide different types of financial incentives to attract and retain doctors in underserved areas, including one-time subsidies to help them set up their practice and recurrent payments such as income guarantees and bonus payments. A number of countries have also introduced measures to encourage students from underserved regions to enrol in medical schools. The effectiveness and cost of different policies to promote a better distribution of doctors can vary significantly, with the impact depending on the characteristics of each health system, the geography of the country, physician behaviours, and the specific policy and programme design. Policies should be designed with a clear understanding of the interests of the target group in order to have any significant and lasting impact (Ono, Schoenstein and Buchan, 2014[1]).

\section{Definition and comparability}

Regions are classified in two territorial levels. The higher level (Territorial Level 2) consists of large regions corresponding generally to national administrative regions. These broad regions may contain a mix of urban, intermediate and rural areas. The lower level is composed of smaller regions classified as predominantly urban, intermediate or rural regions, although there are variations across countries in the classification of these regions. Note that overseas territories are generally excluded from calculations. All data on geographic distributions come from the OECD Regional Database.

\section{References}

[2] OECD (2016), Health Workforce Policies in OECD Countries: Right Jobs, Right Skills, Right Places, OECD Health Policy Studies, OECD Publishing, Paris, https://dx.doi.org/ 10.1787/9789264239517-en.

[1] Ono, T., M. Schoenstein and J. Buchan. (2014), "Geographic Imbalances in Doctor Supply and Policy Responses", OECD Health Working Papers, No. 69, OECD Publishing, Paris, http:// dx.doi.org/10.1787/5jz5sq5ls1wl-en. 
Figure 5.14. Physician density, rural vs urban areas, 2016 (or nearest year)

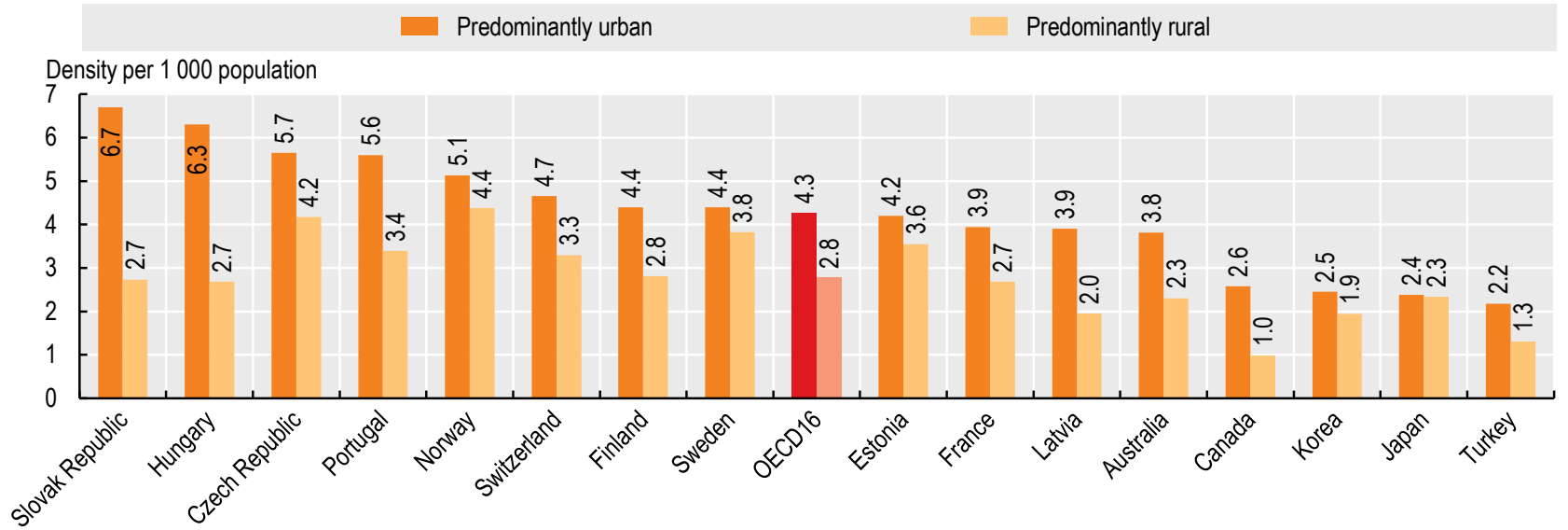

Source: OECD Regional Statistics Database 2019.

StatLink Ants https://doi.org/10.1787/888934015866

Figure 5.15. Physician density across localities, by level 2 regions, 2016 (or nearest year)

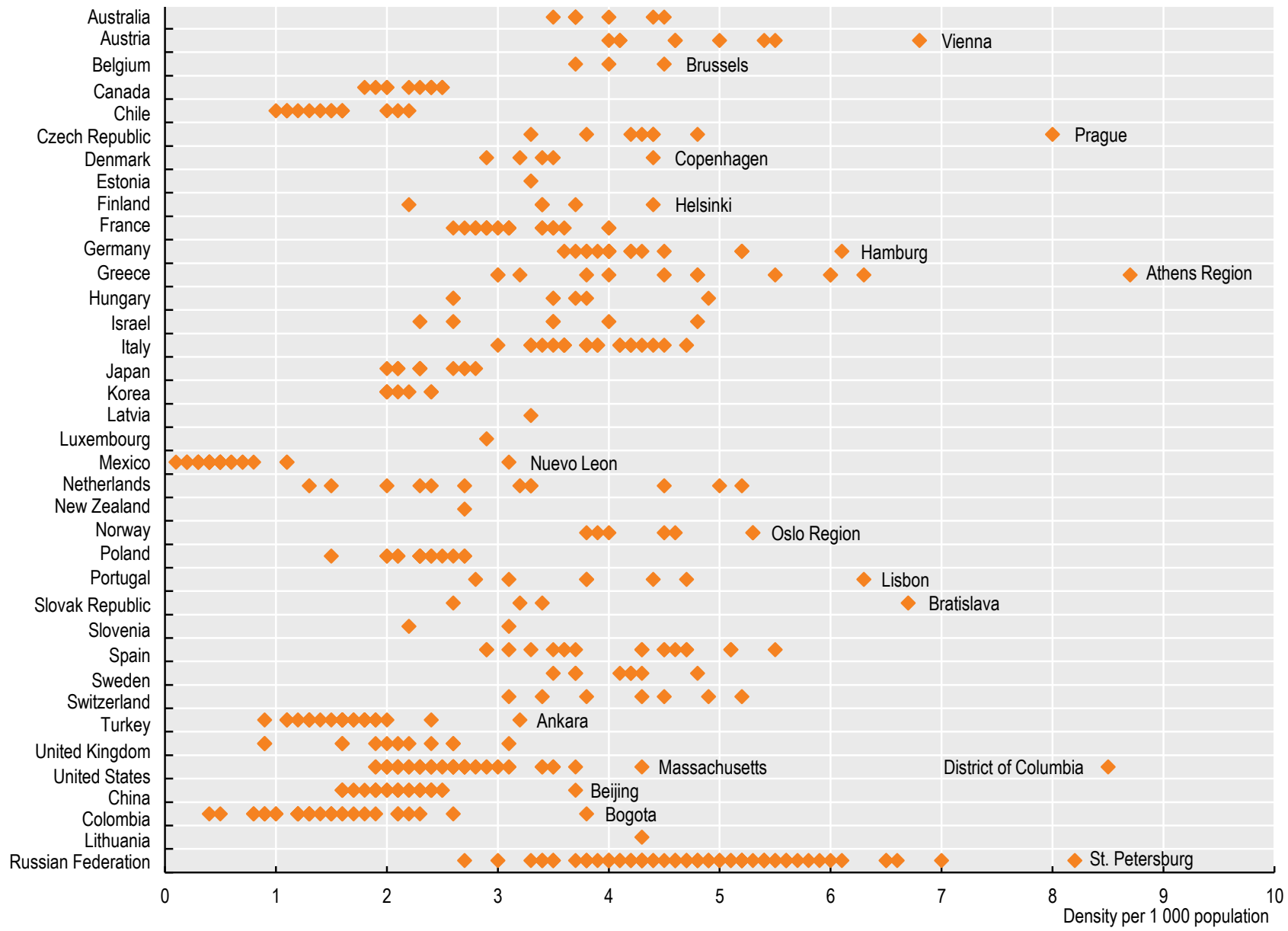

Source: OECD Regional Statistics Database 2019. 
Long waiting times for elective (non-emergency) surgery cause dissatisfaction for patients, because they postpone the expected benefits of treatment, and pain and disability remain. Waiting times are the result of a complex interaction between the demand and supply of health services, with doctors playing a critical role on both sides. Demand for health services and elective surgeries is determined by the health status of the population, progress in medical technologies (including the simplification of many procedures, such as cataract surgery), patient preferences, and the burden of cost-sharing for patients. However, doctors play a crucial role in the decision to operate on a patient or not. On the supply side, the availability of surgeons, anaesthetists and other staff in surgical teams, as well as the supply of the required medical equipment, affect surgical activity rates.

The measure reported here refers to the waiting time from when a medical specialist adds a patient to the waiting list for the procedure, to the moment the patient receives treatment. Both mean and median waiting times are reported. Since a number of patients wait for very long times, the median is consistently and considerably lower than the mean, and might therefore represent a better measure for the central tendency of this indicator. The significant difference between the two measures, especially in countries such as Chile, Estonia, and Poland, highlights the presence of problematic groups of patients who wait significantly longer than others to receive treatment.

In 2017, the median waiting time for cataract surgery was less than 50 days in Italy, Hungary, Denmark, and Sweden (Figure 5.16). Countries with the largest waiting times include Estonia and Poland, with median waits of about seven months and over a year respectively. Over the past decade, waiting times increased in some countries, such as Canada and Portugal; in Spain waits decreased, while in New Zealand they remained relatively stable.

For hip replacement, the median waiting time was less than 50 days in Denmark and Italy (Figure 5.17). There were very long median waiting times of eight months or more in Estonia, Poland and Chile. Over the past five years, some countries, such as Finland, Hungary and Denmark, observed a decline in median waiting times for hip replacement, while Estonia saw a sharp increase.

Waiting times for knee replacement follows the patterns of hip replacement but with higher waiting times on average, with Estonia, Poland and Chile also having by far the longest waiting times (Figure 5.18). The median waiting time across the OECD sample is 114 days, more than 30 days above those of cataract surgery and 20 days above those of hip replacement. In Australia, median waiting times slightly increased over time to reach 200 days, while Portugal remained relatively unchanged since 2007. Hungary and Denmark saw reductions in the past decade.

Waiting time guarantees have become the most common policy tool to tackle long waiting times in several countries, but these guarantees are only effective if well enforced (Siciliani, Borowitz and Moran, 2013[1]).
Denmark has used maximum waiting times, together with patient choice of provider, to reduce waiting times since the late 2000s. The maximum waiting time guarantee was reduced from two months to one month in 2007, combined with free choice of provider. Under this scheme, if the hospital can foresee that the guarantee will not be fulfilled, the patient can choose another public or private hospital. If the treatment is outside of the region's own hospitals, the expenses are covered by the region where the patient lives.

In Hungary, waiting times for many elective surgeries have also been reduced in recent years. Specific objectives were set to reduce waiting times to under 60 days for minor surgery and under 180 days for major surgery, for all patients. To achieve this, the government adopted new laws and regulations on the management of waiting lists, developed an online waiting list system at the national level to monitor the situation in real-time, provided additional payment to reduce waiting times in selected areas or hospitals, and encouraged a reallocation of patients from providers with longer waiting times to those with shorter waiting times.

\section{Definition and comparability}

Two different measures of waiting times for elective procedures are commonly used: 1) measuring the waiting times for patients treated in a given period; or 2) measuring waiting times for patients still on the list at a point in time. The data reported here relate to the first measure (data on the second measure are available in the OECD Health Database). Data come from administrative databases rather than surveys.

Waiting times are reported in terms of both the mean and the median. The median is the value that separates a distribution in two equal parts (i.e. half the patients have longer waiting times, the other half have shorter waiting times). Compared with the average (mean), the median minimises the influence of outliers, i.e. patients with very long waiting times. Waiting times are over-estimated in Norway because they start from the data when a doctor refers a patient for specialist assessment up to the treatment, whereas in other countries they start only when a specialist has assessed the patient and decided to add the person on the waiting list up to the treatment.

\section{References}

[2] National Research Council (US); Institute of Medicine (US) (2013), U.S. Health in International Perspective, National Academies Press, Washington, D.C., http://dx.doi.org/ 10.17226/13497.

[1] Siciliani, L., M. Borowitz and V. Moran (eds.) (2013), Waiting Time Policies in the Health Sector: What Works?, OECD Health Policy Studies, OECD Publishing, Paris, https://dx.doi.org/ 10.1787/9789264179080-en. 
Figure 5.16. Cataract surgery waiting times, averages and selected trends, 2017
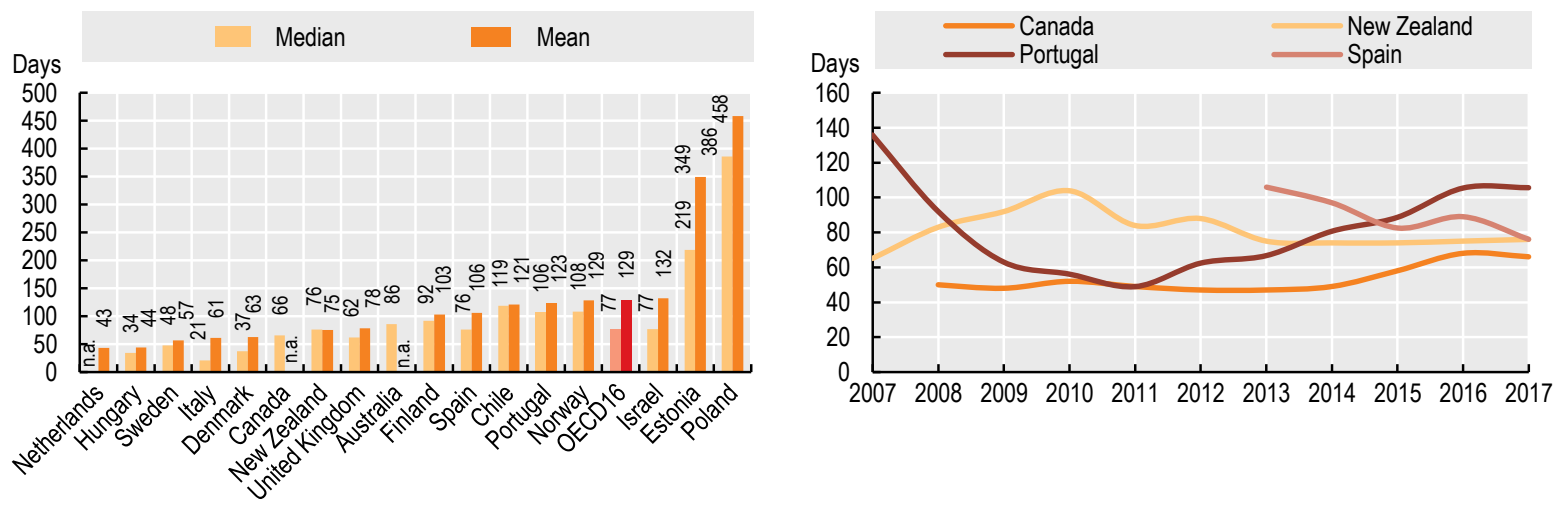

Source: OECD Health Statistics 2019.

StatLink काIs https://doi.org/10.1787/888934015904

Figure 5.17. Hip replacement waiting times, averages and selected trends, 2017
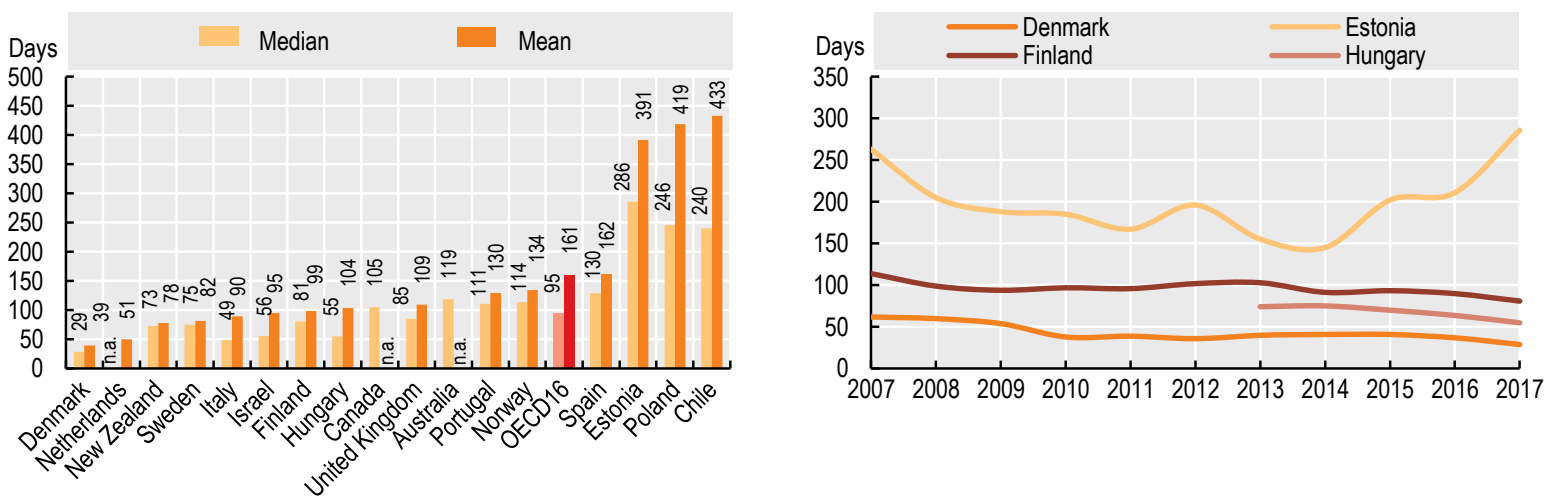

Source: OECD Health Statistics 2019.

Figure 5.18. Knee replacement waiting times, averages and selected trends, 2017
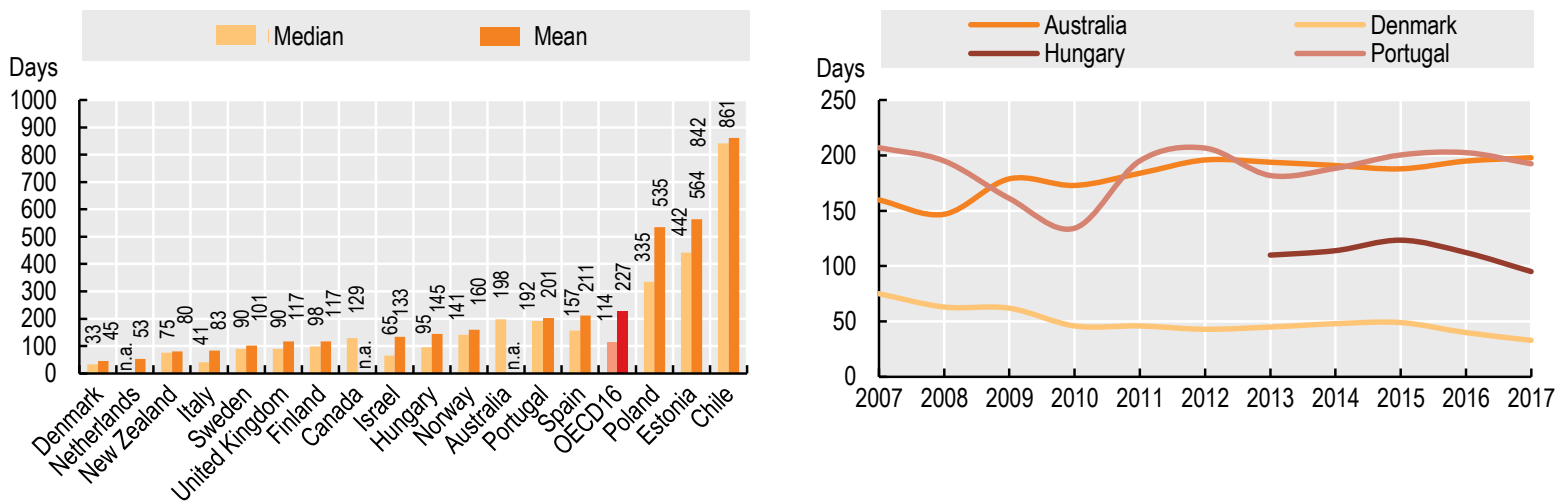

Source: OECD Health Statistics 2019. 


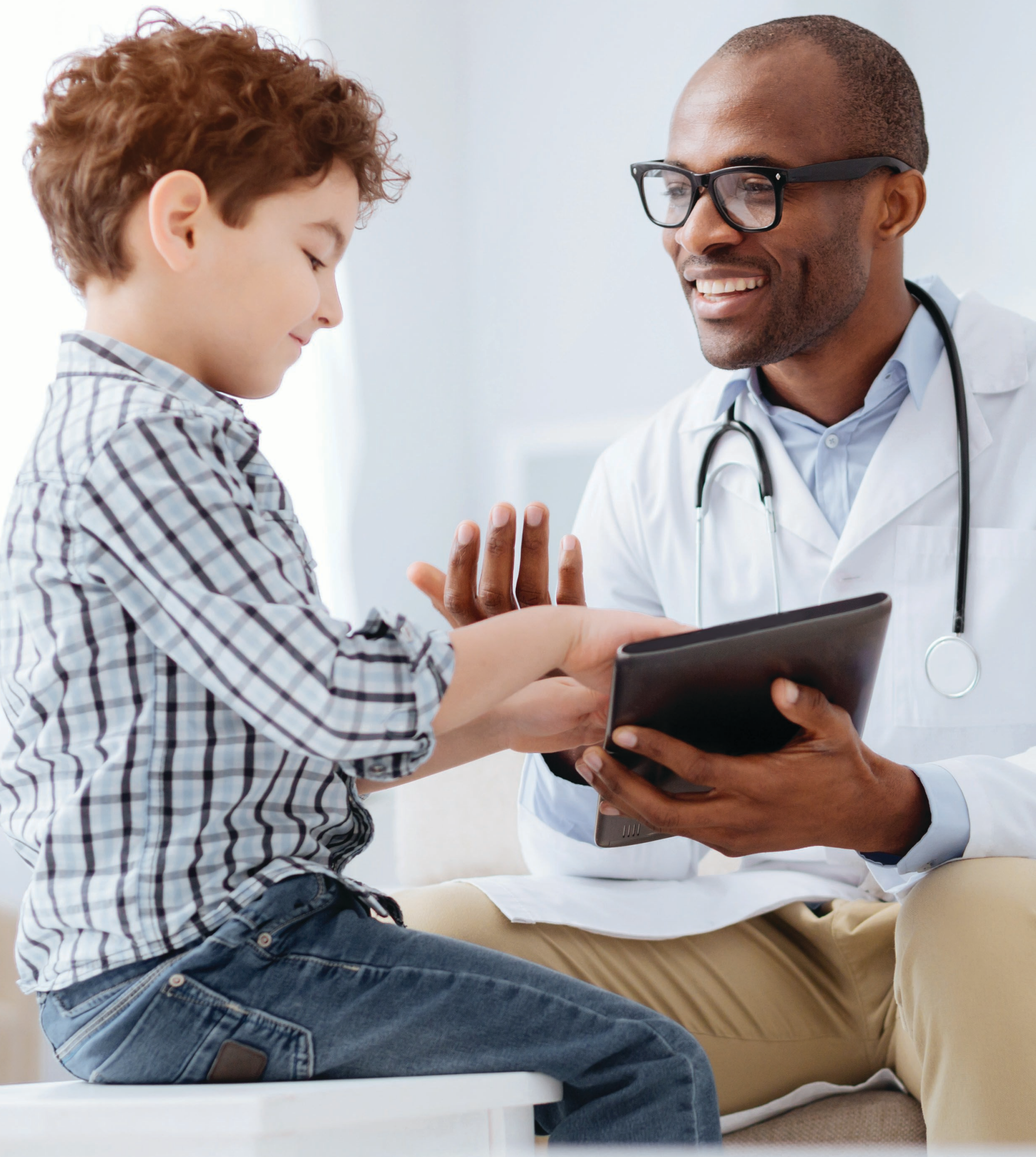


Prescribing can be used as an indicator of health care quality, supplementing consumption and expenditure information (see Chapter 10). The overuse, underuse or misuse of prescription medicines can cause significant hazards to health and lead to wasteful expenditure. This is, for example, the case for opioids and antibiotics.

Opioids are often used to treat acute pain and pain associated with cancer, and over the last decade have been increasingly used to treat chronic pain, despite the risk of dependence, dose increase, shortness of breath and death. Opioid use is now causing an alarming and rising epidemic of overdose deaths in some OECD countries, such as the United States and Canada (OECD, 2019[1]) (see indicator on "Opioids use" in Chapter 4).

Figure 6.1 indicates that, across OECD countries, the average volume of opioids prescribed in primary care in 2017 was more than 16 defined daily doses (DDDs) per 1000 population per day. Iceland and Luxembourg report volumes more than twice the OECD average; Turkey and Korea report the lowest volumes. While these numbers measure prescriptions in primary care, they may reflect conditions on the supply side, as the mean availability of opioids is also low in Turkey (see indicator on "Opioids use" in Chapter 4). On average, more than $2 \%$ of the adult population across OECD countries were chronic users of opioids in 2017 (Figure 6.2). Korea and Italy report the lowest and Iceland reports the highest proportion by a large margin. The large variation can be explained in part by differences in clinical practice in pain management, as well as differences in regulation, legal frameworks for opioids, prescribing policies and treatment guidelines.

Antibiotics should be prescribed only where there is a need that is clearly supported by evidence, to reduce the risk of resistant strains of bacteria (OECD, 2018[2]). For example, quinolones and cephalosporins are considered second-line antibiotics in most prescribing guidelines, which should generally be used only when first-line antibiotics are ineffective. Total volume of antibiotics prescribed and second-line antibiotics as a proportion of total volume have been validated as markers of quality in the primary care setting (OECD, 2017[3]), while overall antibiotic consumption and antimicrobial resistance across OECD countries has been increasing (OECD, 2018[2]).

Figure 6.3 shows the volume of all antibiotics prescribed in primary care in 2017, including second-line antibiotics. Total volumes vary more than three-fold across countries, with the United Kingdom, Estonia and Sweden reporting the lowest volumes, and Italy and Greece reporting the highest. Volumes of second-line antibiotics vary more than 24-fold across countries. The Scandinavian countries and the United Kingdom report the lowest volumes of second-line antibiotics, whereas Greece and Korea report the highest. Variation is likely to be explained, on the supply side, by differences in the guidelines and incentives that govern primary care prescribers and, on the demand side, by differences in attitudes and expectations regarding optimal treatment of infectious illness.

\section{Definition and comparability}

Defined daily dose (DDD) is the assumed average maintenance dose per day for a drug used for its main indication in adults. For instance, the DDD for oral aspirin equals 3 grammes, the assumed maintenance daily dose to treat pain in adults. DDDs do not necessarily reflect the average daily dose actually used in a given country. For more detail, see http:// www.whocc.no/atcddd.

Data for Austria, Latvia, Estonia, Portugal, Spain and Sweden include data for primary care physicians only. Data for Canada, Finland, Italy, Korea and Norway include outpatient care. Data for the Netherlands include prescriptions by primary care doctors and medical specialists in outpatient clinics. Data for Denmark, Ireland and Slovenia include primary care, outpatient care and nursing homes. Data for Belgium and Turkey include primary care, nursing and residential facilities. Data for Iceland include data for primary care, outpatient care, specialists in private practice and nursing homes. Data relate to reimbursed prescriptions, with the exception of Iceland, Slovenia and the Netherlands (for benzodiazepines only), which include non-reimbursed medicines. Data for Denmark, Canada, Finland, Luxembourg, Portugal, the Netherlands and Sweden relate to medicines dispensed in community pharmacies. Data for Germany are based on prescription data of statutory health insurance for the outpatient area. Data for Australia are sourced from the Pharmaceutical Benefits Scheme dataset. Denominators comprise the population held in the national prescribing database, rather than the general population. Further information on sources and methods is at OECD.Stat. Other data in OECD Health Statistics on antibiotics may differ due to differences in data sources and coverage.

For opioids, "chronic users" is defined as the number of adults in the prescribing database with two or more prescriptions for at least 90 days.

\section{References}

[1] OECD (2019), Addressing Problematic Opioid Use in OECD Countries, OECD Health Policy Studies, OECD Publishing, Paris, https://dx.doi.org/10.1787/a18286f0-en.

[2] OECD (2018), Stemming the Superbug Tide: Just A Few Dollars More, OECD Health Policy Studies, OECD Publishing, Paris, https://dx.doi.org/10.1787/9789264307599-en.

[3] OECD (2017), Tackling Wasteful Spending on Health, OECD Publishing, Paris, https://dx.doi.org/10.1787/9789264266414en. 
Figure 6.1. Overall volume of opioids prescribed, 2017 (or nearest year)

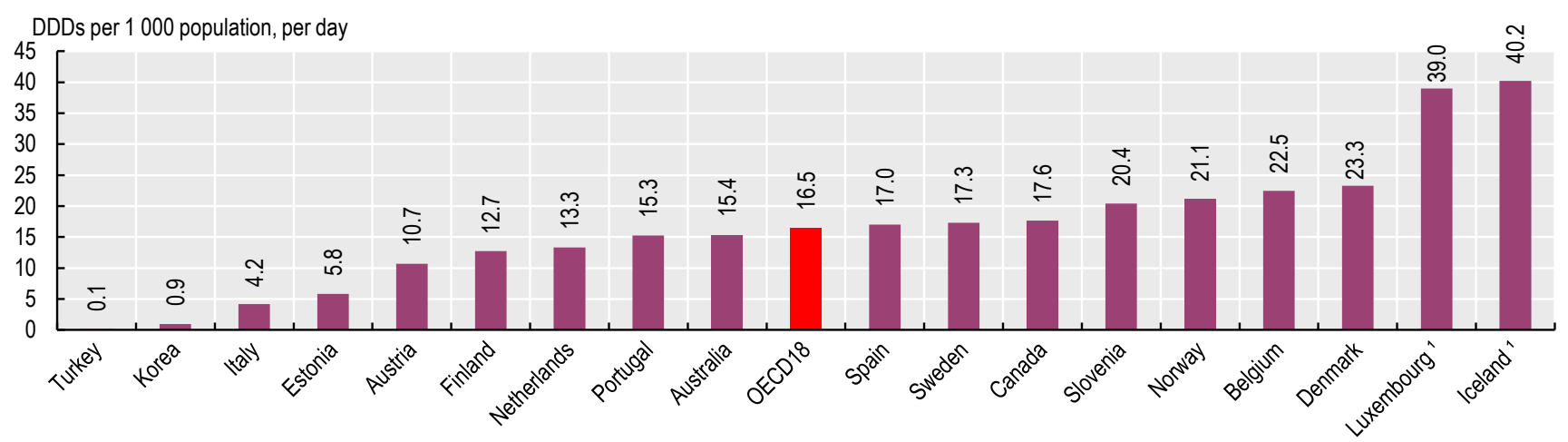

Note: Data exclude products used in the treatment of addiction. 1. Three-year average.

Source: OECD Health Statistics 2019 and Third Australian Atlas of Healthcare Variation 2018.

Figure 6.2. Proportion of chronic opioid users in the adult population, 2017 (or nearest year)

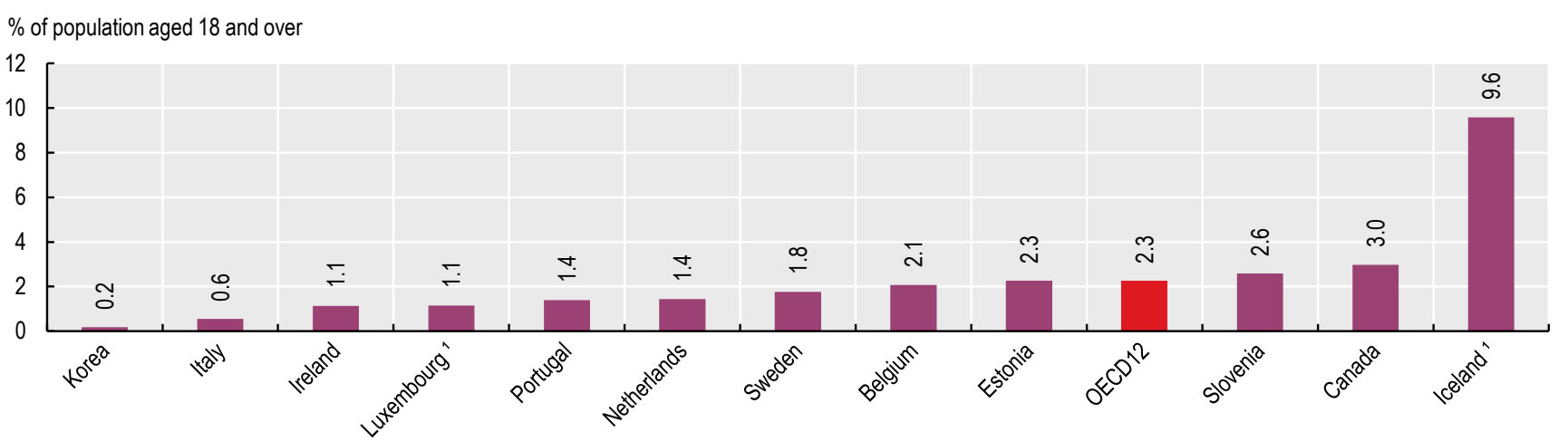

Note: Data exclude products used in the treatment of addiction. 1. Three-year average.

Figure 6.3. Overall volume of antibiotics prescribed, 2017 (or nearest year)

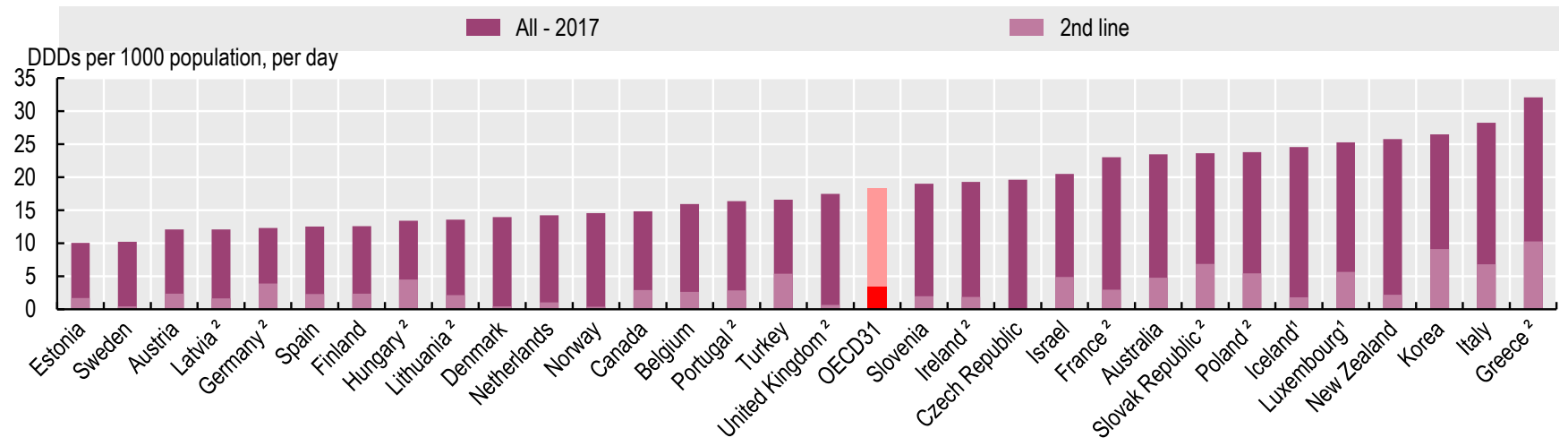

1. Three-year average. 2. Data from European Centre for Disease Prevention and Control as OECD Health Statistics data are not available. Source: OECD Health Statistics 2019. 
Patient safety remains one of the most pressing health issues for public education and further policy action. Over $15 \%$ of hospital expenditure and activity in OECD countries can be attributed to treating patients who experience a safety event, many of which are preventable (Slawomirski, Auraaen and Klazinga, 2018[1]). The World Health Assembly recently endorsed the establishment of an annual World Patient Safety Day to further strengthen awareness and galvanise concerted action for safer care.

Patient safety problems may be categorised as "sentinel" or "never" events: events that should never or very rarely occur; and "adverse" events: events that cannot be fully avoided, but whose incidence could be considerably reduced.

Figure 6.4 illustrates rates for a never event - a foreign body left in during a procedure - using both linked and unlinked data (see the "Definition and comparability" box). The most common risk factors for this never event are emergencies, unplanned changes in procedure, patient obesity and changes in the surgical team. Preventive measures include checklists, counting instruments, methodical wound exploration and effective communication among the surgical team.

Figure 6.5 illustrates rates for an adverse event - the percentage of hospital inpatients with health careassociated infections (HAIs) - in OECD countries, together with the proportion of bacteria causing these infections that are resistanct to antibiotics. HAIs are the single most deadly and costly adverse event, representing up to $6 \%$ of public hospital budgets (Slawomirski, Auraaen and Klazinga, 2018[1]). This impact is increased by antibiotic-resistant bacteria, which can make HAIs difficult or even impossible to treat.

On average, across OECD countries, just under $4.9 \%$ of hospital patients had an HAI in 2015-17. This proportion was $5.2 \%$ in 2011-12. The observed proportion of patients was lowest in Lithuania, Latvia and Germany (around 3\%) and highest in Portugal, Greece and Iceland (more than 7\%). Antibiotic resistance rates ranged from $0 \%$ in Iceland to nearly $70 \%$ in Latvia, although these rates should be interpreted with caution due to small sample sizes in some cases.

Figure 6.6 shows rates for two related adverse events pulmonary embolism (PE) and deep vein thrombosis (DVT) after hip or knee replacement surgery - using both unlinked and linked data definitions (see the "Definition and comparability" box). PE and DVT cause unnecessary pain and in some cases death, but they can be prevented by anticoagulants and other measures. The large variations observed, including an over 25-fold variation in DVT rates, may be explained in part by differences in diagnostic practices across countries.

\section{Definition and comparability}

Indicators using unlinked data rely on information from a patient's admission to the hospital where surgery occurred to calculate rates. The number of discharges with International Classification of Diseases (ICD) codes for the relevant complication in any secondary diagnosis field is divided by the total number of discharges for patients aged 15 and older. The linked data approach expands beyond the surgical admission to include all subsequent related readmissions to any hospital within 30 days after surgery.

Variations in definitions and medical recording practices between countries can affect calculation of rates and limit data comparability in some cases. Higher adverse event rates may signal more developed patient safety monitoring systems and a stronger patient safety culture rather than worse care.

HAI data are based on results of point prevalence studies conducted by the Centers for Disease Control and Prevention (CDC) and the European Centre for Disease Prevention and Control (ECDC) between 2015 and 2017 (Magill et al., 2018[2]; Suetens et al., 2018[3]). HAI rates are unadjusted and may not reflect rates published elsewhere owing to differences in the infections included. See Suetens et al. (2018[3]) and Magill et al. (2018[2]) for more details regarding specific inclusions and exclusions. Country estimates may reflect different levels of variability based on sampling differences.The HAI rate is presented, along with the proportion of patients recruited from intensive care units (ICUs). ICU patients may be at greater risk of developing an HAI. Antibiotic resistance data are based on a composite antibiotic resistance indicator developed by the ECDC (Suetens et al., 2018[3]).

\section{References}

[2] Magill, SS. et al. (2018), "Changes in Prevalence of Health Care-Associated Infections in U.S. Hospitals." New England Journal of Medicine, 1;379(18):1732-1744. doi: http:// dx.doi.org/10.1056/NEJMoa1801550.

[1] Slawomirski, L., A. Auraaen and N. Klazinga (2018), "The Economics of Patient Safety: Strengthening a value-based approach to reducing patient harm at national level," OECD Health Working Papers, No. 96, OECD Publishing, Paris, https:// doi.org/10.1787/5a9858cd-en.

[3] Suetens, C. et al. (2018), "Prevalence of healthcare-associated infections, estimated incidence and composite antimicrobial resistance index in acute care hospitals and long-term care facilities: results from two European point prevalence surveys, 2016 to 2017", Eurosurveillance, http:// dx.doi.org/10.2807/1560-7917.es.2018.23.46.1800516. 
Figure 6.4. Foreign body left in during procedure, 2017 (or nearest year)

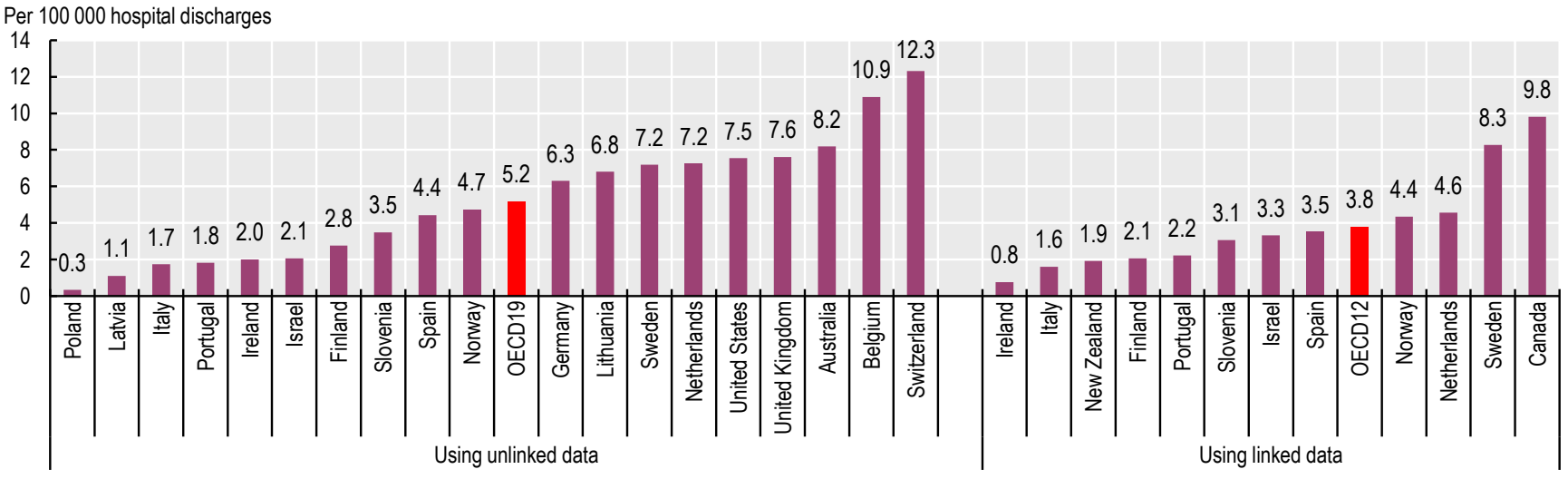

Source: OECD Health Statistics 2019.

Figure 6.5. Percentage of hospitalised patients with at least one health care-associated infection and proportion of bacteria isolated from these infections resistant to antibiotics, 2015-17

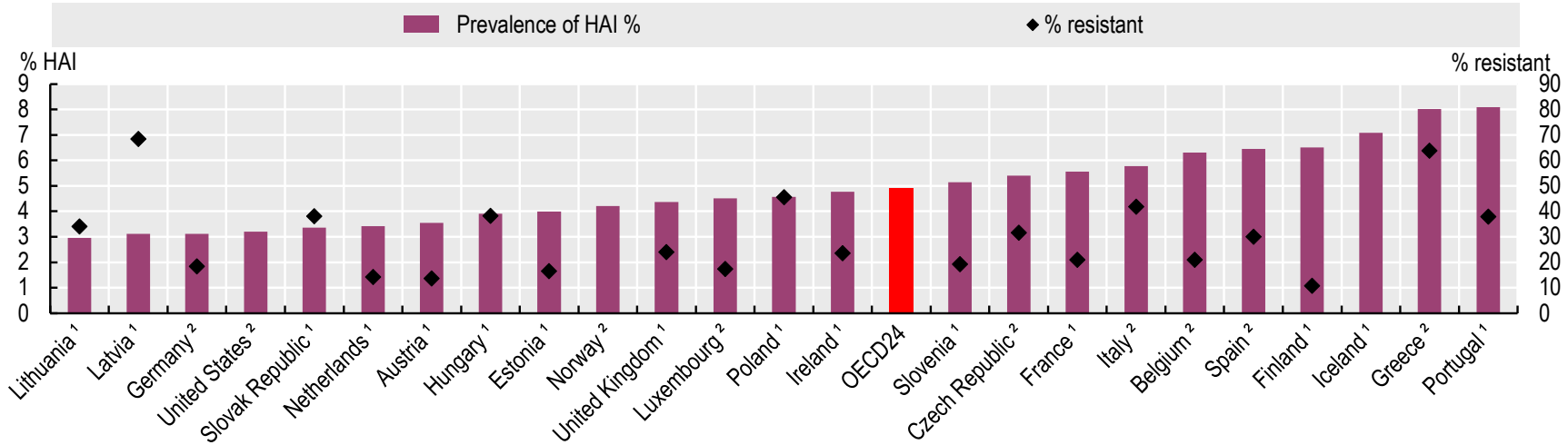

Note: No resistance data available for Iceland, Norway and the United States.

1. Under $5 \%$ of patients from ICUs. 2. Over $5 \%$ of patients from ICUs

Source: ECDC 2016-17 Point prevalence survey. CDC 2015 point prevalence study.

StatLink काIs https://doi.org/10.1787/888934016037

Figure 6.6. Adverse events in hip and knee surgeries: post-operative pulmonary embolism (PE) or deep vein thrombosis (DVT), 2017 (or nearest year)

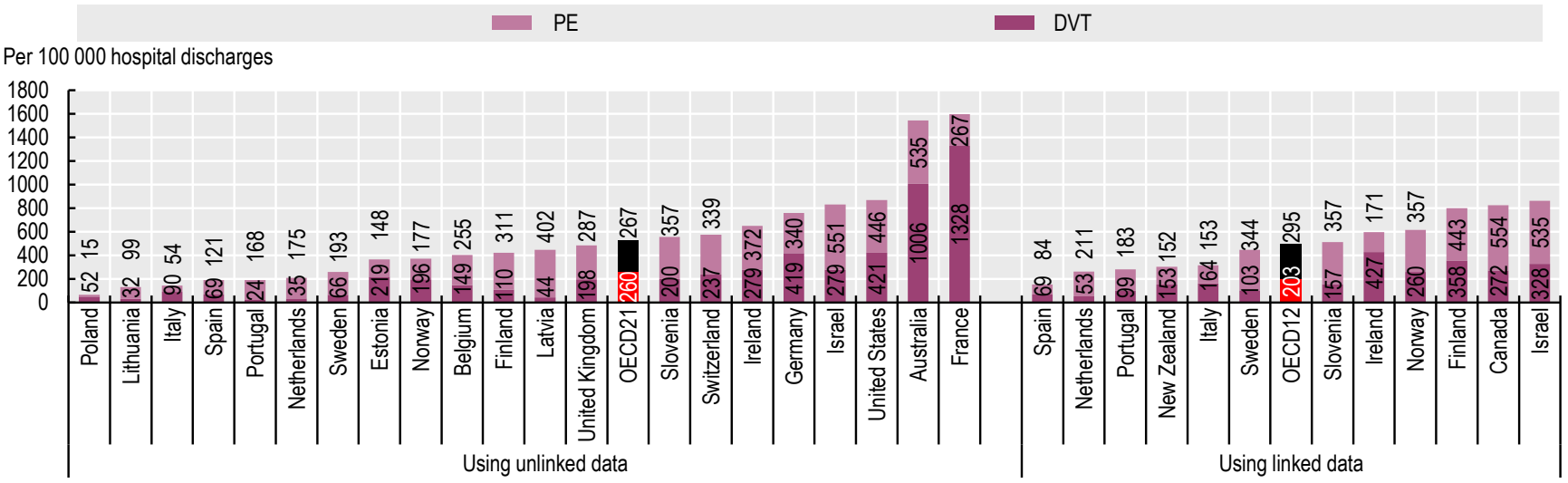

Source: OECD Health Statistics 2019. 
A woman's safety during childbirth can be assessed by looking at potentially avoidable tearing of the perineum during vaginal delivery. Tears that extend to the perineal muscles and bowel wall require surgery. Possible complications include continued perineal pain and incontinence. It is not possible to prevent these types of tear in all cases, but they can be reduced by appropriate labour management and high-quality obstetric care.

The proportion of deliveries involving higher-degree lacerations is considered a useful indicator of the quality of obstetric care. Nevertheless, differences in the consistency with which obstetric units report these complications may make international comparison difficult.

Rates of obstetric trauma may be influenced by other care processes, including the overall national rate of caesarean births, assisted vaginal births (i.e. using forcepts or a vacuum) and episiotomy (i.e. surgical incision of the perineum performed to widen the vaginal opening for delivery of an infant); these remain issues of ongoing research. For example, while the World Health Organization (WHO) (2018[1]) does not recommend routine or liberal use of episiotomy for women undergoing spontaneous vaginal birth, selective use of episiotomy to decrease severe perineal lacerations during delivery remains a matter of debate.

Figure 6.7 shows rates of obstetric trauma with instrument (referring to deliveries using forceps or vacuum extraction) and Figure 6.8 shows rates of obstetric trauma after vaginal delivery without instrument. As the risk of a perineal laceration is significantly increased when instruments are used to assist the delivery, rates for this patient population are reported separately.

High variation in rates of obstetric trauma is evident across countries. Reported rates of obstetric trauma with instrument vary from below $2 \%$ in Poland, Israel, Italy, Slovenia and Lithuania to more than $10 \%$ in Denmark, Sweden, the United States and Canada. The rates of obstetric trauma after vaginal delivery without instrument vary from below 0.5 per 100 deliveries in Poland, Lithuania, Portugal, Latvia and Israel to over 2.5 per 100 deliveries in Denmark, the United Kingdom and Canada.

While the average rate of obstetric trauma with instrument (5.5 per 100 instrument-assisted vaginal deliveries) across OECD countries in 2017 was nearly four times the rate without instrument (1.4 per 100 vaginal deliveries without instrument assistance), there are indications of a relationship between the two indicators, with Israel, Lithuania, Portugal and Poland reporting among the lowest rates and Canada, Denmark and New Zealand reporting among the highest rates for both indicators.

Rates for both indicators reveal noticeable improvements in Denmark and Norway between 2012 and 2017, but no clear trend is evident in the overall rates of obstetric trauma over the five-year period: the OECD average remained relative static for vaginal deliveries both with and without instrument. In some countries, including Estonia, Italy and Slovenia, rates appear to have deteriorated.

In Canada there has been limited action to address the high rates of reported obstetric trauma. One initiative was the Hospital Harm Improvement Resource: Obstetric Trauma by the Canadian Patient Safety Institute to complement measurement of obstetric trauma by the Canadian Institute for Health Information. It links measurement and improvement by providing evidence-informed resources that support patient safety improvement efforts across the health system.

\section{Definition and comparability}

The two obstetric trauma indicators are defined as the proportion of instrument-assisted/non-assisted vaginal deliveries with third- and fourth-degree obstetric trauma codes (ICD-10 codes O70.2-070.3) in any diagnosis and procedure field.

Several differences in data reporting across countries may influence the calculated rates of obstetric patient safety indicators. These relate primarily to differences in coding practices and data sources. Some countries report obstetric trauma rates based on administrative hospital data and others based on obstetric register data.

Careful interpretation of obstetric trauma for instrument-assisted delivery rates over time is required, since the very low number of trauma cases in some countries is likely to give rise to significant yearon-year variation.

Data for 2012 are not available for Latvia and not presented for Belgium, Portugal, Spain and the United States due to a break in the series. Rates for Denmark, the Netherlands and Norway are based on registry data.

\section{References}

[2] Canadian Patient Safety Institute (2018), Hospital Harm Improvement Resource: Obstetric Trauma.

[1] WHO (2018), WHO recommendation on episiotomy policy. 
Figure 6.7. Obstetric trauma, vaginal delivery with instrument, 2012 and 2017 (or nearest year)

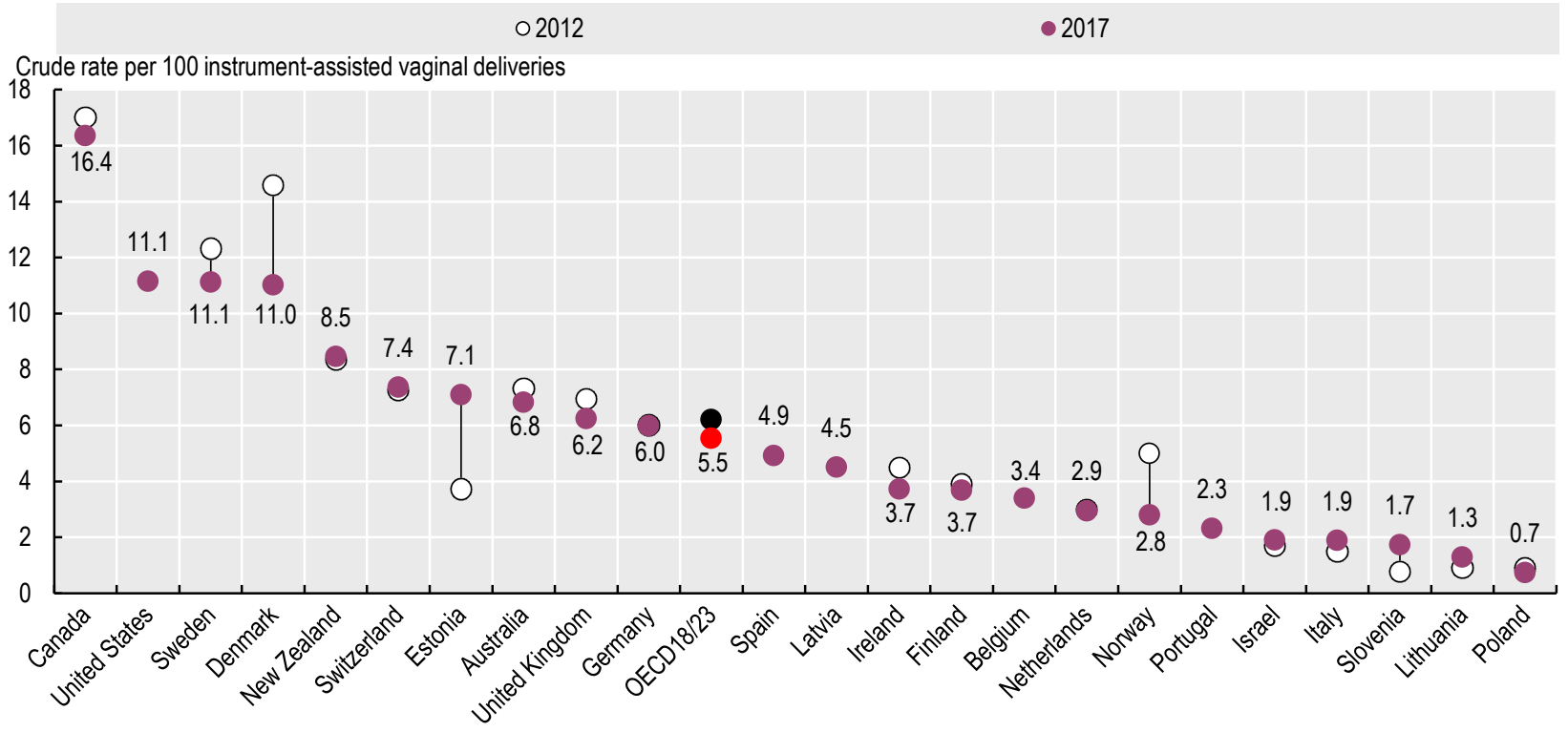

Source: OECD Health Statistics 2019

StatLink ans https://doi.org/10.1787/888934016075

Figure 6.8. Obstetric trauma, vaginal delivery without instrument, 2007 and 2017 (or nearest year)

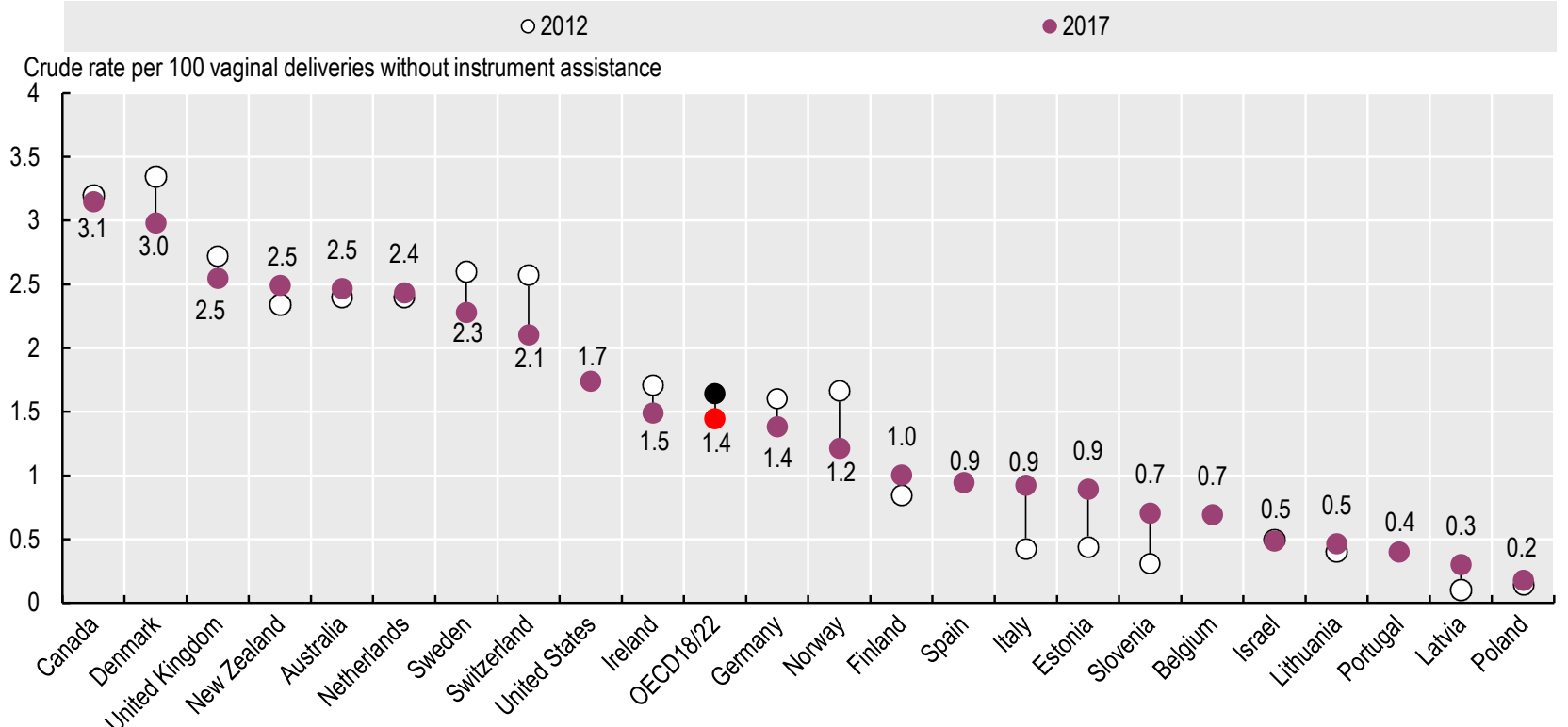

Source: OECD Health Statistics 2019. 
Primary care is expected to serve as the first point contact of people with health systems, and its functions include health promotion and disease prevention, managing new health complaints, treating the majority of uncomplicated cases, managing long-term conditions and referring patients to hospital-based services when appropriate. A key aim of primary care is to keep people well by providing a consistent point of care over the longer term, treating the most common conditions, tailoring and co-ordinating care for those with multiple health care needs and supporting the patient in self-education and self-management. Good primary care has, therefore, the potential to improve health, reduce socio-economic inequalities in health and make health care systems people-centred, while making better use of health care resources (OECD, forthcoming [1]).

Asthma, chronic obstructive pulmonary disease (COPD) and congestive heart failure (CHF) are three widely prevalent long-term conditions. Both asthma and COPD limit the ability to breathe: asthma symptoms are usually intermittent and reversible with treatment, while COPD is a progressive disease that mainly affects current or prior smokers. CHF is a serious medical condition in which the heart is unable to pump enough blood to meet the body's needs. CHF is often caused by hypertension, diabetes or coronary heart disease.

Common to all three conditions is the fact that the evidence base for effective treatment is well established, and much of it can be delivered by primary care. A high-performing primary care system, where accessible and high-quality services are provided, can reduce acute deterioration in people living with asthma, COPD or CHF. This can avoid the need for hospital admissions to treat these conditions, which are used as a marker of quality and access in primary care.

Figure 6.9 shows hospital admission rates for asthma and COPD together, given the physiological relationship between the two conditions. Admission rates specifically for asthma vary 12-fold across OECD countries, with Mexico, Italy and Colombia reporting the lowest rates and Latvia, Turkey and Poland reporting rates over twice the OECD average. International admission rates specifically for COPD vary 15 -fold across OECD countries, with Japan, Italy and Mexico reporting the lowest and Hungary, Turkey and Australia the highest rates. A lower 7-fold variation across countries is seen for the two respiratory conditions combined.

Hospital admission rates for CHF vary 13 -fold, as shown in Figure 6.10. Costa Rica, Mexico and Colombia have the lowest rates, while Poland, Lithuania and the Slovak Republic report rates over twice the OECD average.

Figure 6.11 reveals that in Korea, Lithuania, Mexico and Sweden steady reductions in admission rates for asthma and COPD combined and for CHF have been achieved in recent years, whereas in the Slovak Republic, while rates of admission for asthma and COPD have fallen, rates of admission for CHF have increased. While observed improvements in some countries may represent advances in the quality of primary care, recent reviews undertaken by the OECD indicate that investment in primary care may still not be happening quickly enough (OECD, 2017[2]), potentially resulting in wasteful spending on hospital care (OECD, 2017[3]).

\section{Definition and comparability}

The indicators are defined as the number of hospital admissions with a primary diagnosis of asthma, COPD or CHF among people aged 15 years and over per 100000 population. Rates are age-sex standardised to the 2010 OECD population aged 15 and over. Admissions resulting from a transfer from another hospital and where the patient dies during admission are excluded from the calculation, as these are considered unlikely to be avoidable.

Disease prevalence and availability of hospital care may explain some, but not all, variations in crosscountry rates. Differences in coding practices among countries may also affect the comparability of data. For example, the exclusion of "transfers" cannot be fully complied with by some countries. Differences in data coverage of the national hospital sector across countries may also influence rates.

\section{References}

[1] OECD (forthcoming), Doing Things Differently: Towards better primary care in the 21st century, OECD Publishing, Paris.

[2] OECD (2017), Caring for Quality in Health: Lessons Learnt from 15 Reviews of Health Care Quality, OECD Reviews of Health Care Quality, OECD Publishing, Paris, https://dx.doi.org/ 10.1787/9789264267787-en.

[3] OECD (2017), Tackling Wasteful Spending on Health, OECD Publishing, Paris, https://dx.doi.org/10.1787/9789264266414en. 


\section{QUALITY AND OUTCOMES OF CARE}

Avoidable hospital admissions

Figure 6.9. Asthma and COPD hospital admission in adults, 2017 (or nearest year)

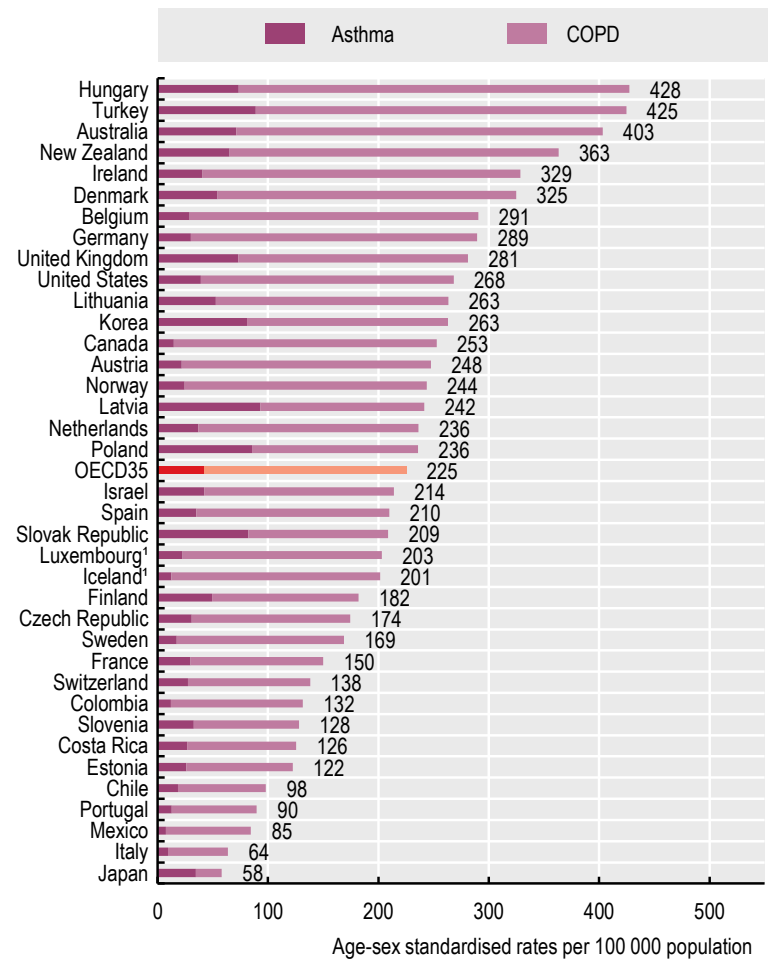

1. Three-year average.

Source: OECD Health Statistics 2019.

StatLink काजा https://doi.org/10.1787/888934016113
Figure 6.10. Congestive Heart Failure (CHF) hospital admission in adults, 2017 (or nearest year)

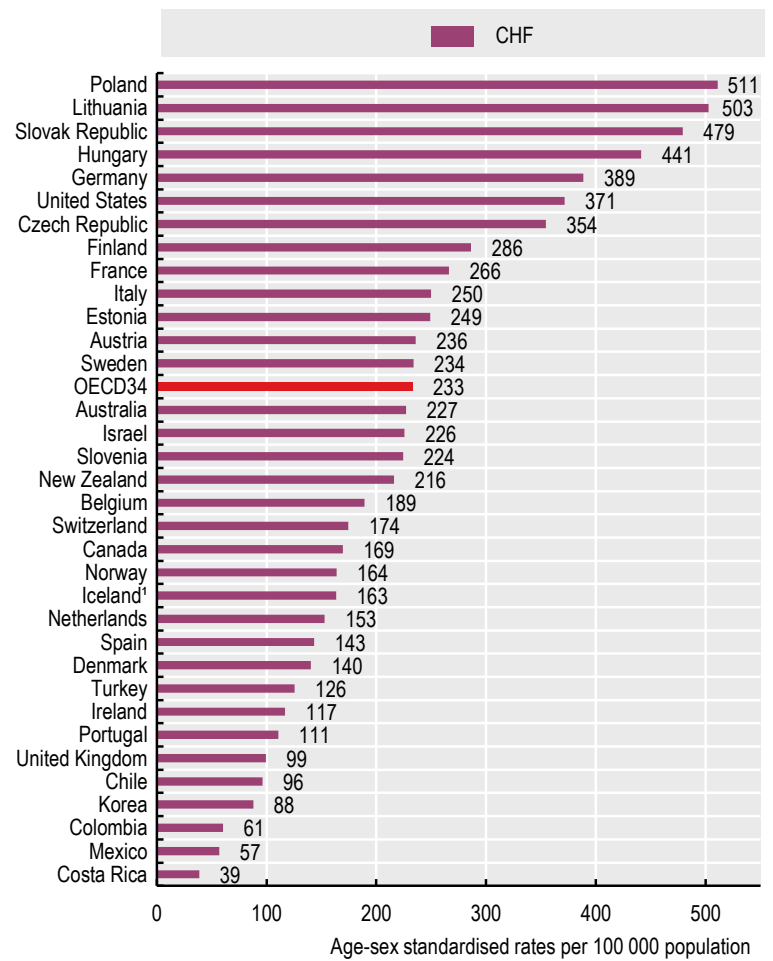

1. Three-year average.

Source: OECD Health Statistics 2019.

StatLink क्गाड https://doi.org/10.1787/888934016132

Figure 6.11. Trends in hospital admission in adults, selected countries 2007-17 (or nearest year)

Asthma and COPD

_... Korea

Lithuania

Age-sex standardised rates per 100000 population

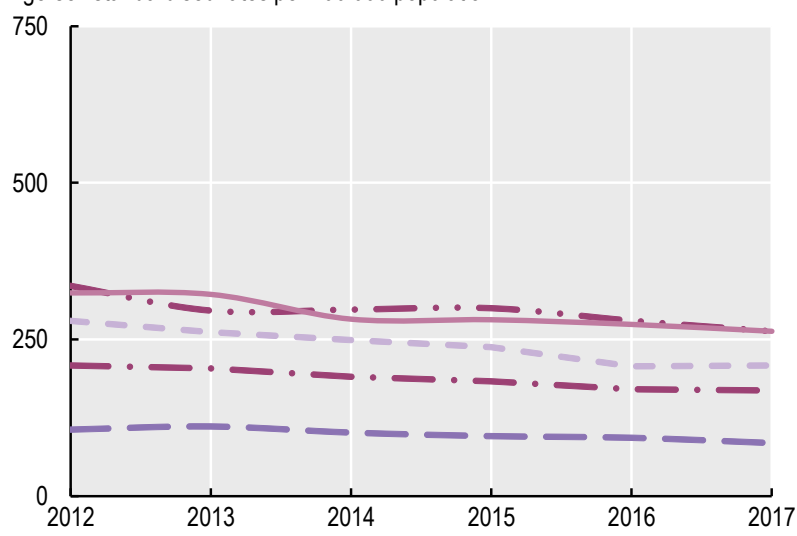

CHF

- Mexico $\quad=-$ Slovak Republic $\quad$ - Sweden

Age-sex standardised rates per 100000 population

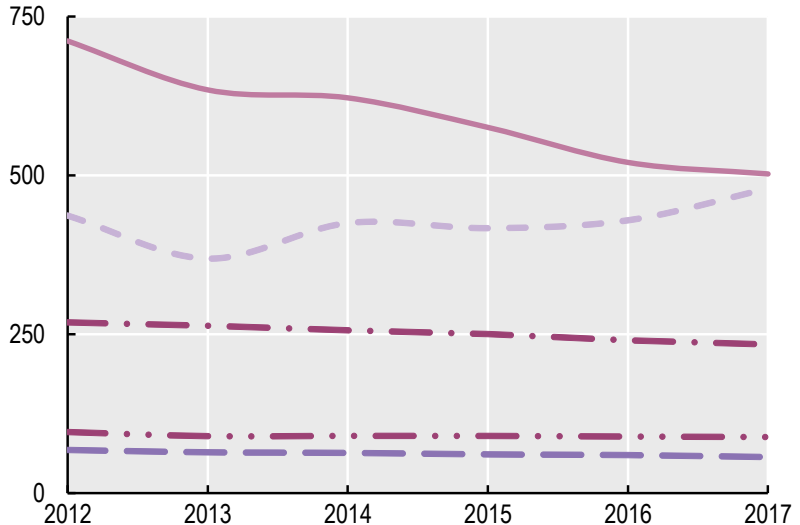

Source: OECD Health Statistics 2019. 
Effective management of diabetes remains a public health priority, with over 425 million people living with the condition worldwide. Diabetes is a chronic disease that occurs when the body's ability to regulate excessive glucose levels in the blood is diminished. It is a leading cause of cardiovascular disease, blindness, kidney failure and lower limb amputation. Diabetes caused 4 million deaths in 2017, and it is projected that by 2045 over 629 million adults will have the condition (IDF, 2017[1]).

Ongoing management of diabetes usually involves a considerable amount of self-care; therefore, advice and education are central to the primary care of people with diabetes (OECD, 2019[2]). Effective control of blood glucose levels through routine monitoring, dietary modification and regular exercise can reduce the onset of serious complications and the need for hospitalisation (Wolters, Braspenning and Wensing, 2017[3]). Management of other key risk factors such as smoking, blood pressure and lipid levels is also important in reducing complications of diabetes.

Figure 6.12 shows avoidable hospital admissions for diabetes. While admissions have fallen in many countries over time, a more than 5 -fold variation in the rates is still evident across countries. Iceland, Italy and Spain report the lowest rates, with Mexico and Korea reporting rates nearly twice the OECD average. Prevalence of diabetes may explain some of this variation. A positive relationship can be demonstrated between overall hospital admissions and admissions for diabetes, providing some indication that access to hospital care can also play a role in explaining international variation (OECD, 2015[4]).

In diabetic individuals with hypertension, angiotensinconverting enzyme inhibitors (ACE-Is) or angiotensin receptor blockers (ARBs) are recommended in most national guidelines as first-line medications to reduce blood pressure. Figure 6.13 reveals broad consistency in the proportion of diabetic patients on recommended antihypertensive medications: only Finland, Belgium and Korea have rates lower than $80 \%$.

Hospital admissions for major lower extremity amputation reflect the long-term quality of diabetes care. Figure 6.14 shows the rates of amputations among adults with diabetes. The international variation is over 20 -fold, with Iceland, Italy, Korea and the United Kingdom reporting rates lower than 3 per 100000 general population and Costa Rica, Israel, Mexico and Austria reporting rates above 13 per 100000.

The relationship between the nature, frequency and duration of primary care for diabetes and the rate of admissions to hospital for related complications is complex and still not well understood. In its ongoing attempts to contribute to reductions in knowledge gaps, the OECD is working to establish an international survey of patients with chronic conditions, including diabetes, to capture their selfreported health outcomes and better understand their primary care context. This survey is central to the PatientReported Indicators Surveys (PaRIS) initiative (https:// www.oecd.org/health/paris.htm).

\section{Definition and comparability}

Diabetes avoidable admission is based on the sum of three indicators: admissions for short-term and longterm complications and for uncontrolled diabetes without complications. The indicator is defined as the number of hospital admissions with a primary diagnosis of diabetes among people aged 15 years and over per 100000 population.

The denominator of people with diabetes who have recommended antihypertensive medication prescriptions is based on people with diabetes (i.e. who are long-term users of glucose-regulating medication) who also have one or more prescriptions per year from a range of medications often used in the management of hypertension. The numberator is the number of these people who have one or more prescriptions of an angiotensin converting enzyme inhibitor (ACE-I) or angiotensin receptor blocker (ARB).

Major lower extremity amputation in adults with diabetes is defined as the number of discharges of people aged 15 years and over per 100000 population. Rates for these indicators have been directly agestandardised to the 2010 OECD population.

Differences in data definition, coding practices and indicator calculation methods between countries may affect comparability of data. Differences in data coverage of the national hospital sector across countries may also influence indicator rates.

In all instances, national data are reported. Variations in the coverage and national representativeness of the indicators for countries are documented in the sources and methods information in OECD.Stat.

\section{References}

[1] IDF (2017), IDF Diabetes Atlas Eighth Edition 2017, International Diabetes Federation, http://www.diabetesatlas.org.

[2] OECD (2019), "Realising the Full Potential of Primary Health Care", OECD, Paris, http://www.oecd.org/health/healthsystems/OECD-Policy-Brief-Primary-Health-Care-May-2019.pdf.

[4] OECD (2015), Cardiovascular Disease and Diabetes: Policies for Better Health and Quality of Care, OECD Health Policy Studies, OECD Publishing, Paris, https://dx.doi.org/10.1787/9789264 233010-en.

[3] Wolters, R., J. Braspenning and M. Wensing (2017), "Impact of primary care on hospital admission rates for diabetes patients: A systematic review", Diabetes Research and Clinical Practice, Vol. 129, pp. 182-196, http://dx.doi.org/10.1016/ j.diabres.2017.05.001. 
Figure 6.12. Diabetes hospital admission in adults, 2012 and 2017 (or nearest year)

O 2012

2017

Age-sex standardised rates per 100000 population

400

350

300

250

150

100

50

$e^{e^{x^{2}}}$ भ.

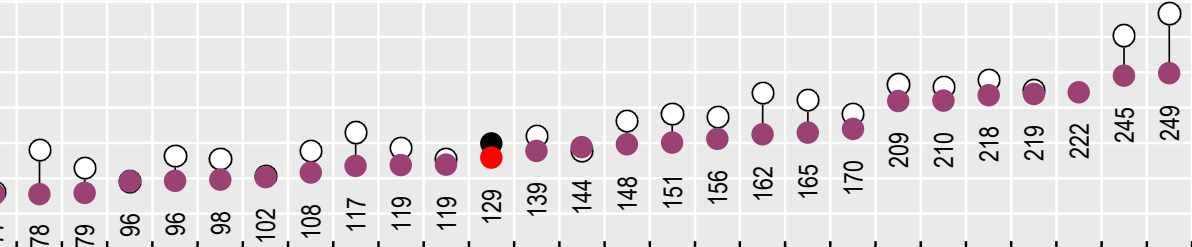

$e^{x^{n^{0}}}$

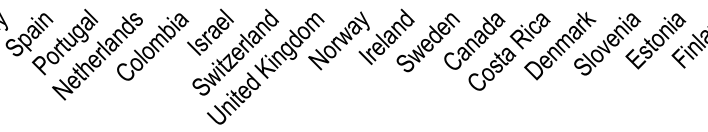

1. Three-year average.

Source: OECD Health Statistics 2019.

StatLink काजा https://doi.org/10.1787/888934016170

Figure 6.13. People with diabetes prescribed recommended antihypertensive medication in the past year, 2017 (or nearest year)

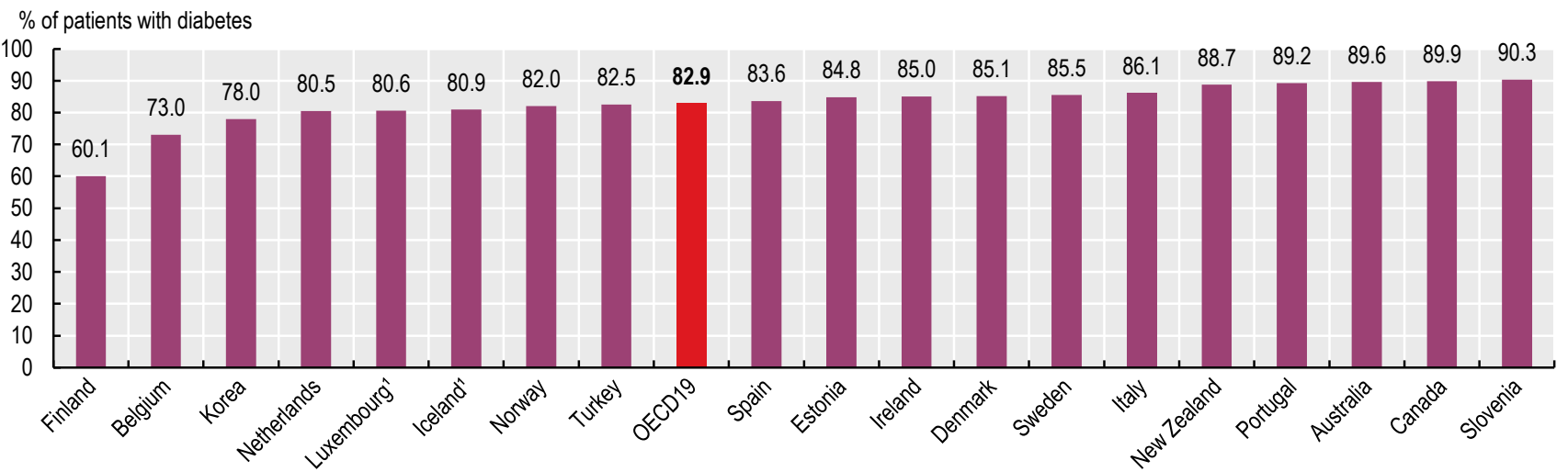

1. Three-year average.

Source: OECD Health Statistics 2019.

StatLink क्ञाइ https://doi.org/10.1787/888934016189

Figure 6.14. Major lower extremity amputation in adults with diabetes, 2012 and 2017 (or nearest year)

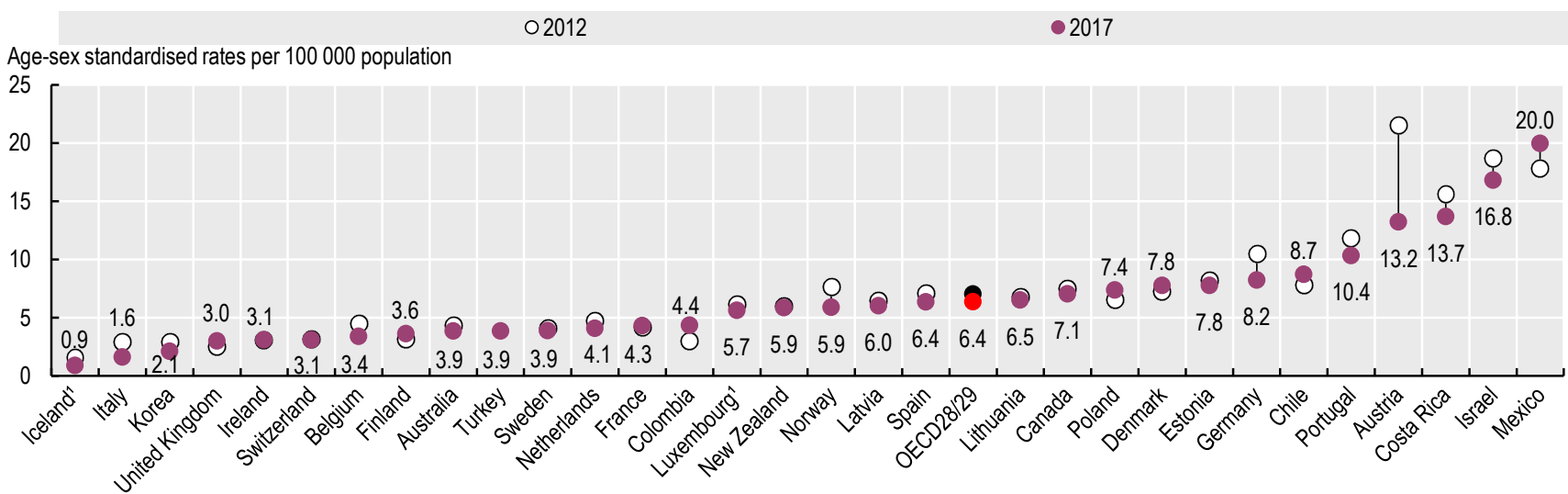


Stroke is the second leading global cause of death behind heart disease and accounted for over $10 \%$ of total deaths worldwide in 2013 (American Heart Association, 2017[1]). A stroke occurs when the blood supply to a part of the brain is interrupted, leading to necrosis (cell death) of the affected part. Of the two types of stroke, about $85 \%$ are ischaemic (caused by clotting) and 15\% are haemorrhagic (caused by bleeding).

Figure 6.15 shows the case-fatality rates within 30 days of hospital admission for ischaemic stroke where the death occurred in the same hospital as the initial admission (unlinked data). Figure 6.16 shows the case-fatality rate where deaths are recorded regardless of where they occurred, including in another hospital or outside the hospital where the stroke was first recorded (linked data). The indicator using linked data is more robust because it captures fatalities more comprehensively than the samehospital indicator, but it requires a unique patient identifier and linked data, which are not available in all countries.

Across OECD countries, 7.7\% of patients in 2017 died within 30 days of hospital admission for ischaemic stroke using unlinked data (Figure 6.15). The case-fatality rates were highest in Slovenia, Poland, Lithuania and Latvia, all with mortality rates over $12 \%$. Rates were less than $4 \%$ in Norway, Korea, Japan and Costa Rica. Low rates in Japan are due in part to recent efforts dedicated to improving the treatment of stroke patients in hospitals, through systematic blood pressure monitoring, major material investment in hospitals and establishment of stroke units (OECD, 2015[2]).

Across the 23 countries that reported linked data rates, $12.3 \%$ of patients died within 30 days of being admitted to hospital for stroke (Figure 6.16). This figure is higher than the same-hospital indicator because it only counts each patient once and captures all deaths.

Treatment for ischaemic stroke has advanced dramatically over the last decade, with systems and processes now in place in many OECD countries to identify suspected ischaemic stroke patients as early as possible and to deliver acute reperfusion therapy quickly. Between 2007 and 2017, case-fatality rates for ischaemic stroke decreased substantially across OECD countries: from $10.1 \%$ to $7.7 \%$ for unlinked data rates and from $14.6 \%$ to $12.6 \%$ for linked data rates.

National measures of ischaemic stroke are affected by within-country variations in performance at the hospital level. Reducing this variation is key to providing equitable care and reducing overall mortality rates. Figure 6.17 presents the dispersion of ischaemic stroke 30-day casefatality rates across hospitals within countries, using both unlinked and linked data.

Reducing this variation requires high-quality stroke care for all, including timely transportation of patients, evidence- based medical interventions and access to high-quality specialised facilities such as stroke units (OECD, 2015[3]). Timely care is particularly important, and advances in technology are leading to new models of care to deliver reperfusion therapy in an even more speedy and efficient manner, whether through pre-hospital triage via telephone or administering the therapy in the ambulance (Chang and Prabhakaran, 2017[4]).

\section{Definition and comparability}

National case-fatality rates are defined in indicator "Mortality following acute myocardial infarction".

Hospital-level stroke mortality rates use a different methodology from national rates. Hospital rates are adjusted for age, sex, co-morbidity, stroke severity and previous stroke (linked data only). The reference population for hospital rates is constructed from data from participating countries. The hospital-level ischaemic stroke definition also differs from the national indicator, using only ICD-10 code I63 (cerebral infarction).

Figure 6.17 is a turnip plot that graphically represents the relative dispersion of rates. A limitation of this type of representation is the inability to detect statistically significant variations. Countries are ordered according to ascending level of dispersion as measured by the interquartile range (between the 25th and 75th percentile) of rates. Hospitals with fewer than 50 ischaemic stroke admissions were excluded from both figures to improve data reliability.

\section{References}

[1] American Heart Association (2017), Heart Disease and Stroke Statistics 2017 at-a-Glance.

[4] Chang, P. and S. Prabhakaran (2017), "Recent advances in the management of acute ischemic stroke", F1000Research, http://dx.doi.org/10.12688/f1000research.9191.1.

[3] OECD (2015), Cardiovascular Disease and Diabetes: Policies for Better Health and Quality of Care, OECD Health Policy Studies, OECD Publishing, Paris, https://dx.doi.org/ 10.1787/9789264233010-en.

[2] OECD (2015), OECD Reviews of Health Care Quality: Japan 2015: Raising Standards, OECD Reviews of Health Care Quality, OECD Publishing, Paris, https://dx.doi.org/ 10.1787/9789264225817-en. 


\section{QUALITY AND OUTCOMES OF CARE}

Mortality following ischaemic stroke

Figure 6.15. Thirty-day mortality after admission to hospital for ischaemic stroke based on unlinked data, 2007 and 2017 (or nearest year)

02007

2017

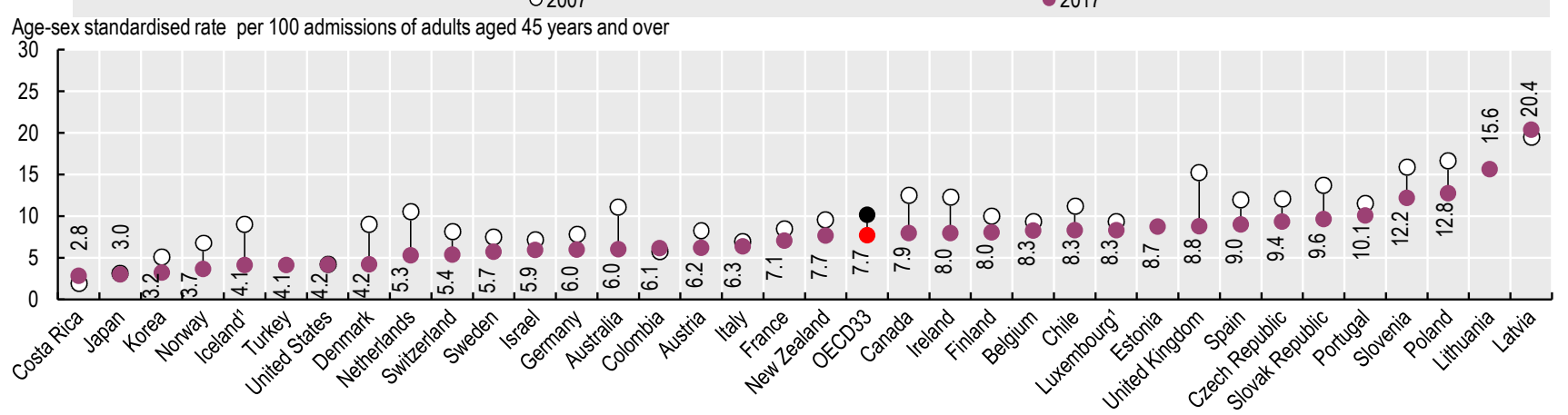

1. Three-year average.

Source: OECD Health Statistics 2019.

StatLink लाइ https://doi.org/10.1787/888934016227

Figure 6.16. Thirty-day mortality after admission to hospital for ischaemic stroke based on linked data, 2007 and 2017 (or nearest year)

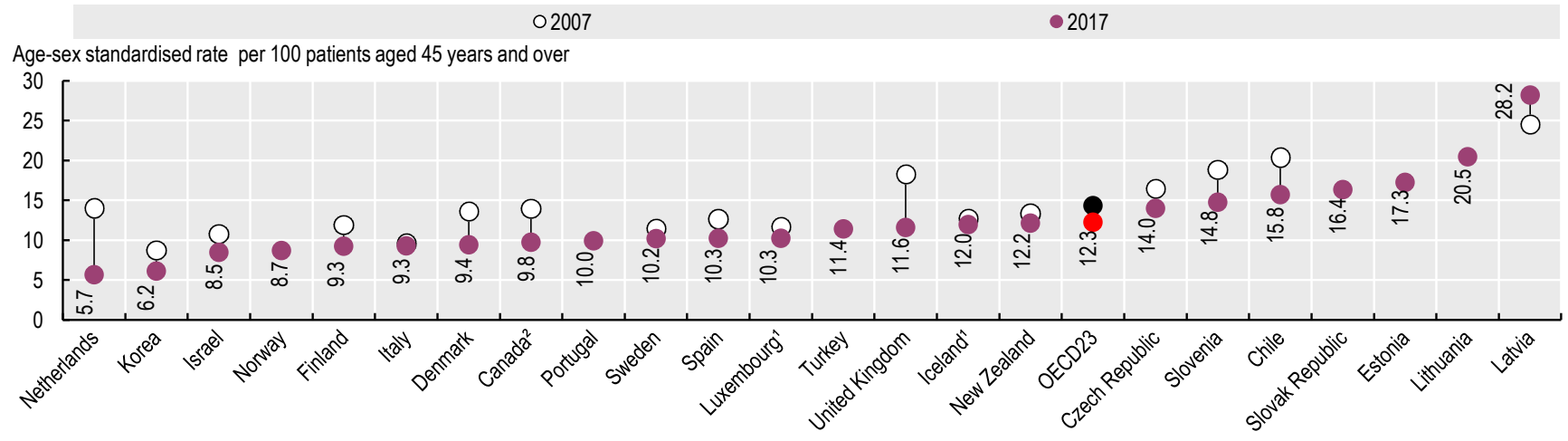

1. Three-year average. 2. Results for Canada do not include deaths outside acute care hospitals.

Source: OECD Health Statistics 2019.

StatLink 엔 https://doi.org/10.1787/888934016246

Figure 6.17. Variations across hospitals in 30-day mortality after admission for ischaemic stroke using linked and unlinked data, 2015-17

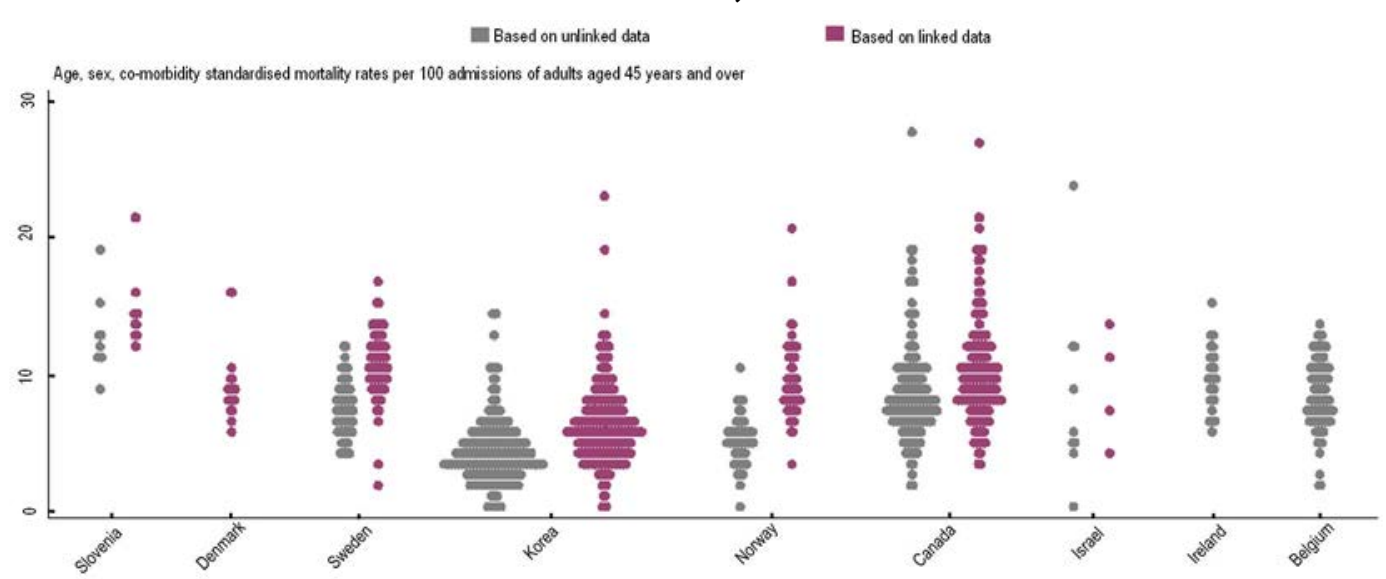

Note: The width of each line in the figure represents the number of hospitals (frequency) with the corresponding rate. Source: OECD Hospital Performance Data Collection 2019. 
Mortality due to coronary heart disease has declined substantially since the 1970s (see indicator "Mortality from circulatory diseases" in Chapter 3). Important advances in both prevention policies, such as for smoking (see indicator "Smoking among adults" in Chapter 4), and treatment of cardiovascular diseases have contributed to these declines (OECD, 2015[1]). A good indicator of acute care quality is the 30-day AMI case-fatality rate. The measure reflects the processes of care, including timely transport of patients and effective medical interventions.

Figure 6.18 shows the case-fatality rates within 30 days of admission for AMI where the death occurs in the same hospital as the initial AMI admission. This method of calculating the indicator is influenced by not only the quality of care provided in hospitals but also differences in hospital transfers and average length of stay. The lowest rates are found in Iceland, Denmark, Norway, the Netherlands, Australia and Sweden (all $4 \%$ or less). The highest rates are in Latvia and Mexico, suggesting that AMI patients do not always receive recommended care in these countries. In Mexico, the absence of a co-ordinated system of care between primary care and hospitals may contribute to delays in reperfusion and low rates of angioplasty (Martínez-Sánchez et al., 2017[2]).

Figure 6.19 shows 30-day case-fatality rates where fatalities are recorded regardless of where they occur (including after transfer to another hospital or after discharge). This is a more robust indicator because it records deaths more widely than the same-hospital indicator, but it requires a unique patient identifier and linked data, which are not available in all countries. The AMI case-fatality rate in 2017 ranged from $4.0 \%$ in the Netherlands to $16.5 \%$ in Latvia.

Case-fatality rates for AMI decreased substantially between 2007 and 2017 (Figure 6.18 and Figure 6.19). Across OECD countries, case fatalities fell from $9.5 \%$ to $6.9 \%$ when considering same-hospital deaths and from $12.5 \%$ to $9.1 \%$ when considering deaths in and out of hospital.

Variations in AMI 30-day case-fatality rates at the national level are influenced by the dispersion of rates across hospitals within countries, as represented in Figure 6.20. The interquartile range of rates within countries varies markedly. The differences between the upper and lower rates are 1.9 deaths per 100 admissions for Sweden and 4.1 deaths per 100 admissions for Korea (based on linked data).

Multiple factors contribute to variations in outcomes of care, including hospital structure, processes of care and organisational culture. Recent research points to higher total numbers of hospital patients as being significantly related to higher performance; this may support national movements towards concentration of care services (Lalloué et al., 2019[3]).

\section{Definition and comparability}

The case-fatality rate measures the percentage of people aged 45 and over who die within 30 days following admission to hospital for a specific acute condition. Rates based on unlinked data only consider deaths occurring in the same hospital as the initial admission. Rates based on linked data consider deaths that occurred anywhere including in or outside hospital. While the linked data-based method is considered more robust, it requires a unique patient identifier to link the data across the relevant datasets, which is not available in all countries.

National rates are age-sex standardised to the 2010 OECD population aged 45 and over admitted to hospital for AMI (ICD-10 codes I21-I22) and ischaemic stroke (ICD-10 codes I63-I64).

Hospital-level AMI mortality rates use a different methodology from national rates. Hospital rates are adjusted for age, sex, co-morbidity and previous AMI (linked data only). The reference population for hospital rates is constructed from data from participating countries (Padget, forthcoming[4]).

Figure 6.20 is a turnip plot that graphically represents the relative dispersion of rates. A limitation of this type of representation is the inability to detect statistically significant variations. Countries are ordered according to ascending level of dispersion as measured by the interquartile range (between the 25th and 75th percentile) of rates. Hospitals with fewer than 50 AMI admissions were excluded from both figures to improve data reliability.

\section{References}

[4] Padget, M. (forthcoming), "OECD Hospital Performance Project: Methodological Development of International Measurement of Acute Myocardial Infraction 30-Day Mortality Rates at the Hospital Level", OECD Health Working Papers, OECD Publishing, Paris.

[3] Lalloué, B. et al. (2019), "Does size matter? The impact of caseload and expertise concentration on AMI 30-day mortality-A comparison across 10 OECD countries.", Health policy (Amsterdam, Netherlands), Vol. 123/5, pp. 441-448, http:// dx.doi.org/10.1016/j.healthpol.2019.03.007.

[2] Martínez-Sánchez, C. et al. (2017), "Reperfusion therapy of myocardial infarction in Mexico: A challenge for modern cardiology.", Archivos de cardiologia de Mexico, Vol. 87/2, pp. 144-150, http://dx.doi.org/10.1016/j.acmx.2016.12.007.

[1] OECD (2015), Cardiovascular Disease and Diabetes: Policies for Better Health and Quality of Care, OECD Health Policy Studies, OECD Publishing, Paris, https://dx.doi.org/ 10.1787/9789264233010-en. 
Figure 6.18. Thirty-day mortality after admission to hospital for AMI based on unlinked data, 2007 and 2017 (or nearest year)

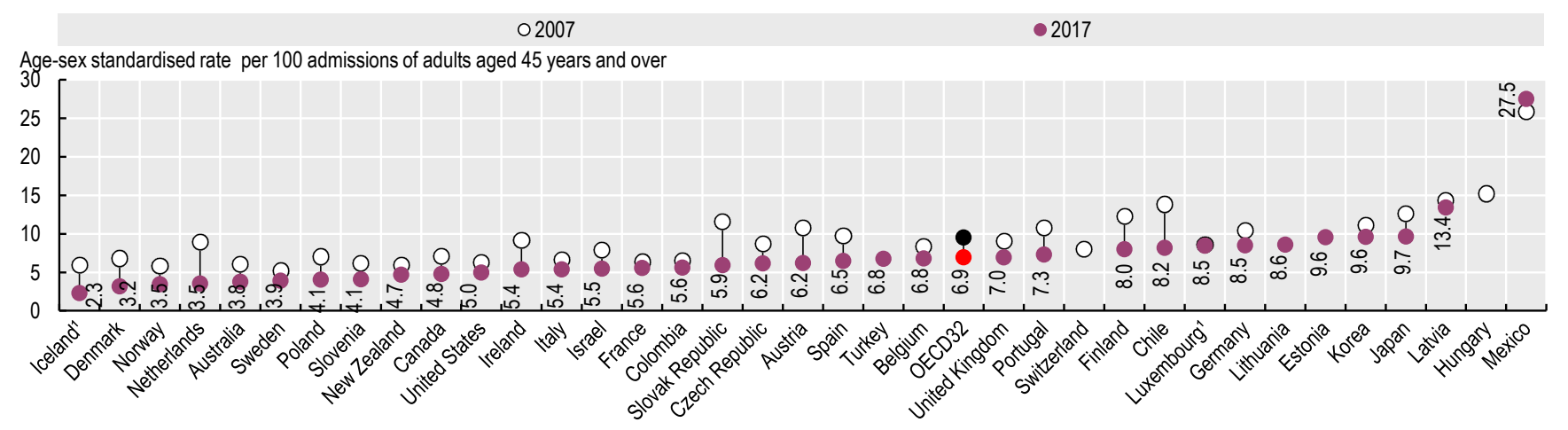

1. Three-year average.

Source: OECD Health Statistics 2019.

Figure 6.19. Thirty-day mortality after admission to hospital for AMI based on linked data, 2007 and 2017 (or nearest year)

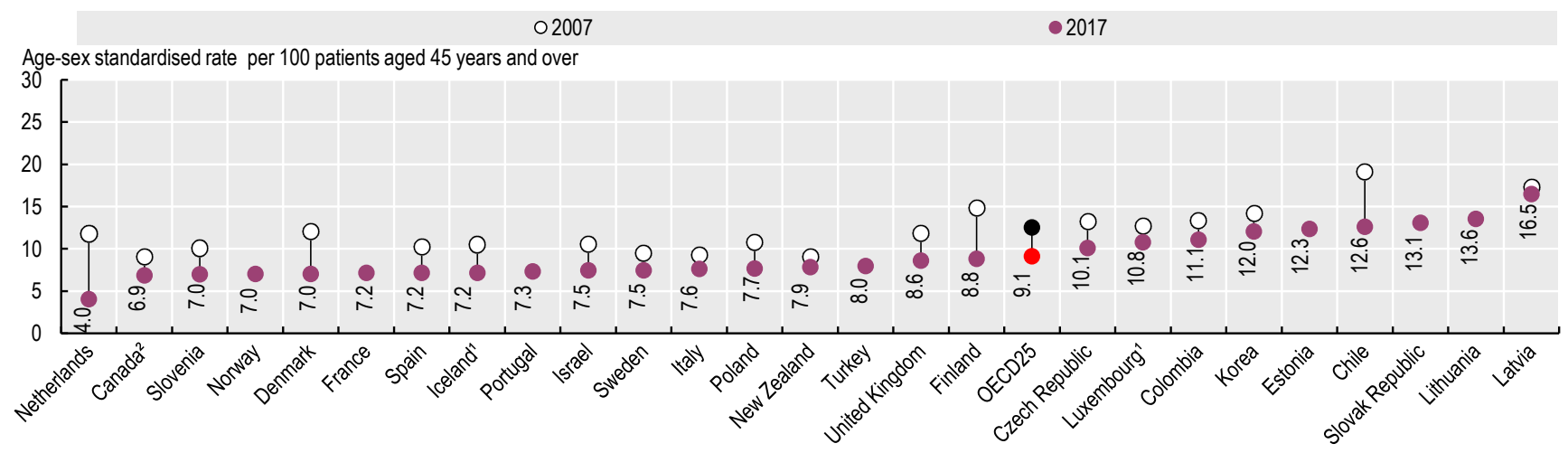

1. Three-year average. 2. Results for Canada do not include deaths outside acute care hospitals.

Source: OECD Health Statistics 2019.

StatLink 部更 https://doi.org/10.1787/888934016303

Figure 6.20. Variations across hospitals in 30-day mortality after admission for AMI using linked and unlinked data, 2015-17

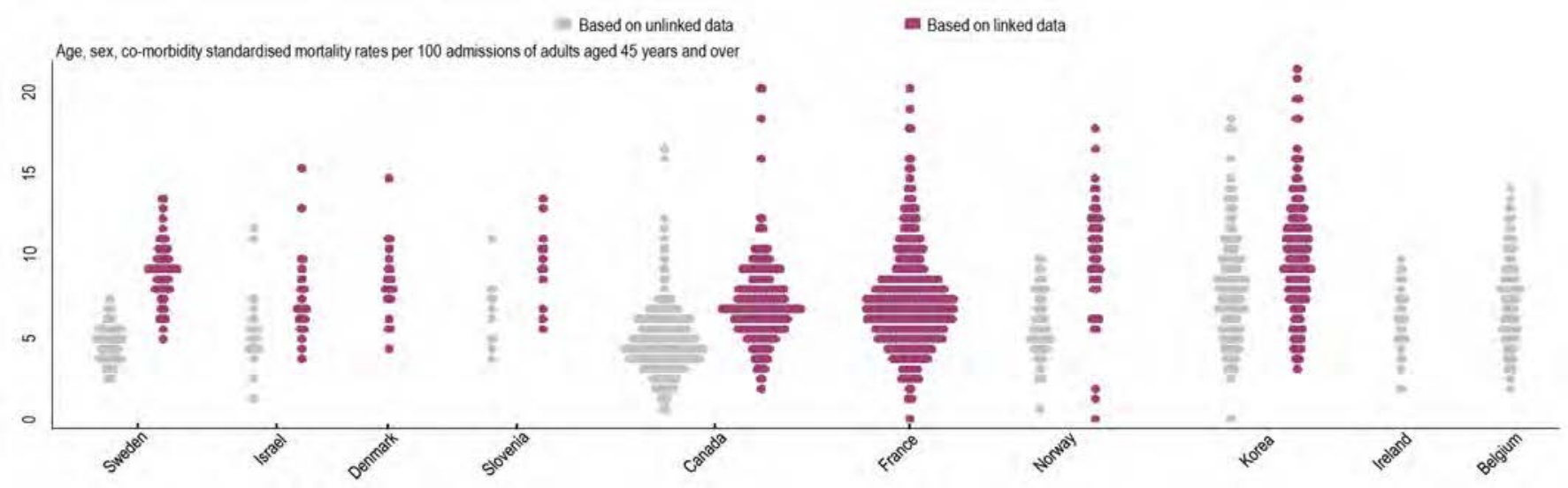

Note: The width of each line in the figure represents the number of hospitals (frequency) with the corresponding rate.

Source: OECD Hospital Performance Data Collection 2019. 
Hip and knee replacement surgeries can be effective treatments for patients with chronic conditions such as osteoarthritis (OA). Surgeries to repair hip fractures are also common and effective. Ageing and a loss of skeletal strength from osteoporosis are the main risk factors associated with a hip fracture, typically sustained during a fall. In most instances, surgical intervention is required to repair or replace the fractured hip joint.

Treatment of patients with hip and knee OA aims to reduce the patient's joint pain and improve their function, mobility and quality of life (QOL). Surgery is generally recommended if symptoms substantially affecting QoL persist after exhausting non-surgical treatment (NICE, 2014[1]). Agestandardised hip and knee replacement rates have risen over the past decade, and vary up to five-fold within and between countries (OECD, 2014[2]).

Figure 6.21 shows the crude mean scores submitted by patients before and at 6 or 12 months after elective hip replacement surgery for $\mathrm{OA}$ in a set of national or subnational joint replacement programmes using the Oxford Hip Score and HOOS-PS, which are validated patientreported outcome measures (PROMs) that have been developed specifically for hip and knee pain. In all programmes, the average patient reported a higher score following surgery, suggesting a positive outcome on average.

Figure 6.22 shows the crude mean scores submitted by patients before and 6 or 12 months after elective knee replacement surgery for $\mathrm{OA}$ in national and sub-national programmes using the Oxford Knee Score and KOOS-PS instruments. On average, knee replacement patients also reported improvement after surgery in all programmes. The amount of improvement for knee replacement was, on average, more modest than that reported by hip replacement patients. However, patients recovering from knee arthroplasty may take longer to recover. Further results and analysis of these measures are provided in Chapter 2.

While a hip replacement for OA is an elective procedure, hip fracture repair is usually an emergency procedure. Evidence suggests that early surgical intervention improves patient outcomes and minimises the risk of complication. There is general agreement that surgery should occur within two days (48 hours) of hospital admission (National Clinical Guideline Centre, 2011[3]).

Time-to-surgery (TTS) is considered a clinically meaningful process indicator of the quality of acute care for patients with hip fracture. However, TTS is influenced by many factors, including hospitals' surgical theatre capacity, flow and access, and targeted policy interventions, including public reporting and monitoring of performance (Siciliani, Borowitz and Moran, 2013[4]).

In 2017 , on average across OECD countries, over $80 \%$ of patients admitted for hip fracture underwent surgery within two days (Figure 6.23) This represents a modest increase of 2.7 percentage points (from 78.2\% to 80.9\%) since 2012.
The biggest improvement was observed in Israel (from 68\% to $89 \%$ ). Targeted policies that effectively incentivise timely surgery following hip fracture admission could partly explain this result. Iceland, the Czech Republic, Portugal and Latvia reported a decline in the proportion over this period, suggesting a need for policy interventions.

\section{Definition and comparability}

The PROM results are based on data from adult patients undergoing elective hip or knee replacement with a principal diagnosis of OA, who completed an Oxford Hip/Knee Score and/or H/KOOS questionnaire pre- and post-operatively (OECD, forthcoming[5]). On both scales, a higher score denotes better outcomes. Data collection at 6 months versus 12 months influences the results. The size of participating programmes varied from entire countries to single hospitals. For further details of the methodological approach and issues regarding comparability, refer to Chapter 2.

Hip fracture indicator is defined as the proportion of patients aged 65 years and over admitted to hospital in a specified year with a diagnosis of upper femur fracture, who had surgery initiated within two calendar days of their admission to hospital. The capacity to capture time of admission and surgery in hospital administrative data varies across countries, resulting in the inability to precisely record surgery within 48 hours in some countries.

While cases where the hip fractures occurred during the admission to hospital should be excluded, not all countries have a 'present on admission' flag in their datasets to enable them to identify such cases accurately.

\section{References}

[3] National Clinical Guideline Centre (2011), The management of hip fracture in adults, National Clinical Guideline Centre, London, http://www.ncgc.ac.uk.

[1] NICE (2014), Osteoarthritis: care and management, The National Institute for Health and Care Excellence.

[2] OECD (2014), Geographic Variations in Health Care: What Do We Know and What Can Be Done to Improve Health System Performance?, OECD Health Policy Studies, OECD Publishing, Paris, https://dx.doi.org/10.1787/9789264216594-en.

[5] OECD (forthcoming), "Patient-reported outcome indicators in joint replacement and breast cancer surgery", OECD Health Working Papers, OECD Publishing, Paris.

[4] Siciliani, L., M. Borowitz and V. Moran (eds.) (2013), Waiting Time Policies in the Health Sector: What Works?, OECD Health Policy Studies, OECD Publishing, Paris, https://dx.doi.org/ 10.1787/9789264179080-en. 
Figure 6.21. Crude mean pre- and post-operative Oxford Hip Score and HOOS-PS, 2013-16 (or nearest year)
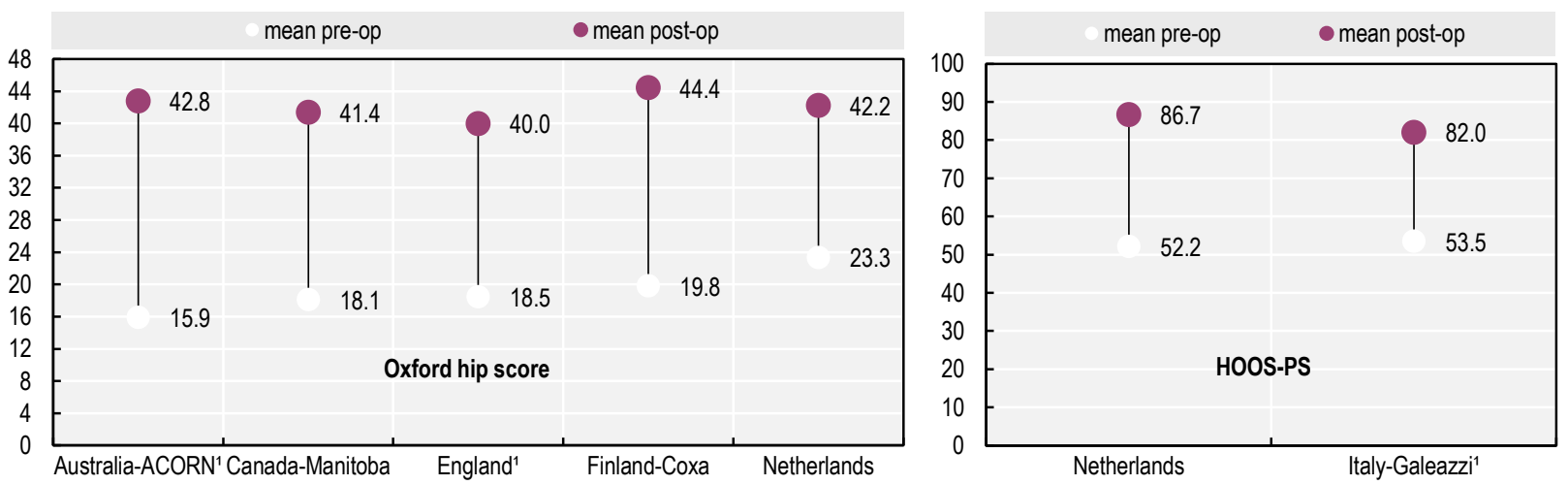

1. Post-operative measurement at six months.

Source: PaRIS Hip/Knee Replacement Pilot Data Collection.

Figure 6.22. Crude mean pre- and post-operative Oxford Knee Score and KOOS-PS, 2013-16 (or nearest year)
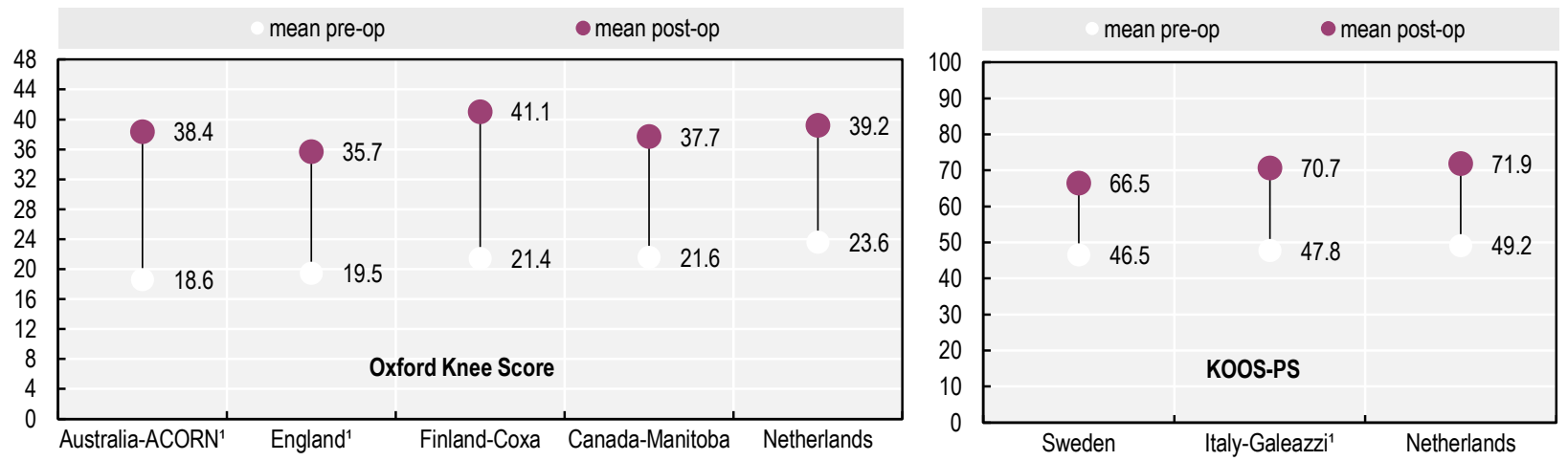

1. Post-operative measurement at six months.

Source: PaRIS Hip/Knee Replacement Pilot Data Collection.

StatLink 에내 https://doi.org/10.1787/888934016360

Figure 6.23. Hip fracture surgery initiation within two days of admission to hospital, 2012 and 2017 (or nearest year)

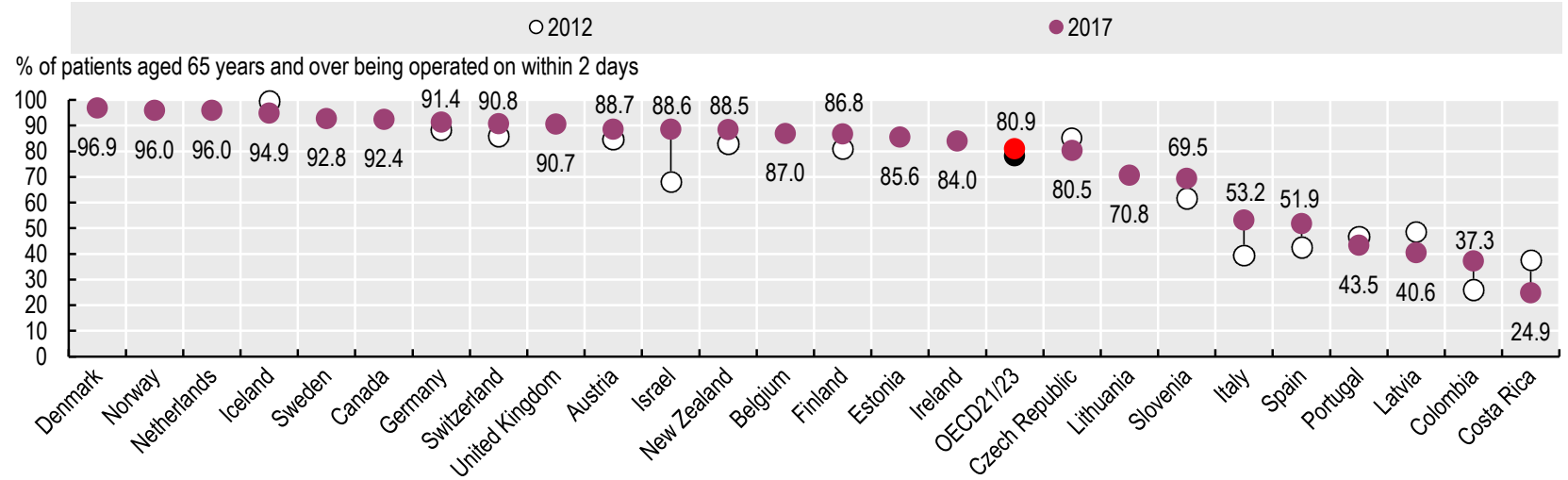

Source: OECD Health Statistics 2019. 
The burden of mental illness is substantial, affecting an estimated one in five people among the population of OECD countries at any given time, and one in two across the life course (see indicator "Mental health" in Chapter 3). The total cost of mental ill health is estimated at between 3.5\% and $4 \%$ of GDP in OECD countries (OECD, 2018[1]). High-quality, timely care has the potential to improve outcomes and may help reduce suicide and excess mortality for individuals with mental disorders.

High-quality care for mental disorders in inpatient settings is vital, and inpatient suicide is a "never" event, which should be closely monitored as an indication of how well inpatient settings are able to keep patients safe from harm. Most countries report inpatient suicide rates below 10 per 10000 patients, but Denmark is an exception, with rates of over 10 (Figure 6.24). Steps to prevent inpatient suicide include identification and removal of likely opportunities for self-harm, risk assessment of patients, monitoring and appropriate treatment plans. While inpatient suicide should be considered a never event, some practices that reduce risk of inpatient suicide - such as use of restraints - may impede high-quality care.

Suicide rates after hospital discharge can indicate the quality of care in the community, as well as co-ordination between inpatient and community settings. Across OECD countries, suicide rates among patients who had been hospitalised in the previous year was as low as 10 per 10000 patients in Iceland and the United Kingdom but higher than 50 per 10000 in the Netherlands, Slovenia and Lithuania (Figure 6.25). Patients with a psychiatric illness are particularly at risk immediately following discharge from hospital, but it is known that suicide in the high-risk days following discharge can be reduced by good discharge planning and follow-up, and enhanced levels of care immediately following discharge.

Individuals with a psychiatric illness have a higher mortality rate than the general population. An "excess mortality" value that is greater than one implies that people with mental disorders face a higher risk of death than the rest of the population. Figure 6.26 shows the excess mortality for schizophrenia and bipolar disorder, which is above two in most countries. In order to reduce their high mortality, a multifaceted approach is needed for people with mental disorders, including primary care prevention of physical ill health, better integration of physical and mental health care, behavioural interventions and changing professional attitudes (OECD, 2014[2]).

Patient experiences can also shed light on the quality of care provided to individuals diagnosed with a mental problem. On average across OECD countries, patients diagnosed with a mental health problem are less likely to report that they were treated with courtesy and respect by doctors and nurses during hospitalisation than hospitalised patients never diagnosed with a mental health problem (Figure 6.27).
In addition, in several countries including Australia, Sweden and France, people diagnosed with a mental health problem are more likely to have received conflicting information from different health care professionals (see Chapter 2). This suggests that there is a room to improve the quality of care for people with mental health problems.

\section{Definition and comparability}

The inpatient suicide indicator is composed of a denominator of patients discharged with a principal diagnosis or first two secondary diagnosis code of mental health and behavioural disorders (ICD-10 codes F10-F69 and F90-99) and a numerator of these patients with a discharge code of suicide (ICD-10 codes X60$\mathrm{X84}$ ). Data should be interpreted with caution due to a very small number of cases. Reported rates can vary over time, so where possible a three-year average has been calculated to give more stability to the indicator, except for New Zealand.

Suicide within 30 days and within one year of discharge is established by linking discharge following hospitalisation with a principal diagnosis or first two listed secondary diagnosis code of mental health and behavioural disorders (ICD-10 codes F10-F69 and F90-99) with suicides recorded in death registries (ICD-10 codes X60-X84).

For the excess mortality indicators, the numerator is the overall mortality rate for persons aged between 15 and 74 diagnosed with schizophrenia or bipolar disorder. The denominator is the overall mortality rate for the general population in the same age group. The relatively small number of people with schizophrenia or bipolar disorder dying in any given year can cause substantial variations from year to year, so three-year averages were presented.

For information on patient experience monitoring see the 2016 Commonwealth Fund International Health Policy Survey of Adults. Differences between countries should be interpreted with care, given the heterogeneity in nature and the size of country samples.

\section{References}

[1] OECD (2018), Health at a Glance: Europe 2018: State of Health in the EU Cycle, OECD Publishing, Paris/European Union, Brussels, https://dx.doi.org/10.1787/health_glance_eur-2018-en.

[2] OECD (2014), Making Mental Health Count: The Social and Economic Costs of Neglecting Mental Health Care, OECD Health Policy Studies, OECD Publishing, Paris, https://dx.doi.org/ 10.1787/9789264208445-en. 
Figure 6.24. Inpatient suicide among patients with a psychiatric disorder, 2015-17 (or nearest year)

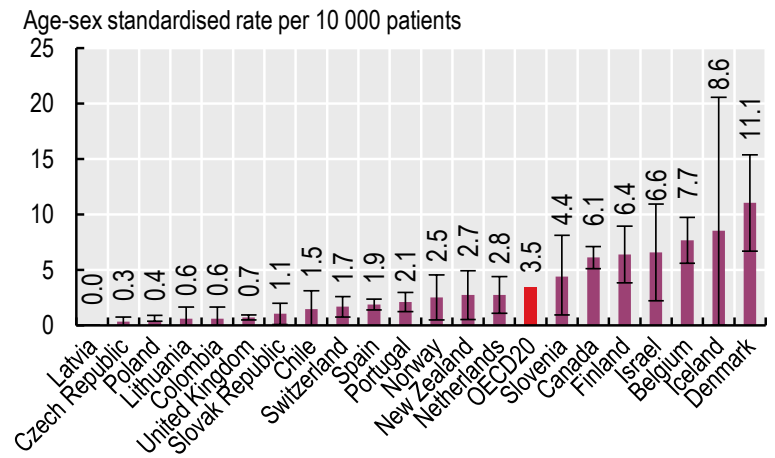

Note: $\mathrm{H}$ lines show $95 \%$ confidence intervals. Source: OECD Health Statistics 2019.

StatLink तारा https://doi.org/10.1787/888934016398
Figure 6.25. Suicide following hospitalisation for a psychiatric disorder, within 30 days and one year of discharge, 2017 (or nearest year)

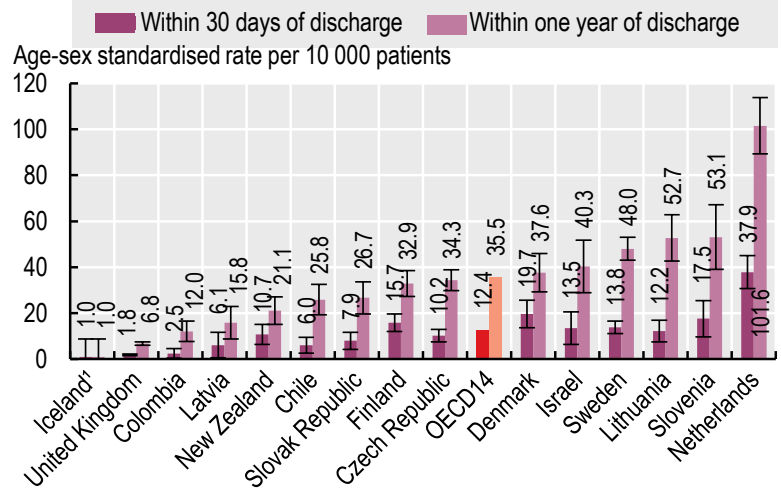

1. Three-year average.

Source: OECD Health Statistics 2019.

StatLink 게s https://doi.org/10.1787/888934016417

Figure 6.26. Excess mortality from bipolar disorder and schizophrenia, 2015-17

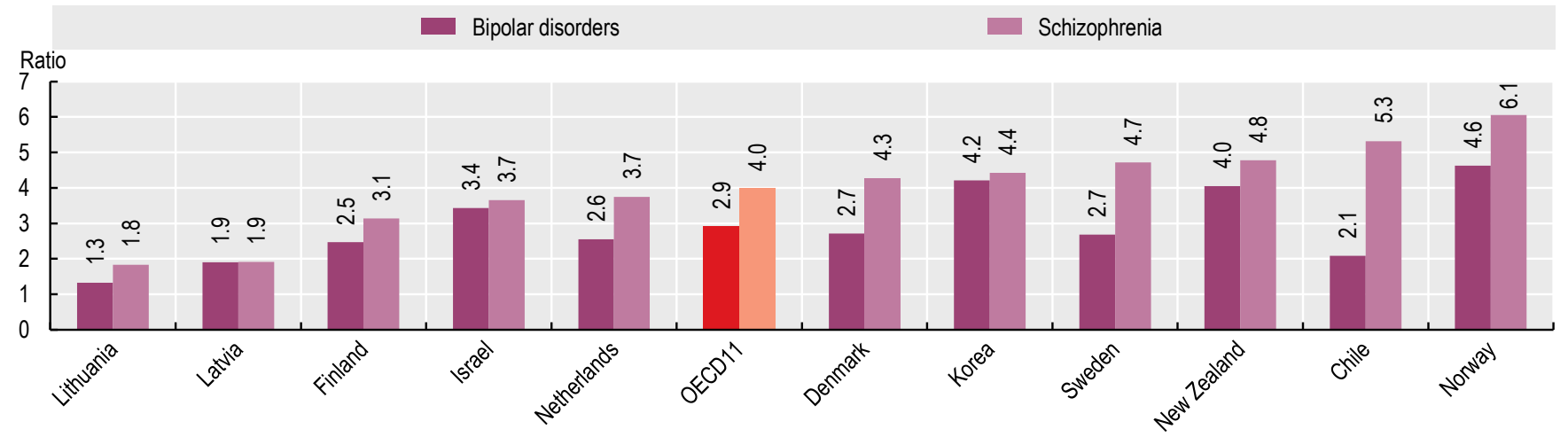

Note: Data represent a three-year average except for the Netherlands (two-year average).

Source: OECD Health Statistics 2019.

StatLink 게s https://doi.org/10.1787/888934016436

Figure 6.27. Share of people who were treated with courtesy and respect by doctors and nurses during hospitalisation, 2016

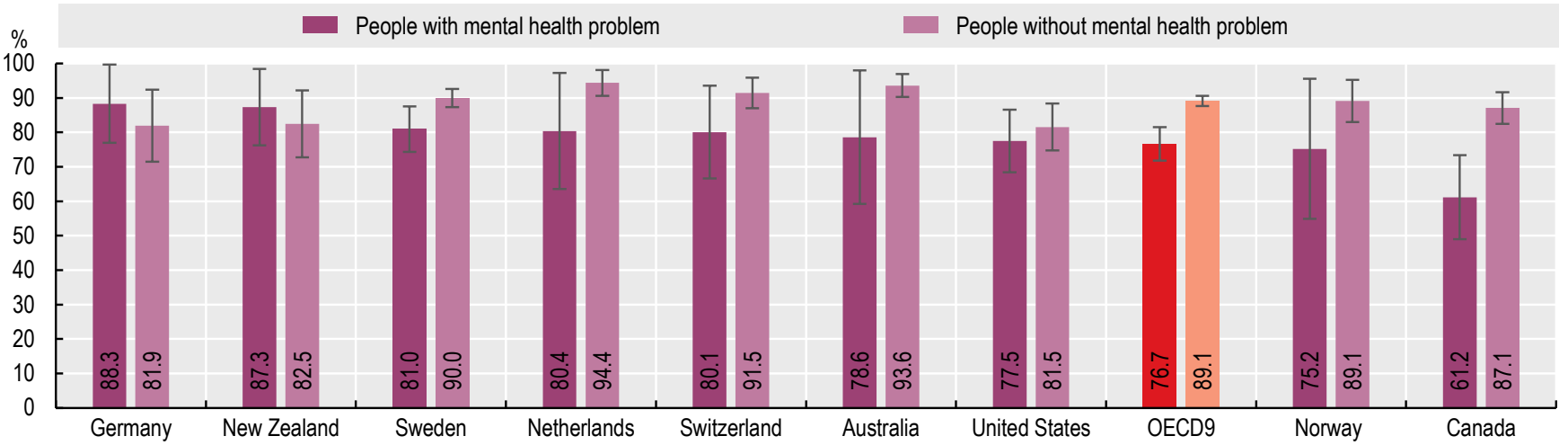

Note: $\mathrm{H}$ lines show $95 \%$ confidence intervals.

Source: Commonwealth Fund International Health Policy Survey 2016. 
Breast cancer is the cancer with the highest incidence among women in all OECD countries, and the second most common cause of cancer death among women (see indicator "Cancer incidence and mortality" in Chapter 3).

During 2010-14, an average of $51.5 \%$ of women with breast cancer were diagnosed at an early stage of disease in OECD countries, while $8.6 \%$ of women were diagnosed at an advanced stage (Figure 6.28). Countries with a high proportion of women diagnosed at an early stage, such as the United States and Japan, have a correspondingly low proportion of women diagnosed at an advanced stage. Since the 1980s, most OECD countries have adopted breast cancer screening programmes as an effective way of detecting the disease early (OECD, 2013[1]). This has contributed to higher proportions of women being diagnosed at an early stage.

In most OECD countries, five-year net survival for women with breast cancer has improved in recent years, reflecting overall improvement in the quality of cancer care (Allemani et al., 2018[2]). In all OECD countries, for women diagnosed at early or localised stage, the cumulative probability of surviving their cancer for at least five years is $90 \%$ and the international variation is small (Figure 6.29). However, net survival for women diagnosed at an advanced stage is still low and ranges widely, from about 30\% in Austria and Lithuania to over 50\% in Israel and Finland.

Motivated providers and patients across OECD countries are increasingly using patient-reported outcome measures (PROMs) for breast cancer to help inform difficult clinical decisions. Figure 6.30 presents crude outcome scores at 6-12 months following breast surgery (breast-conserving therapy and breast reconstruction) for 11 clinical sites from eight countries. Outcomes were measured using the relevant post-operative breast satisfaction scales from the BREAST-Q tool, an internationally validated instrument used to measure breast surgery outcomes reported by patients (Pusic et al., 2009[3]). Further results and analysis of this measure are provided in Chapter 2.

Figure 6.31 presents the proportion of women undergoing implant and autologous reconstruction surgery in the sample from each site. Consolidated crude scores from the participating sites indicate that women are about $6 \%$ (6 percentage points) more satisfied with their breasts after autologous reconstruction surgery than after implant reconstruction (see Figure 2.9 in Chapter 2). This outcome aligns with existing evidence (Matros et al., 2015[4]) and can be an important consideration if choice of surgical intervention is possible.

These PROMs results are not representative for each country but do show the capacity for metrics of this kind to be reported internationally. Some OECD countries are now scaling up efforts to measure breast cancer PROMs as their utility becomes more fully appreciated. For example, in the Netherlands, breast cancer has been identified as a possible priority area as part of a current national policy effort to measure PROMs systematically.

\section{Definition and comparability}

The stage at diagnosis for breast cancer is categorised according to the Tumour, Nodes, Metastasis (TNM) staging system. In this analysis, "early or localised stages" refers to tumours without lymph node involvement or metastasis (T1-3, N0, M0), "intermediate stage" refers to tumours with lymph node involvement but no metastasis (T1-3, N1-3, M0), and "advanced stage" refers to large tumours with ulceration or involvement of the chest wall, and those that have metastasised to other organs (T4, any N, M0 or M1).

Five-year net survival refers to the cumulative probability that the cancer patients would have lived five years after diagnosis if the cancer was the only possible cause of death. The period approach is used to allow estimation of five-year survival where five years of follow-up are not available. Cancer survival estimates are age-standardised with the International Cancer Survival Standard weights.

Cancer patient data were provided by national or regional cancer registries. Quality control and analysis for stage distribution and age-standardised five-year net survival were performed centrally as part of CONCORD, the global programme for the surveillance of cancer survival, led by the London School of Hygiene and Tropical Medicine (Allemani et al., 2018[2]).

See Box 2.3 in Chapter 2 for more details regarding the BREAST-Q breast satisfaction scale used to measure the breast cancer PROMs. Data are only presented for selected sites and are not representative for each country. Note that measurement extended beyond 12 months after surgery for sites in Sweden and Switzerland.

\section{References}

[2] Allemani, C. et al. (2018), "Global surveillance of trends in cancer survival 2000-14 (CONCORD-3): analysis of individual records for 37513025 patients diagnosed with one of 18 cancers from 322 population-based registries in 71 countries", The Lancet, Vol. 391/10125, pp. 1023-1075, http:// dx.doi.org/10.1016/s0140-6736(17)33326-3.

[4] Matros, E. et al. (2015), "Cost-Effectiveness Analysis of Implants versus Autologous Perforator Flaps Using the BREAST-Q", Plastic and Reconstructive Surgery, Vol. 135/4, pp. 937-946, http://dx.doi.org/10.1097/prs.0000000000001134.

[1] OECD (2013), Cancer Care: Assuring Quality to Improve Survival, OECD Health Policy Studies, OECD Publishing, Paris, https:// dx.doi.org/10.1787/9789264181052-en.

[3] Pusic, A. et al. (2009), "Development of a New PatientReported Outcome Measure for Breast Surgery: The BREASTQ", Plastic and Reconstructive Surgery, Vol. 124/2, pp. 345-353, http://dx.doi.org/10.1097/prs.0b013e3181aee807. 
Figure 6.28. Breast cancer stage distribution, 2010-14

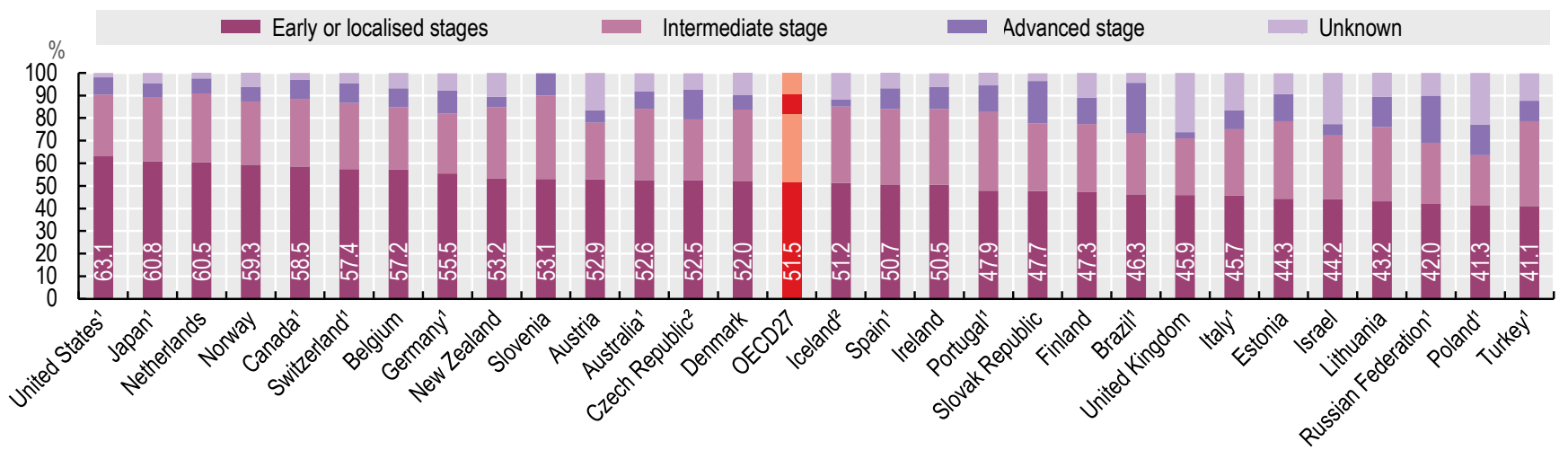

1. Data represent coverage of less than $100 \%$ of the national population. 2. Data for 2004-09.

Source: CONCORD programme, London School of Hygiene and Tropical Medicine.

Figure 6.29. Breast cancer five-year net survival by stage of breast cancer at diagnosis, 2010-14

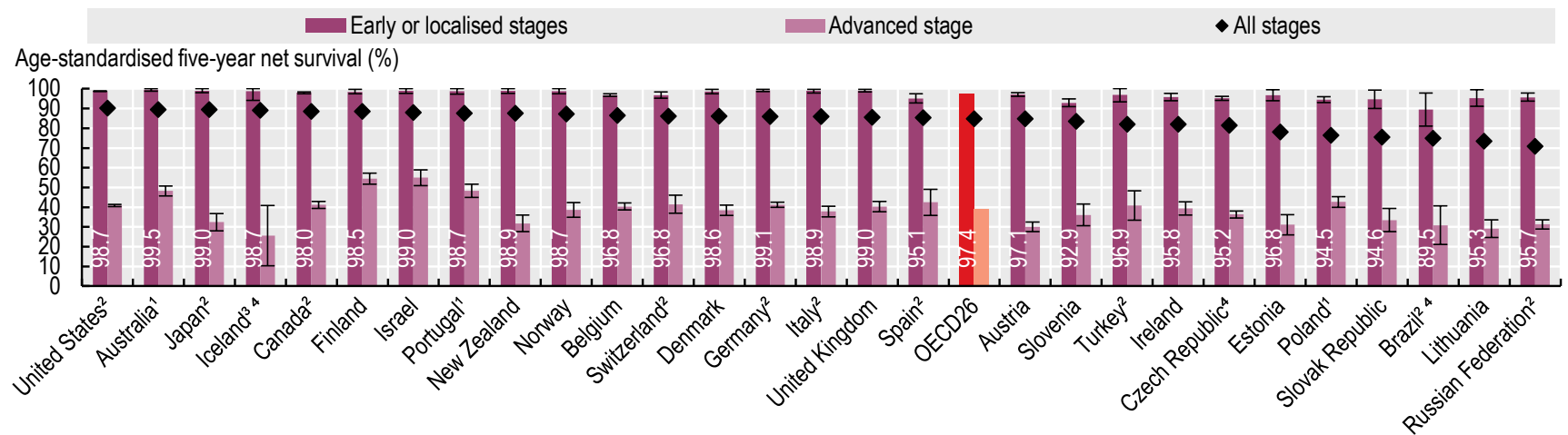

Note: $\mathrm{H}$ line shows $95 \%$ confidence intervals. 1. Coverage is less than $100 \%$ of the national population for stage-specific survival estimates. 2. Coverage is less than $100 \%$ of the national population. 3. Survival estimates for advanced stage are not age-standardised. 4. Data for 2004-09.

Source: CONCORD programme, London School of Hygiene and Tropical Medicine.

Figure 6.30. Self-reported satisfaction with breast surgery: crude scores 6-12 months after surgery, 2017-18 (or nearest year)

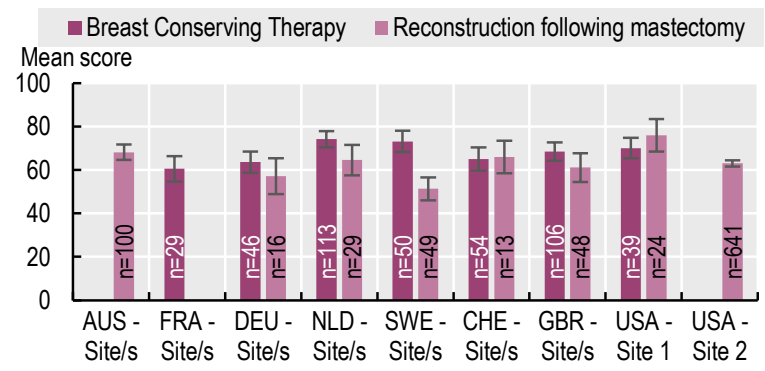

Note: $\mathrm{H}$ line shows $95 \%$ confidence intervals. Data labels at the base of the histogram refer to the sample size at each site.

Source: PaRIS Breast Cancer PROMs pilot data collection 2019. StatLink तiाs https://doi.org/10.1787/888934016512
Figure 6.31. Type of breast reconstruction surgery, proportion of total, 2017-18 (or nearest year)

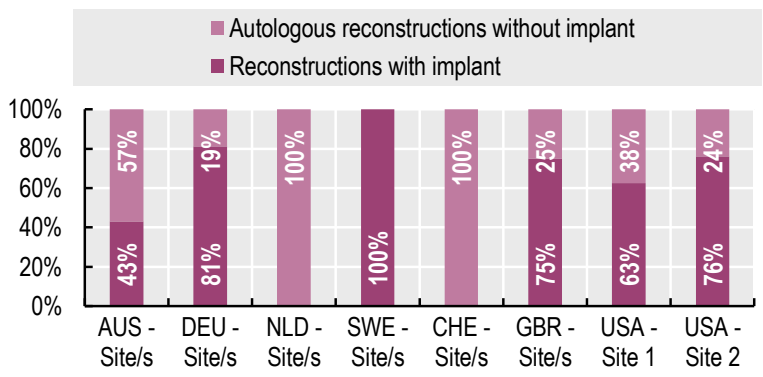

Source: PaRIS Breast Cancer PROMs pilot data collection 2019. StatLink ताIs https://doi.org/10.1787/888934016531 
Colorectal cancer is the third most commonly diagnosed cancer after breast and prostate cancers in OECD countries, and the third most common cause of death from cancer (see indicator "Cancer incidence and mortality" in Chapter 3) (GLOBOCAN, 2018[1]). Several factors increase the risk of developing colorectal cancer, including older age, ulcerative colitis, previous colorectal polyps or a family history of colorectal cancer, as well as lifestyle factors such as a diet high in fat and low in fibre, lack of physical activity, obesity and tobacco and alcohol consumption. Incidence is significantly higher for men than women in most countries. Rectal cancer is often more difficult to treat than colon cancer due to a higher probability of spreading to other tissue, recurrence and post-operative complications.

A growing number of OECD countries have introduced free population-based screening, targeting men and women in their 50s and 60s at either the national or regional levels (OECD, 2013[2]). In most countries that offer the faecal occult blood test, screening is available every two years. The screening/follow-up periodicity schedule is less frequent with colonoscopy and flexible sigmoidoscopy - generally every ten years. These differences complicate international comparisons of screening coverage.

In 2014 , an average $40.4 \%$ of people aged between 50 and 74 in OECD countries had had a faecal occult blood test at least once in their life (Figure 6.32), and $18.4 \%$ of people of all ages had undergone colonoscopy at least once in their life. Population coverage of screening for colorectal cancer is still much lower than for breast and cervical cancer in many OECD countries.

Advances in the diagnosis and treatment of colorectal cancer - including improved surgical techniques, radiation therapy and combined chemotherapy, combined with wider and more timely access to treatments - have contributed to higher survival over the last decade in OECD countries. On average, age-standardised five-year net survival for patients diagnosed during $2010-14$ reached $62.1 \%$ for colon cancer and $60.6 \%$ for rectal cancer (Figure 6.33 and Figure 6.34). Some countries have shown a considerable improvement over the last 10 years, including Denmark, Korea and Lithuania for colon cancer, and the Czech Republic, Denmark, Korea, Ireland, Latvia, Lithuania and Slovenia for rectal cancer.

International variation in age-standardised five-year net survival for cancers of the colon and rectum between OECD countries is very wide. For example, five-year net survival is much higher in Korea than in Chile, for both colon cancer (71.8\% versus $43.9 \%)$ and rectal cancer $(71.1 \%$ versus $32.7 \%$ ). Countries where survival from colon cancer is low also tend to have low survival for rectal cancer, including Chile, the
Czech Republic, Latvia, Poland, the Slovak Republic and Turkey. In recent years, some of these countries have made progress in strengthening their systems to reduce the burden of colorectal cancer. For example, in 2013, Chile included treatment for colorectal cancer as part of its guaranteed health care coverage plan (OECD, 2019[4]).

In order to tackle poor outcomes for other cancers (see indicator "Survival for other major cancers"), several OECD countries have taken a more comprehensive approach to strengthening their cancer care systems. In Latvia, cancer care delivery has been centralised and expertise concentrated in specialised institutions to improve both quality and efficiency of care delivery. A national plan was also adopted in 2017 to improve cancer care through prevention, better access to early diagnosis and optimal treatment, as well as rehabilitation and palliative care (OECD/European Observatory on Health Systems and Policies, 2017[6]).

\section{Definition and comparability}

Net survival is defined in indicator "Breast cancer outcomes". Survival estimates are based on cancer patient records with ICD-10 codes C18-C19 (International Classification of Diseases for Oncology, third edition) for colon cancer and ICD-10 codes C20C21 for rectal cancer.

\section{References}

[5] Allemani, C. et al. (2018), "Global surveillance of trends in cancer survival 2000-14 (CONCORD-3): analysis of individual records for 37513025 patients diagnosed with one of 18 cancers from 322 population-based registries in 71 countries", The Lancet, Vol. 391/10125, pp. 1023-1075, http:// dx.doi.org/10.1016/s0140-6736(17)33326-3.

[1] GLOBOCAN (2018), Cancer Today, https://gco.iarc.fr/today/home.

[3] OECD (2019), OECD Reviews of Public Health: Chile: A Healthier Tomorrow, OECD Publishing, Paris, https://dx.doi.org/ 10.1787/9789264309593-en.

[2] OECD (2013), Cancer Care: Assuring Quality to Improve Survival, OECD Health Policy Studies, OECD Publishing, Paris, https:// dx.doi.org/10.1787/9789264181052-en.

[4] OECD/European Observatory on Health Systems and Policies (2017), Latvia: Country Health Profile 2017, State of Health in the EU, OECD Publishing, Paris/European Observatory on Health Systems and Policies, Brussels, https://dx.doi.org/ 10.1787/9789264283466-en. 
Figure 6.32. People aged 50-74 years who have had faecal occult blood test at least once in their life, 2014

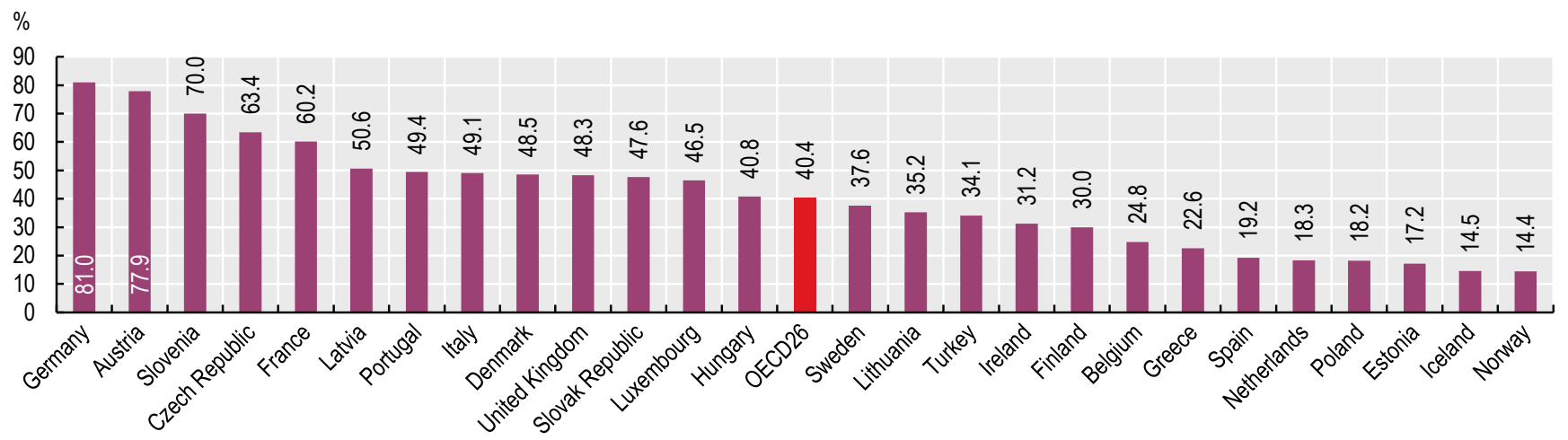

Source: European Health Interview Survey 2014.

Figure 6.33. Colon cancer five-year net survival, 2010-14

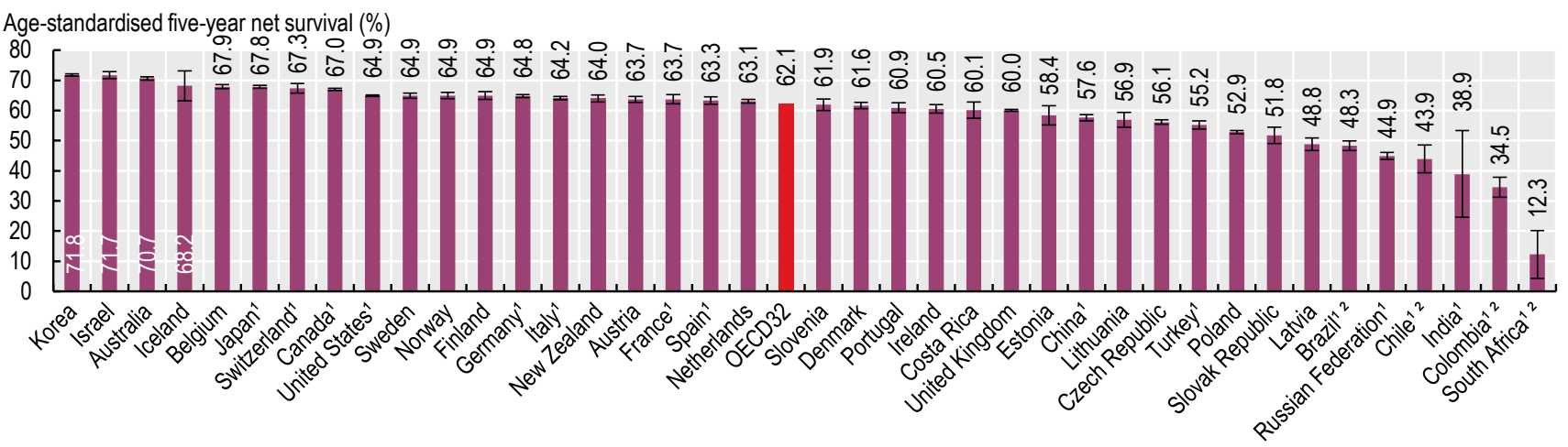

Note: $\mathrm{H}$ line shows $95 \%$ confidence intervals. 1. Data represent coverage of less than $100 \%$ of the national population. 2. Survival estimates are considered less reliable: see Allemani et al. (2018) for more information.

Source: CONCORD programme, London School of Hygiene and Tropical Medicine.

Figure 6.34. Rectal cancer five-year net survival, 2010-14

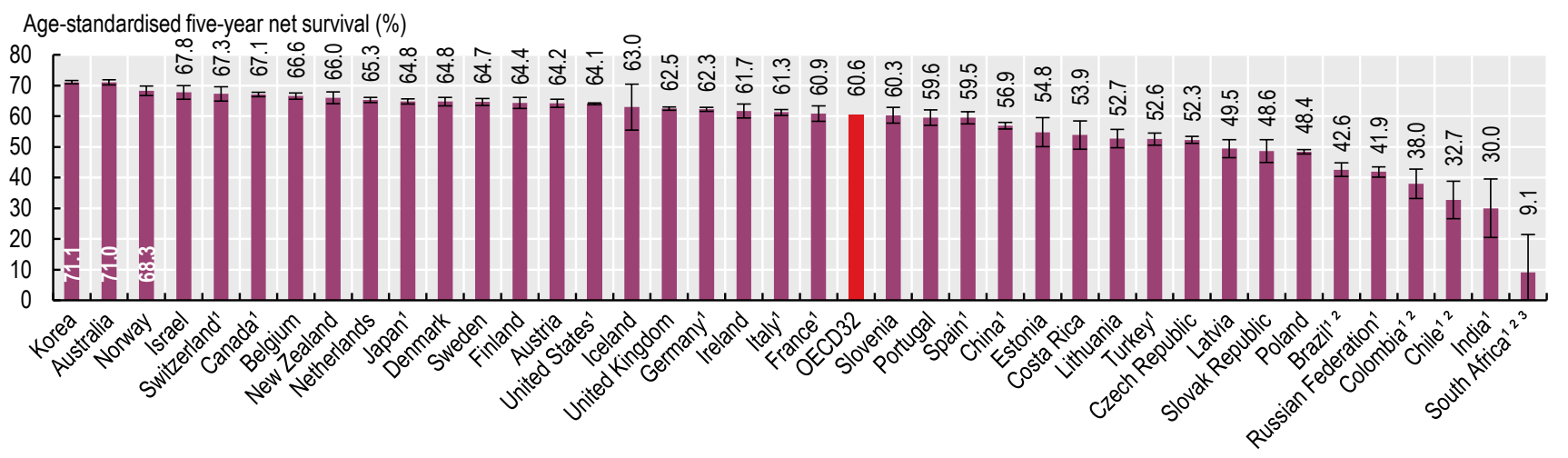

Note: $\mathrm{H}$ line shows $95 \%$ confidence intervals. 1. Data represent coverage of less than $100 \%$ of the national population. 2. Survival estimates are considered less reliable. 3. Survival estimates are not age-standardised.

Source: CONCORD programme, London School of Hygiene and Tropical Medicine. 
Lung cancer is the main cause of cancer death for both men and women in OECD countries (see indicator "Cancer incidence and mortality" in Chapter 3). The main risk factors for lung cancer are smoking; passive smoking; exposure to radon and/or certain chemicals and substances such as arsenic, asbestos, beryllium, cadmium, coal and coke fumes, silica and nickel; air pollution; and a family history of lung cancer. Following the declining trend of smoking in recent decades (see indicator "Smoking among adults" in Chapter 4), incidence rates of lung cancer have declined across OECD countries. However, together with ischaemic heart disease, road accidents and alcohol-related deaths, lung cancer continues to be one of the main causes of preventable mortality in OECD countries.

Compared to other cancers such as breast and colorectal cancers (see indicators "Breast cancer outcomes" and "Screening and survival for colorectal cancer"), lung cancer continues to be associated with very poor survival. On average in OECD countries, for patients diagnosed with lung cancer, the cumulative probability of surviving their cancer for at least five years is less than 20\% (Figure 6.35). Across OECD countries, age-standardised five-year net survival ranged from $32.9 \%$ in Japan to $4.6 \%$ in Chile in $2010-14$, and is low in Lithuania, the Czech Republic, the Slovak Republic, Finland and the United Kingdom. In recent years, agestandardised five-year net survival has increased substantially in Denmark, Ireland, Korea and France. Lung cancer screening is not common in OECD countries, but in Japan, an annual chest X-ray is recommended for people aged 40 and over, and sputum cytology is also recommended for smokers aged 50 and over who have smoked more than 600 cigarettes over their lifetime (OECD, 2019[1]) while the English National Health Service is launching its Targeted Lung Health Checks Programme.

Stomach cancer is another commonly diagnosed cancer and fifth highest cause of cancer death in OECD countries (GLOBOCAN, 2018[2]). The main risk factors for stomach cancer include age, gender, smoking, Helicobacter pylori infection, diet, genetic predisposition, pernicious anaemia, peptic stomach ulcer and stomach surgery. WHO recommends that countries with a high burden of stomach cancer should explore the introduction of population-based $H$. pylori screening and treatment based on local contexts, such as health priorities and cost-effectiveness (IARC, 2014[3]). Incidence of stomach cancer is high in some OECD countries, such as Chile, Korea and Japan; in these countries, stomach cancer screening is available for people in certain age groups (OECD, 2019[1]; OECD, 2019[4]).

Age-standardised five-year net survival for stomach cancer is particularly high in Korea and Japan (60\% or higher), while it ranges between $20 \%$ and $40 \%$ in other OECD countries (Figure 6.36). Net survival is low in Chile, suggesting that there is room to improve stomach cancer screening strategies through stronger stakeholder engagement, better communication strategies to increase public awareness and better access to cancer screening (OECD, 2019[4]).
Leukaemia is the most common cancer among children aged $0-14$; it accounts for over $30 \%$ of all cancers diagnosed in children worldwide (GLOBOCAN, 2018[2]). The causes of leukaemia are not well known, but some known risk factors include inherited factors, such as Down syndrome and a family history of leukaemia, and non-inherited factors, such as exposure to ionising radiation. There are different types of leukaemia but about three-quarters of cases among children are acute lymphoblastic leukaemia (ALL). The prognosis for leukaemia depends on various factors including age, initial white blood cell count, gender, initial reaction to induction treatment and type of leukaemia. Children with acute leukaemia who are free of the disease for five years are considered to have been cured, as remission after five years is rare.

Age-standardised five-year net survival for ALL among children was on average $83.7 \%$ during 2010-14 in OECD countries (Figure 6.37), and it improved over the period, mainly due to progress in chemotherapy and stem cell transplantation technology. However, countries have not benefited equally from progress in medical technologies. Survival estimates are high in Finland and Denmark but low in Chile and Mexico. Chile is making progress in improving access and quality of care for childhood cancer - for example, by including access to care for childhood cancer as part of its guaranteed health care coverage plan (OECD, 2019[4]).

\section{Definition and comparability}

Net survival is defined in indicator "Screening and survival for breast cancer".

\section{References}

[5] Allemani, C. et al. (2018), "Global surveillance of trends in cancer survival 2000-14 (CONCORD-3): analysis of individual records for 37513025 patients diagnosed with one of 18 cancers from 322 population-based registries in 71 countries", The Lancet, Vol. 391/10125, pp. 1023-1075, http:// dx.doi.org/10.1016/s0140-6736(17)33326-3.

[2] GLOBOCAN (2018), Cancer Today, https://gco.iarc.fr/today/home.

[3] IARC (2014), Helicobacter pylori Eradication as a Strategy for Preventing Gastric Cancer.

[4] OECD (2019), OECD Reviews of Public Health: Chile: A Healthier Tomorrow, OECD Publishing, Paris, https://dx.doi.org/ 10.1787/9789264309593-en.

[1] OECD (2019), OECD Reviews of Public Health: Japan: A Healthier Tomorrow, OECD Publishing, Paris, https://dx.doi.org/ 10.1787/9789264311602-en. 
Figure 6.35. Lung cancer five-year net survival, 2010-14

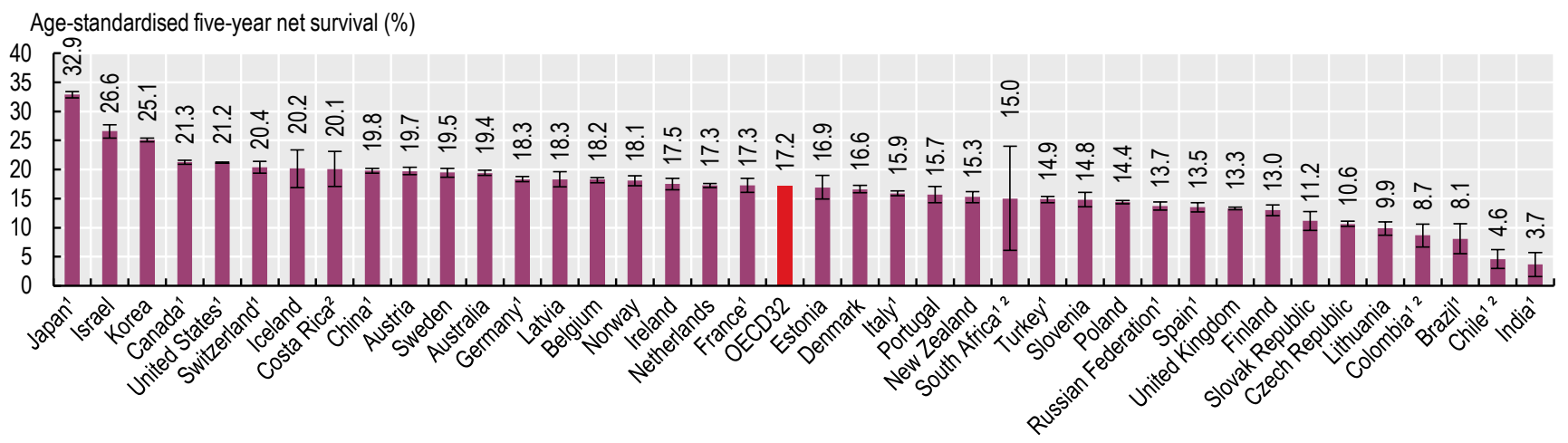

Note: $\mathrm{H}$ line shows $95 \%$ confidence intervals.1. Data represent coverage of less than $100 \%$ of the national population. 2 . Survival estimates are considered less reliable: see Allemani et al. (2018) for more information.

Source: CONCORD programme, London School of Hygiene and Tropical Medicine.

Figure 6.36. Stomach cancer five-year net survival, 2010-14

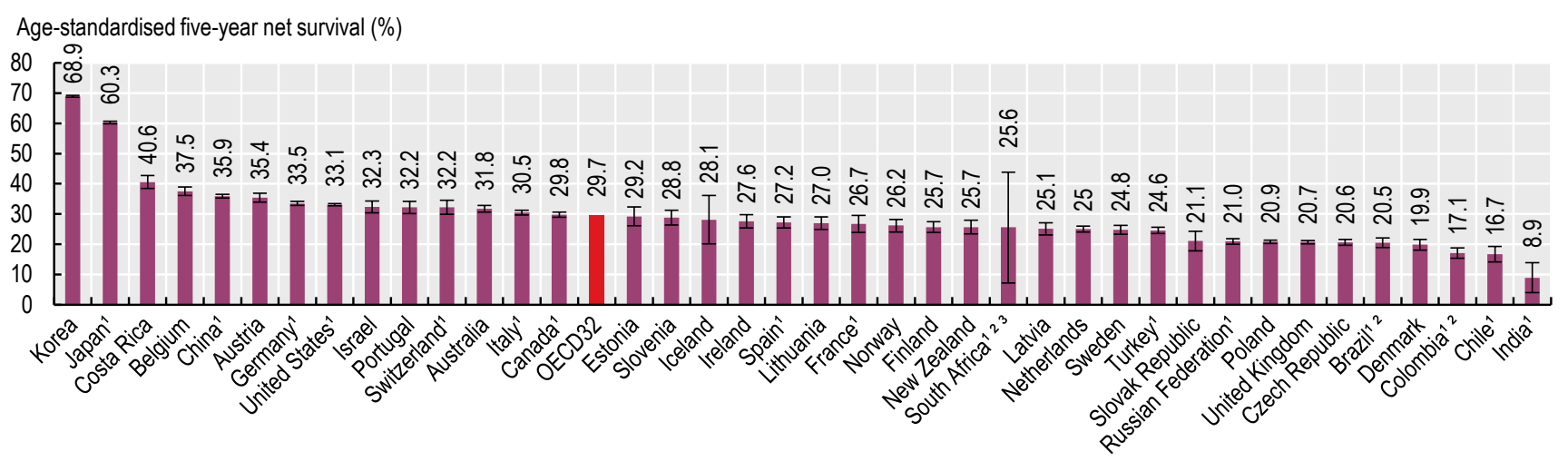

Note: $\mathrm{H}$ line shows $95 \%$ confidence intervals. 1. Data represent coverage of less than $100 \%$ of the national population. 2 . Survival estimates are considered less reliable. 3. Survival estimates are not age-standardised.

Source: CONCORD programme, London School of Hygiene and Tropical Medicine.

Figure 6.37. Childhood acute lymphoblastic leukaemia five-year net survival, 2010-14

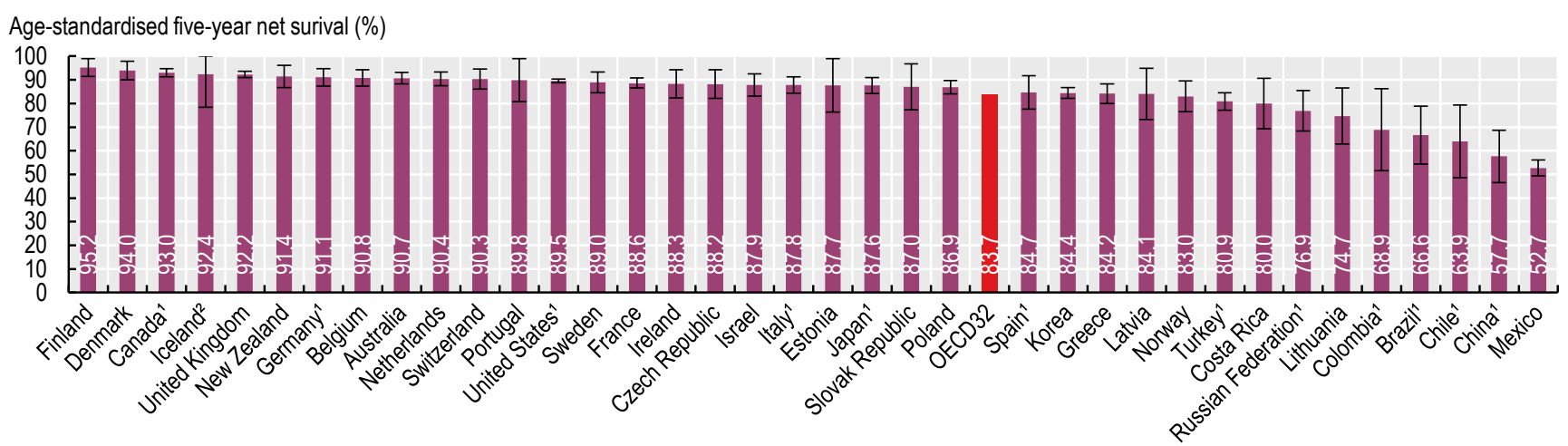

Note: $\mathrm{H}$ line shows $95 \%$ confidence intervals. 1. Data represent coverage of less than $100 \%$ of the national population. 2. Survival estimates are not agestandardised.

Source: CONCORD programme, London School of Hygiene and Tropical Medicine. 
Vaccines are an effective and cost-effective tool for protecting against infectious diseases. The WHO estimates that vaccines prevent between 2 million and 3 million deaths each year worldwide through direct protection of those vaccinated and prevention of the spread of disease to those unvaccinated.

Figure 6.38 shows vaccination coverage for diphtheria, tetanus and pertussis (DTP), measles and hepatitis B at 1 year of age. Across OECD countries, vaccination levels are high, with around $95 \%$ of children receiving the recommended DTP or measles vaccinations and 91\% receiving the recommended hepatitis $B$ vaccination.

Despite high overall rates, however, nearly half of countries fall short of attaining the minimum immunisation levels recommended by the WHO to prevent the spread of measles (95\%) and nearly $15 \%$ of countries fail to meet this target for DTP (90\%). Furthermore, high national coverage rates may not be sufficient to stop disease spread, as low coverage in local populations can lead to outbreaks. In the United States, 1123 individual cases of measles were reported for the period 1 January to 11 July 2019 - the highest number since 1992. Between March 2018 and February 2019 OECD countries in Europe reported 10564 cases of measles. (CDC, 2019[1]; ECDC, 2019[2]).

Over the last decade, rates of vaccination across OECD have increased by six percentage points for hepatitis B and by half a percentage point for measles, but have decreased by one percentage point for DTP. Some countries, however, have experienced important reductions. Coverage for DTP has decreased by four or more percentage points in Mexico, Iceland, Lithuania, Poland, Slovenia and Spain, while rates have dropped at least three percentage points for measles coverage in Estonia, Lithuania, Poland, Canada, Chile, Iceland, the Netherlands, the Slovak Republic and Slovenia.

Figure 6.39 shows trends of vaccination from 2008 to 2018 by country and vaccine type. Countries listed in green boxes increased vaccination rates over the time period while countries in red boxes had declining rates. Roughly onethird of countries had declining levels for each vaccine.

Eroding public confidence in the safety and efficacy of vaccination, despite the lack of scientific evidence to support this, may play a role in declining coverage in some countries. In North America, only $72 \%$ of the population agreed that vaccines are safe; this number was only $59 \%$ in western Europe. In France, one in three people disagree that vaccines are safe (Gallup, 2019[3]).

Influenza is a common infectious disease responsible for 3-5 million severe cases worldwide, along with up to 650000 deaths, including 72000 in the WHO Europe Region (WHO, 2019 [4]). The WHO recommends that $75 \%$ of elderly people be vaccinated against seasonal influenza.

Figure 6.40 shows vaccination rates among adults over 65 for 2007 and 2017. Over this period, the average vaccination rate against influenza among the elderly population decreased among OECD countries from $49 \%$ to $42 \%$. Large decreases can be seen in Germany, Slovenia and Italy. Some countries did show increased vaccination over this period, including Mexico, Israel, the United States, Portugal, Denmark, Greece and New Zealand. Only Korea attained the $75 \%$ target, with coverage of $83 \%$.

\section{Definition and comparability}

Vaccination rates reflect the percentage of people that receive the respective vaccination in the recommended timeframe. The age of complete immunisation differs across countries due to different immunisation schedules. For those countries recommending the first dose of a vaccine after 1 year of age, the indicator is calculated as the proportion of children less than 2 years of age who have received that vaccine. Thus, these indicators are based on the actual policy in a given country.

Some countries administer combination vaccines (e.g. DTP), while others administer the vaccinations separately. Some countries ascertain whether a vaccination has been received based on surveys and others based on encounter data, which may influence the results. In Canada, only four provinces and three territories include vaccination against hepatitis B in their infant immunisation programmes. Other Canadian jurisdictions do this at school age.

Influenza vaccination rates refer to the number of people aged 65 and over who have received an annual influenza vaccination, divided by the total number of people over 65. In some countries, the data are for people over 60 . The main limitation in terms of data comparability arises from the use of different data sources, whether survey or programme, which are susceptible to different types of errors and biases. For example, data from population surveys may reflect some variation due to recall errors and irregularity of administration.

\section{References}

[1] CDC (2019), Measles Cases and Outbreaks.

[2] ECDC (2019), Monthly measles and rubella monitoring report April 2019,.

[3] Gallup (2019), Wellcome Global Monitor - First Wave Findings.

[4] WHO (2019), Seasonal influenza. 
Figure 6.38. Percentage of children at 1 year of age vaccinated for diphtheria, tetanus and pertussis (DTP), measles and hepatitis B, 2018 (or nearest year)

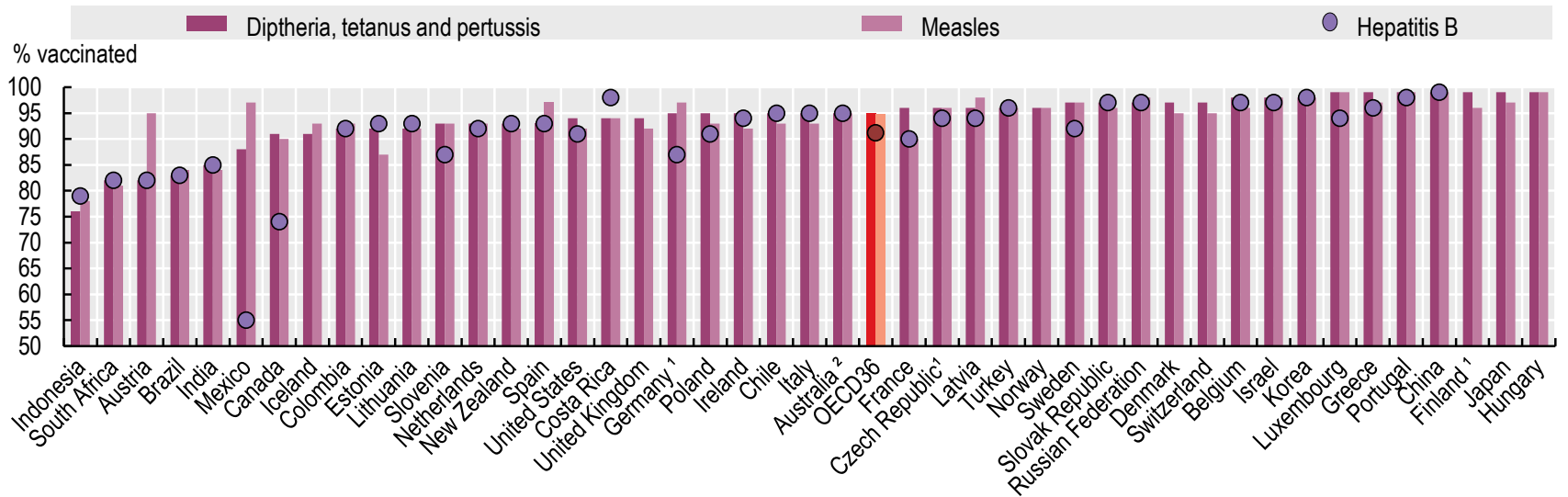

1. DTP data are estimated. 2. Measles data are estimated.

Source: OECD Health Statistics 2019.

StatLink तारा https://doi.org/10.1787/888934016664

Figure 6.39. Trends in vaccination coverage for DTP, measles and hepatitis B, 2008-18 (or nearest year)

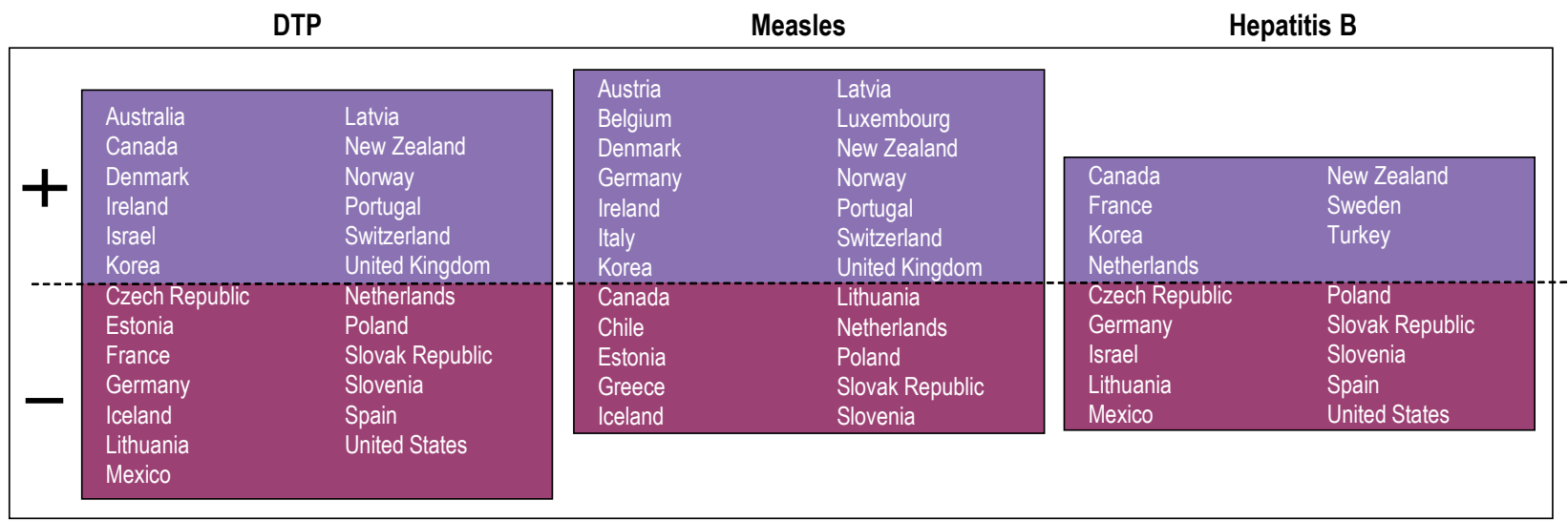

Note: Countries above the dashed line have increased and those below have decreased vaccination coverage by at least two percentage points over the last decade.

Figure 6.40. Percentage of population aged 65 and over vaccinated for influenza, 2007 and 2017

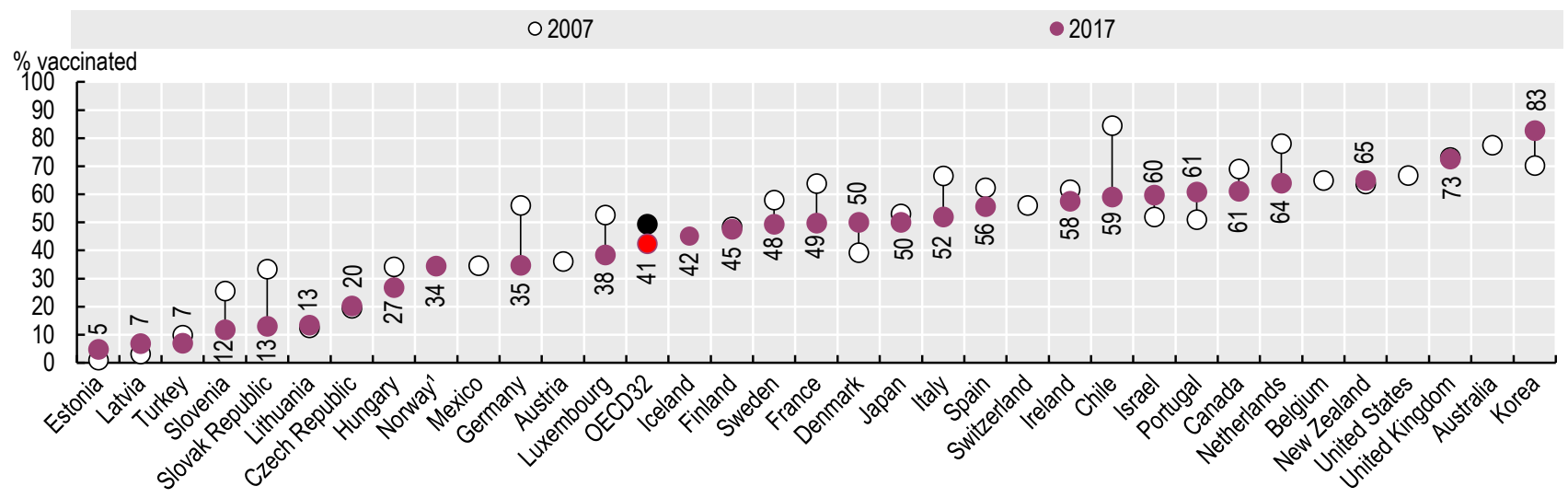

1. 2017 data are estimated.

Source: OECD Health Statistics 2019. 
Given the importance of incorporating people's voices into the development of health systems and improving quality of care, national efforts to develop and monitor patientreported measures have been intensified in recent years (see Chapter 2). In many countries, specific organisations have been established or existing institutions have been identified and made responsible for measuring and reporting patient experiences. These organisations develop survey instruments for regular collection of patient experience data and standardise procedures for analysis and reporting.

Countries use patient-reported data differently to drive quality improvements in health systems. To promote quality of health care through increased provider accountability and transparency, many countries report patient experience data in periodic national health system reports and/or on public websites, showing differences across providers, regions and over time. Canada, the Czech Republic, Denmark, France and the United Kingdom use patient experience measures to inform health care regulators for inspection, regulation and/or accreditation. Patient-reported measures are also used in some Canadian jurisdictions, Denmark, the Netherlands and the United Kingdom to provide specific feedback for providers to support quality improvement (Fujisawa and Klazinga, 2017[1]; Desomer et al., 2018[2]). Germany plans to use patient surveys as part of external quality assurance in the hospital sector.

Across OECD countries, the majority of patients reported that they spent enough time with a doctor during consultation (Figure 6.41), and that a doctor provided easyto-understand explanations (Figure 6.42) and involved them in care and treatment decisions (Figure 6.43). For all three aspects of patient experience, Belgium and Luxembourg score highly at above $95 \%$ of patients with positive experiences; Poland has lower rates, but patient experiences have improved significantly over the past decade. Patient experiences also improved in Estonia in recent years.

Japan has a low rate for patients' perception of the time spent with a doctor, and this is likely to be associated with a high number of consultations per doctor (see indicator "Consultations with doctors" in Chapter 9). However, in Korea, which has by far the highest number of consultations per doctor in OECD countries, a higher proportion of patients report that their doctors spent enough time during consultation.

Patients' income level is associated not only with access to care (see indicator "Unmet needs for health care" in Chapter 5) but also with their experiences with health care. On average across 11 OECD countries, patients with aboveaverage income report a better health care experience than patients with below-average income. Patient experiences also vary by health condition (see indicator "Care for people with mental health disorders").

In order to ensure delivery of people-centred health care across population groups, health care professionals in OECD countries are under increasing pressure to address patient needs, but measures of patient-reported experiences and health outcomes are still limited across countries. The OECD's PaRIS initiative aims to collect key people-reported outcomes and experiences to improve the performance of health care providers and to drive changes in health systems, based on people's voices (OECD, 2018[3]) (see https://www.oecd.org/health/paris.htm).

\section{Definition and comparability}

To monitor general patient experiences in the health system, the OECD recommends collecting data on patient experiences with any doctor in ambulatory settings. An increasing number of countries have been collecting patient experience data based on this recommendation through nationally representative population surveys, while Japan and Portugal collect them through nationally representative service user surveys. About half of the countries presented, including Poland, however, collect data on patient experiences with a regular doctor or regular practice, not data on patient experiences with any doctor in ambulatory care. National data refer to years up to 2018.

In 11 countries, the Commonwealth Fund's International Health Policy Surveys 2010 and 2016 were used as a data source, even though there are limitations relating to the small sample size and low response rates. Data from this survey refer to patient experiences with a GP rather than any doctor, including both GPs and specialists.

Patient experience indicators are not age-standardised to the 2010 OECD population because high-quality health care needs to be provided to all patients regardless of age, and patient experiences are not consistently associated positively with age across countries.

\section{References}

[2] Desomer, A. et al. (2018), Use of patient-reported outcome and experience measures in patient care and policy - Short report, http://www.kce.fgou.be.

[1] Fujisawa, R. and N. Klazinga (2017), "Measuring patient experiences (PREMS): Progress made by the OECD and its member countries between 2006 and 2016", OECD Health Working Papers, No. 102, OECD Publishing, Paris, https:// dx.doi.org/10.1787/893a07d2-en.

[3] OECD (2018), Patient-Reported Indicators Surveys (PaRIS) - OECD, http://www.oecd.org/health/paris.htm. 
Figure 6.41. Doctor spending enough time with patient during consultation, 2010 and 2017 (or nearest year)

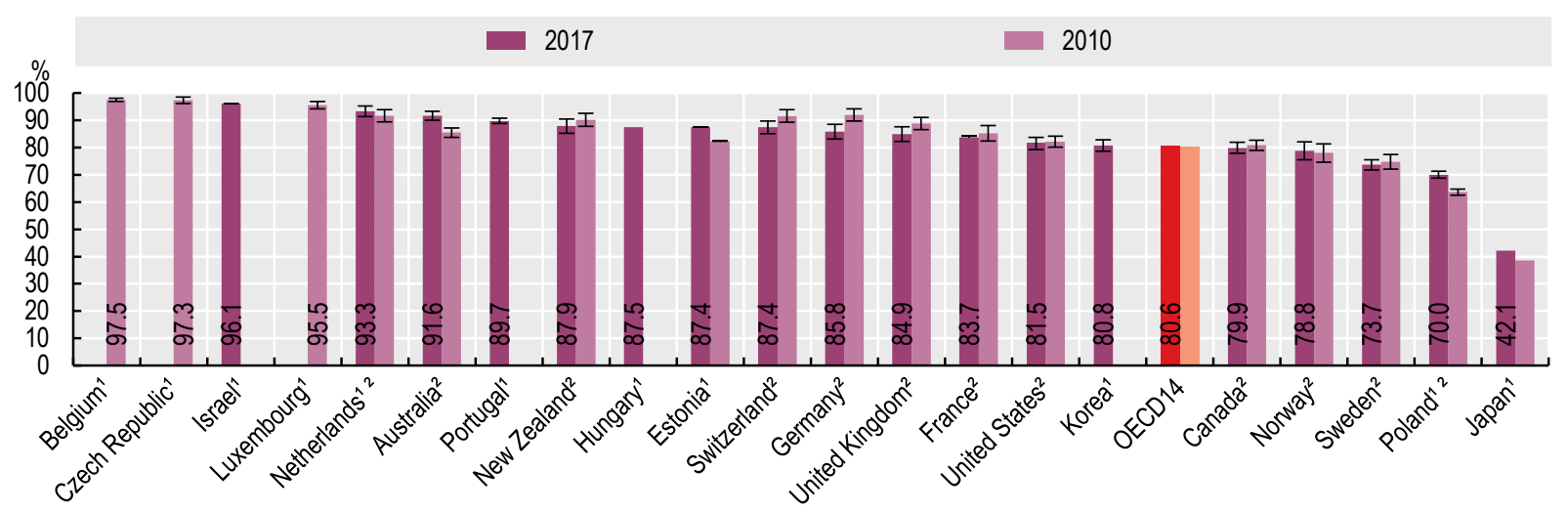

Note: $\mathrm{H}$ line shows $95 \%$ confidence intervals. 1. National sources. 2. Data refer to patient experiences with regular doctor or regular practice. Source: Commonwealth Fund International Health Policy Survey 2016 and other national sources.

Figure 6.42. Doctor providing easy-to-understand explanations, 2010 and 2017 (or nearest year)

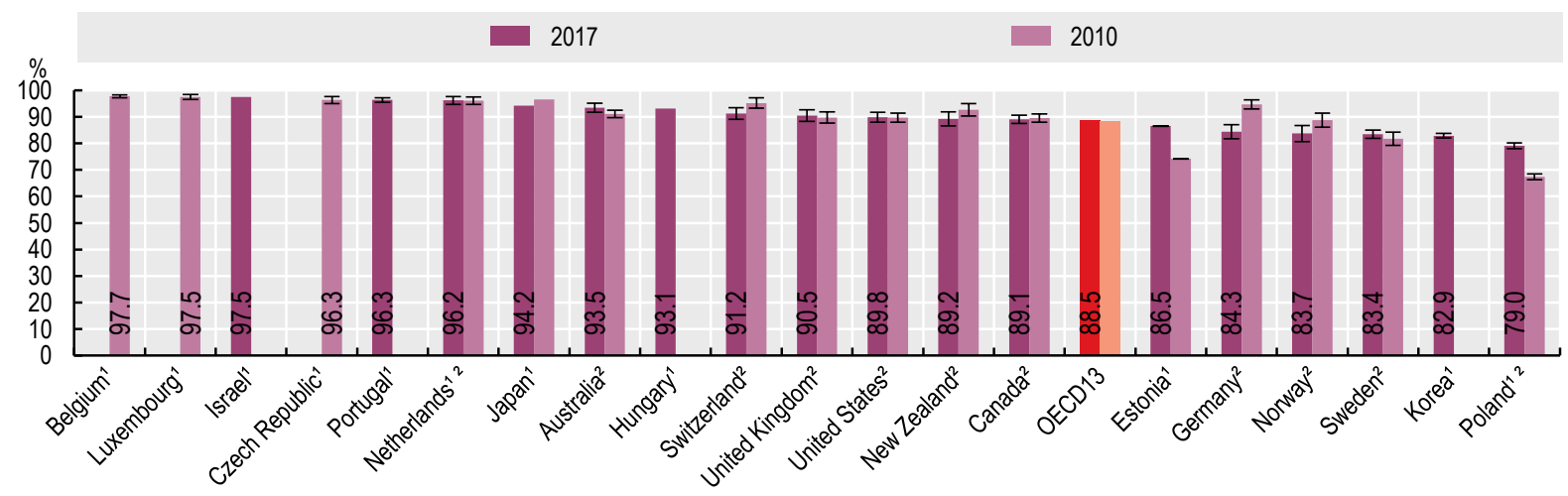

Note: $\mathrm{H}$ line shows $95 \%$ confidence intervals. 1. National sources. 2. Data refer to patient experiences with regular doctor or regular practice. Source: Commonwealth Fund International Health Policy Survey 2016 and other national sources.

Figure 6.43. Doctor involving patient in decisions about care and treatment, 2010 and 2017 (or nearest year)

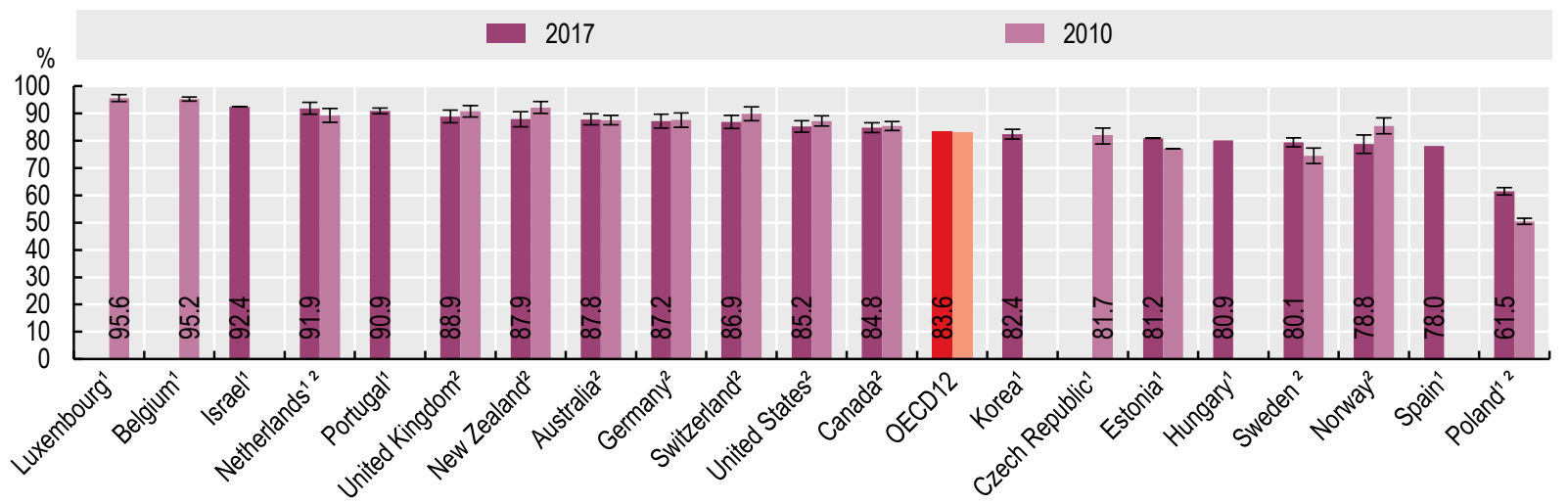

Note: $\mathrm{H}$ line shows $95 \%$ confidence intervals. 1. National sources. 2. Data refer to patient experiences with regular doctor or regular practice. Source: Commonwealth Fund International Health Policy Survey 2016 and other national sources. 


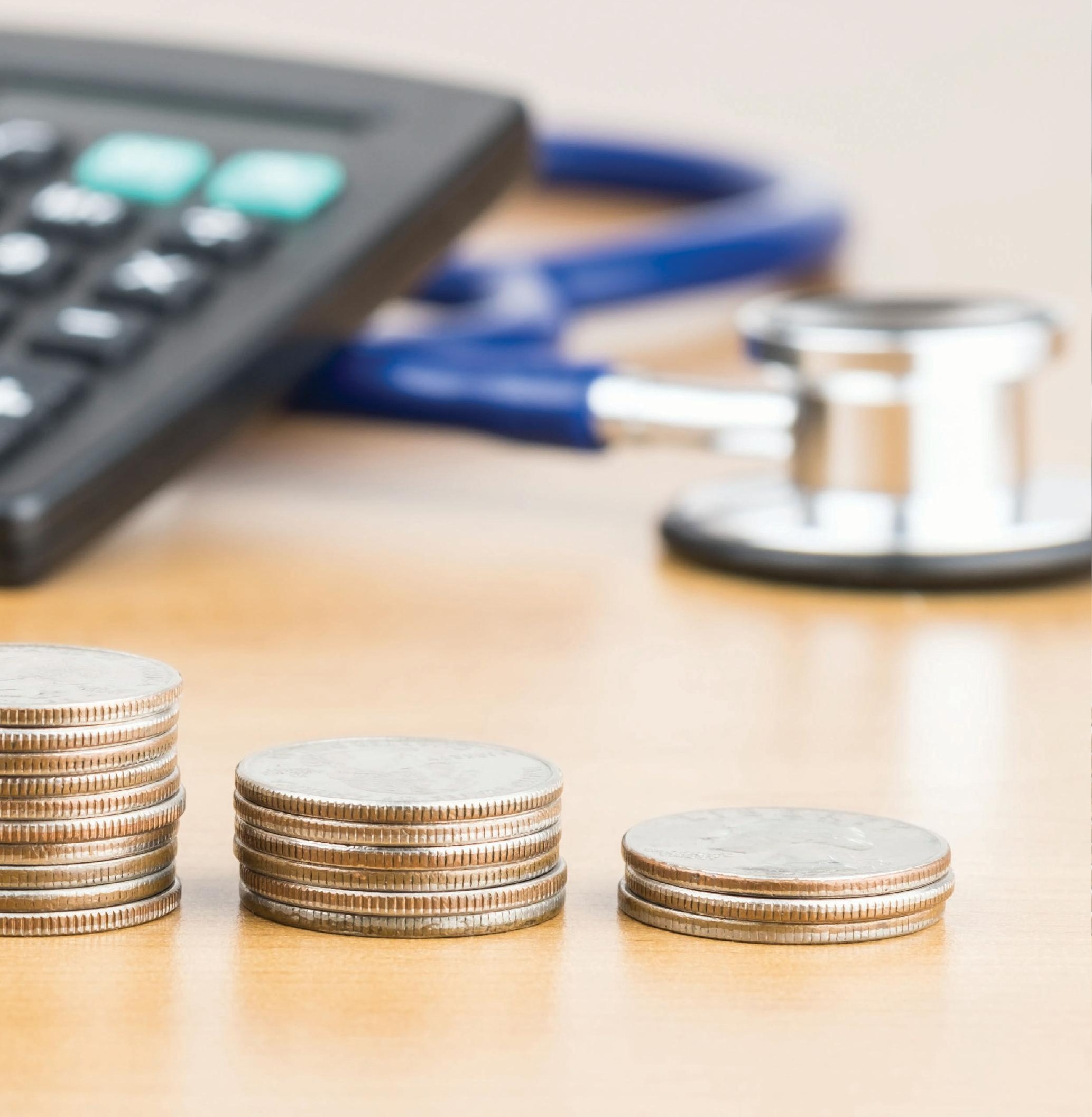



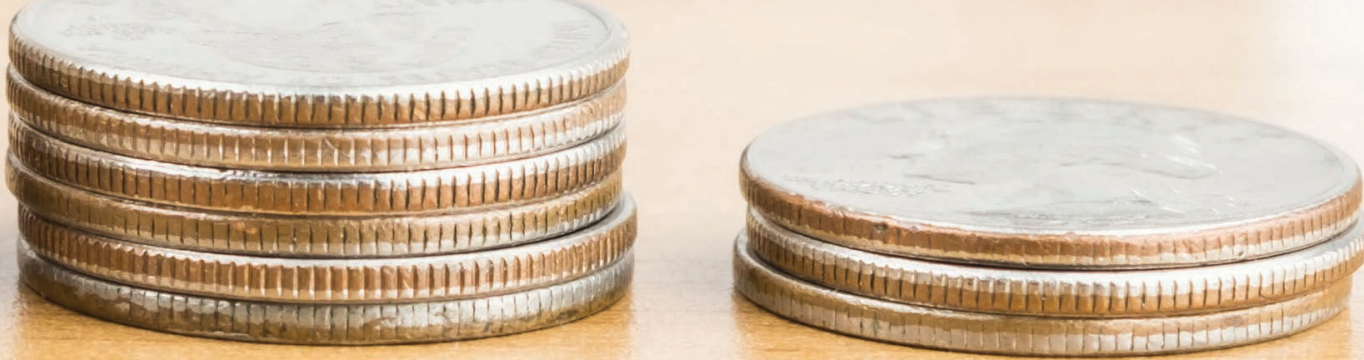

\section{HEALTH EXPENDITURE}

Health expenditure per capita

Health expenditure in relation to GDP

Prices in the health sector

Health expenditure by financing scheme

Public funding of health spending

Health expenditure by type of service

Health expenditure by provider

Capital expenditure in the health sector

Projections of health expenditure

The statistical data for Israel are supplied by and under the responsibility of the relevant Israeli authorities. The use of such data by the OECD is without prejudice to the status of the Golan Heights, East Jerusalem and Israeli settlements in the West Bank under the terms of international law. 
The level of health spending in a country, covering both individual needs and population health as a whole, and how this changes over time is dependent on a wide range of demographic, social and economic factors, as well as the financing and organisational arrangements of the health system.

In 2018, overall spending on health care in the United States was estimated to be the equivalent of more than 10000 dollars for each US resident. This amount of expenditure (when adjusted for different purchasing power in countries) was higher than all other OECD countries by a considerable margin. Switzerland, the next highest spender in the OECD, spent less than $70 \%$ of this amount, while the overall average of all OECD countries was less than $40 \%$ of the US figure (USD 3 994) (Figure 7.1). Many high-income OECD countries, such as Germany, France, Canada and Japan spend only around a half or less of the US per capita spending on health, while the United Kingdom and Italy were around the OECD average. Lowest per capita spenders on health in the OECD were Mexico and Turkey with health expenditure at around a quarter of the OECD average, and levels similar to the key emerging economies such as the Russian Federation, South Africa and Brazil. Latest available figures show that China spent around 20\% of the OECD per capita spending level, while both India and Indonesia spent less than $10 \%$.

Figure 7.1 also shows the split of health spending based on the type of health care coverage, either organised through government health schemes or some kind of compulsory insurance, or through a voluntary arrangement such as private health insurance or direct payments by households (see also indicator "Health expenditure by financing schemes"). Across OECD countries, 76\% of all health spending is financed by government schemes or compulsory insurance (with a cross-country range of $51 \%$ to $85 \%)$. In the United States, since the introduction of the Affordable Care Act in 2014, this share stands at $85 \%$, reflecting the existence of an individual mandate to purchase health insurance. Federal and state programmes such as Medicaid and Medicare continue to play an important role in purchasing health care.

In 2017, OECD per capita spending on health care grew by an average of $2.0 \%$ - a marked slowdown from the 3.3\% growth observed in 2015 and 2016, and significantly below the growth rates experienced before the onset of the global financial and economic crisis. Preliminary estimates for 2018 point to growth having strengthened in 2018. On average, since 2013, annual per capita health spending growth across the OECD has been $2.4 \%$ compared with $1.0 \%$ in the five years up to 2013, in the period following the crisis (Figure 7.2).

In a number of European countries, there have been significant turnarounds in health spending. In Greece, the strong annual decreases in growth halted after 2013, even if growth in health spending has been close to zero overall since 2013 (-9.4\% in the time period $2008-13$ vs. $0.2 \%$ in the time period 2013-18), and real per capita spending in 2018 remained almost a third below the 2009 level. A similar if less dramatic picture can also be seen in Iceland $(-3.0 \%$ vs. $4.0 \%)$. In other European countries, such as Germany and Norway, health spending remained relatively stable over the ten-year period, with annual growth of between 2.0-2.5\%. Overall, health spending growth has picked up in the majority of European countries in most recent years.

Outside of Europe, Korea and Chile have continued to report annual health spending increases above $5 \%$ in real terms since 2008. A provisional estimate for 2018 suggests further strong spending growth of $9.0 \%$ in Korea. In the United States, health spending is estimated to have grown by $1.4 \%$ in real terms in 2018, which along with similar growth in 2017 shows health spending in the United States growing slower than the overall economy.

\section{Definition and comparability}

Expenditure on health gives a measure of the final consumption of health goods and services (i.e. current health expenditure). This includes spending by all types of financing arrangements (e.g. governmentbased programmes, social insurance or out-of-pocket spending) on medical services and goods, population health and prevention programmes, as well as administration of the health system. The split of spending combines government and compulsory financing schemes, the latter including private insurance of a mandatory nature (for example in Switzerland and the Netherlands). Due to data limitations, voluntary private insurance in the United States is included with employer-based private insurance, which is currently mandated under the Affordable Care Act.

To compare spending levels between countries, per capita health expenditures are converted to a common currency (US dollar) and adjusted to take account of the different purchasing power of the national currencies, in order to compare spending levels, Actual Individual Consumption (AIC) PPPs are used as the most available and reliable conversion rates. For the calculation of growth rates in real terms, AIC deflators are used for all countries, where available.

Note that data for 2018 are based on provisional figures provided by the country or estimated by the OECD Secretariat. 
Figure 7.1. Health expenditure per capita, 2018 (or nearest year)

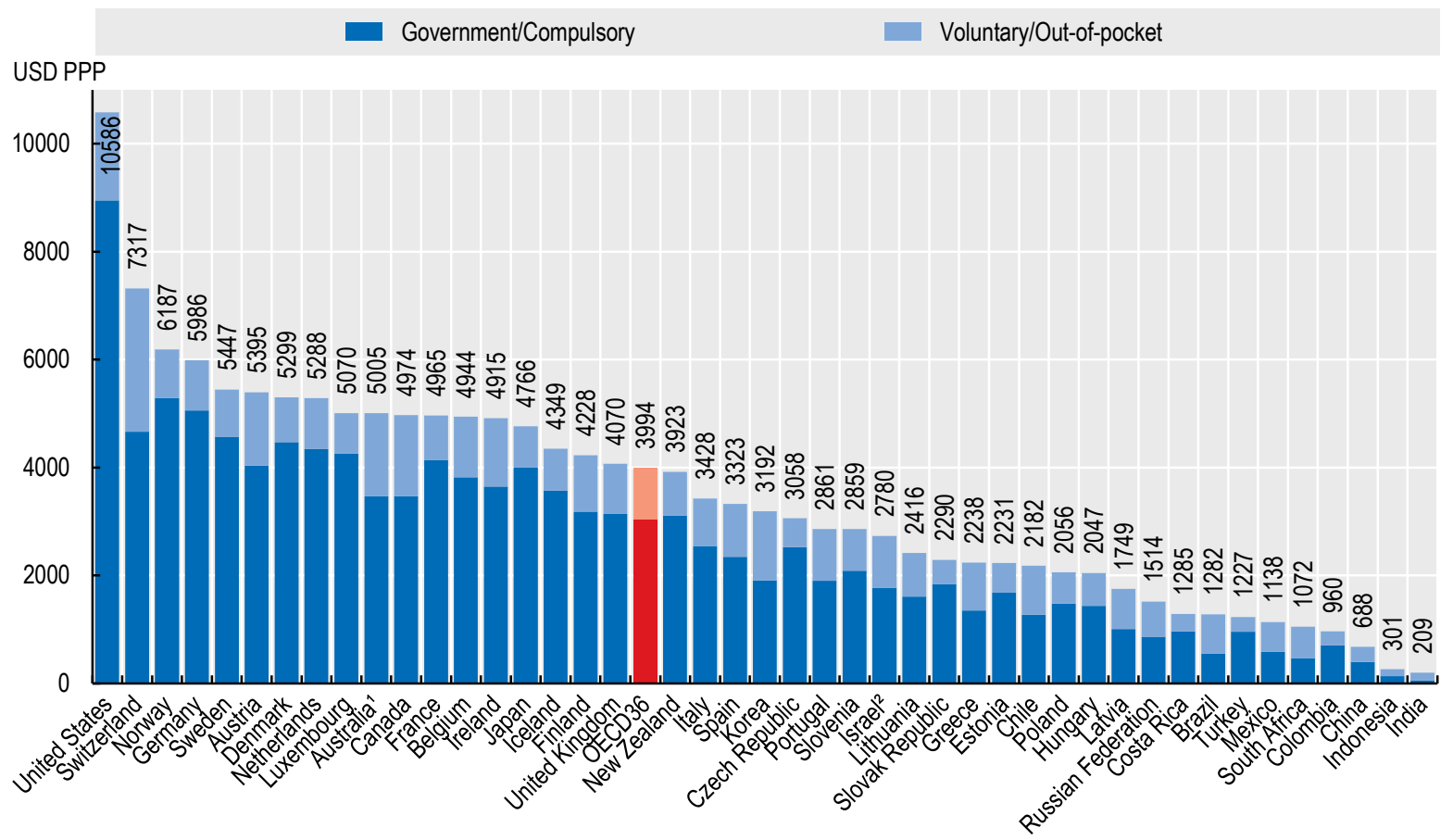

Note: Expenditure excludes investments, unless otherwise stated.

1. Australia expenditure estimates exclude all expenditure for residential aged care facilities in welfare (social) services. 2. Includes investments. Source: OECD Health Statistics 2019, WHO Global Health Expenditure Database.

Figure 7.2. Annual growth in health expenditure per capita (real terms), 2008 to 2018 (or nearest year)

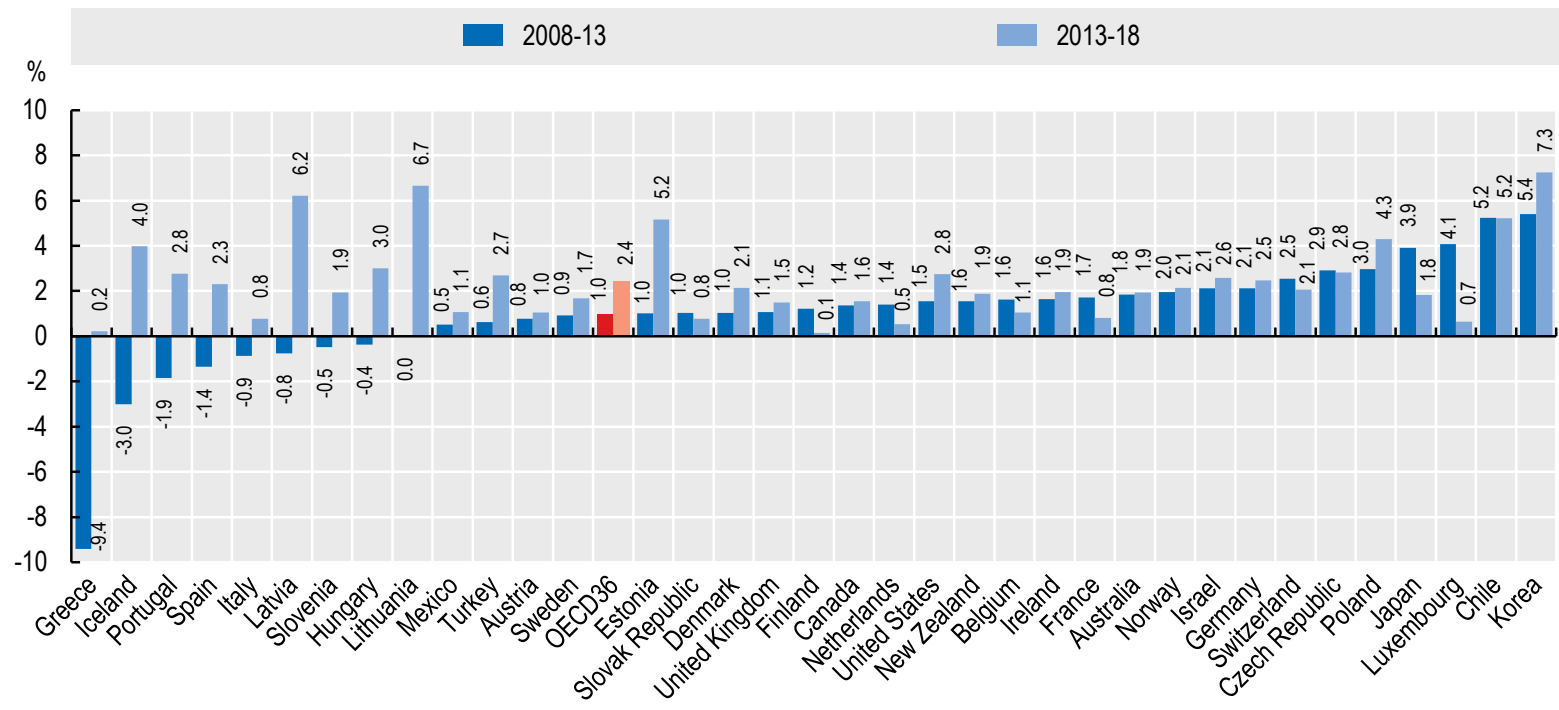

Source: OECD Health Statistics 2019. 
The ratio of spending on health care goods and services compared to total spending in the economy can vary over time due to differences in the growth of health spending compared to overall economic growth. During the 1990s and early 2000s, health spending in OECD countries was generally growing at a faster pace than the rest of the economy, leading to an almost continual rise in the health expenditure to GDP ratio. After a period of volatility during the economic crisis, the average share has remained relatively stable in recent years, as growth in health spending across the OECD has broadly matched overall economic growth.

On average, OECD countries are estimated to have spent $8.8 \%$ of GDP on health care in 2018 , a figure more or less unchanged since 2013 (Figure 7.3). The United States spent by far the most on health care, equivalent to $16.9 \%$ of its GDP - well above Switzerland, the next highest spending country, at $12.2 \%$ (Figure 7.3). After the United States and Switzerland, a group of high-income countries, including Germany, France, Sweden and Japan, all spent close to $11 \%$ of their GDP on health care. A large group of OECD countries spanning Europe, but also Australia, New Zealand, Chile and Korea, fit within a band of health spending of between 8-10\% of GDP. Many of the Central and Eastern European OECD countries, such as Lithuania and Poland, as well as key partner countries, allocated between $6-8 \%$ of their GDP to health care. Finally, a few OECD countries spent less than $6 \%$ of their GDP on health care, including Mexico, Latvia, Luxembourg, and Turkey at $4.2 \%$. Turkey's health spending as a share of GDP sits between that of China and India.

Looking in more detail at trends over the last decade, the average share of GDP related to health care jumped sharply in 2009 as overall economic conditions rapidly deteriorated in many countries, but health spending growth was generally maintained (Figure 7.4). Subsequently, growth in health spending also significantly declined - on average, growth fell to around zero between 2009 and 2011 - as a range of different policy measures to rein in public spending on health kicked in. Since 2011, the average rate of health spending growth has tended to closely track growth in the overall economy, largely maintaining the increased ratio of health spending to GDP at its present level of around 8.8\%.

On a country-by-country basis there have been differing patterns in the health-to-GDP ratio in recent years. In the United States, after a number of years (2009-14) when the ratio of health spending to GDP remained stable at around $16.4 \%$, this rapidly increased to $17.1 \%$ with the onset of a number of coverage changes, before falling to $16.9 \%$ in 2018 as overall economic growth in the US economy outpaced the growth in health spending (Figure 7.5). Korea, due to its rapidly increasing wealth and ongoing government policy to increase health coverage for the population, has seen substantial increases in the share of economic resources allocated to health. In 2003, health spending in Korea accounted for only $4.6 \%$ of GDP compared with 2018 when the ratio was estimated to have reached $8.1 \%$. Chile has also seen its health spending to GDP ratio increase from $7.3 \%$ to $9.0 \%$ over the same time, due to an expansion in the coverage of health care for the population.

In Europe, France has seen the health spending to GDP ratio fluctuate - increasing during the financial crisis to reach a peak of $11.6 \%$ in 2014 - before a gradual decline to $11.2 \%$ by 2018. Health spending in France continued to outpace economic growth until 2016, but then stagnated due to a number of measures to contain costs including for example price negotiations for pharmaceuticals. The Netherlands has seen the proportion of GDP relating to health decrease from a high of $10.6 \%$ in 2014 to an estimated 9.9\% in 2018, relating to reforms in health and long-term care insurance aimed at limiting spending growth within predefined levels.

\section{Definition and comparability}

See indicator "Health expenditure per capita" for a definition of current expenditure on health.

Gross Domestic Product (GDP) is the sum of final consumption, gross capital formation (investment) and net exports. Final consumption includes goods and services used by households or the community to satisfy their individual needs. It includes final consumption expenditure of households, general government and non-profit institutions serving households.

In countries such as Ireland and Luxembourg, where a significant proportion of GDP refers to repatriated profits and thus not available for national consumption, Gross National Income (GNI) may be a more meaningful measure than GDP. However, for consistency, GDP is maintained as the denominator for all countries.

Note that data for 2018 are based on provisional figures provided by the country or preliminary estimates made by the OECD Secretariat. 
Figure 7.3. Health expenditure as a share of GDP, 2018 (or nearest year)

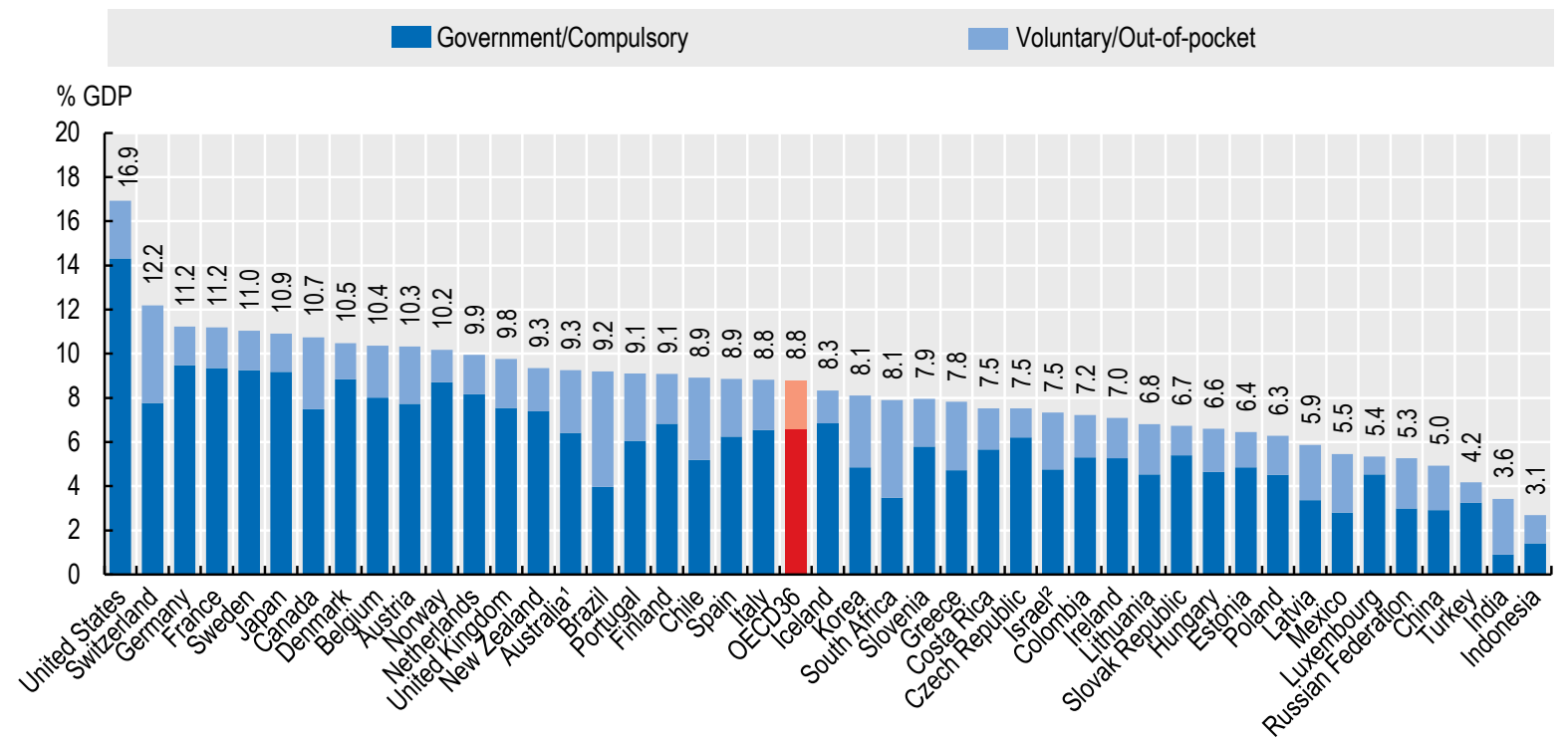

Note: Expenditure excludes investments, unless otherwise stated.

1. Australia expenditure estimates exclude all expenditure for residential aged care facilities in welfare (social) services. 2. Includes investments. Source: OECD Health Statistics 2019, WHO Global Health Expenditure Database.

StatLink Ailst https://doi.org/10.1787/888934016816

Figure 7.4. Annual growth in health expenditure and GDP per capita, OECD average, 2003-18

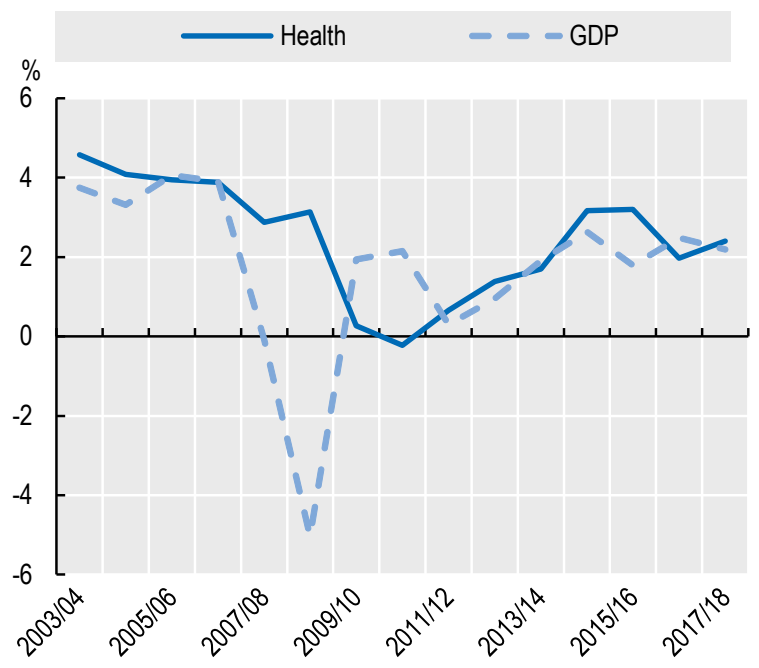

Source: OECD Health Statistics 2019.

StatLink ताs https://doi.org/10.1787/888934016835
Figure 7.5. Health expenditure as a share of GDP, selected OECD countries, 2003-18

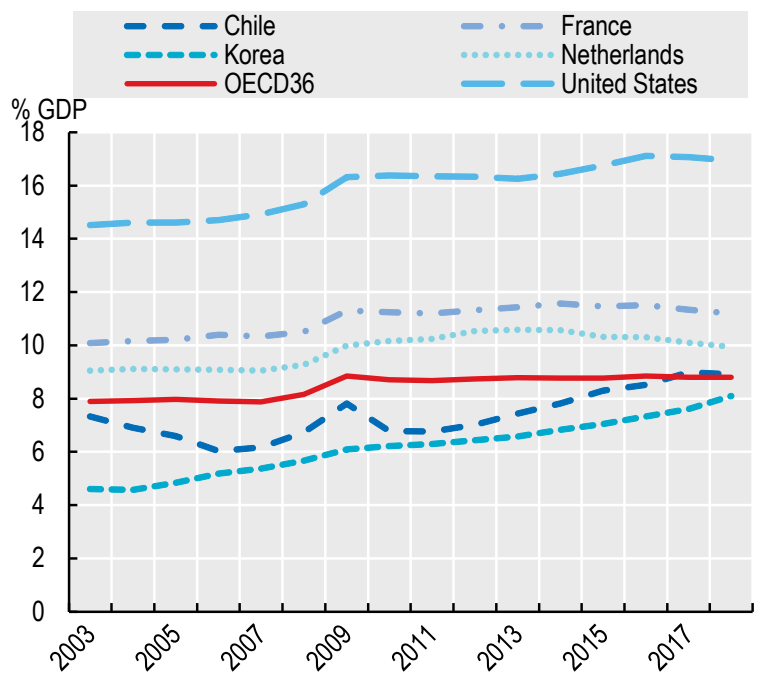

Source: OECD Health Statistics 2019.

StatLink क्ञाज https://doi.org/10.1787/888934016854 
Variations in per capita health spending can be the result of differences in prices for health care goods and services, and in the quantity of care that individuals are using ("volume"). Breaking down health spending into these two components gives policymakers a better understanding of what is driving the differences, and therefore guides them to what responses can be put in place to increase value for money. Depending on what explains high spending, the options can differ.

Comparing spending across countries requires data to be expressed in a common currency. The choice of the currency conversion measure, however, can significantly influence the results and interpretation. Whilst market exchange rates are commonly used, they are not ideal for sectors such as health care. First, exchange rates are determined by the supply and demand for currencies, which can be influenced by speculation and interest rates, among other factors. Second, for predominantly non-traded sectors, such as health care, exchange rates are unlikely to reflect the relative purchasing power of currencies in their national markets (Eurostat/OECD, 2012[1]).

Purchasing power parities (PPPs), on the other hand, are conversion rates that show the ratio of the prices in national currencies for the same basket of goods and services. When PPPs are used, the results are valued at a uniform price level and reflect only differences in volumes of goods and services consumed. Traditionally, health care expenditures have been compared using broad economy-wide PPPs (see indicator "Health expenditure per capita"). This gives an indication of the level of spending on health adjusted to take account of differences in the overall price levels between countries. To assess differences solely in health volumes requires the use of health-specific PPPs. Health and hospital PPPs have been developed and can be used to calculate health price level indices (PLI), a ratio of PPPs to exchange rates, to indicate the number of units of a common currency needed to purchase the same volume of health care.

Figure 7.6 shows a comparison of prices for a basket of health goods and services compared with the price level in the United States. This shows that prices in the health sector based on the same set of goods and services are estimated to be about $10 \%$ more in Sweden, $20 \%$ more in Norway and up to $39 \%$ higher in Switzerland. Prices across all OECD countries are on average around $28 \%$ lower than in the United States. Health care prices in France and Germany are around a third cheaper than in the United States and half that of their neighbour, Switzerland. The lowest prices for health care are in Turkey at $17 \%$ of the US level and less than a quarter of the OECD average.

In general, there is a high correlation between prices in a country and its level of wealth. Prices of durable goods (e.g., cars) vary less than the prices of services (e.g., education and health). In different countries durable goods are frequently traded, which tends to equalise their price levels, while services are often purchased locally, with higher wages in advanced countries leading to higher service prices. The variation in prices in the health sector, which is relatively labour-intensive, therefore tends to be greater than the economy as a whole, with high-income countries having even higher prices for health care compared to lowerincome countries.

By removing the price differences for health goods and services between countries, we can get an idea of volume of health services being consumed (Figure 7.7). The overall effect is to reduce the differences between countries with relatively higher prices compared to those with lower prices. For example, taking the relatively high health prices in the United States into account means that they are still the highest consumers of health services but the gap with the OECD average decreases. It also shows that the difference in volume of health care consumed in the United States compared to countries with lower prices, such as Australia and France, is getting smaller. The very low prices in the Turkish health sector means that on average the population still consumes around 54\% of the OECD average in term of health care, but spends only $30 \%$ of the average.

\section{Definition and comparability}

Purchasing power parities (PPPs) are conversion rates that show the ratio of the prices in national currencies of the same basket of goods and services in different countries. Thus, they can be used as both currency converter and price deflators. When PPPs are used to convert expenditure to a common unit, the results are valued at a uniform price level and should reflect only differences in volumes of goods and services consumed.

To assess differences in health volumes requires health-specific PPPs. Eurostat and the OECD calculate PPPs for GDP and some 50 product groups, including health, on a regular and timely basis. In recent years, a number of countries have worked towards outputbased measures of prices of health care goods and services. The output-based methodology has then been used to produce both health and hospitals PPPs, which are now incorporated into the overall calculation of GDP PPPs. Such PPPs can be used to calculate health price level indices (PLI) to compare price levels and volumes across countries. These indices are calculated as ratios of health PPPs to exchange rates, and indicate the number of units of a common currency needed to purchase the same volume.

\section{References}

[2] Eurostat (2001), Handbook on Price and Volume Measures in National Accounts, European Union, Luxembourg.

[1] OECD/Eurostat (2012), Eurostat-OECD Methodological Manual on Purchasing Power Parities (2012 Edition), OECD Publishing, Paris, https://dx.doi.org/10.1787/9789264189232-en. 
Figure 7.6. Comparative price levels for health, 2017, US=100

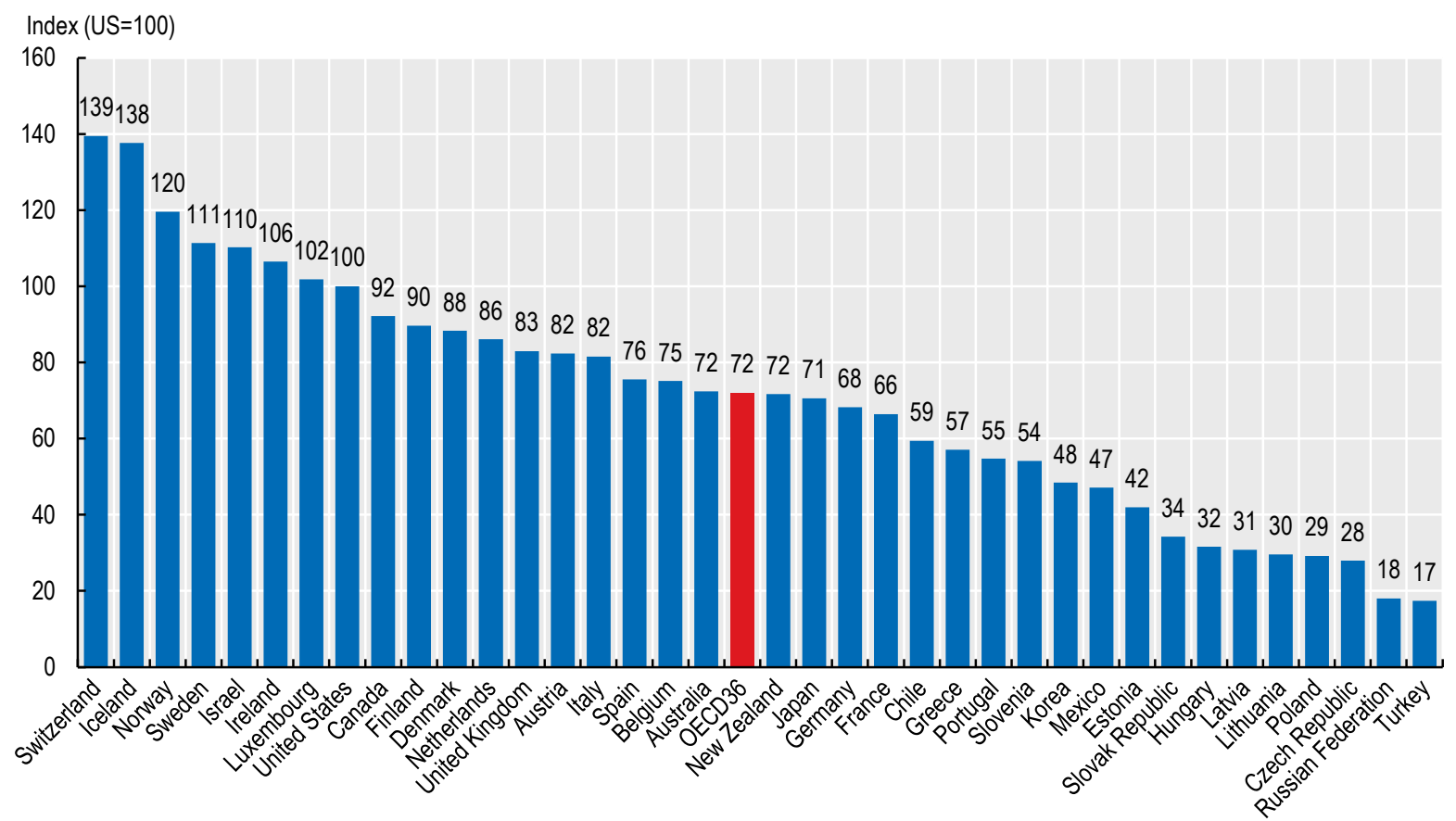

Source: OECD estimates (unpublished).

StatLink क्ञाs https://doi.org/10.1787/888934016873

Figure 7.7. Indices of per capita spending and volume of health care, 2017, US=100

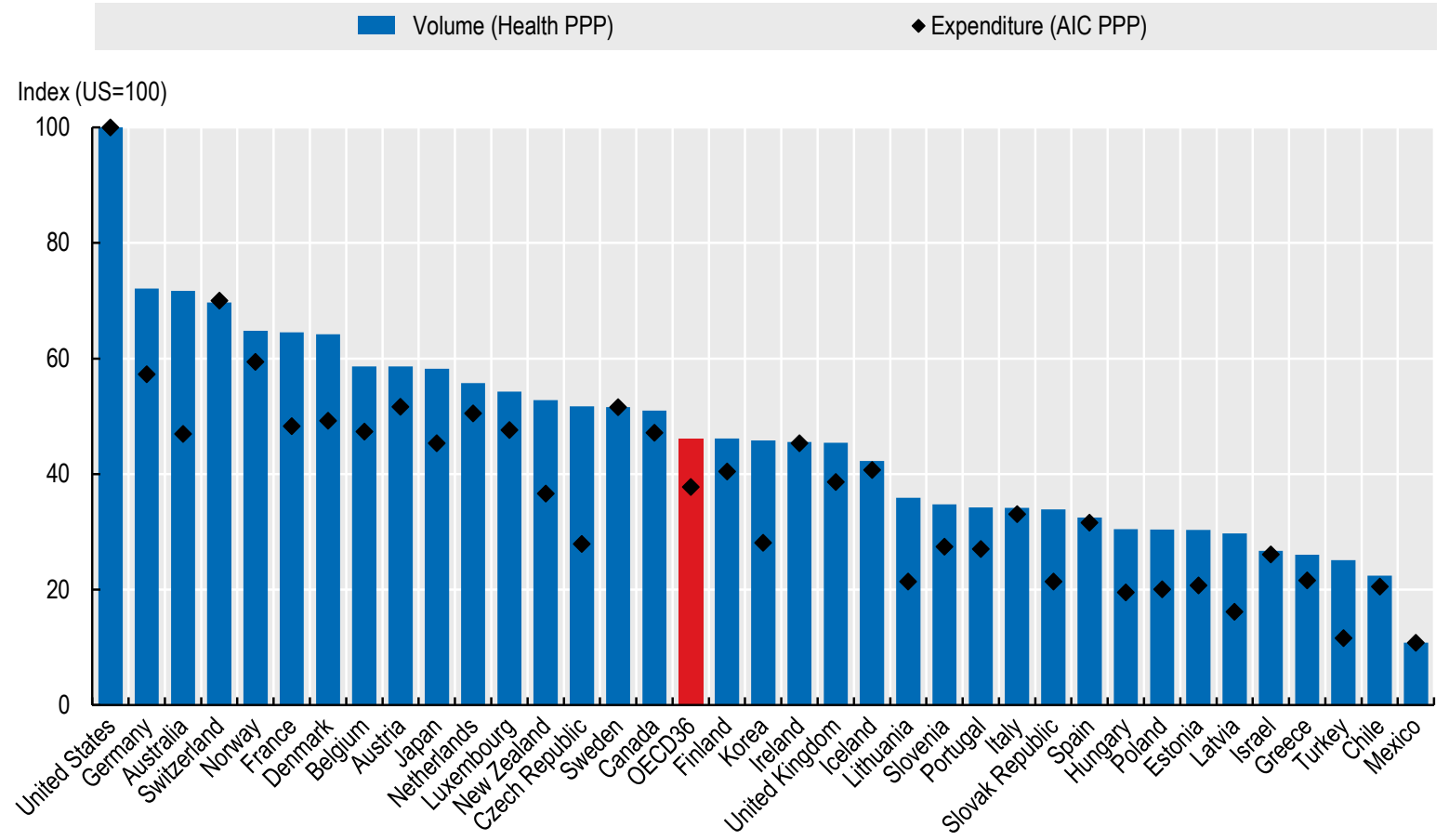

Note: AIC refers to actual individual consumption.

Source: OECD estimates (unpublished). 
A variety of financing arrangements, broadly classified according to their compulsory or voluntary nature, provide coverage against the cost of health care by purchasing health care services. Government financing schemes, organised at a national or regional level or for specific population groups, automatically entitle individuals to care based on residency, and form the principle mechanism by which health care expenses are covered in a number of OECD countries. The main alternative is for residents to be enrolled in a compulsory health insurance scheme (through public or private entities) which then covers the bulk of their health care use. Despite near universal health care coverage in many OECD countries, direct expenditure by households (out-of-pocket spending) in the form of standalone payments or as part of some co-payment arrangement remain an important element of health financing but the extent can vary considerably. Finally, among the other types of discretionary health care financing, voluntary health insurance, in its various forms, can play an important funding role in some countries.

Taken together, government schemes and compulsory health insurance form the principal financing arrangement in all OECD countries (Figure 7.8). On average, around threequarters of all health care spending across the OECD is currently covered through these types of mandatory financing schemes. In Norway, Denmark, Sweden and the United Kingdom, central, regional or local government schemes account for around $80 \%$ or more of all health spending, with out-of-pocket payments making up most of the remainder. Compulsory health insurance schemes are the dominant source of health care financing in Germany, Japan, France, Luxembourg and the Netherlands, typically covering about three-quarters of all health spending. While Germany and Japan rely on a system of social health insurance, France supplements the public health insurance coverage ("assurance maladie") with a system of different private health insurance arrangements (e.g. "mutuelles"), which have become compulsory under certain employment conditions in 2016.

In the United States, federal and state programmes, such as Medicaid, make up around a quarter of all US health care spending. Another $22 \%$ is covered by social health insurance schemes (e.g. Medicare). Most private health insurance, which, since the introduction of the Affordable Care Act (ACA) in 2014, is considered compulsory due to the current existence of an individual mandate for individuals to buy health insurance or pay a penalty, covers more than a third of total health spending.

Out-of-pocket payments generally constitute the next important source of funding. On average private households directly financed more than a fifth of all health spending in 2017, but with substantial variation across the OECD.
Whereas this share is above $30 \%$ in Latvia $(42 \%)$, Mexico $(41 \%)$, Greece (35\%), Korea (34\%) and Chile (34\%) it is below $10 \%$ in France. Out-of-pocket spending on health care was greater than $30 \%$ in India, Russia and China.

With the aim to move towards universal health coverage, a number of OECD countries have increased spending by government or compulsory insurance schemes in recent decades. As a result, there have been some significant decreases in the share of health care costs payable by individuals and voluntary insurance schemes in some countries. Yet, while the proportion of health spending covered by those two schemes across the OECD has slightly decreased from around $28 \%$ in 2003 to $26 \%$ in 2017 , there is notable variability within countries.

Among those countries where voluntary health insurance plays a more important role, this share has been growing in Korea and Australia in recent years while it remained more or less flat in Slovenia and Canada (Figure 7.9). The share of expenditure covered by out-of-pocket payments rose substantially between 2009 and 2017 in several European countries, such as Greece (5\%), Spain (5\%) and Portugal (3\%), though this proportion has stabilised in recent years (Figure 7.10). This is the result of policies introduced in a number of countries to balance public budgets following the global financial and economic crisis, such as introducing or increasing co-payments for primary care and hospitals, raising reimbursement thresholds or reducing benefits for pharmaceuticals and dental care, or removing public coverage for particular groups.

\section{Definition and comparability}

The financing of health care can be analysed from the point of view of financing schemes (financing arrangements through which health services are paid for and obtained by people, e.g. social health insurance), financing agents (organisations managing the financing schemes, e.g. social insurance agencies, and types of revenues of financing schemes (e.g. social insurance contributions). Here "financing" is used in the sense of financing schemes as defined in the System of Health Accounts (OECD, Eurostat and WHO, 2011) and includes government schemes, compulsory health insurance as well as voluntary health insurance and private funds such as households' out-of-pocket payments, NGOs and private corporations. Out-ofpocket payments are expenditures borne directly by patients. They include cost-sharing and, in certain countries, estimations of informal payments to health care providers. 
Figure 7.8. Health expenditure by type of financing, 2017 (or nearest year)

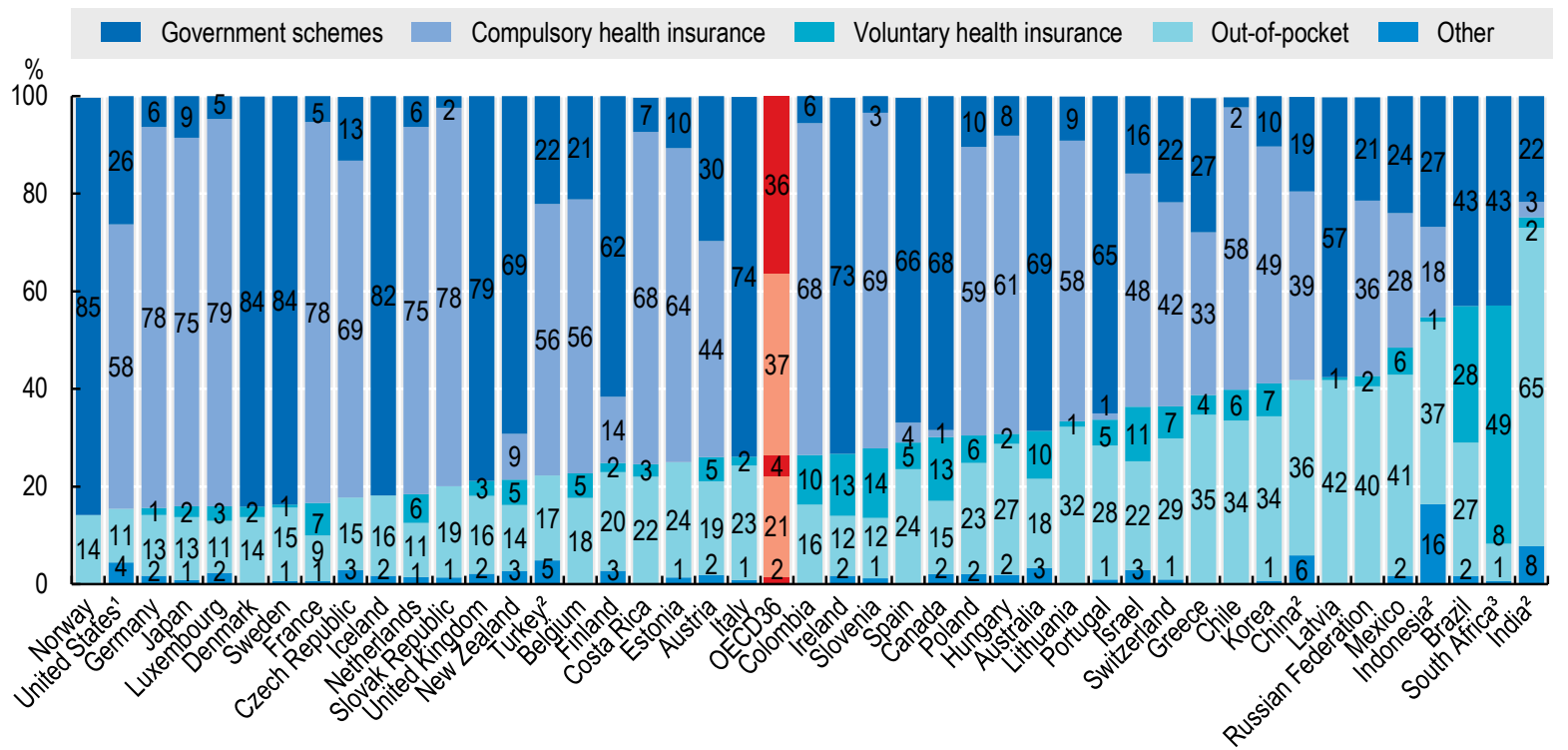

1. All spending by private health insurance companies in the United States is reported under compulsory health insurance. 2. Health payment schemes unable to be disaggregated into voluntary health insurance, NPISH and enterprise financing are reported under other. 3 . Voluntary payment schemes unable to be disaggregated are reported under voluntary health insurance.

Source: OECD Health Statistics 2019.

Figure 7.9. Voluntary health insurance expenditure as a proportion of total, selected countries, 2003-17

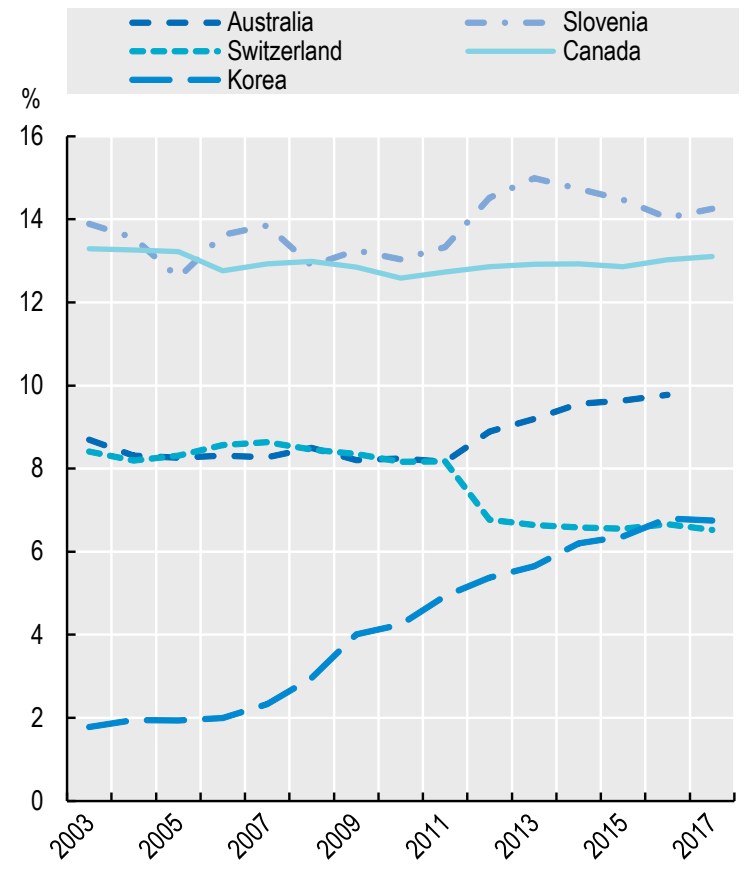

Source: OECD Health Statistics 2019.

StatLink लाাs https://doi.org/10.1787/888934016930
Figure 7.10. Out-of-pocket health expenditure as a proportion of total, selected countries, 2003-17

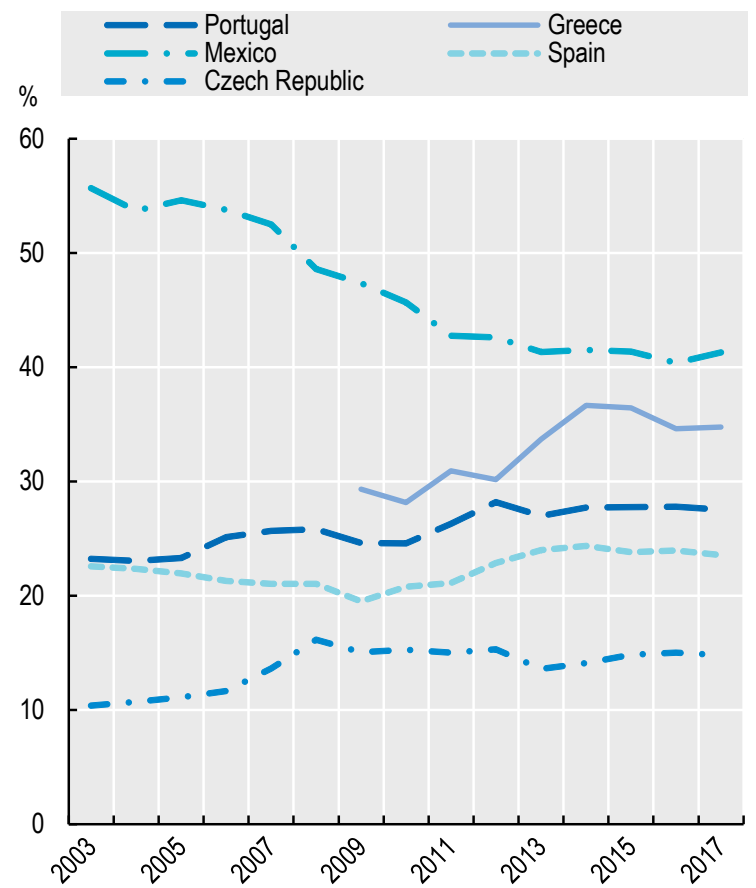

Source: OECD Health Statistics 2019.

StatLink क्ञाs https://doi.org/10.1787/888934016949 
While health care goods and services are purchased through different financing schemes (see indicator "Health expenditure by financing scheme"), these in turn need to mobilise revenues to fund the spending, often relying on a number of different sources. Analysing the financial flows from sources through to the schemes gives a more comprehensive understanding of how health services are ultimately funded and the overall burden on different sectors of the economy.

Funding of government schemes comes mainly from general revenues, primarily through taxation, which are then allocated through a budgetary process across the various levels of government. However, governments might also contribute towards other schemes, such as social health insurance, by covering the contributions of particular population groups or providing general budget support to the insurance fund. Individuals can purchase private health insurance, which means paying regular premiums into a pool, which then pays their medical needs. A proportion of the premium may be paid by their employer or subsidised by government. Individuals also finance care directly, using household income to pay for services in their entirety, or as part of a cost-sharing arrangement. Other health financing schemes (e.g. non-profit or enterprise schemes) can receive donations, or income from investments or other commercial operations. Finally, funds can be received from international sources through bilateral agreements between foreign governments or development partners, though this is limited in most OECD countries.

Government transfers and social contributions paid by employers, employees and others constitute public revenues. Private sources comprise the premiums for voluntary and compulsory insurance policies, as well as any other funds from households or corporations. On average, public sources fund around $71 \%$ of health care spending across OECD countries (Figure 7.11). Where government financing schemes are the principal mechanism, such as in Denmark, public funding is the major source for health care expenditure (84\%). In other countries, governments do not directly pay for the majority of health services but provide transfers and subsidies to other schemes (Mueller and Morgan, 2017[1]). In Japan, only about 9\% of spending on health was directly from government schemes, but transfers and social insurance contributions means that a large proportion of expenditure is still publicly funded (84\% of the total).

Governments are responsible for funding a range of public services, and health care is competing with other sectors such as education, defence and housing. The level of public funding of health is determined by factors such as the type of health system in place, the demographic composition of the population, and government policy. Budget priorities can also shift from year to year due to political decisionmaking and economic effects. Public funding of health spending (via government transfers and social insurance contributions) accounted for an average of $15 \%$ of total government expenditure across the OECD (Figure 7.12).
Around $20 \%$ or more of public spending was linked to health care spending in Japan, the United States, New Zealand, Ireland and Germany. On the other hand, Greece and Hungary allocated around $10 \%$ of government spending to health care, a level similar to that in Russia and Brazil.

Many countries have a system of compulsory health insurance - either social health insurance or through private coverage. There is more diversity in the composition of revenues for these type of schemes (Figure 7.13). The importance of government transfers as a source of revenue can differ significantly. On average, around three-quarters of financing comes from social contributions (or premiums) - primarily split between employees and employers - but around a quarter still comes from government transfers, either on behalf of certain groups (e.g. the poor or unemployed) or as general support. In Hungary, governmental transfers funded $68 \%$ of the health spending of the social health insurance. In Poland, Slovenia and Estonia the share was less than $5 \%$, with social insurance contributions being the main funding source.

\section{Definition and comparability}

Health financing schemes raise revenues to pay for health care for the population they are covering. In general, financing schemes can receive transfers from the government, social insurance contributions, voluntary or compulsory prepayments (e.g. insurance premiums), other domestic revenues and revenues from abroad (e.g. as part of development aid).

Revenues of a financing scheme are rarely equal to expenses in any given year leading to a surplus or deficit of funds. In practice, most countries use the composition of revenues per scheme to apply on a prorata basis to the scheme's expenditure thus providing a picture of how spending was financed in the accounting period.

Total government expenditure is as defined in the System of National Accounts. Public spending on health from the System of Health Accounts is equal to the sum of FS.1 Transfers from government (domestic), FS.2 Transfers from government (foreign) and FS.3 Social insurance contributions. In the absence of information from the revenue side, the sum of HF.1.1 Government financing schemes and HF1.2.1 Social health insurance is taken as a proxy.

\section{References}

[1] Mueller, M. and D. Morgan (2017), "New insights into health financing: First results of the international data collection under the System of Health Accounts 2011 framework", Health Policy, Vol. 121/7, pp. 764-769, http://dx.doi.org/10.1016/ j.healthpol.2017.04.008. 
Figure 7.11. Health expenditure from public sources as share of total, 2017 (or nearest year)

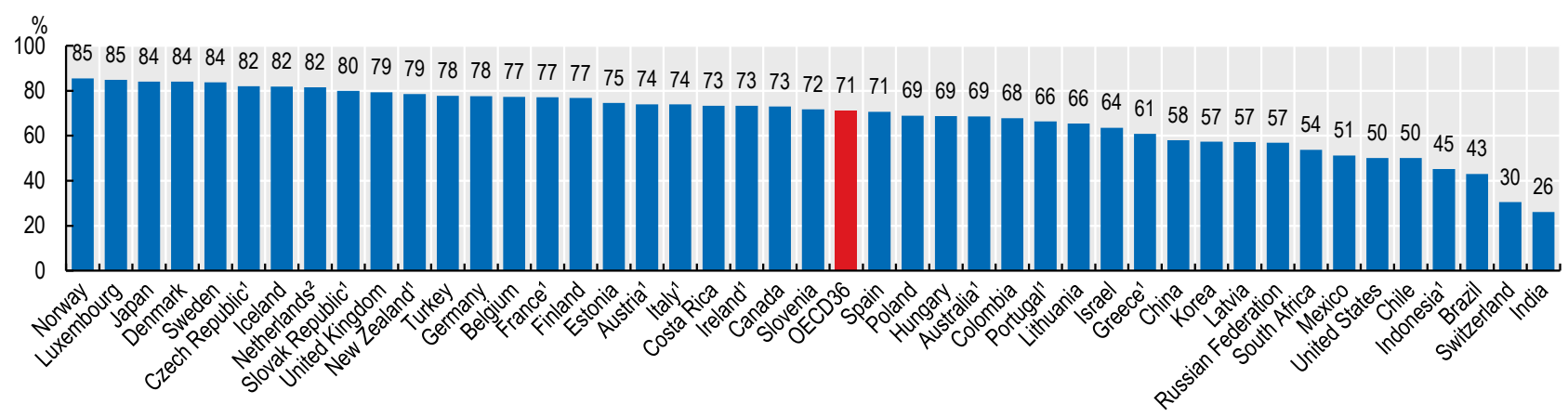

1. Public is calculated using spending by government schemes and social health insurance.

2. Public is calculated using spending by government schemes, social health insurance and compulsory private insurance.

Source: OECD Health Statistics 2019.

Figure 7.12. Health expenditure from public sources as a share of total government expenditure, 2017 (or nearest year)

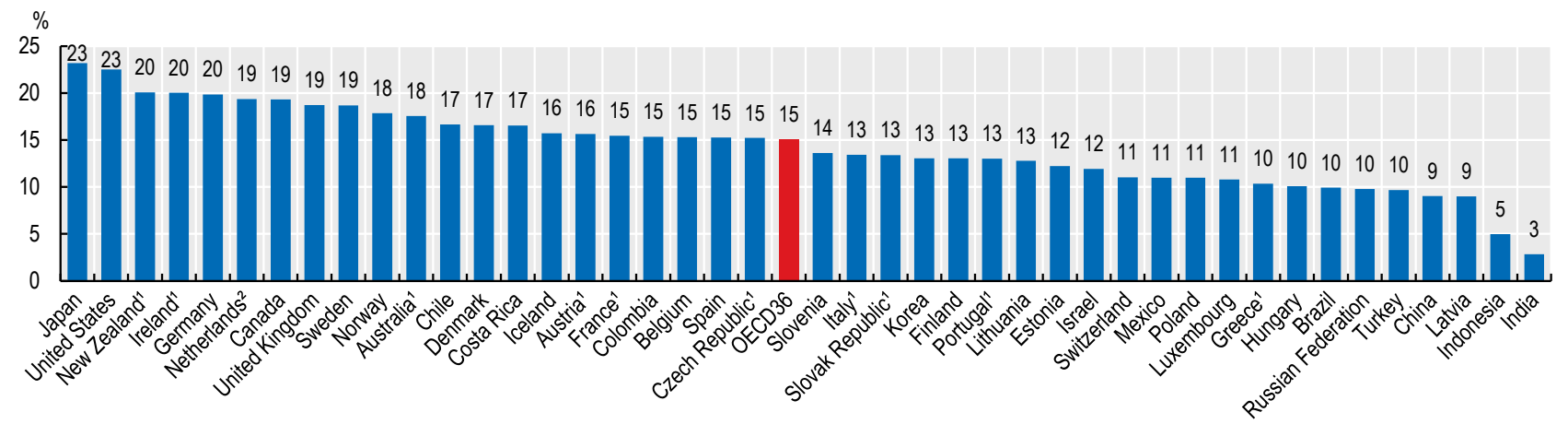

1. Government expenditure includes expenditure by government schemes and social health insurance.

2. Government expenditure includes expenditure by government schemes, social health insurance, and compulsory private insurance.

Source: OECD Health Statistics 2019.

Figure 7.13. Financing sources of compulsory health insurance, 2017 (or nearest year)

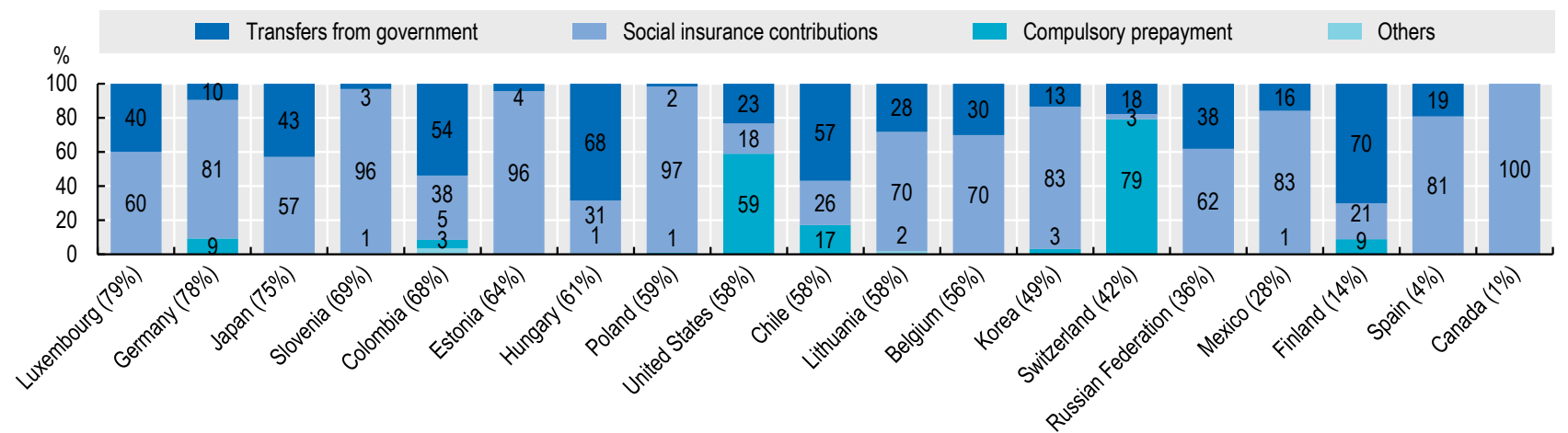

Note: Numbers in brackets indicate the contribution of compulsory health insurance to total expenditure. "Other" includes voluntary prepayments and other domestic revenues.

Source: OECD Health Statistics 2019. 
Factors such as how care is organised and prioritised across providers, input costs and population needs all affect the level of spending across different services. Inpatient and outpatient services comprise the greatest share - typically accounting for around $60 \%$ of all health spending across OECD countries (Figure 7.14). Medical goods (mostly pharmaceuticals) take up a further $20 \%$, followed by a growing share on long-term care, which in 2017 averaged around $14 \%$ of health spending. Administration and overall governance of the health system, together with preventive care covered the remainder.

The structure of spending across the various types of care can vary considerably by country. About $42 \%$ of health spending in Greece can be attributed to inpatient (curative and rehabilitative) care services. This is by far the highest share and some 14 percentage points above the OECD average. At the other end of the scale, many of the Nordic countries, but also Canada and the Netherlands, saw inpatient services account for a quarter or less of all spending. Outpatient care, covering generalist and specialist consultations, was particularly high in Portugal and Israel relative to the OECD average of $32 \%$. Greece and Belgium spent the lowest proportion on outpatient services.

Spending on medical goods comprises the third largest category. Prices of goods generally tend to be less variable across countries than services (see indicator on Prices in the health sector). This means that spending on pharmaceuticals and medical devices often accounts for a higher share of health spending in lower income countries. As such, medical goods accounted for more than a third of all health spending in the Slovak Republic. By contrast, in Denmark, Norway, the Netherlands and Sweden, the share was much lower, at between 10 and $12 \%$.

Where formal arrangements are in place for the care of the elderly and the dependent population such as in Norway, Sweden and the Netherlands, a quarter or more of all health spending can relate to long-term care services. In countries with a more informal long-term care sector such as in many Southern, Central and Eastern European countries, spending on long-term care is much lower - around 5\% or less in Greece, Portugal, Hungary and Latvia.

A vital component of any health system that stretches across the different types of services described above is primary care. As a proxy for this complex concept, primary care is here defined to include a variety of different services such as general outpatient care, preventive services, dental care services and home-based curative services when provided by ambulatory care providers. Using this proxy measure, primary care accounts for around $13 \%$ of all health spending across the OECD, ranging from around $10 \%$ in Switzerland, the Slovak Republic, the Netherlands and Austria to $18 \%$ in Australia and Estonia (Figure 7.15).

Growth in health expenditure resumed across all areas following the general slowdown after the economic crisis (Figure 7.16). During the years of the economic downturn, some governments introduced policies to protect expenditure for primary care and front-line services while looking to make cost savings elsewhere in the health system. Reducing wages in public hospitals, postponing staff replacement and delaying investment in hospital infrastructure were among the most frequent measures taken in OECD countries to balance health budgets. While outpatient care and long-term care continued to grow annually during the period 2009-13, spending on inpatient care and administration stalled in many countries, and decreased for pharmaceuticals and prevention services.

These cuts have since been reversed, and prevention was the fastest growing area between 2013-17 at 3.2\% on average, annually. The rate of growth for outpatient care has more than doubled ( $2.8 \%$ vs $1.1 \%)$, and inpatient care grew by $2.4 \%$. Spending on pharmaceuticals and administration increased more modestly at $1.6 \%$ and $2.0 \%$ per year, respectively. Finally, spending on long-term care has continued to grow at a consistent rate since 2003.

\section{Definition and comparability}

The System of Health Accounts (OECD, Eurostat and WHO, 2017[1]) defines the boundaries of the health care system from a functional perspective, with health care functions referring to the different types of health care services and goods. Current health expenditure comprises personal health care (curative care, rehabilitative care, long-term care, ancillary services and medical goods) and collective services (prevention and public health services as well as administration referring to governance and administration of the overall health system rather than at the health provider level). Curative, rehabilitative and long-term care can also be classified by mode of provision (inpatient, day care, outpatient and home care).

A key health service that has been notably missing in the SHA framework is primary care. Efforts have been made in recent years to develop a methodology using the SHA framework to develop a proxy indicator for primary care spending (Mueller and Morgan, 2018[2]). Comparability of primary care figures is mainly affected by the extent to which countries are able to distinguish between generalist and specialist services and the methods used to implement such a split.

For the calculation of growth rates in real terms, AIC deflators are used.

\section{References}

[2] Mueller, M. and D. Morgan (2018), "Deriving preliminary estimates of primary care spending under the SHA 2011 framework"; http://www.oecd.org/health/health-systems/ Preliminary-Estimates-of-Primary-Care-Spending-underSHA-2011-Framework.pdf.

[1] OECD/Eurostat/WHO (2017), A System of Health Accounts 2011: Revised edition, OECD Publishing, Paris, https://doi.org/ 10.1787/9789264270985-en. 
Figure 7.14. Health expenditure by type of service, 2017 (or nearest year)

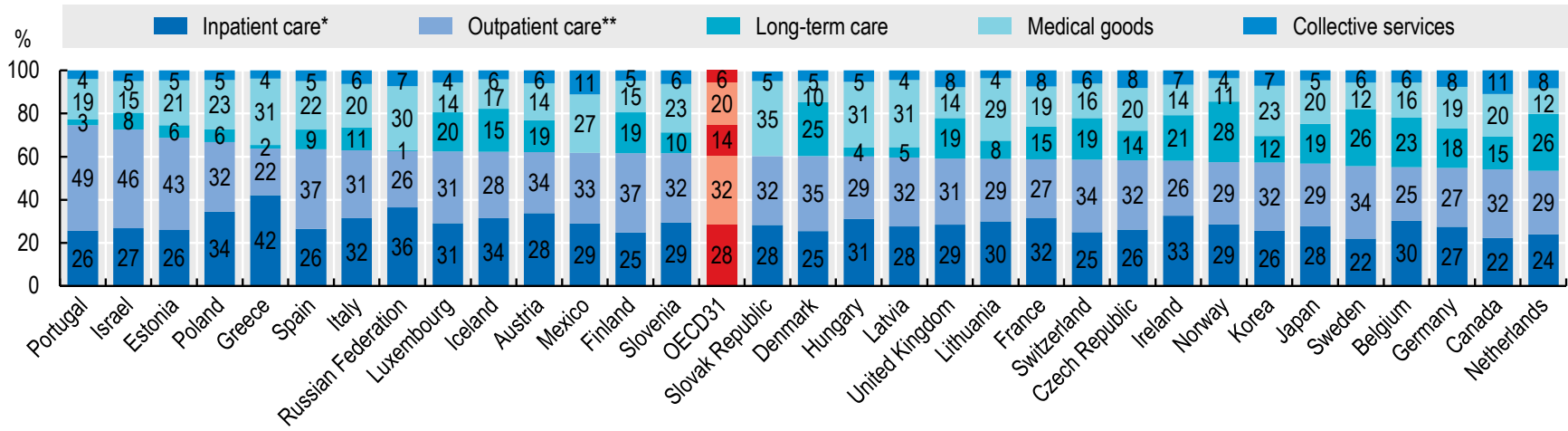

Note: Countries are ranked by curative-rehabilitative care as a share of current expenditure on health.

${ }^{*}$ Refers to curative-rehabilitative care in inpatient and day care settings. ${ }^{* *}$ Includes home care and ancillary services.

Source: OECD Health Statistics 2019.

Figure 7.15. Spending on primary care as a share of current health expenditure, 2017 (or nearest year)

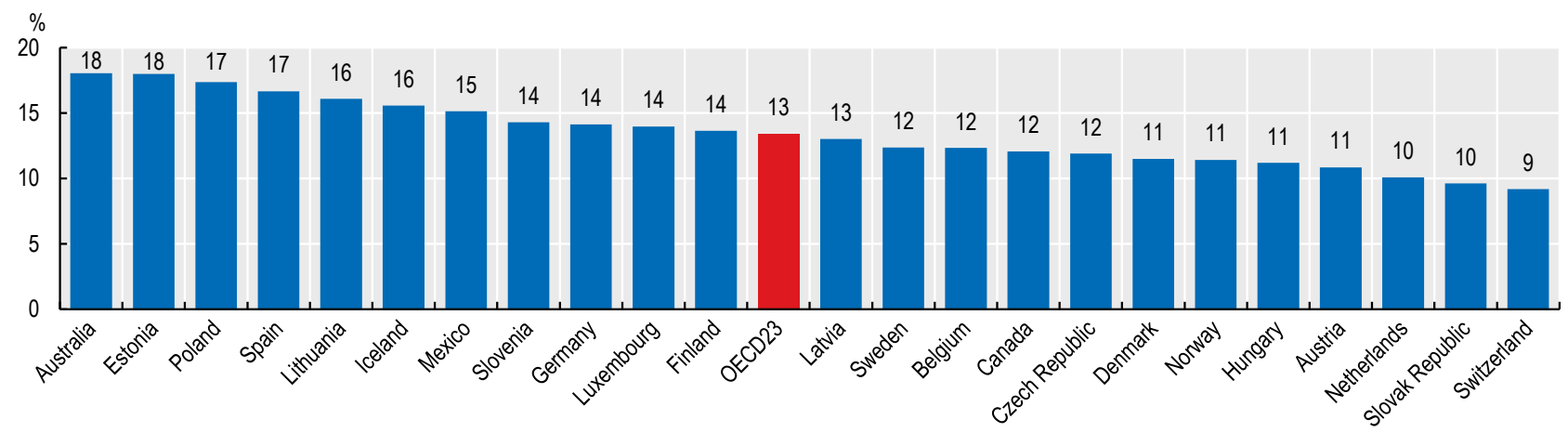

Source: OECD Health Statistics 2019.

Figure 7.16. Annual growth in health expenditure for selected services (real terms), OECD average, 2009-13 and 2013-17

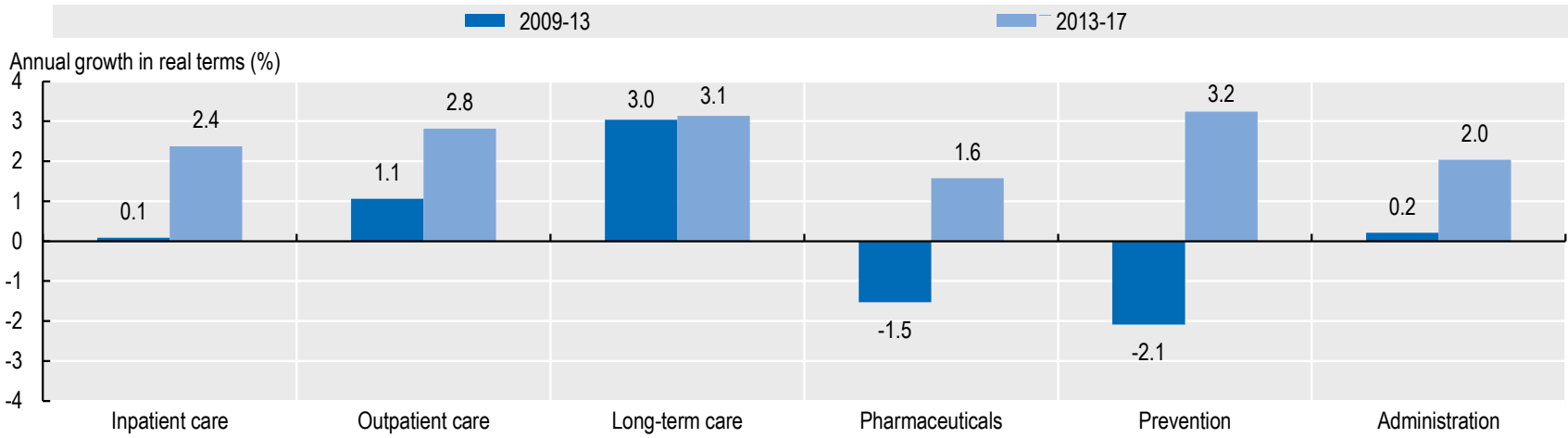

Source: OECD Health Statistics 2019. 
How and where health care is delivered can have a significant impact on spending for different goods and services. Health care can be provided in many different organisational settings, ranging from hospitals and medical practices to pharmacies and even private households caring for family members. Analysing health spending by provider can be particularly useful when considered alongside the functional breakdown of health expenditure, giving a fuller picture of the organisation of health systems (see indicator "Health expenditure by type of service").

Activities delivered in hospitals account for the largest proportion of health care expenditure in almost all OECD countries, even though each country organises their system to provide funding and care in different ways. On average, hospitals receive $38 \%$ of health system funding, but receive more than half of all financial resources in Turkey (Figure 7.17). Estonia, Korea and Italy also have significant hospital sectors, where spending accounts for around $45 \%$. Only Germany and Mexico spend less than $30 \%$ of the total on hospitals.

After hospitals, the largest provider category are ambulatory providers. This category covers a wide range of facilities and depending on the country-specific organisation of health service delivery, most spending relates either to medical practices including offices of GPs and specialists (e.g. Austria, France and Germany) or ambulatory health care centres (e.g. Finland, Ireland and Sweden). Across OECD countries, care delivered by ambulatory providers accounts for around a quarter of all health spending. This share stands above $30 \%$ in Israel, Belgium, the United States, Luxembourg, Mexico and Germany, but is less than $20 \%$ in Turkey, Greece, the Netherlands and the Slovak Republic. Around two-thirds of all spending on ambulatory providers relate to GP and specialist practices together with ambulatory health care centres, and roughly one-fifth to dental practices.

Other main provider categories include retailers (mainly pharmacies selling prescription and over-the-counter medicines) - accounting for $18 \%$ of all health spending - and residential long-term care facilities (mainly providing inpatient care to long-term dependent people), to which $9 \%$ of the total health spending bill can be attributed.

There is a large variation in the range of activities that may be performed by the same category of provider across countries, depending on the structure and organisation of the health system. This variation is most pronounced in hospitals (Figure 7.18). Although inpatient curative and rehabilitative care defines most of the hospital expenditure in almost all OECD countries, hospitals can also be important providers of outpatient care in many countries, for example through accident and emergency departments, specialist outpatient units, or laboratory and imaging services provided to outpatients. In Germany and Greece, hospitals are generally mono-functional with the vast majority (93\%) of spending on inpatient care services, and very little outpatient and day care spending. On the other hand, outpatient care accounts for over $40 \%$ of hospital expenditure in Denmark, Sweden, Estonia, Finland and Portugal. In those countries, specialists are typically receiving outpatients in hospital outpatient departments.

Many countries have shifted some medical services from inpatient to day care settings in recent years (see indicator on "Ambulatory surgery" in Chapter 9). The main motivation behind this is the generation of efficiency gains and a reduction of waiting times. Moreover, for some interventions day care procedures are now the most appropriate treatment method. Hence, in a number of countries day care now accounts for more than $10 \%$ of all hospital expenditure. Furthermore, the provision of longterm care in hospital makes up a sizeable share of hospital expenditure in some countries (e.g. Korea, Japan and Israel).

\section{Definition and comparability}

The universe of health care providers is defined in the System of Health Accounts (OECD, Eurostat and WHO, 2017) and encompasses primary providers, i.e. organisations and actors that deliver health care goods and services as their primary activity, as well as secondary providers for which health care provision is only one among a number of activities.

The main categories of primary providers are hospitals (acute and psychiatric), residential long-term care facilities, ambulatory providers (practices of GPs and specialists, dental practices, ambulatory health care centres, providers of home health care services), providers of ancillary services (e.g. ambulance services, laboratories), retailers (e.g. pharmacies), and providers of preventive care (e.g. public health institutes).

Secondary providers include residential care institutions whose main activities might be the provision of accommodation but provide nursing supervision as secondary activity, supermarkets that sell over-the-counter medicines, or facilities that provide health care services to a restricted group of the population such as prison health services. Secondary providers also include providers of health care system administration and financing (e.g. government agencies, health insurance agencies) and households as providers of home health care. 
Figure 7.17. Health expenditure by provider, 2017 (or nearest year)

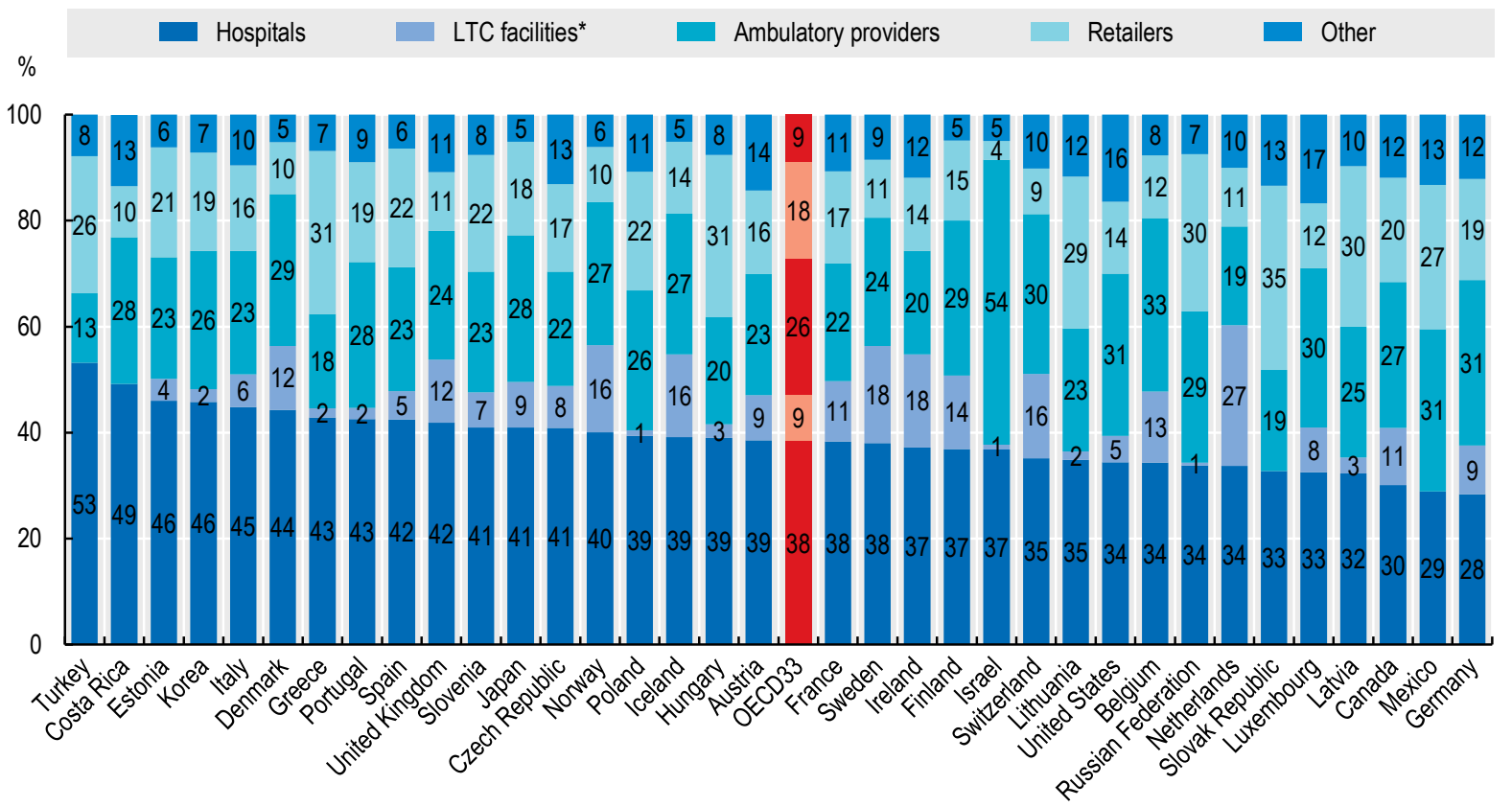

Note: Countries ranked by hospitals as a share of current expenditure on health. ${ }^{*}$ Refers to long-term care facilities.

Source: OECD Health Statistics 2019.

Figure 7.18. Hospital expenditure by type of service, 2017 (or nearest year)

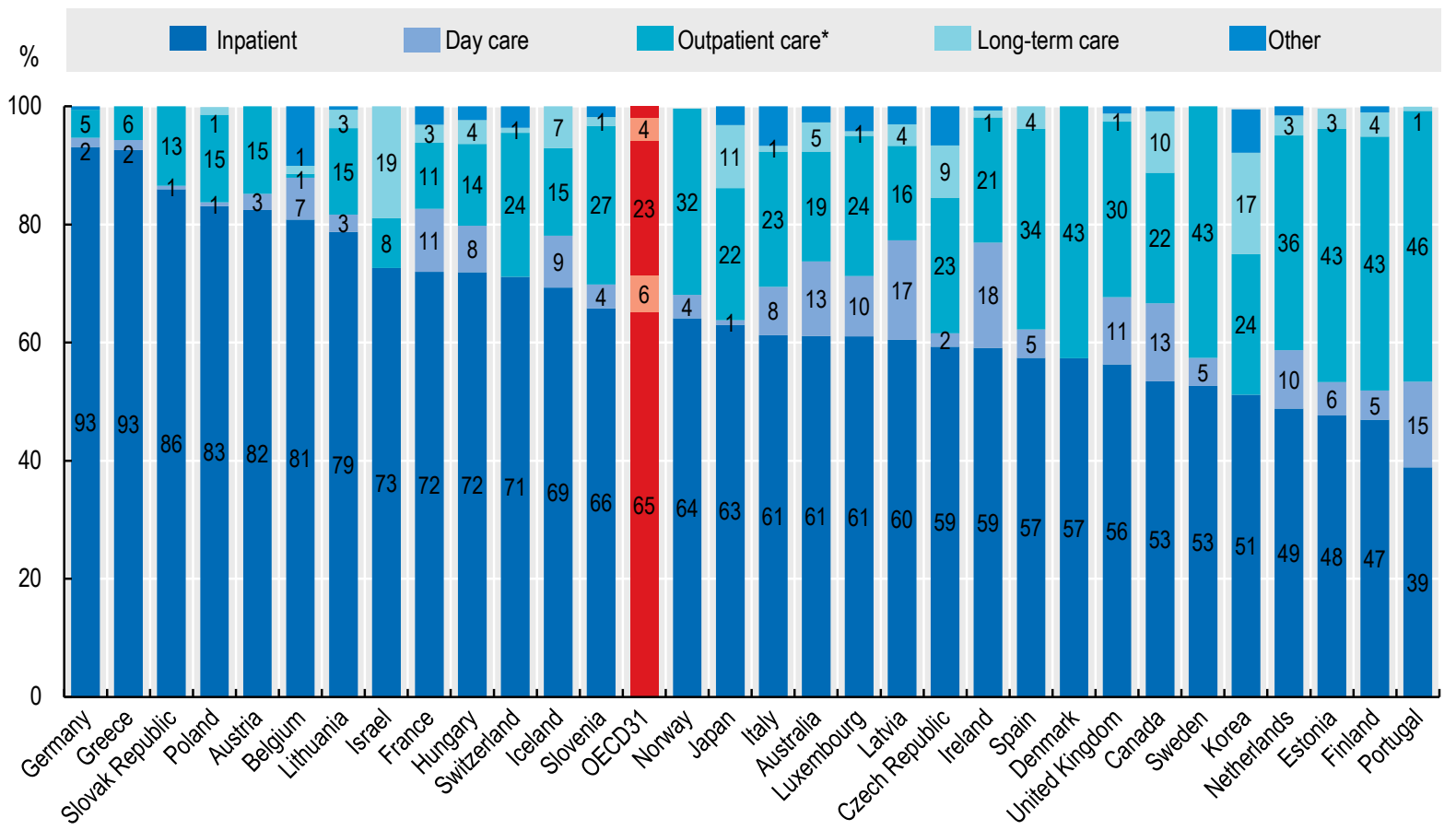

Note: Countries ranked by inpatient curative-rehabilitative care as a share of hospital expenditure. ${ }^{*}$ Includes ancillary services.

Source: OECD Health Statistics 2019. 
The health and long-term care sectors remain highly dependent on labour inputs, but capital is also a key factor in the production of health services. How much a country invests in new health facilities, the latest diagnostic and therapeutic equipment and information and communications technology (ICT) can have an important impact on the capacity of a health system to meet the health needs of the population and thus contribute to better outcomes. For example, a low level of MRI and CT scanners (see indicator "Medical technologies" in Chapter 5) can have consequences on the ability to detect diseases at an early stage. However, the level of capital expenditure tends to fluctuate more from year to year than current spending on health services, as investment decisions can be much more dependent on economic circumstances and political or business choices as well as reflecting future needs and past levels of investment. In making such decisions, policymakers and providers need to weigh up not only the shortterm costs, but also the potential benefits in the short, medium and longer-term. As with any industry, a lack of investment spending in the present can lead to an accumulation of problems and bigger costs in the future as current equipment and facilities deteriorate.

For the most recent year available, the average capital expenditure in OECD countries was equivalent to around $5.6 \%$ of current spending on health (that is, on medical care, pharmaceuticals, etc.) and around $0.5 \%$ of GDP compared to $8.8 \%$ of GDP for current spending on health (see indicator "Health expenditure as a share of GDP") (Figure 7.19). As is the case with current spending, there are significant differences in the levels of investment expenditure between countries and over time, especially as a result of the economic crisis.

In relation to their current spending, Luxembourg and Japan were the highest spenders in 2017 with the equivalent of more than $10 \%$ going on new construction, equipment and technology in the health and social sector, although in relation to its GDP, Luxembourg is closer to the average. A number of European countries - including Germany, Belgium, and the Netherlands - were also relatively high capital spenders, corresponding to around 9\% of current spending on health. Both Japan and Germany spent more than 1\% of GDP on capital investment in the health sector in 2017. The United States and the United Kingdom spent less than the average compared to current spending at $3.5 \%$ and $3.2 \%$, although because of the very high expenditure on health services, this translated into a relatively high share of GDP in the case of the United States. Turkey, by contrast, allocated only $0.3 \%$ of GDP to capital spending in 2017 but this appears relatively high compared to its low current spending on health.
Capital spending fluctuates more than current spending from year to year, particularly in small economies, as capital projects on construction (i.e. building of hospitals and other health care facilities) and investment programmes on new equipment (e.g. medical and ICT equipment) are implemented. Decisions on capital spending also tend to be more affected by economic cycles, with spending on health system infrastructure and equipment often a prime target for reduction or postponement during downturns. Figure 7.20 shows an index of capital spending in real terms over a ten-year period for a selection of European and North American countries. While France maintained a constant level of capital investment over the period, both the United Kingdom and, in particular, Greece reported a sharp drop in capital spending in the wake of the global financial and economic crisis, and expenditure remains at levels well below that of 2007. Both the United States and Canada have current capital spending similar to the levels (in real terms) before the crisis. There was a marked increase in capital expenditure in Canada in 2010/11 as a counter-cyclical measure, which was even more pronounced in Mexico from 2008-12, as the public health insurance (Seguro Popular) was significantly expanded.

\section{Definition and comparability}

Gross fixed capital formation in the health sector is measured by the total value of the fixed assets that health providers have acquired during the accounting period (less the value of the disposals of assets) and that are used repeatedly or continuously for more than one year in the production of health services. The breakdown by assets includes infrastructure (e.g. hospitals, clinics, etc.), machinery and equipment (including diagnostic and surgical machinery, ambulances, and ICT equipment), as well as software and databases.

Gross fixed capital formation is reported by many countries under the System of Health Accounts. It is also reported under the National Accounts broken down by industrial sector according to the International Standard Industrial Classification (ISIC) Rev. 4 using Section Q: Human health and social work activities or Division 86: Human health activities. The former is normally broader than the SHA boundary while the latter is narrower. 
Figure 7.19. Capital expenditure on health as a share of current health expenditure, 2017 (or nearest year)

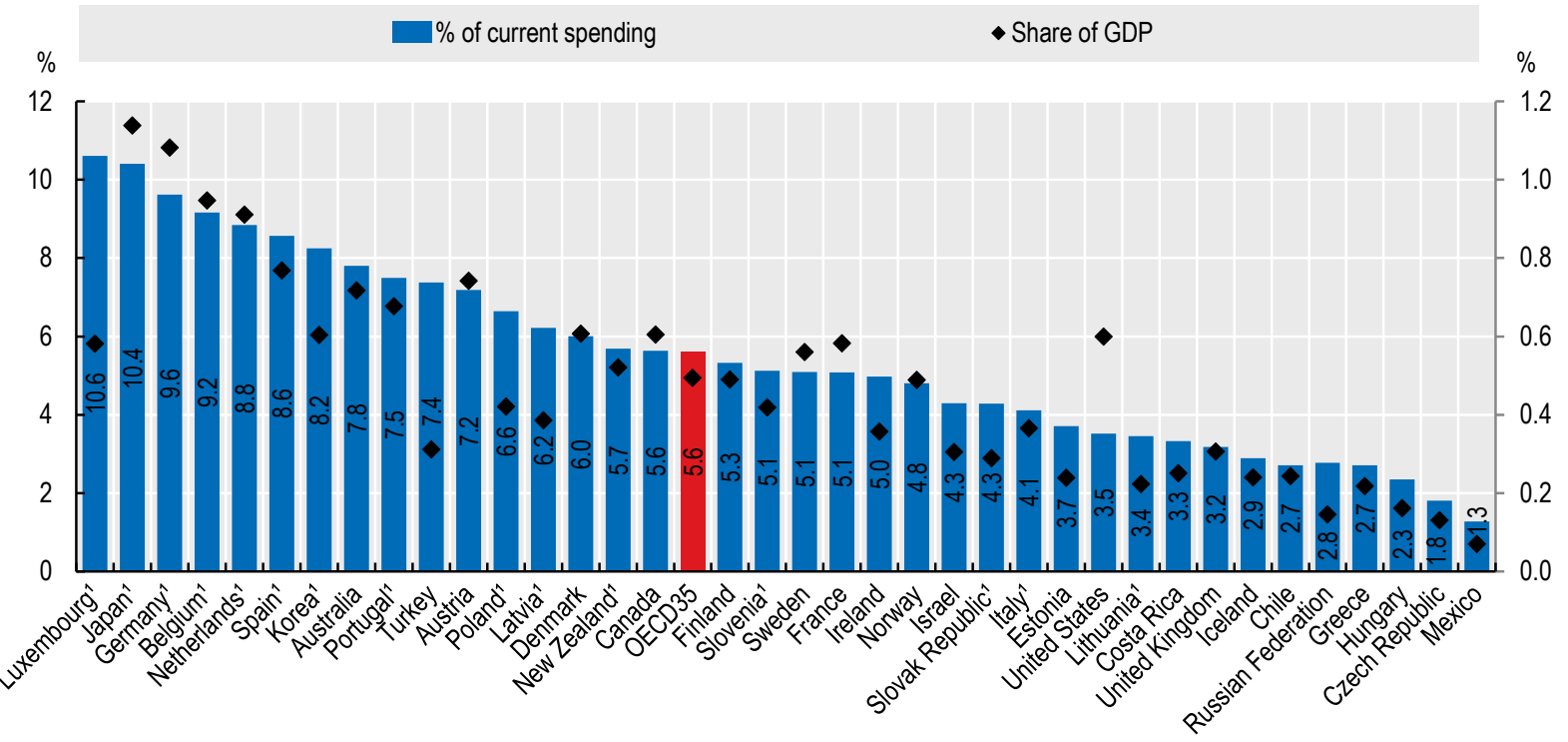

1. Refers to gross fixed capital formation in ISIC Q: Human health and social work activities (ISIC Rev. 4)

Source: OECD Health Statistics 2019, OECD National Accounts.

Figure 7.20. Trends in capital expenditure (constant prices), selected countries, 2007-17
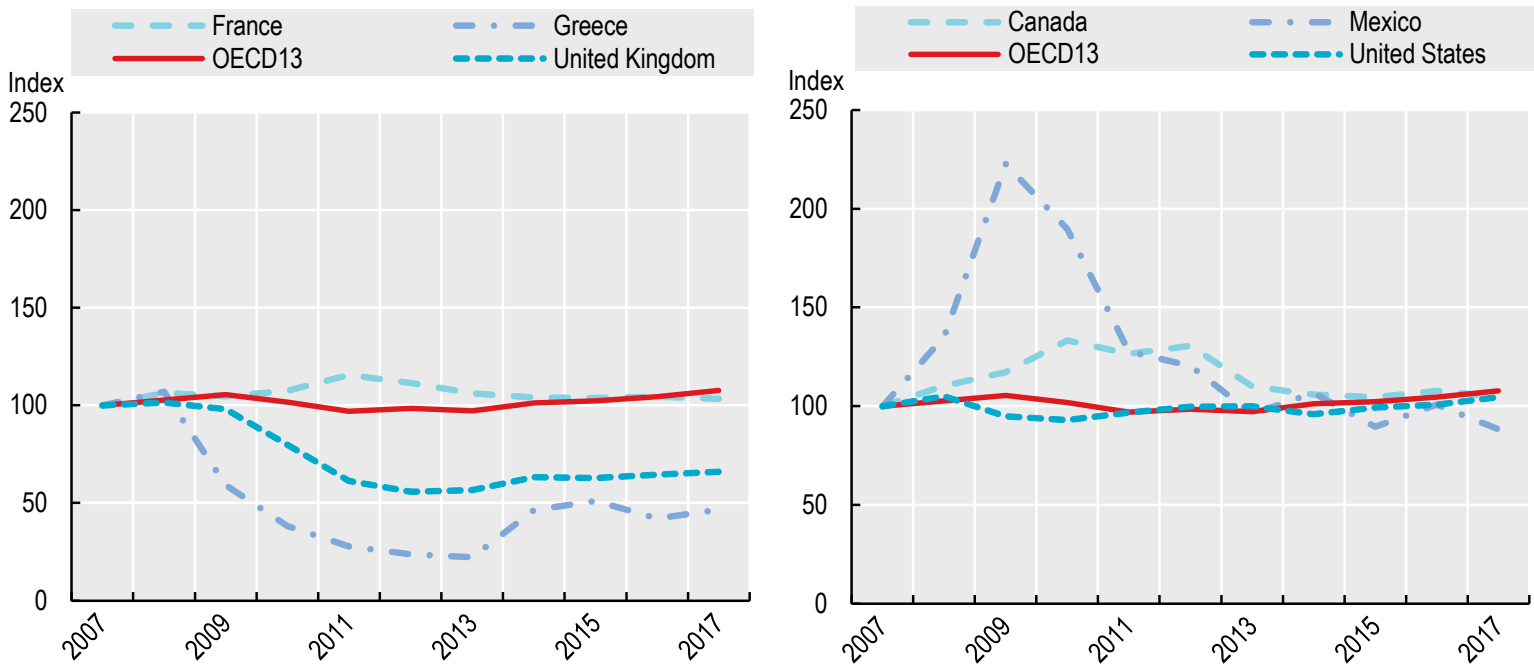

Source: OECD Health Statistics 2019, OECD National Accounts. 
Health expenditure has outpaced economic growth across OECD countries over most of the past half century. This additional spending has contributed to improvements in health outcomes and been an important source of economic growth and jobs. Nevertheless, financial sustainability is becoming an increasing concern, as most countries draw their funding largely from public sources (OECD, 2015[1]). Projections of health expenditure growth can give countries a perspective regarding how quickly, and by how much, health expenditure could rise compared to general economic growth, or with respect to a country's population (Lorenzoni et al., 2019[2]).

Over the long run, health expenditure has largely outpaced GDP growth across all OECD countries, even taking into account the volatility following the financial crisis of 2007-08 (Figure 7.21). Over the period 2000-15, annual health spending growth across the OECD was $3.0 \%$, compared to GDP growth of $2.3 \%$. By comparison, for the period 2015-2030, health expenditure per capita is projected to grow at an average annual rate of $2.7 \%$ across the OECD under a base scenario (with GDP growth averaging 2.1\%). Average growth is projected to be as low as $2.2 \%$ with greater cost control, but as high as $3.1 \%$ in a cost pressure scenario. These scenarios reflect diverging assumptions such as countries' economic growth, productivity and healthy ageing. However, across OECD countries health expenditure is projected to outpace GDP growth in the next 15 years in all scenarios.

Looking at country-specific projections, health spending per capita in 2015-30 is projected to grow more than $4 \%$ per year in the Slovak Republic, Turkey and Korea, while in Belgium, Germany, Italy, Lithuania, Japan and Portugal projected growth is less than $2 \%$ per year (Figure 7.22). In 20 out of 36 OECD countries, growth is projected to be within \pm 1 percentage points growth compared to 2000 -15. In the six countries - Iceland, Hungary, Mexico, Israel, Portugal and Turkey - where per capita growth is projected to be more than one percentage point higher than that observed for 2000-2015, most experienced a slowdown in health spending growth in the aftermath of the global economic and financial crisis. In contrast, in Lithuania, Korea, Chile, Latvia and Estonia, growth rates are projected to be over two percentage points lower than historical rates. These countries also reported some of the highest growth rates in health spending per capita from 2000 to 2015.

Across the OECD, under the base scenario, health expenditure as a share of GDP is projected to rise to $10.2 \%$ by
2030, compared to $8.8 \%$ in 2015 (Figure 7.23). The only countries for which a slight decrease in this ratio is expected are Latvia, Hungary and Lithuania, largely due to projected decreases in population size over the coming decades. Most countries are expected to experience moderate increases in health expenditure as a share of GDP, with only the United States seeing growth of more than three percentage points.

\section{Definition and comparability}

The underlying model for projecting health expenditure in the future includes several countryspecific determinants. It is based on age-specific health expenditure curves for total health expenditure (in real terms), which are projected in the future by using population changes, mortality rates, expected costs associated with dying, and the share of survivors and non-survivors in any given year. These are further adjusted for GDP growth, productivity and wages growth, time effects, individual and collective shares of expenditure and technological change. This modelling is applied to both total and public current health expenditure (excluding capital expenditure), and a range of scenarios are constructed based on parameters gathered from the literature, regressionbased sensitivity analysis, and assumptions in line with specific theories in the literature (i.e. time-todeath, healthy ageing). A detailed breakdown of the theoretical framework and the methodological assumptions underlying the projections presented in this column are available in the References section.

\section{References}

[2] Lorenzoni, L. et al. (2019), "Health Spending Projections to 2030: New results based on a revised OECD methodology", OECD Health Working Papers, No. 110, OECD Publishing, Paris, https://doi.org/10.1787/5667f23d-en.

[3] Marino, A. et al. (2017), "Future trends in health care expenditure: A modelling framework for cross-country forecasts", OECD Health Working Papers, No. 95, OECD Publishing, Paris, https://doi.org/10.1787/247995bb-en.

[1] OECD (2015), Fiscal Sustainability of Health Systems: Bridging Health and Finance Perspectives, OECD Publishing, Paris, https://doi.org/10.1787/9789264233386-en. 
Figure 7.21. Health expenditure per capita vs GDP growth trends, observed and projected, 2000-30

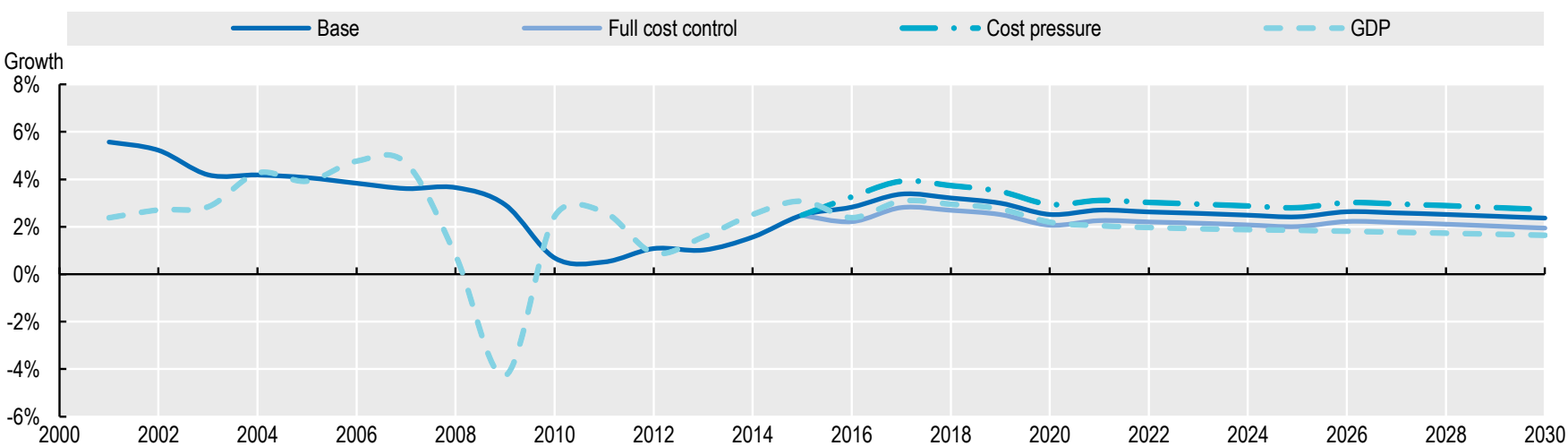

Source: OECD Health Division projections, 2019.

Figure 7.22. Average per capita health expenditure growth, 2000-15 and 2015-30

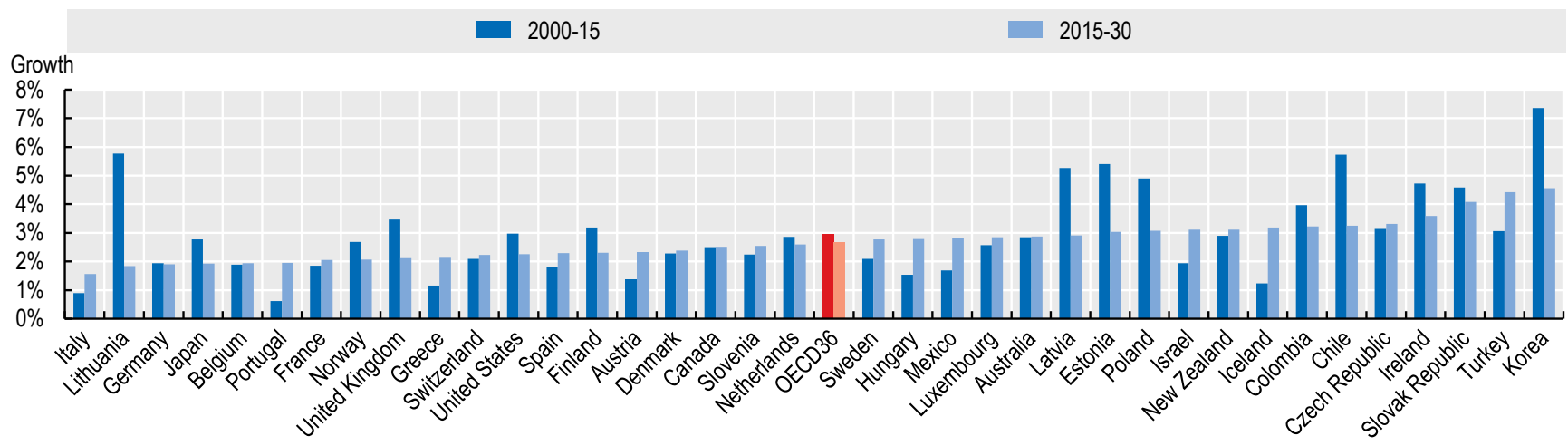

Source: OECD Health Division projections, 2019.

StatLink 세재 https://doi.org/10.1787/888934017177

Figure 7.23. Health expenditure as a share of GDP, projection to 2030

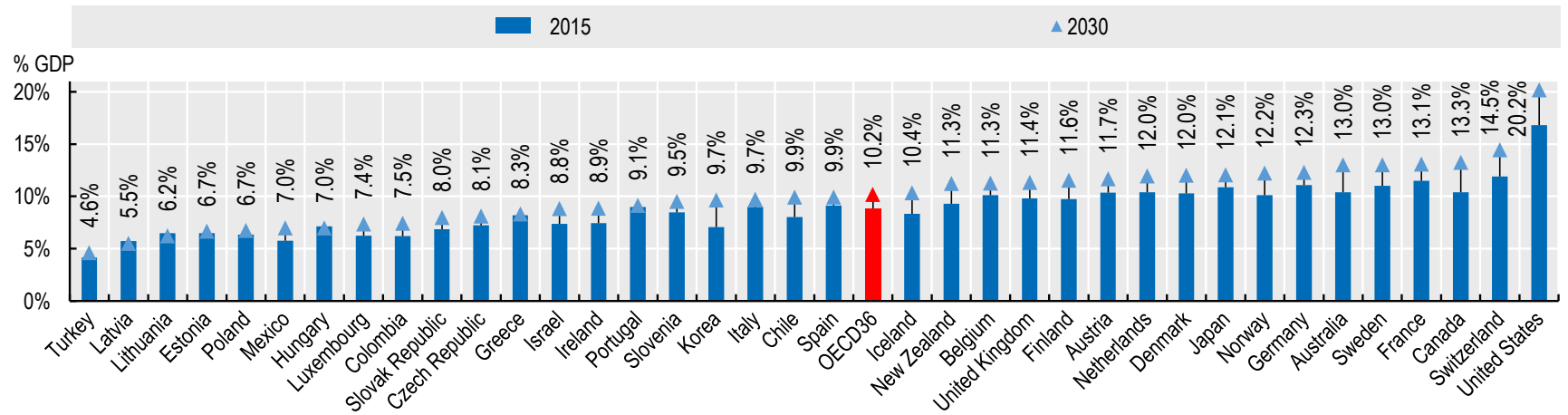

Source: OECD Health Division projections, 2019. 


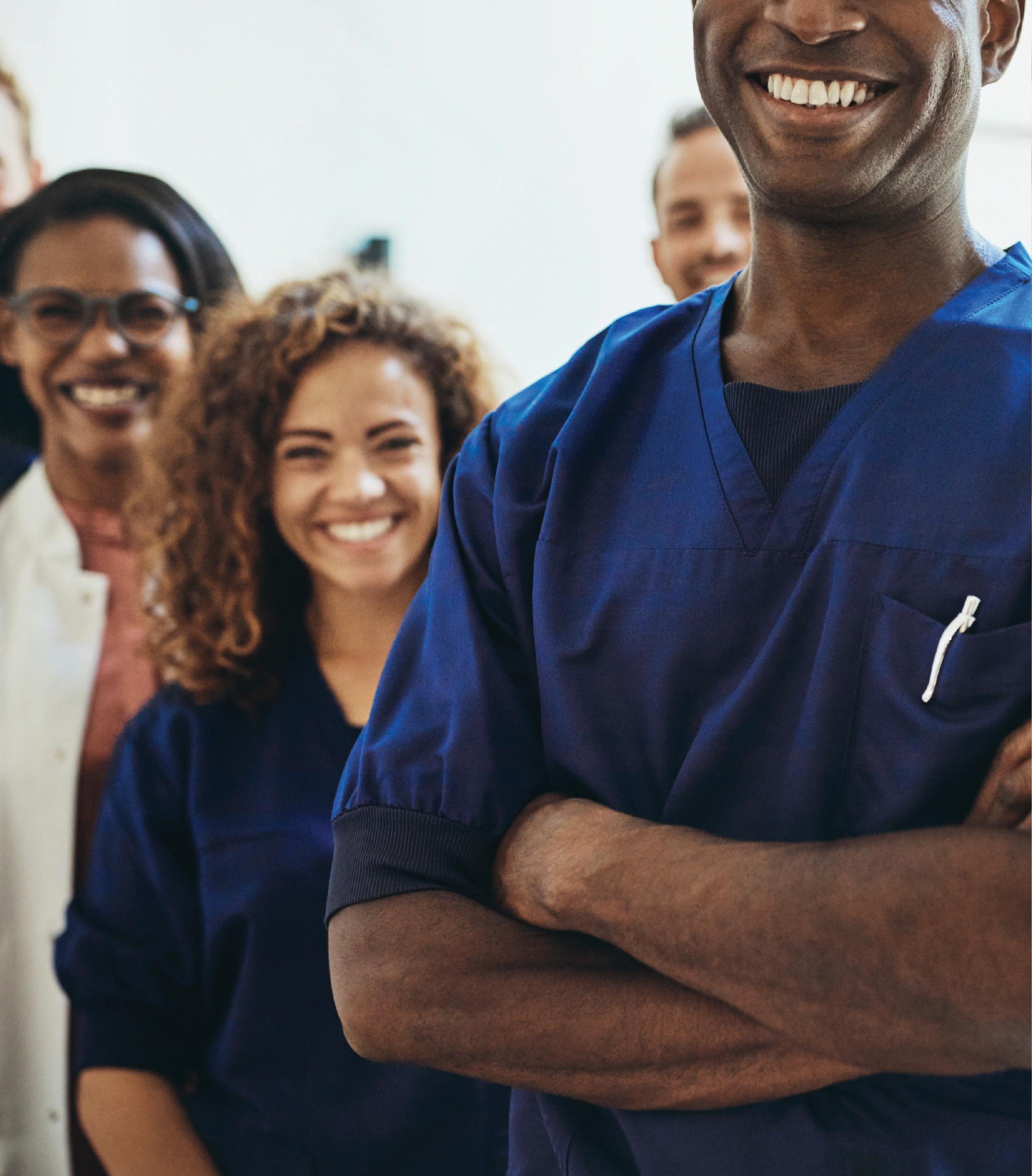




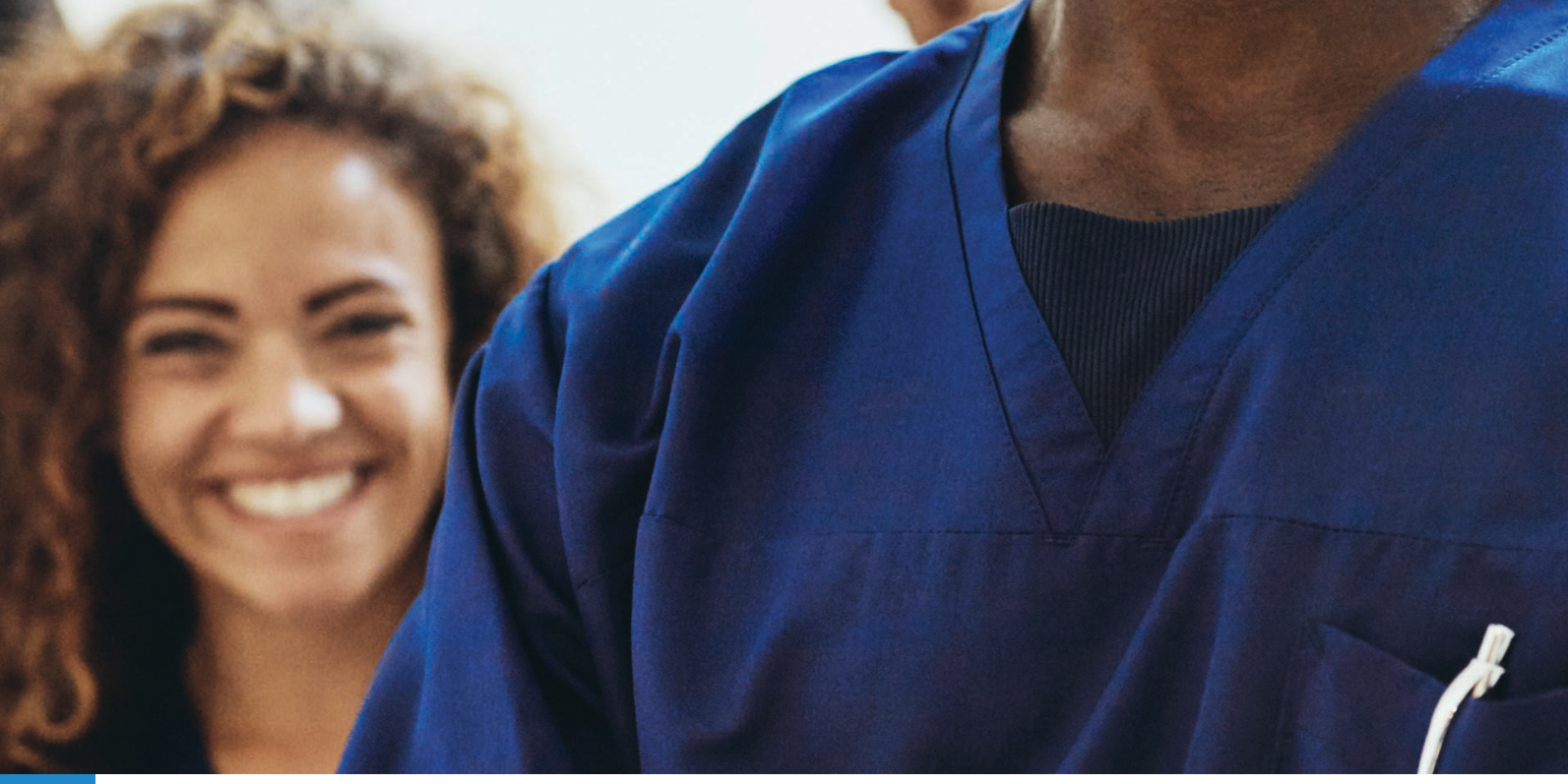

\section{HEALTH WORKFORCE}

Health and social care workforce

Doctors (overall number)

Doctors (by age, sex and category)

Remuneration of doctors (general practitioners and specialists)

Nurses

Remuneration of nurses

Medical graduates

Nursing graduates

International migration of doctors and nurses

The statistical data for Israel are supplied by and under the responsibility of the relevant Israeli authorities. The use of such data by the OECD is without prejudice to the status of the Golan Heights, East Jerusalem and Israeli settlements in the West Bank under the terms of international law. 
In OECD countries, health and social systems employ more workers now than at any other time in history. In 2017, about one in every ten jobs was found in health or social care (Figure 8.1), which amounts to a nearly two percentagepoint increase since 2000. In Nordic countries and the Netherlands, more than $15 \%$ of all jobs are in health and social work. From 2000 to 2017 the share of health and social care workers remained steady or increased in all countries except the Slovak Republic (where it decreased in the 2000s and has remained stable since 2010). In some countries, notably Japan, Ireland and Luxembourg, the share of health and social care workers increased considerably.

The health and social care sector is critical for the effective functioning of OECD societies and economies, and as a result the sector is not directly aligned with general workforce trends. Specifically, in OECD countries from 2000 to 2017, employment in the health and social sector increased on average by $42 \%$ (with a median increase of $38 \%$ ), outpacing even the growth in the service sector and trends in total employment, while employment in agriculture and industry declined sharply across the same period (Figure 8.2). At the same time, the health and social care sector also tends to be more robust to cyclical employment fluctuations. For example, while total employment declined in the United States and other OECD countries during the economic recessions of the early 1990s and, in particular, 2008-09, employment in the health and social care sector continued to grow steadily throughout.

Looking forward, employment in the health and social care sector is likely to continue to increase. Investment in health systems, including in workforce development, can promote economic growth by securing a healthy population, as well as along other pathways such as innovation and health security (UN High-Level Commission on Health Employment and Economic Growth, 2016[1]). The distribution of health and social care workers' skills and roles, however, is expected to change, driven in large part by ageing populations. With more older people, the pattern of demand for health and social services will shift towards greater demand for long-term care and related social services, which are particularly labour-intensive (OECD, 2019[2]). In response to, or in anticipation of, this demographic shift, many countries have begun to introduce new care delivery models that integrate health and social services. Policies such as expanding the roles of non- physician providers (such as nurse practitioners, pharmacists and community health workers), or introducing more multi-professional teams and treatment structures, can increase the productivity of the health workforce, as well as improving continuity and quality of care for patients.

New health technologies are a further factor driving rapid change in the health and social care sector, and their development and impact can be hard to predict. Technological shifts are expected in information technology and big data, automation and artificial intelligence; these may generate demand for new specialities or skills for health and social care workers, while reducing the importance of other professional roles (OECD, 2019[3]).

\section{Definition and comparability}

Health and social work is one of the economic activities defined according to the major divisions of the International Standard Industrial Classification of All Economic Activities (ISIC). Health and social work is a sub-component of the Services sector, and is defined as a composite of human health activities, residential care activities (including long-term care), and social work activities without accommodation. The employment data are taken from the OECD National Accounts database for the 36 OECD member countries, except for Turkey where the source is the OECD Annual Labour Force Statistics database.

\section{References}

[2] OECD (2019), Who Cares? Attracting and Retaining Care Workers for the Elderly, OECD Publishing, Paris, https://doi.org/ 10.1787/92c0ef68-en.

[3] OECD (2019), "Engaging and transforming the health workforce", in Health in the 21st Century: Putting Data to Work for Stronger Health Systems, OECD Publishing, Paris.

[1] UN High-Level Commission on Health Employment and Economic Growth (2016), Working for Health and Growth: Investing in the Health Workforce, World Health Organization, Geneva, http://www.who.int/hrh/com-heeg/reports. 


\section{HEALTH WORKFORCE}

\section{Health and social care workforce}

Figure 8.1. Employment in health and social work as a share of total employment, 2000 and 2017 (or nearest year)

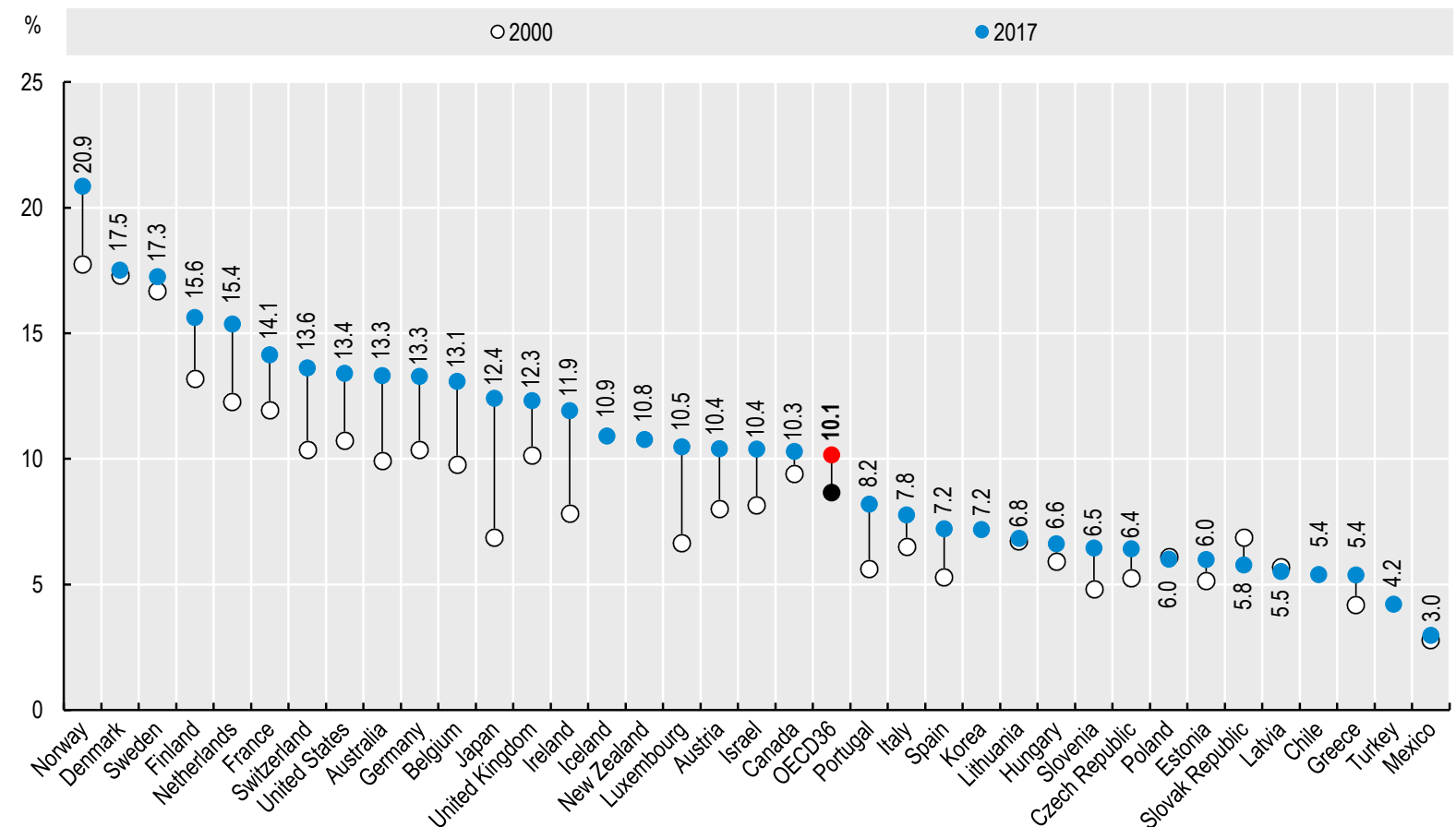

Source: OECD National Accounts; OECD Annual Labour Force Statistics for Turkey.

Figure 8.2. Employment growth by sector, OECD average ${ }^{1}$, 2000-17 (or nearest year)

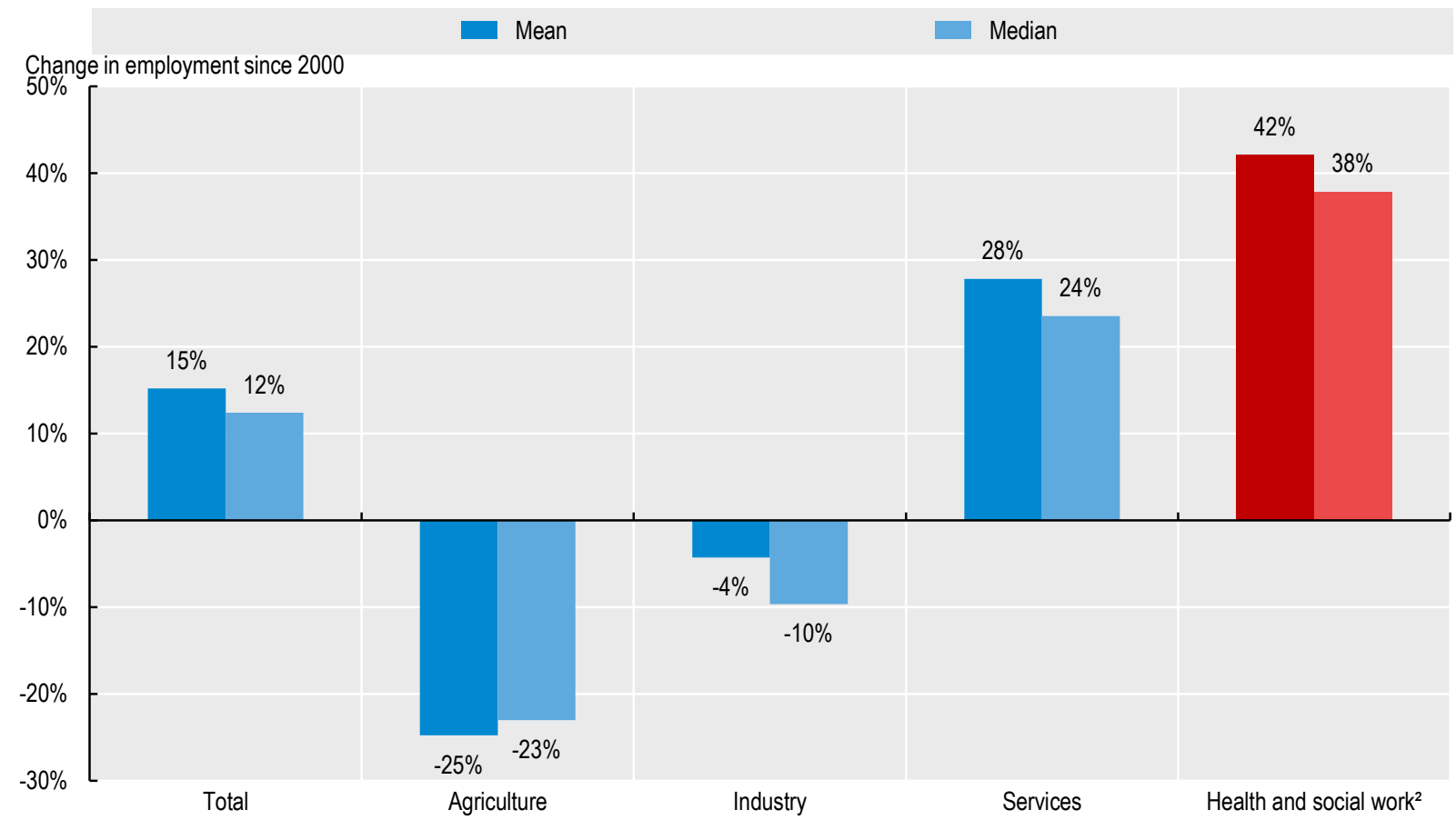

1. Average of 30 OECD countries (excluding Chile, Iceland, Korea, New Zealand, Switzerland and Turkey).

2. Health and social work is classified as a sub-component of the services sector.

Source: OECD National Accounts. 
Across OECD countries in 2017 the number of doctors ranged from 2.5 or less per 1000 population in Turkey, Korea, Poland, Mexico, Japan and Chile, to five or more in Portugal, Austria, and Greece. However, numbers in Portugal and Greece are over-estimated as they include all doctors licensed to practise. On average, there were 3.5 doctors per 1000 population (Figure 8.3). In Indonesia, India and South Africa there were significantly fewer doctors per 1000 population - less than one - while in China the number of doctors increased rapidly from 1.25 per 1000 population in 2000 to 2 per 1000 population in 2017.

Targeted education and training policies, as well as greater retention rates and in some countries immigration of doctors, have meant that both the absolute and per capita numbers of doctors have increased in almost all OECD countries since 2000 . The only exception is Israel, where a $25 \%$ increase in the absolute number of doctors was still not enough to keep pace with total population growth of about $40 \%$ between 2000 and 2017. Overall, in most OECD countries the number of doctors increased steadily between 2000 and 2017, and did not appear vulnerable to external shocks. However, the 2008-09 recession had a profound impact in Greece, where the number of doctors increased until 2008 before stagnating from 2012.

In some countries there were particularly rapid expansions in the number of doctors between 2000 and 2017 (Figure 8.4). This was the case in Korea, Mexico and the United Kingdom, where despite outpacing average per capita growth in doctors, there were still fewer doctors per 1000 than the OECD average in 2017. In other countries, such as Australia,Denmark and Austria, increases both outpaced OECD average growth, and left these countries with more doctors per capita than the OECD average. In Australia, where the number of doctors per capita went from below the OECD average in 2000, to above it in 2017, this increase was driven by a significant rise in the number of graduates from domestic medical education programmes (see indicator on "Medical graduates").

At the other end of the spectrum, the number of doctors per capita grew much more slowly or remained stable since 2000 in Belgium, France, Poland, and the Slovak Republic. In these four countries, the number of domestic students admitted to medical schools has increased in recent years. This should contribute towards replacing those doctors who will be retiring in the coming years, as long as new doctors end up working in their country of training (OECD, 2019[1]).

Concerns about shortages of health professionals are not new in OECD countries, but these concerns have grown in many countries, especially as the "baby-boom" generation of doctors and nurses starts to retire. Over the past decade, concerns about the ageing medical workforce moving towards retirement have prompted many OECD countries to increase the number of students in medical and nursing education programmes (OECD, 2016[2]). While some countries, such as Australia, have already started to see the benefits of earlier increases in medical education places, the long duration of doctors' training means that it takes a decade or more to feel the impact of increasing intake into medical education.

In most OECD countries, there are also concerns about shortages of general practitioners (see the indicator on "Doctors by age, sex and category") and an undersupply of doctors in rural and remote regions (see the indicator on "Geographic distribution of doctors" in Chapter 5). These issues have been driven or exacerbated by the ageing of general practitioners and of the population in general.

\section{Definition and comparability}

The data for most countries refer to practising doctors, defined as the number of doctors providing care directly to patients. In many countries, the numbers include interns and residents (doctors in training). The numbers are based on head counts. The Slovak Republic and Turkey also include doctors who are active in the health sector even though they may not provide direct care to patients, adding another $5-10 \%$ of doctors. Chile, Greece and Portugal report the number of physicians entitled to practice, resulting in an even larger over-estimation of the number of practising doctors. Belgium sets a minimum threshold of activities for general practitioners to be considered to be practising (500 consultations per year), thereby resulting in an under-estimation compared with other countries that do not set such minimum thresholds. Data for India may be over-estimated as they are based on medical registers that are not updated to account for migration, retirement or death; nor do they take into account doctors registered in multiple states.

\section{References}

[1] OECD (2019), Recent Trends in International Migration of Doctors, Nurses and Medical Students, OECD Publishing, Paris, https:// dx.doi.org/10.1787/5571ef48-en.

[2] OECD (2016), "Education and training for doctors and nurses: What's happening with numerus clausus policies?", in Health Workforce Policies in OECD Countries: Right Jobs, Right Skills, Right Places, OECD Publishing, Paris, https://dx.doi.org/ 10.1787/9789264239517-6-en. 
Figure 8.3. Practising doctors per 1000 population, 2000 and 2017 (or nearest year)

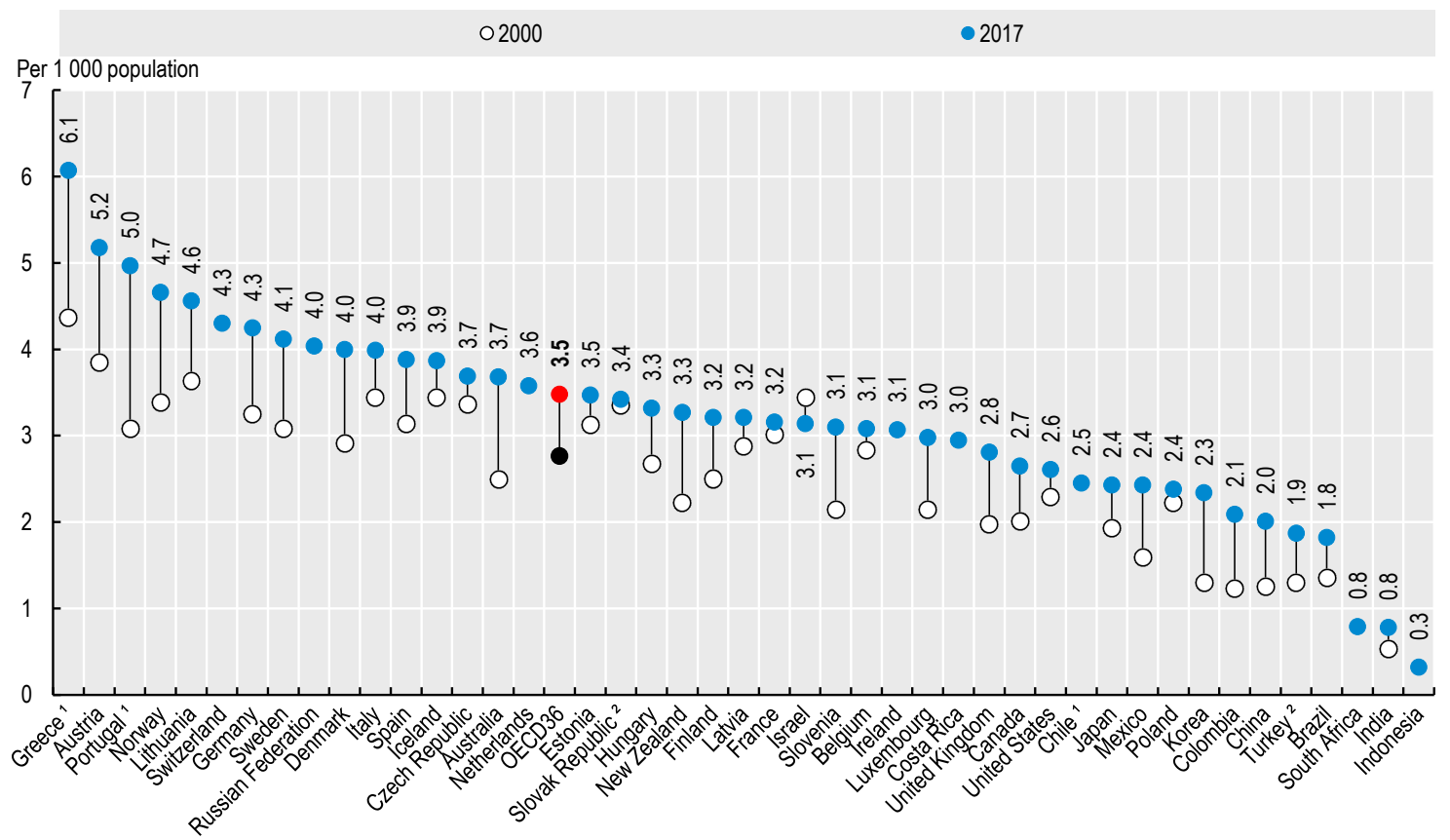

1. Data refer to all doctors licensed to practice, resulting in a large over-estimation of the number of practising doctors (e.g. of around $30 \%$ in Portugal). 2. Data include not only doctors providing direct care to patients but also those working in the health sector as managers, educators, researchers, etc. (adding another $5-10 \%$ of doctors).

Source: OECD Health Statistics 2019.

Figure 8.4. Evolution in the number of doctors, selected countries, 2000-17 (or nearest year)
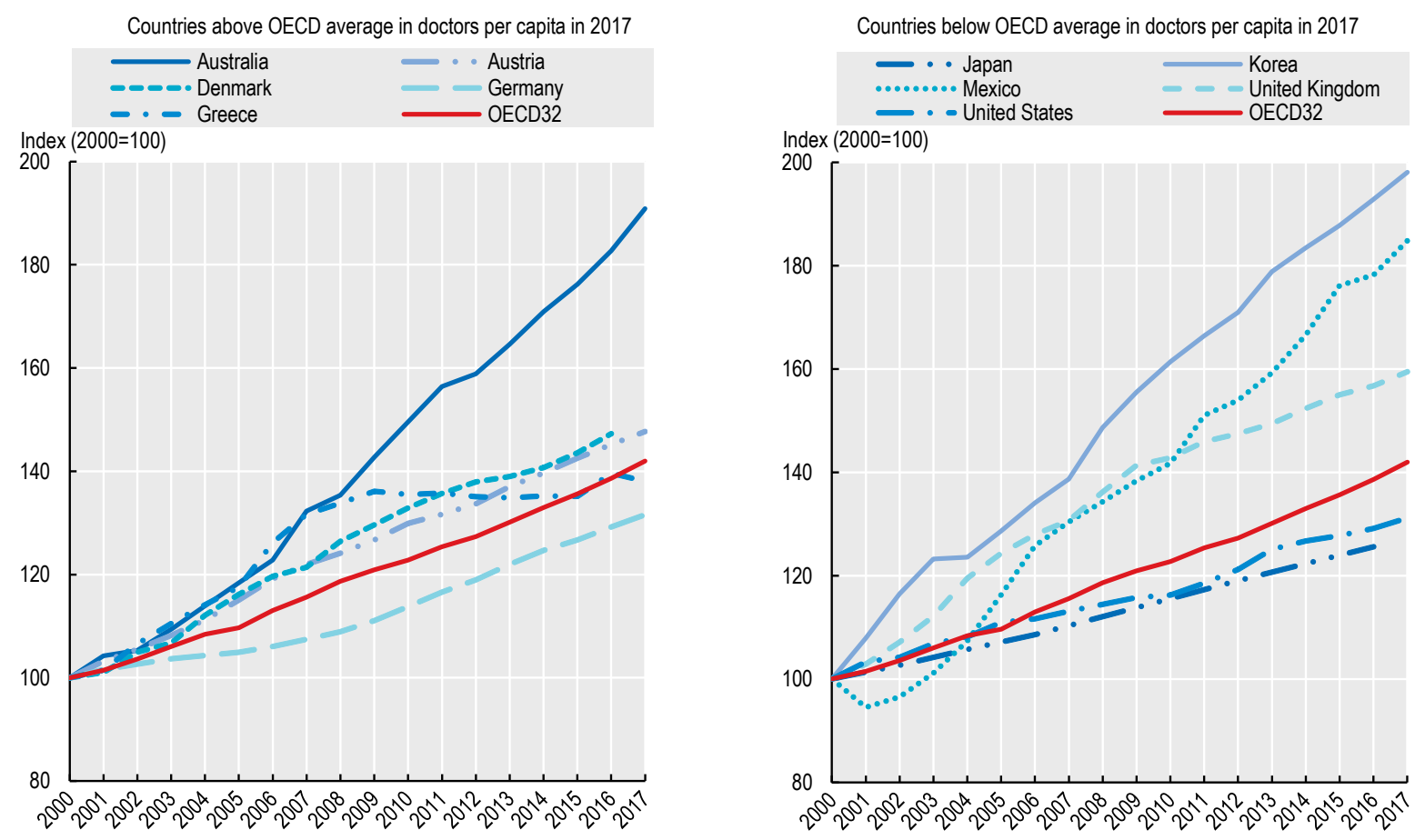

Source: OECD Health Statistics 2019. 
In 2017, more than one third of all doctors in OECD countries were over 55 years of age, up from one-fifth in 2000 (Figure 8.5). The share of doctors over 55 increased in all countries between 2000 and 2017\%.

While some countries saw only a small increase, such as Norway (+2 percentage points), Australia (+3 percentage points), and the United Kingdom (+4 percentage points), others saw a dramatic ageing of their medical workforce. In Italy, the share of older doctors increased by $36 \%$, with $55 \%$ of all doctors aged 55 or over by 2017. In France the doctor population is ageing almost as rapidly, with a $30 \%$ increase in older doctors between 2000 and 2017; other countries such as Israel, Spain and Austria are not far behind (Figure 8.5).

Ageing of the medical workforce is a concern, as doctors aged 55 and over are generally expected to retire in the following decade and need to be replaced in order to prevent a decline in overall physician numbers. Many doctors do keep working beyond age 65, and several OECD countries have reformed their pension systems and increased the retirement age to take into account the longer average life expectancy (OECD, 2016[1]). While few studies have examined the impact of these pension reforms specifically on doctors, it is possible that such steps will prolong the working lives of doctors, which could have a significant impact on future replacement needs.

In 2017 almost half of all doctors in OECD countries were female, at between one-third and two-thirds of all doctors in most OECD countries. In some countries, the gender balance was skewed more dramatically: in Japan and Korea only one-fifth of doctors were women in 2017, while in Latvia and Estonia three quarters of doctors were female (Figure 8.6). In most OECD countries the share of female doctors increased between 2000 and 2017, while in countries such as Lithuania, Estonia and Latvia - which traditionally have far more female than male doctors - the ratio of male-to-female doctors remained stable. The most significant increases in the share of female doctors were reported for the Netherlands (+19 percentage points) and Spain (+18 percentage points). In countries where the number of female doctors has increased, this is probably driven by rising female labour force participation and higher numbers of young women enrolling in medical school, but may also be affected by the retirement of older and more commonly male generations of doctors.

Up to and including the 2015 issue of Health at a Glance, the category 'generalist' did not distinguish between general practitioners/family doctors and non-specialised physicians who work in hospitals and other settings. It is now possible to distinguish between these two categories of phyisicans, and as of 2017, general practitioners/family doctors represented $23 \%$ of all physicians. The share of general practitioners/family doctors as a percentage of all doctors ranged from around half in Chile, Canada and Portugal, to just 5\% in Greece and Korea (Figure 8.7). The numbers of generalists remains difficult to compare, however, due to variation between countries in the ways doctors are categorised. For example, in the United States, general internal medicine doctors often play a role similar to that of general practitioners/family doctors in other countries, yet they are categorised as specialists. In other countries, such as Japan, general practitioners/family doctors are very uncommon, and the majority of physician consultations are with specialists.

In many countries, general practitioners/family doctors play a key role in guaranteeing good access to health care, managing chronic conditions and keeping people out of hospital (see indicator on "Avoidable hospital admissions" in Chapter 6). Accordingly, many countries have taken steps to increase the number of training places in general medicine in response to concerns about shortages of general practitioners. However, in most OECD countries, specialists earn more than general practitioners, which provides financial incentives for doctors to specialize (see indicator on the "Remuneration of doctors").

\section{Definition and comparability}

The definition of doctors is provided under the previous indicator. In some countries, the data are based on all doctors licensed to practice, not only those practising (Chile, Greece and Portugal; and also Israel and New Zealand for doctors by age and gender). Not all countries are able to report all their physicians in the two broad categories of specialists and generalists. This may be due to the fact that specialty-specific data are not available for doctors in training or for those working in private practice. A distinction is made in the generalists category between general practitioners/family doctors and non-specialist doctors working in hospital or in other settings. In Switzerland, general internal medicine doctors and other generalists are included under general practitioners.

\section{References}

[1] OECD (2016), Health Workforce Policies in OECD Countries: Right Jobs, Right Skills, Right Places, OECD Health Policy Studies, OECD Publishing, Paris, https://dx.doi.org/ 10.1787/9789264239517-en. 
Figure 8.5. Share of doctors aged 55 and older, 2000 and 2017 (or nearest year)

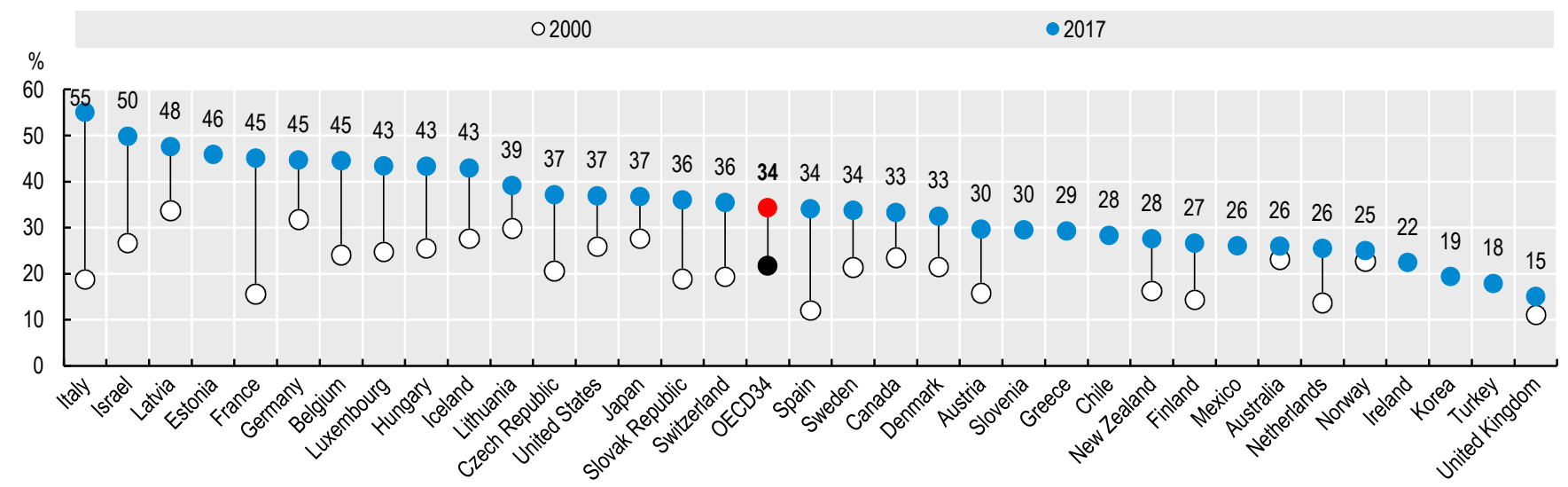

Source: OECD Health Statistics 2019.

StatLink क्ञाs https://doi.org/10.1787/888934017291

Figure 8.6. Share of female doctors, 2000 and 2017 (or nearest year)

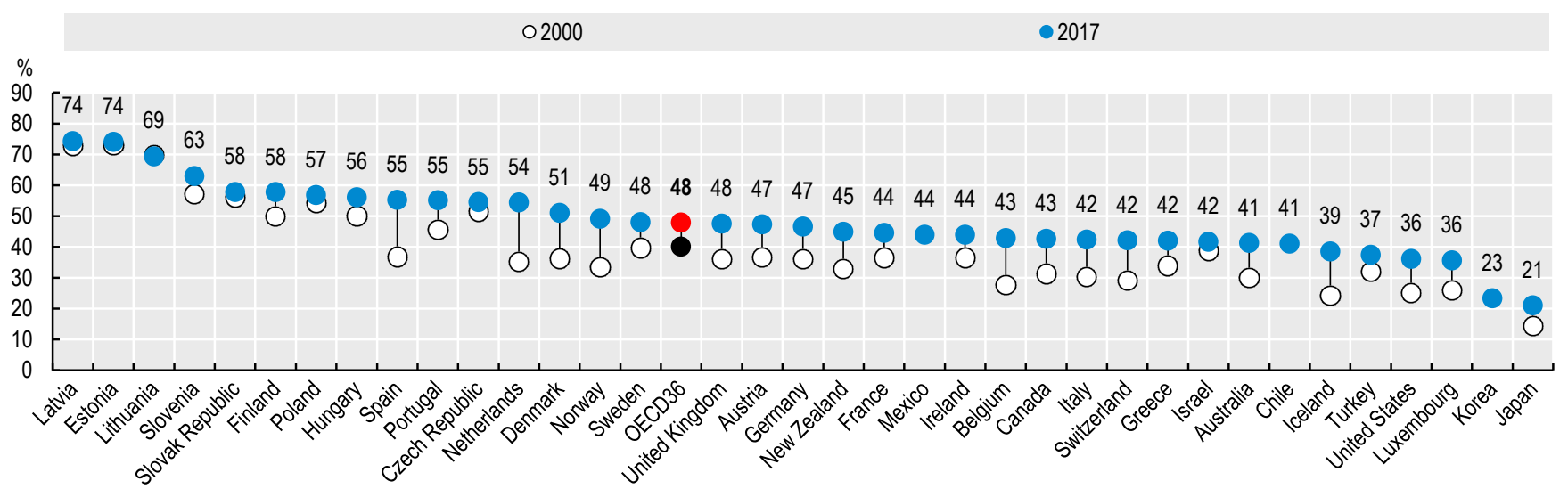

Source: OECD Health Statistics 2019.

StatLink क्ञाs https://doi.org/10.1787/888934017310

Figure 8.7. Share of different categories of doctors, 2017 (or nearest year)

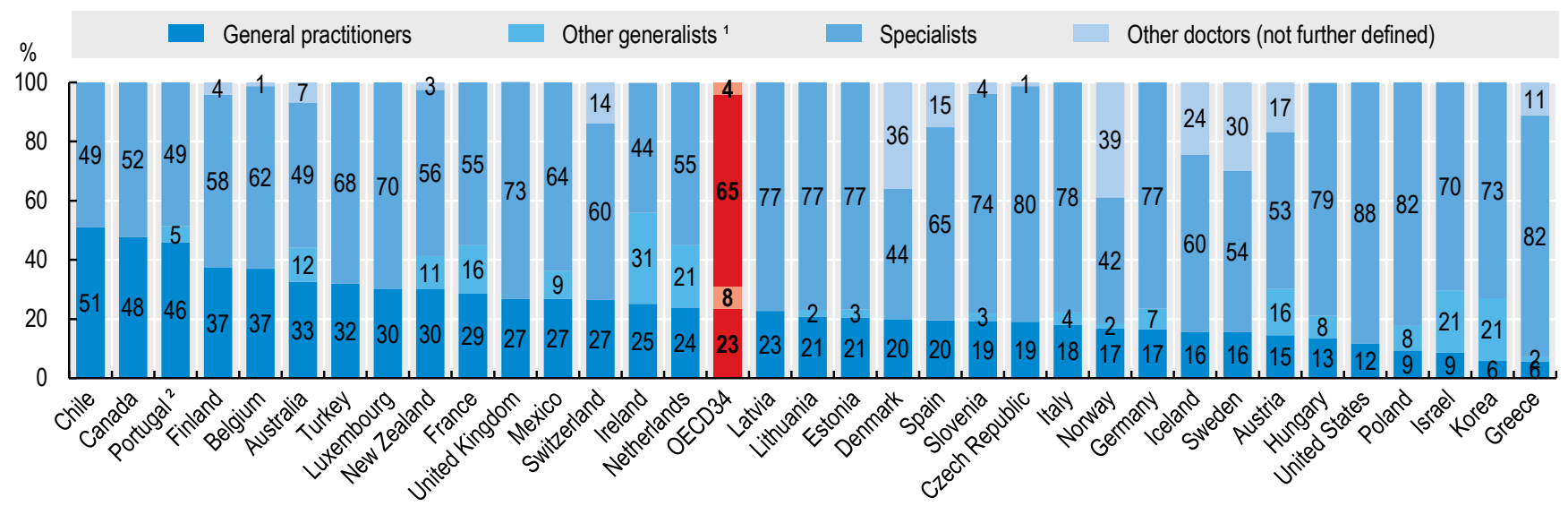

1. Includes non-specialist doctors working in hospital and recent medical graduates who have not yet started post-graduate specialty training. 2. In Portugal, only about 30\% of doctors employed by the public sector work as GPs in primary care, the other $70 \%$ work in hospitals.

Source: OECD Health Statistics 2019. 
The remuneration level and structure for the different categories of doctors affects the (relative) financial attractiveness of these specialities. In many countries, governments can determine or influence the level and structure of physician remuneration by regulating their fees or by setting salaries when doctors are employed in the public sector. As for any other category of workers, differences in remuneration levels of doctors across countries can be a push or pull factor when it comes to physician migration (OECD, 2019[1]).

Across OECD countries, the remuneration of doctors (both general practitioners and specialists) is substantially higher than the average wages (Figure 8.8). In most countries, general practitioners earn two to four times more than the average wage in each country, while specialists earn two to six times more.

In most countries, specialists earned more than general practitioners (Figure 8.8). In Australia, Belgium, and Luxembourg self-employed specialists earned at least twice as much as self-employed general practitioners. In Germany the difference between specialists and general practitioners is much smaller, at only $20 \%$. Among salaried physicians, specialists in Israel and the United Kingdom earned twice as much as general practitioners in 2017. In Poland, however, salaried specialists earned $40 \%$ less than salaried general practitioners.

The remuneration of physicians has generally increased since 2010, but at different rates across countries and between general practitioners and specialists (Figure 8.9). Both generalists and specialists in Hungary and Estonia have obtained substantial pay raises in recent years. To reduce shortages and emigration of doctors, the Hungarian government has substantially increased their remuneration since 2010, with the income of general practitioners increasing by about $80 \%$ between 2010 and 2017 and that of specialists nearly doubling. These pay raises have started to have a measurable impact on the intention of Hungarian doctors to leave the country: between 2017 and 2018, the number of doctors asking for foreign work certificates decreased by over $10 \%$.

In several countries, the remuneration of specialists has risen faster than those of generalists since 2010, thereby increasing the remuneration gap. However, in Austria and Belgium, the gap has narrowed slightly, as the income of general practitioners grew a little more than that of specialists (Figure 8.9).

\section{Definition and comparability}

The remuneration of doctors refers to average gross annual income, including social security contributions and income taxes payable by the employee. It should normally exclude practice expenses for self-employed doctors (in Belgium, practice expenses are included). OECD data on physician remuneration makes the distinction between salaried and self-employed physicians. In some countries this distinction is blurred, since some salaried physicians are allowed to have a private practice and some self-employed doctors receive part of their remuneration through salaries. The OECD data also distinguish between general practitioners and all other medical specialists combined, although especially the latter may be a rather inhomogeneous group.

A number of data limitations contribute to an underestimation of remuneration levels in some countries: 1) payments for overtime work, bonuses, other supplementary income or social security contributions are excluded in some countries (in Austria for GPs, Ireland for salaried specialists, and Italy); 2) incomes from private practices for salaried doctors are not included in some countries (e.g. Czech Republic, Hungary, Iceland, Ireland and Slovenia); 3) informal payments, which may be common in certain countries (e.g. Greece and Hungary), are not included; 4) data relate only to public sector employees, who tend to earn less than those working in the private sector in Chile, Denmark, Greece, Hungary, Iceland, Ireland, Norway, the Slovak Republic and the United Kingdom; and 5) physicians in training are included in Australia.

The income of doctors is compared to the average wage of full-time employees in all sectors in the country. The average wage of workers in the economy is from the OECD Employment Database.

\section{References}

[1] OECD (2019), Recent Trends in International Migration of Doctors, Nurses and Medical Students, OECD Publishing, Paris, https:// doi.org/10.1787/5571ef48-en. 
Figure 8.8. Remuneration of doctors, ratio to average wage, 2017 (or nearest year)
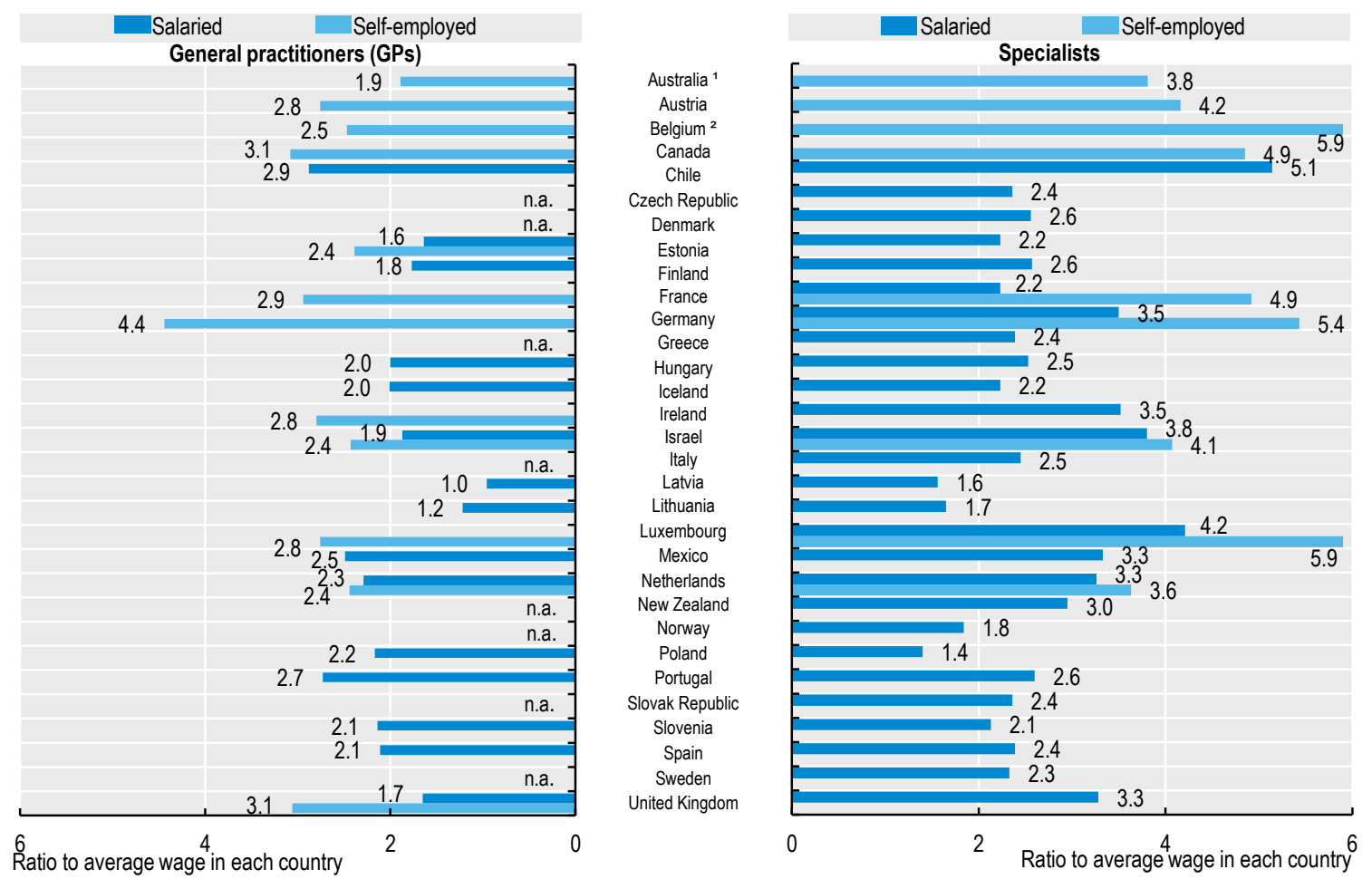

1. Physicians in training included (resulting in an under-estimation). 2. Practice expenses included (resulting in an over-estimation). Source: OECD Health Statistics 2019 and OECD Employment Database 2019.

Figure 8.9. Growth in remuneration of GPs and specialists, 2010-17 (or nearest year)

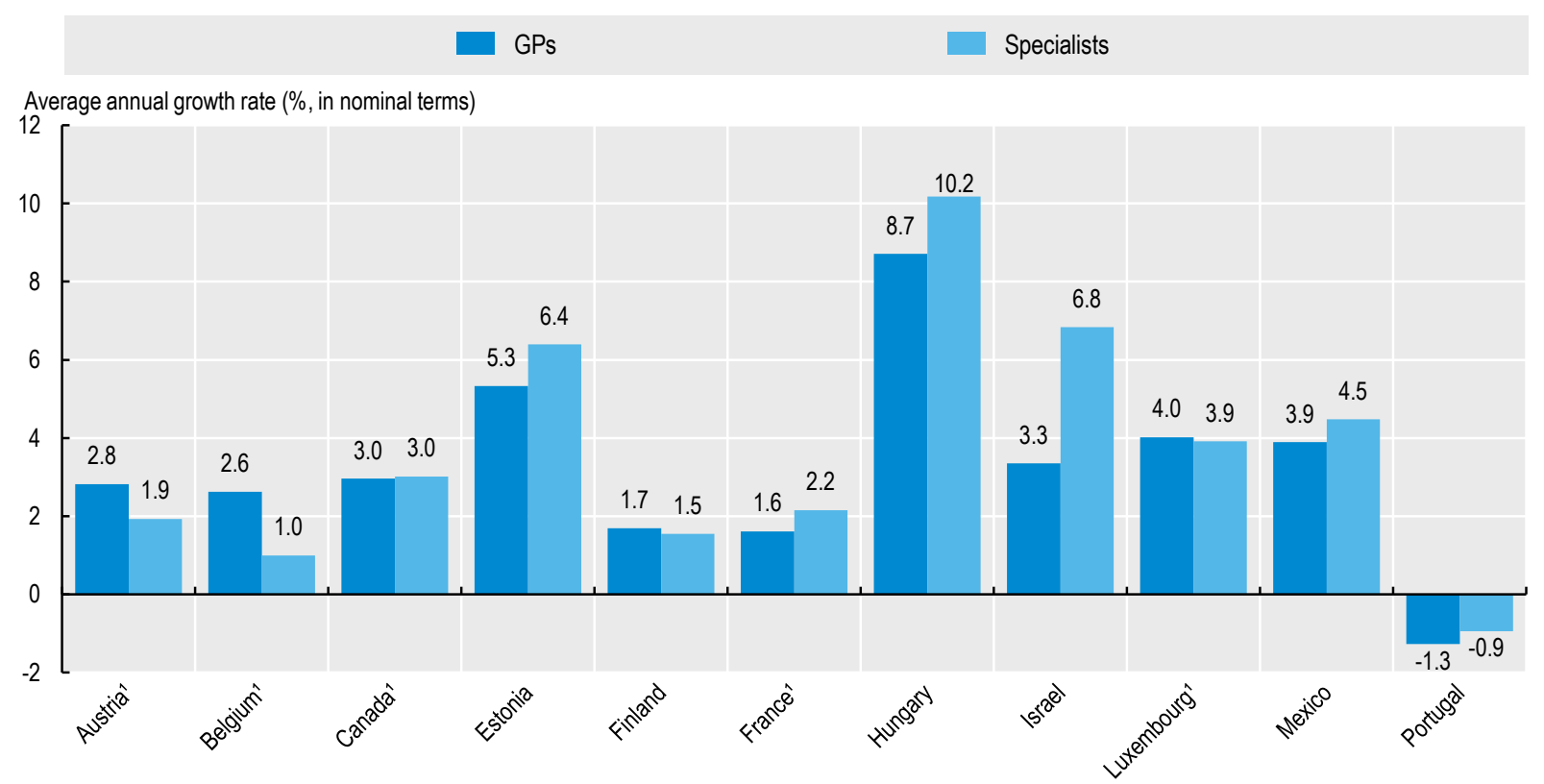

1. The growth rate is for self employed GPs and specialists.

Source: OECD Health Statistics 2019. 
There were just under nine nurses per 1000 population in OECD countries in 2017, ranging from about two per 1000 in Turkey to more than 17 per 1000 in Norway and Switzerland. Between 2000 and 2017 the number of nurses per capita grew in almost all OECD countries, and the average rose from 7.4 per 1000 population in 2000 to 8.8 per 1000 population in 2017. In the Slovak Republic, Israel, the United Kingdom and Ireland, however, the number of nurses per capita fell over that period (Figure 8.10).

The decreases in Israel and Ireland are due to the rapid growth of the population, with the increase in the number of nurses not keeping up. In Ireland, the growth in the number of nurses outpaced population growth until 2008, when it peaked at 13.6 per 1000 population, but has since fallen behind population increases. In the Slovak Republic, the number of nurses declined both in absolute and per capita numbers, mainly during the 2000s, while in the United Kingdom the number of nurses per capita increased rapidly between 2000 and 2006 and then declined until 2017.

No clear pattern emerges from the rate of increase of nurses: significant increases were seen in both countries which already have high numbers of nurses per capita, such as Switzerland, as well as countries with lower numbers of nurses, such as France, Slovenia and Korea. In most countries, growth in the number of both doctors and nurses has been driven by growing numbers of domestic nursing and medical school graduates, although in some countries immigration of foreign-trained doctors and nurses also played an important role (see indicator on "International migration of doctors and nurses").

Nurses outnumber physicians in most OECD countries, and on average there are three nurses to every doctor. The ratio of nurses to doctors ranges from about one nurse per doctor in Chile, Turkey and Greece, to more than four nurses per doctor in Japan, Ireland, Finland and the United States (Figure 8.11).

In response to shortages of doctors, and to ensure proper access to care, some countries have developed more advanced roles for nurses, including "nurse practitioner" roles. Evaluations of nurse practitioners from the United States, Canada and the United Kingdom show that advanced practice nurses can improve access to services and reduce waiting times, while delivering the same quality of care as doctors for a range of patients, including those with minor illnesses and those needing routine follow-ups. These evaluations find a high patient satisfaction rate, while the impact on cost is either cost-reducing or cost-neutral. The implementation of new advanced practice nursing roles can require changes to legislation or regulation (Maier, Aiken and Busse, 2017[1]).

\section{Definition and comparability}

The number of nurses includes those employed in public and private settings providing services directly to patients ("practising") and in some cases also those working as managers, educators or researchers. The numbers are based on head counts.

In countries where different nurses can hold different levels of qualification or role, the data include both "professional nurses" who have a higher level of education and perform more complex or skilled tasks, and "associate professional nurses" who have a lower level of education but are nonetheless recognised and registered as nurses. Health care assistants (or nursing aides) who are not recognised as nurses are excluded. The number of nurses in Denmark and Austria is lower than reported in previous editions because "caring personnel" (nursing aides) were formerly included for these two countries. Midwives are excluded, except in some countries where they are included at least in part because they are considered as specialist nurses, or for other categorisation reasons (Australia, Ireland and Spain).

Austria and Greece report only nurses working in hospitals, resulting in an under-estimation.

\section{References}

[1] Maier, C., L. Aiken and R. Busse (2017), "Nurses in advanced roles in primary care: Policy levers for implementation", OECD Health Working Papers, No. 98, OECD Publishing, Paris, https://dx.doi.org/10.1787/a8756593-en. 
Figure 8.10. Practising nurses per 1000 population, 2000 and 2017 (or nearest year)

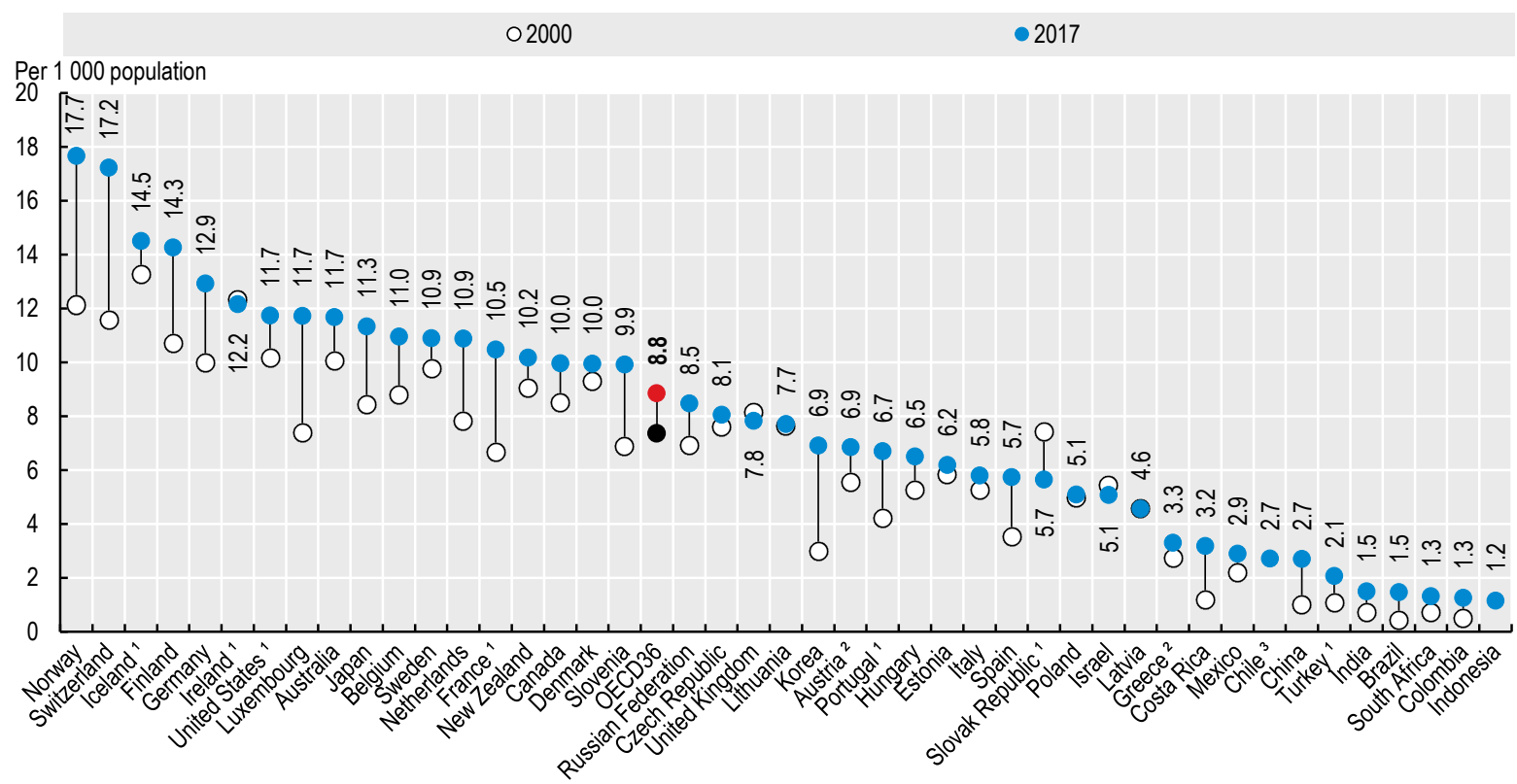

1. Data include not only nurses providing direct care to patients, but also those working in the health sector as managers, educators, researchers, etc. 2. Austria and Greece report only nurses employed in hospital. 3. Data in Chile refer to all nurses who are licensed to practice.

Source: OECD Health Statistics 2019.

StatLink क्ञाज् https://doi.org/10.1787/888934017386

Figure 8.11. Ratio of nurses to doctors, 2017 (or nearest year)

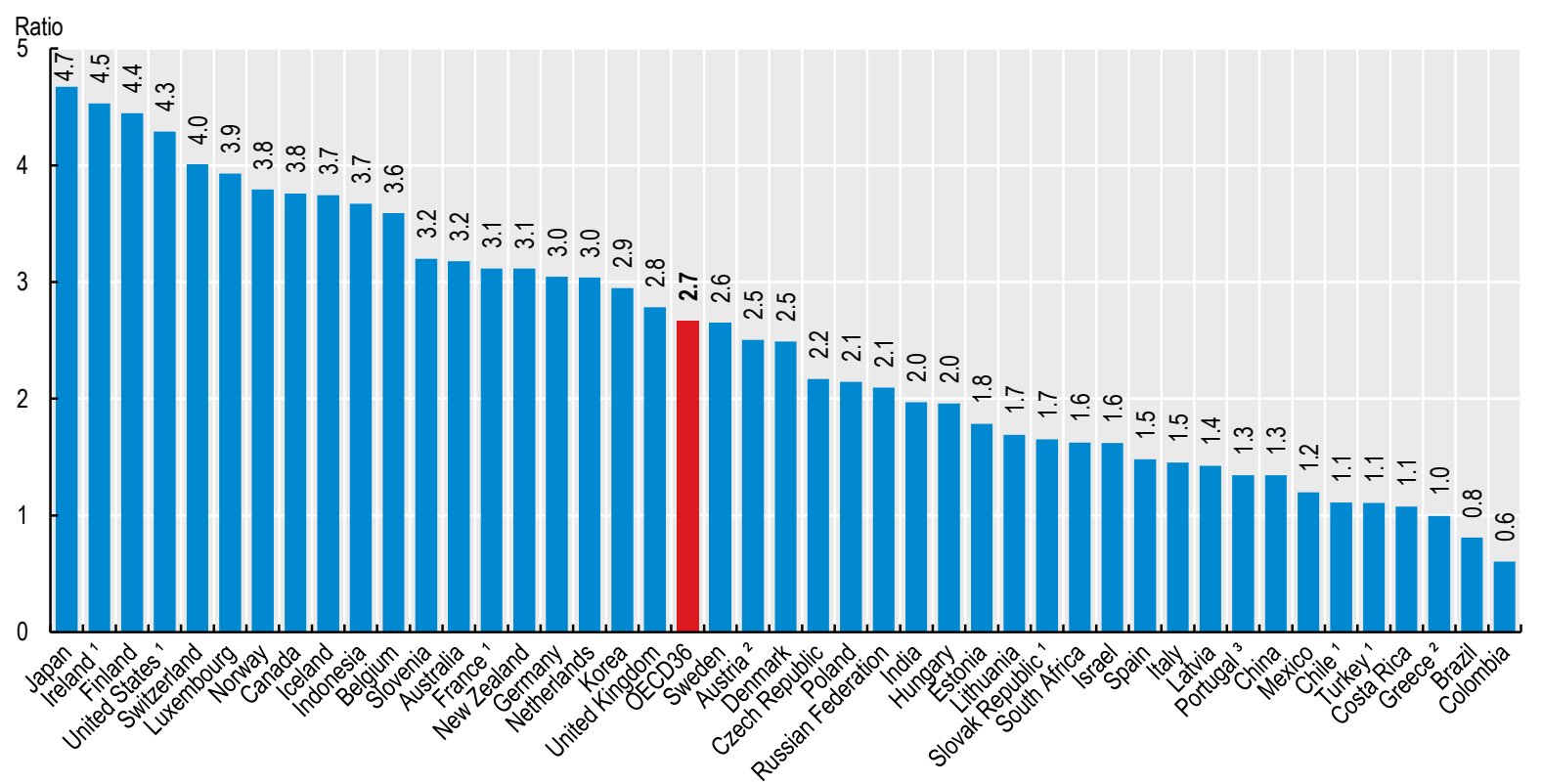

1. For countries that have not provided data for practising nurses and/or practising doctors, the numbers relate to the "professionally active" concept for both nurses and doctors (except Chile, where numbers include all nurses and doctors licensed to practise). 2. For Austria and Greece, the data refer to nurses and doctors employed in hospitals. 3. The ratio for Portugal is underestimated because the numerator refers to professionally active nurses while the denominator includes all doctors licensed to practise.

Source: OECD Health Statistics 2019. 
On average across OECD countries, the remuneration of hospital nurses was slightly above the average wage of all workers in 2017. In most countries, their remuneration was in the range of being about $10 \%$ lower than the average wage to $20 \%$ higher. However, in some countries like Lithuania and Latvia, nurses earn much less than the average wage of all workers, while in other countries like Chile, Mexico, Israel and Luxembourg, they earn much more (Figure 8.12). .

Converting the remuneration of hospital nurses to a common currency (here US dollars) and adjusting for purchasing power parity (PPP) reveals a sizeable variation in the income of hospital nurses across countries. In 2017, nurses in Luxembourg had remuneration levels six times higher than those working in Latvia and Lithuania (Figure 8.13). In general, nurses working in Central and Eastern European countries have the lowest levels of remuneration, explaining at least partly that many of them migrate to other EU countries (OECD, 2019[1]).

The remuneration of nurses in the United States is higher than in most other OECD countries, explaining why the United States is able to attract several thousands of nurses from other countries every year.

In most countries, the remuneration of nurses has increased since 2010, albeit at different rates (Figure 8.14). In some countries, like the Czech Republic and the Slovak Republic, nurses have obtained substantial pay raises in recent years. In the Czech Republic, nurses benefitted from a pay increase following protests of hospital workers in 2011 (although their pay rise was lower than that given to doctors), accompanied by improvements in other areas of their working conditions (OECD, 2016[2]). The remuneration of nurses in the Slovak Republic increased by about $40 \%$ between 2010 and 2017, and in 2018 the Slovak government also announced a further increase of at least $10 \%$ in the salaries of nurses and other non-medical health professionals.

In other countries, like Portugal and Spain, the remuneration of nurses fell after the 2008-09 economic crisis due to remuneration cuts in the public sector and have only recovered slowly in recent years. . This was also the case in Greece where the salaries of nurses decreased by about $25 \%$ between 2009 and 2015.

\section{Definition and comparability}

The remuneration of nurses refers to average gross annual income, including social security contributions and income taxes payable by the employee. It should normally include all extra formal payments, such as bonuses and payments for night shifts and overtime. In most countries, the data relate specifically to nurses working in hospitals, although in Canada the data also cover nurses working in other settings. In some federal states, such as Australia, Canada and the United States, the level and structure of nurse remuneration is determined at the sub-national level, which may contribute to variations across jurisdictions.

Data refer only to registered ("professional") nurses in Canada, Chile, Ireland and the United States, resulting in an over-estimation compared to other countries where lower-level ("associate professional") nurses are also included. Data for New Zealand include all nurses employed by publicly funded district health boards, registered and otherwise, and includes health assistants who have a different and significantly lower salary structure than registered nurses.

The data relate to nurses working full time, with the exception of Belgium, where the data provided also include part-time nurses (resulting in an underestimation). The data for some countries do not include additional income such as overtime payments and bonuses (e.g. Italy and Slovenia). Informal payments, which in some countries represent a significant part of total income, are not reported.

The income of nurses is compared to the average wage of full-time employees in all sectors in the country. The source for the average wage of workers in the economy is the OECD Employment Database.

\section{References}

[1] OECD (2019), Recent Trends in International Migration of Doctors, Nurses and Medical Students, OECD Publishing, Paris, https:// dx.doi.org/10.1787/5571ef48-en.

[2] OECD (2016), Health Workforce Policies in OECD Countries: Right Jobs, Right Skills, Right Places, OECD Health Policy Studies, OECD Publishing, Paris, https://dx.doi.org/10.1787/9789264239517-en. 
Figure 8.12. Remuneration of hospital nurses, ratio to average wage, 2017 (or nearest year)

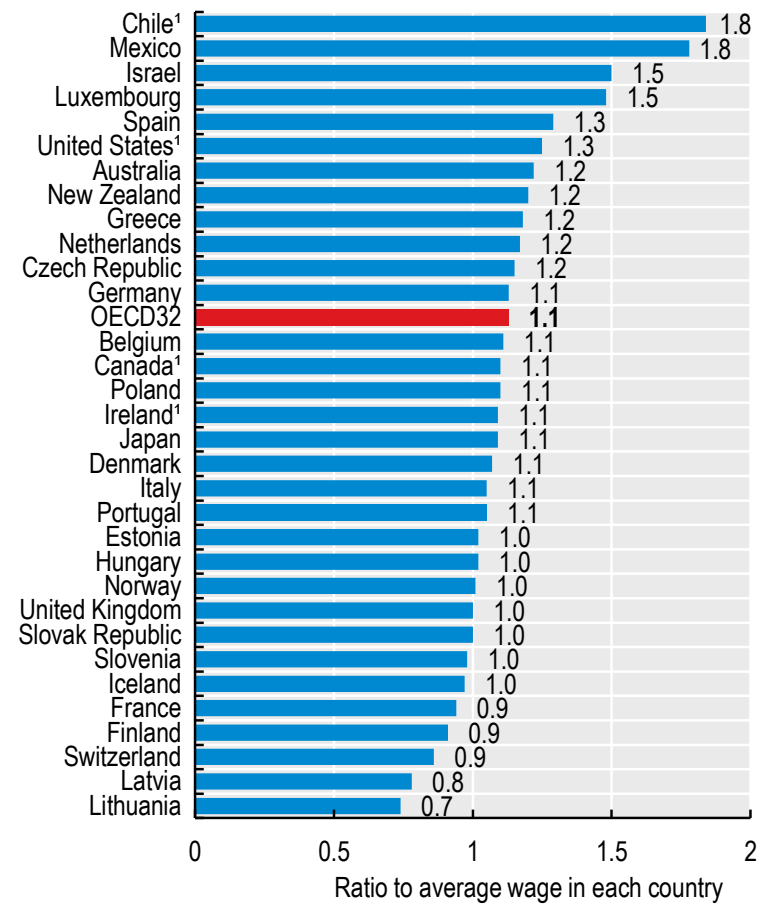

1. Data refer to registered ("professional") nurses in the United States, Canada, Ireland and Chile (resulting in an over-estimation).

Source: OECD Health Statistics 2019.

StatLink काist https://doi.org/10.1787/888934017424
Figure 8.13. Remuneration of hospital nurses, USD PPP, 2017 (or nearest year)

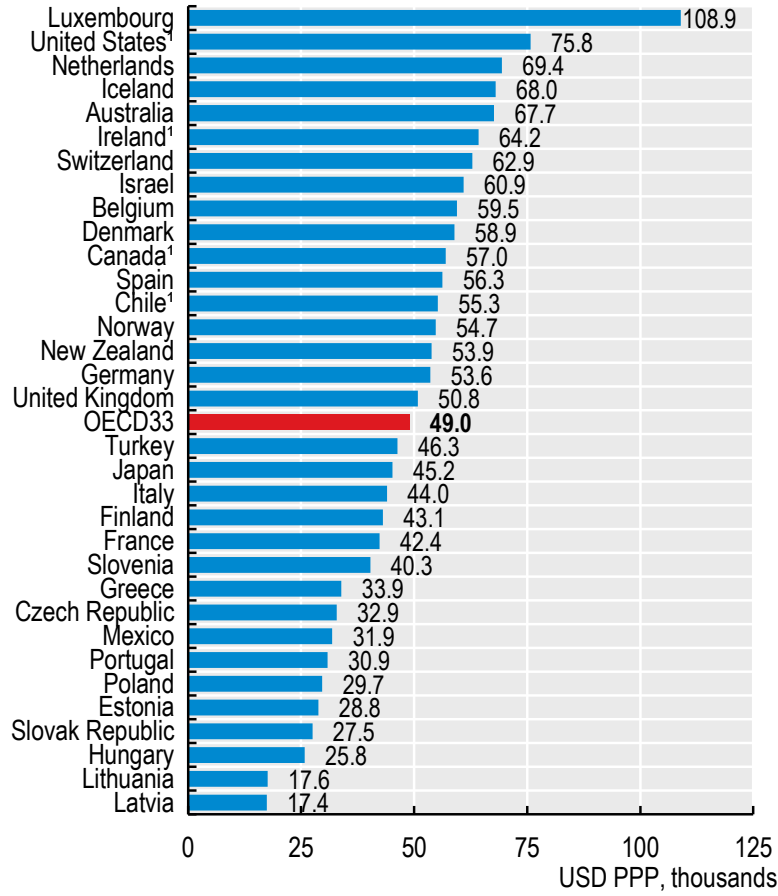

1. Data refer to registered ("professional") nurses in the United States, Canada, Ireland and Chile (resulting in an over-estimation).

Source: OECD Health Statistics 2019.

StatLink ans https://doi.org/10.1787/888934017443

Figure 8.14. Trends in the remuneration of hospital nurses in nominal terms, selected OECD countries, 2010-17
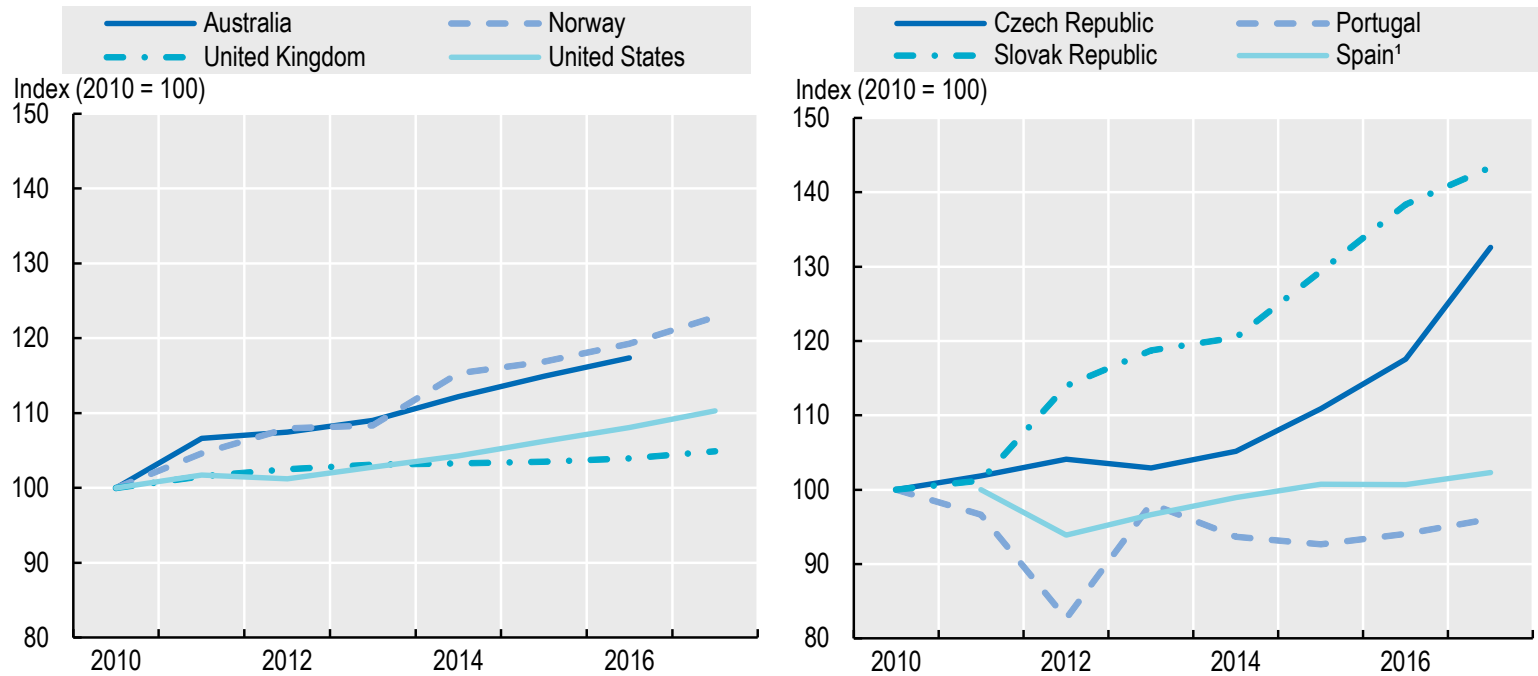

1. Index for Spain, $2011=100$.

Source: OECD Health Statistics 2019. 


\section{HEALTH WORKFORCE}

\section{Medical graduates}

On average across OECD countries in 2017, there were 13 new medical graduates per 100000 population (up from 12 in 2015). This ranges from about seven in countries such as Japan and Israel to more than 20 in Ireland and Denmark (Figure 8.15).

In Israel, the low number of domestic medical graduates is compensated by the high number (about 60\%) of foreigntrained doctors. Increasingly however, foreign-trained doctors consist of Israeli-born people returning after completing studies abroad. In contrast, Japan does not currently rely on foreign-trained doctors. However, Japan recently took action to increase the number of students admitted to medical schools (the numerus clausus), but this is not yet reflected in the number of new medical graduates due to lags. In Ireland, the high number of medical graduates reflects the large share of international medical students. In the academic year 2017/18, this share made up half of all medical students, with the majority coming from outside the OECD area. However, after obtaining their first medical degree, international medical students often leave Ireland due to difficulties in securing an internship - the last stage in medical education prior to postgraduate training. At the same time, Ireland compensates for its shortage of doctors by importing doctors trained in other countries (OECD, 2019[1]).

In all OECD countries except Greece, the number of new medical graduates per capita has risen since 2000. However increases have not been steady, with numbers falling to less than $90 \%$ of levels in 2000 (mostly during the 2000s) in Belgium, the Slovak Republic and Switzerland (countries close to the OECD average), as well as in Turkey, France, and Israel, with numbers considerably below the OECD average (OECD, 2019[1]).

In Latvia, Slovenia, Portugal and Australia, where annual numbers of new medical graduates per capita are above the OECD average, the number increased up to fourfold between 2007 and 2017. Twofold increases are common, and are found in countries with high, medium, and low numbers of new medical graduates per capita (Figure 8.16). In total, the number of medical graduates across OECD countries increased from less than 100000 in 2006 to nearly 120000 in 2017.
The growth of the number of doctors in the majority of the OECD countries since 2000 has been fueled predominantly by a rise in the number of domestic medical graduates. In most cases, this rise reflects goal-oriented policy decisions taken a few years earlier to raise the number of students admitted to medical schools. This was in response to concerns about current or possible future shortages of doctors. In some countries like Poland, as well as other central, and eastern European countries, the strong increase in recent years also reflects the growing number of international medical students and graduates. Polish medical schools, for example, offer medical studies in English, and $25 \%$ of all medical students are foreigners (OECD, 2019[1]).

In reply to the OECD Health System Characteristics Survey 2016, none of the responding OECD countries other than Italy and Spain reported that they had reduced admission rates for medical schools and most countries declared increases (OECD, 2016[2]). Hence, the number of new medical graduates can be expected to continue to increase in most countries in the coming years.

\section{Definition and comparability}

Medical graduates are defined as students who have graduated from medical schools in a given year. The data for Australia, Austria and the Czech Republic include foreign graduates, but other countries may exclude them.

\section{References}

[1] OECD (2019), Recent Trends in International Migration of Doctors, Nurses and Medical Students, OECD Publishing, Paris, https:// dx.doi.org/10.1787/5571ef48-en.

[2] OECD (2016), OECD Health System Characteristics Survey 2016, http://www.oecd.org/els/health-systems/characteristics.htm. 


\section{HEALTH WORKFORCE}

Figure 8.15. Medical graduates, 2017 (or nearest year)

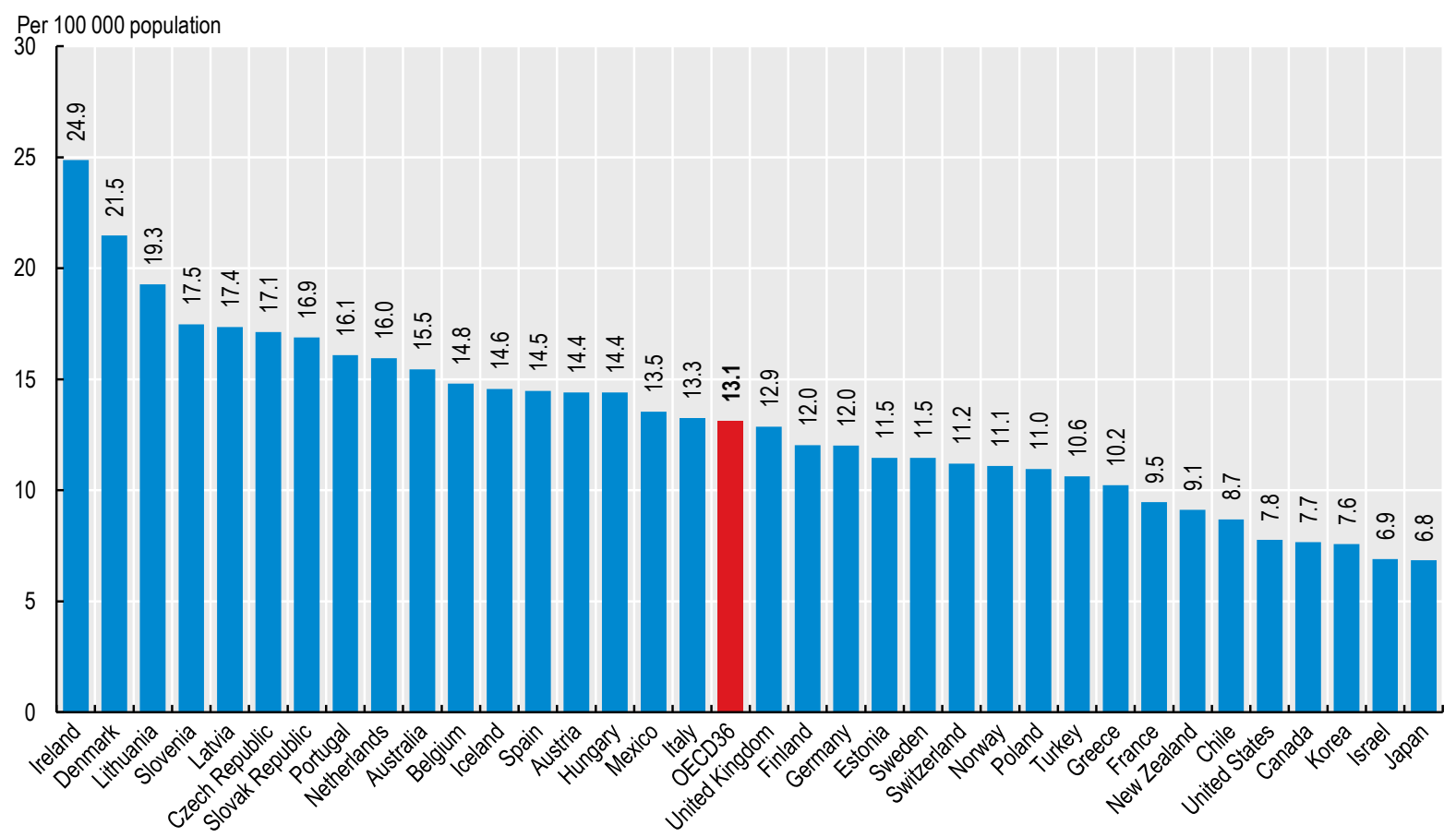

Source: OECD Health Statistics 2019.

StatLink बताs https://doi.org/10.1787/888934017481

Figure 8.16. Evolution in the number of medical graduates, selected OECD countries, 2000-17 (or nearest year)

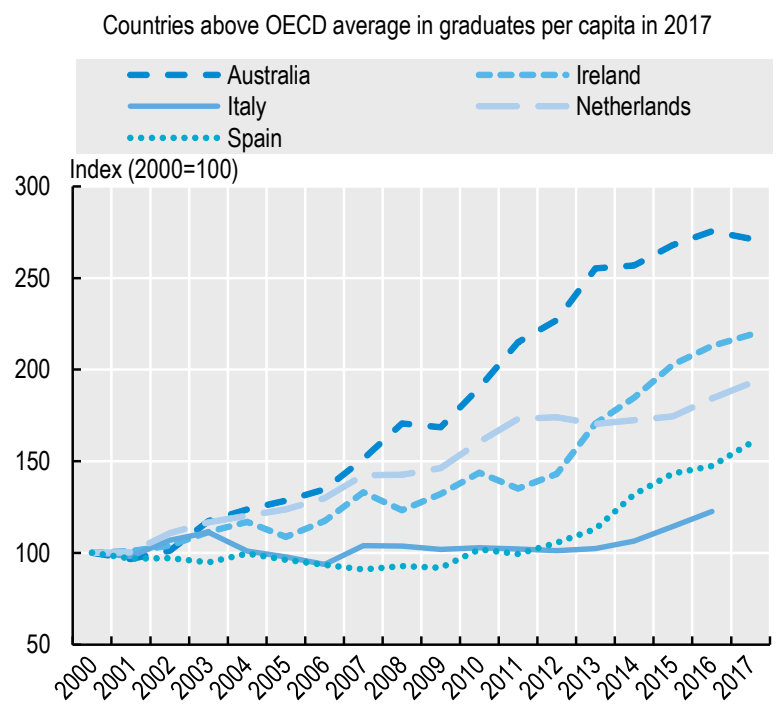

Countries below OECD average in graduates per capita in 2017

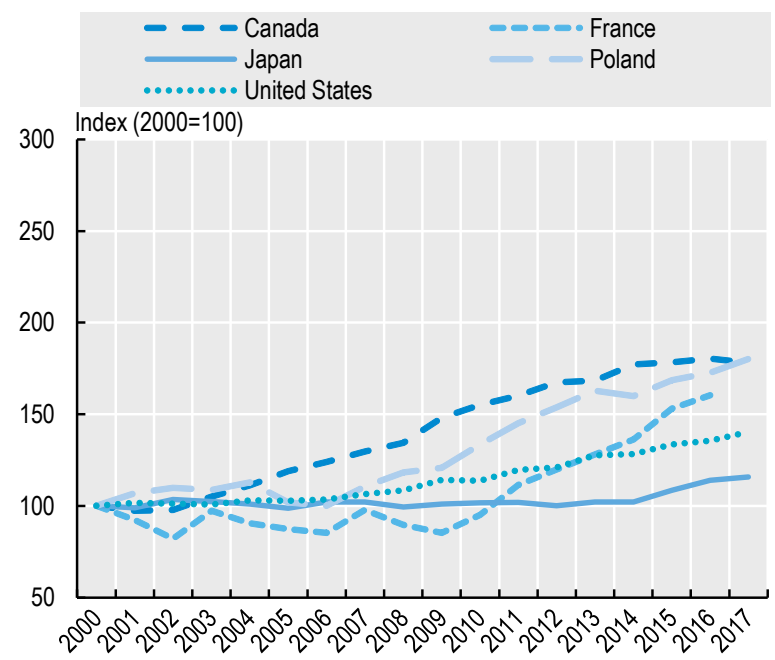

Source: OECD Health Statistics 2019. 


\section{HEALTH WORKFORCE}

\section{Nursing graduates}

On average across OECD countries in 2017, there were around 44 new nurse graduates per 100000 population, with a range from around 14 in the Czech Republic and Mexico to about 100 in Switzerland and Korea (Figure 8.17). This wide range may be explained by differences in the current number and age structure of the nursing workforce, in the capacity of nursing schools to take on more students, and in the future employment prospects of nurses.

Since 2000, the number of nursing graduates has increased in most OECD countries, with the exception of Luxembourg, Japan, the Czech Republic, Lithuania and Ireland. Of these countries, only Japan has maintained a number above the OECD average. In Finland, Hungary and Belgium, the number of nursing graduates has recently returned to numbers above the level in 2000 and is now well above the OECD average, after experiencing intermittent declines.

Despite a more than tenfold increase in the annual number of nursing graduates since 2000 in Poland, Turkey and Mexico, the numbers in these countries remain well below the OECD average. At least 50\% increases between 2000 and 2017 are common and are seen across countries with high, medium and low numbers of nurse graduates per capita (Figure 8.18). In total, the number of nurse graduates across OECD countries increased from about 450000 in 2006 to more than 550000 in 2017.

The increase in the number of nursing graduates in most casesreflects deliberate policy decisions taken a few years earlier to increase the number of students admitted to nursing schools, in response to concerns about current or possible future shortages (OECD, 2016[1]). In reply to the OECD Health System Characteristics Survey 2016, none of the responding OECD countries reported that they had reduced admission rates for nursing schools and many declared increases (OECD, 2016[2]). Hence, the number of nursing graduates can be expected to continue to increase in most countries in the coming years.

In Norway, the number of students admitted to and graduating from nursing education programmes has grown particularly since 2010, and the number of new nursing graduates in 2017 was one-third higher than in 2000, which should contribute to increasing the supply of nurses. However, as many as one in five recently graduated nurses work outside the health sector. This has led to the implementation of a series of measures in recent years to improve the working conditions of nurses to increase retention rates, including pay raises.

In Italy, the number of nurse graduates increased fairly rapidly in the 2000s but has levelled off and even decreased slightly in recent years. While the number of students admitted to nursing education programmes has remained more or less stable during this decade, there has been a sharp drop in the number of applicants (with the number cut by half), signalling reduced student interest in the profession.

\section{Definition and comparability}

Nursing graduates refer to students who have obtained a recognised qualification required to become a licensed or registered nurse. They include graduates from both higher-level and lower-level nursing programmes. They exclude graduates from Masters or PhD degrees in nursing to avoid double-counting nurses acquiring further qualifications.

The data for the United Kingdom are based on the number of new nurses receiving an authorisation to practise.

\section{References}

[1] OECD (2016), Health Workforce Policies in OECD Countries: Right Jobs, Right Skills, Right Places, OECD Health Policy Studies, OECD Publishing, Paris, https://dx.doi.org/10.1787/9789264239517-en.

[2] OECD (2016), OECD Health System Characteristics Survey 2016, http://www.oecd.org/els/health-systems/characteristics.htm. 


\section{HEALTH WORKFORCE}

\section{Figure 8.17. Nursing graduates, 2017 (or nearest year)}

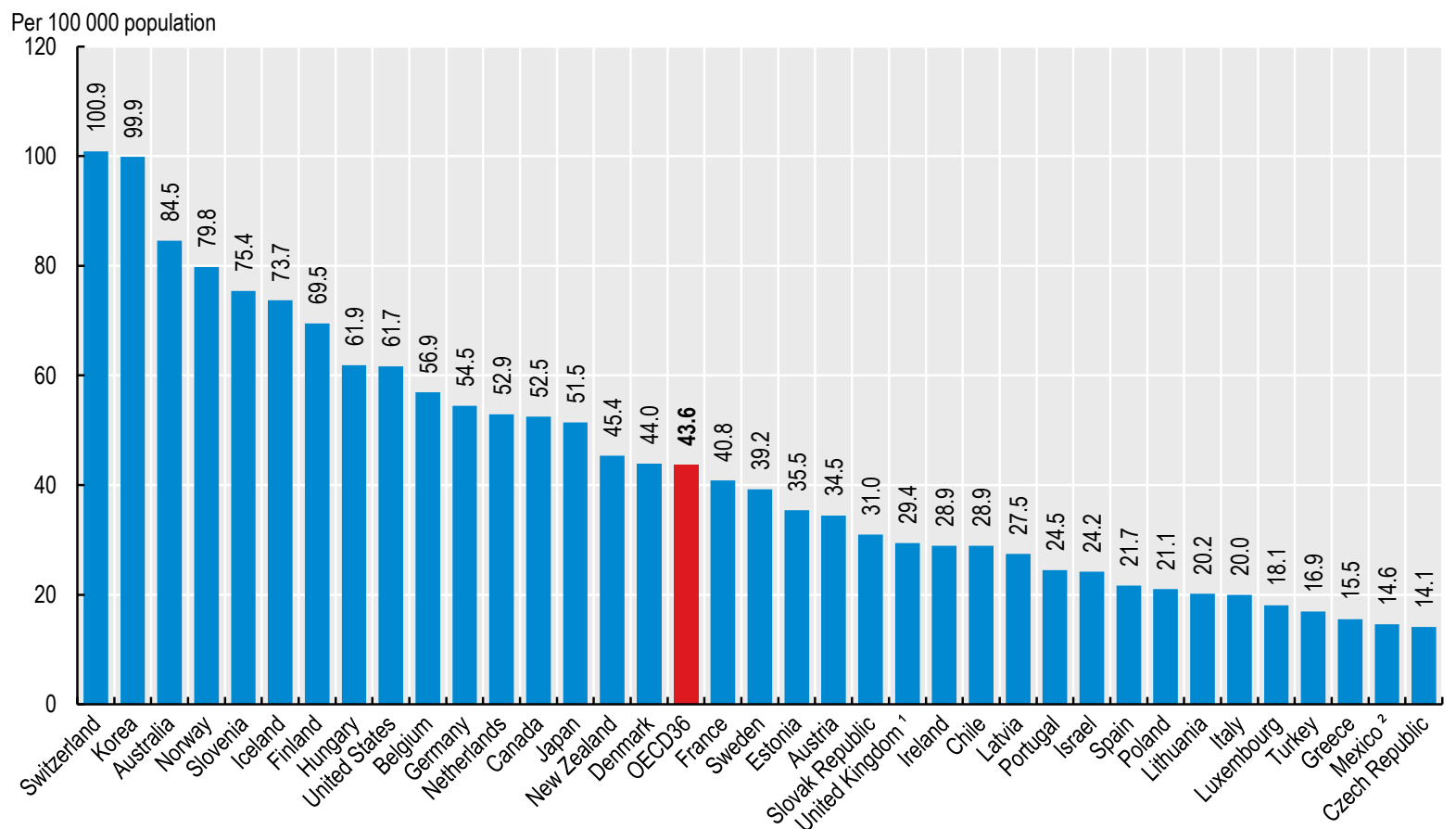

1. In the United Kingdom, the numbers refer to new nurses receiving an authorisation to practise, which may result in an over-estimation if these include foreign-trained nurses. 2. In Mexico, the data include professional nursing graduates only.

Source: OECD Health Statistics 2019.

StatLink त्ञाs https://doi.org/10.1787/888934017519

Figure 8.18. Evolution in the number of nursing graduates, selected OECD countries, 2000-17

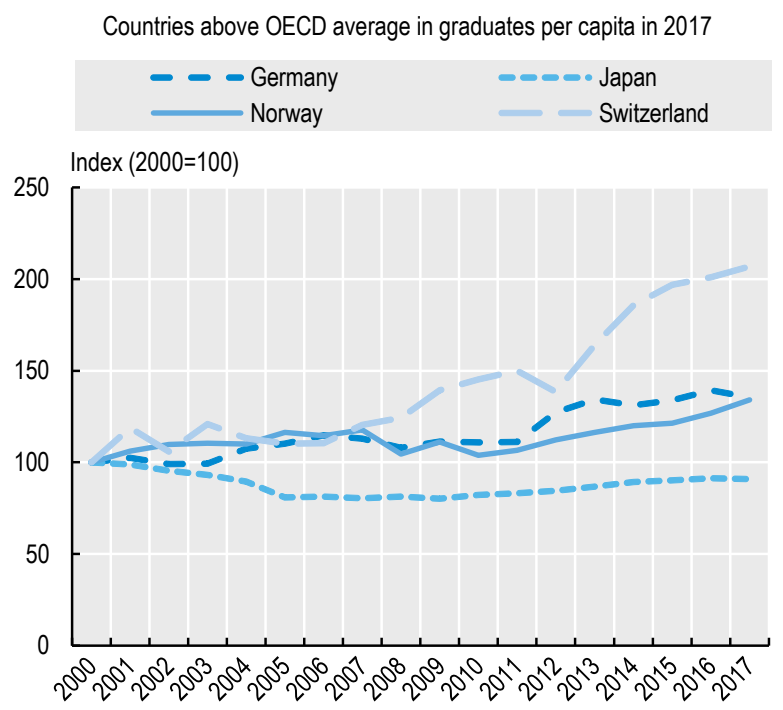

Countries below OECD average in graduates per capita in 2017

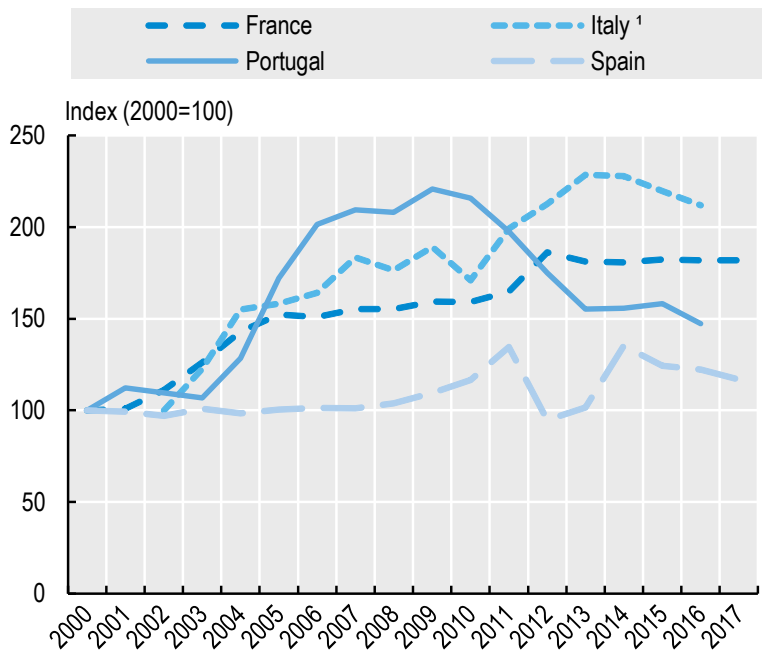

1. Index for Italy, $2002=100$

Source: OECD Health Statistics 2019. 
The number and share of foreign-trained doctors - and in some countries foreign-trained nurses - working in OECD countries has continued to rise over the past decade (OECD, 2019[1]). In 2017, more than one in six doctors working in OECD countries had obtained at least their first medical degree in another country (Figure 8.19), up from one in seven a decade earlier. For nurses, on average, one in 17 had obtained a nursing degree in another country in 2017 (Figure 8.20). These developments occurred in parallel with a significant increase in the numbers of domestically trained medical and nursing graduates in nearly all OECD countries (see also indicators on "Medical graduates" and "Nursing graduates"), which is indicative of substantial demand for these professionals.

In 2017, the share of foreign-trained doctors ranged from less than 3\% in Turkey, Lithuania, Italy, the Netherlands and Poland, to around 40\% in Norway, Ireland and New Zealand, and to nearly $60 \%$ in Israel. In most OECD countries, the share of foreign-trained nurses is below 5\%, but Australia, Switzerland and New Zealand have proportions of around or above $20 \%$. However, in some cases, foreign-trained doctors and nurses consists of people born in the country who studied abroad but have returned. In a number of countries (including Israel, Norway, Sweden and the United States), this share is large and growing. These foreign-trained but native-born doctors and nurses frequently paid the full cost of their studies abroad. In 2017 in Israel, for example, around $40 \%$ of foreign-trained doctors and nurses are native-born.

The share of foreign-trained doctors in various OECD countries evolved between 2000 and 2017 (Figure 8.21). The share remained relatively stable in the United States, with the number of foreign and domestically trained doctors increasing at a similar rate. However, among the medical graduates with a foreign degree who obtained certification to practise in the United States in 2017, one-third were American citizens, up from 17\% in 2007 (OECD, 2019[1]). In Europe, the share of foreign-trained doctors increased rapidly in Norway and Sweden. However, in Norway more than one half of foreign-trained doctors are Norwegianborn, returning after studying abroad. In Sweden, the number of foreign-trained but native-born doctors quadrupled since 2006, accounting for nearly one-fifth of foreign-trained doctors in 2015. In France and Germany, the number and share of foreign-trained doctors has also increased steadily over the past decade (with the share doubling from $5-6 \%$ of all doctors in 2007 to $11-12 \%$ in 2017). Conversely, in the United Kingdom, the share of foreigntrained doctors decreased slightly, as the number of domestically-trained doctors increased more rapidly.

The share of foreign-trained nurses has increased steadily over the past decade in Australia, Canada and New Zealand, although in New Zealand, a slight decline occurred between
2016 and 2017 (Figure 8.22). In Israel, the share of foreigntrained nurses has decreased over time, but has stagnated at around 9\% since 2015. In France, while the share of foreigntrained nurses is relatively low, the number has nearly doubled over the past decade. In Italy, the number of foreign-trained nurses increased sharply between 2007 and 2015 (driven mainly by the arrival of nurses trained in Romania following its accession to the EU in 2007), but the number and share have started to decrease in recent years.

\section{Definition and comparability}

The data relate to foreign-trained doctors and nurses working in OECD countries measured in terms of total stocks. The OECD health database also includes data on the annual flows for most of the countries shown here, as well as by country of origin. The data sources in most countries are professional registries or other administrative sources.

The main comparability limitation relates to differences in the activity status of doctors and nurses. Some registries are regularly updated, making it possible to distinguish doctors and nurses who are still actively working in health systems, while other sources include all doctors and nurses licensed to practice, regardless of whether they are still active. The latter will tend to over-estimate not only the number of foreign-trained doctors and nurses, but also the total number of doctors and nurses (including those trained domestically), making the impact on the share unclear.

The data source in some countries includes interns and residents, while these physicians in training are not included in other countries. Because foreigntrained doctors are often over-represented in the categories of interns and residents, this may result in an under-estimation of the share of foreign-trained doctors in countries where they are not included (such as Austria, France and Switzerland).

The data for Germany (on foreign-trained doctors) and for some regions in Spain are based on nationality (or place of birth in the case of Spain), not on the place of training.

\section{References}

[1] OECD (2019), Recent Trends in International Migration of Doctors, Nurses and Medical Students, OECD Publishing, Paris, https:// dx.doi.org/10.1787/5571ef48-en. 
Figure 8.19. Share of foreign-trained doctors, 2017 (or nearest year)

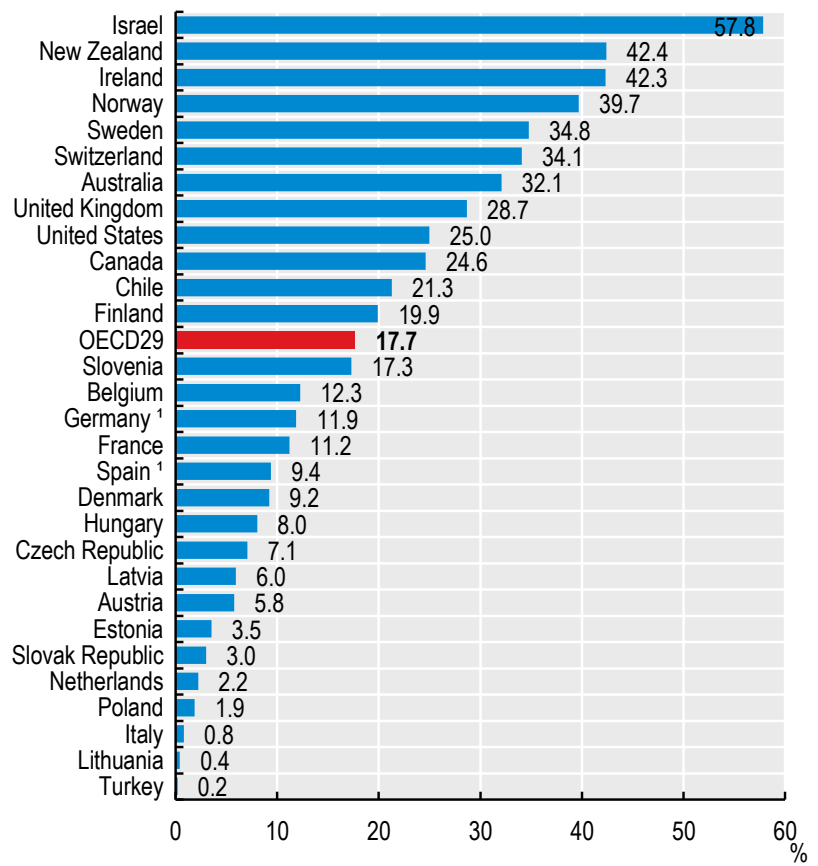

1. In Germany and some regions in Spain data based on nationality (or place of birth in the case of Spain), not on the place of training. Source: OECD Health Statistics 2019.

StatLink 게개 https://doi.org/10.1787/888934017557

Figure 8.21. Evolution in the share of foreign-trained doctors, selected OECD countries, 2000-17

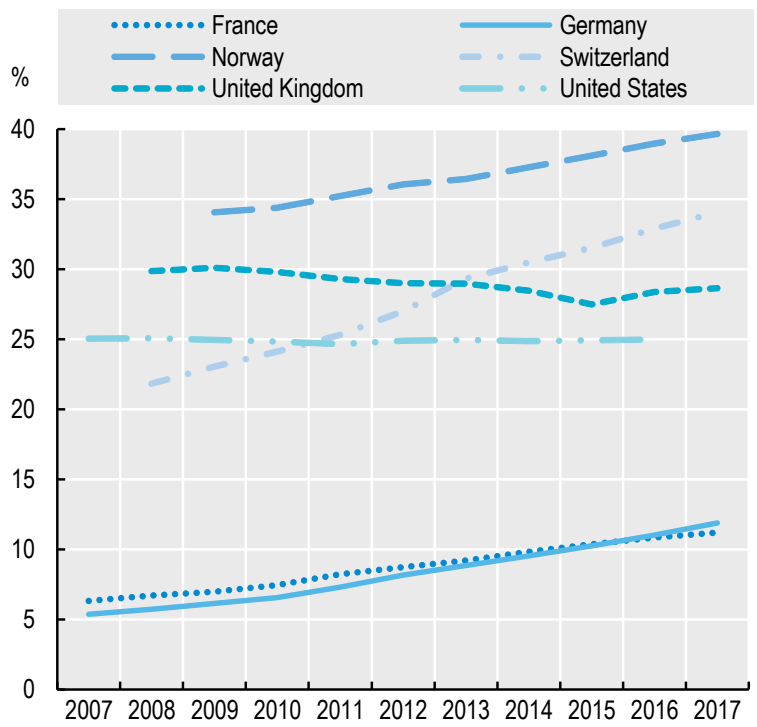

Source: OECD Health Statistics 2019.

StatLink तiाs
Figure 8.20. Share of foreign-trained nurses, 2017 (or nearest year)

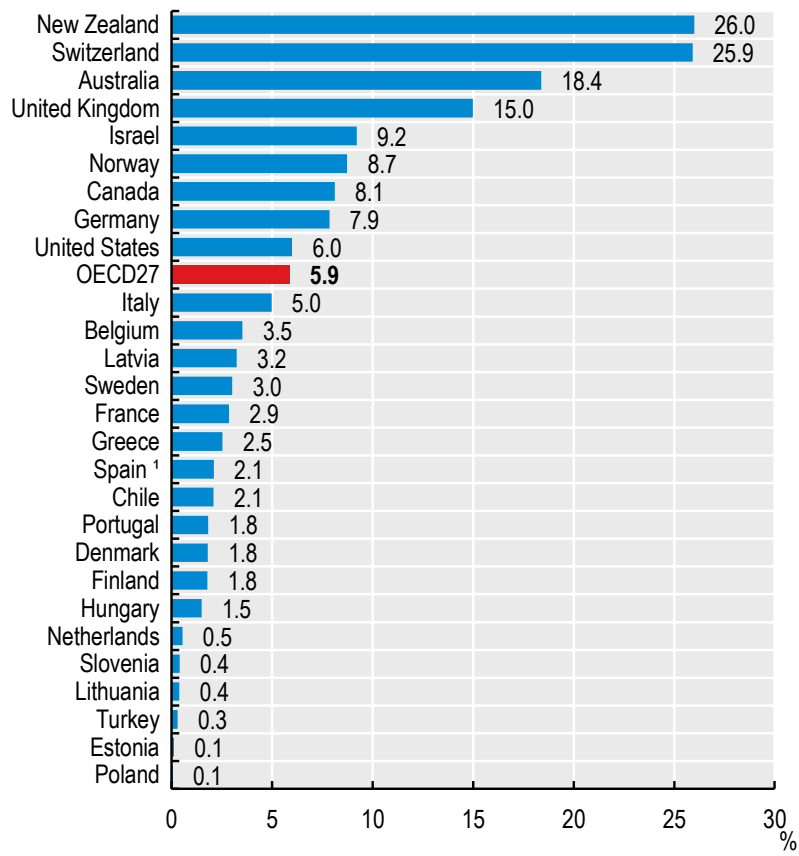

1. Data for some regions in Spain based on nationality or place of birth, not on the place of training.

Source: OECD Health Statistics 2019

StatLink काIs https://doi.org/10.1787/888934017576

Figure 8.22. Evolution in the share of foreign-trained nurses, selected OECD countries, 2000-17

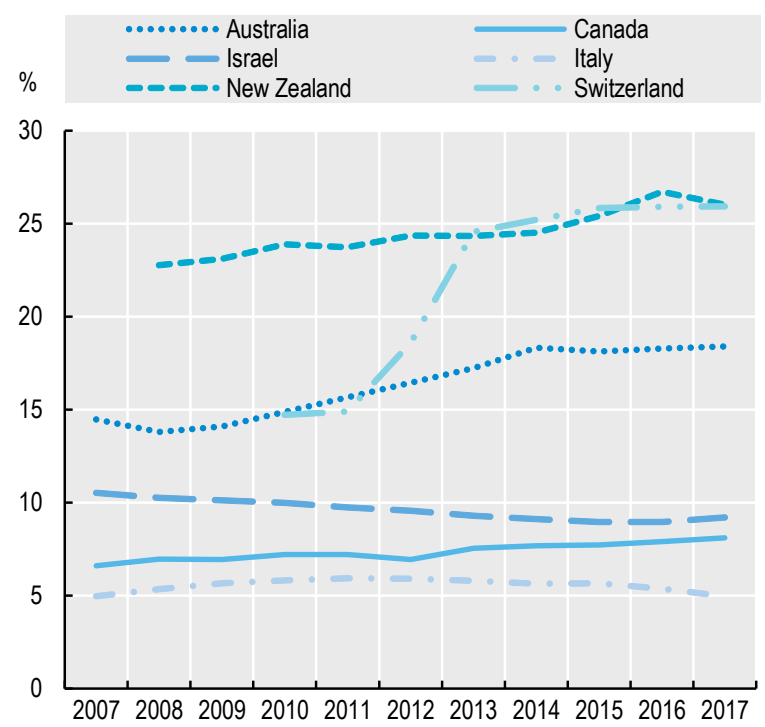

Source: OECD Health Statistics 2019

StatLink AाIs https://doi.org/10.1787/888934017614 


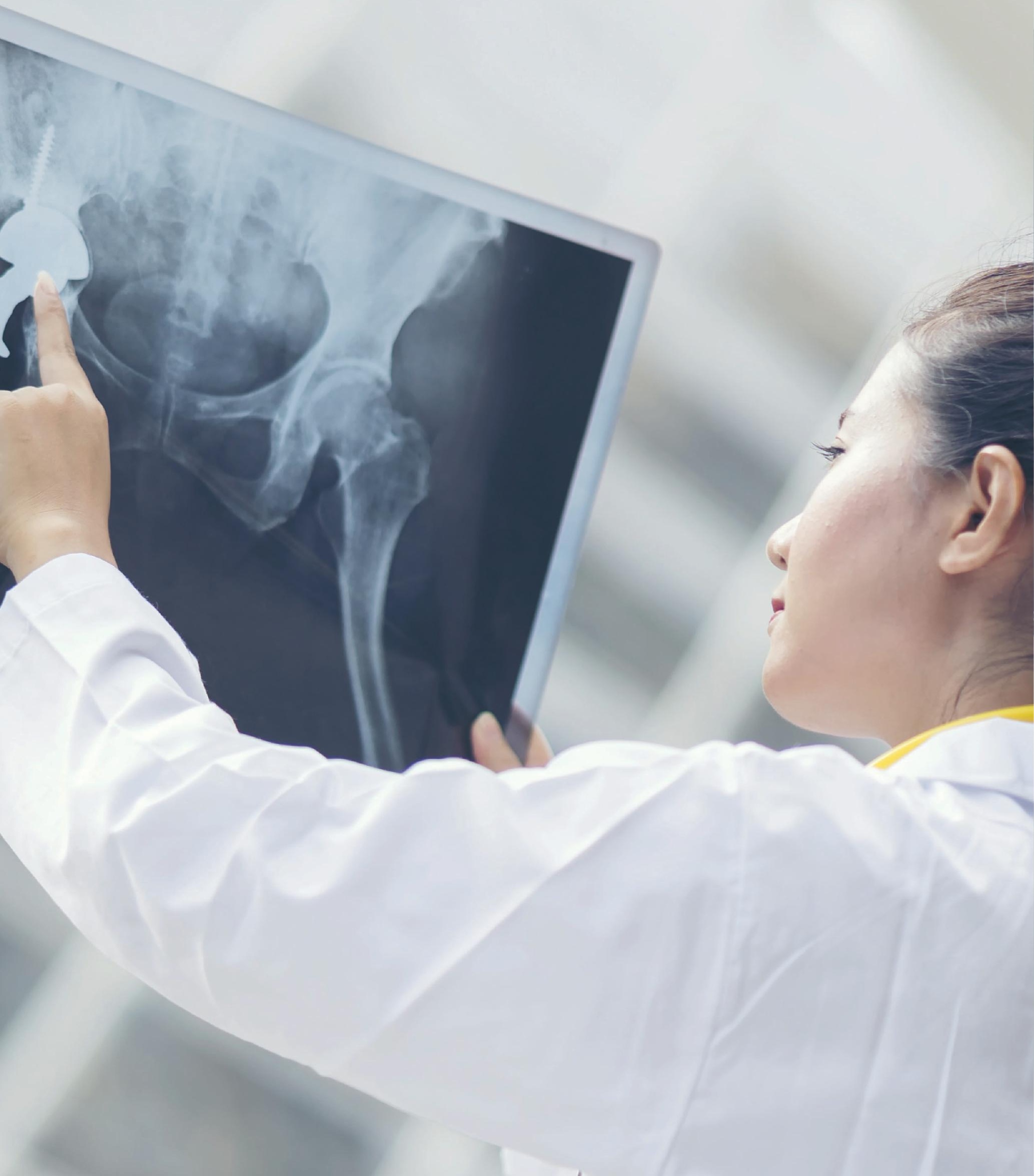




\section{HEALTH CARE ACTIVITIES}

Consultations with doctors

Medical technologies

Hospital beds and discharge rates

Average length of stay in hospitals

Hip and knee replacement

Caesarean sections

Ambulatory surgery

The statistical data for Israel are supplied by and under the responsibility of the relevant Israeli authorities. The use of such data by the OECD is without prejudice to the status of the Golan Heights, East Jerusalem and Israeli settlements in the West Bank under the terms of international law. 
Consultations with doctors are, for many people, the most frequent contact with health services, and often provide an entry point for subsequent medical treatment. Consultations can take place in doctors' clinics, hospital outpatient departments or, in some cases, patients' own homes. Increasingly, consultations occur online or by video call, to improve access for remote populations, or for consultations after regular hours.

In 2017, the number of doctor consultations per person ranged from less than 3 in Mexico and Sweden, to almost 17 in Korea (Figure 9.1). The OECD average was 6.8 consultations per person per year, with most countries reporting between four and ten. Among key partners, consultation rates were also less than 3 in Colombia, Costa Rica, South Africa and Brazil.

Cultural factors play a role in explaining some of the variations across countries, but incentive structures also matter. Provider payment methods and the levels of copayments are particularly relevant. For example, in Korea and Japan, health providers are paid through fee-for-service, thus creating incentives for overprovision of services, while countries with mostly salaried doctors tend to have belowaverage rates (e.g. Mexico, Finland and Sweden). However, in Switzerland and the United States, doctors are paid mainly by fee-for-service but consultation rates are below average. In these countries, patient co-payments can be high, which may result in patients not consulting a doctor because of the cost of care (see indicators in Chapter 5 on access).

Recent reforms to expand the role of nurses across many OECD countries can also partially explain low rates of consultations with doctors. This may involve nurses working as generalists to support GPs, focusing on health promotion, or as single-disease specialists. In many cases, nurses also have the authority to prescribe pharmaceuticals and order medical tests and exams. In Canada, Finland, Ireland, New Zealand, Sweden, the United Kingdom and the United States, nurses are authorised to work at high levels of advanced practice in primary care - in all these countries doctor consultation rates are below the OECD average (Maier, Aiken and Busse, 2017[1]).

The average number of doctor consultations per person across OECD countries has remained relatively stable since 2000 (between 6.5 and 6.8). However, some countries have seen large increases over time (Germany, Korea, Lithuania and Turkey), while in a few countries, numbers have fallen. This was the case in Japan and Spain, although consultations remain above the OECD average in both countries.

Information on the number of doctor consultations per person can be used to estimate the annual numbers of consultations per doctor. This indicator should not be taken as a measure of doctors' productivity, since consultations vary in length and effectiveness; and because it excludes services doctors deliver for hospital inpatients, as well as time spent on research and administration. Keeping these comparability issues in mind, the estimated number of consultations per doctor is highest in Korea, Japan and
Turkey (Figure 9.2). Numbers were lowest in Sweden and Norway, where consultations with doctors in both primary care and hospital settings tend to be focused towards patients with more severe and complex cases.

The number and type of doctor consultations can vary among different socio-economic groups. Wealthier individuals are more likely to see a doctor than individuals in the lowest income quintile, for a comparable level of need (see indicator on "Use of primary care services" in Chapter 5). Income inequalities in accessing doctors are much more marked for specialists than for general practitioner consultations (OECD, 2019 [2]).

\section{Definition and comparability}

Consultations with doctors refer to the number of contacts with physicians, including generalists and specialists. There are variations across countries in the physicians counted (e.g. physicians on parental or sick leave) and in the coverage of these consultations, notably in outpatient departments of hospitals. Data come mainly from administrative sources, although in some countries (Ireland, Italy, Netherlands, New Zealand, Spain and Switzerland) the data come from health interview surveys. Estimates from administrative sources tend to be higher than those from surveys because of problems with recall and nonresponse rates.

In Hungary, figures include consultations for diagnostic exams such as CT and MRI scans (resulting in an over-estimation). Figures for the Netherlands exclude contacts for maternal and child care. Data for Portugal exclude visits to private practitioners (resulting in an under estimation). In Germany, data include only the number of cases of physicians' treatment according to reimbursement regulations of the social health insurance scheme. This may lead to both underestimation (a case only counts the first contact over a three-month period, even if the patient consults a doctor more often) and overestimation (contacts that are not face-to-face, such as laboratory testing, are counted). Telephone contacts are included in a few countries (e.g. Spain). In Turkey, the most consultations with doctors occur in outpatient departments in hospitals.

\section{References}

[1] Maier, C., L. Aiken and R. Busse (2017), "Nurses in advanced roles in primary care: Policy levers for implementation", OECD Health Working Papers, No. 98, OECD Publishing, Paris, https://dx.doi.org/10.1787/a8756593-en.

[2] OECD (2019), Health for Everyone? Social Inequalities in Health and Health Systems, OECD Publishing, Paris, https://doi.org/ 10.1787/3c8385d0-en. 
Figure 9.1. Number of doctor consultations per person, 2000 and 2017 (or nearest year)

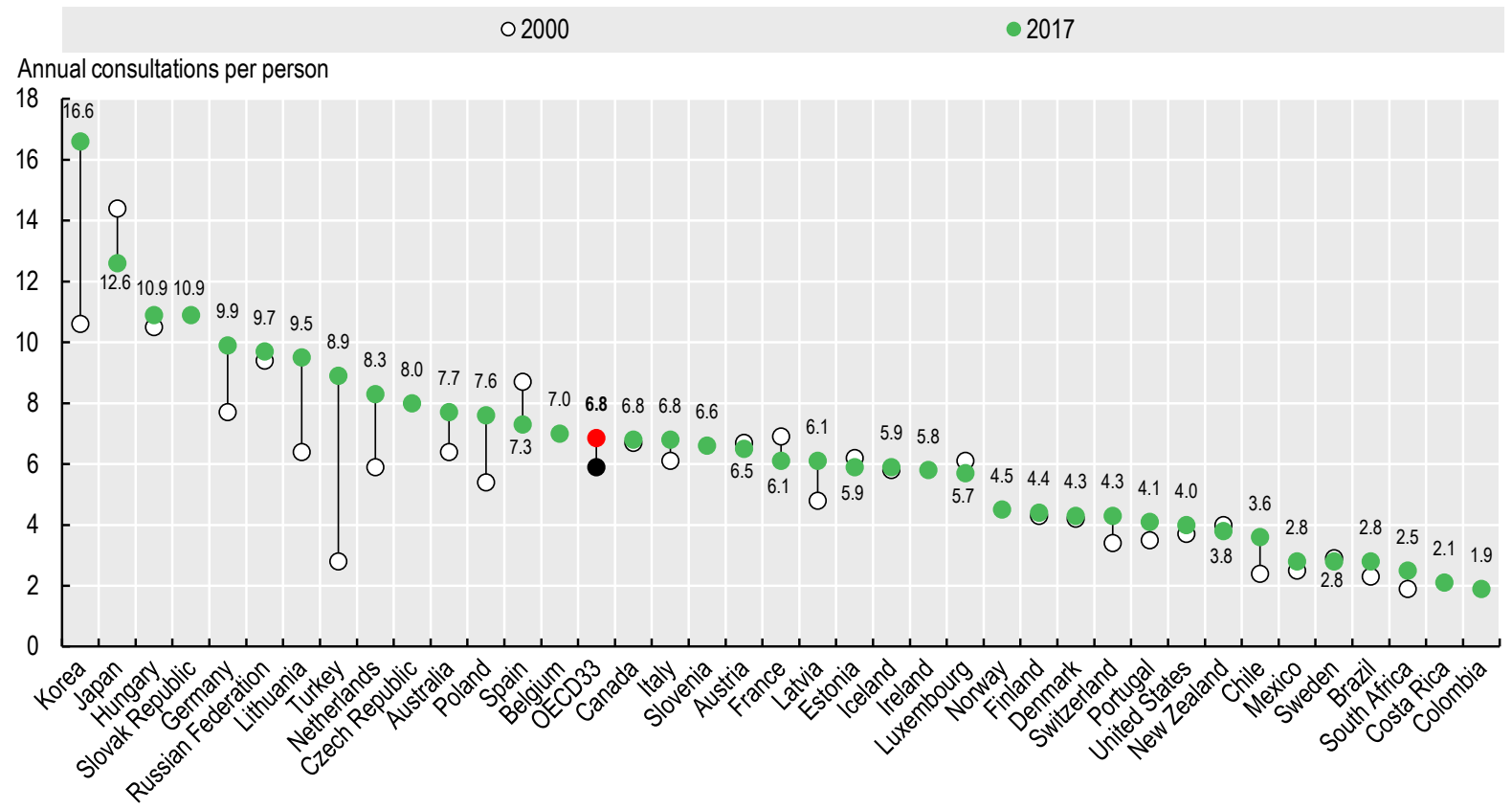

Source: OECD Health Statistics 2019.

Figure 9.2. Estimated number of consultations per doctor, 2017 (or nearest year)

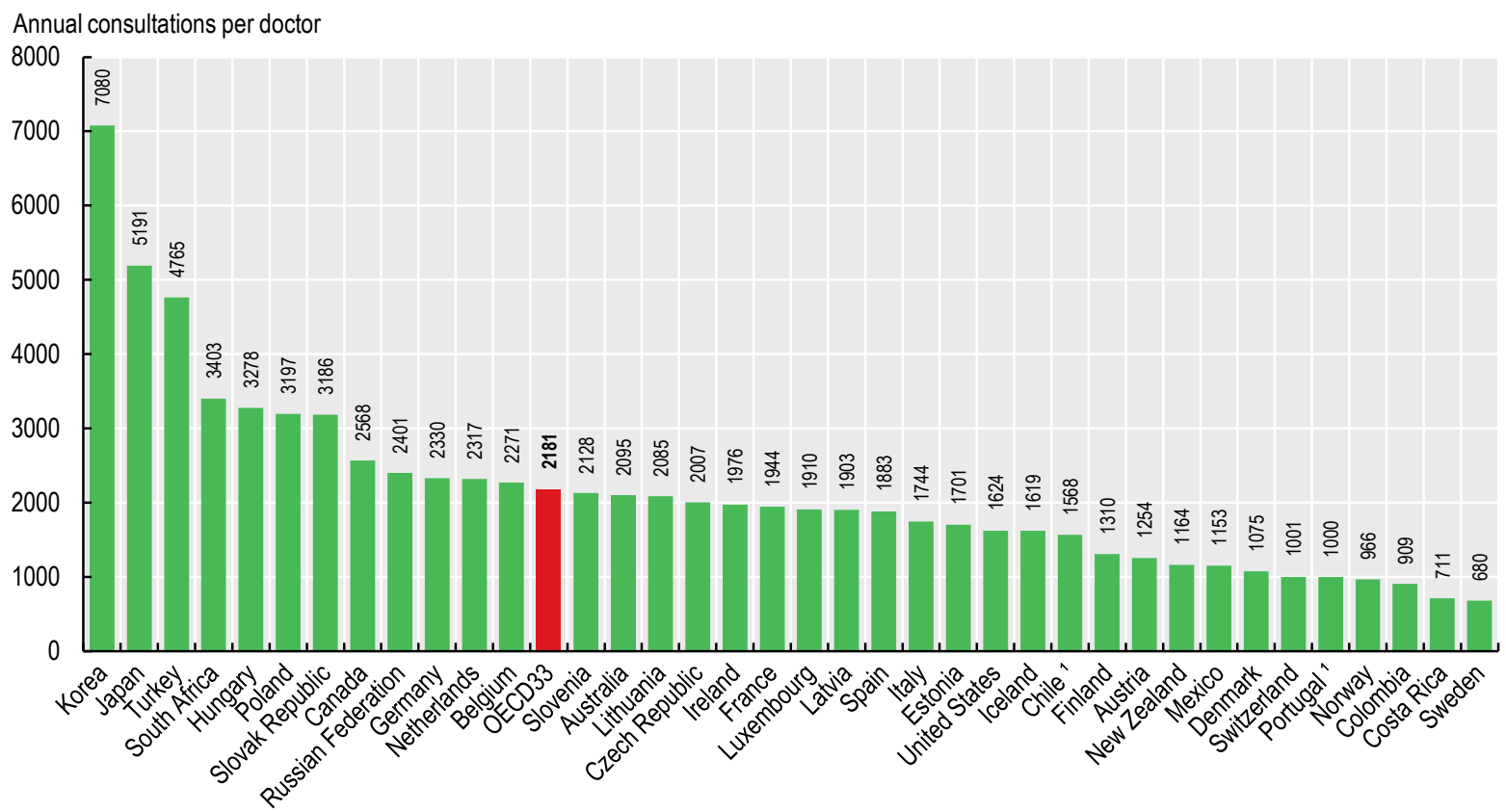

1. In Chile and Portugal, data for the denominator include all doctors licensed to practice. Source: OECD Health Statistics 2019. 
Technology plays an important role in the health system, allowing physicians to better diagnose and treat patients. However, new technologies can also drive up costs, and are commonly acknowledged to be one of the main causes behind increases in health spending (Lorenzoni et al 2019[1]). This section presents data on the availability and use of two diagnostic imaging technologies: computed tomography (CT) scanners and magnetic resonance imaging (MRI) units. CT and MRI exams help physicians diagnose a range of conditions.

The availability of CT scanners and MRI units has increased rapidly in most OECD countries over the past two decades. Japan has by far the highest number of MRI units and CT scanners per capita, followed by the United States for MRI units and by Australia for CT scanners (Figure 9.3). Austria, Germany, Greece, Iceland, Italy, Korea and Switzerland also have significantly more MRI and CT scanners per capita than the OECD average. The number of MRI units and CT scanners per population is the lowest in Mexico, Hungary, Israel and the United Kingdom. It is also comparatively low in Colombia, Costa Rica and the Russian Federation.

There is no general guideline or international benchmark regarding the ideal number of CT scanners or MRI units per million population. However, too few units may lead to access problems in terms of geographic proximity or waiting times. If there are too many, this may result in overuse of these costly diagnostic procedures, with little if any benefits for patients.

Data on the use of these diagnostic scanners are available for most OECD countries. The number of MRI examinations per capita is highest in Germany, the United States, Japan and France, all of which have more than 100 MRI exams per 1000 population (Figure 9.4). In France, the (absolute) number of MRI exams more than doubled between 2007 and 2017. The number of CT exams per capita is highest in the United States, followed by Japan and Iceland (Figure 9.5). There are large variations in the use of CT scanners and MRI units not only across but also within countries - for example, in Belgium, recent analysis shows a $50 \%$ variation in the use of diagnostic exams of the spine across provinces in 2017, and this variation is even larger across smaller areas (INAMI/RIVIZ, 2019[2]), .

Clinical guidelines exist in several OECD countries to promote more rational use of MRI and CT exams. Through the Choosing Wisely campaign, which began in the United States in 2012 and has been emulated in a growing number of countries since, some medical societies have identified cases when an MRI or CT exam is not necessary. For example, the Royal College of Physicians in the United Kingdom recommends, based on evidence from the National Institute for Health and Care Excellence (NICE), that patients with low back pain or suspected migraine do not routinely need an imaging test (Choosing Wisely UK, 2018[3]).

\section{Definition and comparability}

The data in most countries cover MRI units and CT scanners installed both in hospitals and the ambulatory sector, but coverage is more limited in some countries. MRI units and CT scanners outside hospitals are not included in Belgium, Portugal, Sweden and Switzerland (for MRI units). For the United Kingdom, the data only include equipment in the public sector. For Australia and Hungary, the number of MRI units and CT scanners includes only those eligible for public reimbursement.

Similarly, MRI and CT exams performed outside hospitals are not included in Austria, Portugal, Switzerland and the United Kingdom. In Australia, the data only include exams for private patients (in or out of hospitals); while in Korea and the Netherlands they only include publicly financed exams.

\section{References}

[3] Choosing Wisely UK (2018), "Clinical Recommendations: Royal College of Physicians", http:// www.choosingwisely.co.uk/.

[2] INAMI/RIVIZ (2019), "Medical Practice Variations", https:// www.healthybelgium.be/en/medical-practice-variations.

[1] Lorenzoni, L. et al. (2019), "Health Spending Projections to 2030: New results based on a revised OECD methodology", OECD Health Working Papers, No. 110, OECD Publishing, Paris, https://doi.org/10.1787/5667f23d-en.

[4] OECD (2014), Geographic Variations in Health Care: What Do We Know and What Can Be Done to Improve Health System Performance?, OECD Health Policy Studies, OECD Publishing, Paris, http://dx.doi.org/10.1787/9789264216594-en. 
9. HEALTH CARE ACTIVITIES

Medical technologies

Figure 9.3. CT scanners and MRI units, 2017 (or nearest year)

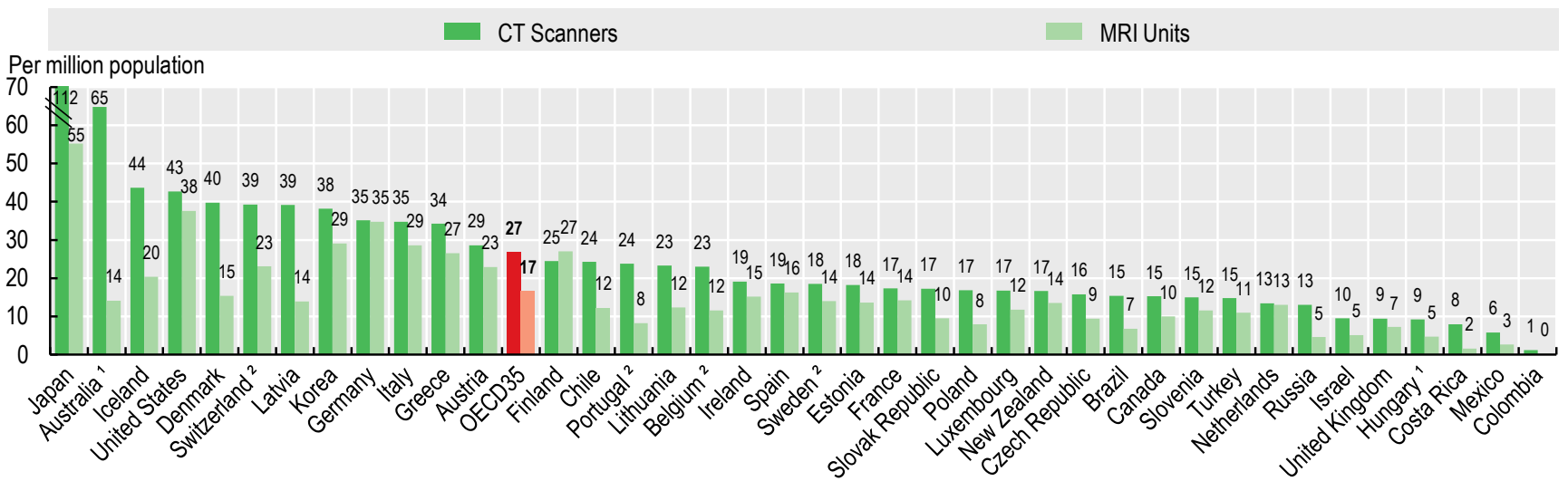

1. Only equipment eligible for public reimbursement. 2. Equipment outside hospital not included. For Switzerland, this only applies for MRI units. Source: OECD Health Statistics 2019.

StatLink Aisk https://doi.org/10.1787/888934017671

Figure 9.4. MRI exams, 2007 and 2017 (or nearest year)

○ 2007

2017

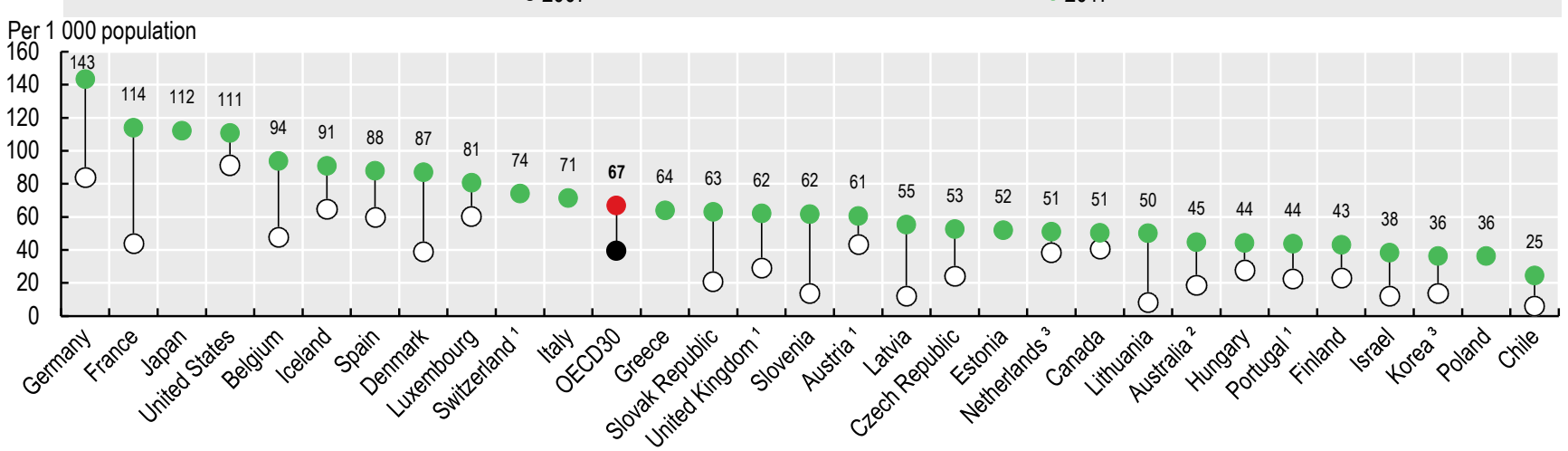

1. Exams outside hospital not included. 2. Exams on public patients not included. 3. Exams privately funded not included.

Source: OECD Health Statistics 2019.

StatLink Ails https://doi.org/10.1787/888934017690

Figure 9.5. CT exams, 2007 and 2017 (or nearest year)

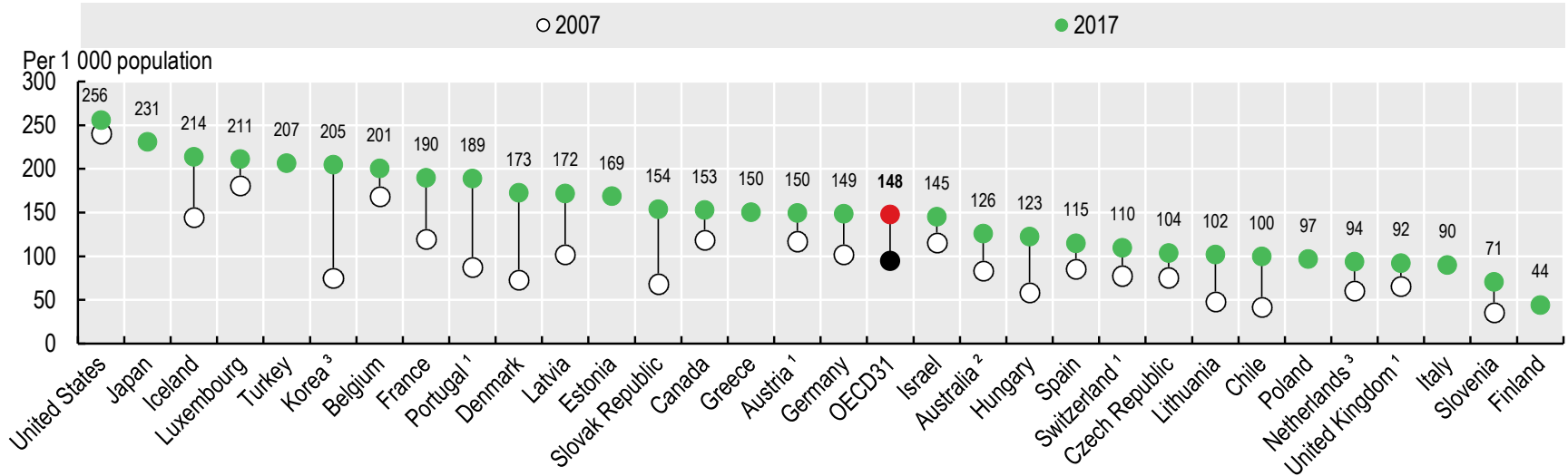

1. Exams outside hospital not included. 2. Exams on public patients not included. 3. Exams privately funded not included. Source: OECD Health Statistics 2019. 
The number of hospital beds provides an indication of the resources available for delivering services to inpatients. The influence of the supply of hospital beds on admission rates has been widely documented, confirming that a greater supply generally leads to higher admission numbers (Roemer's Law that a "built bed is a filled bed"). Therefore policymakers are recognising that simply increasing the number of hospital beds will not solve problems of overcrowding or delays in hospitals.

Across OECD countries, there were on average 4.7 hospital beds per 1000 people in 2017. In Japan and Korea, rates were much higher (13.1 and 12.3 beds per 1000 people respectively). Two-thirds of OECD countries reported between 3 and 8 hospital beds per 1000 population, with rates lowest in Mexico, Chile and Sweden.

Since 2000, the number of beds per capita has decreased in nearly all OECD countries. The largest reduction occurred in Finland, with a fall of more than $50 \%$ (from 7.5 beds per 1000 population in 2000 to 3.3 in 2017), mainly affecting long-term care beds and psychiatric care beds. Several other countries reduced capacity by 2 beds or more per 1000 population (Estonia, France, Latvia, Lithuania and the Slovak Republic). Part of the decrease can be attributed to advances in medical technology, allowing more surgery to be performed on a same-day basis, or as part of a broader policy strategy to reduce the number of hospital admissions. On the other hand, the number of beds has strongly increased in Korea (+164\%), with a significant number of these dedicated to long-term care.

Hospital discharge rates measure the number of patients who leave a hospital after staying at least one night. Improving timely discharge of patients can help the flow of patients through a hospital, allowing hospitals to reduce the number of beds. Both premature and delayed discharges not only worsen health outcomes, but also increase costs: premature discharges can lead to costly readmissions; delayed discharges use up limited hospital resources.

On average across OECD countries, there were 154 hospital discharges per 1000 population in 2017. Hospital discharge rates were highest in Germany, Austria and Lithuania (over 200 per 1000 population) and lowest in Mexico, Canada, Chile and the Netherlands (less than 100). The number of discharges fell in the majority of OECD countries, with some of the largest reductions observed in countries where there were also large decreases in the number of beds (e.g. Italy, Finland, Estonia, Sweden and Latvia). On the other hand, hospital discharge rates doubled in Korea, Turkey and China.

High occupancy rates of curative (acute) care beds can be symptomatic of a health system under pressure, and may lead to bed shortages and higher rates of infection. Overly low occupancy rates may reflect underutilised resources. The National Institute of Health and Care Excellence (NICE) in the United Kingdom recommend that health care providers should plan capacity to minimise the risks associated with occupancy rates exceeding 90\% (NICE, 2018[1]). The occupancy rate was over 90\% in Ireland, Israel and Canada in 2017. In Ireland, this represents a ten percentage point increase since 2000 (from 85\% to 95\%). Occupancy rates were comparatively low in Greece, the United States, the Netherlands and Hungary (around $65 \%$ or less). Around half of OECD countries have bed occupancy rates of $70-80 \%$, and the OECD average is $75 \%$.

\section{Definition and comparability}

Hospital beds include all beds that are regularly maintained and staffed and are immediately available for use. They include beds in general hospitals, mental health and substance abuse hospitals, and other specialty hospitals. Beds in residential long-term care facilities are excluded. In the United Kingdom, data are restricted to public hospitals. Data for Sweden exclude private beds that are privately financed. Beds for use by patients recovering from day surgery but released the same day may be included in countries where they cannot be distinguished from inpatient beds (e.g. Austria, Luxembourg and the Netherlands). Cots for healthy infants are included for a few countries (e.g. Canada, the Netherlands and Poland).

Discharge is defined as the release of a patient who has stayed at least one night in hospital. It includes deaths in hospital following inpatient care. Same-day discharges are usually excluded, with the exceptions of Chile, Japan, Norway and the United States which include some same-day discharges. Healthy babies born in hospitals are excluded from hospital discharge rates in several countries (Australia, Austria, Canada, Chile, Estonia, Finland, France, Greece, Ireland, Lithuania, Luxembourg, Mexico, the Netherlands and Norway). These comprise around $3-10 \%$ of all discharges. Data for some countries do not cover all hospitals. For instance, data for Mexico, New Zealand and the United Kingdom are restricted to public or publicly funded hospitals. Data for Ireland cover public acute and psychiatric (public and private) hospitals. Data for Canada, the Netherlands and the United States include only acute care/short-stay hospitals.

The occupancy rate for curative (acute) care beds is calculated as the number of hospital bed-days related to curative care divided by the number of available curative care beds (multiplied by 365 ).

\section{References}

[1] NICE (2018), "Bed occupancy", https://www.nice.org.uk/ guidance/ng94/evidence/39.bed-occupancy-pdf-172397464704.

[2] OECD (2014), Geographic Variations in Health Care: What Do We Know and What Can Be Done to Improve Health System Performance?, OECD Health Policy Studies, OECD Publishing, Paris, https://dx.doi.org/10.1787/9789264216594-en. 
9. HEALTH CARE ACTIVITIES

Hospital beds and discharge rates

Figure 9.6. Hospital beds, 2000 and 2017 (or nearest year)

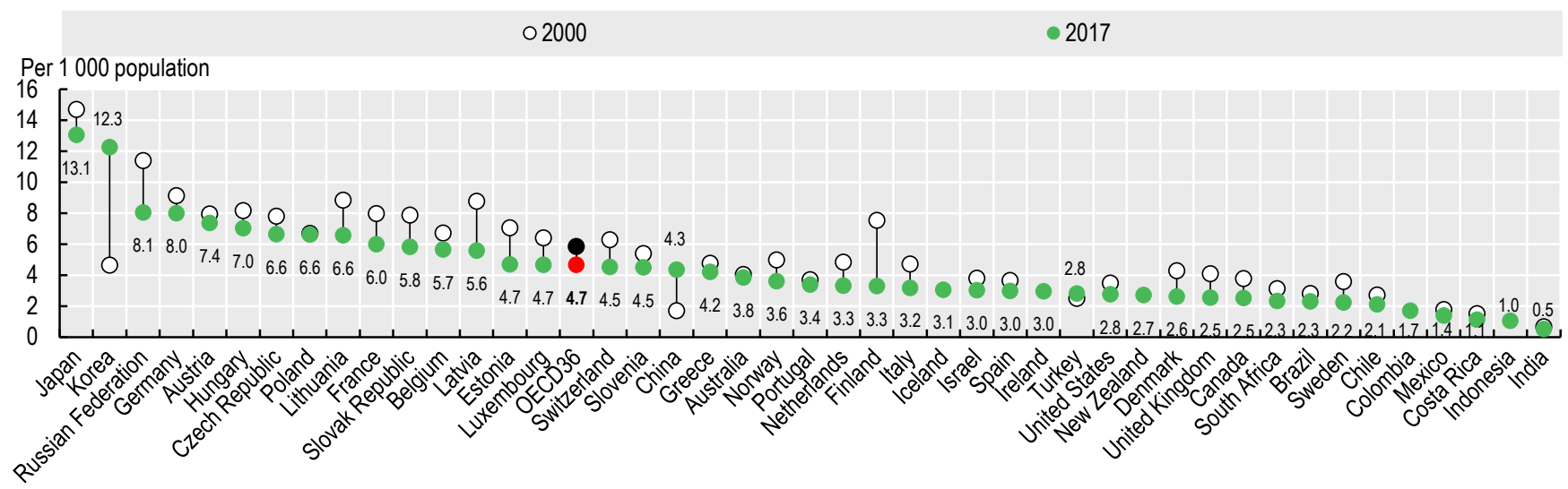

Source: OECD Health Statistics 2019.

StatLink क्ञाज https://doi.org/10.1787/888934017728

Figure 9.7. Hospital discharge rates, 2000 and 2017 (or nearest year)

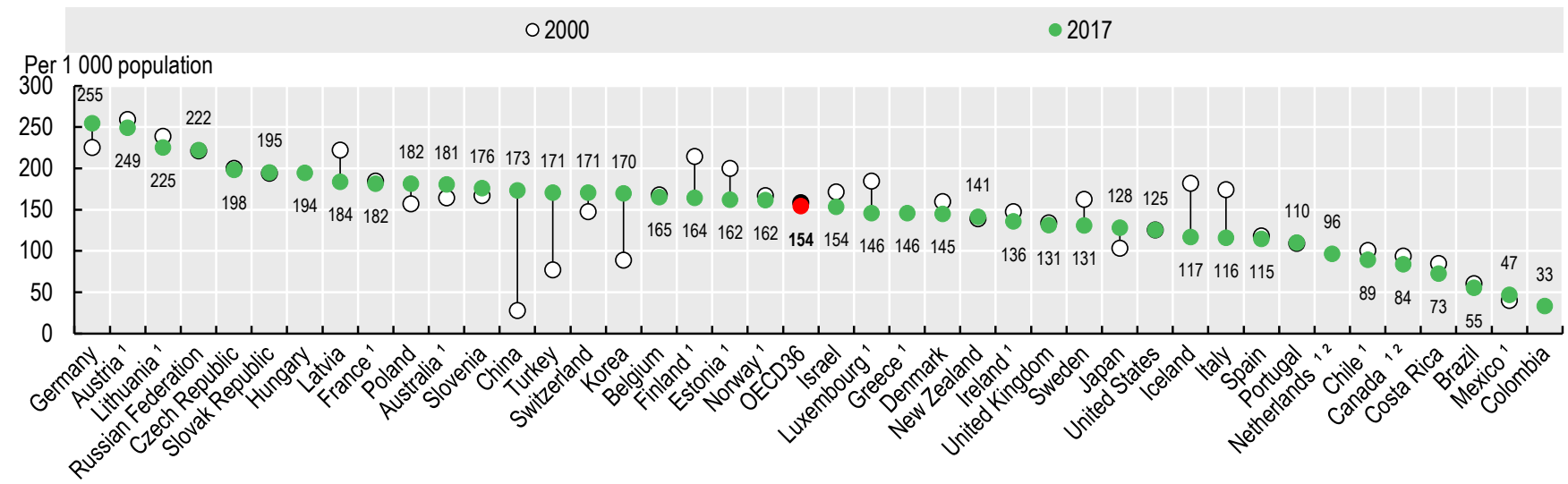

1. Data exclude discharges of healthy babies born in hospital (3-10\% of all discharges).

2. Data include discharges for curative (acute) care only.

Source: OECD Health Statistics 2019.

StatLink AाIs https://doi.org/10.1787/888934017747

Figure 9.8. Occupancy rate of curative (acute) care beds, 2000 and 2017 (or nearest year)

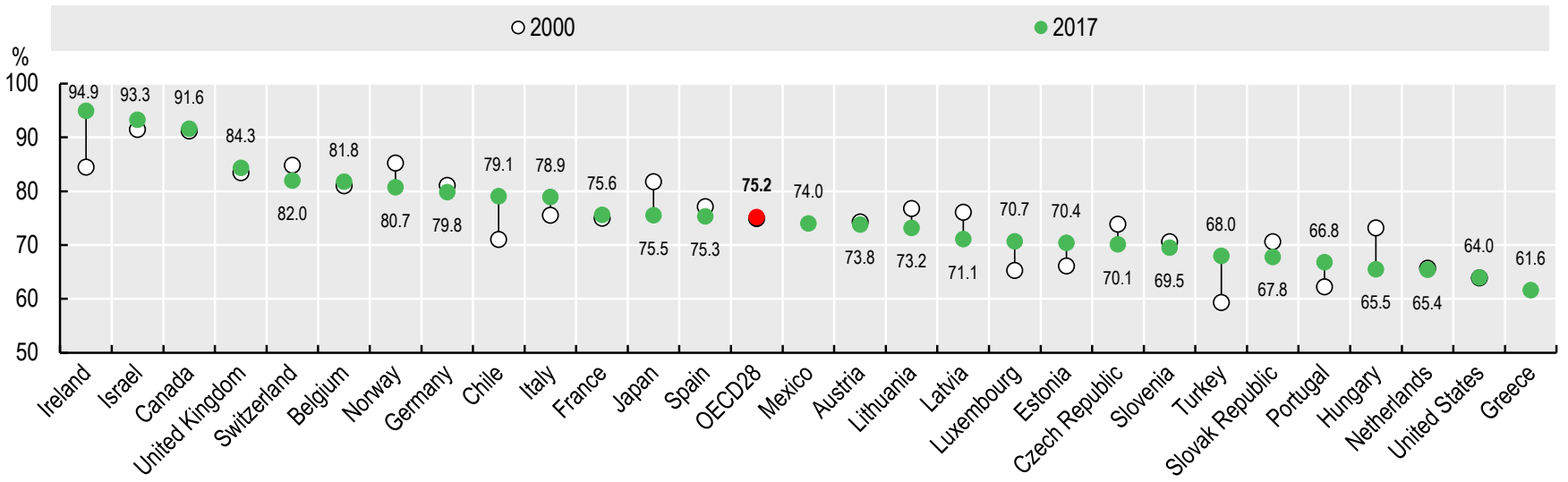

Source: OECD Health Statistics 2019. 
The average length of stay in hospitals is often regarded as an indicator of efficiency in health service delivery. All else being equal, a shorter stay will reduce the cost per discharge and will shift care from inpatient to less expensive settings. Longer stays can be a sign of poor care coordination, resulting in some patients waiting unnecessarily in hospital until rehabilitation or long-term care can be arranged. At the same time, some patients may be discharged too early, when staying in hospital longer could have improved their health outcomes or reduced chances of re-admission.

In 2017, the average length of stay in hospitals was slightly less than 8 days across OECD countries (Figure 9.9). Mexico and Turkey had the shortest stays, with patients staying for about 4 days on average in hospitals. Korea and Japan had the longest stays, averaging over 16 days per patient. Since 2000 , the average length of stay has decreased in most countries; the most significant declines occurred in Japan, Finland, Switzerland, the United Kingdom, Israel and the Netherlands. The only country with a large increase was Korea (from around 15 days in 2002 to 18.5 in 2017) - but this reflects in part an increase in the role of 'long-term care' hospitals whose function is similar to nursing homes or long-term care facilities.

Focusing on specific diseases or conditions can remove some of the effect of different case mix and severity. Across OECD countries, the average length of stay for a normal delivery was 2.9 days in 2017 (Figure 9.10). It reached over 4 days in Hungary, the Slovak Republic and the Czech Republic, and was less than 2 in Mexico, the United Kingdom, Canada, Iceland and the Netherlands. Length of stay for normal deliveries has decreased since 2000 in most countries, most notably in those with long stays such as the Slovak Republic and Czech Republic.

For acute myocardial infarction (AMI), the average length of stay ranged from 11 days or over in Chile and Korea to about 4 or under in Norway, Denmark and Sweden (Figure 9.11). The OECD average stood at 6.6 days, three days shorter than in 2000. The average length of stay for AMI decreased in all countries except Chile (where it increased by more than 3 days).

Apart from disparities in the average length of stay due to case mix, other factors including payment structures can explain cross-country variations. In particular, the introduction of prospective payment systems that encourage providers to reduce the cost of episodes in care, such as diagnosis-related groups (DRG), has been credited for the reduction in the average length of stay in hospitals.
France, Austria and Sweden are among the countries that have moved to DRG payment structures, and in doing so have experienced a decrease in the average length of stay.

Results from a recent OECD study highlight the significance of a number of hospital characteristics on the average length of stay in hospitals. Specifically, hospitals with many beds (higher than 200) are associated with a longer length of stay, while a bed occupancy rate of $70 \%$ or more is associated with a shorter length of stay (Lorenzoni and Marino, 2017[1]).

\section{Definition and comparability}

Average length of stay refers to the average number of days patients spend in hospital. It is generally measured by dividing the total number of days stayed by all inpatients during a year by the number of admissions or discharges. Day cases are excluded.

Data cover all inpatient cases (including not only curative/acute care cases) for most countries, with the exceptions of Canada, Japan and the Netherlands, where data refer to average length of stay for curative/ acute care or in acute care hospitals only (resulting in an under estimation).

Healthy babies born in hospitals are excluded from hospital discharge rates in several countries (e.g. Australia, Austria, Canada, Chile, Estonia, Finland, France, Greece, Ireland, Lithuania, Luxembourg, Mexico and Norway), resulting in a slight overestimation of the length of stay (e.g. the inclusion of healthy newborns would reduce the average length of stay by 0.5 days in Canada). These comprise around 3$10 \%$ of all discharges.

Data for normal delivery refer to ICD-10 code O80, and for AMI to ICD-10 codes I21-I22.

\section{References}

[1] Lorenzoni, L. and A. Marino (2017), "Understanding variations in hospital length of stay and cost: Results of a pilot project", OECD Health Working Papers, No. 94, OECD Publishing, Paris, https://dx.doi.org/10.1787/ae3a5ce9-en.

[2] OECD (2017). Tackling Wasteful Spending in Health, OECD Publishing, Paris, http://dx.doi.org/10.1787/9789264266414-en. 
Figure 9.9. Average length of stay in hospital, 2000 and 2017 (or nearest year)

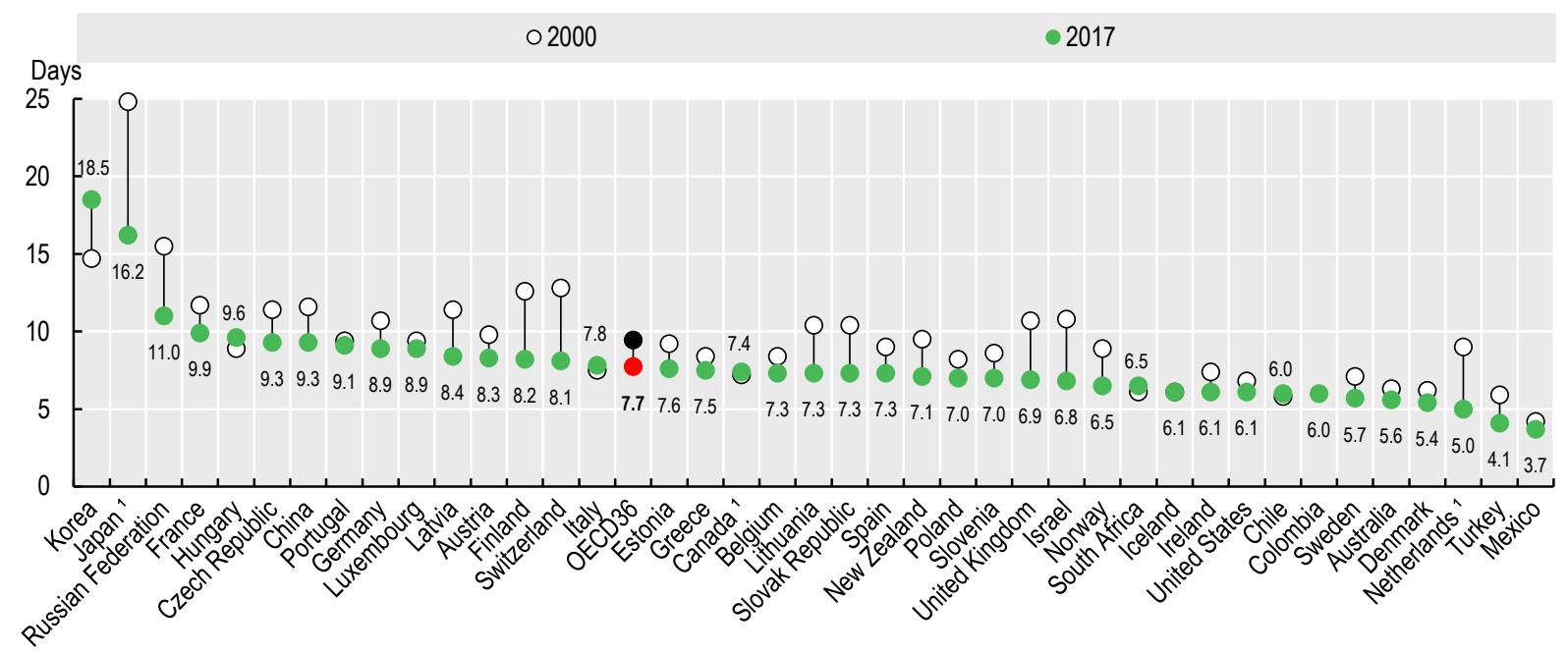

1. Data refer to average length of stay for curative (acute) care (resulting in an under-estimation). In Japan, the average length of stay for all inpatient care was 28 days in 2017 (down from 39 days in 2000).

Source: OECD Health Statistics 2019.

Figure 9.10. Average length of stay for normal delivery, 2017 (or nearest year)

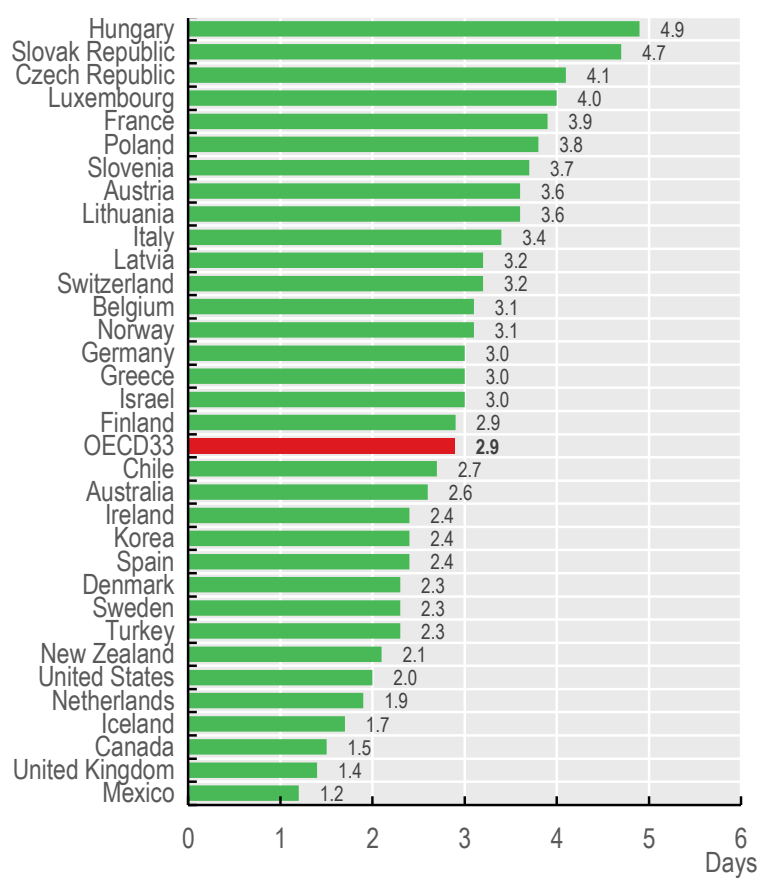

Source: OECD Health Statistics 2019.

StatLink गाIs https://doi.org/10.1787/888934017804

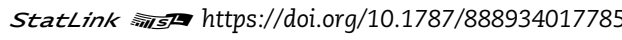

Figure 9.11. Average length of stay for acute myocardial infarction, 2017 (or nearest year)

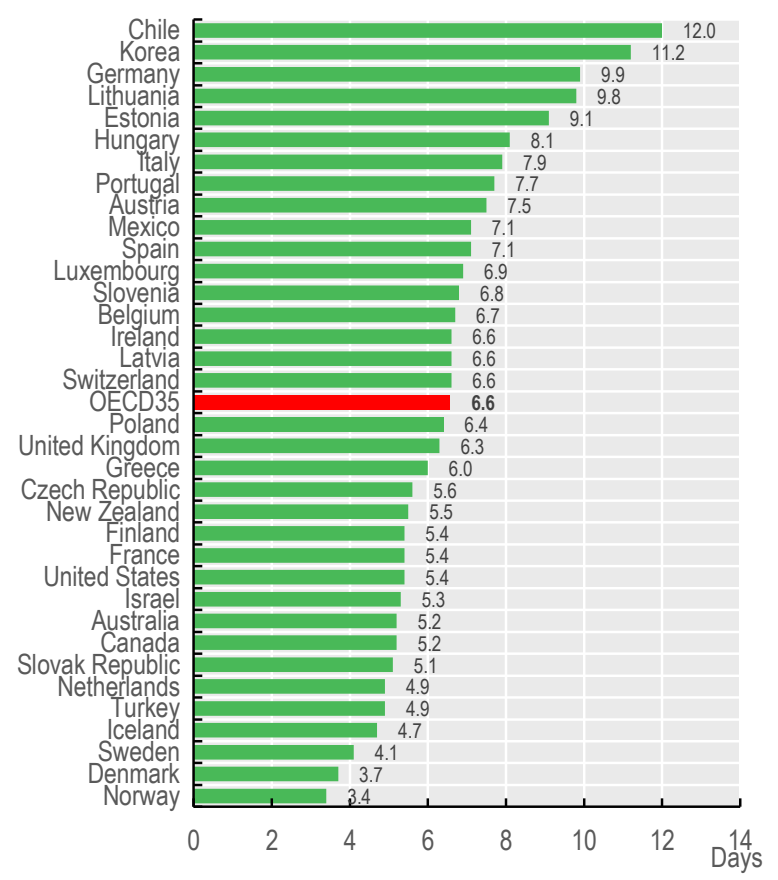

Source: OECD Health Statistics 2019.

StatLink काls https://doi.org/10.1787/888934017823 
Hip and knee replacements are some of the most frequently performed and effective surgeries worldwide. The main indication for hip and knee replacement (joint replacement surgery) is osteoarthritis, which leads to reduced function and quality of life.

Osteoarthritis is a degenerative form of arthritis characterised by the wearing down of cartilage that cushions and smooths the movement of joints - most commonly for the hip and knee. It causes pain, swelling and stiffness resulting in a loss of mobility and function. Osteoarthritis is one of the ten most disabling diseases in developed countries. Worldwide, estimates show that $10 \%$ of men and $18 \%$ of women aged over 60 years have symptomatic osteoarthritis, including moderate and severe forms (WHO, 2014[1]).

Age is the strongest predictor of the development and progression of osteoarthritis. It is more common in women, increasing after the age of 50 especially in the hand and knee. Other risk factors include obesity, physical inactivity, smoking, excessive alcohol consumption and injuries. While joint replacement surgery is mainly carried out among people aged 60 and over, it can also be performed on people at younger ages.

In 2017, Germany, Austria, Switzerland, Finland, Luxembourg and Belgium were among the countries with the highest rates for hip and knee replacement (Figure 9.12 and Figure 9.13). The OECD averages are 182 per 100000 population for hip replacement, and 135 per 100000 for knee replacement. Mexico, Portugal, Israel, Ireland and Korea have low hip and knee replacement rates. Differences in population structure may explain part of this variation across countries, and age standardisation reduces it to some extent. Nevertheless, large differences persist and the country ranking does not change significantly after age standardisation (McPherson, Gon and Scott, 2013[2]).

National averages can mask important variation in hip and knee replacement rates within countries. In Australia, Canada, Germany, France and Italy, the rate of knee replacement is more than twice as high in some regions than others, even after age-standardisation (OECD, 2014[3]). Alongside the number of operations, the quality of hip and knee surgery (see indicator on "Hip and knee surgery" in Chapter 6) and waiting times (see indicator on "Waiting times for elective surgery" in Chapter 5) are also critical for patients.

Since 2000, the number of hip and knee replacements has increased rapidly in most OECD countries (Figure 9.14 and Figure 9.15). On average, hip replacement rates increased by $30 \%$ between 2007 and 2017 and knee replacement rates by $40 \%$. This aligns with the rising incidence and prevalence of osteoarthritis, caused by ageing populations and growing obesity rates in OECD countries. For example, in the United States, the prevalence of knee osteoarthritis has more than doubled since the mid-20 th century (Wallace et al., 2017[4]).

Most OECD countries show increasing trends of varying degrees, but Ireland and Luxembourg show slower growth than the average, these are also the only OECD countries to show a decrease in hip replacements rates from 2007.

\section{Definition and comparability}

Hip replacement is a surgical procedure in which the hip joint is replaced by a prosthetic implant. It is generally conducted to relieve arthritis pain or treat severe physical joint damage following hip fracture.

Knee replacement is a surgical procedure to replace the weight-bearing surfaces of the knee joint in order to relieve the pain and disability of osteoarthritis. It may also be performed for other knee diseases such as rheumatoid arthritis.

Classification systems and registration practices vary across countries, which may affect the comparability of the data. While most countries include both total and partial hip replacement, some countries only include total replacement. In Ireland, Mexico, New Zealand and the United Kingdom, the data only include activities in publicly funded hospitals, thereby underestimating the number of total procedures presented here (for example, approximately $15 \%$ of all hospital activity in Ireland is undertaken in private hospitals). Data for Portugal relate only to public hospitals on the mainland. Data for Spain only partly include activities in private hospitals.

\section{References}

[2] McPherson, K., G. Gon and M. Scott (2013), "International Variations in a Selected Number of Surgical Procedures", OECD Health Working Papers, No. 61, OECD Publishing, Paris, https://dx.doi.org/10.1787/5k49h4p5g9mw-en.

[3] OECD (2014), Geographic Variations in Health Care: What Do We Know and What Can Be Done to Improve Health System Performance?, OECD Health Policy Studies, OECD Publishing, Paris, https://dx.doi.org/10.1787/9789264216594-en.

[4] Wallace, I. et al. (2017), "Knee osteoarthritis has doubled in prevalence since the mid-20th century", Proceedings of the National Academy of Sciences, Vol. 114/35, pp. 9332-9336, http:// dx.doi.org/10.1073/pnas.1703856114.

[1] WHO (2014), "Chronic Rheumatic Conditions", Fact Sheet, http://www.who.int/chp/topics/rheumatic/en/. 
9. HEALTH CARE ACTIVITIES

Hip and knee replacement

Figure 9.12. Hip replacement surgery, 2017 (or nearest year)

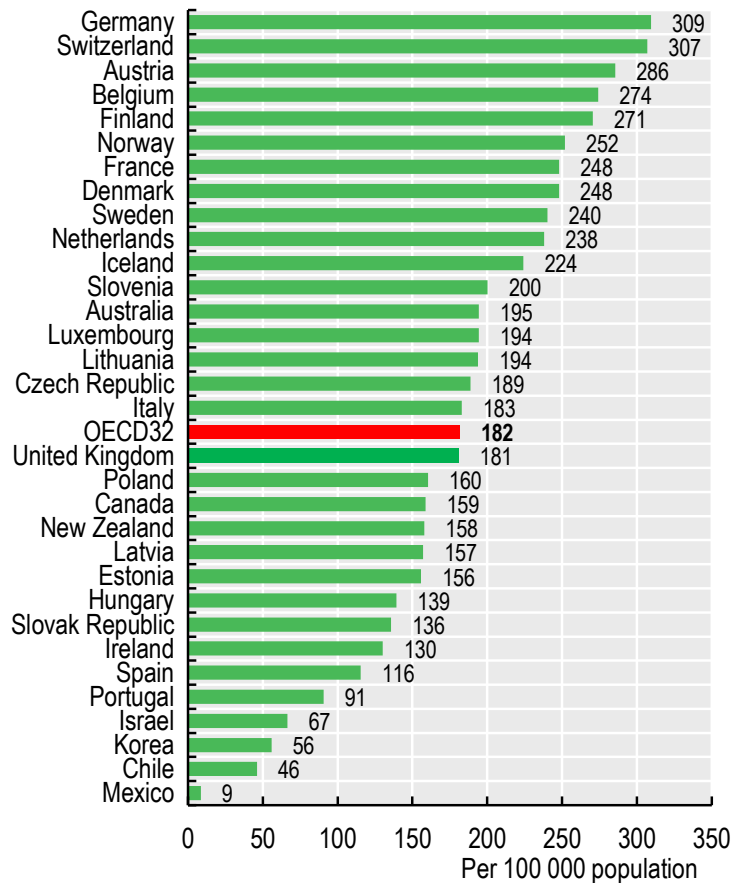

Source: OECD Health Statistics 2019.

StatLink काIs https://doi.org/10.1787/888934017842

Figure 9.14. Hip replacement surgery trends in selected OECD countries, 2007-17

$\begin{array}{cc}- \text { Canada } & \text { Germany }- \text { - Italy } \\ -\infty-\infty \text { Sweden } & \text { OECD30 }\end{array}$

Per 100000 population

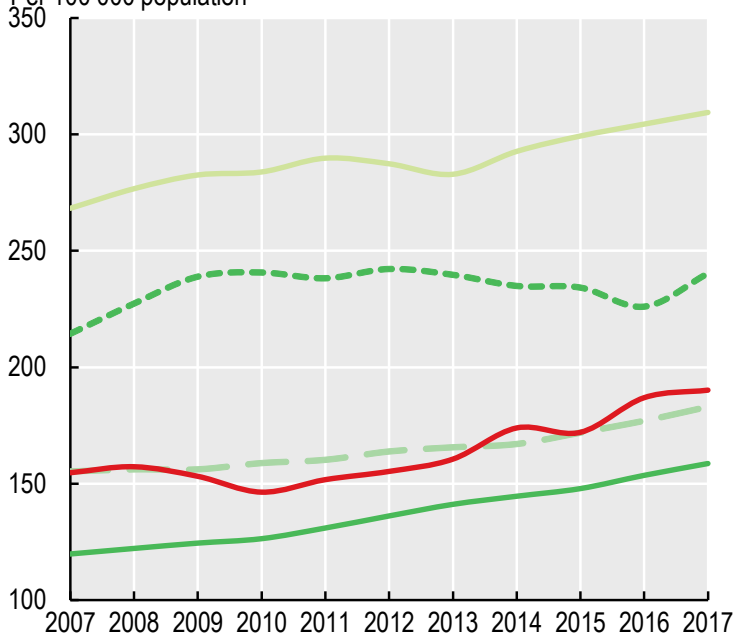

Source: OECD Health Statistics 2019

StatLink AाIs https://doi.org/10.1787/888934017880
Figure 9.13. Knee replacement surgery, 2017 (or nearest year)

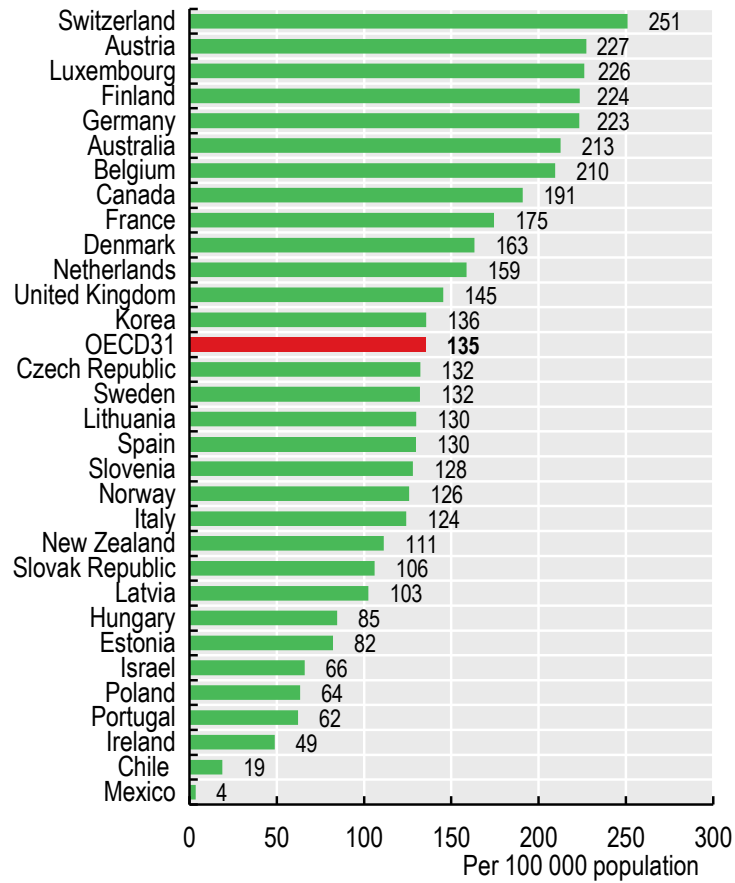

Source: OECD Health Statistics 2019.

StatLink ला1s https://doi.org/10.1787/888934017861

Figure 9.15. Knee replacement surgery trends in selected OECD countries, 2007-17

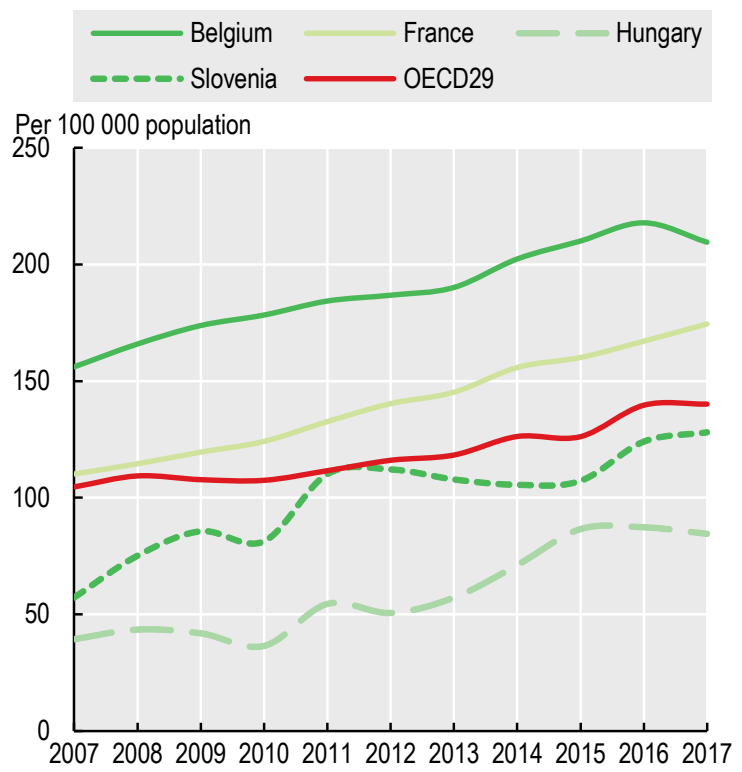

Source: OECD Health Statistics 2019.

StatLink ताड का https://doi.org/10.1787/888934017899 
Caesarean sections can be a lifesaving and necessary procedure. Nonetheless, caesarean delivery continues to result in increased maternal mortality, maternal and infant morbidity, and increased complications for subsequent deliveries. This raises concerns over the growing rates of caesarean sections performed across OECD countries since 2000 , in particular among women at low risk of a complicated birth who have their first baby by caesarean section for non-medical reasons. The World Health Organization concludes that caesarean sections are effective in saving maternal and infant lives, but that caesarean section rates higher than $10 \%$ are not associated with reductions in maternal and newborn mortality rates at the population level. Nevertheless, caesarean sections should be provided based on need, rather than striving to achieve a specific rate.

In 2017, caesarean section rates remain lowest in Nordic countries (Iceland, Finland, Sweden and Norway), Israel and the Netherlands, with rates ranging from $15 \%$ to $17 \%$ of all live births (Figure 9.16). They were highest in Korea, Chile, Mexico and Turkey, with rates ranging from $45 \%$ to $53 \%$ of all births. Across OECD countries, $28 \%$ of live births were performed as caesarean sections.

Caesarean rates have increased since 2000 in most OECD countries, with the average rising from $20 \%$ in 2000 to $28 \%$ in 2017 , although the rate of growth has slowed over the past five years (Figure 9.17). Growth rates have been particularly rapid in the Slovak Republic and Czech Republic, Slovenia and Austria, which have historically had relatively low rates. There have also been large increases over the past decade in Chile, Korea, Mexico and Turkey - countries that already had high caesarean rates. In Italy, caesarean rates have come down significantly in recent years, although they remain among the highest in Europe.

Variations in caesarean section rates across countries have been attributed to a number of factors, including financial incentives, malpractice liability concerns, differences in the availability and training of midwives and nurses, and the proportion of women who access private maternity care. For example, there is evidence that private hospitals tend to perform more caesarean sections than public hospitals. In Switzerland, caesarean sections were found to be substantially higher in private clinics (41\%) than in public hospitals (30.5\%) (OFSP, 2013[1]).

Furthermore, divergences exist for preferences among women for a caesarean section for a healthy birth across countries, which can be linked to the institutional arrangements of the maternal health system and cultural attitudes towards labour and birth. For example, in Iceland, the rate of preference for a caesarean section in the context of a healthy birth was $9.2 \%$ of women, compared to $16 \%$ in
Australia. Preference for a caesarean section in young women can also be linked to psychological reasons, including fear of uncontrollable pain and fear of physical damage (Stoll et al., 2017[2]).

Public reporting, provider feedback, the development of clearer clinical guidelines, and adjustments to financial incentives have been used to try to reduce the inappropriate use of caesarean sections. In Australia, where caesarean section rates are high relative to most OECD countries, a number of states have developed clinical guidelines and required reporting of hospital caesarean section rates, including investigation of performance against the guidelines. These measures have discouraged variations in practice and contributed to slowing down the rise in caesarean sections. Other countries have reduced the gap in hospital payment rates between a caesarean section and a normal delivery, with the aim of discouraging the inappropriate use of caesareans (OECD, 2014[3]).

\section{Definition and comparability}

The caesarean section rate is the number of caesarean deliveries performed per 100 live births.

In Ireland, Mexico, New Zealand and the United Kingdom, the data only include activities in publicly funded hospitals (though for Ireland all maternity units are located in publicly funded hospitals and for New Zealand the number of privately funded births is negligible). This may lead to an underestimation of caesarean section rates in these countries, since there is some evidence that private hospitals tend to perform more caesarean sections than public hospitals.

\section{References}

[3] OECD (2014), Geographic Variations in Health Care: What Do We Know and What Can Be Done to Improve Health System Performance?, OECD Health Policy Studies, OECD Publishing, Paris, https://dx.doi.org/10.1787/9789264216594-en.

[1] OFSP - Office fédéral de la santé publique (2013), Accouchements par césariennes en Suisse [Births by caesareans in Switzerland], Bern.

[2] Stoll, K. et al. (2017), "International Childbirth Attitudes- Prior to Pregnancy (ICAPP) Study Team - Preference for cesarean section in young nulligravid women in eight OECD countries and implications for reproductive health education", Reproductive Health, Vol. 14/1, http://dx.doi.org/10.1186/ s12978-017-0354-x. 
Figure 9.16. Caesarean section rates, 2017 (or nearest year)

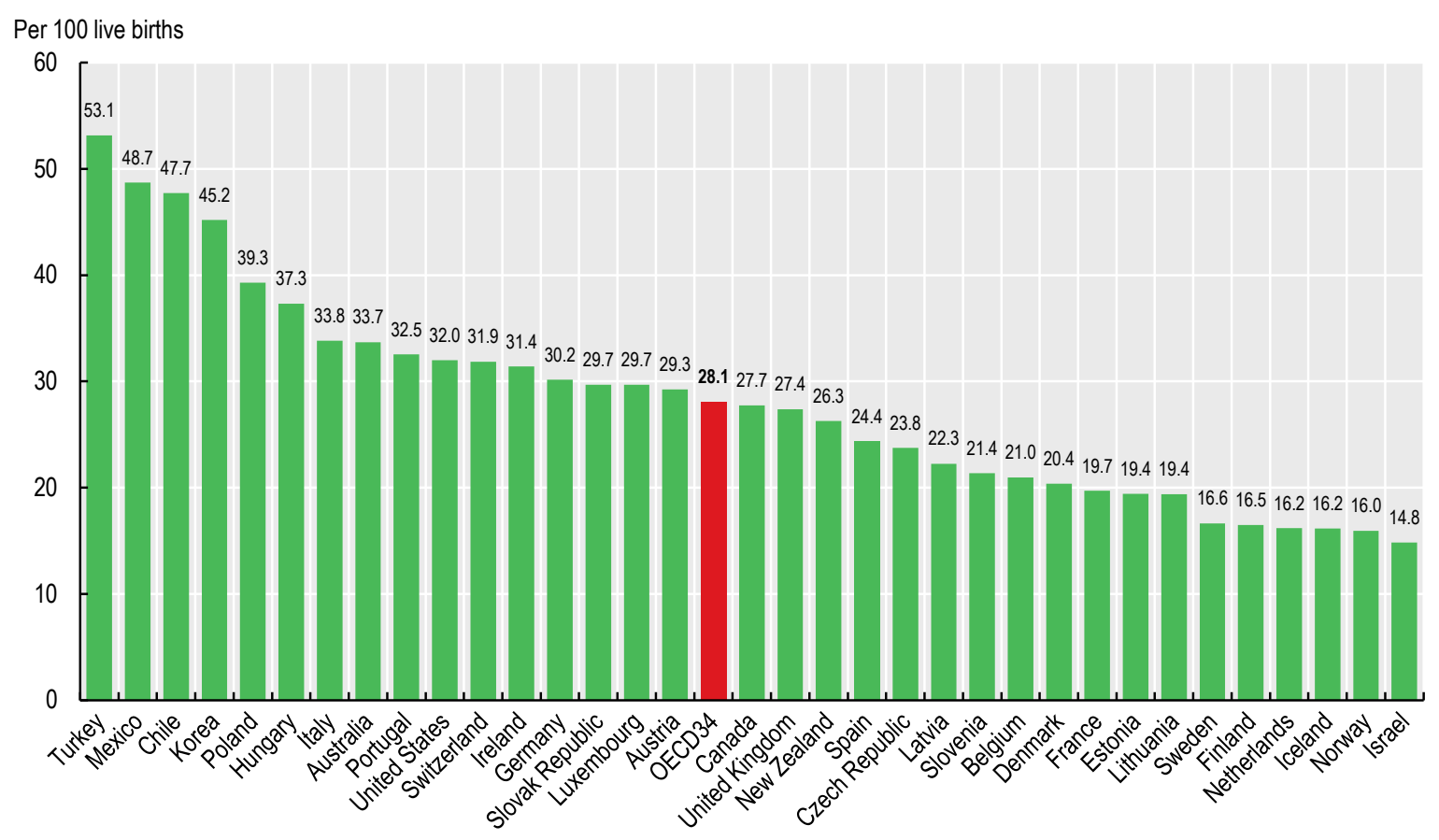

Source: OECD Health Statistics 2019.

StatLink AाIs/ https://doi.org/10.1787/888934017918

Figure 9.17. Caesarean section trends in selected OECD countries, 2000-17
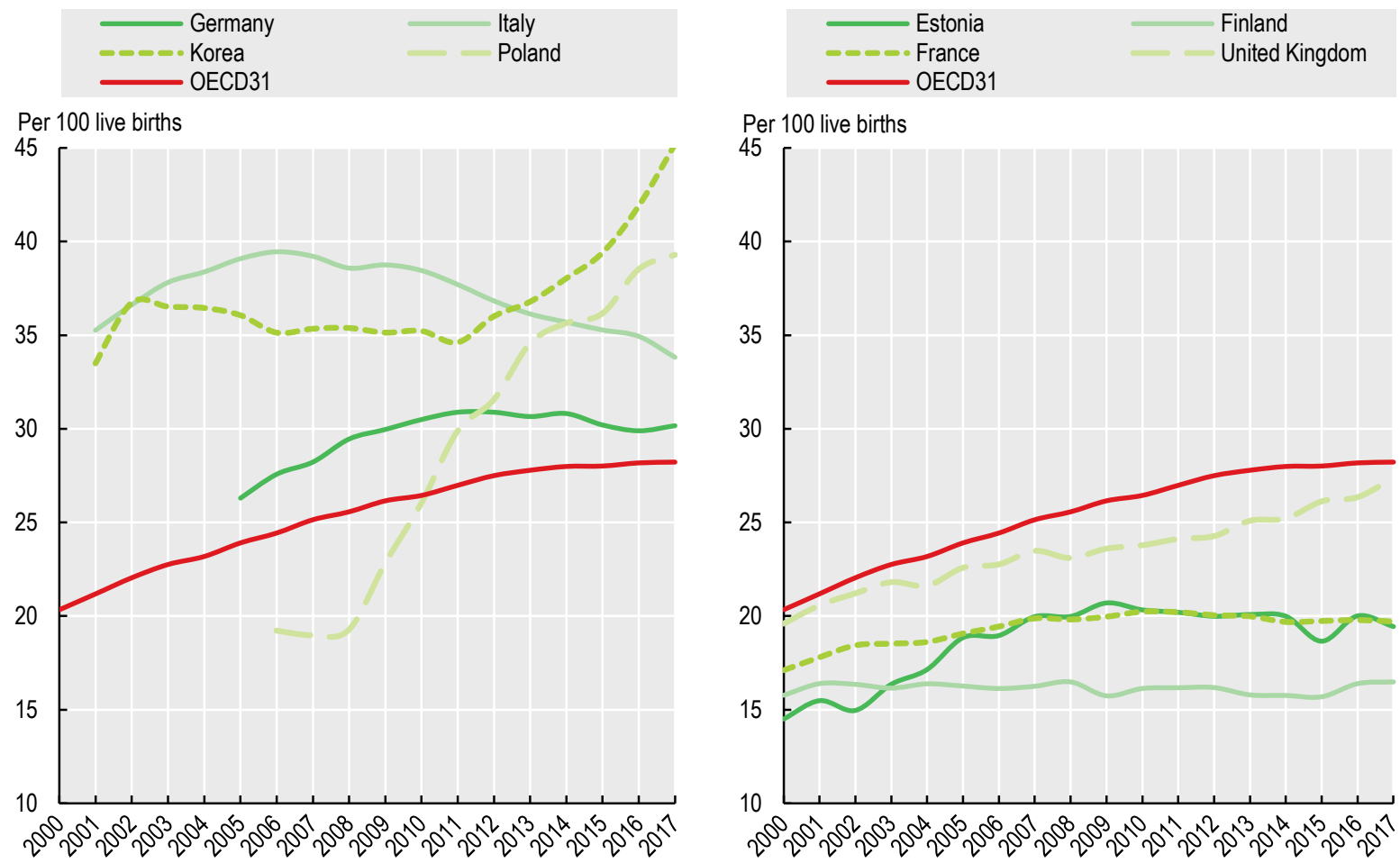

Source: OECD Health Statistics 2019. 
In the past few decades, the number of surgical procedures carried out on a same-day basis has markedly increased in OECD countries. Advances in medical technologies - in particular the diffusion of less invasive surgical interventions - and better anaesthetics have made this development possible. These innovations have improved patient safety and health outcomes. Further, by shortening the treatment episode, same-day surgery can save important resources without any adverse effects on quality of care. It also frees up capacity within hospitals to focus on more complex cases or to reduce waiting lists. However, the impact of the rise in same-day surgeries on overall health spending may not be straightforward since the reduction in unit costs (compared to inpatient surgery), may be offset by overall growth in the volume of procedures performed. Any additional cost related to post-acute care and community health services following the interventions also need to be considered.

Cataract surgeries and tonsillectomies (the removal of tonsils - glands at the back of the throat - mainly performed on children) provide good examples of high-volume surgeries that are now mainly carried out on a same-day basis in many OECD countries.

Day surgery accounts for $90 \%$ or more of all cataract surgeries in the majority of OECD countries (Figure 9.18). In several countries, nearly all cataract surgeries are performed as day cases. However, the use of day surgery is low in Poland, Lithuania, Turkey and Hungary, with less than $60 \%$ of surgeries performed as day cases). While this may be explained partly by limitations in the data coverage of outpatient activities in hospital or outside hospital, it may also reflect higher reimbursement for inpatient stays or constraints on the development of day surgery.

The number of cataract surgeries performed on a same-day basis has grown significantly since 2007 in many countries, including Austria, France, Hungary, Ireland, Poland, Portugal and Slovenia (Figure 9.18). In Austria, the share of cataract surgeries performed as day cases increased from only $10 \%$ in 2007 to almost $85 \%$ in 2017.

Tonsillectomies are one of the most frequent surgical procedures performed on children, usually those suffering from repeated or chronic infections of the tonsils, breathing problems or obstructive sleep apnoea due to large tonsils. Although the operation is performed under general anaesthesia, it is now carried out predominantly as sameday surgery in 10 of 29 OECD countries with comparable data, with children returning home the same day (Figure 9.19). However, the proportion of day cases is not as high as for cataract surgery, at $34 \%$ of tonsillectomies, on average across OECD countries. Day surgery rates are relatively high in Iceland, Finland and Sweden (75\% of cases or higher) but remain less than $10 \%$ of cases in 10 OECD countries. In Slovenia, Hungary, the Czech Republic and Austria, practically no tonsillectomies are undertaken as day cases. These large differences in the share of same-day surgery may reflect variations in the perceived risks of postoperative complications, or simply clinical traditions of keeping children for at least one night in hospital after the operation.

Financial incentives can also affect the extent to which minor surgery is conducted on a same-day basis. In Denmark and France, diagnostic-related group (DRG) systems have been adjusted to incentivise same-day surgery. In the United Kingdom, a financial incentive of approximately GBP 300 per case is awarded for selected surgical procedures if the patient was managed on a daycase basis (OECD, 2017[1]).

\section{Definition and comparability}

Cataract surgery consists of removing the lens of the eye because of the presence of cataracts partially or completely clouding the lens, and replacing it with an artificial lens. It is mainly performed on elderly people. Tonsillectomy consists of removing the tonsils glands at the back of the throat. It is mainly performed on children.

The data for several countries do not include outpatient cases in hospital or outside hospital (i.e. patients who are not formally admitted and discharged), leading to some under-estimation. In Ireland, Mexico, New Zealand and the United Kingdom, the data only include cataract surgeries carried out in public or publicly funded hospitals, excluding any procedures performed in private hospitals (in Ireland, it is estimated that approximately $15 \%$ of all hospital activity is undertaken in private hospitals). Data for Portugal relate only to public hospitals on the mainland. Data for Spain only partly include activities in private hospitals.

\section{References}

[1] OECD (2017), Tackling Wasteful Spending in Health, OECD Publishing, Paris, http://dx.doi.org/10.1787/9789264266414-en. 
Figure 9.18. Share of cataract surgery carried out as ambulatory cases, 2007 and 2017 (or nearest year)

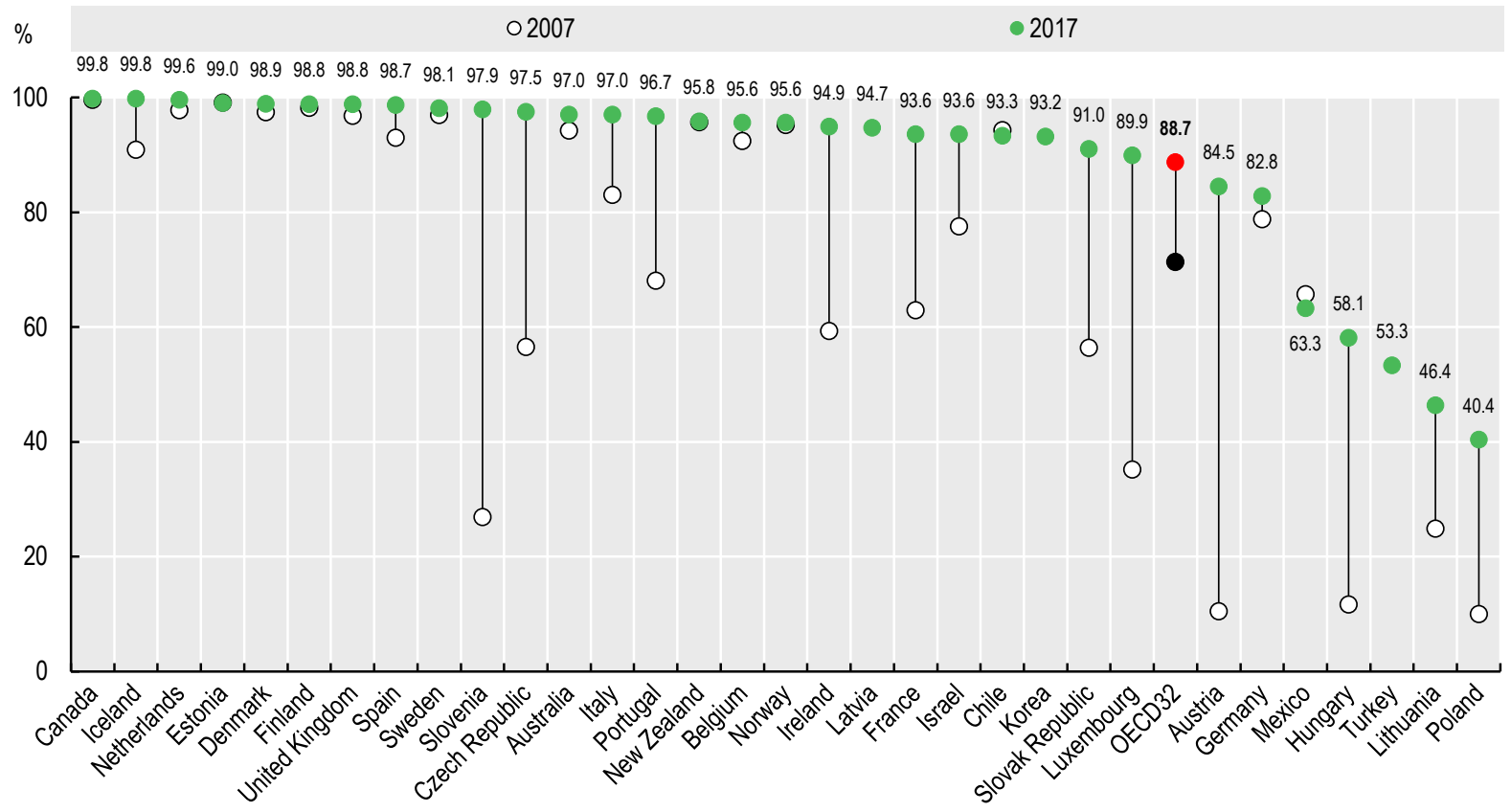

Source: OECD Health Statistics 2019.

Figure 9.19. Share of tonsillectomy carried out as ambulatory cases, 2007 and 2017 (or nearest year)

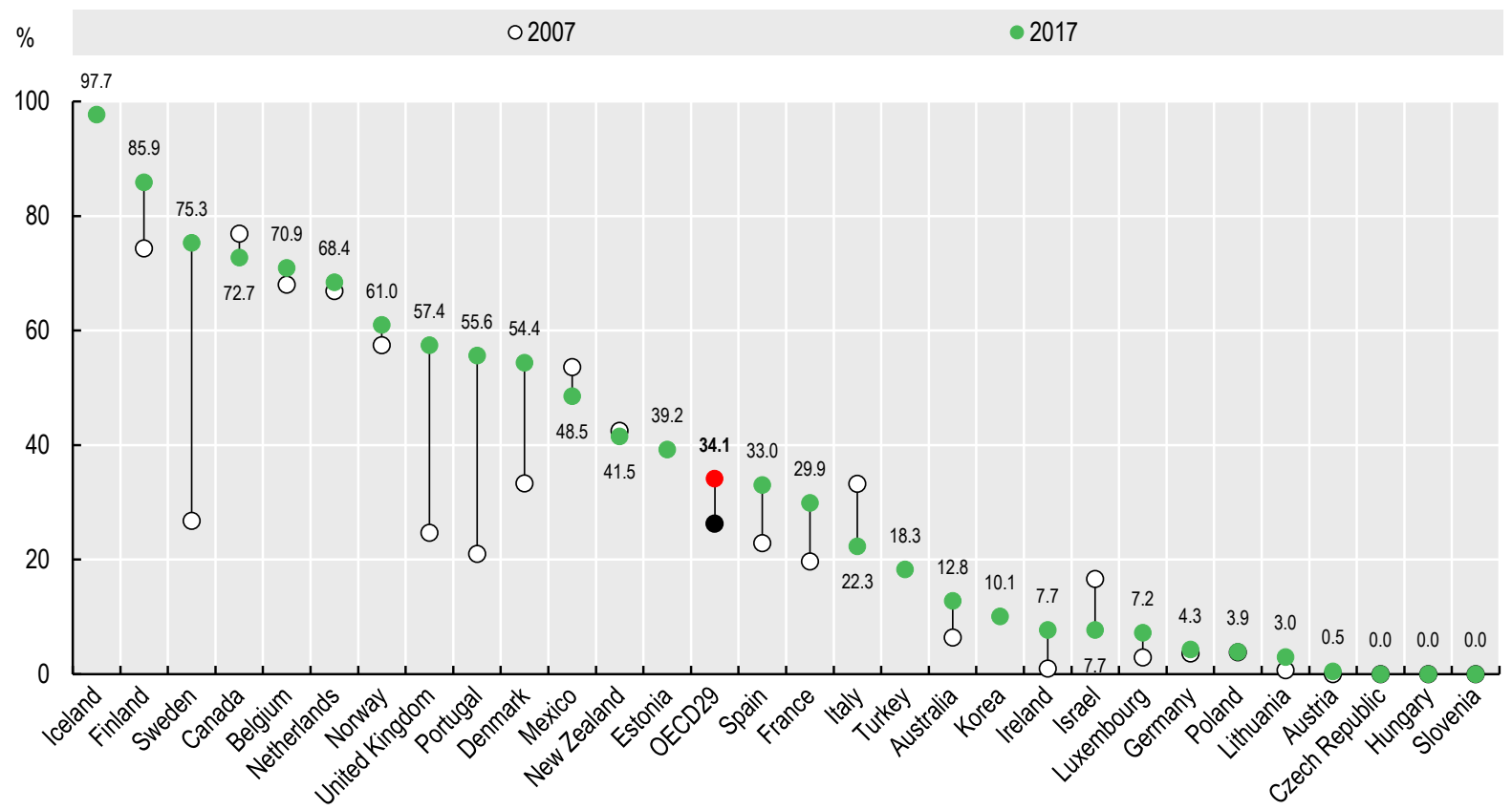

Source: OECD Health Statistics 2019. 


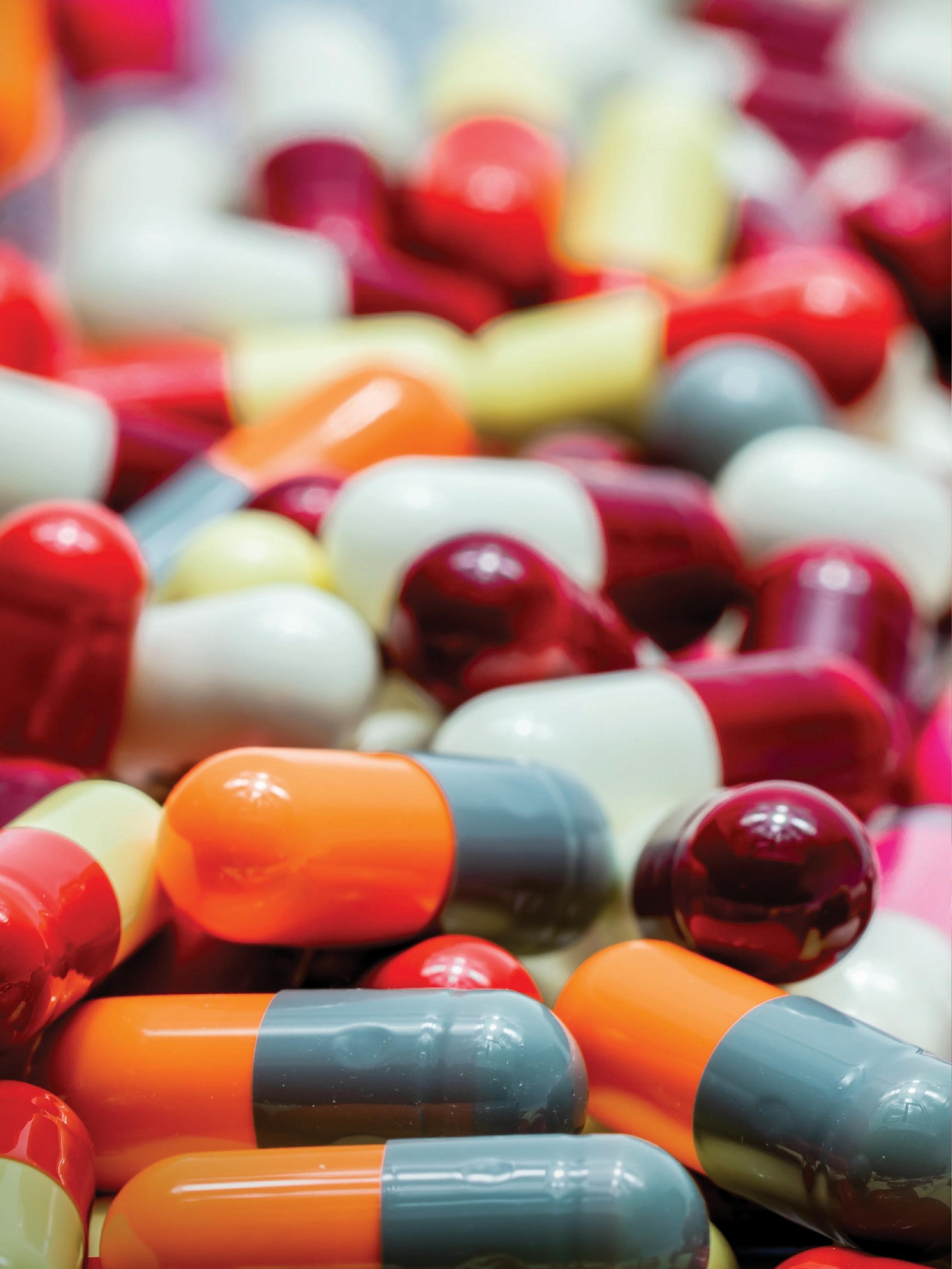




\title{
10. PHARMACEUTICAL SECTOR
}

\author{
Pharmaceutical expenditure \\ Pharmacists and pharmacies \\ Pharmaceutical consumption \\ Generics and biosimilars
}

Research and development in the pharmaceutical sector

The statistical data for Israel are supplied by and under the responsibility of the relevant Israeli authorities. The use of such data by the OECD is without prejudice to the status of the Golan Heights, East Jerusalem and Israeli settlements in the West Bank under the terms of international law. 
Pharmaceutical care is constantly evolving, with many novel drugs entering the market. These offer alternatives to existing treatments, and in some cases, the prospect of treating conditions previously considered incurable. However, the costs of new drugs can be very high, with significant implications for health care budgets. In 2017, retail pharmaceuticals accounted for almost one-fifth of all health care expenditure, and represented the third largest spending component in OECD countries after inpatient and outpatient care.

Across OECD countries, funding from governments and compulsory insurance schemes played the largest role in purchasing pharmaceuticals (Figure 10.1). On average, these schemes covered $58 \%$ of spending on retail pharmaceuticals. Most of the remainder is financed from household out-of-pocket payments; only $3 \%$ of spending is covered by voluntary insurance. In Germany and France, government and compulsory schemes cover $80 \%$ or more of pharmaceutical costs. By contrast, in Latvia, Poland and Lithuania, almost two-thirds of pharmaceutical spending was through out-of-pocket payments.

Spending for retail pharmaceuticals averaged USD 564 per person across OECD countries in 2017, adjusted for differences in purchasing power (Figure 10.2). Cross-country differences are marked, with spending more than double the average in the United States, followed by Switzerland and Japan. Per capita spending was lowest in Mexico and Denmark, at around half or less of the OECD average. Crosscountry differences in spending reflect differences in distribution and dispensing patterns, the uptake of both generic and novel medicines, as well as pricing and procurement policies.

Most spending on retail pharmaceuticals is for prescription medicines $(75 \%)$, with the remainder spent on over-thecounter (OTC) medicines (19\%) and medical non-durables (5\%). The costs of OTC medicines are typically borne by patients, though occasionally public payers or mandatory insurance schemes may contribute. Depending on countryspecific legislation, some OTC medicines can be sold outside pharmacies, for example, in supermarkets, other retail stores or via the internet. Expenditure on OTC medicines in Poland is almost equal to that on prescription medicines, and accounted for almost a third of the total in Spain, Latvia and Australia.

Growth in retail pharmaceutical spending has fluctuated over the past decade across OECD countries, declining in the years during and after the financial crisis, but increasing again in recent years (see indicator on "Health expenditure by type of service" in Chapter 7). This reflects the actions of many governments in introducing cost-control measures such as de-listing of products (excluding them from reimbursement), cutting manufacturer prices and margins for pharmacists and wholesalers, and introducing or increasing user charges for retail prescription drugs (Belloni et al., 2016[1]).

Figure 10.3 compares growth rates of pharmaceutical spending in the retail sector and in hospitals for a selection of OECD countries. In Greece, where a policy to reduce wasteful use of drugs was introduced, retail spending on pharmaceuticals has decreased substantially. Growth over the last decade has been positive in some countries, such as Germany and Canada, partly due to new high cost treatments - notably oncology treatments and hepatitis C drugs. Yet analysing retail pharmaceuticals only gives a partial picture of spending: the costs of pharmaceuticals used for hospital inpatient care can also be high, accounting on average for an additional $20 \%$ on top of retail spending. Growth in spending on hospital pharmaceuticals has generally been higher than that for retail medicines, with the highest rates in Korea and Iceland. Several countries, including Denmark, Finland and Portugal, experienced growth in hospital pharmaceutical expenditure at the same time as spending on retail drugs declined.

\section{Definition and comparability}

Pharmaceutical expenditure covers spending on prescription medicines and self-medication, the latter often referred to as over-the-counter products. Other medical non-durable goods (such as first aid kits and hypodermic syringes) are also included. It further includes pharmacists' remuneration when the latter is separate from the price of medicines. Retail pharmaceuticals are provided outside hospital care, such as those dispensed through a pharmacy or bought from a supermarket. Hospital pharmaceuticals include drugs administered or dispensed during an episode of hospital care.

Expenditure on retail pharmaceuticals includes wholesale and retail margins and value-added tax. Total pharmaceutical spending refers in most countries to "net" spending - i.e. adjusted for possible rebates payable by manufacturers, wholesalers or pharmacies. Pharmaceuticals consumed in hospitals and other health care settings as part of an inpatient or day-case treatment are excluded (available data suggests that their inclusion would add another $10-20 \%$ to retail pharmaceutical spending). Comparability issues exist regarding the administration and dispensing of pharmaceuticals for outpatients in hospitals. In some countries, the costs are included under curative care; in others, under pharmaceuticals.

\section{References}

[1] Belloni, A., D. Morgan and V. Paris (2016), "Pharmaceutical Expenditure And Policies: Past Trends And Future Challenges", OECD Health Working Papers, No. 87, OECD Publishing, Paris, https://doi.org/10.1787/5jm0q1f4cdq7-en. 
Figure 10.1. Expenditure on retail pharmaceuticals ${ }^{1}$ by type of financing, 2017 (or nearest year)

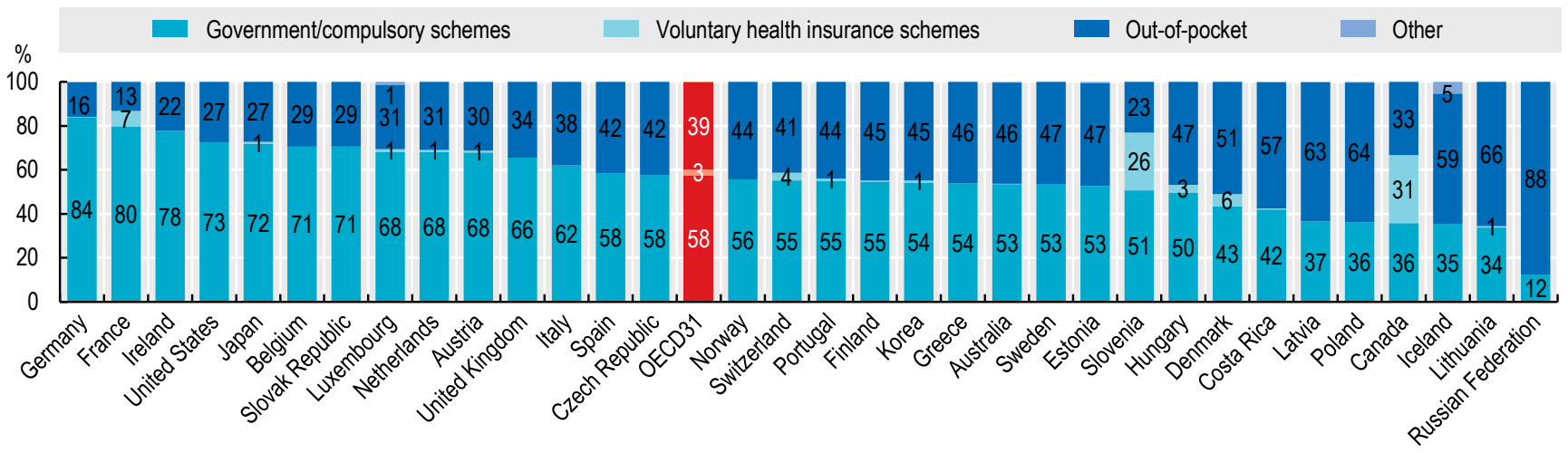

Note: "Other" includes financing from non-profit-schemes, enterprises and the rest of the world.

1. Includes medical non-durables.

Source: OECD Health Statistics 2019.

Figure 10.2. Expenditure on retail pharmaceuticals per capita, 2017 (or nearest year)

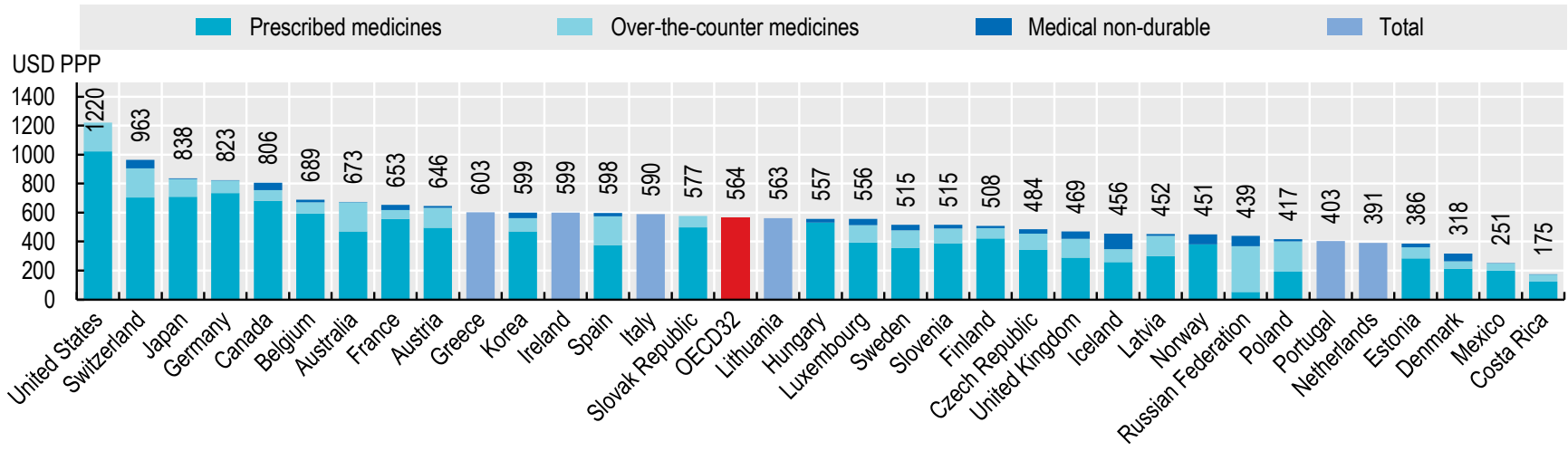

Source: OECD Health Statistics 2019.

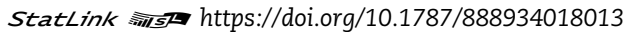

Figure 10.3. Annual average growth in retail and hospital pharmaceutical expenditure, in real terms, 2008-18 (or nearest year)

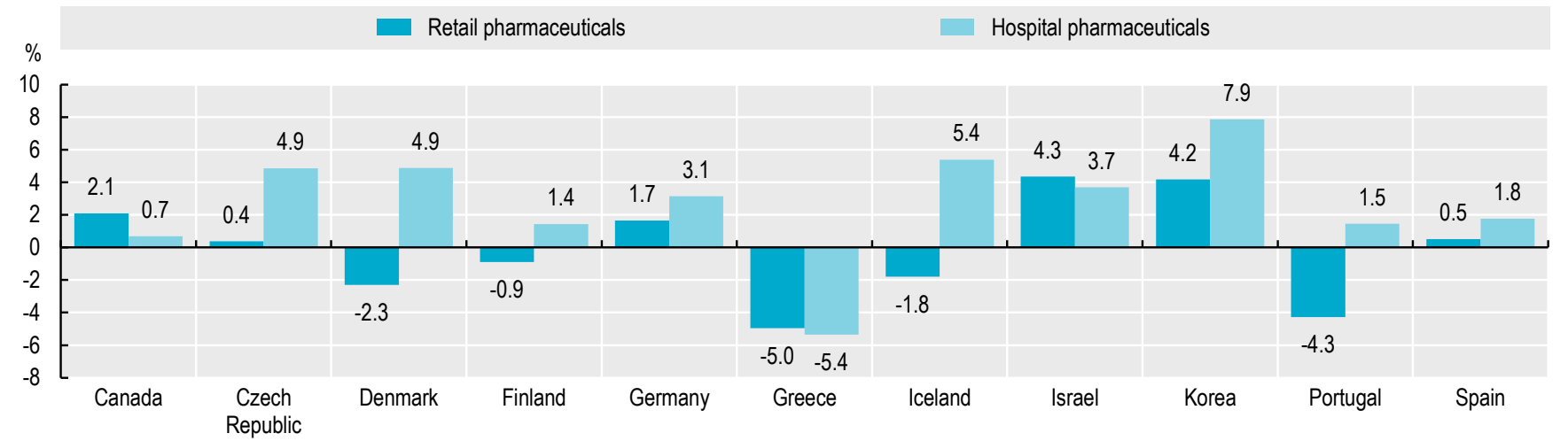

Note: OECD estimates for Portugal exclude expenditure on other medical products from retail spending. Source: OECD Health Statistics 2019. 
Pharmacists are trained health care professionals who manage the distribution of medicines to consumers/ patients and help ensure their safe and efficacious use. Between 2000 and 2017, the density of practising pharmacists increased by $33 \%$ on average in OECD countries, to 83 pharmacists per 100000 inhabitants (Figure 10.4). The number of pharmacists per capita is highest in Japan (181 pharmacists per 100000 people), and lowest in the Netherlands (21 pharmacists).

The number of pharmacists per capita increased in all OECD countries for which time series are available. Pharmacist density increased most rapidly in Japan, Portugal and Slovenia. In Japan, increased numbers of pharmacists are largely attributable to the government's efforts to more clearly separate drug prescribing by doctors from drug dispensing by pharmacists (the Bungyo system).

Across the OECD, most pharmacists work in community retail pharmacies, but some also work in hospital, industry, research and academic settings. In Canada, for example, in 2016 more than three-quarters of practising pharmacists worked in community pharmacies, while about $20 \%$ worked in hospitals and other health care facilities (CIHI, 2017[1]). In Japan, around $57 \%$ of pharmacists worked in community pharmacies in 2016, while around 19\% worked in hospitals or clinics and the remaining $24 \%$ in other settings (Ministry of Health, Labour and Welfare, 2017[2]).

The number of community pharmacies per 100000 people ranges from 7 in Denmark to 88 in Greece; with an average of 29 across OECD countries (Figure 10.5). This variation can be explained in part by differences in common distribution channels. Some countries rely more on hospital pharmacies to dispense medicines to outpatients; others still have doctors dispensing medicines to their patients (e.g. in the Netherlands). Denmark has fewer community pharmacies, but these are often large, and include branch pharmacies and subsidiary pharmacy units attached to the main pharmacy. The range of products and services provided by pharmacies also varies between countries. In most European countries, for example, pharmacies also sell cosmetics, food supplements, medical devices and homeopathic products.
The role of the community pharmacist has changed over recent years. Although their main role is to dispense medications, pharmacists are increasingly providing direct care to patients (e.g. flu vaccinations in Australia, Ireland and New Zealand; medicine adherence support in Australia, Japan, New Zealand and the United Kingdom), both in community pharmacies and as part of integrated health care provider teams.

\section{Definition and comparability}

Practising pharmacists are defined as the number of pharmacists who are licensed to practice and provide direct services to clients/patients. They can be either salaried or self-employed, and work in community pharmacies, hospitals and other settings. Assistant pharmacists and other employees of pharmacies are normally excluded.

In Ireland, the figures include all pharmacists registered with the Pharmaceutical Society of Ireland, possibly including some pharmacists who are not actively working. Assistant pharmacists are included in Iceland.

Community pharmacies are premises which, in accordance with the local legal provisions and definitions, may operate as a facility for the provision of pharmacy services in community settings. The number of community pharmacies reported are the number of premises where medicines are dispensed under the supervision of a pharmacist.

\section{References}

[1] CIHI (2017), Pharmacists in 2016, Health Workforce, https:// www.cihi.ca/en/pharmacists (accessed on 19 July 2019).

[2] Ministry of Health, Labour and Welfare (2017), Summary of Survey of Physicians, Dentists and Pharmacists, Health Statistics Office, Director-General for Statistics and Information Policy, Ministry of Health, Labour and Welfare, Tokyo. 
Figure 10.4. Practising pharmacists, 2000 and 2017 (or nearest year)

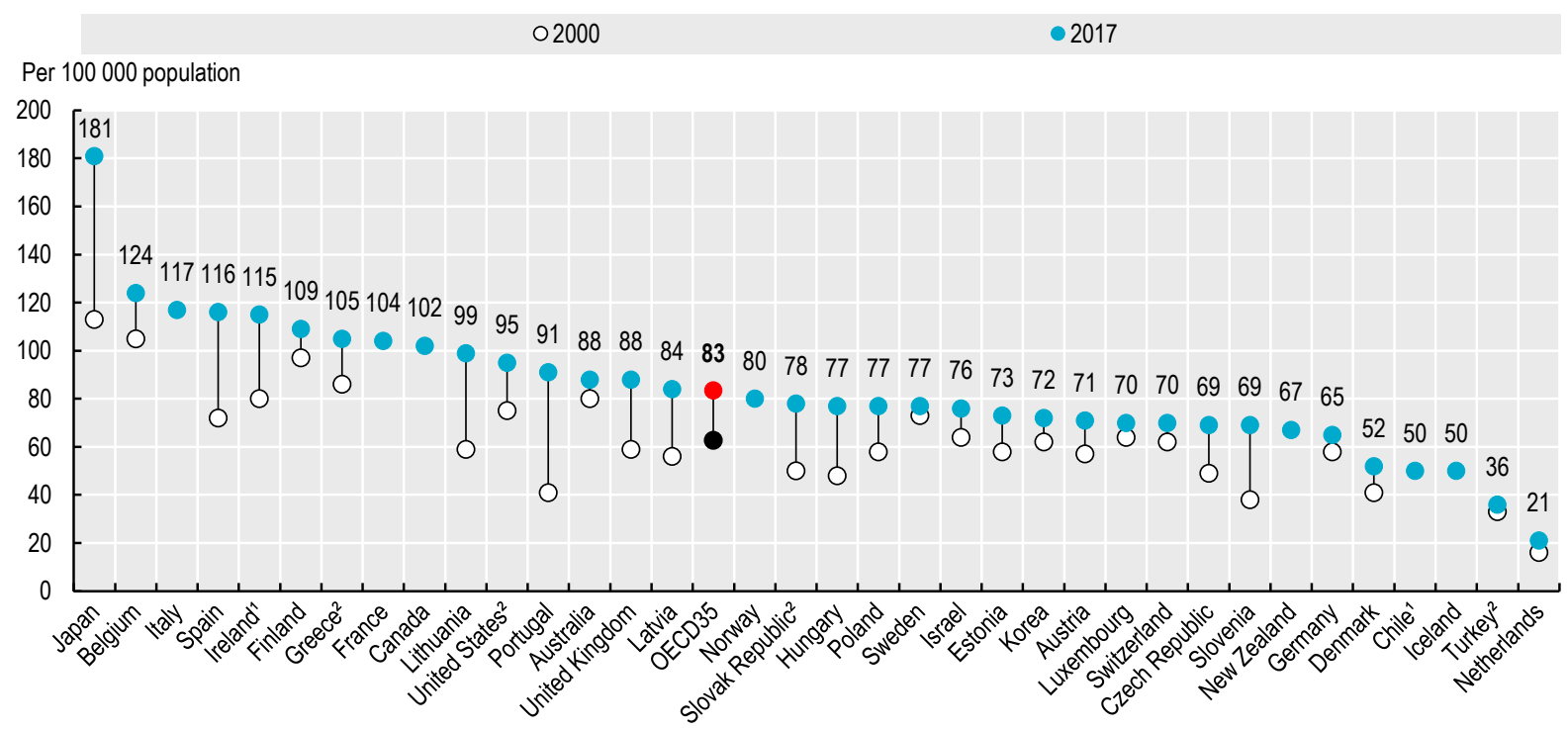

1. Data refer to all pharmacists licensed to practice. 2. Data include not only pharmacists providing direct services to patients, but also those working in the health sector as researchers, for pharmaceutical companies, etc.

Source: OECD Health Statistics 2019.

Figure 10.5. Community pharmacies, 2017 (or nearest year)

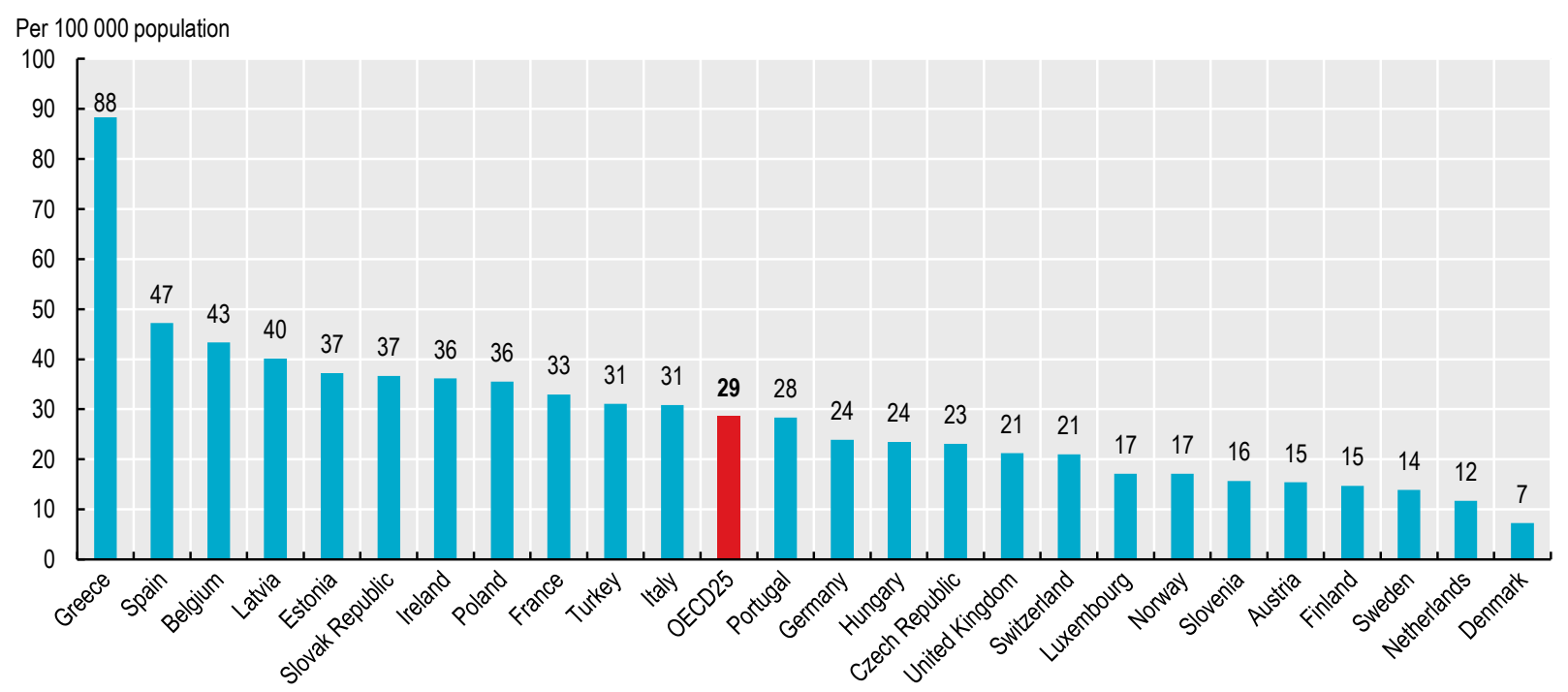

Source: Pharmaceutical Group of the European Union database 2017 or national sources. 
Pharmaceutical consumption has been increasing for decades, driven by a growing need for drugs to treat agerelated and chronic diseases, and by changes in clinical practice. This section examines consumption of four categories of medicines: anti-hypertensives, cholesterollowering agents, anti-diabetics, and anti-depressants. These medicines address illnesses for which the prevalence has increased markedly across OECD countries in recent decades.

Consumption of anti-hypertensive drugs in OECD countries increased by $70 \%$ on average between 2000 and 2017, nearly quadrupling in Luxembourg and Estonia (Figure 10.6). It remains highest in Germany and Hungary, which report almost five times the levels of consumption seen in Korea and Turkey. These variations probably reflect both differences in the prevalence of hypertension and variations in clinical practice.

Even greater growth was seen in the use of cholesterollowering agents, with consumption in OECD countries increasing by a factor of three between 2000 and 2017 (Figure 10.7). The United Kingdom, Denmark and Belgium report the highest levels of consumption per capita in 2017, with a seven-fold variation in consumption levels across the OECD.

The use of anti-diabetic drugs also grew dramatically, almost doubling in OECD over the same period (Figure 10.8). This growth can be explained in part by the rising prevalence of diabetes, which is largely linked to the increasing prevalence of obesity (see indicator on "Overweight and obesity" in Chapter 4), a major risk factor for the development of type 2 diabetes. In 2017, consumption of anti-diabetic drugs was highest in Finland and lowest in Latvia, with a two-fold variation.

Consumption of anti-depressant drugs doubled in OECD countries between 2000 and 2017 (Figure 10.9). This may reflect improved recognition of depression, availability of therapies, evolving clinical guidelines and changes in patient and provider attitudes (Mars et al., 2017[1]). However, there was significant variation between countries, with Iceland reporting the highest level of consumption in 2018, at a rate ten times that of Latvia.

\section{Definition and comparability}

The defined daily dose (DDD) is the assumed average maintenance dose per day for a drug used for its main indication in adults. DDDs are assigned to each active ingredient in a given therapeutic class by international expert consensus. For example, the DDD for oral aspirin is 3 grammes, which is the assumed maintenance daily dose to treat pain in adults. DDDs do not necessarily reflect the average daily dose actually used in a given country. They can be aggregated within and across therapeutic classes of the Anatomic-Therapeutic Classification (ATC). For more detail, see http://www.whocc.no/atcddd.

The volume of anti-hypertensive drugs consumption presented in Figure 10.6 refers to the sum of five ATC2 categories, which may all be prescribed for hypertension (C02-anti-hypertensives, C03-diuretics, C07-beta blocking agents, C08-calcium channel blockers, C09-agents acting on the renin-angiotensin system). ATC codes for other classes are: C10 for cholesterol lowering drugs; A10 for antidiabetic drugs; and N06A for anti-depressants.

Data generally refer to outpatient consumption only, except for Chile, the Czech Republic, Estonia, Finland, Iceland, Italy, Korea, Norway, the Slovak Republic and Sweden, where data also include hospital consumption. The data for Canada relate to three provinces only (British Columbia, Manitoba and Saskatchewan). The data for Luxembourg and Spain refer to outpatient consumption for prescribed drugs covered by the national health system (public insurance). Data for Luxembourg are underestimated due to incomplete consideration of products with multiple active ingredients.

\section{References}

[1] Mars, B. et al. (2017), "Influences on antidepressant prescribing trends in the UK: 1995-2011", Social Psychiatry and Psychiatric Epidemiology, Vol. 52/2, pp. 193-200, http:// dx.doi.org/10.1007/s00127-016-1306-4. 
Figure 10.6. Anti-hypertensive drug consumption, 2000 and 2017 (or nearest year)

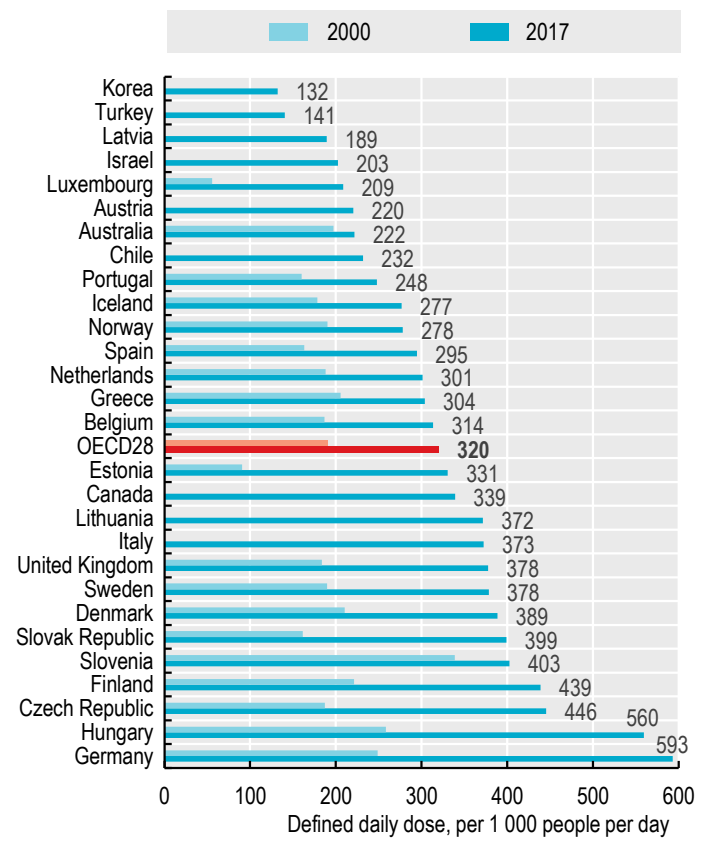

Note: Data refer to the sum of classes: C02-antihypertensives, C03diuretics, C07-beta blocking agents, C08-calcium channel blockers, C09agents acting on the renin-angiotensin system.

Source: OECD Health Statistics 2019.

StatLink कीजम https://doi.org/10.1787/888934018089

Figure 10.8. Anti-diabetic drug consumption, 2000 and 2017 (or nearest year)

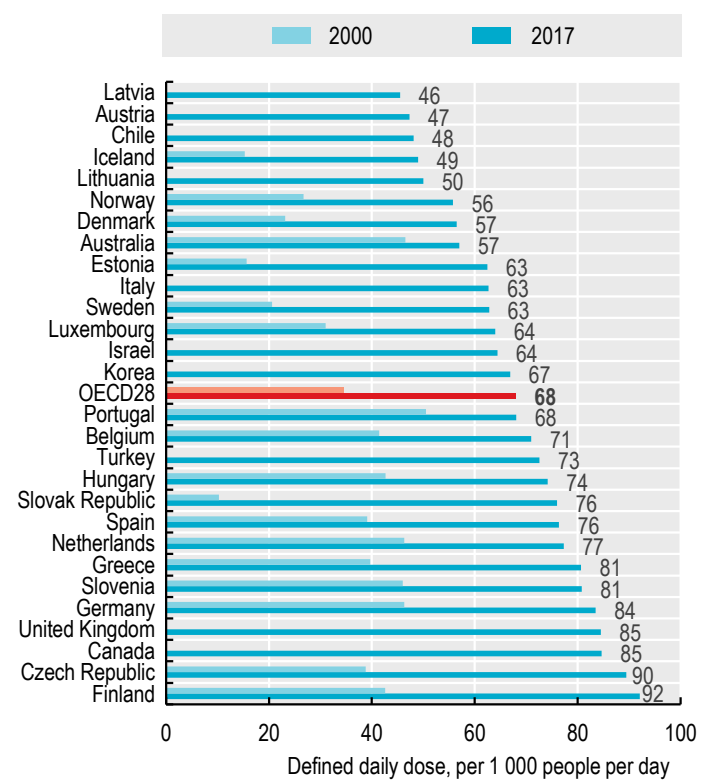

Note: Data refer to class A10-drugs used in diabetes.

Source: OECD Health Statistics 2019.

StatLink काज्ञा https://doi.org/10.1787/888934018127
Figure 10.7. Cholesterol-lowering drug consumption, 2000 and 2017 (or nearest year)

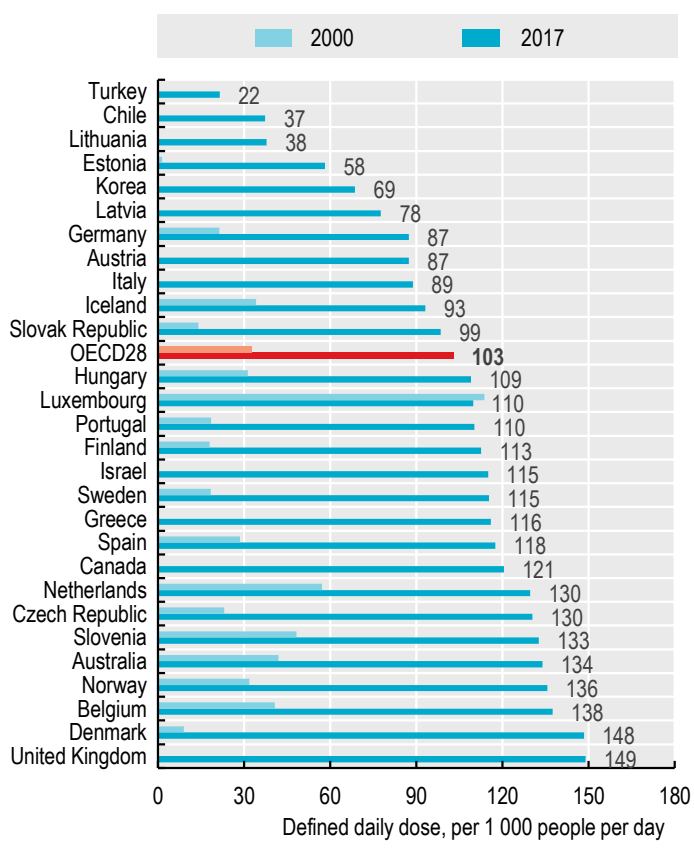

Note: Data refer to class C10-lipid modifying agents. Source: OECD Health Statistics 2019.

StatLink Aाs https://doi.org/10.1787/888934018108

Figure 10.9. Anti-depressant drug consumption, 2000 and 2017 (or nearest year)

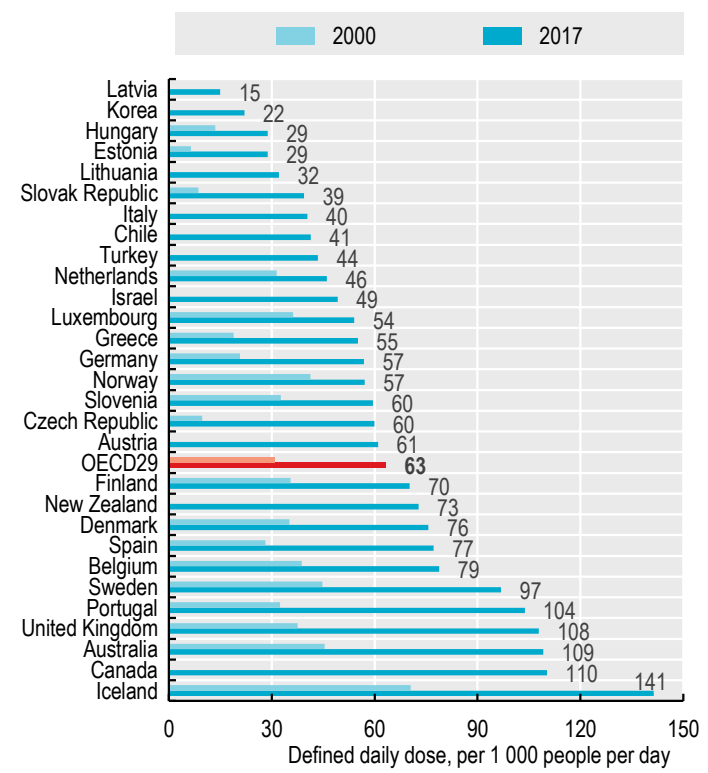

Note: Data refer to class N06A-antidepressants.

Source: OECD Health Statistics 2019.

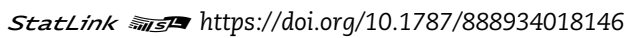


All OECD countries view generic and biosimilar markets as an opportunity to increase efficiency in pharmaceutical spending, but many do not fully exploit their potential. In 2017, generics accounted for more than three-quarters of the volume of pharmaceuticals sold in the United Kingdom, Chile, Germany and New Zealand, but less than one-quarter in Luxembourg and Switzerland (Figure 10.10). Differences in market structures (notably the number of off-patent medicines) and prescribing practices explain some crosscountry differences, but generic uptake also depends on policies (OECD, 2018[1]; Socha-Dietrich, James and Couffinhal, 2017[2]). In Austria, for example, generic substitution by pharmacists is still not allowed. In Luxembourg, generic substitution by pharmacists is set by law but is limited to selected medicines.

Many countries have implemented incentives for physicians, pharmacists and patients to boost generic markets. Over the last decade, France and Hungary, for example, have introduced incentives for GPs to prescribe generics through pay-for-performance schemes. In Switzerland, pharmacists receive a fee for generic substitution; in France, pharmacies receive bonuses if their substitution rates are high. In many countries, third-party payers fund a fixed reimbursement amount for a given medicine, allowing the patient a choice between the originator and a generic, but with responsibility for any difference in price. In Greece, patients choosing originator over generic drugs are also required to directly pay the difference.

Biological medicines contain active substances from a biological source, such as living cells or organisms. When such medicines no longer have monopoly protection, 'copies' ("biosimilars") of these products can be approved. Biosimilars have increased price competition and improved affordability. In 2017, biosimilars accounted for more than $70 \%$ of the volume of the 'accessible market' for erythropoietin (used to treat anaemia) in Finland, Germany, the Slovak Republic and Greece (Figure 10.11). In most European countries, prices of erythropoietin fell between $30 \%$ and $80 \%$ after biosimilar entry. In Norway and Denmark, known for their effective procurement policies, data show zero or small biosimilar uptake and no price reduction in 2017. In Denmark, the tender process had already triggered competition between originator products, leading to price reductions with which biosimilars could not compete. In Norway, the originator product won the nationwide tender in 2017, with confidential rebates that affected the list price. These examples highlight the inherent problems of lack of price transparency.

For tumour necrosis factor (TNF) inhibitors (used to treat autoimmune and immune-mediated disorders), biosimilars have over $90 \%$ of the accessible market in Denmark and Norway, but less than $10 \%$ in Switzerland, Ireland and Greece (Figure 10.11). Price reductions since biosimilar entry are more modest than for erythropoietin, ranging from $4 \%$ in Switzerland to $45 \%$ in Poland. For both biosimilars, actual price reductions may be higher than what appears in figures, which only report list prices.

\section{Definition and comparability}

A generic medicine is defined as a pharmaceutical product which has the same qualitative and quantitative composition in active substances and the same pharmaceutical form as the reference product, and whose bioequivalence with the reference product has been demonstrated. Generics may be either branded (generics with a specific trade name) or unbranded (identified using the international nonproprietary name and the name of the company).

Countries were requested to provide data for the whole of their respective markets. However, many countries provided data covering only the community pharmaceutical market or the reimbursed pharmaceutical market (see figure notes). The share of generic market expressed in value can be the turnover of pharmaceutical companies, the amount paid for pharmaceuticals by third-party payers, or the amount paid by all payers (third party and consumers). The share of the generic market by volume can be expressed in DDDs or as a number of packages/boxes or standard units.

A biosimilar medicinal product (a biosimilar) is a product granted regulatory approval by demonstrating sufficient similarity to the reference medicinal product (biological) in terms of quality characteristics, biological activity, safety and efficacy.

Biosimilar market shares and changes in prices are measured for the 'accessible market', i.e. the market composed of originator products that are no longer protected and their biosimilars. Market share is computed as biosimilar treatment days as a share of the total volume of biosimilar and referenced product(s). Price change is measured as the difference between prices in 2017 and in the year before entry of the first biosimilar.

\section{References}

[1] OECD (2018), "Strategies to reduce wasteful spending: Turning the lens to hospitals and pharmaceuticals", in Health at a Glance: Europe 2018: State of Health in the EU Cycle, OECD Publishing, Paris, https://dx.doi.org/10.1787/ health_glance_eur-2018-5-en.

[3] QuintilesIMS (2017), The Impact of Biosimilar Competition in Europe, QuintilesIMS Institute, Parsippany, http:// www.quintilesims.com.

[2] Socha-Dietrich, K., C. James and A. Couffinhal (2017), "Reducing ineffective health care spending on pharmaceuticals", in Tackling Wasteful Spending on Health, OECD Publishing, Paris, https://dx.doi.org/ 10.1787/9789264266414-7-en. 
Figure 10.10. Share of generics in the total pharmaceutical market, 2017 (or nearest year)

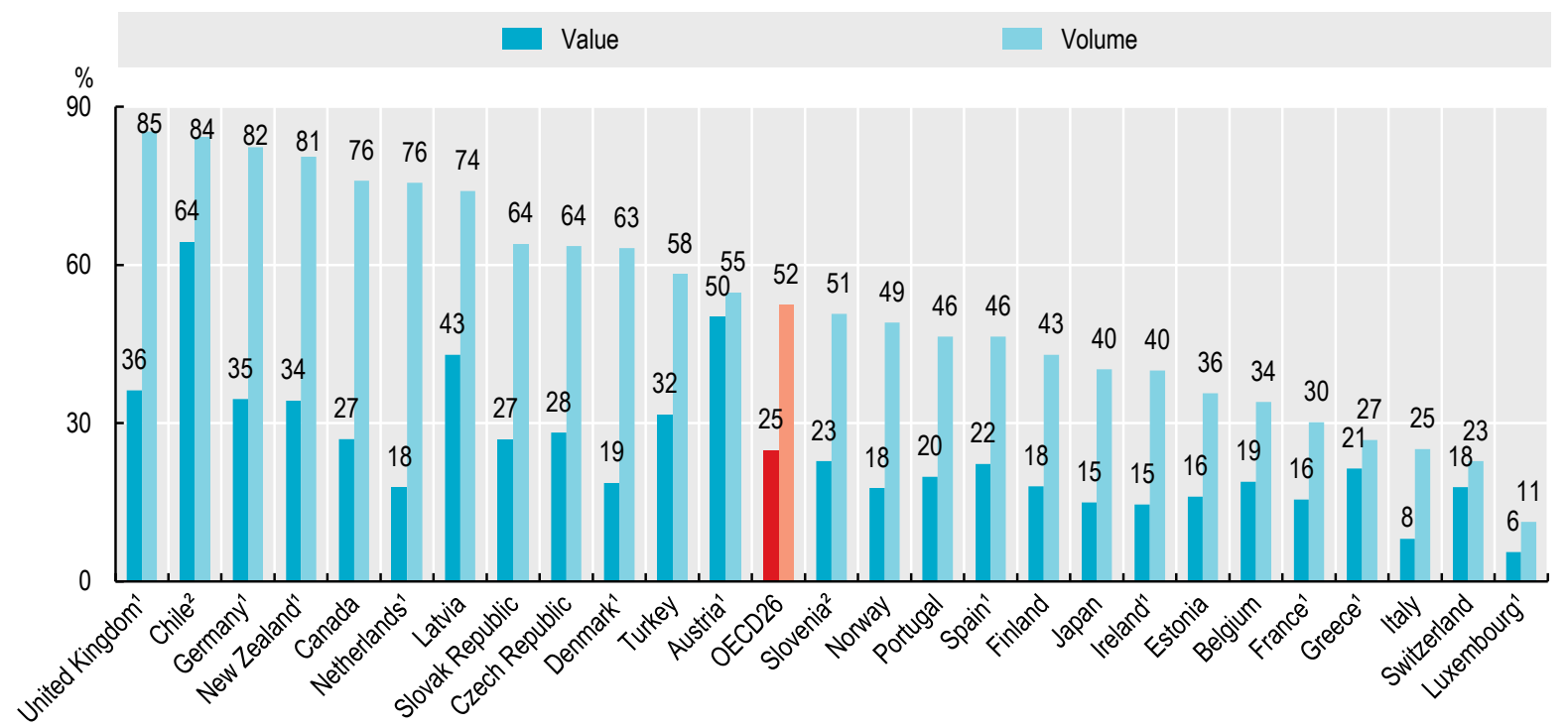

1. Reimbursed pharmaceutical market. 2. Community pharmacy market.

Source: OECD Health Statistics 2019.

Figure 10.11. Biosimilar market share in treatment days for anti-TNF alfas and erythropoietin vs accessible market, 2017 (or nearest year), in European countries
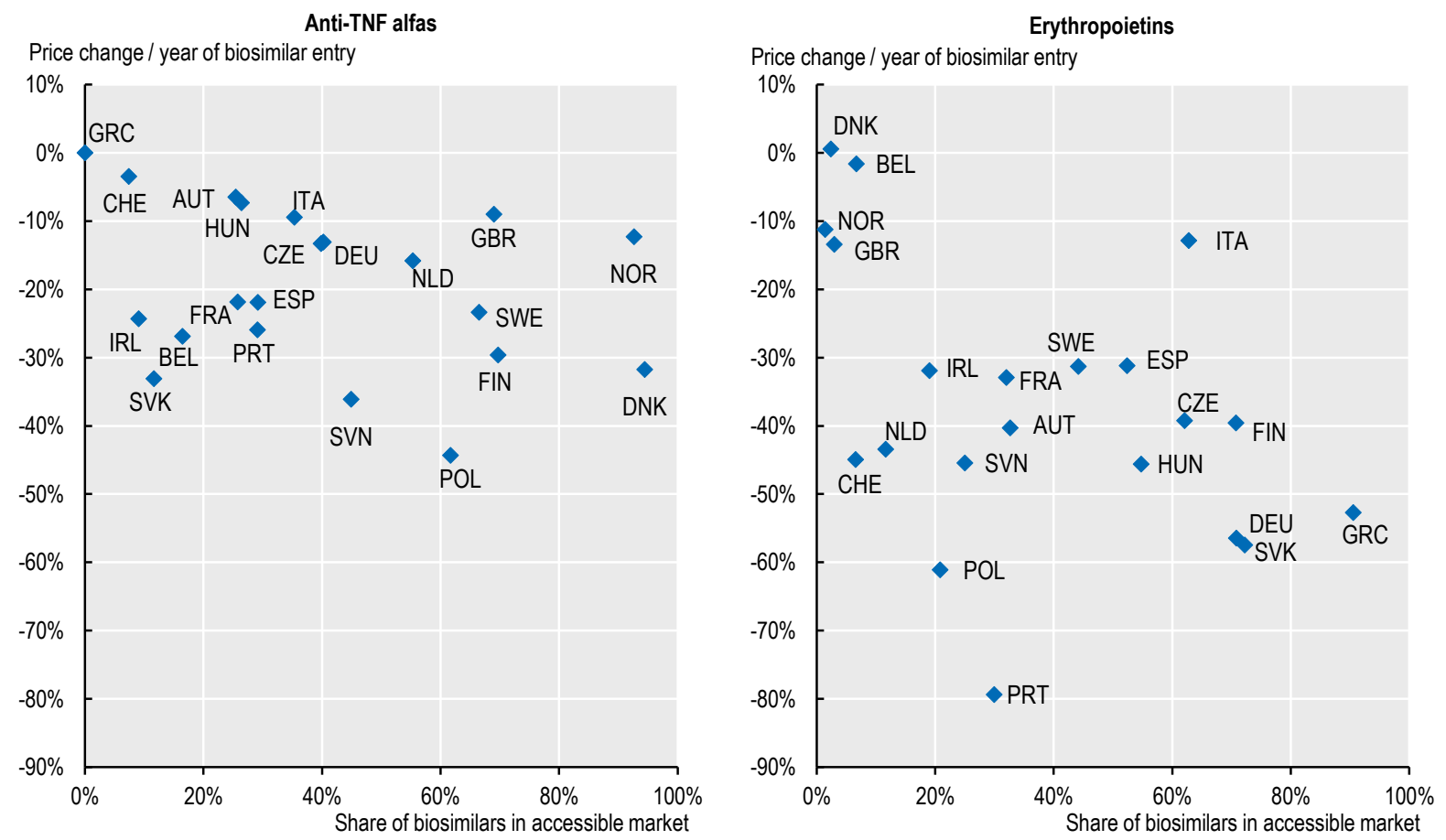

Source: IQVIA MIDAS MAT December 2017. 
Pharmaceutical research and development (R\&D) is funded from a complex mix of private and public sources. Governments mainly support basic and early-stage research through direct budget allocations, research grants, publicly owned research institutions and higher education institutions. The pharmaceutical industry is active across all phases of $R \& D$ but makes the largest contribution to translating and applying knowledge to develop products. Clinical trials required to gain market approval are largely funded by industry. However, industry also receives direct R\&D subsidies or tax credits in many countries.

In 2016, governments of 31 OECD countries from which data are available collectively budgeted about USD 53 billion for health-related R\&D (a broader category than pharmaceuticals). This figure understates total government support because it excludes most tax incentives and funding for higher education and publicly-owned corporations. Meanwhile, the pharmaceutical industry spent approximately USD 101 billion on R\&D across OECD countries.

Most pharmaceutical industry R\&D expenditure comes from OECD countries but the share from non-OECD countries is increasing (EFPIA, 2018[1]). Growth has been particularly rapid in China, where the industry spent USD 14 billion on R\&D in 2016 (0.07\% of GDP) - a more than 2.5-fold increase since 2010 (in real terms) (OECD, 2019[2]). Nearly two-thirds of the spending in OECD countries (Figure 10.12) occurs in the United States, where the industry spent about USD 65 billion ( $0.35 \%$ of GDP), and government budgets on health-related R\&D were USD 36 billion ( $0.19 \%$ of GDP). The industry spent USD 20 billion (0.1\% of GDP) and governments budgeted USD 11 billion ( $0.06 \%$ of GDP) in Europe; the figures were USD 13 billion ( $0.25 \%$ of GDP) and USD 1.4 billion $(0.03 \%$ of GDP) respectively in Japan. As a share of GDP, industry spending is highest in Switzerland (0.85\%), Denmark (0.46\%) and Slovenia $(0.45 \%)$, smaller countries with relatively large pharmaceutical sectors.

The pharmaceutical industry is highly R\&D intensive. On average across OECD countries, the industry spent nearly $12 \%$ of its gross value added on R\&D. This is almost as high as in the electronics and optical and air and spacecraft industries, and considerably higher than across manufacturing as a whole (Figure 10.13).

Expenditure on R\&D in the pharmaceutical industry in OECD countries grew by 14\% in real terms between 2010 and 2016. The number of new drug approvals has also increased since 2010, following a decline after the 1980s. In the United States, for example, the annual number of approvals is now back to a similar level to that seen in the 1980s (Figure 10.14). However, given the increase in R\&D expenditure, the number of approvals per inflation-adjusted R\&D spending has declined steadily.

This pattern of decreasing productivity despite advances in technology is driven by a complex combination of factors. These include growing requirements to obtain market approval, which have increased clinical trial costs, and an ever-increasing base of effective drugs that has shifted efforts to drugs for more complex conditions. Rising R\&D costs can be both a cause and a result of higher drug prices, as the acceptance of higher prices by payers can make increasingly expensive $R \& D$ and acquisitions of $R \& D$ projects financially viable. Increasing $R \& D$ and acquisition costs can, in turn, drive up prices.

\section{Definition and comparability}

Business enterprise expenditure on R\&D (BERD) covers $R \& D$ carried out by corporations, regardless of the origin of the funding, which can include government subsidies. BERD is recorded in the country where the R\&D activity took place, not the country providing funding. National statistical agencies collect data primarily through surveys and according to the Frascati Manual but there is some variation in national practices. Pharmaceutical R\&D refers to BERD by businesses classified in the pharmaceutical industry. Europe includes $21 \mathrm{EU}$ member states that are also OECD countries, Iceland, Norway and Switzerland (with no data available for Lithuania and Luxembourg). Government budgets for R\&D (GBARD) capture R\&D performed directly by government and amounts paid to other institutions for R\&D. Health-related R\&D refers to GBARD aimed at protecting, promoting and restoring human health, including all aspects of medical and social care. It does not cover spending by public corporations or general university funding that is subsequently allocated to health.

The gross value added (GVA) of a sector equals gross output less intermediate consumption. It includes the cost of wages, consumption of fixed capital and taxes on production. Because GVA does not include intermediate consumption, it is less sensitive than gross output to sector-specific reliance on raw materials. The OECD average in Figure 10.13 is an unweighted mean of R\&D intensity across 18 countries with data available for air and spacecraft; and 29-33 countries for all other industries.

Data in Figure 10.14 include approvals of new molecular entities (NMEs) and other new drug applications (NDAs) and new biologic license application (BLAs) and other BLAs.

\section{References}

[1] EFPIA (2018), The Pharmaceutical Industry in Figures, https:// www.efpia.eu/media/361960/efpia-pharmafigures2018_v07hq.pdf.

[2] OECD (2019), Analytical Business Enterprise Research and Development (ANBERD) Database, http://oe.cd/anberd. 
10. PHARMACEUTICAL SECTOR

Figure 10.12. Business enterprise expenditure for pharmaceutical R\&D (BERD) and government outlays for health-related R\&D (GBARD), 2016 (or nearest year)
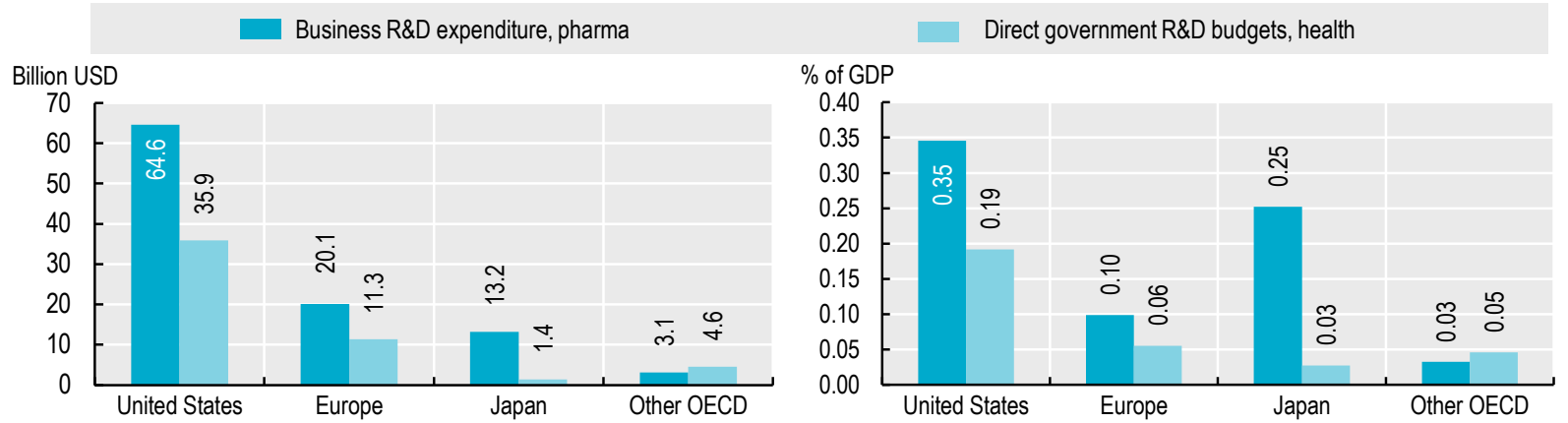

Source: OECD Main Science and Technology Indicators and Research and Development Statistics databases.

StatLink AाIS https://doi.org/10.1787/888934018203

Figure 10.13. R\&D intensity by industry: business enterprise R\&D expenditure as a share of gross valued added, 2016 (or nearest year)

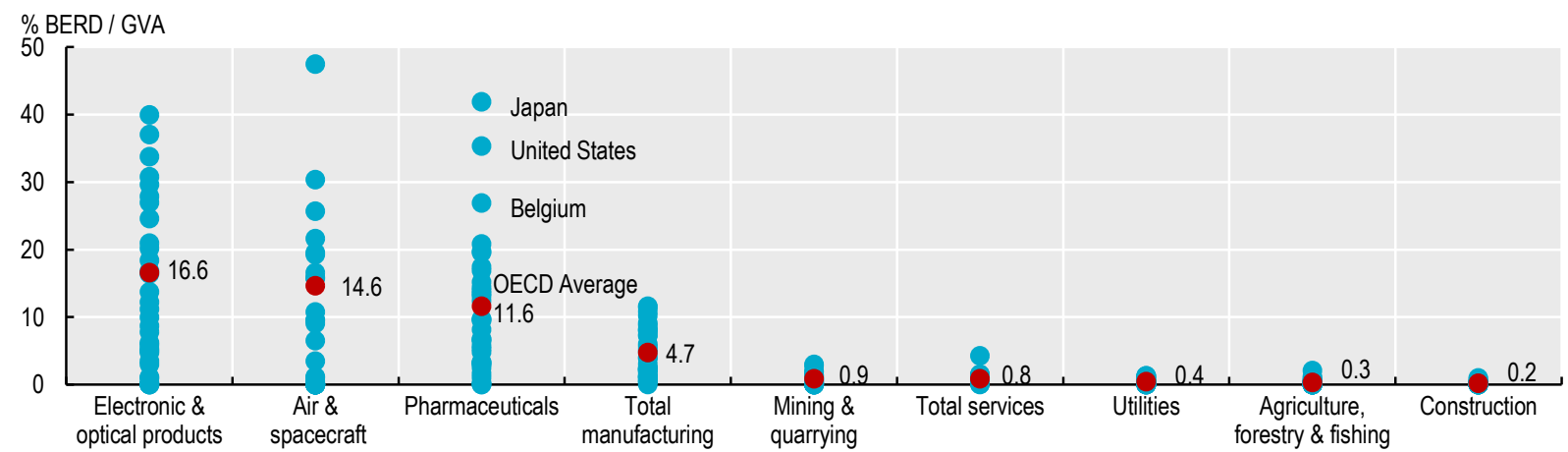

Source: OECD Analytical Business Enterprise R\&D, Structural Analysis and System of National Accounts databases.

StatLink AाIs https://doi.org/10.1787/888934018222

Figure 10.14. Annual approvals of new medicines per billion USD pharmaceutical business expenditure on R\&D in the United States, inflation-adjusted, 1980 to 2017

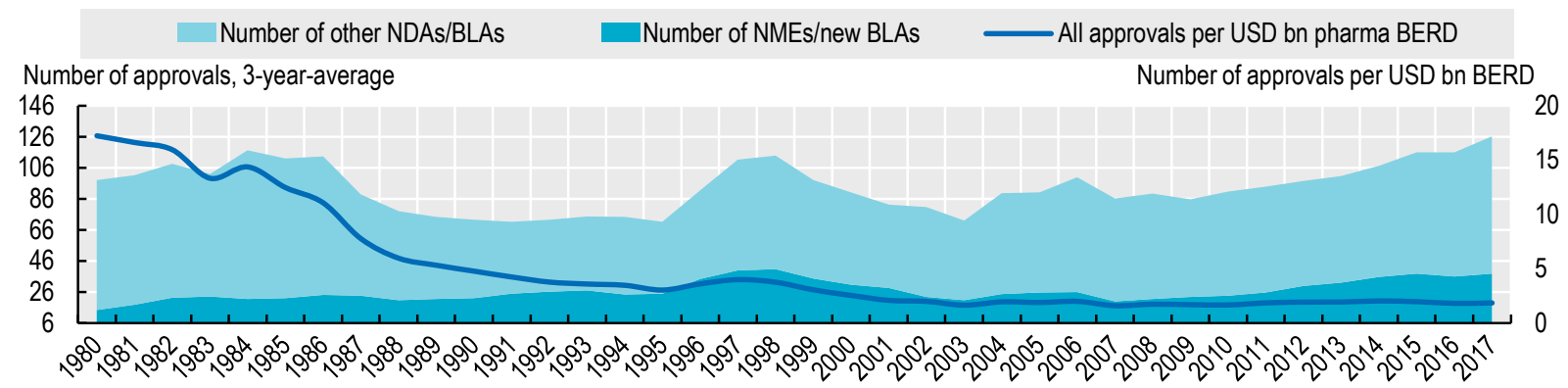

Source: United States Food and Drug Administration; Pharmaceutical Research and Manufacturers of America.

StatLink क्गाड https://doi.org/10.1787/888934018241 


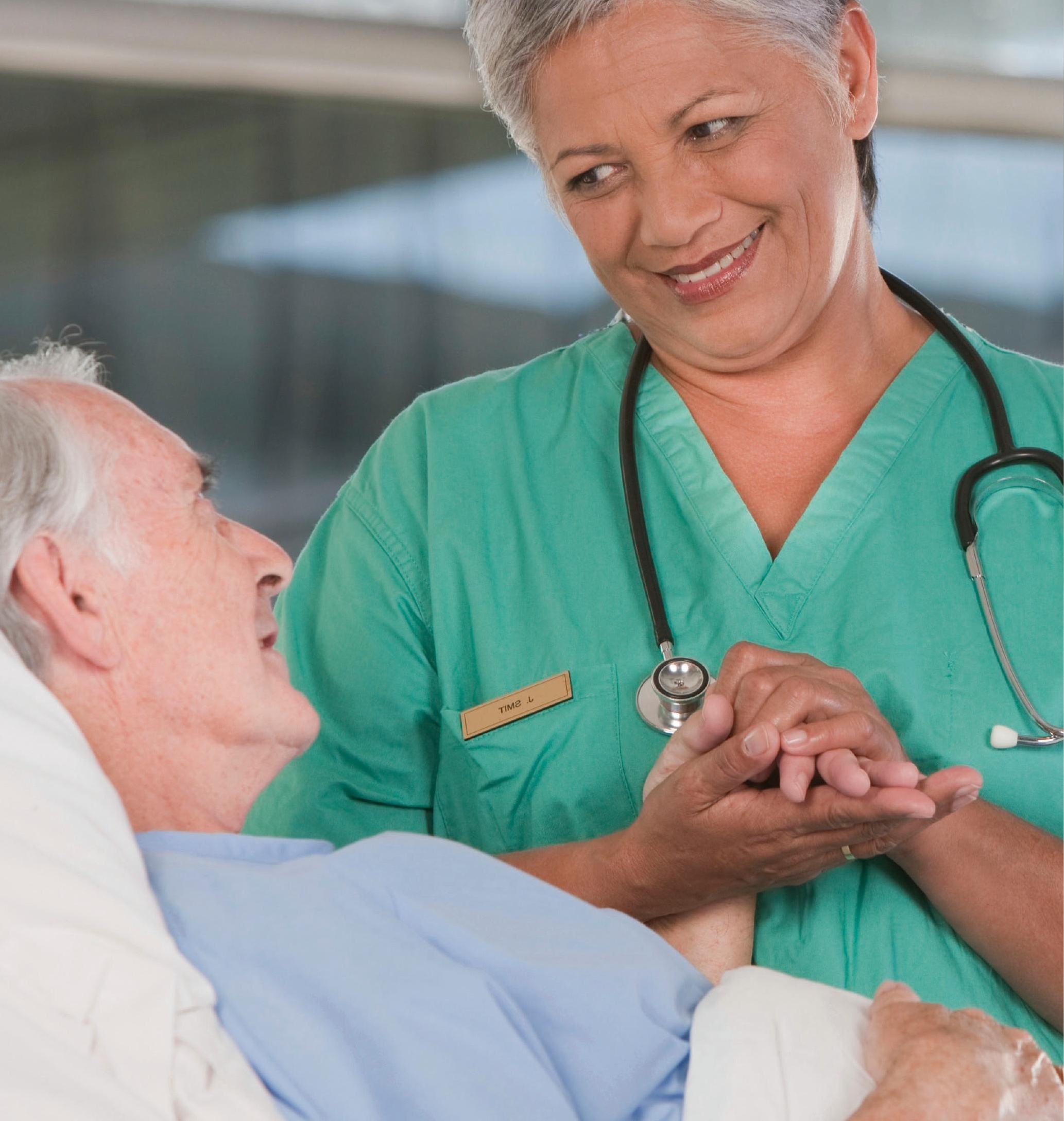



In recent decades, the share of the population aged 65 years or older has nearly doubled on average across OECD countries. The proportion of the population aged 65 years or over increased from less than $9 \%$ in 1960 to more than $17 \%$ in 2017. Declining fertility rates and longer life expectancies (see indicator on "Life expectancy" in Chapter 3) have meant that older people make up an increasing proportion of the population in OECD countries.

Across OECD countries on average, the share of the population aged 65 and over is projected to continue increasing in the coming decades, rising from $17.4 \%$ in 2017 to $27.1 \%$ by 2050 (Figure 11.1). In five OECD countries (Italy, Portugal, Greece, Japan, and Korea), the share of the population aged 65 and over will exceed one-third by 2050 . At the other end of the spectrum, the population aged 65 and over in Israel, Mexico and Australia will represent less than $20 \%$ of the population in 2050 , due to higher fertility and migration rates.

While the rise in the population aged 65 and over has been striking across OECD countries, the increase has been particularly rapid among the oldest - people 80 years of age and older. Between 2017 and 2050, the share of the population 80 and above will more than double on average in OECD countries, from $4.6 \%$ to $10.1 \%$. At least one in ten people will be 80 or older in nearly half (17) of OECD countries by 2050, while in six countries (Lithuania, Portugal, Italy, Greece, Korea and Japan), more than one in eight people will be 80 or older.

While most OECD partner countries have a younger age structure than many OECD members, population ageing will nonetheless occur rapidly in the coming years - sometimes at a faster pace than among OECD countries. In China, the share of the population aged 65 and over will increase much more rapidly than in OECD countries, more than doubling from $10.6 \%$ in 2017 to $26.3 \%$ in 2050 . The share of the Chinese population aged 80 and above will rise even more quickly, increasing more than three-fold from $1.8 \%$ in 2017 to $8.1 \%$ in 2050. Brazil - whose population aged 65 and over was barely half of the OECD average in 2017 - will see similarly rapid growth, with nearly $22 \%$ of the population projected to be aged 65 or over by 2050 .

The speed of population ageing has varied markedly across OECD countries, with Japan in particular experiencing rapid ageing over the past three decades (Figure 11.2). In the coming years, Korea is projected to undergo the most rapid population ageing among OECD members, with the share of the population 80 and above quintupling from well below the OECD average in 2017 (3\% versus 4.6\%), to 15.1\% (well above the OECD average of $10.1 \%$ ) by 2050. Among OECD partner countries, the speed of ageing has been slower than OECD members, though rapid ageing in large countries including Brazil and China will accelerate in the coming decades.

One of the major implications of rapid population ageing is the decline in the potential supply of labour in the economy, even despite recent efforts by countries to extend working lives. Moreover, despite the gains in healthy life expectancy seen in recent years (see indicator on "Life expectancy and healthy life expectancy"), population ageing will likely lead to greater demand for labour-intensive long-term care. Between 2015 and 2030, the number of older people in need of care around the world is projected to increase by 100 million (ILO and OECD 2019[1]). Countries such as the United States are already facing shortages of long-term care workers, and in the coming years, more will find themselves under pressure to recruit and retain skilled long-term care staff (see indicator on "Long-term care workers").

\section{Definition and comparability}

Data on the population structure have been extracted from the OECD historical population data and projections (1950-2050). The projections are based on the most recent "medium-variant" population projections from the United Nations, World Population Prospects-2019 Revision.

\section{References}

[2] Muir, T. (2017), "Measuring social protection for long-term care", OECD Health Working Papers, No. 93, OECD Publishing, Paris, https://dx.doi.org/10.1787/a411500a-en.

[1] OECD and ILO (2019), "New job opportunities in an ageing society", https://www.oecd.org/g20/summits/osaka/ILO-OECDG20-Paper-1-3-New-job-opportunities-in-an-ageing-society.pdf.

[3] OECD (2015), Fiscal Sustainability of Health Systems: Bridging Health and Finance Perspectives, OECD Publishing, Paris, http:// dx.doi.org/10.1787/9789264233386-en.

[4] OECD (2017), Pensions at a Glance 2017: OECD and G20 Indicators, OECD Publishing, Paris, https://doi.org/10.1787/ pension_glance-2017-en.

[5] United Nations (2019), "2019 Revision of World Population Prospects", United Nations, https://esa.un.org/unpd/wpp/. 
Figure 11.1. Share of the population aged over 65 and 80 years, 2017 and 2050

Population aged 65 years and over

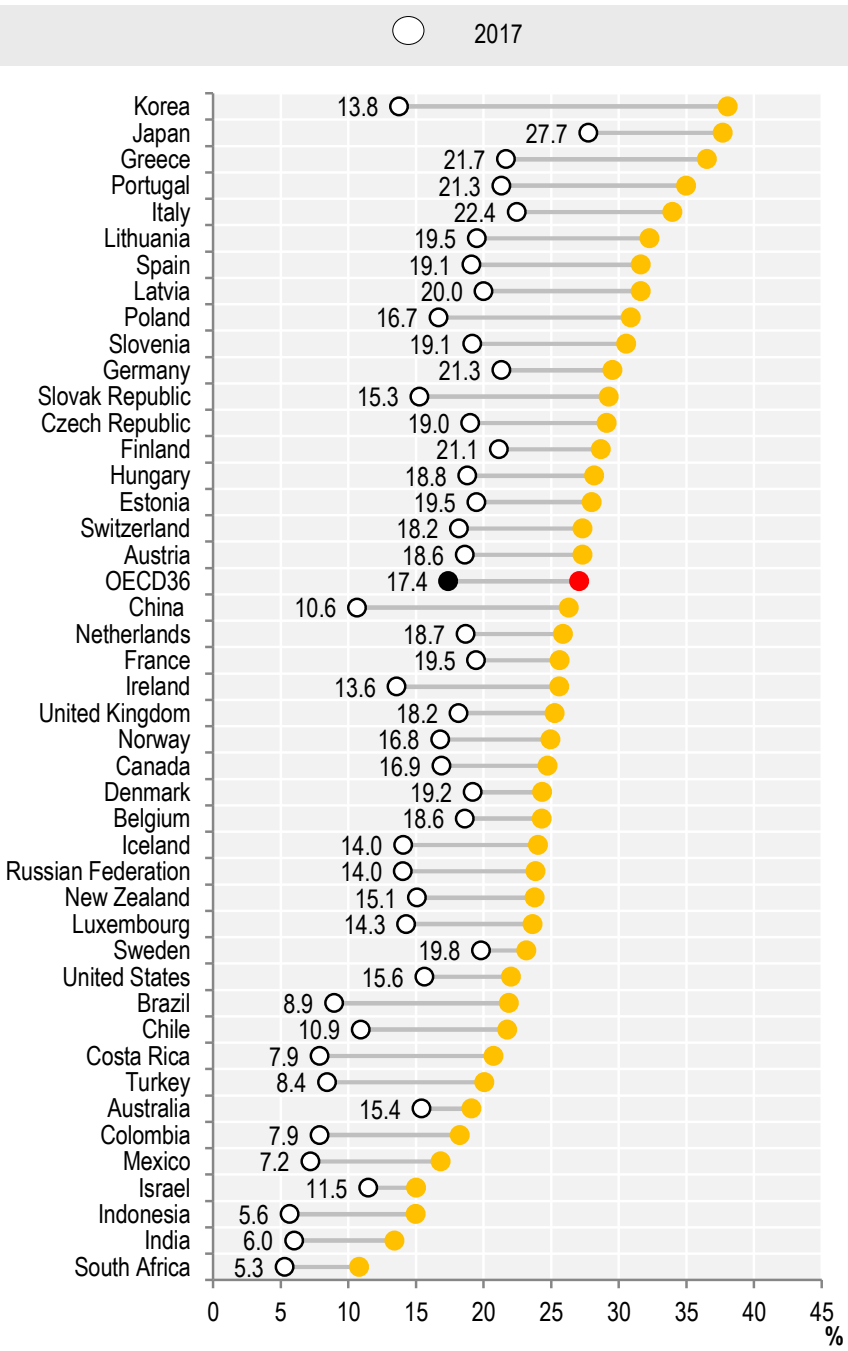

Population aged 80 years and over

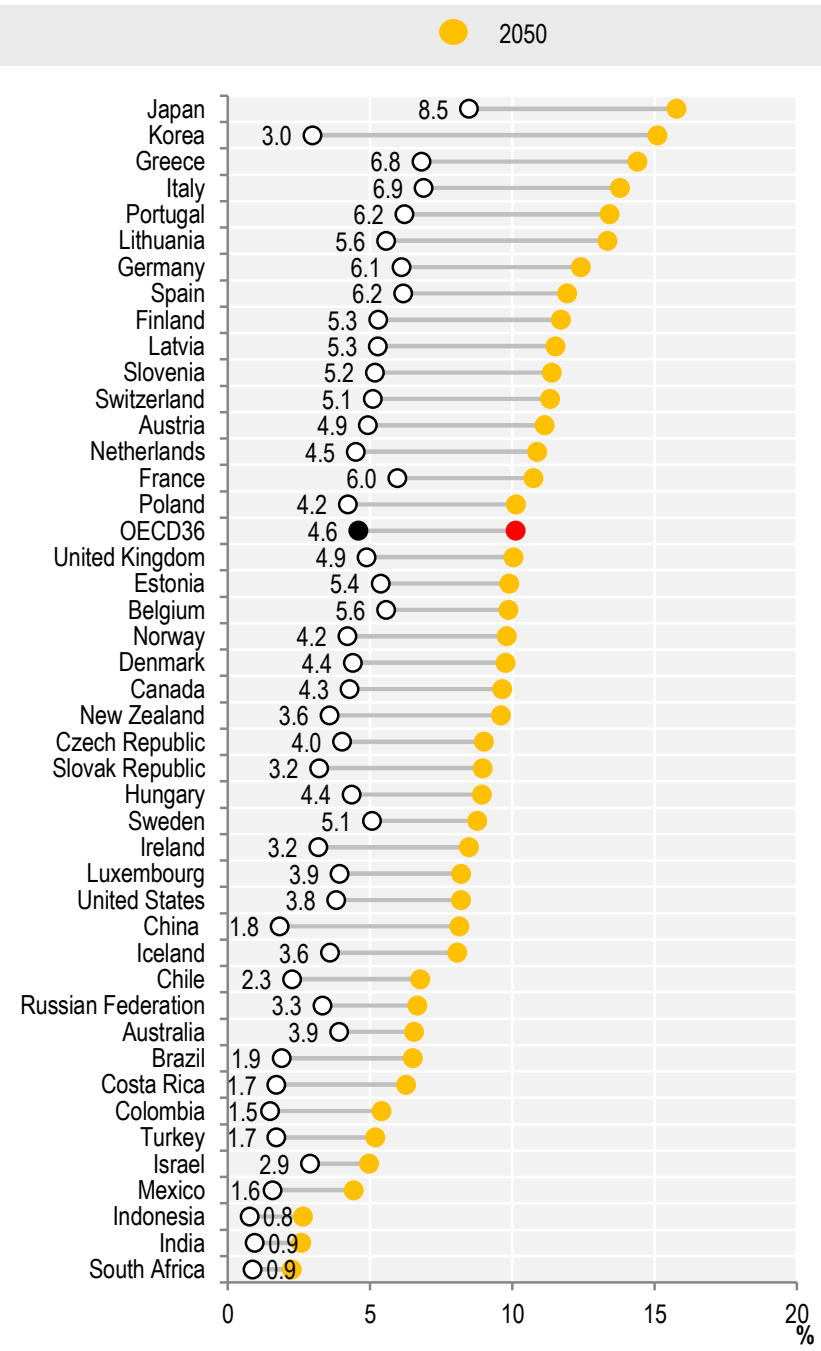

Source: OECD Health Statistics 2019, OECD Historical Population Data and Projections Database, 2019.

StatLink 겐 https://doi.org/10.1787/888934018260

Figure 11.2. Trends in the share of the population aged over 80 years, 1990-2050

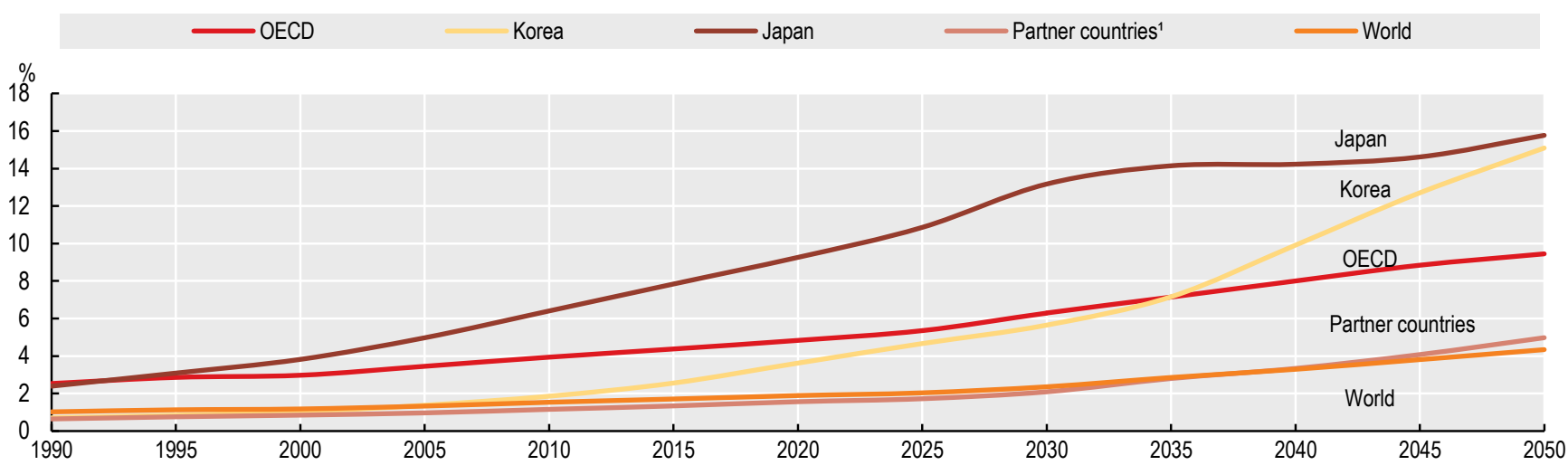

1. Partner countries include Brazil, China, Colombia, Costa Rica, India, Indonesia, the Russian Federation and South Africa. Source: OECD Historical Population Data and Projections Database, 2019. 
All OECD countries have experienced tremendous gains in life expectancy at age 65 for both men and women in recent decades. On average across OECD countries, life expectancy at age 65 increased by 5.5 years between 1970 and 2017 (Figure 11.3). Four countries (Australia, Finland, Korea, and Japan) enjoyed gains of more than seven years over the period; only one country (Lithuania) experienced an increase in life expectancy at age 65 of less than two years between 1970 and 2017.

On average across OECD countries, people at age 65 could expect to live a further 19.7 years. Life expectancy at age 65 is more than 2.5 years higher for women than for men of the same age. This gender gap has not changed substantially since 1970, when life expectancy at age 65 was 2.9 years longer for women than men. Life expectancy at age 65 was highest for women in Japan (24.4 years) and for men in Switzerland (20 years). Among OECD countries, life expectancy at age 65 in 2017 was lowest for women in Hungary (18.4 years), and for men in Latvia (14.1 years).

While all OECD countries experienced gains in life expectancy at age 65 between 1970 and 2017, not all additional years are lived in good health. The number of healthy life years at age 65 varies substantially across OECD countries (Figure 11.4). In Europe, an indicator of disabilityfree life expectancy known as "healthy life years" is calculated regularly, based on a general question about disability in the European Union Statistics on Income and Living Conditions (EU-SILC) survey. On average across OECD countries participating in the survey, the number of healthy life years at age 65 was 9.6 for women and 9.4 for men - a markedly smaller difference than that of general life expectancy at age 65 between men and women. Healthy life expectancy at age 65 was above 15 years for both men and women in Norway, Sweden and Iceland; for men, this was nearly three years above the next-best performing countries (Ireland and Spain). Healthy life expectancy at 65 was less than five years for both men and women in the Slovak Republic and Latvia. In the Slovak Republic and Latvia, women spend nearly $80 \%$ of additional life years in poor health, compared with less than $30 \%$ in Norway, Sweden and Iceland.

Gains in life expectancy at age 65 have slowed in recent years (Figure 11.5). Life expectancy at age 65 increased by 11 months on average in OECD countries between 2002 and 2007; between 2012 and 2017, countries added just over seven months to life expectancy at age 65. Gains in life expectancy at age 65 accelerated in just eight OECD countries (Chile, Greece, Israel, Japan, Latvia, Lithuania, Slovak Republic and Turkey) between 2012-2017 compared with 2002-2007; in Iceland, life expectancy at age 65 declined between 2012 and 2017. The slowdown in life expectancy at age 65 in 2012-2017 compared with 2002-2007 may be partially explained by the severe influenza epidemic of 2014-2015, which affected frail and older populations in particular. As population ageing continues, OECD countries will need to anticipate health challenges, like flu outbreaks, that can disproportionately affect older populations, and be prepared to address them, including by ensuring high influenza vaccination rates.

\section{Definition and comparability}

Life expectancy measures how long on average a person of a given age can expect to live, if current death rates do not change. However, the actual agespecific death rate of any particular birth cohort cannot be known in advance. If rates are falling, as has been the case over the past decades in OECD countries, actual life spans will be higher than life expectancy calculated using current death rates. The methodology used to calculate life expectancy can vary slightly between countries. This can change a country's estimates by a fraction of a year. Life expectancy at age 65 is the unweighted average of the life expectancy at age 65 of women and men. Gains in life expectancy were calculated as the difference in the number of years gained in life expectancy between the periods 2002-2007 and 2012-2017.

Disability-free life expectancy (or "healthy life years") is defined as the number of years spent free of activity limitation. In Europe, this indicator is calculated annually by Eurostat for EU countries and some EFTA countries. The disability measure is based on the Global Activity Limitation Indicator (GALI) question, which comes from the EU-SILC survey. The question asks: "For at least the past six months, have you been hampered because of a health problem in activities people usually do? Yes, strongly limited / Yes, limited / No, not limited". While healthy life years is the most comparable indicator to date, there are still problems with translation of the GALI question, although it does appear to satisfactorily reflect other health and disability measures (Jagger et al., 2010[1]).Data on the population structure have been extracted from the OECD historical population data and projections (1950-2050). The projections are based on the most recent "medium-variant" population projections from the United Nations, World Population Prospects - 2019 Revision.

\section{References}

[1] Jagger, C. et al. (2010), “The Global Activity Limitation Indicator (GALI) Measured Function and Disability Similarly across European Countries", Journal of Clinical Epidemiology, Vol. 63, pp. 892-899.

[2] Mäki, N. et al. (2013), "Educational Differences in Disabilityfree Life Expectancy: A Comparative study of Long-standing Activity Limitation in Eight European Countries", Social Science \& Medicine, Vol. 94, pp. 1-8. 
11. AGEING AND LONG-TERM CARE

Figure 11.3. Life expectancy at age 65, 1970 and 2017 (or nearest year)

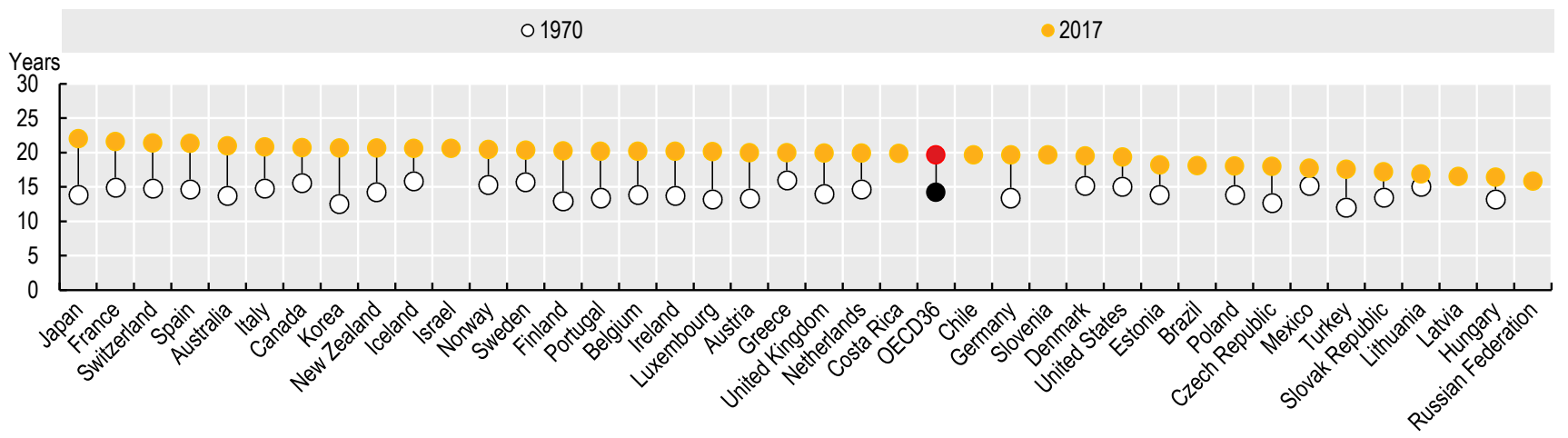

Source: OECD Health Statistics 2019.

StatLink (त)

Figure 11.4. Life expectancy and healthy life years at age 65, by sex, 2017 (or nearest year)

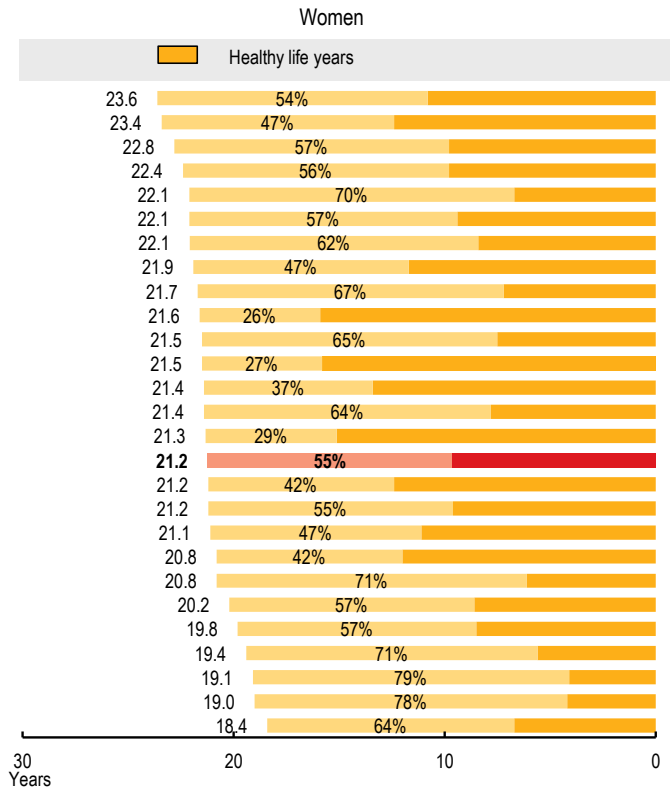

France
Spain
Switzerland
Italy
Portugal
Finland
Luxembourg
Belgium
Slovenia
Norway
Austria
Sweden
Ireland
Greece
Iceland
OECD26
Germany
Netherlands
United Kingdom
Denmark
Estonia
Poland
Czech Republic
Lithuania
Slovak Republic
Latvia
Hungary

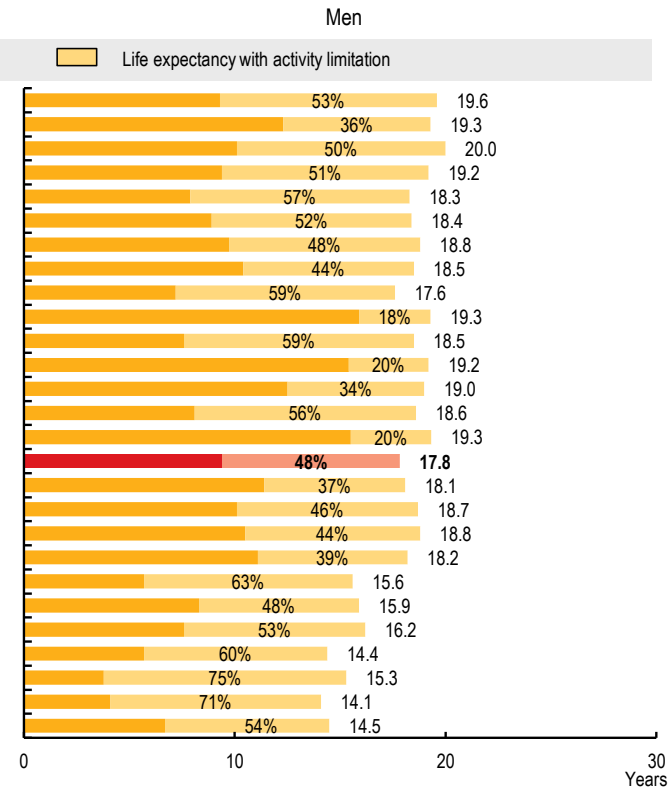

Note: Data comparability is limited because of cultural factors and different formulations of question in EU-SILC.

1. Three-year average (2015-17).

Source: Eurostat Database.

StatLink (iाls https://doi.org/10.1787/888934018317

Figure 11.5. Slowdown in life expectancy gains

○ 2002-2007

2012-2017

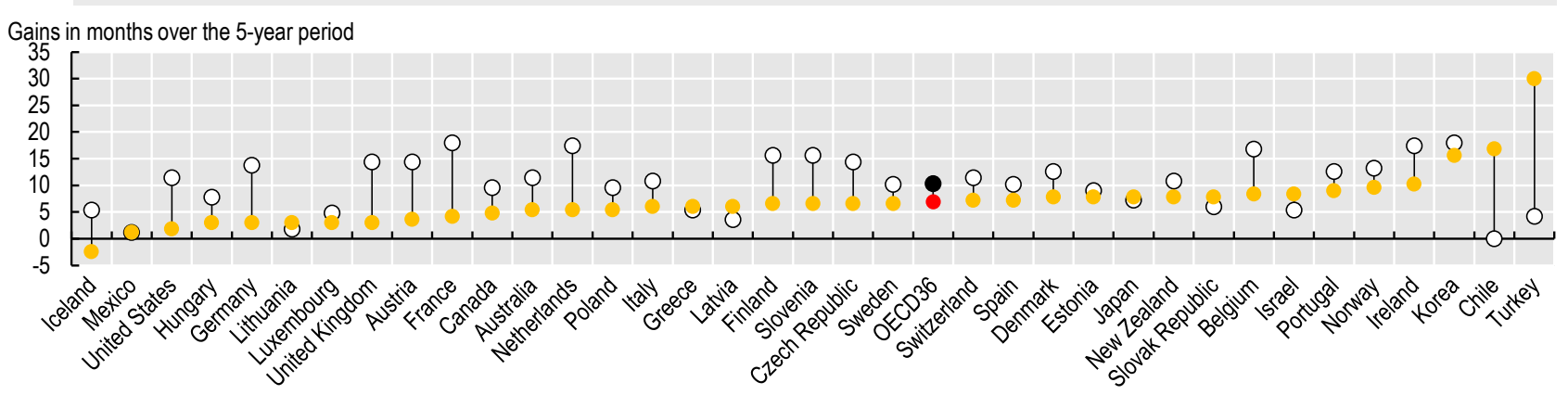

Source: OECD Health Statistics 2019. 
Even as life expectancy at age 65 has increased across OECD countries, many adults spend a high proportion of their older lives in poor or fair health (see indicator on "Life expectancy and healthy life expectancy"). In 2017, more than half the population aged 65 and over in 35 OECD countries reported being in poor or fair health (Figure 11.6). Older people in eastern European OECD countries report some of the highest rates of poor or fair health, with more than three-quarters of people aged 65 and over reporting their health to be fair, bad or very bad in Lithuania, Latvia, Estonia, Hungary, Poland, and the Slovak Republic. High rates of poor health are also reported in Portugal and Korea. Women are slightly more likely to report being in poor or fair health than men: $59 \%$ of women report their health to be fair, bad or very bad on average across OECD countries, compared with $54 \%$ of men. Less than $40 \%$ of the total population aged 65 and over reported being in poor or fair health in five European countries (Norway, Ireland, Switzerland, Sweden and the Netherlands). The lowest rate of poor or fair health for women was reported in Ireland (31\%), while men reported the lowest rate of poor or fair health in Norway (also 31\%).

In all OECD countries with available data, older people in the lowest income quintile are more likely to rate their health as poor or fair (two in three people) than those in the top income quintile (less than one in two) (Figure 11.7). In every country except Luxembourg, the gap between self-reported poor or fair health among people in the lowest and highest income quintiles is larger than 14 percentage points. In five countries - Iceland, Norway, Finland, Sweden and Ireland adults aged 65 and over in the lowest income quintile are more than twice as likely to report living in poor or fair health, compared with adults in the highest income quintile.

Across 26 European OECD countries, 50\% of people aged 65 and over reported having at least some limitations in their daily activities: $33 \%$ reported some limitations and a further $17 \%$ reported severe limitations (Figure 11.8). Many of the countries reporting the highest rates of self-rated poor health also reported high rates of limitations in daily activities in adults aged 65 and over. In the Slovak Republic and Latvia, three in four adults aged 65 and over reported at least some limitations to activities of daily living, while in Latvia, the Slovak Republic and Estonia one in four adults aged 65 and over reported severe limitations. In contrast, about one in five people aged 65 and over in Sweden (21\%) and Norway (22\%) reported having limitations in their daily activities, with fewer than one in 12 reporting severe limitations in both countries.

\section{Definition and comparability}

Self-reported health reflects people's overall perception of their own health, including both physical and psychological dimensions. Typically, survey respondents are asked a question such as: "How is your health in general: very good, good, fair, poor, very poor?". OECD Health Statistics provides figures related to the proportion of people rating their health to be "fair/bad/very bad" combined.

Caution is required in making cross-country comparisons of perceived health status for at least two reasons. First, people's rating of their health is subjective and can be affected by cultural factors. Second, there are variations in the question and answer categories used to measure perceived health across surveys/countries. In particular, the response scale used in Australia, Canada, New Zealand and the United States is asymmetrical (skewed on the positive side), including response categories: "excellent, very good, good, fair, poor". The data reported in OECD Health Statistics refer to respondents answering one of the two negative responses (fair, poor). By contrast, in most other OECD countries, the response scale is symmetrical, with response categories: "very good, good, fair, poor, very poor". The data reported from these countries refer to the last three categories ("fair, poor, very poor"). This difference in response categories may introduce an upward bias in the results from those countries that use an asymmetrical scale.

The category of limitations in daily activities is measured by the Global Activity Limitation Indicator (GALI) question, which comes from the EUSILC survey. The question is: "For at least the past six months, have you been hampered because of a health problem in activities people usually do: yes, strongly limited; yes, limited; no, not limited?". People in institutions are not surveyed, resulting in an underestimation of disability prevalence. Again, the measure is subjective, and cultural factors may affect survey responses.

\section{References}

[1] Hirosaki, M. et al. (2017), "Self-rated Health Is Associated with Subsequent Functional Decline Among Older Adults in Japan", International Psychogeriatrics, Vol. 29, No. 9, pp. 1475-1483, http://dx.doi.org/10.1017/S1041610217000692.

[2] Schnittker, J. and V. Bacak (2014), “The Increasing Predictive Validity of Self-Rated Health", PLoS ONE, Vol. 9, No. 1, e84933, https://doi.org/10.1371/journal.pone.0084933. 
Figure 11.6. Adults aged 65 and over rating their own health as fair, bad, or very bad, 2017 (or nearest year)

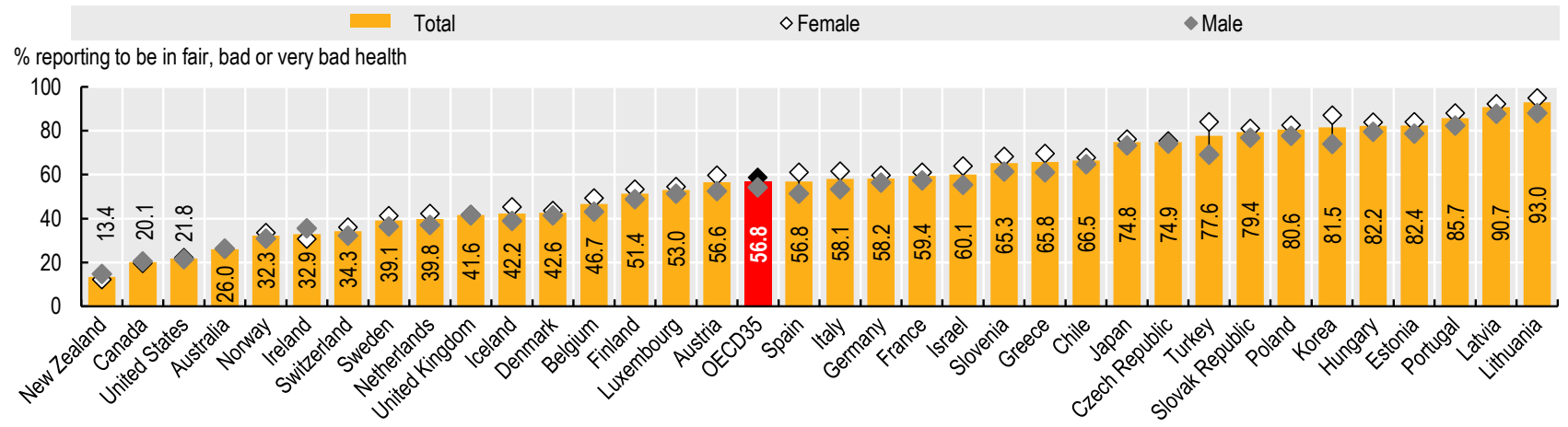

Note: Numbers are close together for males and females for Canada, the United States, Australia, the United Kingdom and the Czech Republic. Data for New Zealand, Canada, the United States and Australia biased downwards relative to other countries and so are not directly comparable.

Source: OECD Health Statistics 2019.

StatLink 에s https://doi.org/10.1787/888934018355

Figure 11.7. Adults aged 65 and over rating their own health as fair, bad, or very bad, by income, European countries, 2017 (or nearest year)

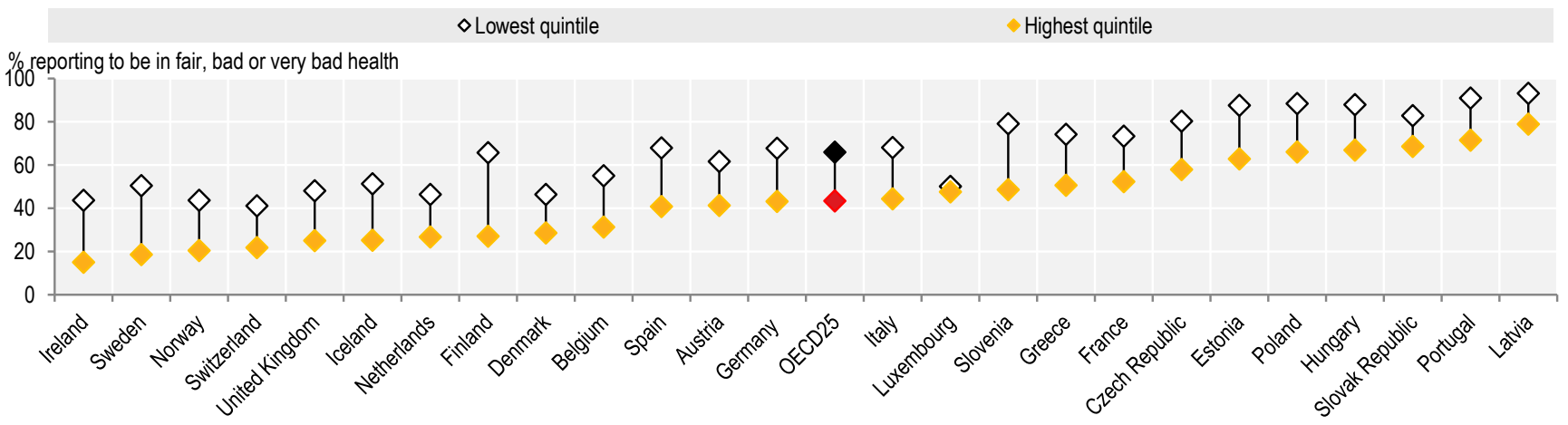

Source: Eurostat Database.

Figure 11.8. Limitations in daily activities in adults aged 65 and over, European countries, 2017 (or nearest year)

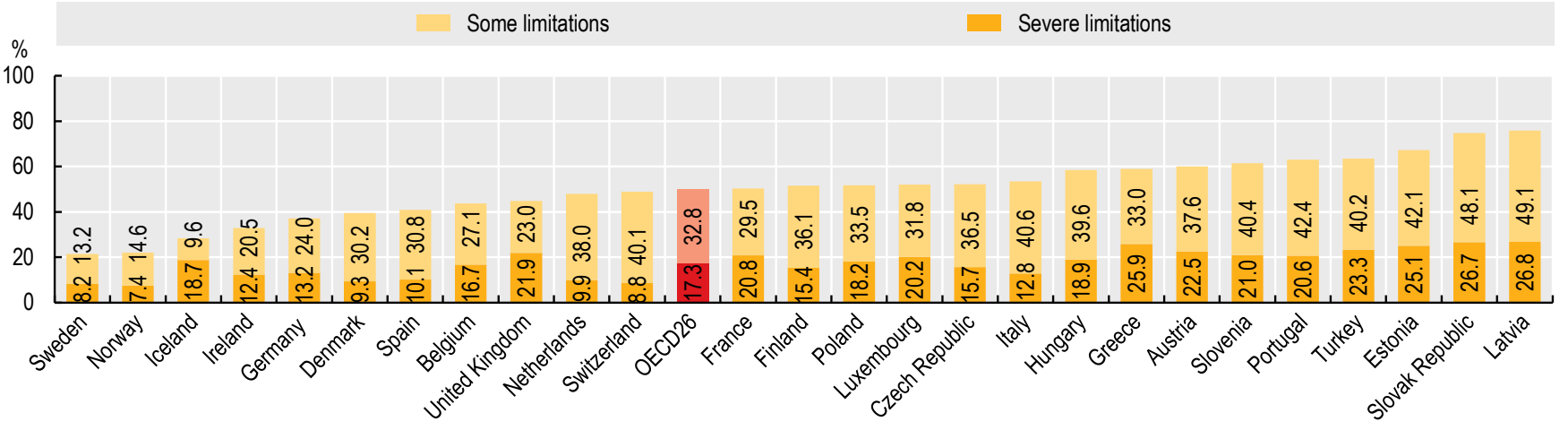

Source: Eurostat Database. 
Dementia represents one of the greatest challenges associated with population ageing. Dementia describes a variety of brain disorders, including Alzheimer's disease, which progressively lead to brain damage and cause a gradual deterioration of a person's functional capacity and social relations. Despite billions of dollars spent on research into dementia-related disorders, there is still no cure or even substantially disease-modifying treatment for dementia.

Nearly 20 million people in OECD countries are estimated to have dementia in 2019. If current trends continue, this number will more than double by 2050, reaching nearly 41 million people across OECD countries. Age remains the greatest risk factor for dementia: across the 36 OECD countries, average dementia prevalence rises from $2.3 \%$ among people aged 65-69 to nearly $42 \%$ among people aged 90 or older. This means that as countries age, the number of people living with dementia will also increase - particularly as the proportion of the population over 80 rises. Already, countries with some of the oldest populations in the OECD including Japan, Italy, and Germany - also have the highest prevalence of dementia. Across OECD countries on average, 15 people per 1000 population are estimated to have dementia (Figure 11.9). In seven countries, more than 20 people per 1000 population are living with a dementia disorder. By 2050, all but three OECD countries (Slovak Republic, Israel and Hungary) will have a dementia prevalence of more than 20 people per 1000 population, while in four countries (Japan, Italy, Portugal and Spain), more than one in 25 people will be living with dementia.

Even without an available treatment, however, there is much that health and social care systems can do to improve care and the quality of life for people living with dementia and their families. In recent years, at least 25 OECD countries have developed or announced national plans or strategies for dementia, and there is growing attention to reducing stigma around dementia and better adapting communities and care facilities to meet the needs of people with dementia (OECD, 2018[1]).

Although antipsychotic drugs can reduce the behavioural and psychological symptoms that affect many people with dementia, the availability of effective non-pharmacological interventions, as well as the associated health risks and ethical issues of antipsychotic medications, means that they are only recommended as a last resort. However, the inappropriate use of these drugs remains widespread and reducing their overuse is a policy priority for many OECD countries. Across 16 OECD countries in 2017, more than 5\% of adults aged 65 and over received a prescription for antipsychotic medicines. This masks the wide variation in prescribing rates between countries. Excluding Latvia, antipsychotic prescribing varies by a factor of three and a half across most OECD countries, from 29 prescriptions per 1000 people aged 65 and over in Sweden, to more than 99 prescriptions per 1000 in Ireland. Moreover, agestandardised rates of antipsychotic prescribing were higher for women than for men in every OECD country. Across 16 OECD countries on average, women were $23 \%$ more likely to be prescribed an antipsychotic medication than men (Figure 11.10).

\section{Definition and comparability}

The prevalence estimates in Figure 11.9 are taken from the World Alzheimer Report 2015, which includes a systematic review of studies of dementia prevalence around the world. Prevalence by country has been estimated by applying these age-specific prevalence rates for the relevant region of the world to population estimates from the United Nations (World Population Prospects: the 2017 Revision). Differences between countries are therefore driven by the age structure of populations - i.e. countries with older populations have more people with dementia. The World Alzheimer Report 2015 analysis includes studies carried out since 1980, with the assumption that agespecific prevalence is constant over time. This assumption is retained in the construction of this indicator, so that fixed age-specific prevalence rates are applied for both 2017 and 2050. Although genderspecific prevalence rates were available for some regions, overall rates were used in this analysis.

Antipsychotics are defined consistently across countries using Anatomical Therapeutic Classification (ATC) codes. The numerator includes all patients on the medications register with a prescription for a drug within ATC subgroup N05A. The denominator is the total number of people on the register. Most countries are unable to identify which prescriptions relate to people with dementia, so the antipsychotics indicator covers all people aged 65 and over. For the Netherlands and Sweden, the denominator covers all people aged 65 and over who have received at least one prescription of any type, so may slightly overestimate the antipsychotics prescription rate in comparison with other countries. In Latvia, the numerator includes only prescriptions made in primary care. Because many antipsychotics prescriptions are made by specialists, this likely undercounts the proportion of people who received a prescription. Some caution is needed when making inferences about the dementia population, since it is not certain that a higher rate of prescribing among all those aged 65 and over translates into more prescriptions for people with dementia. Nonetheless, measuring this indicator, exploring the reasons for variation and reducing inappropriate use can help to improve the quality of dementia care.

\section{References}

[1] OECD (2018), Care Needed: Improving the Lives of People with Dementia, OECD Health Policy Studies, OECD Publishing, Paris, https://dx.doi.org/10.1787/9789264085107-en. 
Figure 11.9. Estimated prevalence of dementia, 2019 and 2050

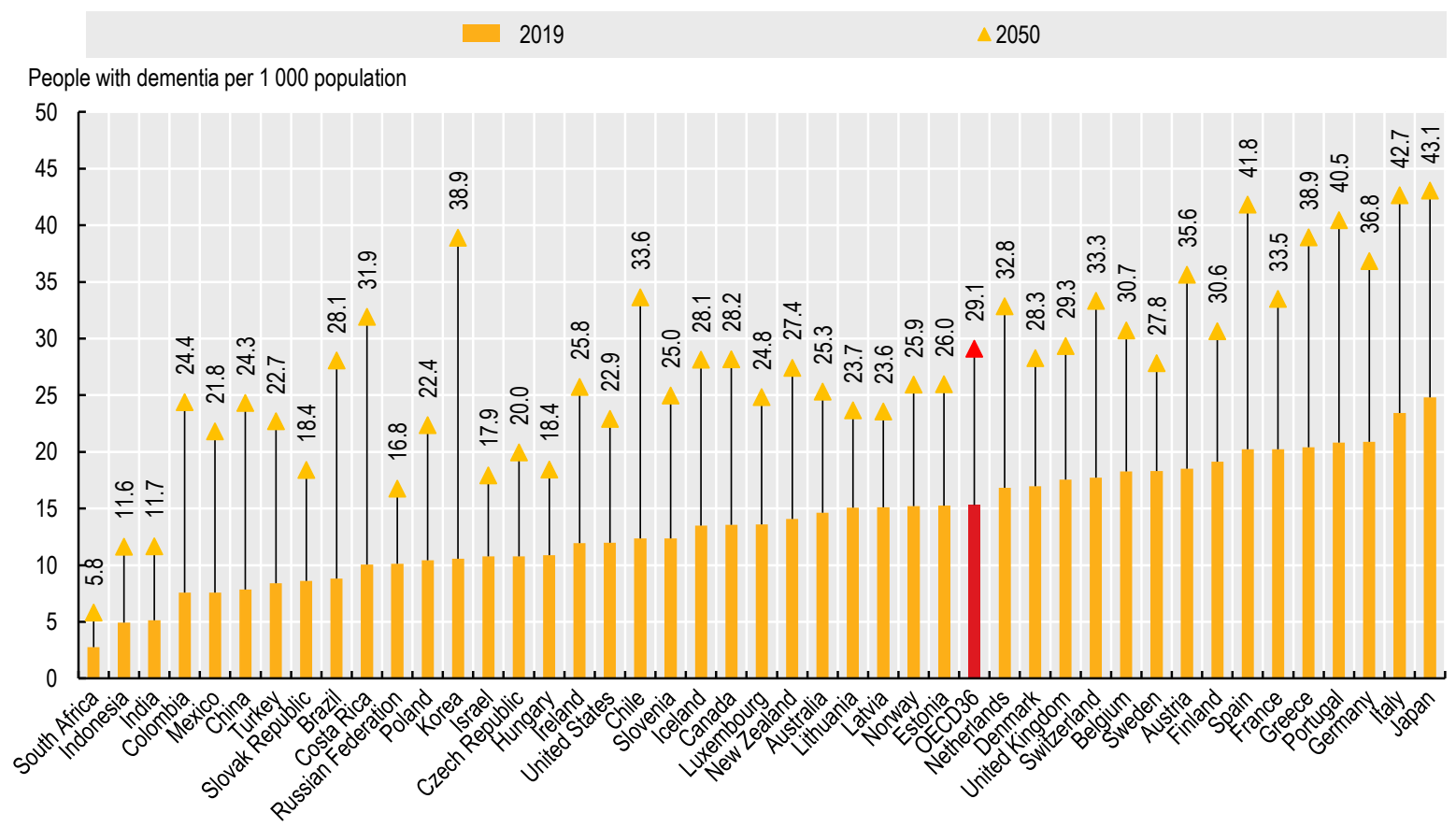

Source: OECD analysis of data from the World Alzheimer Report 2015 and the United Nations.

Figure 11.10. Antipsychotic prescribing rates by sex, 2017 (or nearest year)

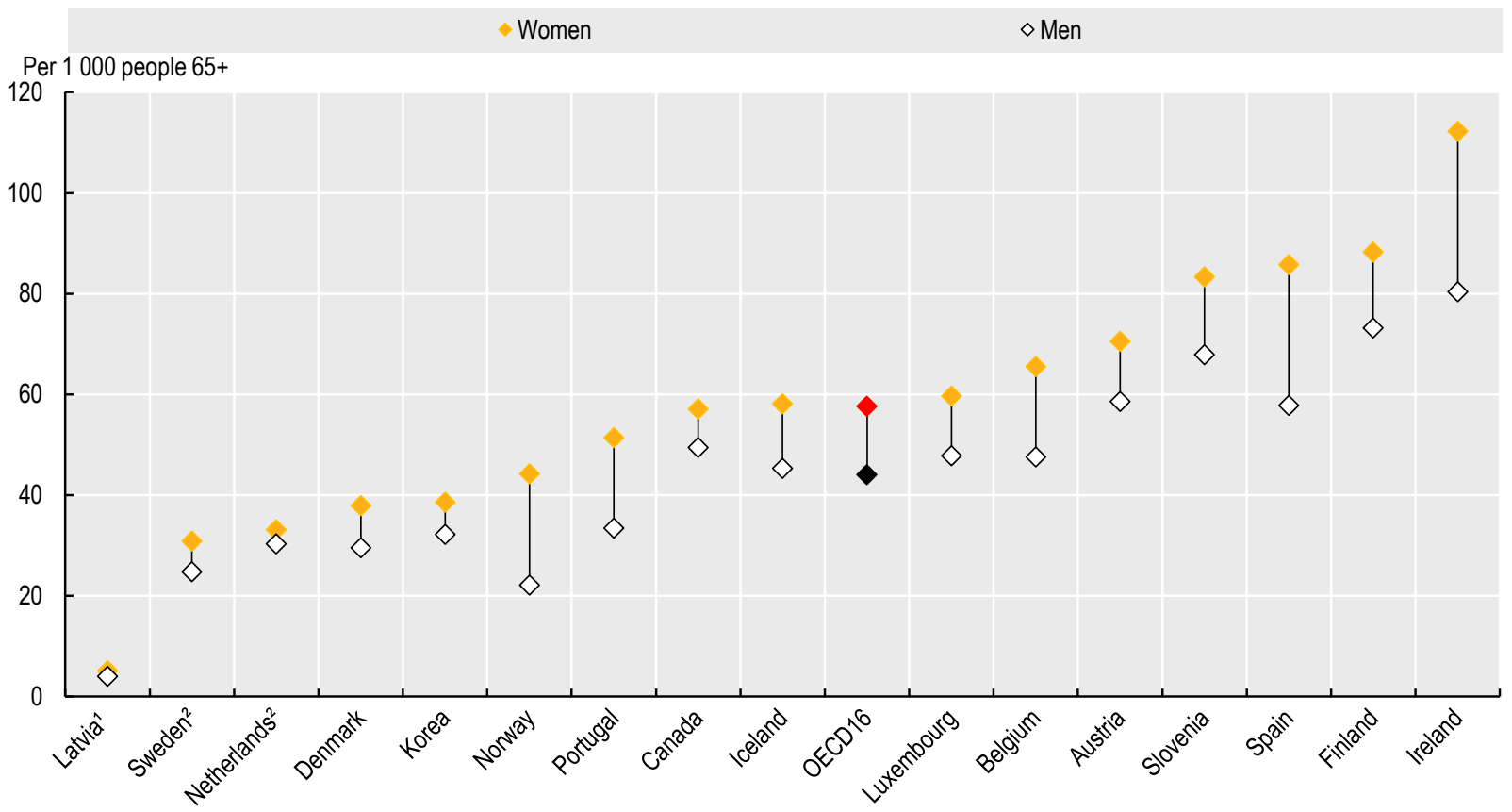

1. Data for Latvia includes only patients receiving a prescription in primary care. 2. Data for the Netherlands and Sweden refers to all people with at least one prescription of any kind.

Source: OECD Health Statistics 2019. 
Prescribing is a critical component of care for older people. Ageing and multimorbidity often require older patients to take multiple medicines (polypharmacy) for long periods of their lives. While polypharmacy is in many cases justified for the management of multiple conditions, inappropriate polypharmacy increases the risk of adverse drug events (ADEs), medication error and harm, resulting in falls, episodes of confusion and delirium. Various initiatives to improve medication safety and prevent harm involve regular medicine reviews and increased coordination between prescribing networks of doctors and pharmacists along the patient care pathway. ADEs cause 8.6 million unplanned hospitalisations in Europe every year (Mair et al., 2017[1]). Polypharmacy is one of the three key action areas of the third WHO Global Patient Safety Challenge (WHO, 2019[2]).

Across a selection of 14 countries with broader data coverage, polypharmacy rates among older people vary more than 11-fold across countries with broader data coverage, with Turkey reporting the lowest rates, and Luxembourg the highest. Among countries with only primary care data, polypharmacy rates vary almost threefold, with Finland reporting the lowest rate and Korea the highest (Figure 11.11). These large variations can be explained in part by the establishment of targeted polypharmacy initiatives in some countries, including related reimbursement and prescribing policies. Countries that cannot separate prescription data from primary and long-term care show higher average and larger variation of polypharmacy rates than countries with only primary care data.

Opioids are often used to treat pain (see indicators "Opioids use" in Chapter 4 and "Safe primary care - prescribing" in Chapter 6) and are associated with high rates of emergency admissions caused by ADEs among older adults (Lown Institute, 2019[3]). Figure 11.12 indicates that across all countries except Canada, the overall volume of opioids consumed is highest among older people. On average across OECD countries, older people consume 1.5 times more than the average volume of those aged 50-69, and nearly five times more than the volume consumed by those aged 18-49. Luxembourg shows the highest opioids volumes among older adults, and Turkey the lowest. This variation can be explained in part by differences in clinical practice in pain management, as well as differences in regulation, legal frameworks of opioids, prescribing policies and treatment guidelines.

Despite the risk of adverse side effects such as fatigue, dizziness and confusion, benzodiazepines are often prescribed for older adults for anxiety and sleep disorders. Long-term use of benzodiazepines can lead to adverse events (falls, road accidents and overdoses), tolerance, dependence and dose escalation. As well as the period of use, there is concern about the type of benzodiazepine prescribed, with long-acting types not recommended for older adults because they take longer for the body to eliminate (OECD, 2017[4]). Inappropriate prescribing of benzodiazepines has been targeted as a priority area to improve the rational use of medicines among older populations by Choosing Wisely (2019[5]).

There was a decline in the use of benzodiazepines between 2012 and 2017 across OECD countries on average (Figure 11.13). The largest decline in chronic usage was seen in Iceland and Finland, and Korea and Norway experienced the largest decline in usage of long-acting benzodiazepines. The large variation can be explained in part by different reimbursement and prescribing policies for benzodiazepines, as well as by differences in disease prevalence and treatment guidelines.

\section{Definition and comparability}

See the "Definition and comparability" box on "Safe primary care - prescribing" in Chapter 6 for more details regarding the definition and comparability of prescription data across countries.

\section{References}

[5] Choosing Wisely (2019), Choosing Wisely, https:// www.choosingwisely.org/.

[3] Lown Institute (2019), Medication Overload: America's Other Drug Problem, https://lowninstitute.org/wp-content/uploads/ 2019/04/medication-overload-lown-web.pdf.

[1] Mair A, F., H. Alonso A and E. al. (2017), The Simpathy consortium. Polypharmacy Management by 2030: a patient safety challenge, SIMPATHY Consortium, Coimbra, http:// www.simpathy.eu/.

[4] OECD (2017), Tackling Wasteful Spending on Health, OECD Publishing, Paris, https://dx.doi.org/10.1787/9789264266414en.

[2] WHO (2019), The Pursuit of Responsible Use of Medicines: Sharing and Learning from Country Experiences, https://www.who.int/ medicines/areas/rational_use/en/. 
Figure 11.11. Polypharmacy in adults aged 75 and over: primary and long-term care, 2017 (or nearest year)
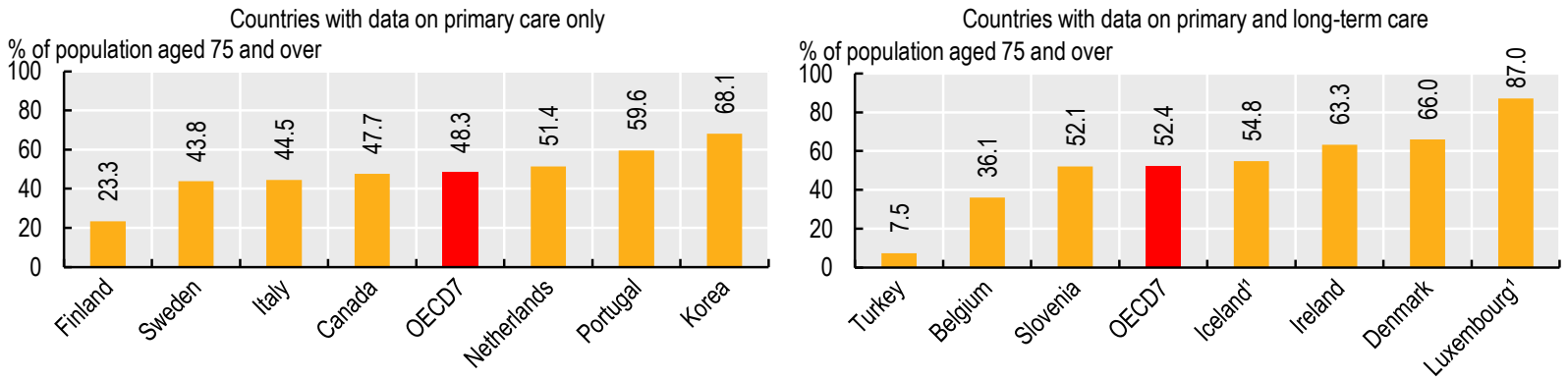

Note: Chronicity defined based on use above 90 DDDs/days in a given year, except in results for Turkey, Ireland, Denmark, Finland and Portugal which instead use number of prescriptions (four and over) in a given year. Dermatologicals for topical use are excluded.

1. Three-year average.

Source: OECD Health Statistics 2019.

StatLink त्ञाड़ https://doi.org/10.1787/888934018450

Figure 11.12. Opioid prescriptions across age groups, 2017 (or nearest year)

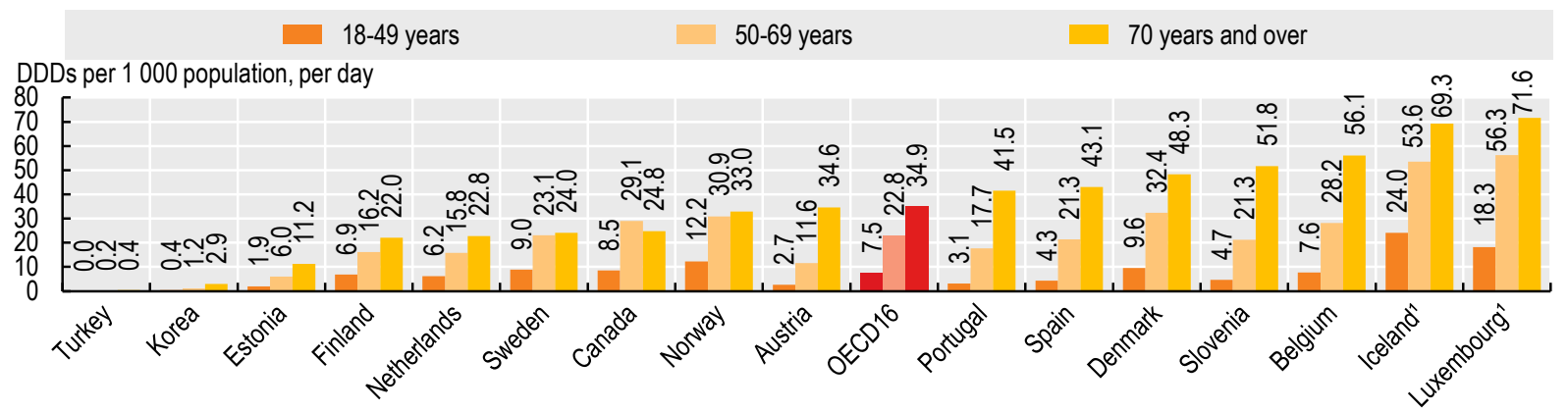

Note: Data excludes products used in the treatment of addiction.

1. Three-year average.

Source: OECD Health Statistics 2019.

StatLink क्ञाs https://doi.org/10.1787/888934018469

Figure 11.13. Trends in benzodiazepine use in adults aged 65 and over, 2012-17 (or nearest years)

Chronic benzodiazepine use

2012

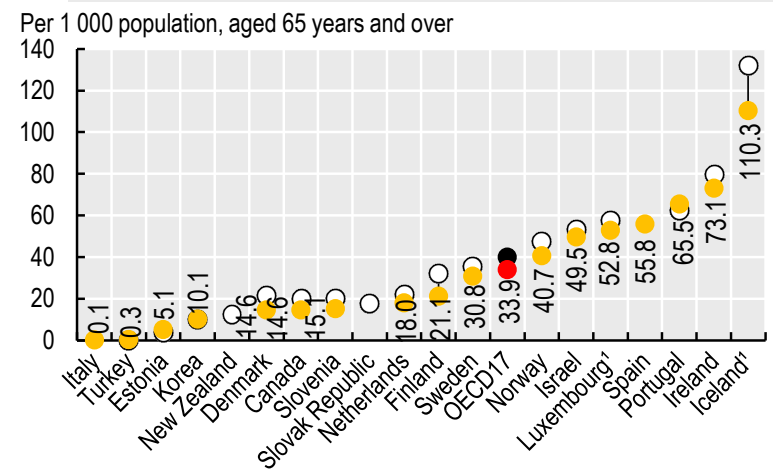

Long-acting benzodiazepine use

2017

Per 1000 population, aged 65 years and over

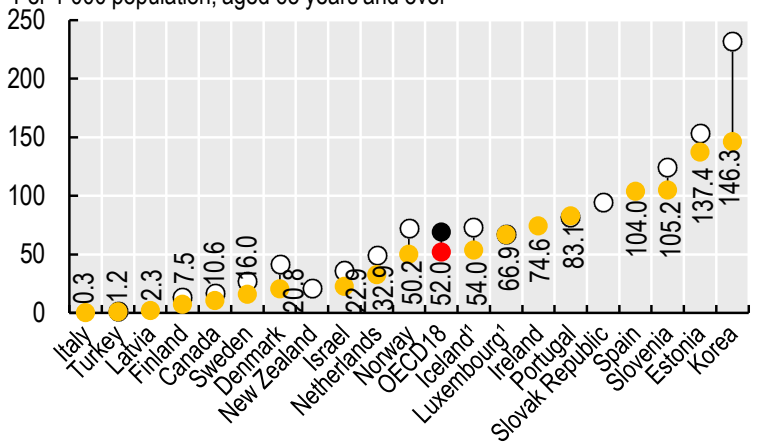

1. Three-year average.

Source: OECD Health Statistics 2019. 
As populations across OECD countries continue to age, an increasing number of people will require support from longterm care (LTC) services, including nursing homes and LTC living facilities (see indicator on "Recipients of long-term care"). Providing safe care for these patients is a key challenge for OECD health systems, as residents of LTC facilities are more frail and sicker, and present a number of other risk factors for the development of patient safety events, including healthcare-associated infections (HAIs) and pressure ulcers (OECD/European Commission, 2013[1]).

HAIs can lead to significant increases in patient morbidity, mortality and cost for the health system. In the acute care sector, HAIs alone are estimated to make up 3-6\% of hospital budgets (Slawomirski et al., 2017[2]). These infections are also generally considered to be preventable through standard prevention and hygiene measures. The most commonly occurring HAIs in LTC facilities include urinary tract infections, lower respiratory tract infections, skin and soft tissue infections (Suetens et al., 2018[3]).

In 2016-17, the average prevalence of HAIs among LTC facility residents in OECD countries was 3.8\% (Figure 11.14). This proportion was lowest in Lithuania, Hungary, Sweden, Germany, and Luxembourg (less than $2 \%$ ), and highest in Denmark, Portugal, Greece and Spain (over 5\%).

The impact of HAIs is increased by the rise of antibioticresistant bacteria, which can lead to infections that are difficult or even impossible to treat. Figure 11.15 shows the proportions of bacteria isolated from LTC residents that are resistant to antibiotics. On average, over one quarter of isolates were resistant to antibiotics. This is nearly equivalent to levels seen in acute care hospitals, where antibiotic resistance is considered a major threat.

Pressures ulcers are another important patient safety concern in LTC facilities. A pressure ulcer is an injury to the skin or underlying tissue resulting from sustained pressure; they occur frequently in patients with limited mobility. Pressure ulcers can lead to complications including infections, and cost up to EUR 170 per patient per day in LTC settings (Demarré et al., 2015[4]).

Across OECD countries on average, the observed prevalence rate of pressure ulcers in selected LTC facilities was 5.35 (Figure 11.16). The highest rates of pressure ulcer prevalence were observed in Spain, Italy and Portugal, at nearly twice the OECD average, while Lithuania, Hungary and Luxembourg reported the lowest rates at under 3\%.

\section{Definition and comparability}

Data came from point-prevalence surveys conducted between 2016 and 2017 by the European Centre for Disease Control and Prevention (ECDC) and the Centers for Disease Control and Prevention (CDC) among participating LTC facilities. Facilities in ECDC data included: general nursing homes, mixed longterm care facilities and residential homes, and excluded specialised long-term care facilities, as defined by the ECDC. Only nursing homes were included in CDC data. Point-prevalence surveys currently represent the best tool for collecting internationally comparable data, but they are subject to possible biases due to facility selection, local recording practices or observer training. Countries noted as having poor data representativeness had low participation among LTC facilities, which may lead to large variance or biased estimates.

Pressure ulcers in prevalence estimates include all grades or categories, including grade I. Accuracy of recognising pressure ulcers may vary considerably, particularly as this measurement was not the core purpose of data collection. HAI data included healthcare-associated pneumonia, urinary tract infections, surgical site infections, Clostridium difficile infections and primary bloodstream infections. Resistance proportion data are based on a composite antibiotic resistance indicator developed by the ECDC (Suetens et al., 2018).

Both HAI and pressure ulcer prevalence data are unadjusted. Many factors - including increased patient age, limited mobility and use of invasive medical devices - may increase the risk of developing either an HAI or a pressure ulcer and may influence the variability of rates between countries. One of these factors - the proportion of residents with limited mobility -is noted by country in the relevant figures.

\section{References}

[4] Demarré, L., et al. (2015). "The cost of prevention and treatment of pressure ulcers: a systematic review", International journal of nursing studies, Vol. 52, No. 11, pp. 1754-1774.

[1] OECD/European Commission (2013), A Good Life in Old Age? Monitoring and Improving Quality in Long-term Care, OECD Health Policy Studies, OECD Publishing, Paris, https://doi.org/ 10.1787/9789264194564-en.

[3] Suetens, C. et al. (2018), "Prevalence of healthcare-associated infections, estimated incidence and composite antimicrobial resistance index in acute care hospitals and long-term care facilities: results from two European point prevalence surveys, 2016 to 2017", Euro Surveill., Vol. 23, No. 46, https://doi.org/10.2807/1560-7917.ES.2018.23.46.1800516.

[2] Slawomirski, L., A. Auraaen and N. Klazinga (2017), "The economics of patient safety: Strengthening a value-based approach to reducing patient harm at national level", OECD Health Working Papers, No. 96, OECD Publishing, Paris, https:// doi.org/10.1787/5a9858cd-en. 
Figure 11.14. Percentage of long-term care facility residents with at least one healthcare-associated infection, 2016-17

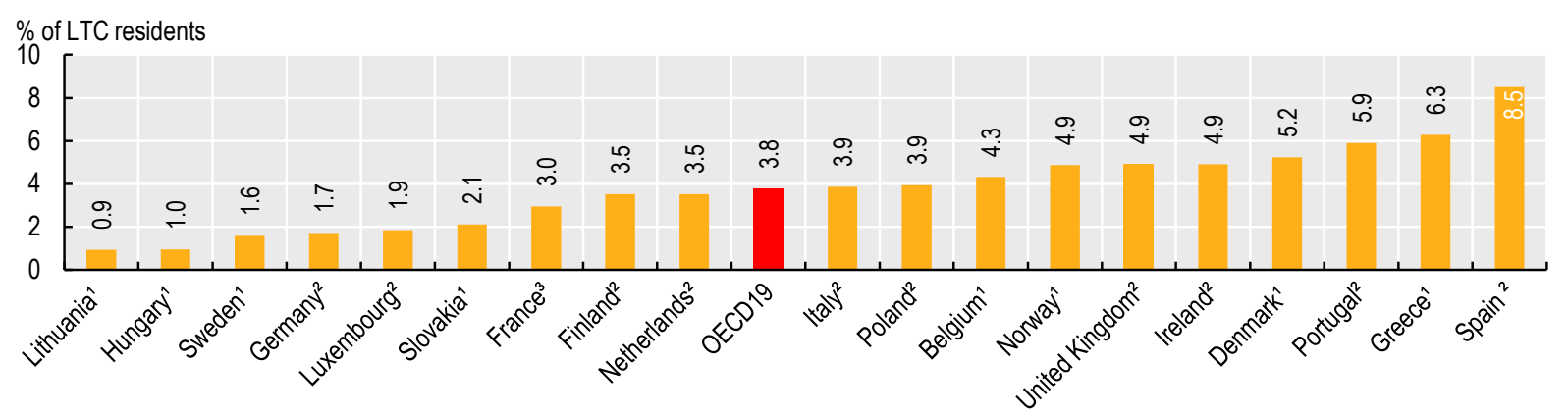

1. Limited country representativeness 2. Under $40 \%$ of residents sampled were wheelchair-bound or bedridden. 3 . Between $40 \%$ and $50 \%$ of residents sampled were wheelchair-bound or bedridden.

Source: ECDC.

StatLink Aाsस https://doi.org/10.1787/888934018507

Figure 11.15. Proportion of antimicrobial-resistant bacterial isolates from healthcare-associated infections in long-term care, 2016-17

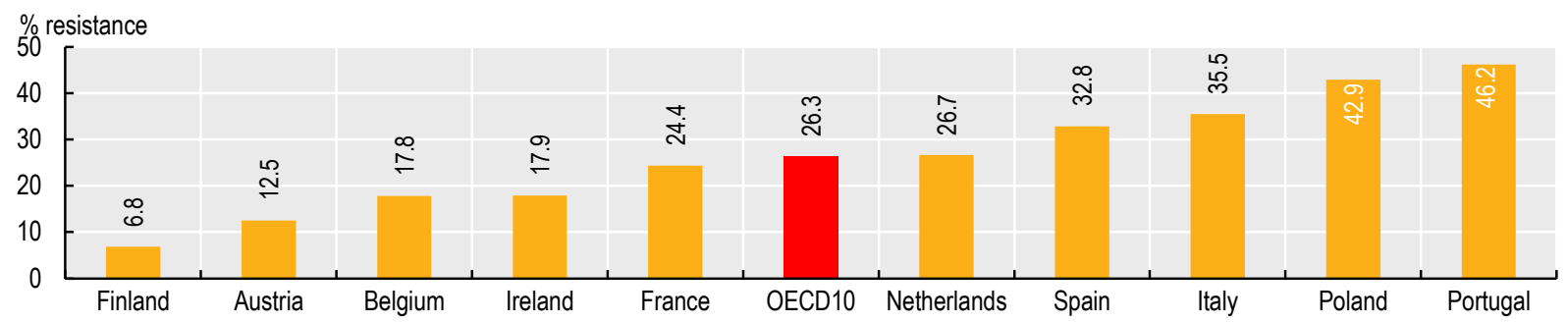

Note: Based on composite antibiotic resistance indicator developed by ECDC. Only countries with over 15 bacterial isolates were included. Source: ECDC.

StatLink 겐 https://doi.org/10.1787/888934018526

Figure 11.16. Percentage of long-term care facility residents with at least one pressure ulcer, 2016-17

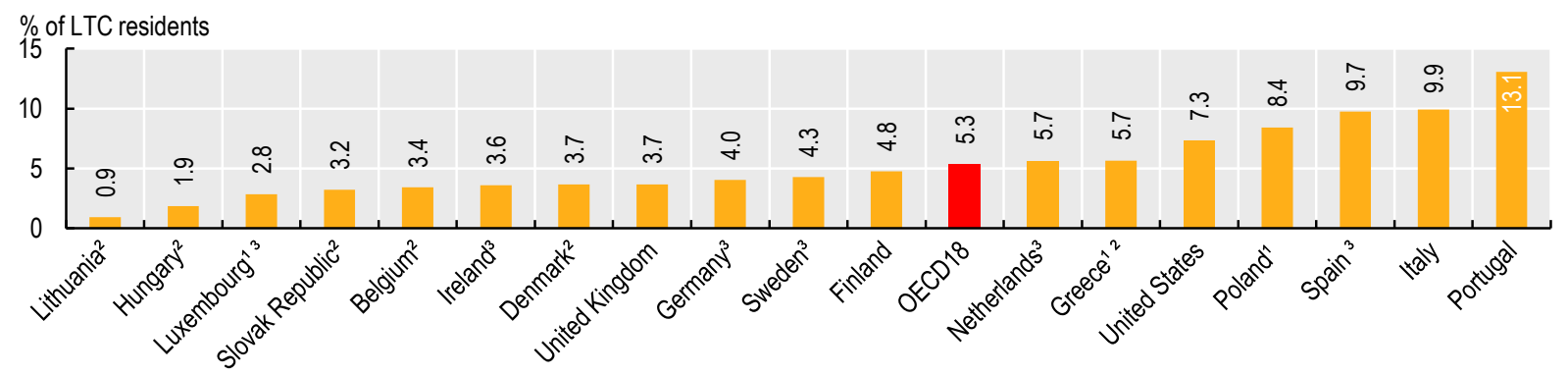

1. Under $45 \%$ of residents sampled were wheelchair-bound or bedridden. 2 . Over $45 \%$ of residents sampled were wheelchair-bound or bedridden. 3. No data available on the proportion of wheelchair-bound or bedridden residents.

Source: ECDC, CDC. 
Across OECD countries, an average of $10.8 \%$ of people aged 65 and over received long-term care (LTC) in 2017. This represents a 5\% increase compared with 2007 (Figure 11.17). More than one in five people aged 65 and over received LTC services in Switzerland (22\%) and Israel (20\%), compared with less than $5 \%$ in the Slovak Republic (4\%), Canada (4\%), Ireland (3\%), Portugal (2\%), and Poland (1\%).

The majority of LTC recipients are older adults (Figure 11.18). Although LTC services are also delivered to younger disabled groups, people are more likely to develop disabilities and need support from LTC services as they age. In 2017, just $21 \%$ of LTC recipients on average across OECD countries were younger than 65 , while a further $27 \%$ were between 65 and 79. Adults aged 80 and over represent the majority of LTC recipients in OECD countries. On average in OECD countries, $51 \%$ of LTC recipients were aged 80 and above in 2017. In Japan, two-thirds of LTC recipients were 80 and over, while people aged 0-64 represented just 3\% of LTC recipients.

While population ageing is a significant driver of the growth in LTC users over time, the cross-country variation in the proportion of older LTC recipients suggests that other drivers - notably publicly funded LTC services - also determine LTC use. For example, Israel has one of the youngest populations among OECD countries but a greater than average proportion receive LTC. Because data on people receiving care outside public systems are more difficult to collect and may be underreported, figures for countries that rely more heavily on privately-funded care may be artificially low. Cultural norms around the degree to which families look after older people may also be an important driver of the utilisation of formal services (see indicator on "Informal carers").

Many people in need of LTC care wish to remain in their homes for as long as possible. In response to these preferences, and the high costs of care facility-based LTC, many OECD countries have developed services to support home-based care for older adults. Between 2007 and 2017, the proportion of LTC recipients who received care at home rose by $4 \%$, from $64 \%$ to $68 \%$ (Figure 11.19 ). Increases have been particularly large in Portugal, Australia, Sweden, Germany and the United States. In Germany, part of the increase was due to policy reforms expanding the definition of long-term care and therefore increasing the number of benefit recipients. While the proportion of LTC recipients living at home has increased over the past decade in most OECD countries, it has declined significantly in Estonia, where there has been a significant increase in the use of institutional care, but an even larger decrease in the number of "curators" appointed by local government to care for people at home.

\section{Definition and comparability}

LTC recipients are defined as people receiving longterm care by paid providers, including non-professionals receiving cash payments under a social programme. They also include recipients of cash benefits such as consumer-choice programmes, care allowances or other social benefits granted with the primary goal of supporting people with LTC needs. LTC institutions refer to nursing and residential care facilities that provide accommodation and LTC as a package. LTC at home is defined as people with functional restrictions who receive most of their care at home. Home care also applies to the use of institutions on a temporary basis, community care and day-care centres and specially designed living arrangements. Data for Poland, Ireland, Canada, the Slovak Republic, Iceland and Belgium are only available for people receiving LTC in institutions, so the total number of recipients will be underestimated. In Estonia, data on recipients of home care refer only to those who have a "curator" appointed by local government. Other social services, without a personal care component, are not included in the data. It is possible that some of the decrease in recipients reflects the replacement of curators with these other services.

Data on LTC services are difficult to collect in many countries and there are some known limitations of the figures. Data for some countries refers only to people receiving publicly funded care, while other countries include people who are paying for their own care.

\section{References}

[1] Colombo, F. et al. (2011), Help Wanted? Providing and Paying for Long-Term Care, OECD Health Policy Studies, OECD Publishing, Paris, http://dx.doi.org/10.1787/9789264097759-en.

[2] Muir, T. (2017), "Measuring social protection for long-term care", OECD Health Working Papers, No. 93, OECD Publishing, Paris, https://dx.doi.org/10.1787/a411500a-en. 
Figure 11.17. Share of adults aged 65 and over receiving long-term care, 2007 and 2017 (or nearest year)

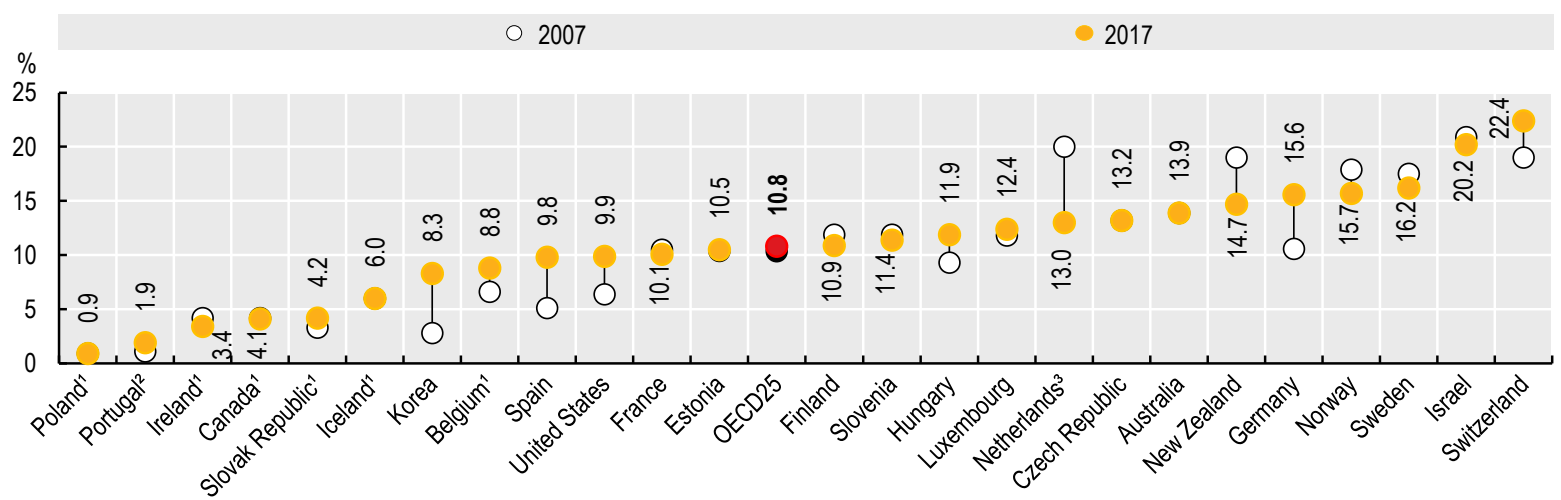

1. Include only recipients of LTC in institutions. 2. Refers to people receiving care through the National Network of Integrated Continuing Care. 3. Refers to social-insurance funded LTC only: the fall in recent years largely reflects the transfer of many LTC services to municipalities in 2015.

Figure 11.18. Long-term care recipients by age, 2017 (or nearest year)

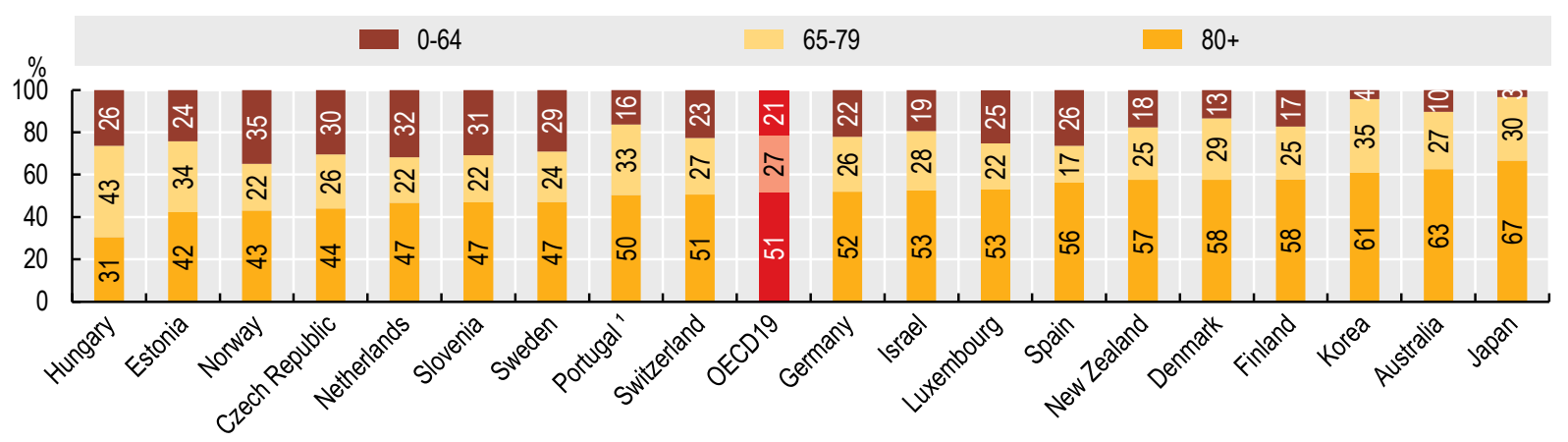

1. Data refer to people receiving care through the National Network of Integrated Continuing Care.

Source: OECD Health Statistics 2019.

StatLink क्गाड़ https://doi.org/10.1787/888934018583

Figure 11.19. Long-term care recipients aged 65 and over receiving care at home, 2007 and 2017 (or nearest year)

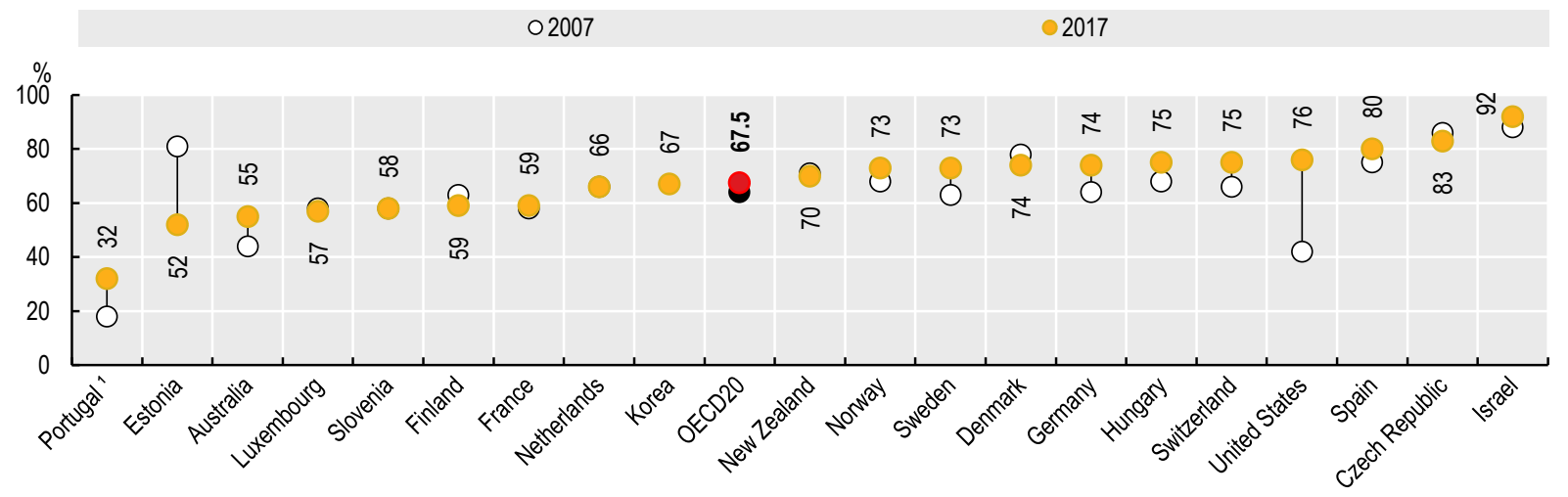

1. Data refer to people receiving care through the National Network of Integrated Continuing Care.

Source: OECD Health Statistics 2019. 
Family and friends are the most important source of care for people with long-term care (LTC) needs in OECD countries. Because of the informal nature of the care they provide, it is not easy to get comparable data on the number of people caring for family and friends across countries, nor on the frequency of their caregiving. The data presented in this section come from national or international health surveys and refer to people aged 50 years and over who report providing care and assistance to family members and friends.

On average across OECD countries for which data is available, around $13 \%$ of people aged 50 and over report providing informal care at least weekly. The share of people aged 50 and over providing informal care is close to $20 \%$ in the Czech Republic, Austria, Belgium, the United Kingdom, France, and Germany, and less than $10 \%$ in Portugal, Sweden, Poland, the United States, Ireland, and Greece (Figure 11.20). There is also variation in the intensity of the care provided. The lowest rates of daily care provision are found in Sweden, Greece, Switzerland, Denmark and the Netherlands - in most of which the formal LTC sector is well developed and public coverage is comprehensive.

Intensive caregiving is associated with a reduction in labour force attachment for caregivers of working age, higher poverty rates, and a higher prevalence of mental health problems. Many OECD countries have implemented policies to support family carers with a view to mitigating these negative impacts. These include paid care leave (e.g. Belgium and France), flexible work schedules (e.g. Australia and the United States), respite care (e.g. Austria, Denmark, France, and Germany) and counselling/training services (e.g. Sweden). Moreover, a number of OECD countries provide cash benefits to family caregivers or cash-for-care allowances for recipients which can be used to pay informal caregivers, or periods of paid leave for informal carers (OECD, 2018[1]). In France, evidence suggests that even short-term respite care solutions for older people with Alzheimer's disease may significantly reduce informal caregivers' psychological burden (Rapp, Apouey and Senik, 2018[2]).

On average across OECD countries, $61 \%$ of those providing daily informal care are women (Figure 11.21). Greece and Portugal have the greatest gender imbalance, with over $70 \%$ of informal carers being women. Around two-thirds of carers are looking after a parent or a spouse, but patterns of caring vary for different age groups. Younger carers (aged between 50 and 65) are much more likely to be caring for a parent (Figure 11.22). They are more likely to be women and may not be providing care every day. Carers aged over 65 are more likely to be caring for a spouse. Caring for a spouse tends to be more intensive, requiring daily care, and men and women are equally likely to take on this role.

The fact that fewer people provide daily care in countries with stronger formal LTC systems suggests that there is a trade-off between informal and formal care. Declining family size, increased geographical mobility and rising participation rates of women in the labour market mean that there is a risk that fewer people will be willing and able to provide informal care in the future. Coupled with the effects of an ageing population, this could lead to higher demand for professional LTC services. Public LTC systems will need adequate resources to meet increased demand while maintaining access and quality.

\section{Definition and comparability}

Informal carers are defined as people providing any help to older family members, friends and people in their social network, living inside or outside their household, who require help with everyday tasks. The data relate only to the population aged 50 and over, and are based on national surveys for Australia (Survey of Disability, Ageing and Carers, SDAC), the United Kingdom (English Longitudinal Study of Ageing, ELSA), the United States (Health and Retirement Survey, HRS) and an international survey for other European countries (Survey of Health, Ageing and Retirement in Europe, SHARE). Data for Ireland were taken from its 2016 census.

Questions about the intensity of care vary between surveys. In SHARE, carers are asked about how often they provided care in the last year; this indicator includes people who provided care at least weekly. It is important to highlight the change of methodology in SHARE wave 7 , in which over four fifths of the respondents answered the SHARELIFE part of the questionnaire only instead of the panel interview. In ELSA, people are asked if they have provided care in the last week, which may be broadly comparable with "at least weekly". Questions in HRS and SDAC are less comparable with SHARE. Carers in HRS are included if they provided more than 200 hours of care in the last year. In SDAC, a carer is defined as someone who has provided ongoing informal assistance for at least six months. People caring for disabled children are excluded for European countries but included in data for the United States and Australia. However, the United States data only include those caring for someone outside their household. Australia and Ireland consider all informal carers together. As a result, data for Australia, Ireland and the United States may not be comparable with other countries' data.

\section{References}

[1] OECD (2018), Care Needed: Improving the Lives of People with Dementia, OECD Health Policy Studies, OECD Publishing, Paris, https://dx.doi.org/10.1787/9789264085107-en.

[2] Rapp, T., B. Apouey and C. Senik (2018), "The impact of institution use on the wellbeing of Alzheimer's disease patients and their caregivers", Social Science and Medicine, http://dx.doi.org/10.1016/j.socscimed.2018.04.014. 


\section{Figure 11.20. Share of informal carers among population aged 50 and over, 2017 (or nearest year)}

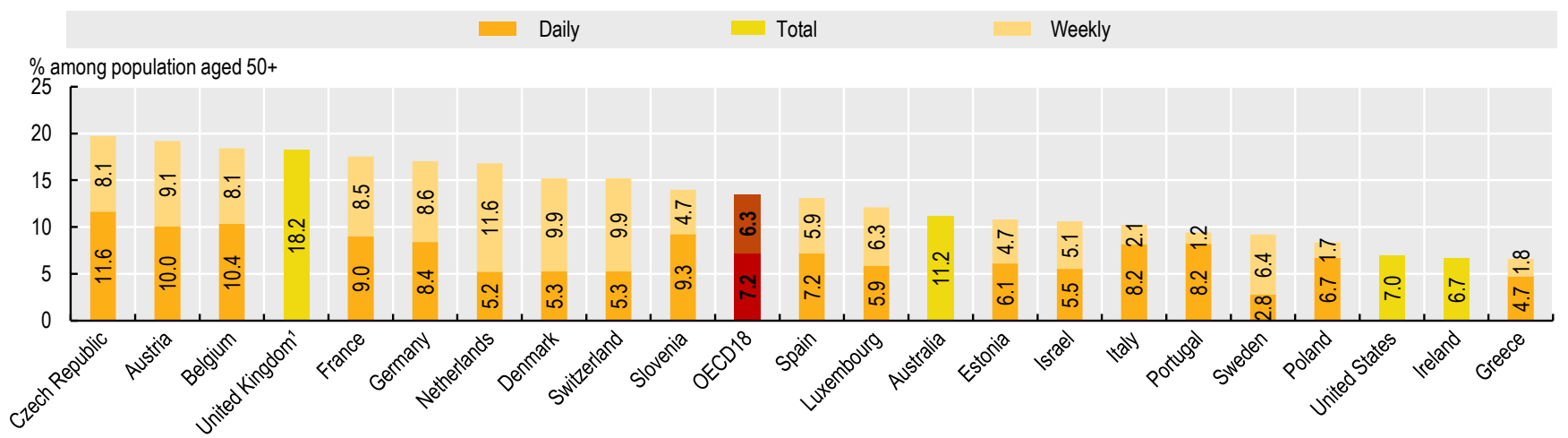

1. The United Kingdom refers to England.

Note: The definition of informal carers differs between surveys (see the "Definition and comparability" box). The United Kingdom and the United States include informal weekly carers. Australia and Ireland include all informal carers together.

Source: Survey of Health, Ageing and Retirement in Europe, wave 7 (2017); Survey of Disability, Ageing and Carers for Australia (2015); English Longitudinal Study of Ageing, wave 8 (2017); Health and Retirement Survey for the United States, wave 13 (2016); Census 2016 for Ireland.

StatLink iाls https://doi.org/10.1787/888934018621

Figure 11.21. Share of women among informal daily carers aged 50 and over, 2017 (or nearest year)

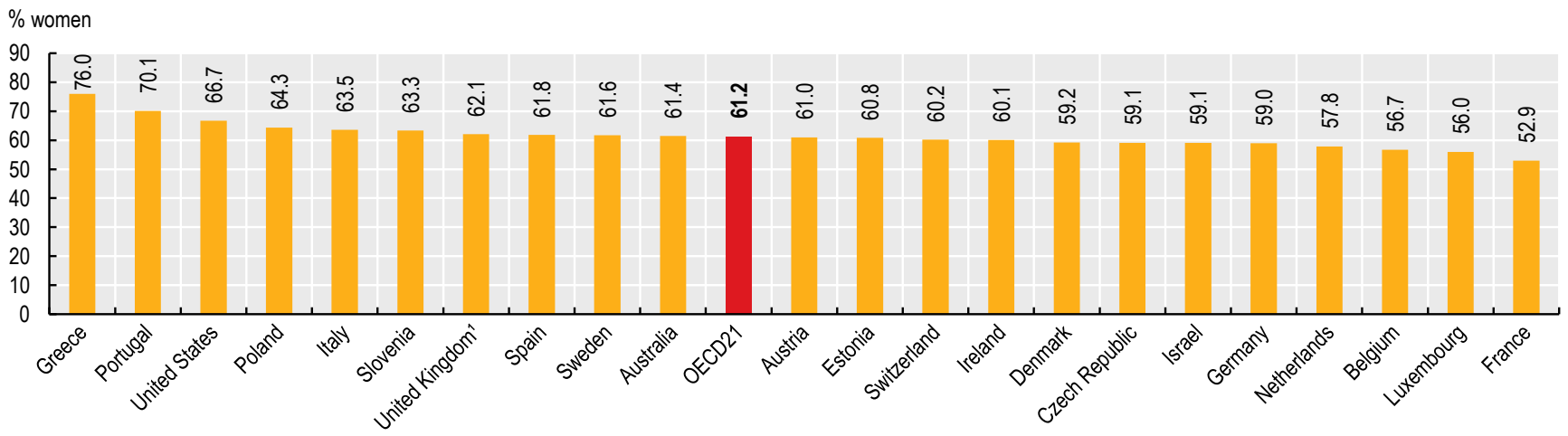

1. The United Kingdom refers to England.

Note: The definition of informal carers differs between surveys (see the "Definition and comparability" box).

Source: Survey of Health, Ageing and Retirement in Europe, wave 7 (2017); Survey of Disability, Ageing and Carers for Australia (2015); English Longitudinal Study of Ageing, wave 8 (2017); Health and Retirement Survey for the United States, wave 13 (2016); Census 2016 for Ireland.

StatLink ants https://doi.org/10.1787/888934018640

Figure 11.22. Share of informal carers in the European population aged 50 and over, by recipients of care and age, daily and weekly, 2017
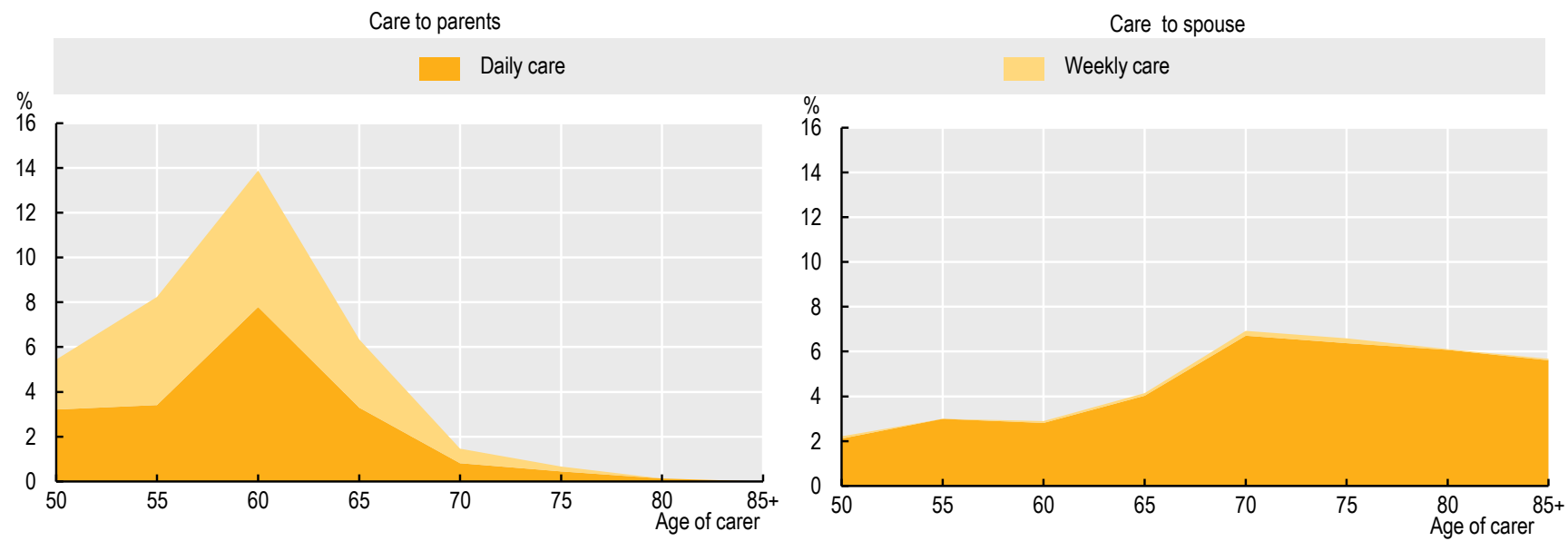

Note: Data refer to the population aged 50 and over for countries included in SHARE wave 7.

Source: Wave 7 of the Survey of Health, Ageing and Retirement in Europe (2017). 
Long-term care (LTC) is a labour-intensive service, and formal care is in many cases a necessary complement to informal, unpaid work in supporting people with LTC needs (see indicators on "Informal carers"). Formal LTC workers are defined as paid staff - typically nurses and personal carers - who provide care and/or assistance to people limited in their daily activities at home or in institutions, excluding hospitals. There are on average five LTC workers per 100 people aged 65 and over across 28 OECD countries, ranging from 13 in Norway to less than one in Greece, Poland, and Portugal (Figure 11.25).

In more than half of OECD countries, population ageing has outpaced the growth of LTC supply. The LTC workforce has stagnated or declined even in countries where the LTC supply is much higher than the OECD average (such as Denmark, the Netherlands, Norway, and Sweden). Nine countries experienced an overall increase in their LTC supply between 2011 and 2016. As populations continue to age, demand for LTC workers is likely to rise. Responding to increasing demand will require policies to improve recruitment; improve retention; and increase productivity.

Less than one-quarter of LTC workers hold tertiary education across OECD countries (see Figure 11.23). This can be explained by the fact that personal care workers represent $70 \%$ of the LTC workforce on average in OECD countries, and up to $90 \%$ in a few countries (Estonia, Switzerland, Korea, Israel, and Sweden). Only Germany, Hungary, and Switzerland have a supply of nurses greater than the supply of personal care workers. Very few countries currently require personal care workers to hold minimum education levels, licences and/or certifications. Despite being mostly staffed by lower-skilled workers, LTC involves spending significant time delivering more complex tasks than basic care. Personal care workers do not always have sufficient knowledge and training, which can affect the quality of care delivered.

Working conditions in this sector tend to be relatively poor. This tends to affect women disproportionately as, on average, women hold about $90 \%$ of the jobs in the LTC sector. For instance, $45.5 \%$ of LTC workers work part-time in OECD countries (Figure 11.24.) In northern and central European countries, more than half of workers are employed on a part-time basis. Part-time work is particularly widespread among personal carers and homebased workers. The fact that basic LTC services are mostly needed for reduced hours at specific times of the day may contribute to explain such high rates. In addition, half of LTC workers experience shift work and almost one quarter are on temporary contracts. Further, while LTC tends to be demanding, both physically and mentally, pay is often low.

\section{Definition and comparability}

LTC workers are defined as paid workers who provide care at home or in institutions (outside hospitals). They include qualified nurses and personal care workers providing assistance with activities of daily living (ADL) and other personal support. Personal care workers include different categories that may be called different names in different countries. Because personal care workers may not be part of recognised occupations, it is more difficult to collect comparable data for this category of LTC workers across countries. LTC workers also include family members or friends who are employed under a formal contract by the care recipient, an agency, or public and private care service companies. They exclude nurses working in administration. The numbers are expressed as head counts, not full-time equivalents. Data refer only to workers employed in the public sector for some countries, but include workers in the private and notfor-profit sectors for others. Data from the Czech Republic and Japan are based on surveys of establishments, meaning that people who work in more than one establishment are double-counted.

\section{References}

[1] OECD (2018), Care Needed: Improving the Lives of People with Dementia, OECD Health Policy Studies, OECD Publishing, Paris, https://dx.doi.org/10.1787/9789264085107-en. 
Figure 11.23. Long-term care workers by education level, 2016

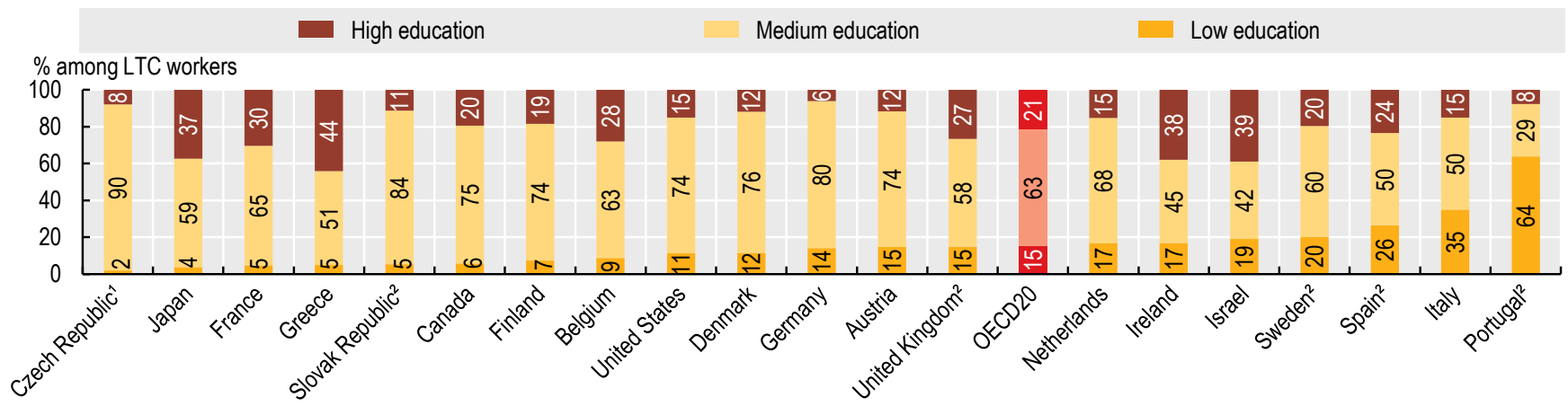

Note: EU-Labour Force Survey (LFS) data based on ISCO 4 digit and NACE 2 digit. 1. Interpret with caution as sample sizes small. 2. Based on ISCO 3 digit and NACE 2 digit.

Source: EU-LFS; ASEC-CPS for the United States; Census 2016 for Canada; LFS for Israel; Survey on Long-term Care Workers FY for Japan.

StatLink iाls https://doi.org/10.1787/888934018678

Figure 11.24. Share of long-term care workers who work part-time, 2016

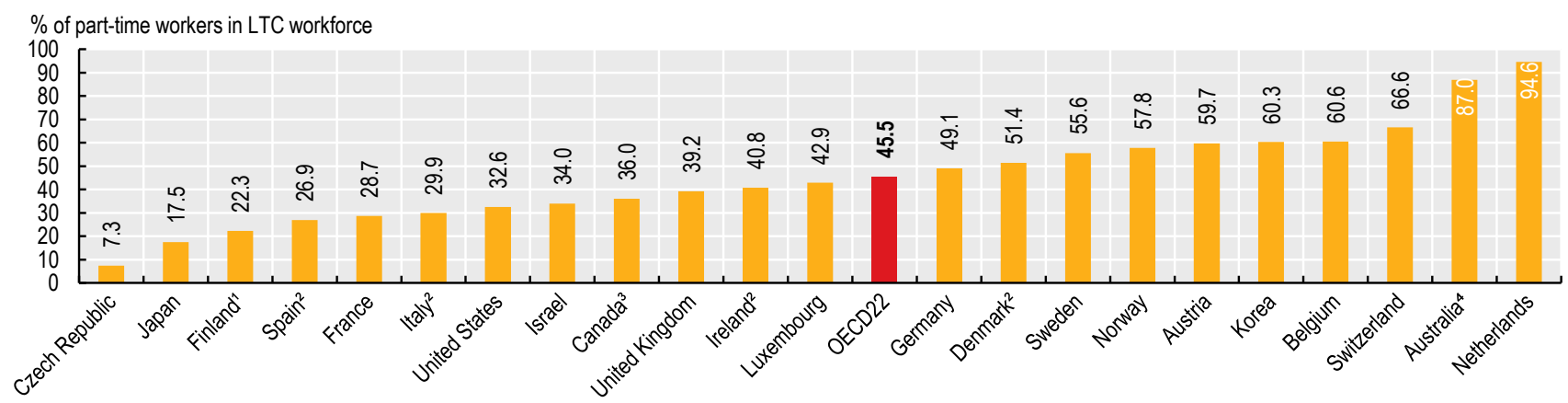

Note: EU-Labour Force Survey (LFS) data based on ISCO 4 digit and NACE 2 digit. 1. Interpret with caution as sample sizes small. 2. Based on ISCO 3 digit and NACE 2 digit. 3. Covers only those working mostly full-time or part-time. 4. Covers only those with a permanent position.

Source: EU-LFS; ASEC-CPS for the United States; Census 2016 for Canada; LFS for Israel; Survey on Long-term Care Workers FY for Japan; National Health Insurance System for Korea; OECD estimate based on national source for Australia.

StatLink AाIs https://doi.org/10.1787/888934018697

\section{Figure 11.25. Long-term care workers per 100 people aged 65 and over, 2011 and 2016 (or nearest year)}

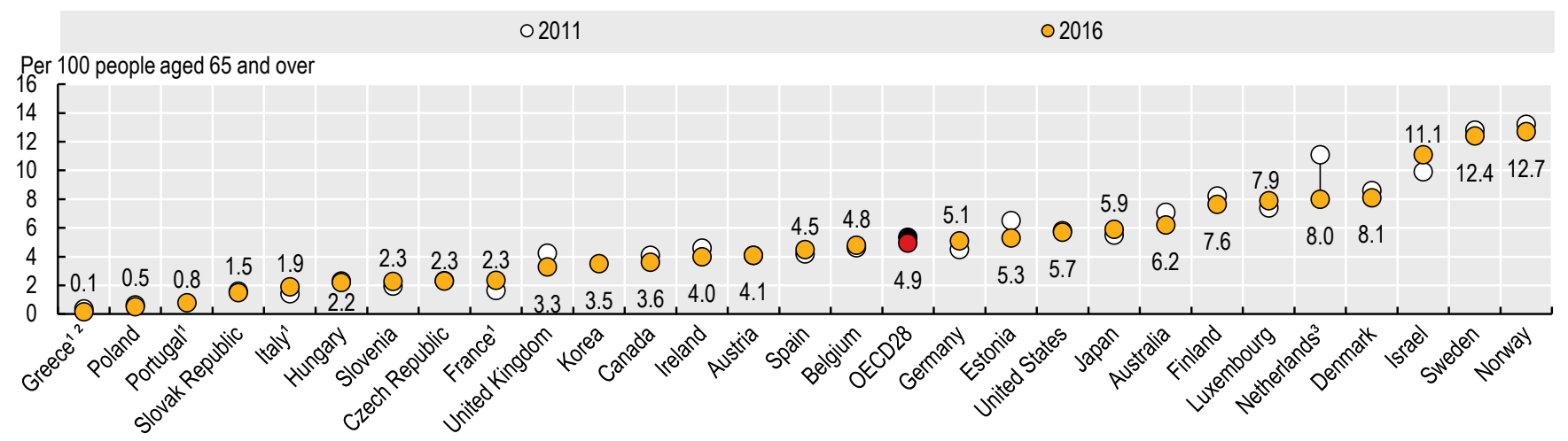

Note: EU-Labour Force Survey (LFS) data based on ISCO 4 digit and NACE 2 digit.1. Based on ISCO 3 digit and NACE 2 digit. 2. Interpret with caution as sample sizes small. 3. The decrease in the Netherlands partly due to a methodological break in 2012, as well as reforms.

Source: EU-LFS and OECD Health Statistics 2018, with the exception of the Quarterly LFS for the United Kingdom and the Current Population Survey (ASEC-CPS) for the United States; Eurostat Database for population demographics. 
While countries have increasingly taken steps to ensure that people in need of long-term care (LTC) services who wish to live at home for as long as possible can do so, many people will at some point require LTC services that cannot be delivered at home. The number of beds in LTC facilities and in LTC departments in hospitals offers a measure of the resources available for delivering LTC services to individuals outside their home.

Across OECD countries, there were 47 beds per 1000 people aged 65 and over in 2017 (Figure 11.26). The vast majority of beds -44 per 1000 people aged 65 and over - were located in LTC facilities, with just three LTC beds per 1000 people in hospitals. The number of LTC beds per 1000 people aged 65 and over varies enormously between OECD countries. Luxembourg, the country with the highest number $(82.8$ beds), had more than 18 times more beds than Greece (4.5 beds), the country with the lowest number in 2017. Five countries - Italy, Latvia, Poland, Turkey and Greece - had fewer than 20 beds per 1000 adults aged 65 and over. Four Luxembourg, the Netherlands, Belgium and Sweden - had more than 70 beds per 1000 adults aged 65 and over.

Between 2007 and 2017, OECD countries reduced the number of LTC beds in facilities by an average of 3.4 beds per 1000 people aged 65 and over (Figure 11.27). However, the change in the number of beds varied significantly between OECD countries. Over the ten-year period, Sweden, Iceland and Finland each reduced the number of beds in LTC facilities by 15 or more per 1000 people aged 65 and over. At the other end of the spectrum, Korea increased the number of LTC beds by 36 over the same period. These substantial changes have been largely driven by changes in policies over the period. Reductions in the number of facility-based LTC beds in Sweden have been driven by a move towards community-based LTC service provision, while in Korea, the massive increase in capacity followed the introduction of a public LTC insurance scheme in 2008.

Providing LTC in facilities can be more efficient than community care for people with intensive needs, owing to economies of scale and the fact that care workers do not need to travel to each person separately. However, it often costs public budgets more, since informal carers make less of a contribution and LTC systems often pick up board, lodging and care costs. Facility-based LTC may also be against the preferences of LTC recipients, many of whom wish to remain at home for as long as possible. Most countries have taken steps in recent years to support this preference and promote community care. However, depending on individual circumstances, a move to LTC facilities may be the most appropriate option - for example for people living alone and requiring round-the-clock care and supervision (Wiener et al., 2009[1]) or people living in remote areas with limited home care support. It is therefore important that countries retain an appropriate level of residential LTC capacity, and that care facilities develop and apply models of care that promote dignity and autonomy. This includes ensuring that staff working in LTC facilities are appropriately trained and receive the support they need to discourage high turnover and facilitate the recruitment and retention of high-quality care workers (see indicator on "Long-term care workers").

\section{Definition and comparability}

LTC facilities refer to nursing and residential care facilities that provide accommodation and LTC as a package. They include specially designed facilities or hospital-like settings where the predominant service component is LTC for people with moderate to severe functional restrictions. They do not include beds in adapted living arrangements for people who require help while guaranteeing a high degree of autonomy and self-control. For international comparisons, they should also not include beds in rehabilitation centres.

However, there are variations in data coverage across countries. Several countries only include beds in publicly funded LTC facilities, while others also include private facilities (both for-profit and not-forprofit). Some countries also include beds in treatment centres for addicted people, psychiatric units of general or specialised hospitals, and rehabilitation centres.

\section{References}

[2] Colombo, F. et al. (2011), Help Wanted? Providing and Paying for Long-Term Care, OECD Health Policy Studies, OECD Publishing, Paris, http://dx.doi.org/10.1787/9789264097759-en.

[3] Muir, T. (2017), "Measuring social protection for long-term care", OECD Health Working Papers, No. 93, OECD Publishing, Paris, https://dx.doi.org/10.1787/a411500a-en.

[1] Wiener, J. et al. (2009), "Why Are Nursing Home Utilization Rates Declining", Real Choice Systems Change Grant Program, US Department of Health and Human Services, Centers for Medicare and Medicaid Services. 


\section{AGEING AND LONG-TERM CARE}

Figure 11.26. Long-term care beds in facilities and hospitals, 2017 (or nearest year)

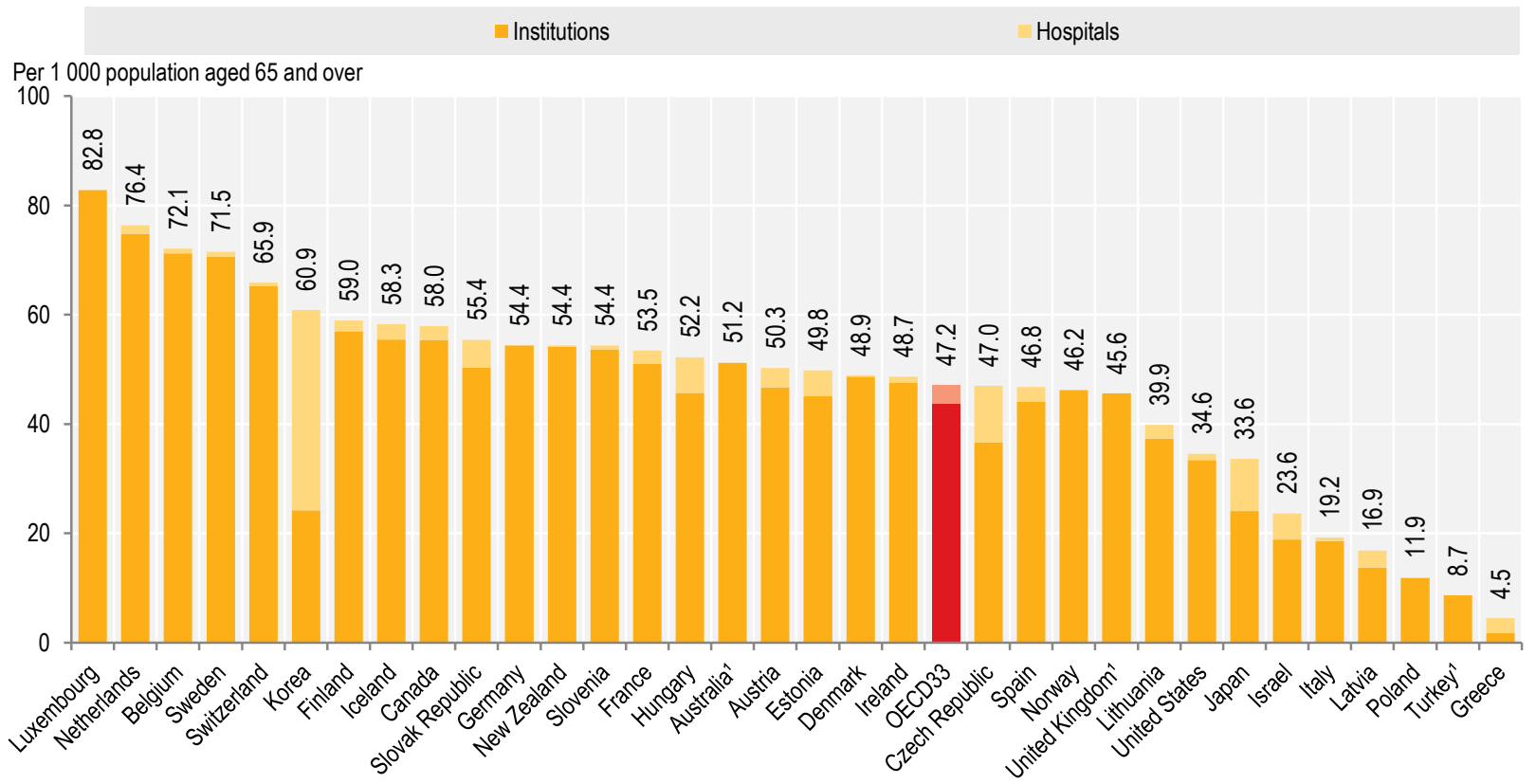

1. The numbers of LTC beds in hospitals are not available for Australia, Turkey and the United Kingdom. Source: OECD Health Statistics 2019.

Figure 11.27. Trends in long-term care beds in facilities and hospitals, 2007-17 (or nearest year)

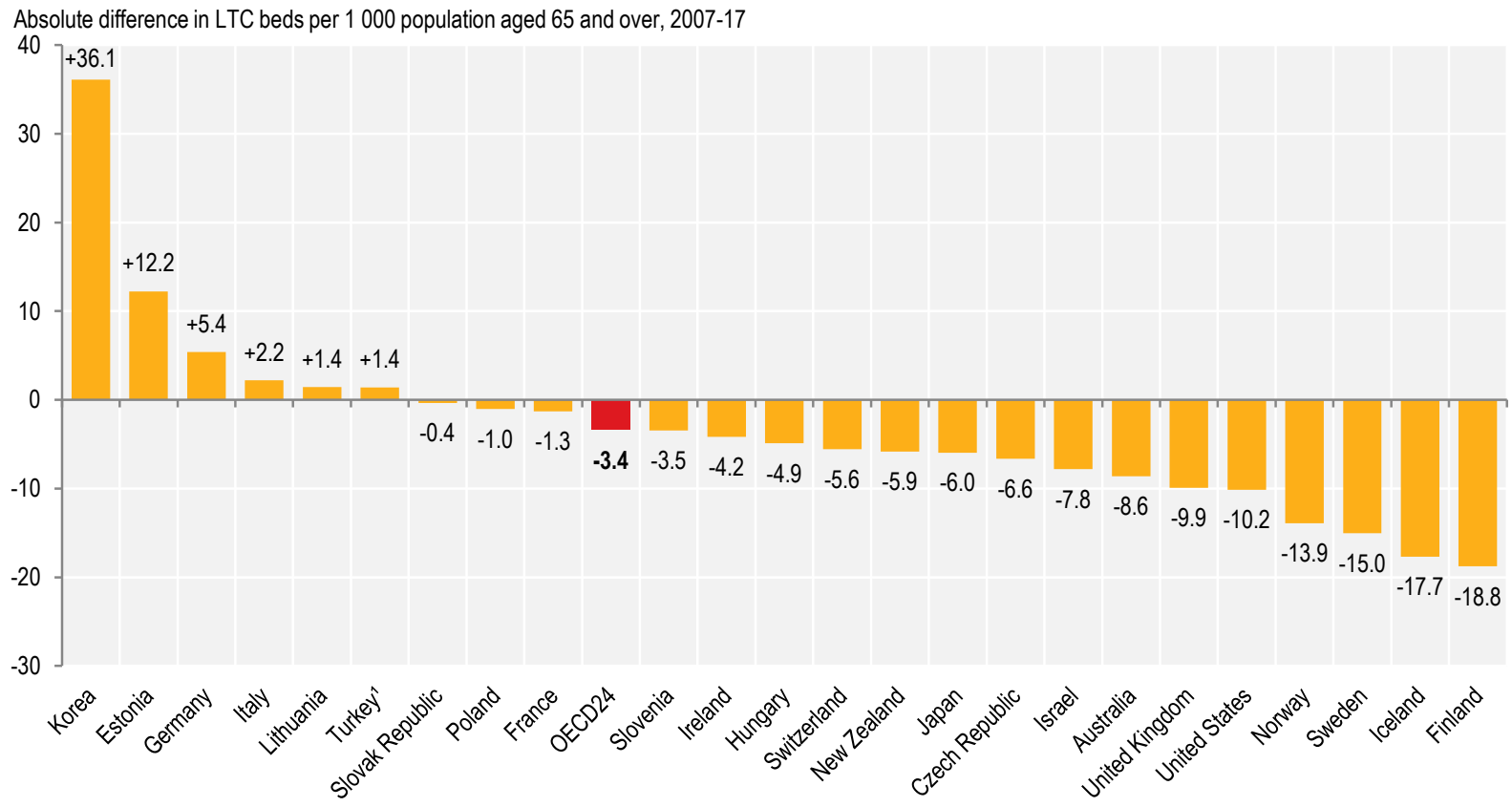

1. 2007 data refer to 2011.

Source: OECD Health Statistics 2019. 
Compared to other areas of health care, spending on longterm care (LTC) has seen the highest growth in recent years (see indicator on "Health expenditure by function" in Chapter 7). Population ageing leads to more people needing ongoing health and social care; rising incomes increase expectations on the quality of life in old age; the supply of informal care is potentially shrinking; and productivity gains are difficult to achieve in such a labour-intensive sector. All these factors create upward cost pressures, and substantial further increases in LTC spending in OECD countries are projected for the coming years.

A significant share of the spending on LTC services is covered by government or compulsory insurance schemes. Total government/compulsory spending on LTC (including both the health and social care components) accounted for $1.7 \%$ of GDP on average across OECD countries in 2017 (Figure 11.28). At 3.7\% of GDP, the highest spender was the Netherlands, followed by Norway (3.3\%) and Sweden (3.2\%). In these countries, public expenditure on LTC was around double the OECD average. At the other end of the scale, Hungary, Estonia, Poland, and Latvia all allocated less than $0.5 \%$ of their GDP to the delivery of LTC services. This variation partly reflects differences in the population structure, but mostly reflects the stage of development of formal LTC systems, as opposed to more informal arrangements based mainly on care provided by unpaid family members. Generally, the health component of LTC represents the vast majority of all LTC expenditure, but some issues remain around properly distinguishing between health and social LTC in some countries.

The way LTC is organised in countries affects the composition of LTC (health) spending and can also have an impact on overall LTC spending. Across OECD countries, around two-thirds of government and compulsory spending on LTC (health) was for inpatient LTC in 2017. These services are mainly provided in residential LTC facilities (Figure 11.29). Yet in Poland, Finland, Denmark, Lithuania, Austria and Germany, spending on home-based LTC accounted for more than $50 \%$ of all LTC spending. Spending for home-based LTC can be on services provided by either professional LTC workers or informal workers, when a care allowance exists that remunerates the caregiver for the LTC services provided.

The important role public schemes play in the financing of LTC can be explained by the substantial costs for care that older people with LTC needs face. These costs vary widely between countries but are always high relative to median incomes among elderly people. For institutional care, for example, the costs for a person with severe LTC needs represent between just under one the median disposable income for individuals of retirement age and more than four times that income (Figure 11.30), depending on the country or region. Compared to the average income, costs are higher in Finland, Ireland and the Netherlands and lower in
Hungary, Slovenia and Croatia. Only in Slovenia and Croatia would an older person with median income be able to afford the costs of institutional care from their income alone. All OECD countries have some form of social protection against these high financial risks, and out-of-pocket costs that older people ultimately face tend to be lower in countries where public expenditure on LTC is higher, such as in the Netherlands and Finland (Muir, 2017[1]).

\section{Definition and comparability}

LTC spending comprises both health and social services to LTC dependent people who need care on an ongoing basis. Based on the System of Health Accounts, the health component of LTC spending relates to nursing care and personal care services (i.e. help with activities of daily living). It also covers palliative care and care provided in LTC institutions (including costs for room and board) or at home. LTC social expenditure primarily covers help with instrumental activities of daily living. Progress has been made in improving the general comparability of LTC spending in recent years but there is still some variation in reporting practices between the health and social components for some LTC activities in some countries. Currently, LTC expenditure funded by governments and compulsory insurance schemes is more suitable for international comparison as there is more variation in the comprehensiveness of reporting of privately funded LTC expenditure across OECD countries. Finally, some countries (e.g. Israel and the United States) can only report spending data for institutional care, and hence underestimate the total amount of spending on LTC services by government and compulsory insurance schemes.

Long-term care institutions refer to nursing and residential care facilities that provide accommodation and long-term care as a package. They are specially designed institutions where the predominant service component is LTC for dependent people with moderate to severe functional restrictions. An older person with severe needs is defined as someone who requires 41.25 hours of care per week. A detailed description of their needs can be found in Muir (2017[1])

\section{References}

[1] Muir, T. (2017), "Measuring social protection for long-term care", OECD Health Working Papers, No. 93, OECD Publishing, Paris, $h$ ttps://dx.doi.org/10.1787/a411500a-en. 
Figure 11.28. Long-term care expenditure (health and social components) by government and compulsory insurance schemes, as a share of GDP, 2017 (or nearest year)

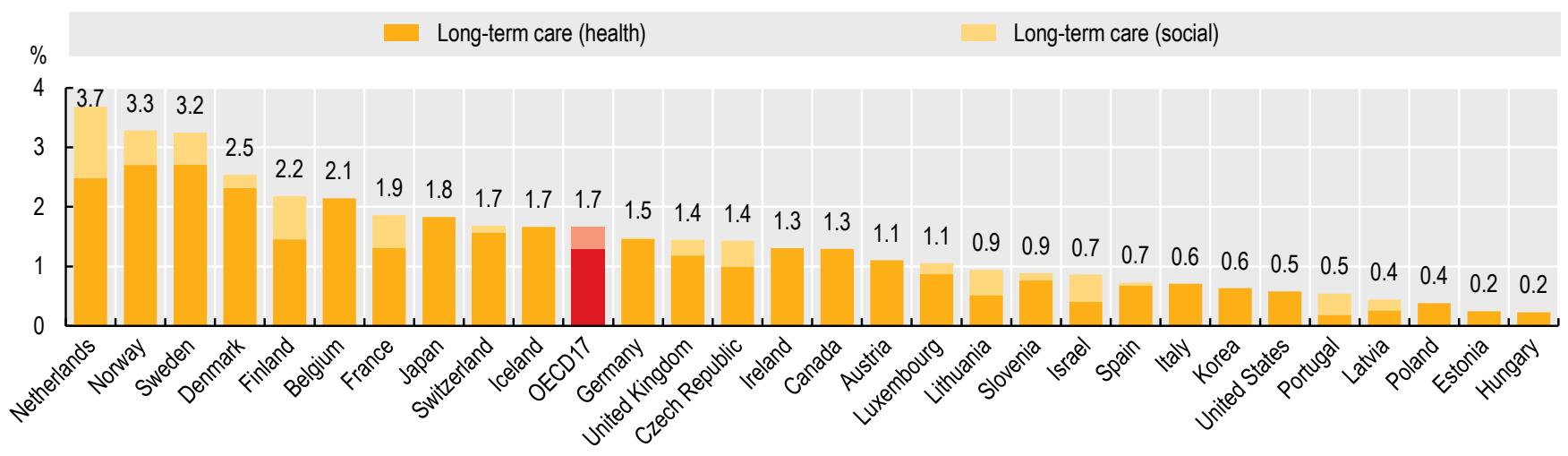

Note: The OECD average only includes 17 countries that report health and social LTC.

Source: OECD Health Statistics 2019.

StatLink iㅔs https://doi.org/10.1787/888934018773

Figure 11.29. Government and compulsory insurance spending on LTC (health) by mode of provision, 2017 (or nearest year)

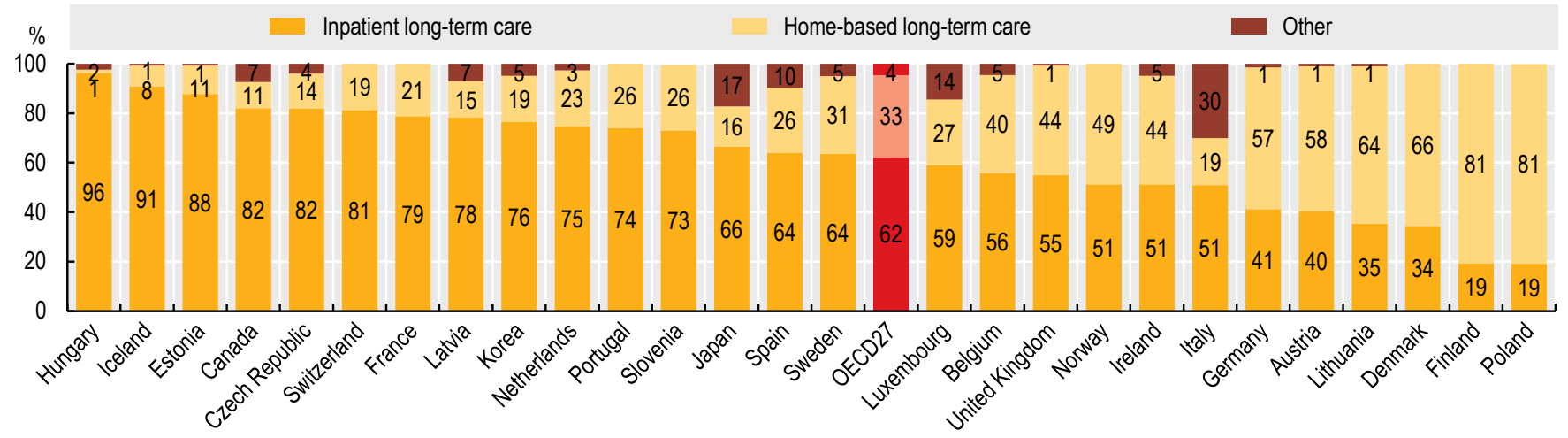

Note: "Other" includes LTC in day care and outpatient settings.

Source: OECD Health Statistics 2019.

StatLink 시느 https://doi.org/10.1787/888934018792

Figure 11.30. Costs of institutional long-term care for an older person with severe needs, as a share of the median income among people of retirement age and older, 2018 (or nearest year)

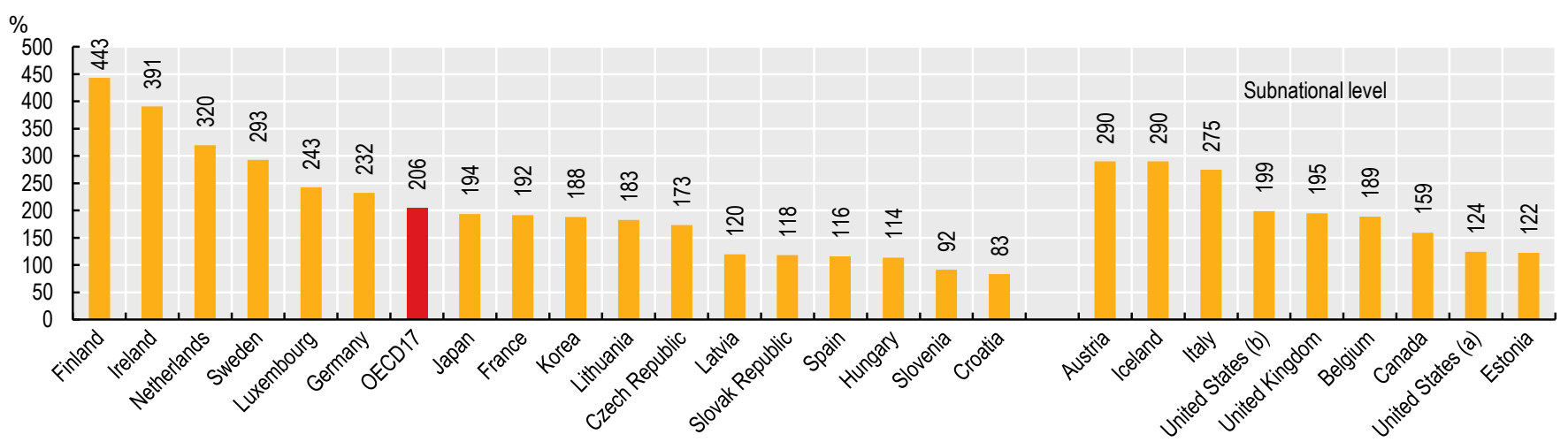

Note: Belgium refers to Flanders, Iceland refers to Reykjavik, Canada refers to Ontario, Estonia refers to Tallinn, Austria refers to Vienna, the United States refers to (a) California and (b) Illinois, Italy refers to South Tyrol, and the United Kingdom refers to England.

Source: OECD Long-Term Care Social Protection questionnaire (2018) and OECD Income Distribution Database (2018). 


\section{ORGANISATION FOR ECONOMIC CO-OPERATION AND DEVELOPMENT}

The OECD is a unique forum where governments work together to address the economic, social and environmental challenges of globalisation. The OECD is also at the forefront of efforts to understand and to help governments respond to new developments and concerns, such as corporate governance, the information economy and the challenges of an ageing population. The Organisation provides a setting where governments can compare policy experiences, seek answers to common problems, identify good practice and work to co-ordinate domestic and international policies.

The OECD member countries are: Australia, Austria, Belgium, Canada, Chile, the Czech Republic, Denmark, Estonia, Finland, France, Germany, Greece, Hungary, Iceland, Ireland, Israel, Italy, Japan, Korea, Latvia, Lithuania, Luxembourg, Mexico, the Netherlands, New Zealand, Norway, Poland, Portugal, the Slovak Republic, Slovenia, Spain, Sweden, Switzerland, Turkey, the United Kingdom and the United States. The European Union takes part in the work of the OECD.

OECD Publishing disseminates widely the results of the Organisation's statistics gathering and research on economic, social and environmental issues, as well as the conventions, guidelines and standards agreed by its members. 


\section{Health at a Glance 2019 OECD INDICATORS}

Health at a Glance compares key indicators for population health and health system performance across OECD members, candidate and partner countries. It highlights how countries differ in terms of the health status and health-seeking behaviour of their citizens; access to and quality of health care; and the resources available for health. Analysis is based on the latest comparable data across 80 indicators, with data coming from official national statistics, unless otherwise stated.

Alongside indicator-by-indicator analysis, an overview chapter summarises the comparative performance of countries and major trends, including how much health spending is associated with staffing, access, quality and health outcomes. This edition also includes a special focus on patient-reported outcomes and experiences, with a thematic chapter on measuring what matters for people-centred health systems.

Consult this publication on line at $\boldsymbol{h t t p s : / / d o i . o r g / 1 0 . 1 7 8 7 / 4 d d 5 0 c 0 9 - e n . ~}$

This work is published on the OECD iLibrary, which gathers all OECD books, periodicals and statistical databases. Visit www.oecd-ilibrary.org for more information.

OECDpublishing www.oecd.org/publishing
ISBN 978-92-64-38208-4

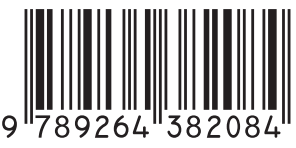

